\title{
Experimental and Computational Investigation of Spoiler Deployment on Wing Stall
}

\author{
by \\ Scott Lindsay, \\ Bachelor of Aerospace Engineering, Ryerson, 2016 \\ A thesis \\ presented to Ryerson University \\ in partial fulfillment of the \\ requirements for the degree of \\ Master of Applied Science \\ in the program of \\ Aerospace Engineering
}

Toronto, Ontario, Canada, 2018

(O)Scott Lindsay, 2018 


\section{AUTHOR'S DECLARATION FOR ELECTRONIC SUBMISSION OF A THESIS}

I hereby declare that I am the sole author of this thesis. This is a true copy of the thesis, including any required final revisions, as accepted by my examiners.

I authorize Ryerson University to lend this thesis to other institutions or individuals for the purpose of scholarly research.

I further authorize Ryerson University to reproduce this thesis by photocopying or by other means, in total or in part, at the request of other institutions or individuals for the purpose of scholarly research.

I understand that my thesis may be made electronically available to the public. 


\section{Experimental and Computational Investigation of Spoiler Deployment on Wing Stall}

Scott Lindsay,

Master of Applied Science, Aerospace Engineering, Ryerson University, Toronto (2018)

\section{ABSTRACT}

Upper surface flaps commonly referred to as spoilers or drag brakes can increase maximum lift, and improve aerodynamic efficiency at high, near-stall angles of attack. This phenomenon was studied experimentally and computationally using a 0.307626 m chord length NACA 2412 airfoil in six different configurations, and one baseline clean configuration. A wind tunnel model was placed in the Ryerson Low Speed Wind Tunnel (atmospheric, closed-circuit, $3 \mathrm{ft} \times 3 \mathrm{ft}$ test section) at a Reynold's number of approximately 780,000 and a Mach number of 0.136.

The wind tunnel study increased the lift coefficient by $0.393 \%-2.497 \%$ depending on the spoiler configuration. A spoiler of $10 \%$ chord length increased the maximum lift coefficient by $2.497 \%$ when deflected $8^{\circ}$, by $2.110 \%$ when deflected $15^{\circ}$, and reduced the maximum lift coefficient by $2.783 \%$ when deflected $25^{\circ}$. A spoiler of $15 \%$ chord length produced smaller maximum lift coefficient gains; $0.393 \%$ when deflected $8^{\circ}$, by $1.760 \%$ when deflected $15^{\circ}$, and reduced the maximum lift coefficient by $4.475 \%$ when deflected $25^{\circ}$. Deflecting the spoiler increased the stall angle between $37.658 \%$ and $87.544 \%$ when compared with the clean configuration. The drag coefficient of spoiler configurations was lower than the clean configuration at angles of attack above $18^{\circ}$. The combination of the increased lift and reduced drag at angles of attack above $18^{\circ}$ created by the spoiler configurations resulted in a higher aerodynamic efficiency than the clean configuration case. A $10 \%$ chord length spoiler deflected at $8^{\circ}$ produced the highest aerodynamic efficiency gains.

At low angles of attack, the computational study produced consistently higher lift coefficients compared with the wind tunnel experiment. The lift-slope was consistent with the wind tunnel experiment 
lift-slope. The spoiler airfoil stall behaviour was inconsistent with the results from the wind tunnel experiment. The drag coefficient results were consistent with the wind tunnel experiment at low angles of attack. However, the spoiler equipped airfoils did not reduce drag at high angles of attack. Therefore, the computational model was not valid for the spoiler configurations at high angles of attack. 


\section{ACKNOWLEDGEMENTS}

I would like to sincerely thank Dr. Paul Walsh for his guidance and support. I would also like to thank Mr. Jerry Karpynczyk and Mr. Peter Bradly for their technical support, and Dr. Bramesfeld and the members of the Ryerson Applied Aerodynamics Laboratory of Flight for their assistance with the wind tunnel. 


\section{TABLE OF CONTENTS}

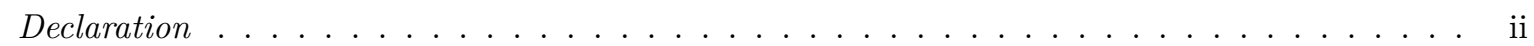

Abstract . . . . . . . . . . . . . . . . . . . . . . iii

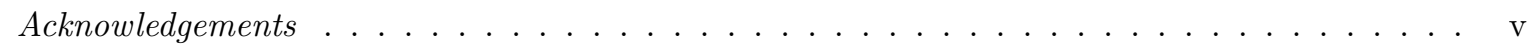

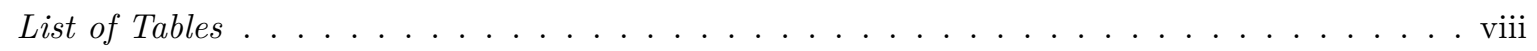

List of Figures . . . . . . . . . . . . . . . . . . . . . . . . ix

List of Appendices . . . . . . . . . . . . . . . . . . . . . . xvii

\begin{tabular}{lll}
\hline & Introduction & 1
\end{tabular}

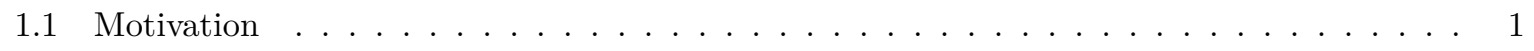

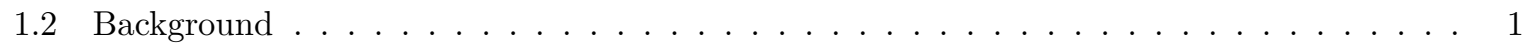

$1.2 .1 \quad$ Spoiler Flow-Field Characteristics $\ldots \ldots \ldots \ldots \ldots \ldots \ldots$

1.2 .2 The lifting spoiler effect $\ldots \ldots \ldots \ldots \ldots \ldots \ldots$

$1.2 .3 \quad$ Experimental Domain $\ldots \ldots \ldots \ldots \ldots \ldots \ldots$

\begin{tabular}{|lrl}
\hline 2 & Wind Tunnel Study & 17
\end{tabular}

2.1 Data Acquisition . . . . . . . . . . . . . . . . . . . . . . . . . . 19

$2.1 .1 \quad$ Airfoil and Wake Pressure Data $\ldots \ldots \ldots \ldots$. . . . . . . . . . . 19

2.1 .2 Temperature Data $\ldots \ldots \ldots \ldots \ldots$. . . . . . . . . . . . . . . . . . 19

$2.1 .3 \quad$ Free-stream Dynamic Pressure Data $\ldots \ldots \ldots \ldots \ldots . \ldots \ldots$

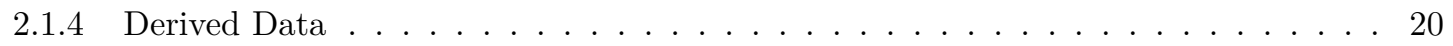

2.1 .5 Data Reduction . . . . . . . . . . . . . . . . . . . . . . . . . 21

$2.1 .6 \quad$ Lift and Moment Coefficient from Pressure Tap Integration . . . . . . . . . . . 21

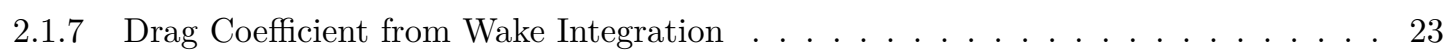

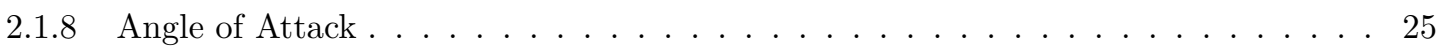

2.1 .9 Wind Tunnel Correction Factors $\ldots \ldots \ldots \ldots \ldots$. . . . . . . . . 26

2.1 .10 Data Flow . . . . . . . . . . . . . . . . . . . . . . 27

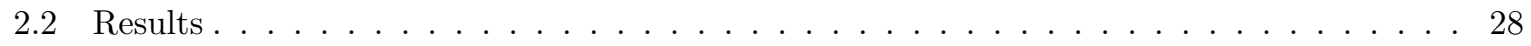

2.3 Comments on Repeatability $\ldots \ldots \ldots \ldots \ldots \ldots \ldots$ 
\begin{tabular}{|lll}
\hline 3 & Computational Study & 38
\end{tabular}

3.1 Computational Setup $\ldots \ldots \ldots \ldots$. . . . . . . . . . . . . . . . . 38

3.2 Grid Independent Study $\ldots \ldots \ldots \ldots$. . . . . . . . . . . . . . . . . . 41

3.2 .1 Method . . . . . . . . . . . . . . . . . . . . . . 41

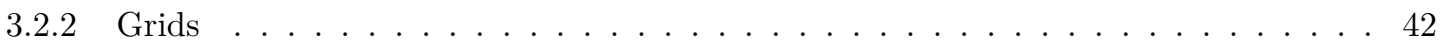

$3.2 .3 \quad$ Clean Configuration Grid Study Results . . . . . . . . . . . . . . . . . . . 45

$3.2 .4 \quad$ Spoiler Configuration Grid Study Results . . . . . . . . . . . . . . . . . . . 45

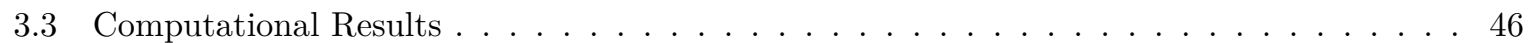

3.3 .1 Force Curves $\ldots \ldots \ldots \ldots \ldots \ldots \ldots$

3.3 .2 Time History of Force Coefficients $\ldots \ldots \ldots$. . . . . . . . . . . 53

$3.3 .3 \quad$ Pressure Coefficient Distribution $\ldots \ldots \ldots \ldots \ldots \ldots$. . . . . . . . . . . 57

3.3 .4 Streamline Plots $\ldots \ldots \ldots \ldots \ldots$

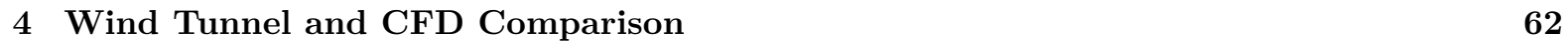

4.1 Lift Coefficient Curves $\ldots \ldots \ldots \ldots$. . . . . . . . . . . . . . . . . . 62

4.2 Drag Coefficient Curves $\ldots \ldots \ldots \ldots \ldots$

$\begin{array}{lll}5 & \text { Conclusion } & 71\end{array}$

\begin{tabular}{lr}
\hline References & 210
\end{tabular} 


\section{LIST OF TABLES}

1.1 Spoiler Configurations Studied $\ldots \ldots \ldots \ldots \ldots \ldots$

2.1 Temperature Probe Specifications $[1.] \ldots \ldots \ldots \ldots$

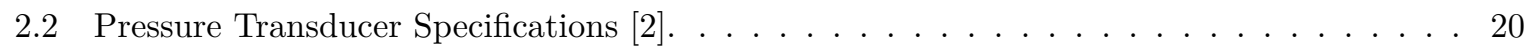

$2.3 \quad$ Wind tunnel $C_{l_{\max }}$ and $\alpha_{\text {stall }}$ change with spoiler configuration $\ldots \ldots \ldots \ldots \ldots$

3.1 Clean Configuration Grid Information $\ldots \ldots \ldots \ldots$. . . . . . . . . . 43

3.2 Spoiler Configuration Grid Information $\ldots \ldots \ldots \ldots \ldots \ldots$

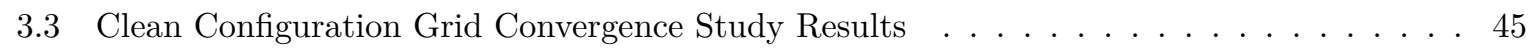

3.4 Clean Configuration Grid Convergence Study Results . . . . . . . . . . . . . . . . . 45

3.5 Spoiler Configuration Grid Convergence Study Results . . . . . . . . . . . . . . . . . 46

3.6 Spoiler Configuration Grid Convergence Study Results . . . . . . . . . . . . . . . . . . 46

3.7 CFD $C_{l_{\max }}$ and $\alpha_{\text {stall }}$ change with spoiler configuration $\ldots \ldots \ldots \ldots \ldots$

3.8 CFD Aerodynamic Efficiency Values of Each Configuration $\ldots \ldots \ldots \ldots$

3.9 CFD Aerodynamic Efficiency Compared with Clean Configuration . . . . . . . . . . . 51

$1.1 \quad$ NACA 2412 airfoil coordiantes $(c=0.307626$ with sharp tip $) \ldots \ldots \ldots \ldots$. . . . . 74

1.2 Spoiler Coordinates by Configuration $\ldots \ldots \ldots \ldots \ldots \ldots$

2.1 Pressure Tap Locations $\ldots \ldots \ldots \ldots \ldots$

$3.1 \quad C_{l}$ values calculated from trapezodial integration of pressure, with and without spline interpolation compared with values from CFX5Post $\ldots \ldots \ldots$. . . . . . . . 92

$3.2 \quad$ Trapezodial integration lift coefficient percent difference (compared with CFX5Post value)

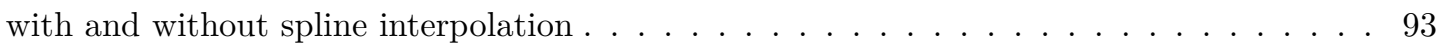

4.1 Trapezodial integration drag coefficient percent difference (compared with CFX5Post

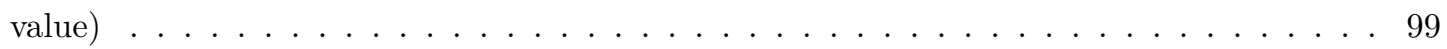

5.1 Body Shape Factor change with Thickness ratio for NACA 4-Digit Airfoils [3] . . . . . . . 101 


\section{LIST OF FIGURES}

$1.1 \quad$ Variation of lift and drag coefficients with angle of attack [4] . . . . . . . . . . . . 2

$1.2 C_{P}$ distribution on upper and lower surfaces $\left(\alpha=2^{\circ}\right)[4] \ldots \ldots \ldots \ldots$

1.3 Time history and Fast Fourier transform (FFT) of $C_{l}$ for the BATR airfoil-spoiler $\alpha=0^{\circ}$

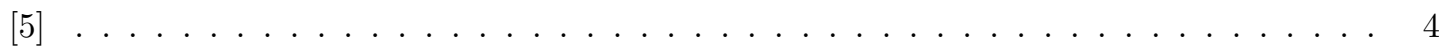

1.4 Streamline periodic patttern for the BATR airfoil-spoiler at $\delta=60^{\circ}, \alpha=0^{\circ}[5]$. . . . . . 5

1.5 Streamline periodic patttern for the BATR airfoil-spoiler at $\delta=60^{\circ}, \alpha=8^{\circ}[\underline{5}$. . . . . . . 5

1.6 Lift and moment coefficients vs angle of attack curves of the baseline S824 airfoil along with those of a single and double effector equipped configurations $[\underline{6}] \ldots \ldots \ldots$

1.7 Lift vs drag coefficients of baseline S824 airfoil along with those of a single and double effector equipped configurations $[6] \ldots \ldots \ldots \ldots \ldots \ldots \ldots$

1.8 Pressure distributions for $\alpha=16^{\circ}$ of baseline S824 airfoil and of configuration having a single effector at $86 \%$ chord. $[6] \ldots \ldots \ldots \ldots \ldots \ldots$

$1.9 \quad$ Flexible spoiler geometry renderings. Spoiler lengths are $12 \%$ of the chord. [7] . . . . . . 9

1.10 Effect of spoiler geometry and location on measured L/D ratio. [7] . . . . . . . . . . . 10

$1.11 C_{l}$ curves for the clean airfoil and the 6 fixed deplyment effectors []$\left._{0}\right] \ldots \ldots \ldots 11$

$1.12 C_{p}$ distribution at $\alpha=4^{\circ}$ for the clean airfoil compared to the effector fixed at $45^{\circ}$

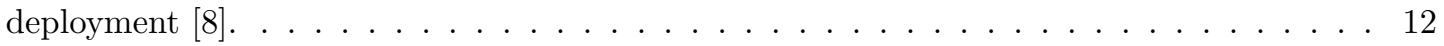

$1.13 C_{p}$ distribution at $\alpha=18^{\circ}$ for the clean airfoil compared to the effector fixed at $30^{\circ}$

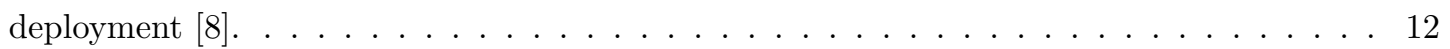

1.14 Velocity Magnitude Streamlines, $\alpha=19^{\circ}, R e=3.79 \times 10^{6} 0[9]$. . . . . . . . . . . . 13

1.15 Aerodynamic efficiency comparisons of NACA 2412 clean airfoil to spoiler-equipped airfoil at $60 \%$ chord at $\delta=4^{\circ}$ and $10^{\circ}[9] . \ldots \ldots \ldots \ldots \ldots \ldots$

1.16 Geometry of a sharp tip NACA 2412 airfoil equipped with a spoiler. . . . . . . . . . 15

2.1 Wind tunnel model fully assembled and mounted in the Ryerson Large Wind Tunnel. . . 18

2.2 Airfoil Geometry in Regards to Pressure Tap Integration. . . . . . . . . . . . . . . . . 22

$2.3 \quad$ Airfoil Pressure Tap Integration Discretized into Finite Points. . . . . . . . . . . . . . 23

2.4 Application of the momentum equation to calculate airfoil drag from wake velocity profile 24

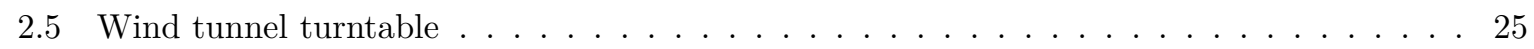

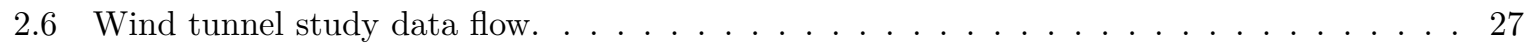


2.7 Wind tunnel $C_{l}-\alpha$ comparison between clean and spoiler configurations. $\ldots \ldots \ldots$

2.8 Wind tunnel $C_{d}-\alpha$ comparison between clean and spoiler configurations. . . . . . . . 30

$2.9 \quad$ Wind tunnel $C_{l} / C_{d}-\alpha$ comparison between clean and spoiler configurations. . . . . . . 31

2.10 Wind tunnel $C_{l}$ vs $C_{d}$ comparison between clean and spoiler configurations. . . . . . . . 32

2.11 Wind tunnel $C_{m_{\frac{1}{4}} c}-\alpha$ comparison between clean and spoiler configurations. . . . . . . . 33

$2.12 C_{p}$ plot comparison of clean and spoiler equipped airfoil at $\alpha \approx 3.7^{\circ} \ldots \ldots . \ldots 34$

$2.13 C_{p}$ plot comparison of clean and spoiler equipped airfoil at $\alpha \approx 17.7^{\circ} \ldots \ldots . \ldots 35$

2.14 Wake plot comparison of clean and spoiler equipped airfoil at $\alpha \approx 3.7^{\circ} \ldots \ldots \ldots$

2.15 Wake plot comparison of clean and spoiler equipped airfoil at $\alpha \approx 15.7^{\circ} \ldots \ldots 37$

3.1 CFD Process Flow $\ldots \ldots \ldots \ldots$. . . . . . . . . . . . . . . . . . . 40

3.2 Clean Configuration Airfoil Grids $\ldots \ldots \ldots$. . . . . . . . . . . . . . 43

3.3 Spoiler Airfoil Grids $\ldots \ldots \ldots \ldots \ldots \ldots \ldots \ldots \ldots$

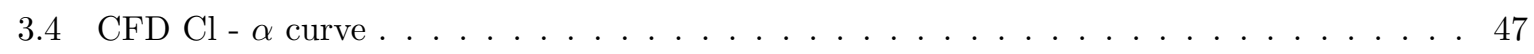

3.5 CFD Cd $-\alpha$ curve $\ldots \ldots \ldots \ldots \ldots \ldots \ldots \ldots$

$3.6 \quad \mathrm{CFD} \mathrm{Cl/Cd}-\alpha$ curve $\ldots \ldots \ldots \ldots \ldots \ldots$

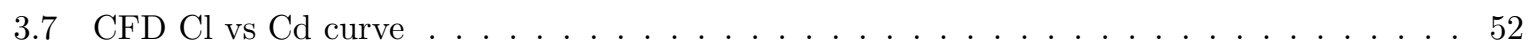

$3.8 \quad$ CFD Cm $-\alpha$ curve $\ldots \ldots \ldots \ldots \ldots \ldots$

3.9 CFD Force coefficient time history - clean configuration $\ldots \ldots \ldots \ldots \ldots$

3.10 CFD Force coefficient time history - configuration $2 \ldots \ldots \ldots \ldots$

3.11 CFD Force coefficient time history - configuration $3 \ldots \ldots \ldots$

3.12 CFD Force coefficient time history - configuration $5 \ldots \ldots \ldots$. . . . . . . 57

3.13 CFD Pressure Coefficient Curve Comparing Configuration 3 and Clean at $\alpha=4^{\circ}$. . . . 58

3.14 CFD Pressure Coefficient Curve Comparing Configuration 3 and Clean at $\alpha=17^{\circ} \ldots$. . 59

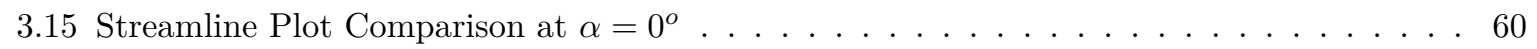

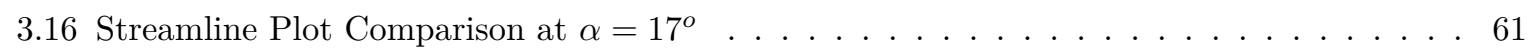

4.1 CFD and Wind Tunnel Comparison $\mathrm{Cl}-\alpha$ curve Configuration $1 \ldots \ldots$. . . . . . . 63

4.2 CFD and Wind Tunnel Comparison $\mathrm{Cl}-\alpha$ curve Configuration $2 \ldots \ldots$. . . . . . . . 64

$4.3 \quad$ CFD and Wind Tunnel Comparison $\mathrm{Cl}-\alpha$ curve Configuration 3 . . . . . . . . . . . 65

4.4 CFD and Wind Tunnel Comparison $\mathrm{Cl}-\alpha$ curve Configuration 5 . . . . . . . . . . . 66

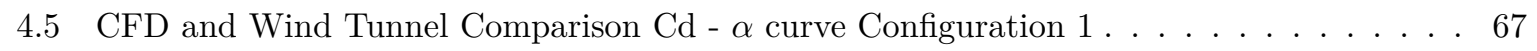

$4.6 \quad$ CFD and Wind Tunnel Comparison $\mathrm{Cd}-\alpha$ curve Configuration $2 \ldots \ldots$. . . . . . . 68

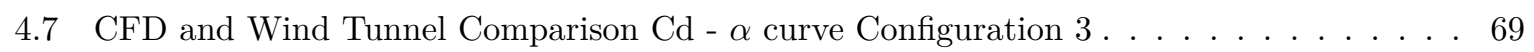

$4.8 \quad$ CFD and Wind Tunnel Comparison Cd $-\alpha$ curve Configuration $5 . \ldots \ldots$. . . . . . 70

1.1 NACA 2412 Airfoil Coordinates from Table 1.1 . . . . . . . . . . . . . . . . . . . 75

1.2 Geometry of a sharp tip NACA 2412 airfoil equipped with a spoiler. . . . . . . . . . 76

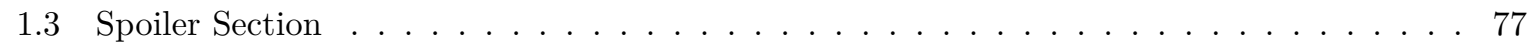

1.4 Sketch of the Configurations Described in Table $1.2 \ldots \ldots \ldots \ldots \ldots$ 
2.1 CATIA V5 Render of Wind Tunnel Model . . . . . . . . . . . . . . . . . . . . . . . 80

2.2 Drawing of Airfoil Section Pieces … . . . . . . . . . . . . . . . . . 83

2.3 Diagram of Model Mount to Wind Tunnel . . . . . . . . . . . . . . . . . . . . . . . . . 84

2.4 Front view drawing of four different spoiler holder insert parts (clean, $\left.8^{\circ}, 15^{\circ}, 25^{\circ}\right)$. . . . 86

2.5 Drawing of spoiler system assembly. $\ldots \ldots \ldots \ldots \ldots$

2.6 Wake rake drawing $\ldots \ldots \ldots \ldots \ldots \ldots$

2.7 Wake rake with polyurethane tubing attached. $\ldots \ldots \ldots$. . . . . . . . . . . . . 89

2.8 Wind tunnel model fully assembled and mounted in the Ryerson Large Wind Tunnel. . . 90

$3.1 \quad C_{l}-\alpha$ curve containing data from Table 3.1 . . . . . . . . . . . . . . . . . . 94

3.2 Comparison between $C_{p}$ plot extracted CFX5Post and spline interpolation of 32 pressure

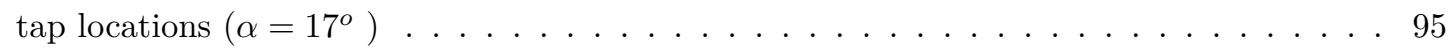

3.3 Comparison between $C_{p}$ plot extracted CFX5Post and spline interpolation of 32 pressure

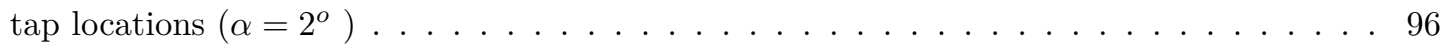

$4.1 \quad C_{d}-\alpha$ curve containing data from Table 4.1$] \ldots \ldots \ldots \ldots$

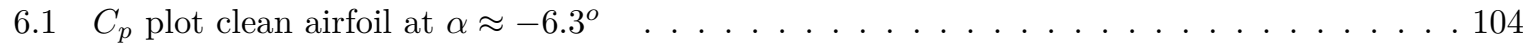

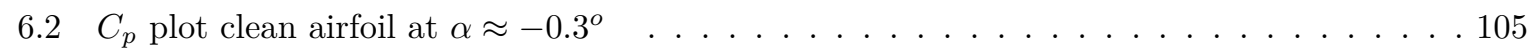

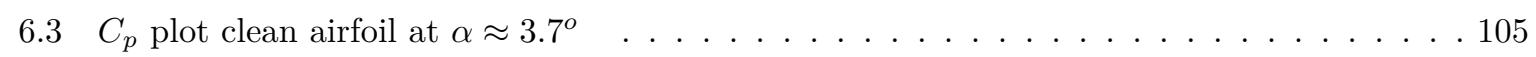

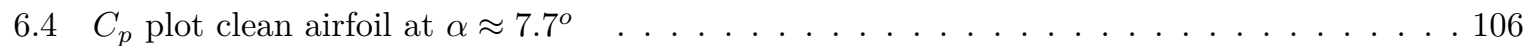

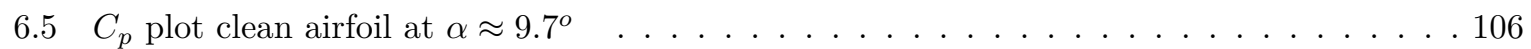

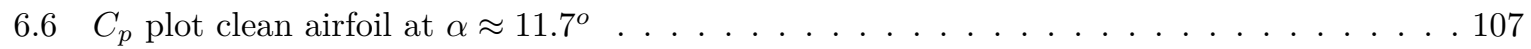

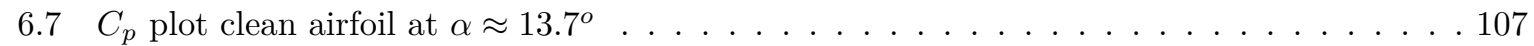

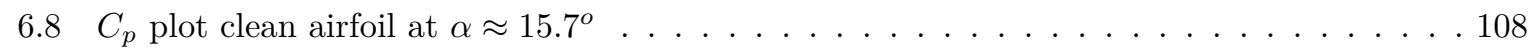

$6.9 C_{p}$ plot clean airfoil at $\alpha \approx 17.7^{\circ} \ldots \ldots \ldots \ldots \ldots \ldots \ldots \ldots \ldots \ldots \ldots \ldots$

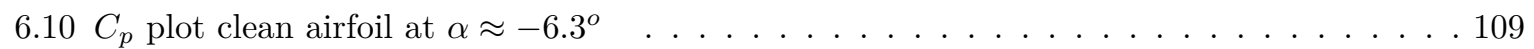

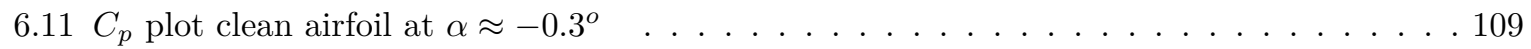

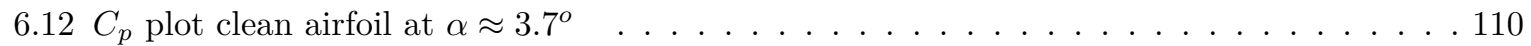

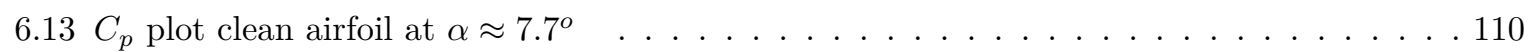

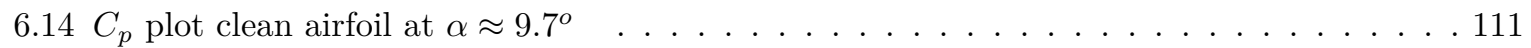

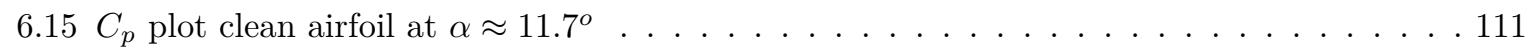

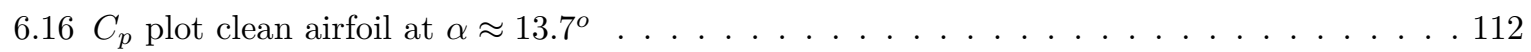

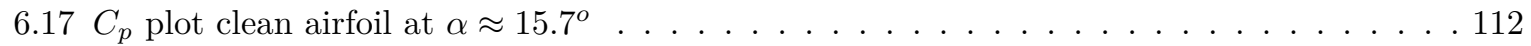

$6.18 C_{p}$ plot clean airfoil at $\alpha \approx 17.7^{\circ} \ldots \ldots \ldots \ldots \ldots \ldots \ldots \ldots \ldots$

$6.19 C_{p}$ plot clean airfoil at $\alpha \approx 18.7^{\circ} \ldots \ldots \ldots \ldots \ldots \ldots \ldots \ldots \ldots \ldots \ldots \ldots$

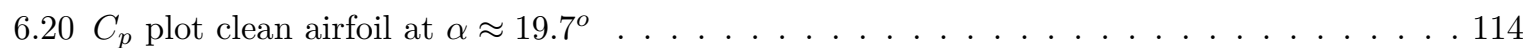

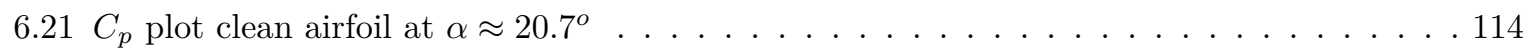

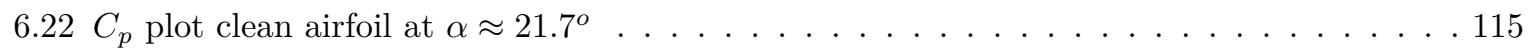

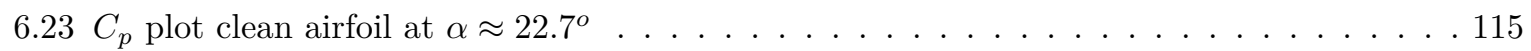

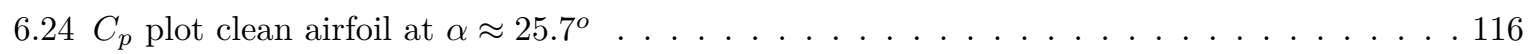




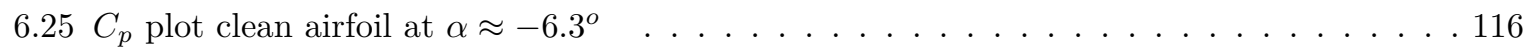

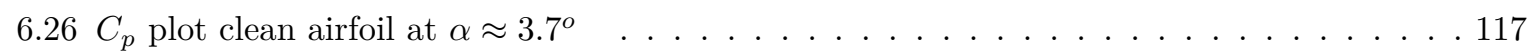

$6.27 C_{p}$ plot clean airfoil at $\alpha \approx 9.7^{\circ} \ldots \ldots \ldots \ldots \ldots \ldots \ldots \ldots \ldots \ldots \ldots \ldots$

$6.28 C_{p}$ plot clean airfoil at $\alpha \approx 13.7^{\circ} \ldots \ldots \ldots \ldots \ldots \ldots \ldots \ldots \ldots \ldots$

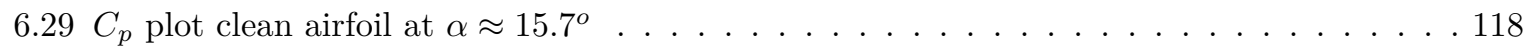

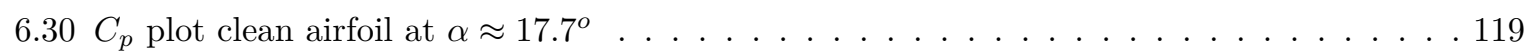

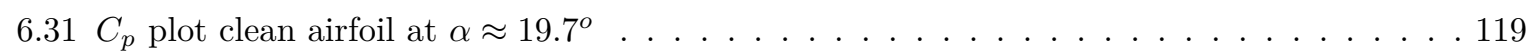

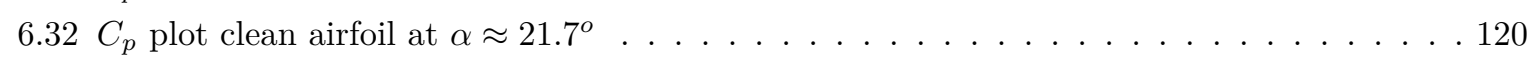

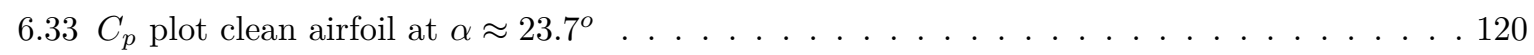

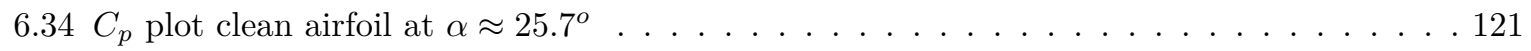

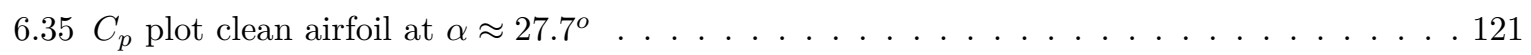

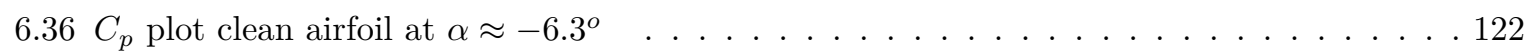

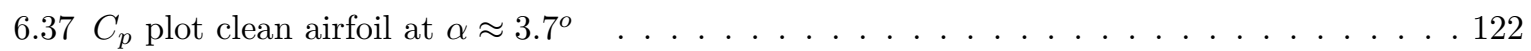

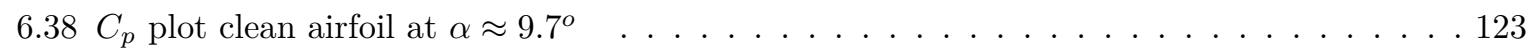

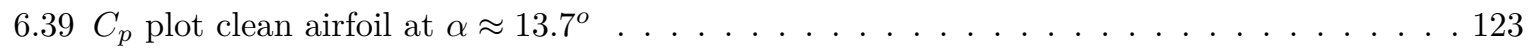

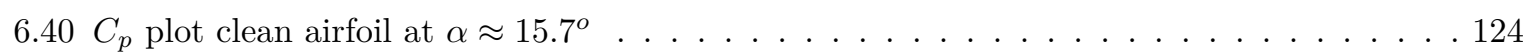

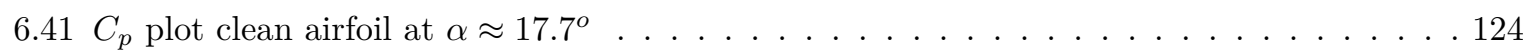

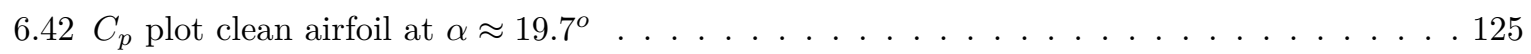

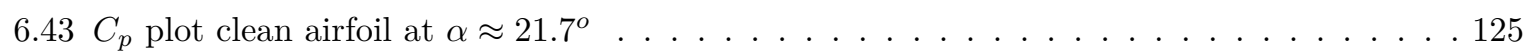

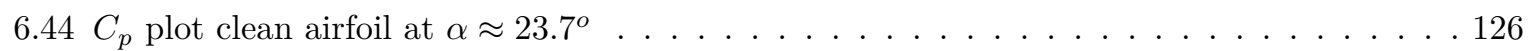

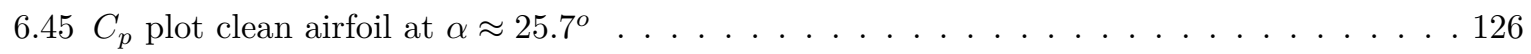

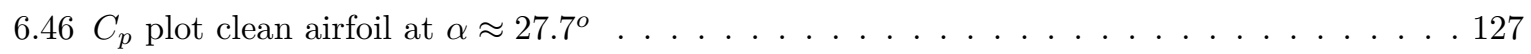

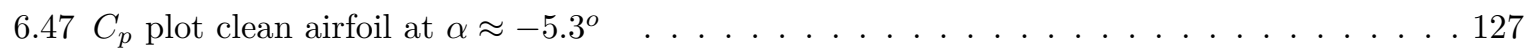

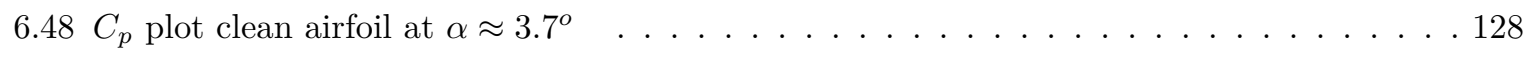

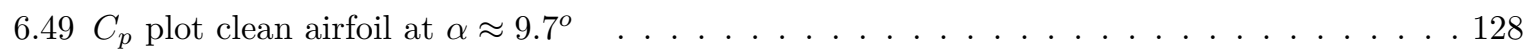

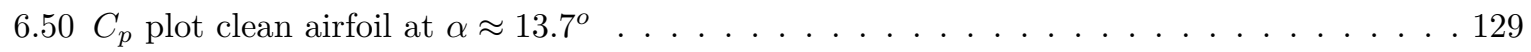

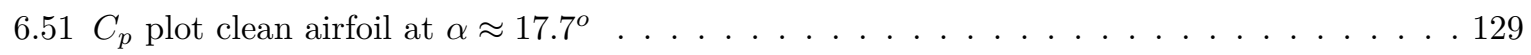

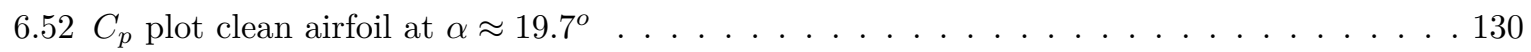

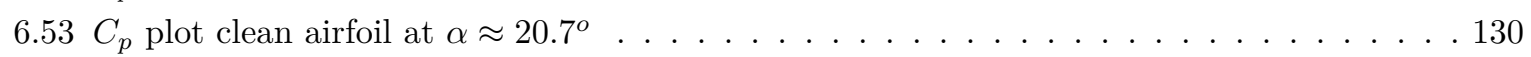

$6.54 C_{p}$ plot clean airfoil at $\alpha \approx 21.7^{\circ} \ldots \ldots \ldots \ldots \ldots \ldots \ldots \ldots \ldots$

$6.55 C_{p}$ plot clean airfoil at $\alpha \approx 22.7^{\circ} \ldots \ldots \ldots \ldots \ldots \ldots \ldots \ldots \ldots \ldots$

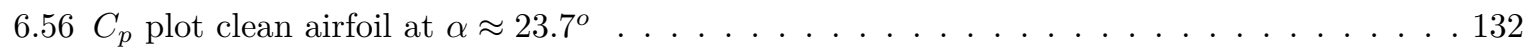

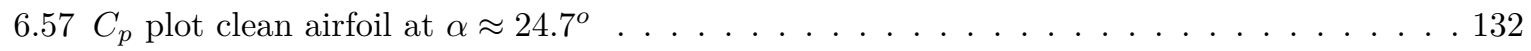

$6.58 C_{p}$ plot clean airfoil at $\alpha \approx 25.7^{\circ} \ldots \ldots \ldots \ldots \ldots \ldots \ldots \ldots \ldots \ldots$

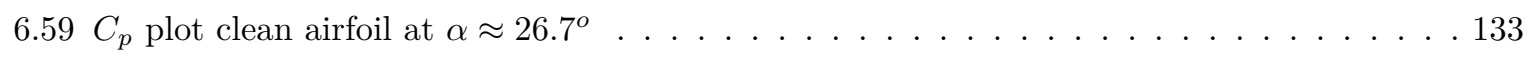

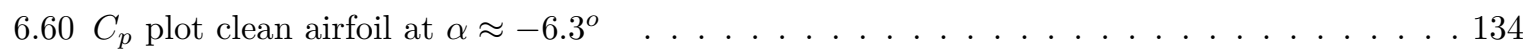

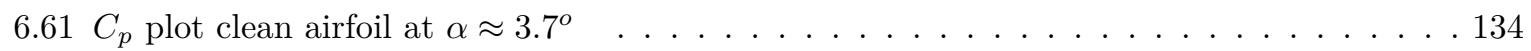

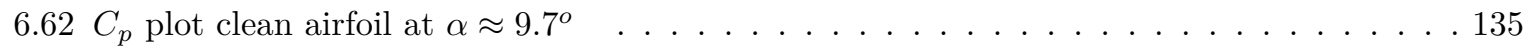

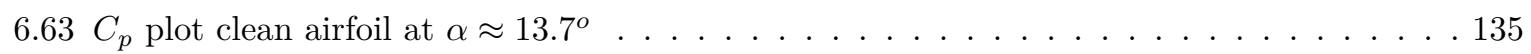

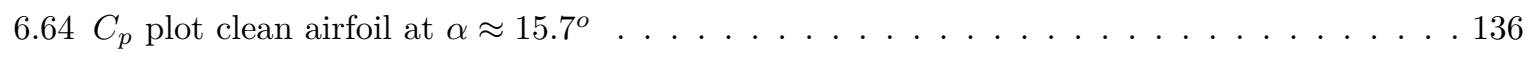


$6.65 C_{p}$ plot clean airfoil at $\alpha \approx 17.7^{\circ} \ldots \ldots \ldots \ldots \ldots \ldots \ldots \ldots \ldots$

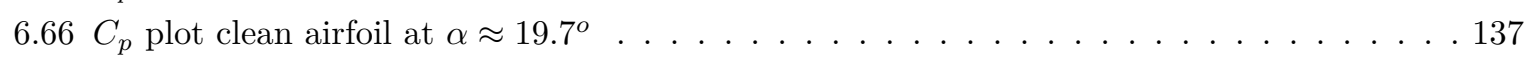

$6.67 C_{p}$ plot clean airfoil at $\alpha \approx 21.7^{\circ} \ldots \ldots \ldots \ldots \ldots \ldots \ldots \ldots \ldots \ldots \ldots$

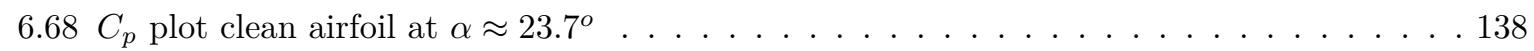

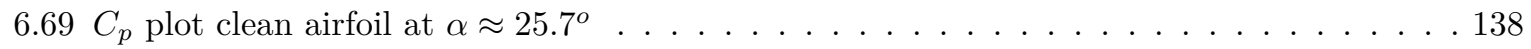

$6.70 C_{p}$ plot clean airfoil at $\alpha \approx 26.7^{\circ} \ldots \ldots \ldots \ldots \ldots \ldots \ldots \ldots$

$6.71 C_{p}$ plot clean airfoil at $\alpha \approx 27.7^{\circ} \ldots \ldots \ldots \ldots \ldots \ldots \ldots \ldots$

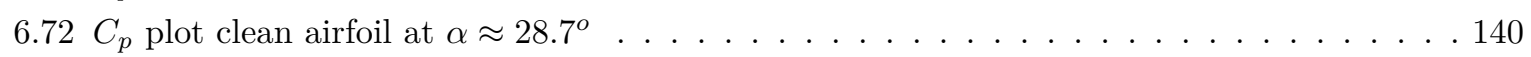

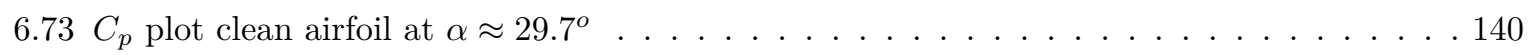

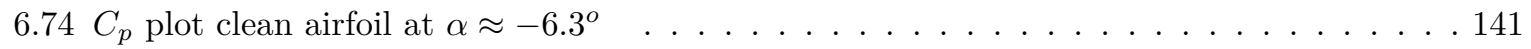

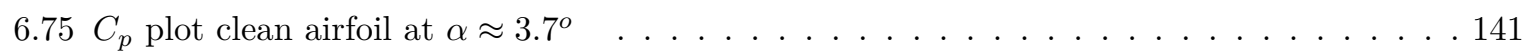

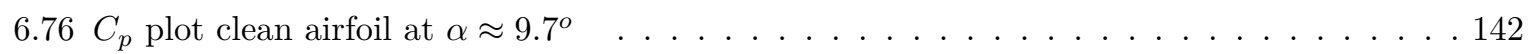

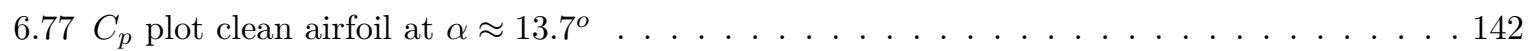

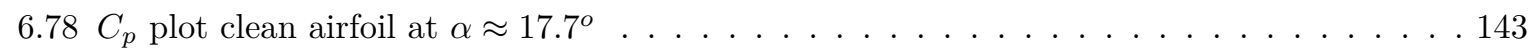

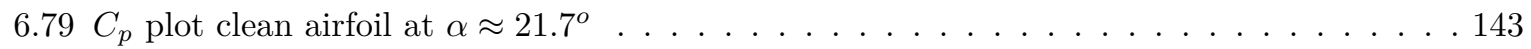

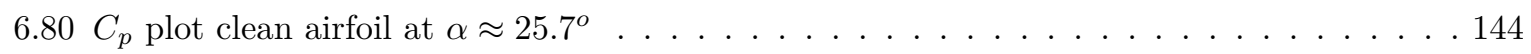

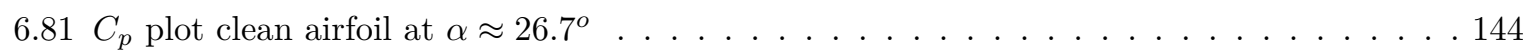

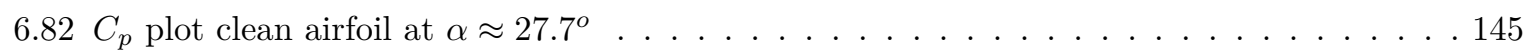

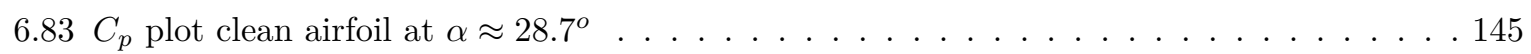

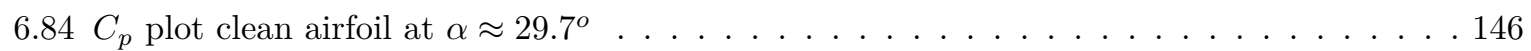

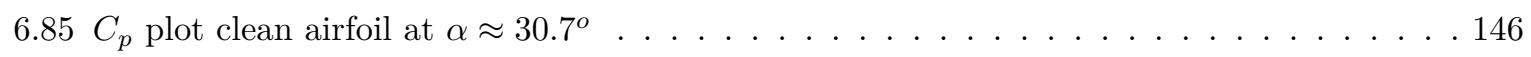

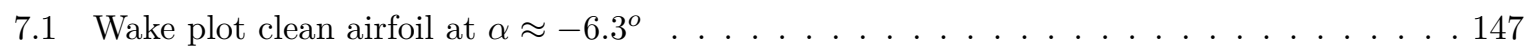

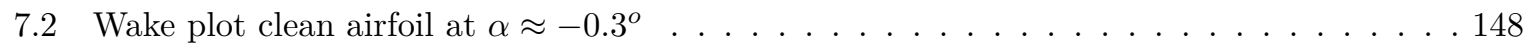

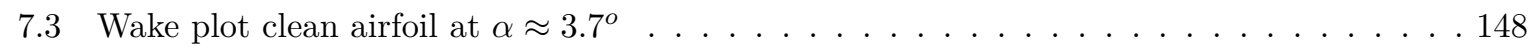

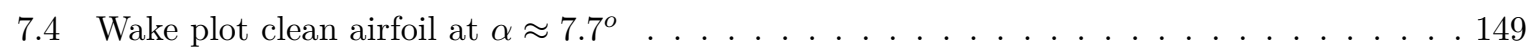

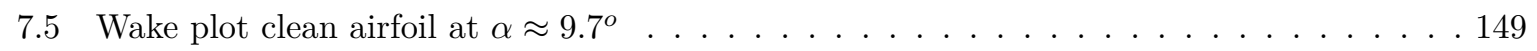

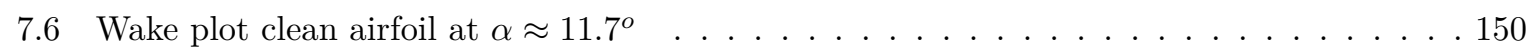

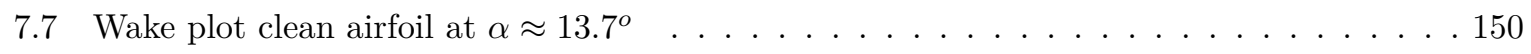

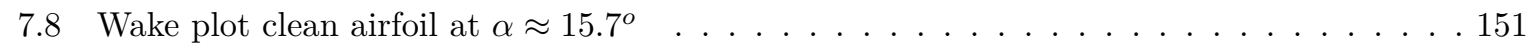

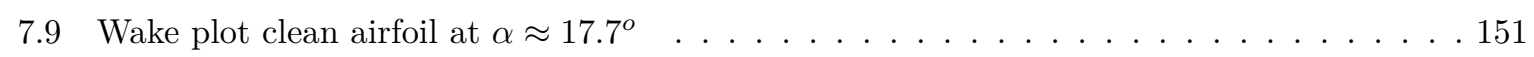

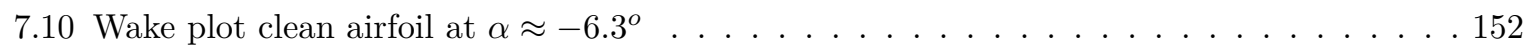

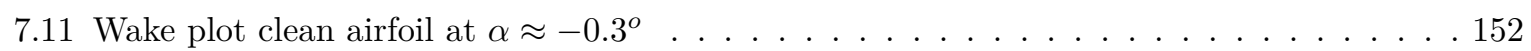

7.12 Wake plot clean airfoil at $\alpha \approx 3.7^{\circ} \ldots \ldots \ldots \ldots \ldots \ldots \ldots \ldots \ldots \ldots$

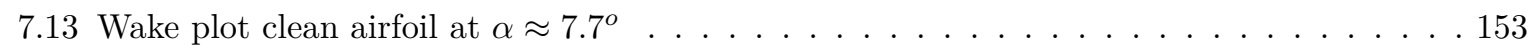

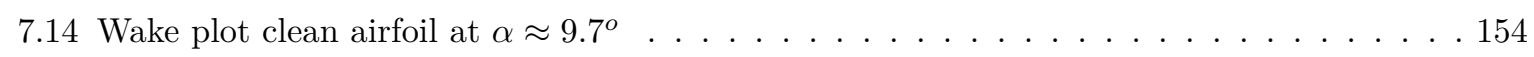

7.15 Wake plot clean airfoil at $\alpha \approx 11.7^{\circ} \ldots \ldots \ldots \ldots \ldots \ldots$

7.16 Wake plot clean airfoil at $\alpha \approx 13.7^{\circ} \ldots \ldots \ldots \ldots \ldots \ldots \ldots$

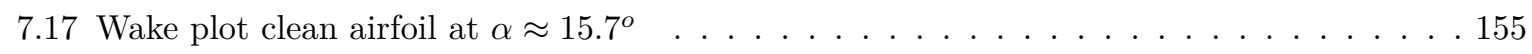

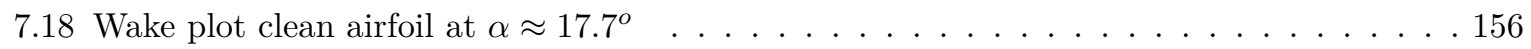




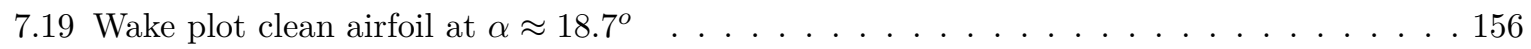

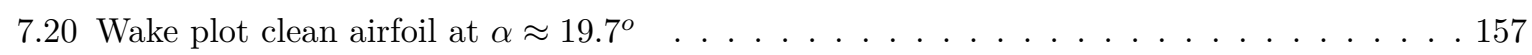

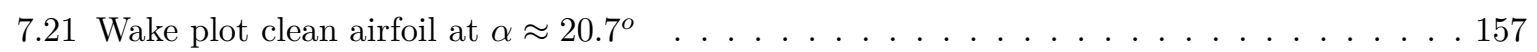

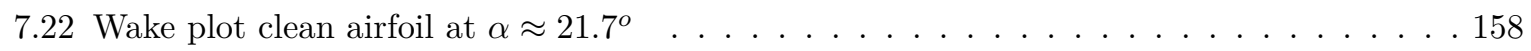

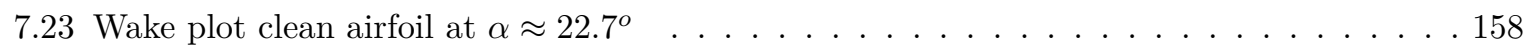

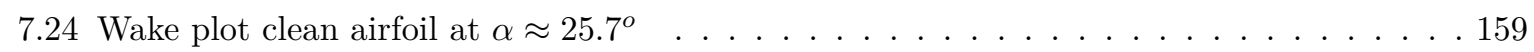

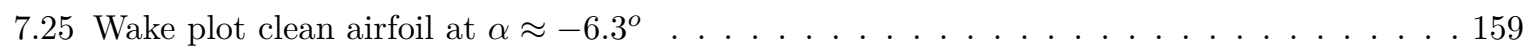

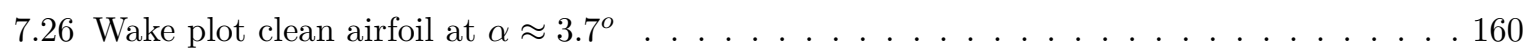

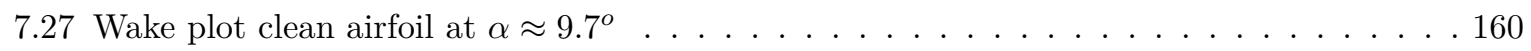

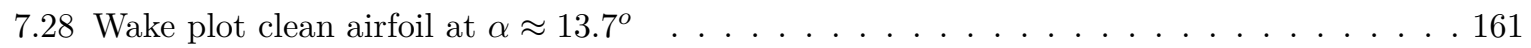

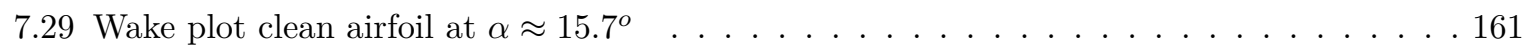

7.30 Wake plot clean airfoil at $\alpha \approx 17.7^{\circ} \ldots \ldots \ldots \ldots \ldots \ldots \ldots \ldots \ldots \ldots \ldots$

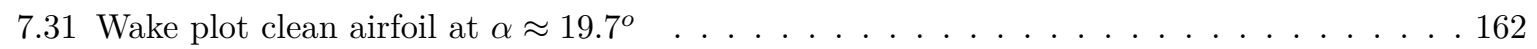

7.32 Wake plot clean airfoil at $\alpha \approx 21.7^{\circ} \ldots \ldots \ldots \ldots \ldots \ldots \ldots \ldots \ldots \ldots \ldots$

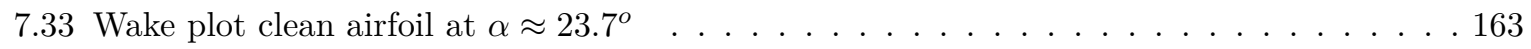

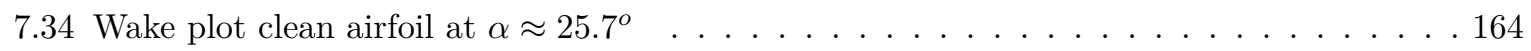

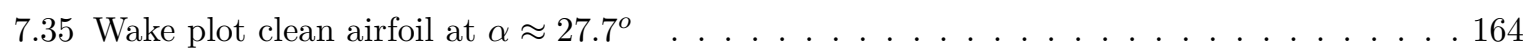

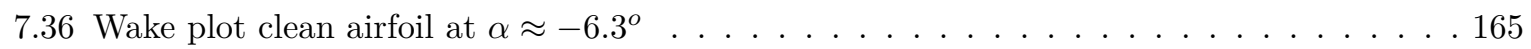

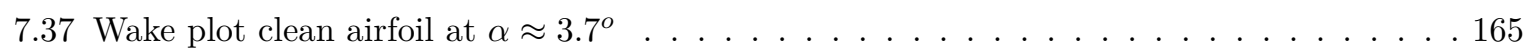

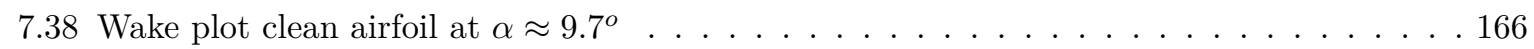

7.39 Wake plot clean airfoil at $\alpha \approx 13.7^{\circ} \ldots \ldots \ldots \ldots \ldots \ldots \ldots \ldots \ldots \ldots$

7.40 Wake plot clean airfoil at $\alpha \approx 15.7^{\circ} \ldots \ldots \ldots \ldots \ldots \ldots$

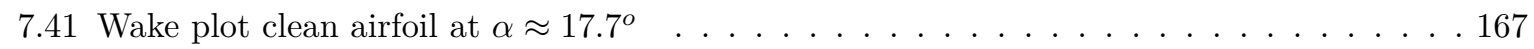

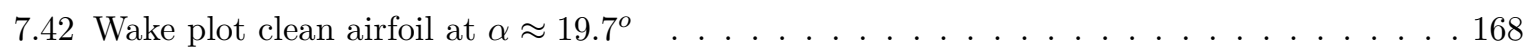

7.43 Wake plot clean airfoil at $\alpha \approx 21.7^{\circ} \ldots \ldots \ldots \ldots \ldots \ldots$

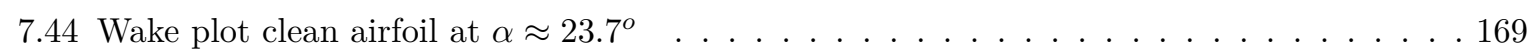

7.45 Wake plot clean airfoil at $\alpha \approx 25.7^{\circ} \ldots \ldots \ldots$. . . . . . . . . . . 169

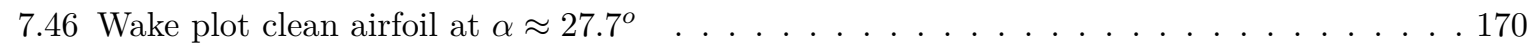

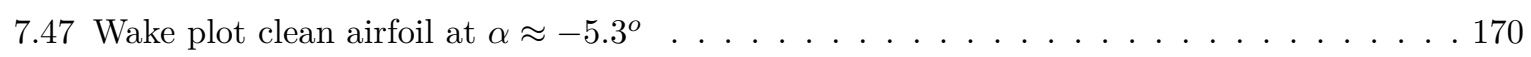

7.48 Wake plot clean airfoil at $\alpha \approx 3.7^{\circ} \ldots \ldots \ldots \ldots \ldots \ldots \ldots \ldots \ldots$

7.49 Wake plot clean airfoil at $\alpha \approx 9.7^{\circ} \ldots \ldots \ldots \ldots \ldots \ldots \ldots$

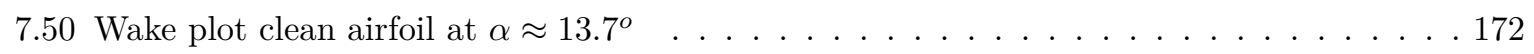

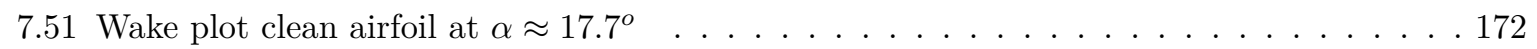

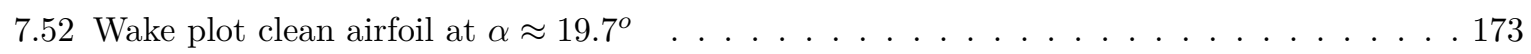

7.53 Wake plot clean airfoil at $\alpha \approx 20.7^{\circ} \ldots \ldots \ldots \ldots \ldots \ldots \ldots \ldots \ldots$

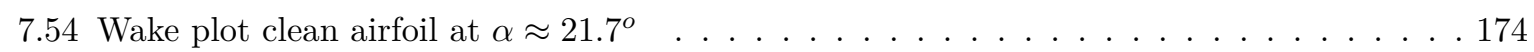

7.55 Wake plot clean airfoil at $\alpha \approx 22.7^{\circ} \ldots \ldots \ldots \ldots \ldots \ldots \ldots$

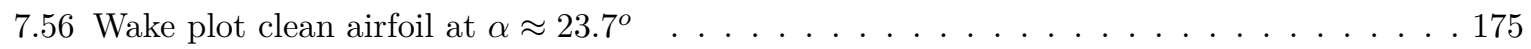

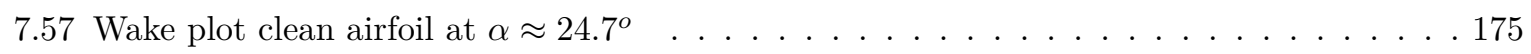

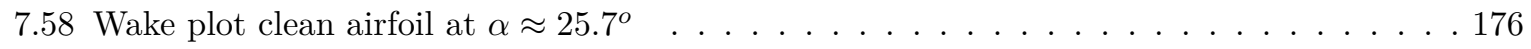


7.59 Wake plot clean airfoil at $\alpha \approx 26.7^{\circ} \ldots \ldots \ldots \ldots \ldots \ldots \ldots \ldots \ldots \ldots$

7.60 Wake plot clean airfoil at $\alpha \approx-6.3^{\circ} \ldots \ldots \ldots \ldots \ldots \ldots \ldots \ldots$

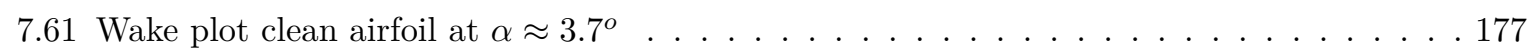

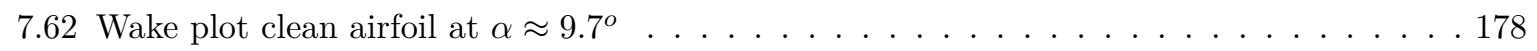

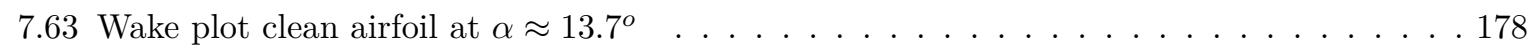

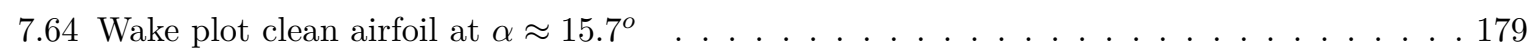

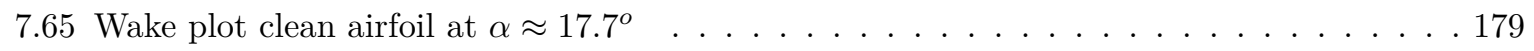

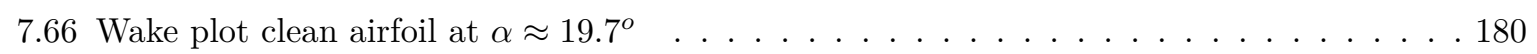

7.67 Wake plot clean airfoil at $\alpha \approx 21.7^{\circ} \ldots \ldots \ldots \ldots \ldots \ldots$

7.68 Wake plot clean airfoil at $\alpha \approx 23.7^{\circ} \ldots \ldots \ldots \ldots$. . . . . . . . . . . 181

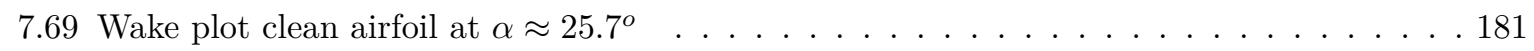

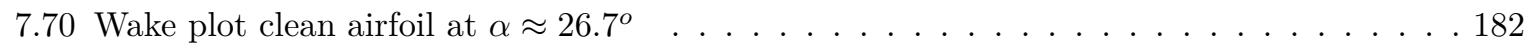

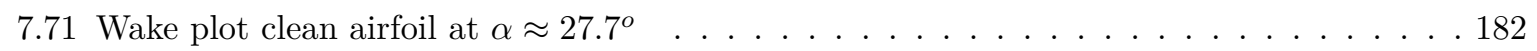

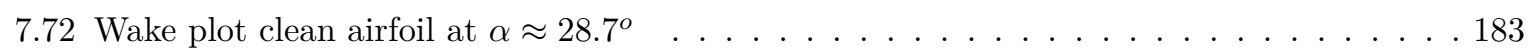

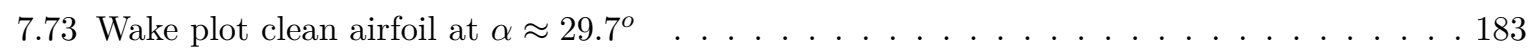

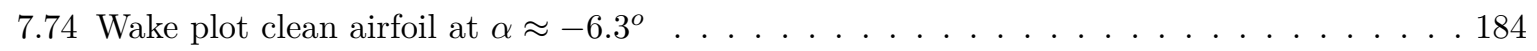

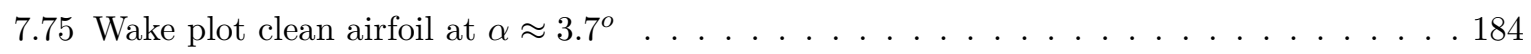

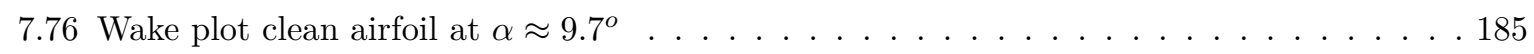

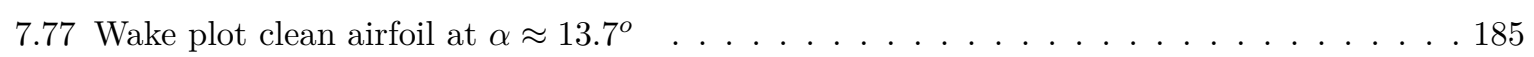

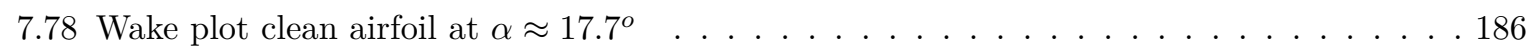

7.79 Wake plot clean airfoil at $\alpha \approx 21.7^{\circ} \ldots \ldots \ldots \ldots \ldots \ldots$

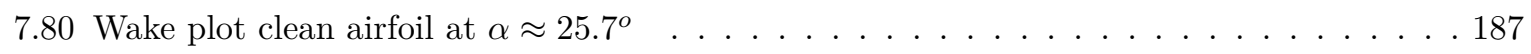

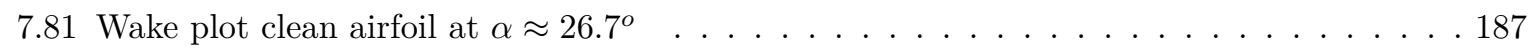

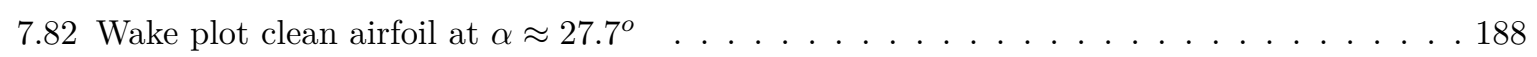

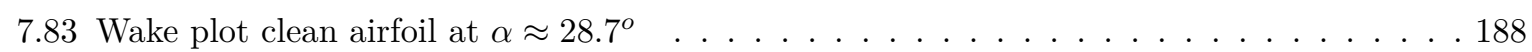

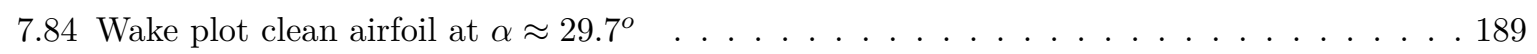

7.85 Wake plot clean airfoil at $\alpha \approx 30.7^{\circ} \ldots \ldots \ldots \ldots \ldots \ldots$

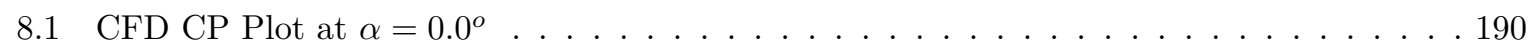

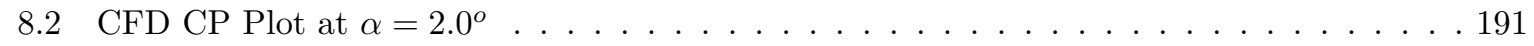

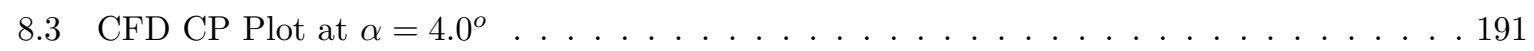

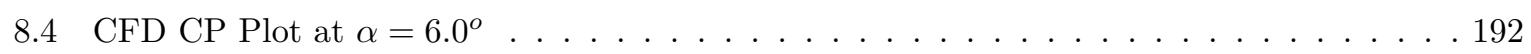

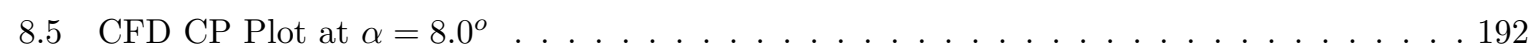

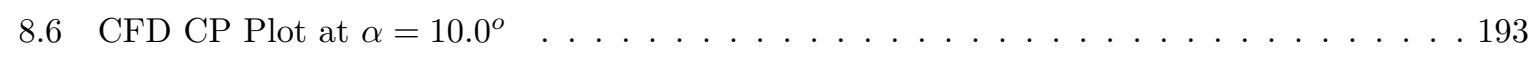

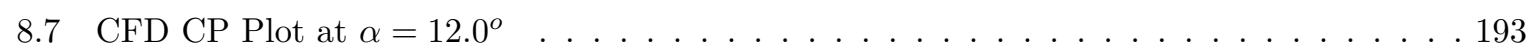

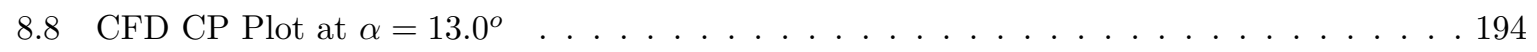

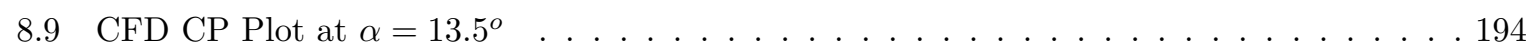

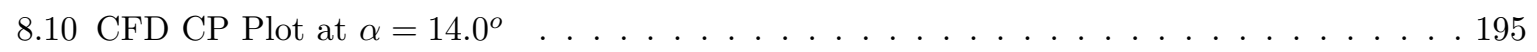

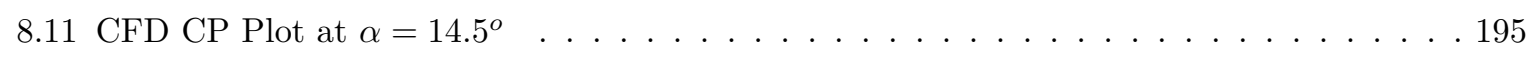

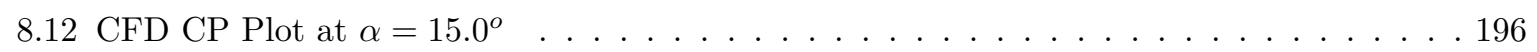




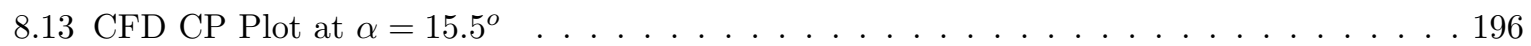

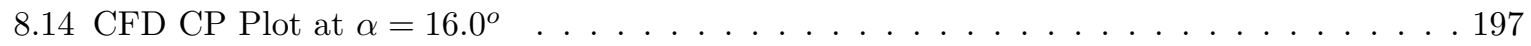

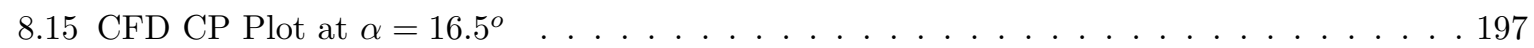

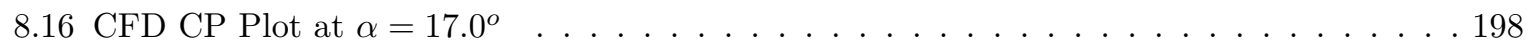

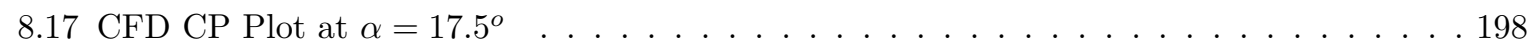

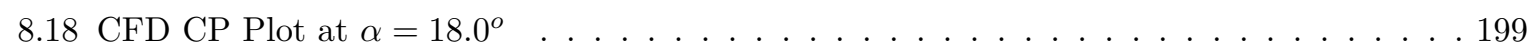

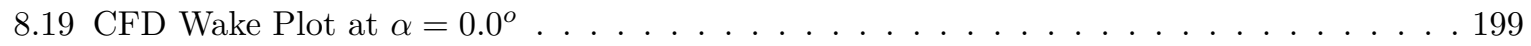

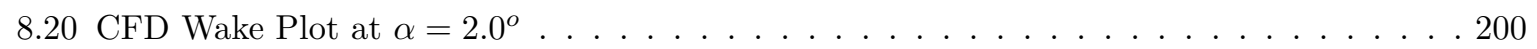

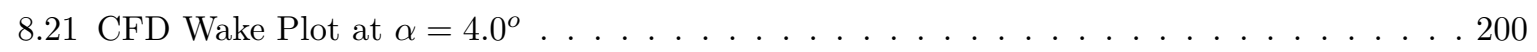

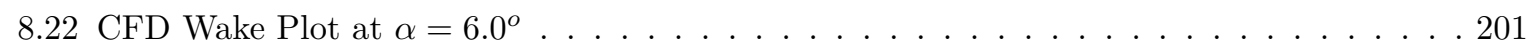

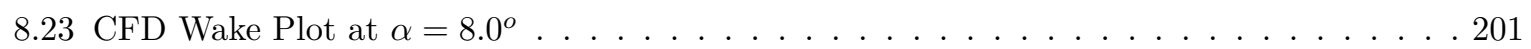

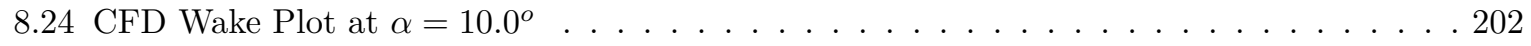

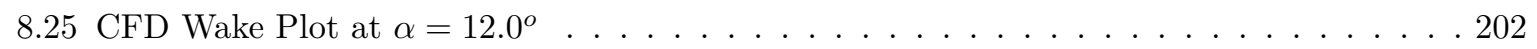

8.26 CFD Wake Plot at $\alpha=13.0^{\circ} \ldots \ldots \ldots \ldots \ldots \ldots \ldots \ldots \ldots \ldots \ldots \ldots \ldots \ldots$

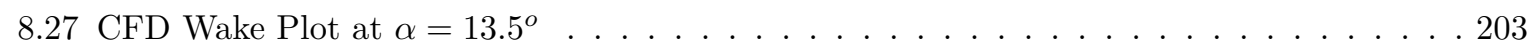

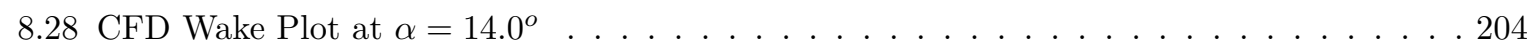

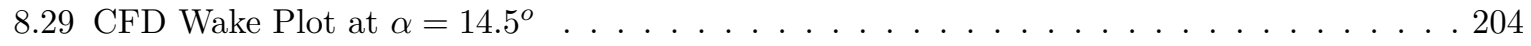

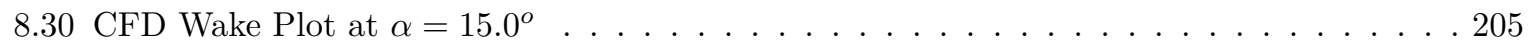

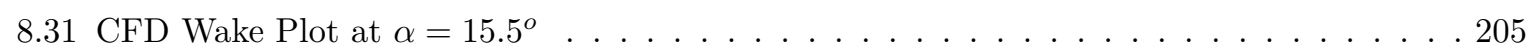

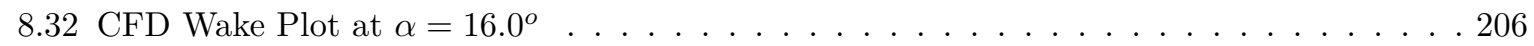

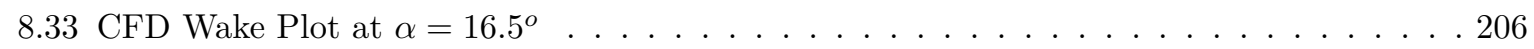

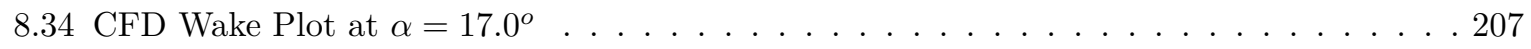

8.35 CFD Wake Plot at $\alpha=17.5^{\circ} \ldots \ldots \ldots \ldots \ldots \ldots \ldots \ldots \ldots \ldots \ldots \ldots \ldots$

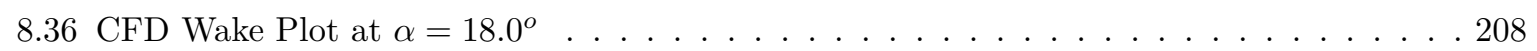




\section{LIST OF APPENDICES}

\begin{tabular}{ll}
\hline Appendix & 73
\end{tabular}

\begin{tabular}{lll}
\hline 1 & Detailed Geometry Information & $\mathbf{7 3}$
\end{tabular}

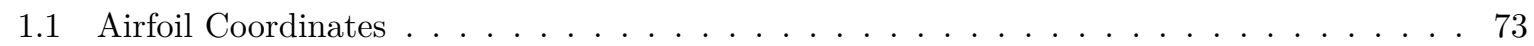

1.2 Spoiler Geometry $\ldots \ldots \ldots \ldots \ldots \ldots \ldots$

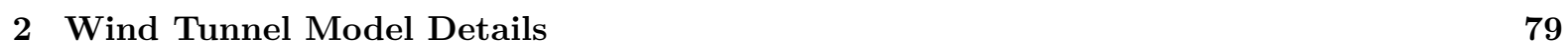

2.1 Requirements . . . . . . . . . . . . . . . . . . . . . . . . 81

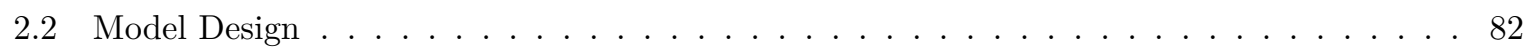

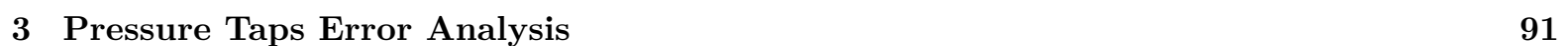

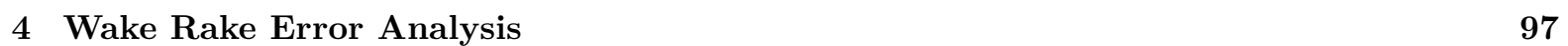

$\begin{array}{llr}5 & \text { Wind Tunnel Correction Factors } & 100\end{array}$

\begin{tabular}{|lll}
6 & Pressure Coefficient Plots from Wind Tunnel Experiment & 104
\end{tabular}

6.1 Clean Configuration . . . . . . . . . . . . . . . . . . . . . . . 104

$6.2 \delta=8^{\circ}, b=10, a=60$ Configuration $\ldots \ldots \ldots \ldots \ldots$

$6.3 \delta=15^{\circ}, b=10, a=60$ Configuration $\ldots \ldots \ldots \ldots \ldots \ldots$

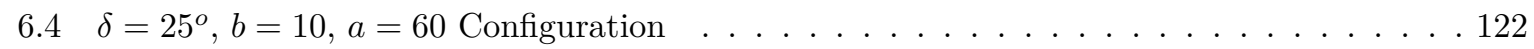

$6.5 \delta=8^{\circ}, b=15, a=60$ Configuration $\ldots \ldots \ldots \ldots \ldots \ldots \ldots \ldots$

$6.6 \quad \delta=15^{\circ}, b=15, a=60$ Configuration $\ldots \ldots \ldots \ldots \ldots \ldots \ldots \ldots \ldots \ldots$

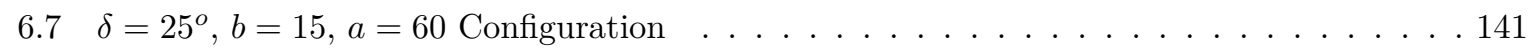

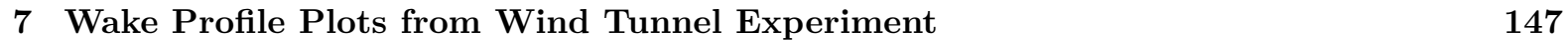

7.1 Clean Configuration . . . . . . . . . . . . . . . . . . . 147

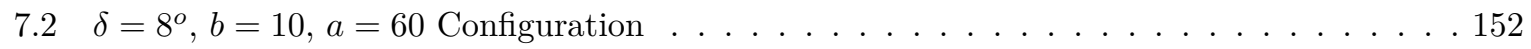

$7.3 \delta=15^{\circ}, b=10, a=60$ Configuration $\ldots \ldots \ldots \ldots \ldots$

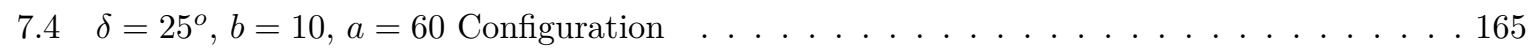

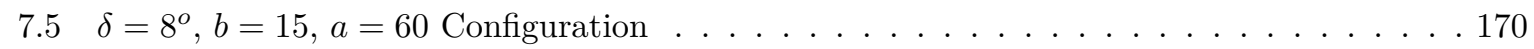

$7.6 \quad \delta=15^{\circ}, b=15, a=60$ Configuration $\ldots \ldots \ldots \ldots \ldots \ldots \ldots \ldots \ldots$ 
$7.7 \delta=25^{\circ}, b=15, a=60$ Configuration $\ldots \ldots \ldots \ldots \ldots \ldots$

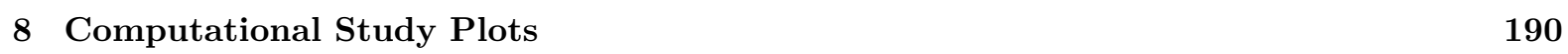

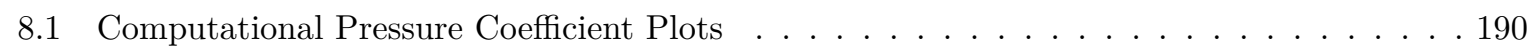

8.2 Computational Wake Plots $\ldots \ldots \ldots \ldots$ 


\section{Chapter 1}

\section{Introduction}

\subsection{Motivation}

Flow separation control over wings and airfoils is an important subject of study due to it's impact on aerodynamic efficiency, and stall. Flow separation provides a limit on the lifting capabilities of wings, thus impacting landing, takeoff, and maneuverability. This provides a strong incentive to research potential stall delaying techniques. Traditionally, leading-edge flaps and/or slats satisfy this role, and newer techniques such as blowing and suction are continuously being studied. One such technique has arisen from an unlikely candidate, spoilers.

Spoilers are upper surface flaps designed to control flow-separation to provide aerodynamic braking, and lift dumping at touchdown. They also function as effective roll control devices when deployed asymmetrically [4. Spoilers achieve this lift-reducing effect by creating an adverse pressure gradient on the upper surface of the airfoil, thus forcing the air to separate. This increases drag, and reduces lift. Contrary to their usual function as lift reducing, drag increasing devices, spoilers have been shown to increase maximum lift coefficient and improve aerodynamic efficiency at high angles of attack by preventing the propagation of the flow separation bubble from moving upstream from trailing edge to leading edge. This potential for performance enhancement could be significant, however it cannot be properly exploited without a thorough understanding of the effect, performance gains, and possible adverse impacts. Thus providing the motivation for this study.

\subsection{Background}

\subsubsection{Spoiler Flow-Field Characteristics}

The flow-field of an airfoil with a spoiler is described by Lee and Bodapti [4. The flow includes separation, reattachment, and vortex shedding. Flow separates from the upper surface due the the adverse pressure gradient created by the spoiler. At pre-stall angles of attack, the separation bubble re-attaches to the airfoil surface. A recirculating bubble called a "hinge-bubble" is formed upstream of the spoiler hinge. 
The flow separates from the spoiler tip and moves downstream as a free-shear layer. The shedding vorticies from the spoiler tip form an unsteady, oscillating wake pattern.

Lee and Bodapti observed the flow-field around a spoiler-equipped Boeing Advanced Transport Research (BATR) airfoil placed in a $45.7 \times 45.7$-cm test section at a Reynold's number of $2.8 \times 10^{5}$. The airfoil had a chord length of $20.3 \mathrm{~cm}$, had a maximum thickness ratio of $11.3 \%$, and had a spoiler of $15.5 \%$ chord length equipped at $73 \%$ chord. Boundary layer trips were placed at $7.5 \%$ chord on both the upper and lower surfaces. Lift coefficient $\left(C_{l}\right)$, drag coefficient $\left(C_{d}\right)$ and pressure coefficient distribution $\left(C_{P}\right)$ was measured for angles of attack $(\alpha)$ ranging from $-10^{\circ}$ to $12^{\circ}$, and spoiler deflection angles $(\delta)$ ranging from $15^{\circ}$ to $60^{\circ}$. The results of the study are shown in figure 1.1 and figure 1.2 below.
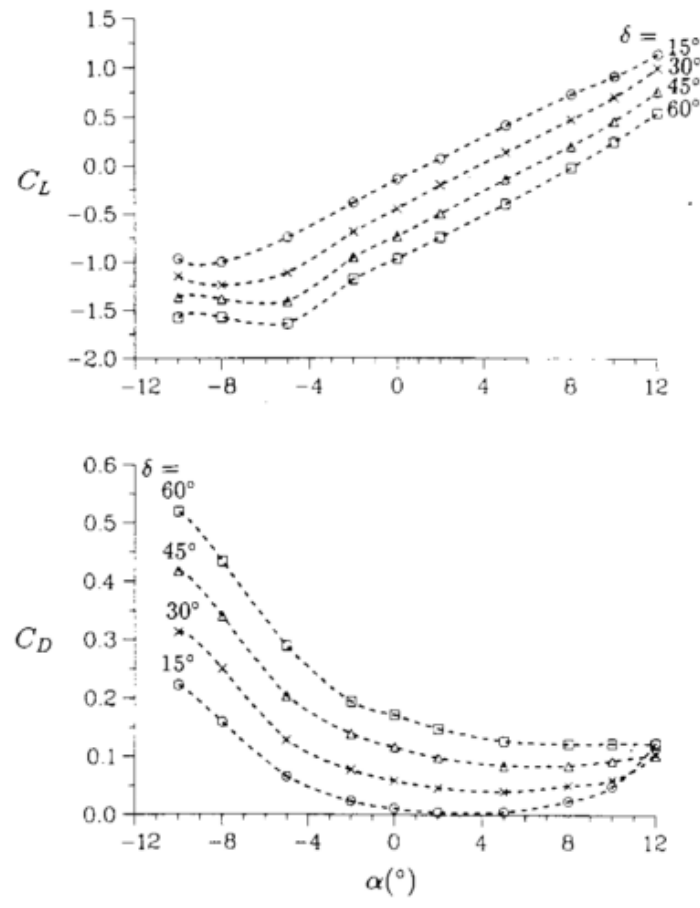

Figure 1.1: Variation of lift and drag coefficients with angle of attack [4]

Figure 1.1 indicates the change the lift and drag curve slopes with changing spoiler deflection angle. As $\delta$ increases, the lift coefficient decreases. The slope of the lift-curve does not experience significant change, thus deflecting the spoiler can be described as effectively de-cambering the airfoil. The lifting spoiler phenomena was not captured by Lee and Bodapti since they did not continue the experiment into stall. The drag coefficient increases with an increase in spoiler deflection angle. However, at $\alpha=12^{\circ}$, the $\delta=15^{\circ}$ case produces lower drag coefficients than cases with greater $\delta$. Although not commented on by Lee and Bodapti, this could represent the higher $\delta$ cases blocking the flow separation bubble from propagating further upstream, thus reducing drag. 

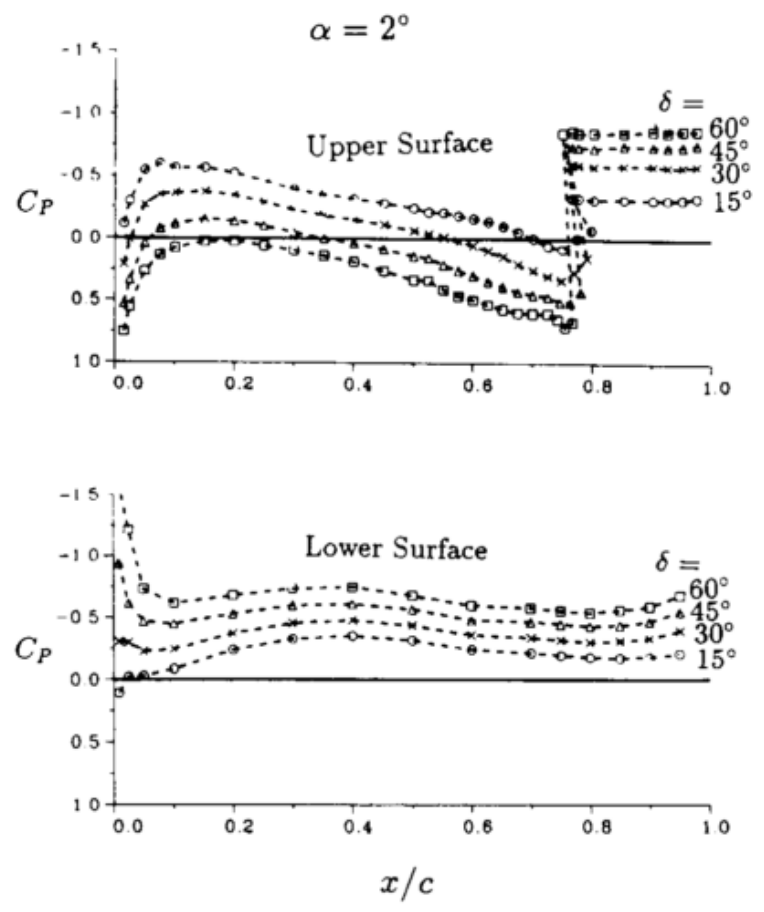

Figure 1.2: $C_{P}$ distribution on upper and lower surfaces $\left(\alpha=2^{\circ}\right)[4$

Figure 1.2 indicates the pressure coefficient distribution over the airfoil chord at $\alpha=2^{\circ}$. Higher spoiler deflection angles decrease the static pressure on the upper surface, upstream of the spoiler. This trend is also seen on the lower surface. The pressure coefficient decreases sharply after the flow encounters the spoiler.

The typical flow field present around a spoiler was described numerically by Alhawwary et al. using a higher order spectral difference method to solve the two-dimensional full Navier-Stokes equations on unstructured quadralatial grids [5. Numerical results were gathered from the BATR airfoil at angles of attack $\alpha=0^{0}, 8^{\circ}$, and spoiler deflection angles $\delta=0^{\circ}, 15^{\circ}, 30^{\circ}, 60^{\circ}$.

Alhawwary et al. found that the flow-field of an airfoil spoiler is characterized by a highly fluctuating pressure over the model surface, and vortex shedding in the wake, resulting in an oscillatory lift and drag behavior. For smaller deflection angles the frequency of the lift fluctuation is high and it's amplitude is low. As the spoiler deflection angle increases, the frequency of the lift decreases and it's amplitude increases. This behaviour is shown in figure 1.3 below. 


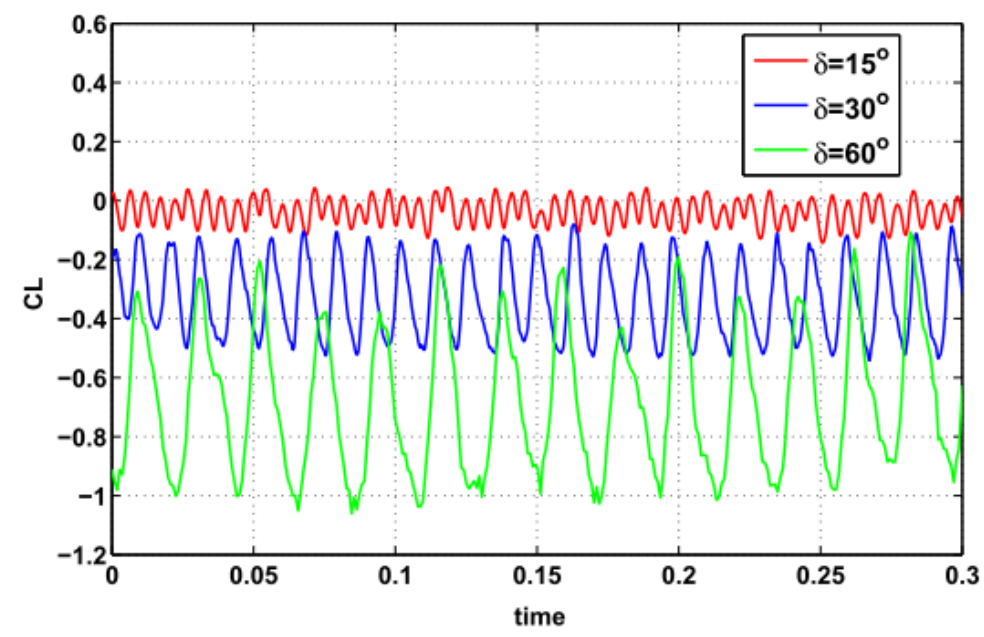

(a) Time history

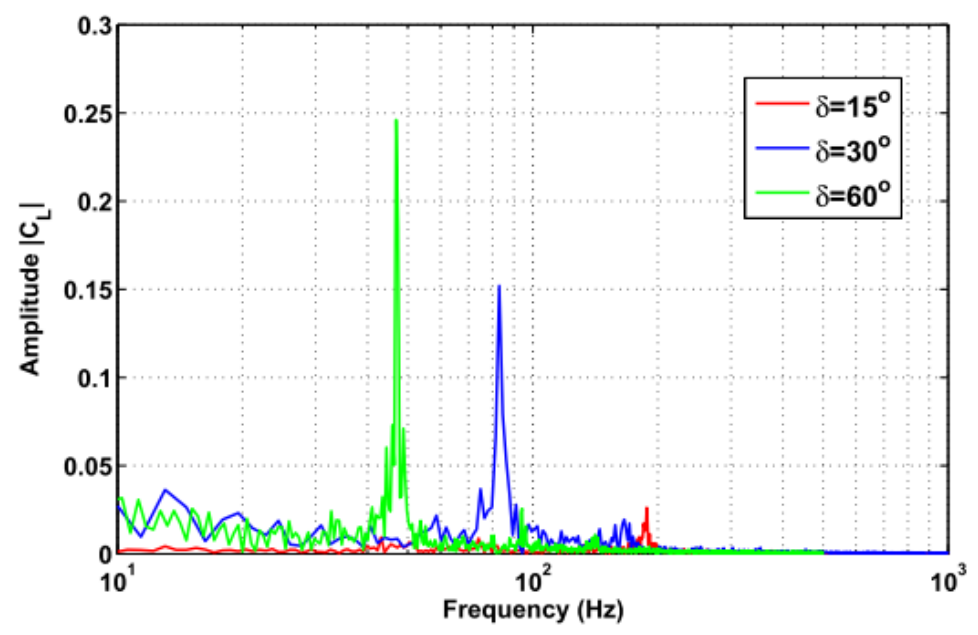

(b) FFT

Figure 1.3: Time history and Fast Fourier transform (FFT) of $C_{l}$ for the BATR airfoil-spoiler $\alpha=0^{\circ}[5]$

Streamline patterns for different times during one period of vortex shedding are shown in the figures 1.4 and 1.5 below. The typical vortex shedding behavior of a spoiler is similar to that of bluff body shedding. The flow field is characterized by a highly turbulent fluctuating wake. The strength and chaos increase with deflection angle. At moderate angles of attack, flow separates ahead of the spoiler forming a hinge bubble, and it's strength increases with angle of attack. 


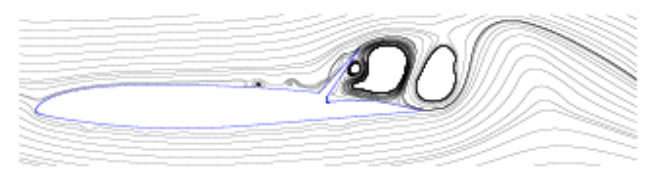

(a) $0 \mathrm{~T}$, zero and increasing lift

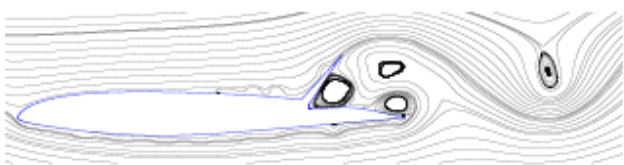

(c) $\frac{1}{2} T$, zero and decreasing lift

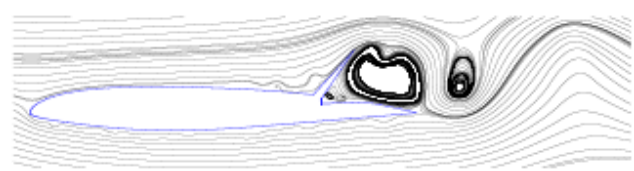

(b) $\frac{1}{4} T$, maximum lift

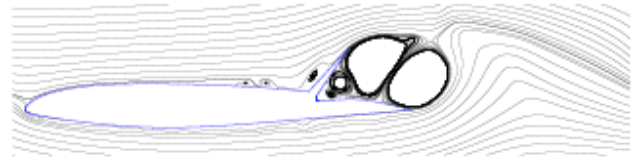

(d) $\frac{3}{4} T$, minimum lift

Figure 1.4: Streamline periodic patttern for the BATR airfoil-spoiler at $\delta=60^{\circ}, \alpha=0^{\circ}$ [5].

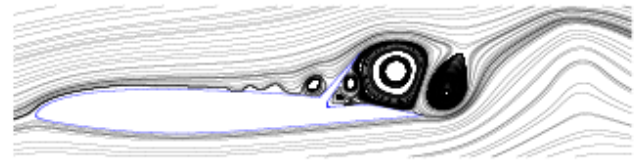

(a) 0T, zero and increasing lift

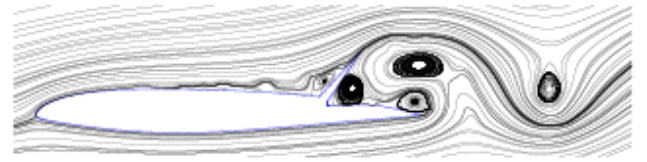

(c) $\frac{1}{2} T$, zero and decreasing lift

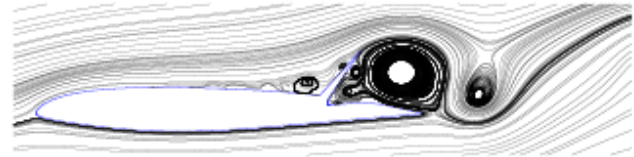

(b) $\frac{1}{4} T$, maximum lift

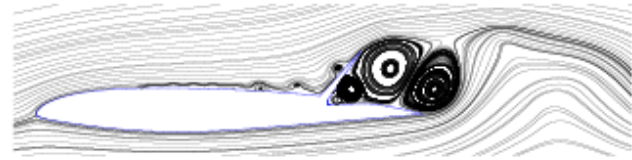

(d) $\frac{3}{4} T$, minimum lift

Figure 1.5: Streamline periodic patttern for the BATR airfoil-spoiler at $\delta=60^{\circ}, \alpha=8^{\circ}[5$.

\subsubsection{The lifting spoiler effect}

The lifting spoiler effect has been described by Bramesfeld and Maughmer [6]. A 17.7\% thick S824 airfoil was used to assess the performance of spoilers that deploy passively under the influence of separated flow (called lift enhancing effectors, or LEE's by Bramesfeld and Maughmer). The effectors were studied experimentally using the Pennsylvania State University Low Speed, Low Turbulence Wind Tunnel. The tunnel is a closed-throat, single return atmospheric tunnel with a $1.0 \times 1.5 \mathrm{~m}$ tunnel. The flow velocity was set to $46 \mathrm{~m} / \mathrm{s}$, thus yielding a Reynold's number of approximately $1.0 \times 10^{6}$. Figure 1.6 below shows the $C_{l}$ vs $\alpha$ curves of the baseline clean configuration, single effector, and double effector airfoils. The maximum lift coefficient was increased by approximately 18-20\% when the airfoil was equipped with LEEs compared to the clean configuration. 


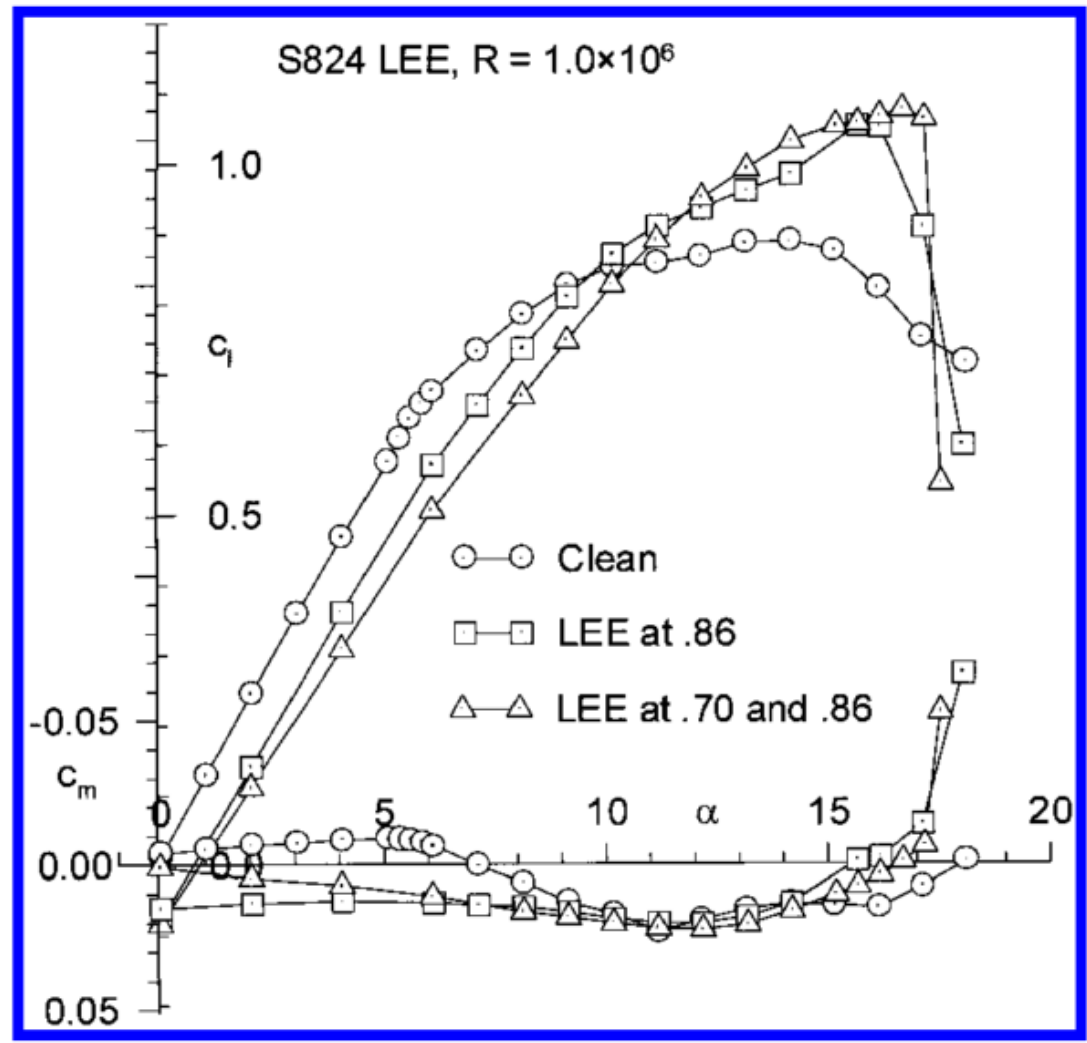

Figure 1.6: Lift and moment coefficients vs angle of attack curves of the baseline S824 airfoil along with those of a single and double effector equipped configurations [6]

Figure 1.7 below shows the $C_{l}$ vs $C_{d}$ curves of the baseline clean configuration, single effector, and double effector airfoils. At high angles of attack, the LEE causes an increase in aerodynamic efficiency. 


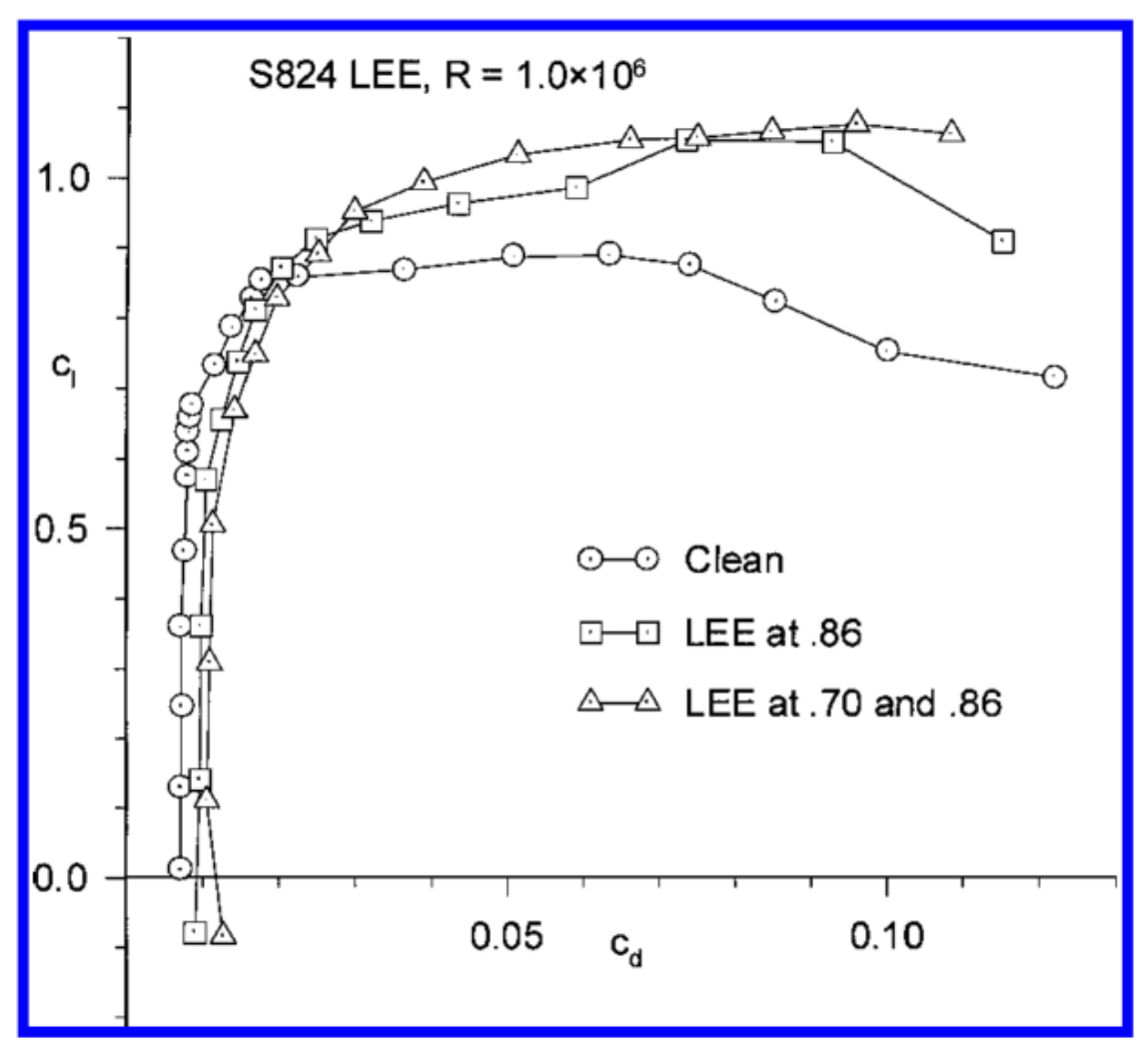

Figure 1.7: Lift vs drag coefficients of baseline S824 airfoil along with those of a single and double effector equipped configurations [6]

The pressure distribution for the clean configuration and the single LEE equipped airfoil at $\alpha=16^{\circ}$ is shown in figure 1.8 below. The most notable difference is the pressure recovery step across the effector location. Upstream of the effector on the upper surface the pressures reach lower values than on the clean airfoil. The upper surface suction peak reaches a value of $C_{p}=-6.97$ for the LEE equipped airfoil versus $C_{p}=-5.54$ for the clean airfoil. The effector causes the pressure downstream to be higher than those on the clean airfoil. This causes a slightly higher pressure on the lower surface. Both these factors increase the overall area under the pressure coefficient plot, thus increasing the resultant lift coefficient compared with the clean airfoil. 


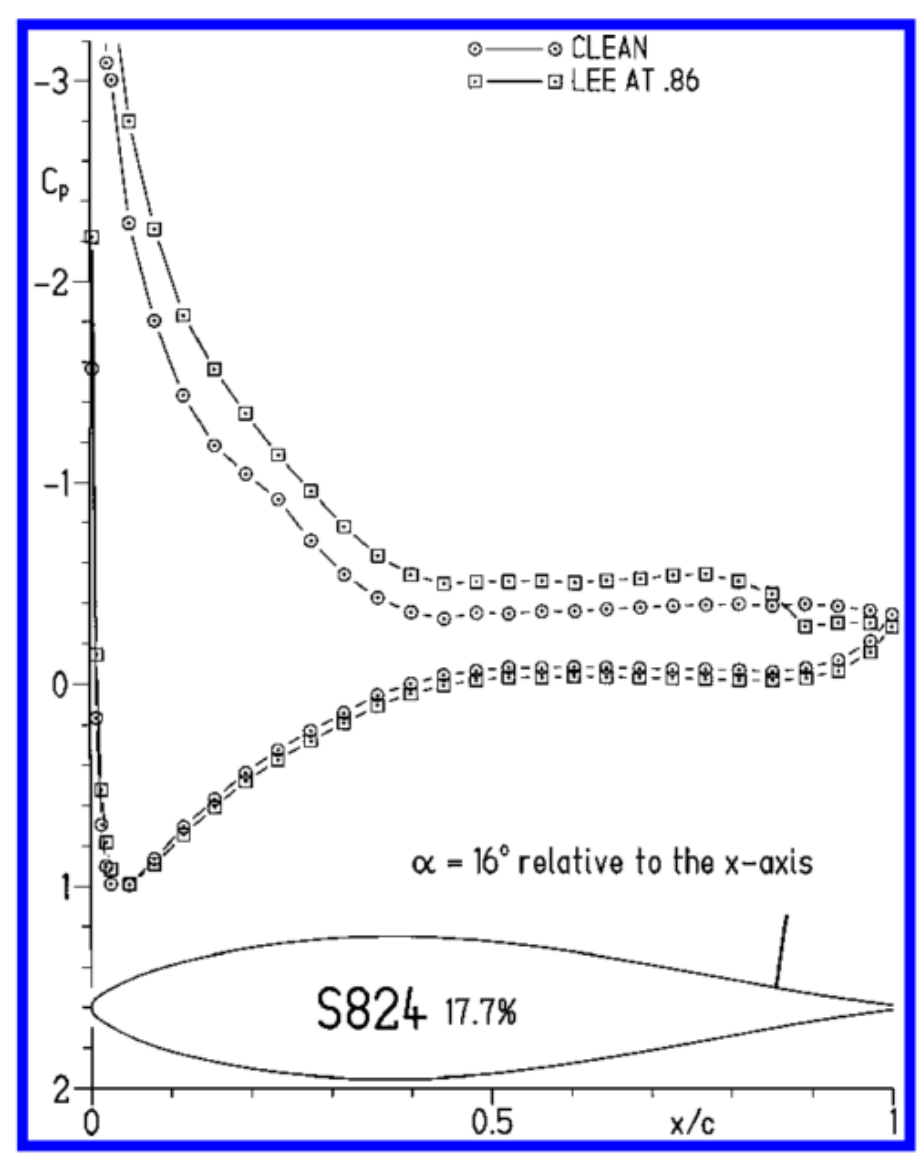

Figure 1.8: Pressure distributions for $\alpha=16^{\circ}$ of baseline S824 airfoil and of configuration having a single effector at $86 \%$ chord. [6]

The lifting spoiler phenomenon was also observed by Traub and Jaybush [7] in a series of wind tunnel experiments. Traub and Jaybush studied the impacts of a self-actuating spoiler. Similarly with Bramesfeld and Maughmer's design, the spoiler actuated passively under the presence of reversed flow. The design of Traub and Jaybush's spoiler configurations are shown in figure 1.9 

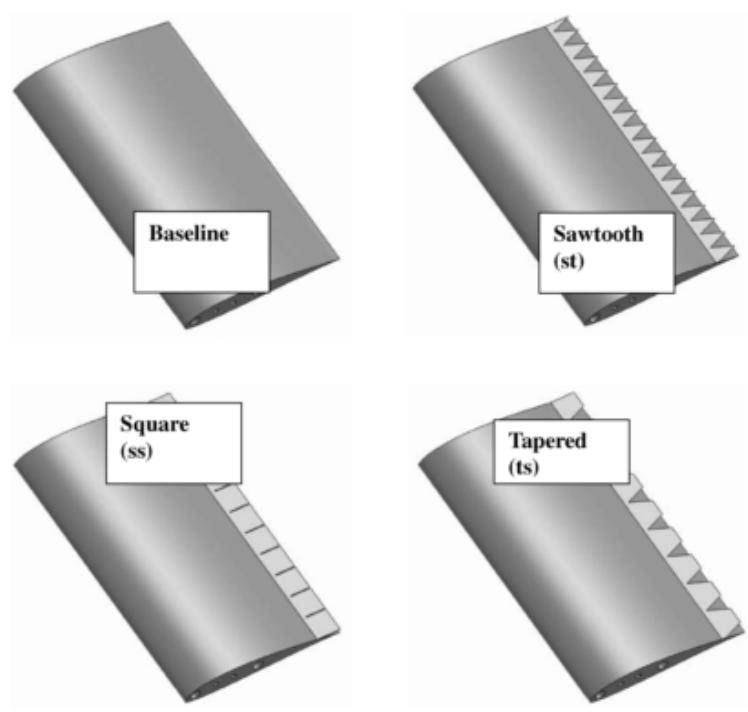

Figure 1.9: Flexible spoiler geometry renderings. Spoiler lengths are $12 \%$ of the chord. [7]

The rectangular model $(\mathrm{SD} 7062$, aspect ratio $=4$ ) was rapid prototyped from ABS (acrylonitrile butadiene styrene) plastic. A transition strip was placed at $5 \%$ chord. The spoilers were contructed using $0.1 \mathrm{~mm}$ plastic sheeting, and was hinged to the airfoil using Scotch tape at 68,78 , and $88 \%$ chord. The model was placed in a $2 \times 2 \mathrm{ft}$ wind tunnel and tested at a Reynolds number of 225,000.

The spoiler-equipped configurations show a moderate drag-coefficient reductions for lift coefficients 0.6 to stall and an increase in the wing's lift-curve slope. The spoilers were found to delay the initial rounding and lessening of the lift-curve slope associated with the onset of trailing edge separation. The combination of lift-enhancement and drag reduction causes an increase in lift-over-drag ratio at higher angles of attack as shown in figure 1.10 below. 

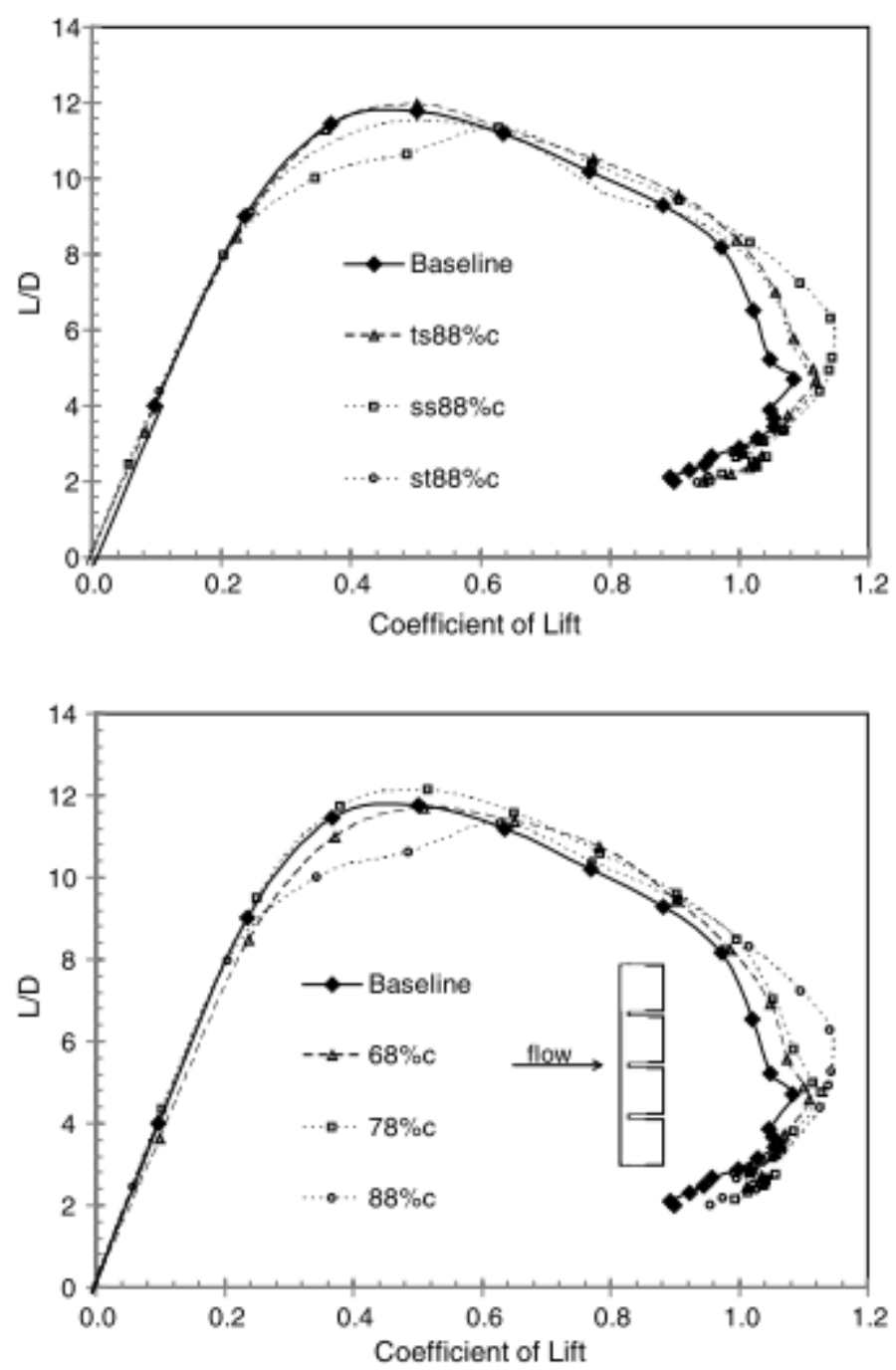

Fig. 3 Effect of spoiler geometry and location on measured $L / D$ ratio.

Figure 1.10: Effect of spoiler geometry and location on measured L/D ratio. [7]

A similar study was conducted by Johnson et al. [8] to experimentally determine the stall mitigation factors of a spoiler. Johnston et al. studied this effect in a closed-circuit low speed tunnel on a $30.5 \mathrm{~cm}$ chord length airfoil section at a Reynolds number of $4 \times 10^{5}$. The results from this study are shown in figure 1.11, which indicates an increase in the maximum angle of attack, and a gentler stall characteristic with an increase in spoiler deflection. The largest gain in $C_{l}$ occurred at a deflection angle of $30^{\circ}$, which resulted in an increase in lift of $30 \%$ when compared to the clean configuration. The stall angle was increased from $12^{\circ}$ to a maximum of $16^{\circ}$. During pre-stall conditions, an increase in spoiler deflection decreased the amount of lift produced by the section. 


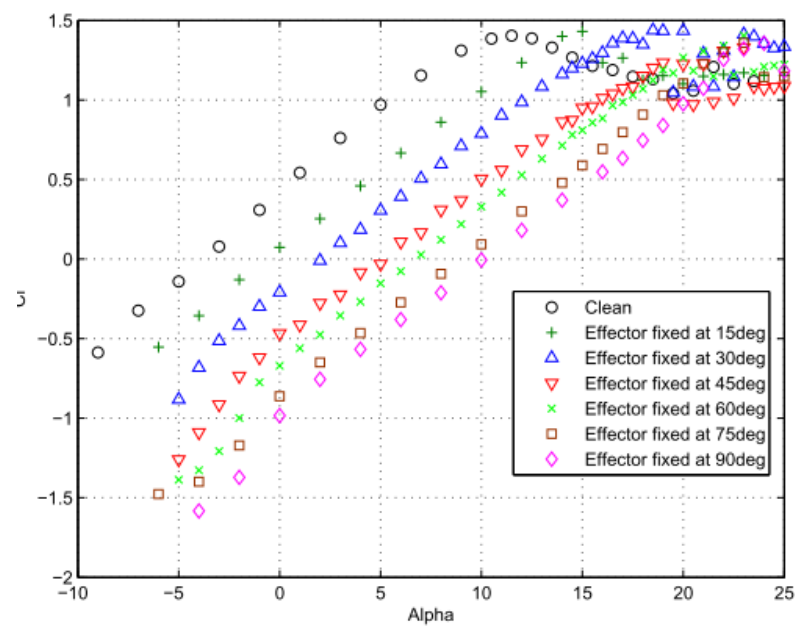

Figure 1.11: $C_{l}$ curves for the clean airfoil and the 6 fixed deplyment effectors $[8$.

The study also produced pressure distribution plots comparing the spoiler to the clean configuration shown in figure 1.12 and figure 1.13 These plots indicate the effect the spoiler had on the airflow, and give insight into how the spoiler caused the observed changes in lift. In figure 1.12 the airfoil is at a low angle of attack was well within the pre-stall region. The spoiler's presence created a large increase in pressure on the upper surface of the airfoil when compared to the clean configuration, and thus reduced lift. However at high angles of attack as shown in figure 1.13 the spoiler decreased the pressure upstream on the upper surface and increased the pressure downstream. The pressure increased slightly on the lower surface, in a similar fashion to the observations by Bramesfeld and Maughmer. The increased suction fore of the spoiler is significantly greater than the decreased suction aft, thus resulting in an overall net gain in lift. 


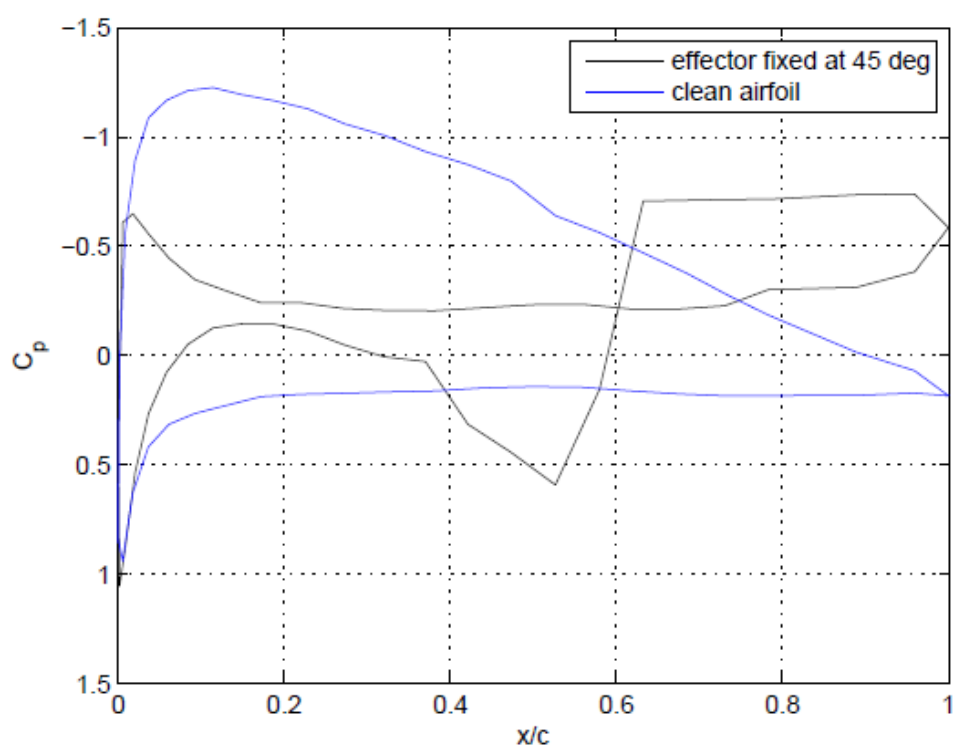

Figure 1.12: $C_{p}$ distribution at $\alpha=4^{\circ}$ for the clean airfoil compared to the effector fixed at $45^{\circ}$ deployment $[8$.

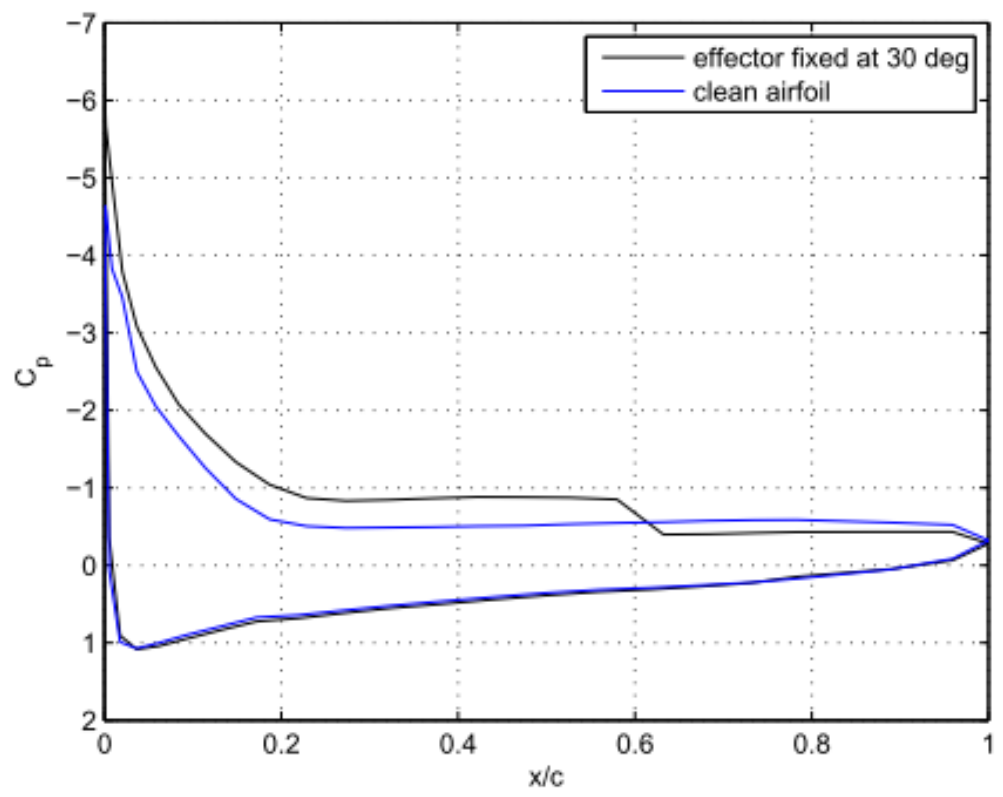

Figure 1.13: $C_{p}$ distribution at $\alpha=18^{\circ}$ for the clean airfoil compared to the effector fixed at $30^{\circ}$ deployment [8]. 
A computational fluid dynamic study was conducted on the effects of spoilers on a two-dimensional NACA 2412 airfoil by Pabla at Ryerson University [9]. Pabla studied a $1 \mathrm{~m}$ chord airfoil with a $10 \%$ spoiler chord length at deflection angles $4^{\circ}$, and $10^{\circ}$, and spoiler positions at $60 \%, 65 \%$ and $70 \%$ chord.

Pabla found that the separation bubble formed by an intensifying pressure gradient at post stall angles $\alpha=17^{\circ}$ to $19^{\circ}$ is influenced by the location and deflection angle of the spoiler. At $19^{\circ}$, the separation bubble is noticeably larger in the clean airfoil case (shown in the streamline plot in figure 1.14 below). Moving the spoiler aft causes the separation bubble to decrease in size. Since large separation bubbles are associated with a lift decrease and a drag increase, it is visually apparent how the spoiler is used to improve aerodynamic efficiency.
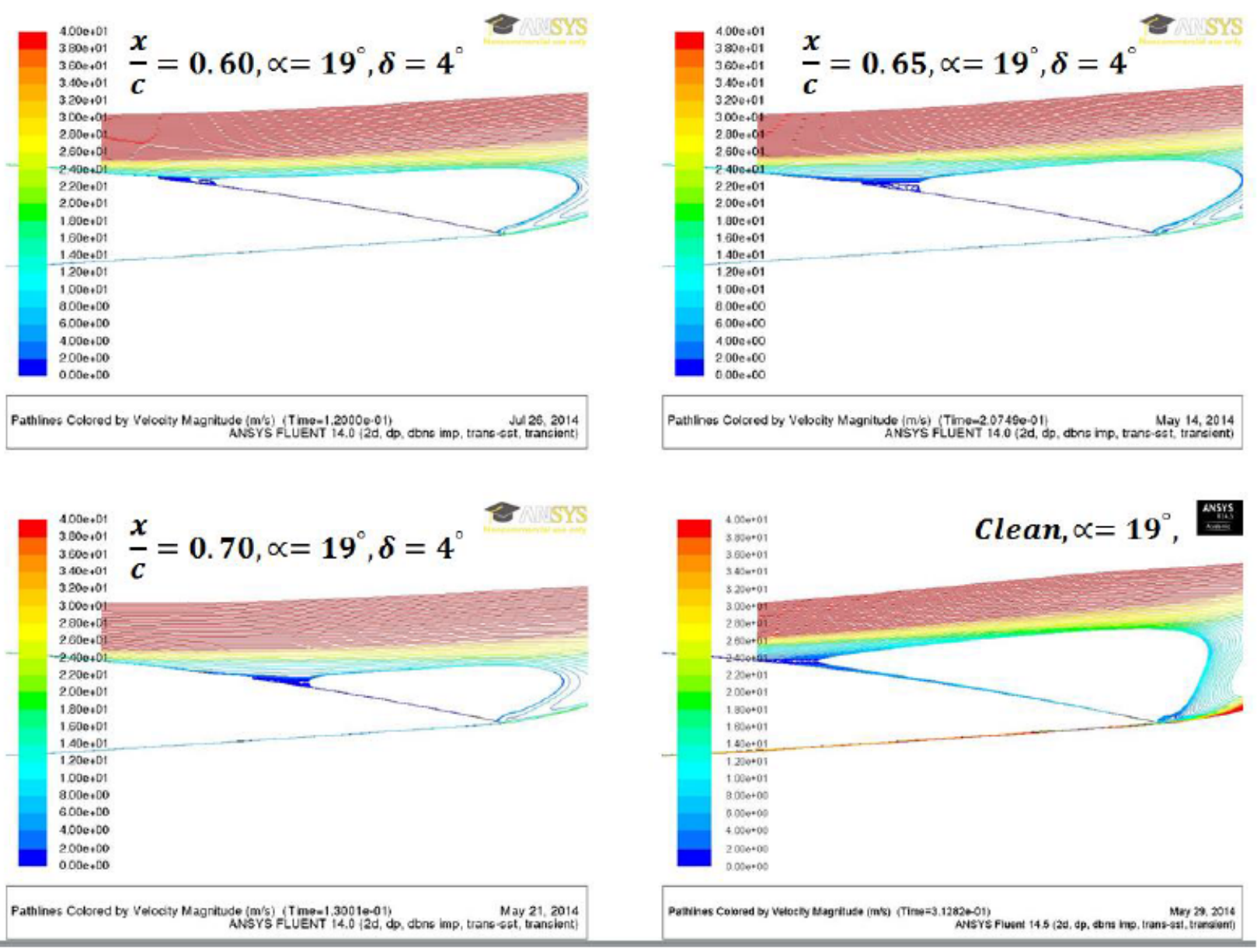

Figure 1.14: Velocity Magnitude Streamlines, $\alpha=19^{\circ}, R e=3.79 \times 10^{6} 0 \underline{9}$.

The clean airfoil lift coefficient at $\alpha=19^{\circ}$ was found to be 1.702 . With the inclusion of the spoiler, the lift coefficient rose to $1.75,1.78$ and 1.80 for the $60 \%, 65 \%$ and $70 \%$ chord spoiler locations respectively. 
Results showed lift coefficient gains for angles of attack greater than $18^{\circ}$ degrees or more.

Similarly, the positive gain in lift coefficient resulted in an increase in aerodynamic efficiency. This is shown in figure 1.15 below. As the angle of attack increased towards stall, there was a sharp drop off in aerodynamic efficiency. The spoiler-equipped airfoils experienced a more gradual drop-off in efficiency. Thus the efficiency of the spoiler-equipped airfoils were higher at high angles of attack.

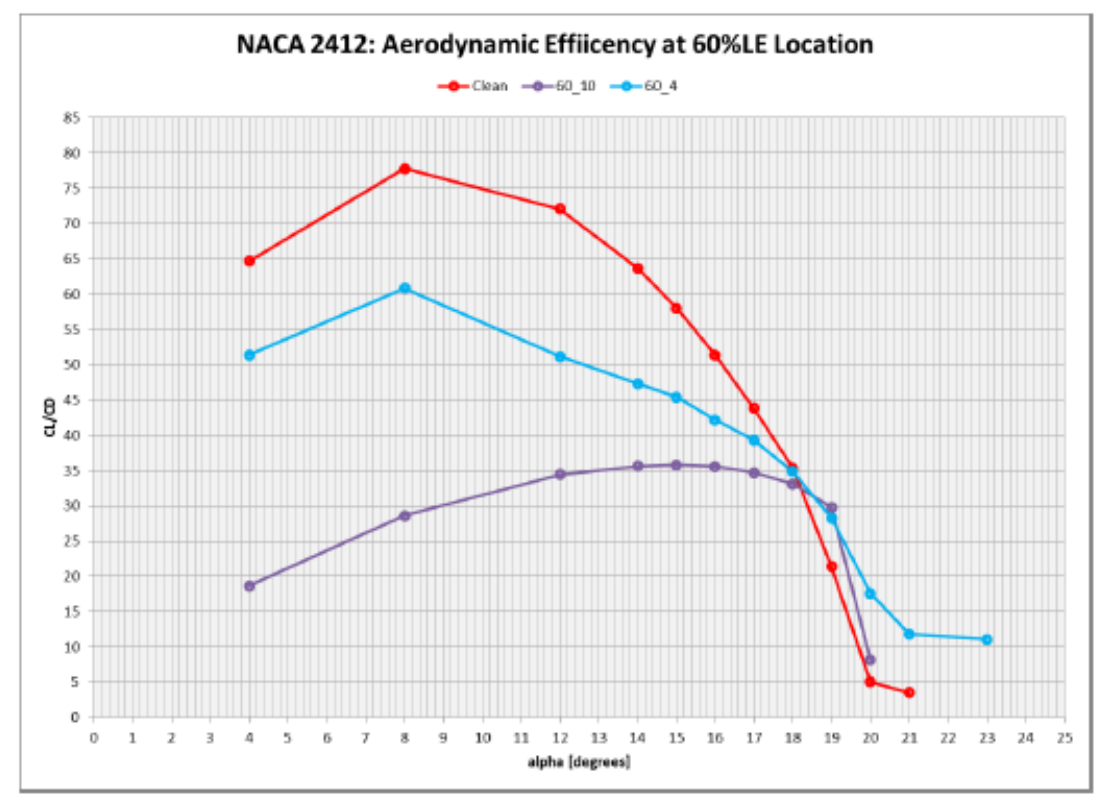

Figure 1.15: Aerodynamic efficiency comparisons of NACA 2412 clean airfoil to spoiler-equipped airfoil at $60 \%$ chord at $\delta=4^{\circ}$ and $10^{\circ}[9]$.

From an analysis of the above articles and theses, it is apparent that the idea of using spoilers as lift-enhancing devices has merit, and justifies further studying. The complexity of the flow has restricted the above studies of spoilers to experimental or computational realms, therefore this study used both techniques to analyze the lift-enhancement capabilities of aircraft spoilers at high angles of attack.

\subsubsection{Experimental Domain}

The objective of this experiment was to capture how the lift, drag and moment about the quarter-chord of an airfoil changed with different spoiler configurations over a range of angles of attack up to stall and into post stall in order to capture the lifting-spoiler phenomenon. Additional data about the pressure distribution and wake profile of the clean and spoiler configurations was also captured. Lift, drag and moment about the quater chord were reported in coefficient form according to the following equations.

$$
C_{l}=\frac{L}{q_{\infty} c}
$$




$$
\begin{gathered}
C_{d}=\frac{D}{q_{\infty} c} \\
C_{m_{\frac{1}{4} c}}=\frac{M_{\frac{1}{4} c}}{q_{\infty} c^{2}}
\end{gathered}
$$

A NACA 2412 airfoil with a sharp trailing edge was selected to determine the impacts of a lifting spoiler's influence on maximum angle of attack. Both the base-line clean configuration airfoil, and spoiler-equipped airfoils of varying spoiler configuration were studied. The airfoil chord length was

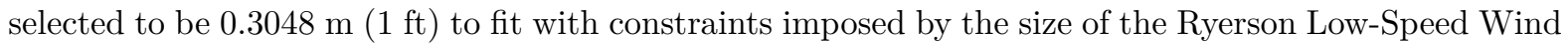
Tunnel. With the addition of the sharp tip to the NACA 2412 airfoil, the chord length was increased to $0.307626 \mathrm{~m}(1.009 \mathrm{ft})$. This combined with the maximum tunnel speed (approximately $47 \mathrm{~m} / \mathrm{s}$ ) limited the Reynold's number to roughly $1.0 \times 10^{6}$ at standard atmospheric conditions. However, the heat generated by the wind tunnel operation significantly reduced the Reynold's number to approximately $7.8 \times 10^{5}$. Figure 1.16 indicates the configuration of the sharp tipped, NACA 2412 airfoil equipped with a spoiler that was tested in this study.

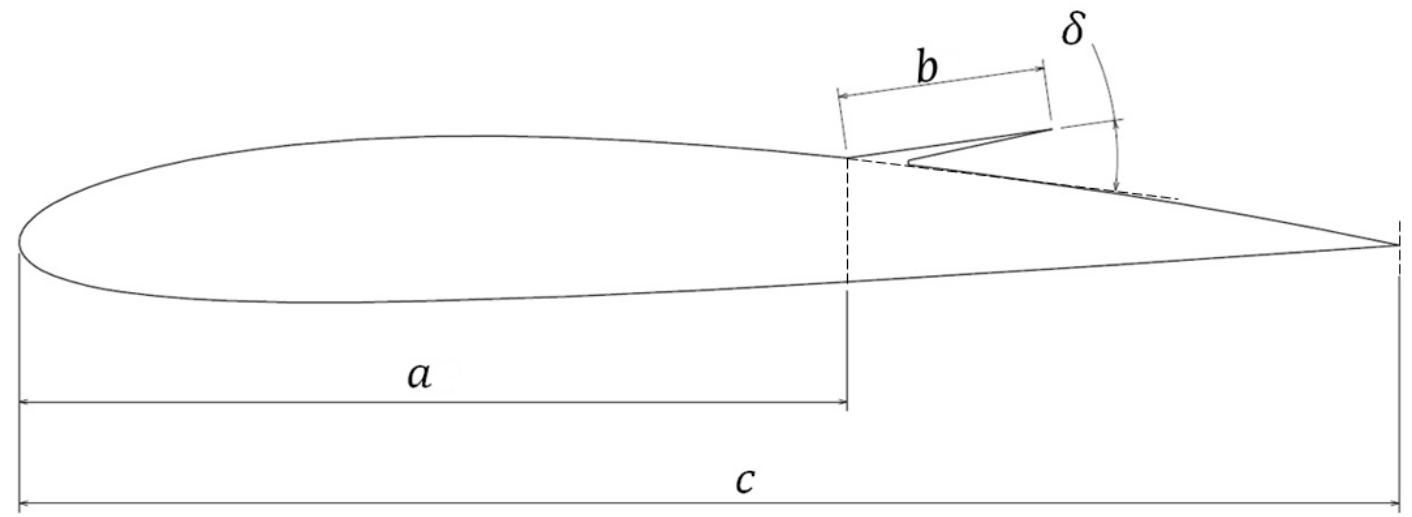

Figure 1.16: Geometry of a sharp tip NACA 2412 airfoil equipped with a spoiler

Where $c$ indicates the airfoil chord (horizontal line from airfoil leading edge to trailing edge). $a$ indicates the spoiler position (distance from the leading edge to the spoiler hinge) in percent chord. $b$ indicates the spoiler length (distance from the spoiler hinge to the spoiler tip) in percent chord. $\delta$ indicates the spoiler deflection angle (angle measured between upstream surface of the spoiler and a straight line tangent with the airfoil curve at the spoiler hinge).

The spoiler configurations that were tested in this study are shown in table 1.1 below: 


\begin{tabular}{c|c|c|c} 
Configuration Number & $a(\% \mathrm{c})$ & $b(\% \mathrm{c})$ & $\delta$ \\
\hline 1 (Clean) & - & - & - \\
2 & 60 & 10 & $8^{\circ}$ \\
3 & 60 & 10 & $15^{\circ}$ \\
4 & 60 & 10 & $25^{\circ}$ \\
5 & 60 & 15 & $8^{\circ}$ \\
6 & 60 & 15 & $15^{\circ}$ \\
7 & 60 & 15 & $25^{\circ}$
\end{tabular}

Table 1.1: Spoiler Configurations Studied 


\section{Chapter 2}

\section{Wind Tunnel Study}

A wind tunnel model was constructed using 3D printed ABS plastic and placed in the Ryerson Low Speed Wind Tunnel to gather all necessary data outlined in Chapter 1.2.3 The model contained a single piece of 12 gauge sheet metal that could be positioned in any configuration shown in Table 1.1. The model contained taps to measure the pressure distribution around the airfoil. A wake rake was located behind the model to capture the wake profile. For a detailed description of the wind tunnel model, it's design, and construction see Appendix 2 


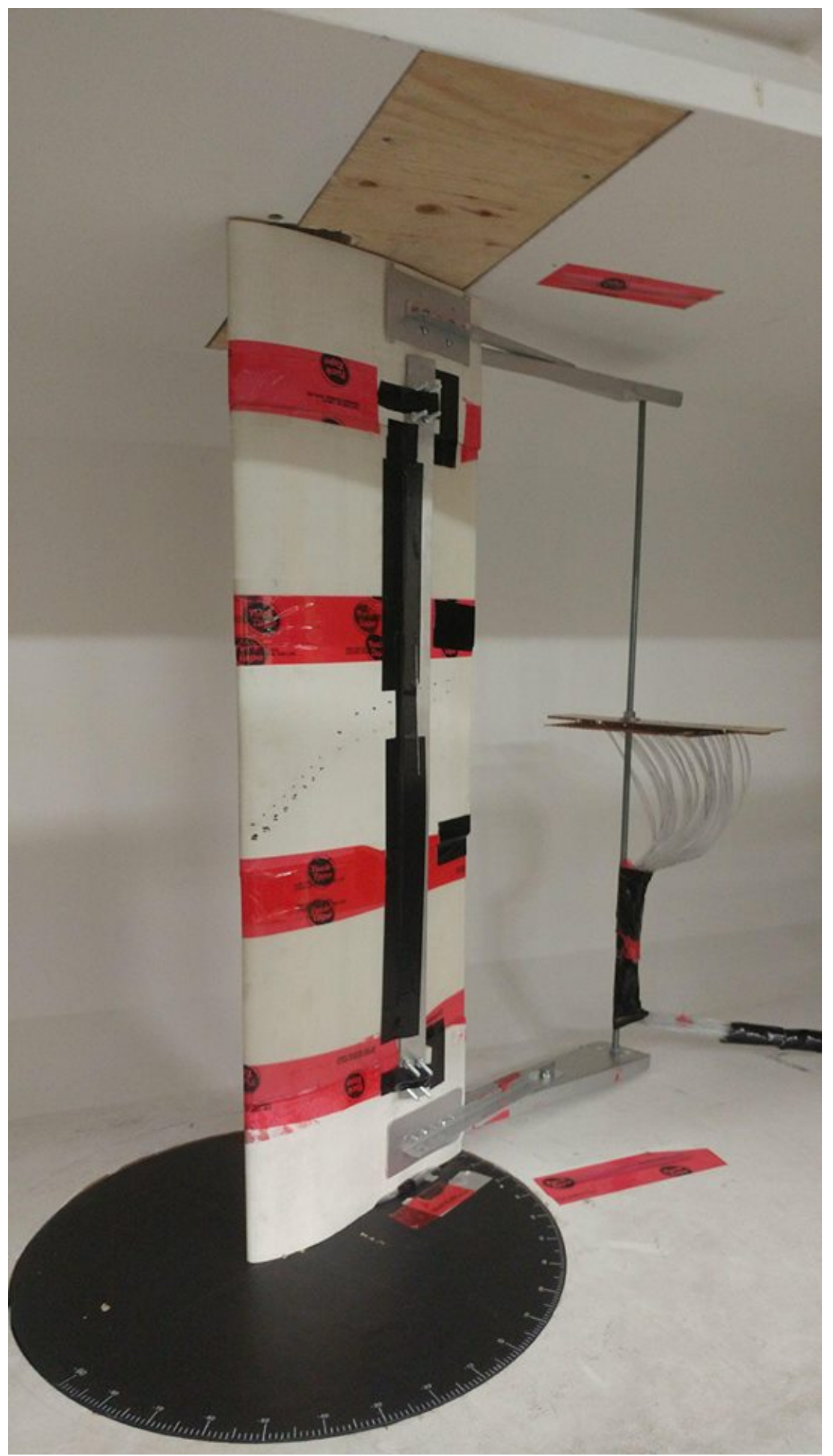

Figure 2.1: Wind tunnel model fully assembled and mounted in the Ryerson Large Wind Tunnel. 


\subsection{Data Acquisition}

The following data was taken from the wind tunnel experiments:

- Pressure $(p)$ from airfoil pressure taps $(\times 31)$

- Total Pressure $\left(p_{t}\right)$ from wake rake pressure taps $(\times 31)$

- Free-stream temperature $\left(T_{\infty}\right)$

- Free-stream dynamic pressure $\left(q_{\infty}\right)$

- Atmospheric pressure $\left(p_{\infty}\right)$

\subsubsection{Airfoil and Wake Pressure Data}

The pressure distribution around the airfoil was measured with two 16-channel DSA3217 ScaniValve units. The pressure taps were connected to the units via standard quick air couplings. The DSA3217 Digital Sensor Array, incorporates 16 temperature compensated piezoresistive pressure transducers with a pneumatic calibration valve, RAM, 16 bit A/D converter, and a microprocessor in a compact self contained module [10]. The microprocessor compensates for temperature changes and performs engineering unit conversion. The microprocessor also controls the actuation of an internal calibration valve to perform on-line zero and multipoint calibrations. This on-line calibration capability virtually eliminates sensor thermal errors with a long term system accuracy of $\pm .05 \%$ FS (5 psi and up) [10].

\subsubsection{Temperature Data}

The free-stream temperature inside the Ryerson Large Wind Tunnel was taken using an EI-1034 Temperature Probe. The EI-1034 is a universal temperature probe that consists of a silicon type temperature sensor mounted in a waterproof 316 stainless steel tube. It uses the LM34CAZ precision silicon temperature sensor with a typical room temperature accuracy of $\pm 0.4^{\circ} \mathrm{F}\left( \pm 1.0^{\circ} \mathrm{F}\right.$ max $)$. Because of the high-level linear voltage output this probe is easy to use and highly accurate in the range of 0 to $230{ }^{\circ} \mathrm{F}$ (temperature range varies with positive supply voltage, negative supply voltage, and LabJack model) [1. 


\begin{tabular}{|c|l|}
\hline & $\pm 0.4^{\circ} \mathrm{F}$ Typical Room Temperature \\
& $\pm 1^{\circ} \mathrm{F}$ Max Room Temperature \\
& $\pm 2^{\circ} \mathrm{F}$ Max to $230^{\circ} \mathrm{F}$ \\
& $\pm 3^{\circ} \mathrm{F} \mathrm{Max}-40^{\circ} \mathrm{F}$ to $0^{\circ} \mathrm{F}$ \\
\hline Sensor device in probe & LM34CAZ \\
\hline Power & +4 to $35 \mathrm{VDC}$ at $100-400 \mu \mathrm{A}$ \\
\hline Output current & $10 \mathrm{~mA}$ \\
\hline Probe Dimensions & $6 \mathrm{in} \times 0.25$ in diameter \\
\hline
\end{tabular}

Table 2.1: Temperature Probe Specifications [1.

\subsubsection{Free-stream Dynamic Pressure Data}

The free-stream dynamic pressure was taken using a PX277-05D5V pressure transducer. The PX274 is a rugged differential preessure transmitter that offers up to 6 field-selectable ranges in one unit. It incorporates a rugged NEMA 4 (IP65) dust-proof and splashproof enclosure with an external mounting bracket [2].

\begin{tabular}{|c|l|}
\hline Excitation & 12 to 35 VDC \\
\hline Output & $\begin{array}{l}0 \text { to } 5 \text { or } \\
0 \text { to } 10 \text { VDC (selectable) }\end{array}$ \\
\hline Accuracy & $\pm 1.0 \% \mathrm{FS}$ \\
\hline Operating Temp & 0 to $175^{\circ} \mathrm{F}$ \\
\hline Compensated Temp Range & 25 to $150^{\circ} \mathrm{F}$ \\
\hline Thermal Effects & $\pm 0.0125 \% \mathrm{FS} /{ }^{\circ} \mathrm{F}$ \\
\hline Proof Pressure & $10 \mathrm{psi}$ \\
\hline
\end{tabular}

Table 2.2: Pressure Transducer Specifications [2].

\subsubsection{Derived Data}

Free-stream air density was derived from the temperature measurement and the atmospheric pressure measurement using the ideal gas law.

$$
\rho_{\infty}=\frac{p_{\infty}}{R T_{\infty}}
$$

Density was then used in conjunction with the dynamic pressure to derive free-stream air speed.

$$
U_{\infty}=\sqrt{\frac{2 q_{\infty}}{\rho_{\infty}}}
$$


Dynamic viscosity was derived from the free-stream temperature data using Sutherland's Law.

$$
\mu_{\infty}=\mu_{r e f}\left(\frac{T_{\infty}}{T_{r e f}}\right)^{3 / 2} \frac{T_{r e f}+S}{T_{\infty}+S}
$$

$T_{\text {ref }}=273.15 \mathrm{~K}$,

$\mu_{\text {ref }}=1.716 \times 10^{-5} \mathrm{~kg} / \mathrm{m} / \mathrm{s}$,

$S=110.4 \mathrm{~K}$

Reynolds number was then calculated.

$$
R e=\frac{\rho_{\infty} U_{\infty} c}{\mu_{\infty}}
$$

Mach number was calculated from the free-stream velocity, free-stream temperature and the definition of speed of sound.

$$
M=\frac{U_{\infty}}{\sqrt{\gamma R T_{\infty}}}
$$

\subsubsection{Data Reduction}

The airfoil pressure tap data taken by the two 16-channel DSA3217 ScaniValve units was non-dimensionalized using the free-stream dynamic pressure taken from the PX277-05D5V pressure transducer.

$$
C_{p}=\frac{p_{t}-p_{\infty}}{q_{\infty}}
$$

DSA3217 ScaniValve units were zeroed to automatically factor out the atmospheric pressure portion of the equation.

The total pressure measurements from the wake rake taken from two 16-channel DSA3217 ScaniValve units and converted into velocities. The velocities were then non-dimensionalized with the free-stream speed.

$$
\frac{u}{U_{\infty}}=\sqrt{\frac{2\left(p-p_{\infty}\right.}{\rho_{\infty}}} / U_{\infty}
$$

\subsubsection{Lift and Moment Coefficient from Pressure Tap Integration}

Pressure taps were installed on the wind tunnel model surface to measure the pressure distribution around the airfoil. The pressure distribution was integrated around the surface of the airfoil $(s)$ to compute lift.

$$
L=\int_{\text {surface }}\left(p_{\infty}-p\right) \sin \theta d s
$$


Where $\theta$ is the angle normal to the airfoil surface relative to the horizontal plane. (see Figure 2.3). Moment about the quarter chord was calculated in a similar fashion.

$$
M_{\frac{1}{4} c}=\int_{\text {surface }}\left(p_{\infty}-p\right)\left(x-\frac{c}{4}\right) \sin \theta d s
$$

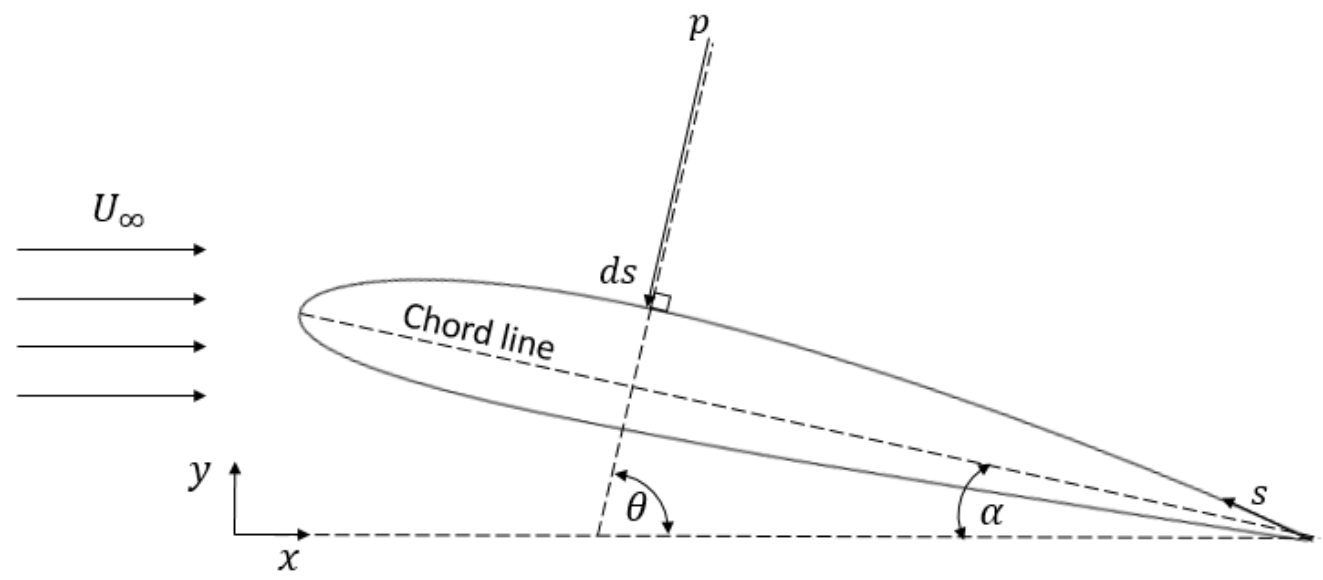

Figure 2.2: Airfoil Geometry in Regards to Pressure Tap Integration.

Or in non-dimensional form using chord as a reference length:

$$
\begin{gathered}
C_{l}=\frac{1}{c} \int_{\text {surface }} C_{p} \sin (\theta) d s \\
C_{m_{\frac{1}{4} c}}=\frac{1}{c^{2}} \int_{\text {surface }} C_{p}\left(x-\frac{c}{4}\right) \sin (\theta) d s
\end{gathered}
$$

Using a trapezoidal numerical integration scheme where each value of $i$ corresponds to a pressure tap number:

$$
\begin{gathered}
C_{l}=\frac{1}{c} \sum_{i=1}^{31} \frac{\left(C_{p_{i+1}}+C_{p_{i}}\right)}{2} \sin \left(\frac{\theta_{i+1}+\theta_{i}}{2}\right) \Delta s \\
C_{m_{\frac{1}{4} c}}=\frac{1}{c^{2}} \sum_{i=1}^{31} \frac{\left(C_{p_{i+1}}+C_{p_{i}}\right)}{2}\left(\frac{x_{i+1}+x_{i}}{2}-\frac{c}{4}\right) \sin \left(\frac{\theta_{i+1}+\theta_{i}}{2}\right) \Delta s
\end{gathered}
$$




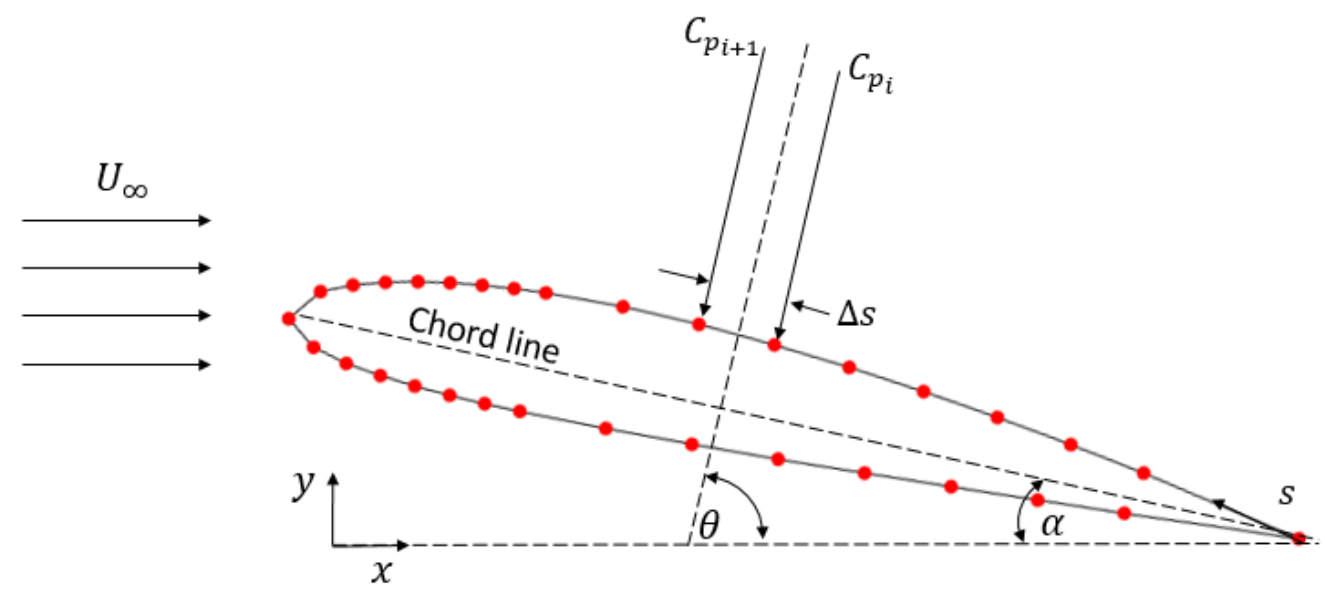

Figure 2.3: Airfoil Pressure Tap Integration Discretized into Finite Points.

The taps were more closely spaced near the leading edge in order to capture the large pressure changes expected in this region. It was desired to measure the pressure at the trailing edge with a $32^{\text {nd }}$ pressure tap, however the sharp tipped airfoil shape prevented this. Instead, the pressure coefficient at the trailing edge was set to zero, allowing for an integration over the entire airfoil surface. Trial runs using data extracted from the computational study indicated that doing so improved the accuracy of the lift calculation (see Appendix 3).

Equation 2.12 and equation 2.13 are more accurate with a greater number of pressure taps. Since only 31 pressure taps could be installed due to space limitations inside the model, a certain degree of error is present in the lift and moment coefficient calculation. This error was reduced by fitting two spline interpolation curves to the pressure tap data; one for the upper surface taps, and one for the lower surface. The error and justification for the spline interpolation is analyzed in Appendix 3.

\subsubsection{Drag Coefficient from Wake Integration}

The profile drag of the airfoil was determined by the integrating wake method. A wake rake measures static pressures and the decrease in total pressure within the wake and compare those values with the free-stream total pressure. The drag force acting on a body moving through a fluid is related to the pressure deficit.

Figure 2.4 depicts the velocity distribution before and aft of the airfoil sufficiently far downstream such that the static pressure $(p)$ is equal to the free-stream static pressure $\left(p_{\infty}\right)$. In this case, the profile drag was determined by the momentum deficit across the control volume.

$$
D=\int_{\text {wake }} \rho u\left(u-U_{\infty}\right) d y
$$




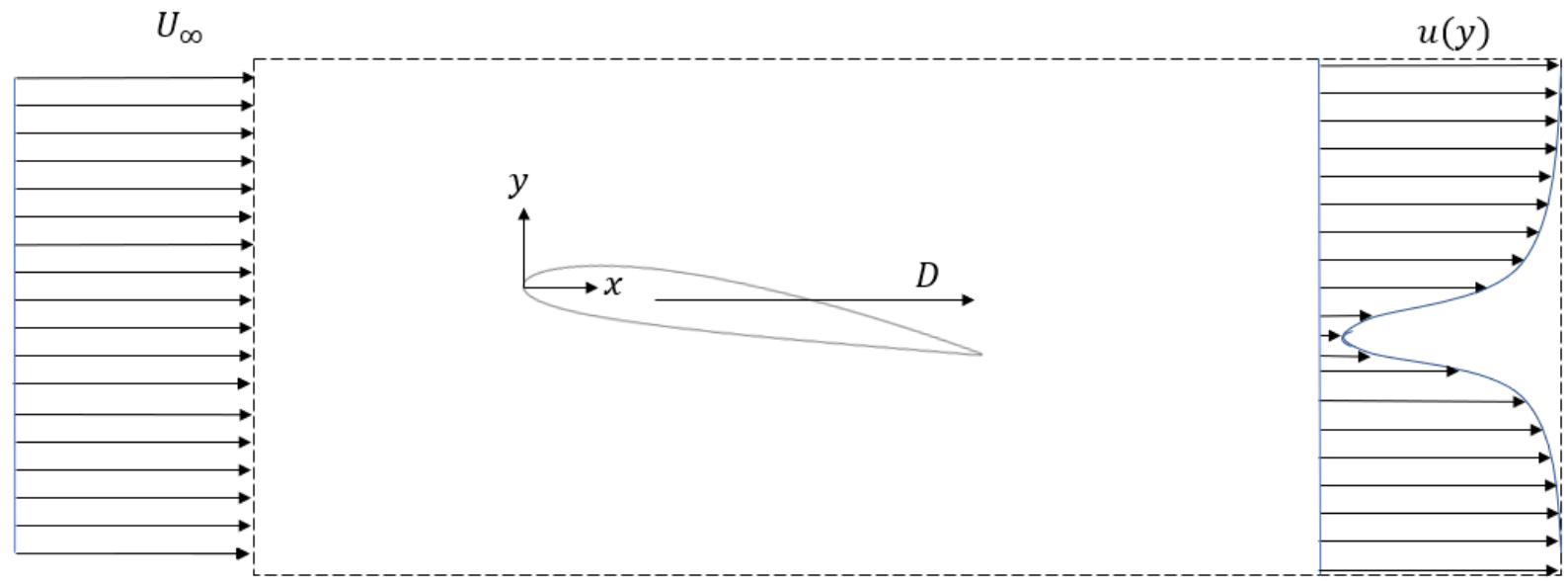

Figure 2.4: Application of the momentum equation to calculate airfoil drag from wake velocity profile

A series of vertically-stacked pressure tubes (a wake survey) was positioned at a distance behind the airfoil trailing edge to capture total pressure $\left(p_{t}\right)$ distribution of the wake. The section drag coefficient was determined by converting the total pressure and measured static pressure to velocity via Bernoulli's equation.

$$
C_{d}=\frac{1}{c q_{\infty}} \int_{\text {wake }}\left(p_{\infty}-p_{t}\right) d y
$$

Or in terms of a trapezoidal numerical integration scheme with a 32 -sample point wake rake:

$$
C_{d}=\frac{1}{q_{\infty} c} \sum_{i=1}^{32}\left[p_{\infty}-\frac{1}{2}\left(p_{t_{i+1}}+p_{t_{i}}\right)\right] \Delta y
$$

However, this equation is only valid if the static pressure has fully recovered, which occurs far from the airfoil trailing edge. Since the wake must be placed at a distance close to the airfoil trailing edge due to the geometry of the Ryerson Low-Speed Wind Tunnel, expressions taking into account the static pressure drop were used. Silverstein and Katzoff derived an empirical equation for the change in the static pressure [11. Plaisance extended the equation to include the effects of changing Reynold's number [12.

$$
\frac{p-p_{\infty}}{q_{\infty}}=\left(-1.33 \times 10^{-6} R e+4.36\right) \frac{t / c}{(0.77+3.1 \xi)^{2}}
$$

Where $t$ is the airfoil thickness and $\xi$ is the distance between the airfoil trailing edge and the wake rake.

Equation 2.16 is more accurate with more pressure tubes to better capture the wake. Since the number of pressure tubes was limited to 32 , a certain degree of error is present in the calculation. To 
mitigate this error, a spline curve was fitted to the data from the wake tubes. For an analysis of the error present in the drag coefficient calculation due to the number of pressure tubes, see Appendix 4 .

\subsubsection{Angle of Attack}

The angle of attack was measured using the angle markings on the turntable in the wind tunnel. These markings increment by $1^{\circ}$. An image of the turntable is shown in figure 2.5 below.

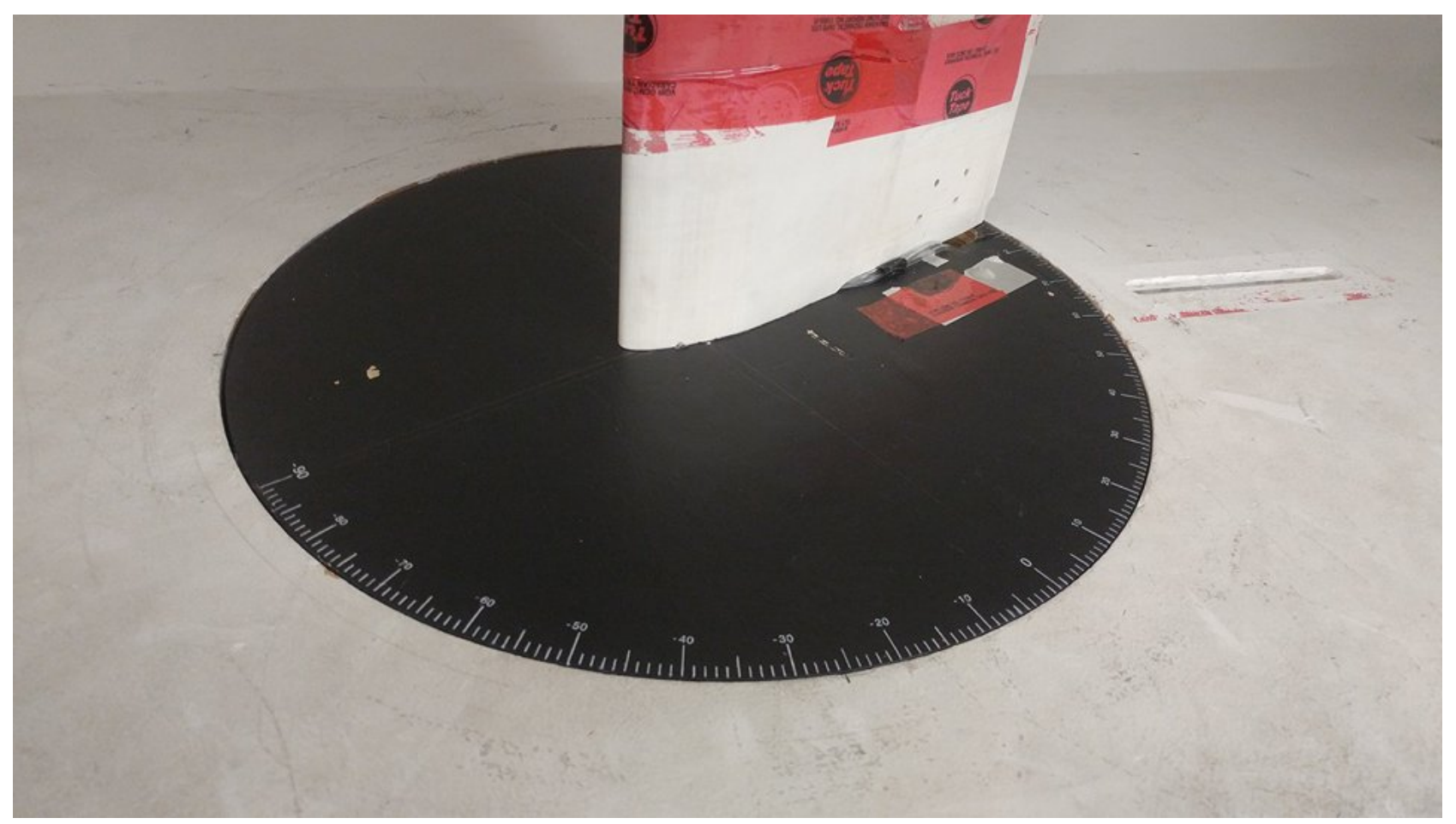

Figure 2.5: Wind tunnel turntable

The airfoil was mounted on the turntable in such a way that the airfoil chord line would align with the free-stream direction, therefore the turntable angle would equal the angle of attack $\left(\alpha_{\text {turntable }}=\alpha\right)$. However, in the absence of precision mounting tools, this was not possible within desired accuracy levels. To correct for the difference between $\alpha_{\text {turntable }}$ and $\alpha$, the $\alpha_{\text {turntable }}$ value was offset such that the clean $\alpha_{L=0}$ from the wind tunnel matched the clean $\alpha_{L=0}$ from the CFD solution. Using this procedure, the model was found to be mounted at $\alpha=-4.3^{\circ}$. Therefore

$$
\alpha=\alpha_{\text {turntable }}-4.3^{\circ}
$$




\subsubsection{Wind Tunnel Correction Factors}

The influence of the wind tunnel walls creates conditions that differ from a true free-stream air flow. Corrections were made for the impacts of the wind tunnel walls on a two-dimensional airfoil study described in Low-Speed Wind Tunnel Testing by Barlow et al [13. For a full detailed description of the correction equations used, see Appendix 5 


\subsubsection{Data Flow}

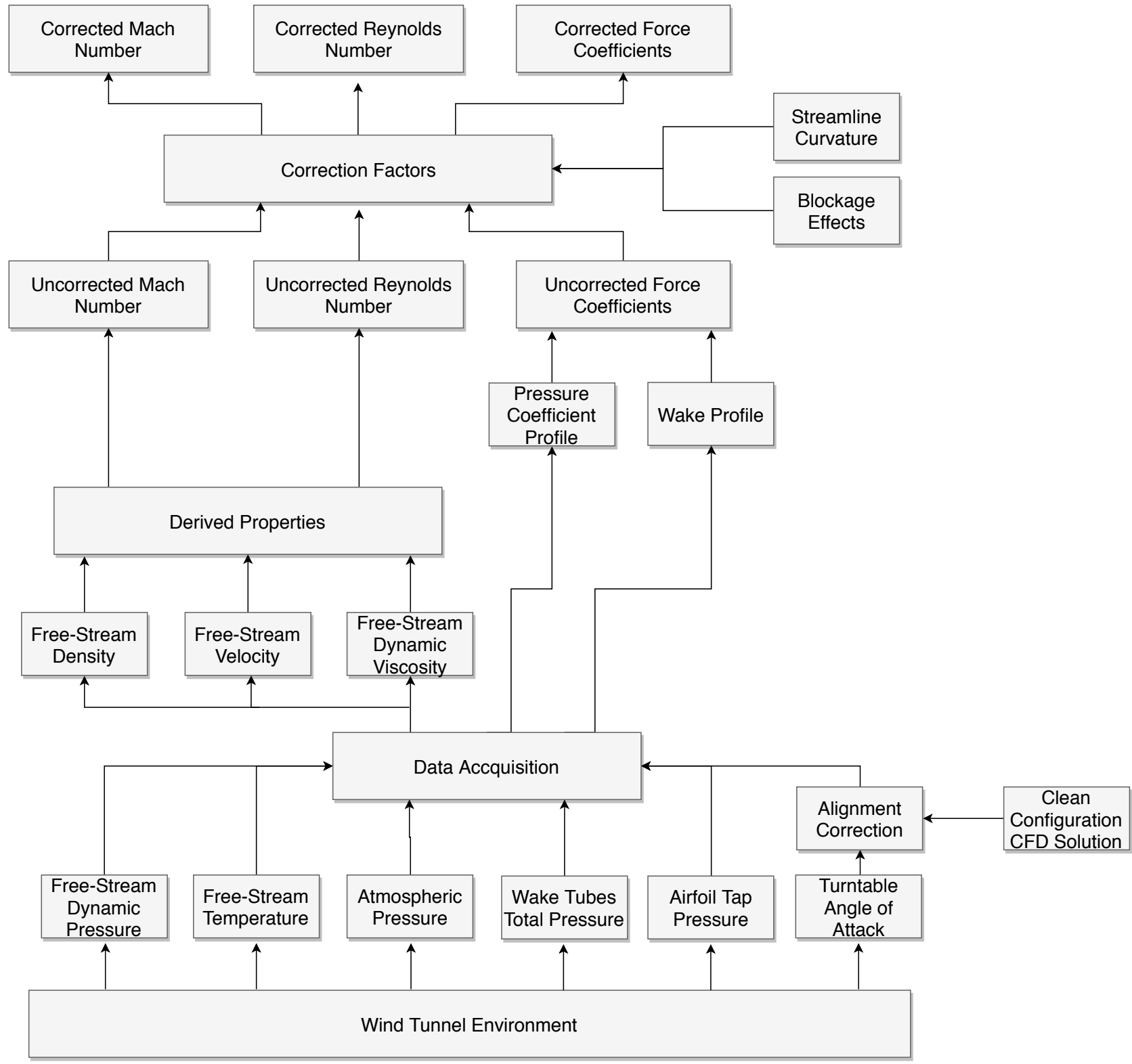

Figure 2.6: Wind tunnel study data flow. 


\subsection{Results}

The $C_{l}-\alpha$ curve for each of the configurations is shown in Figure 2.7 below. The $C_{l_{\max }}$ and $\alpha_{\text {stall }}$ is recorded in Table 2.3 Figure 2.7 indicates that the spoiler acts to effectively de-camber the airfoil. The higher the spoiler deflection angle, the greater the de-cambering effect. The spoiler pushed the lift-curve further to the right, thereby increasing the stall angle of the airfoil. Table 2.3 indicates that spoiler deflection increased the maximum lift coefficient $0.3 \%$ to $2.5 \%$ depending on the specific configuration. In all cases, a spoiler deflection angle of $25^{\circ}$ caused a decrease in the maximum lift coefficient. The b $=10$ spoiler length resulted in greater $C_{l_{\max }}$ gains than the longer $\mathrm{b}=15$ spoiler length. The spoiler configurations tend to flatten out the peak of the $C_{l}-\alpha$ curve, resulting gentler stall onset compared with the clean configuration.

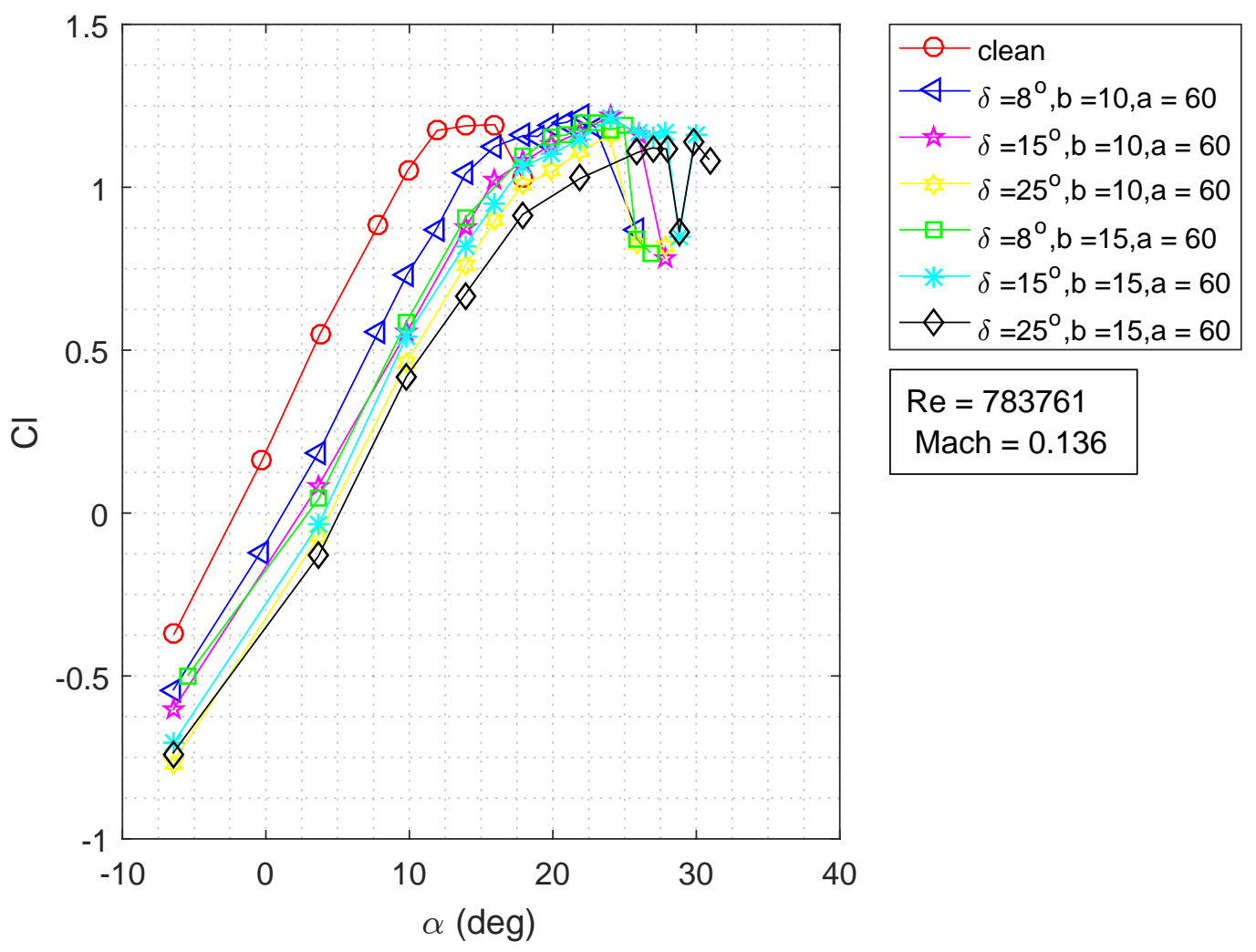

Figure 2.7: Wind tunnel $C_{l}-\alpha$ comparison between clean and spoiler configurations. 


\begin{tabular}{c|c|c|c|c} 
Configuration & $C_{l}$ Max & $C_{l}$ Max \% Difference & Stall $\alpha$ & Stall $\alpha \%$ Difference \\
\hline Clean & 1.192 & - & 15.960 & - \\
$\delta=8^{\circ}, \mathrm{b}=10, \mathrm{a}=60$ & 1.222 & 2.497 & 21.970 & 37.658 \\
$\delta=15^{\circ}, \mathrm{b}=10, \mathrm{a}=60$ & 1.217 & 2.110 & 23.969 & 50.188 \\
$\delta=25^{\circ}, \mathrm{b}=10, \mathrm{a}=60$ & 1.159 & -2.783 & 23.957 & 50.108 \\
$\delta=8^{\circ}, \mathrm{b}=15, \mathrm{a}=60$ & 1.197 & 0.393 & 21.963 & 37.618 \\
$\delta=15^{\circ}, \mathrm{b}=15, \mathrm{a}=60$ & 1.213 & 1.760 & 23.969 & 50.185 \\
$\delta=25^{\circ}, \mathrm{b}=15, \mathrm{a}=60$ & 1.139 & -4.475 & 29.931 & 87.544
\end{tabular}

Table 2.3: Wind tunnel $C_{l_{\max }}$ and $\alpha_{\text {stall }}$ change with spoiler configuration

The $C_{d}-\alpha$ curve for each of the configurations is shown in Figure 2.8 below. The wake rake was incapable of accurately predicting drag once the airfoil had stalled. This is not to do with the theoretical basis, but with the fact that stalled conditions commonly produce a re-circulating region and a wake that does not return to sufficient parallel flow within the tunnel test section for the wake survey assumptions to be valid [13. Therefore the stall drag coefficients are not present in Figure 2.8. An increase in spoiler deflection angle corresponded with an increase in drag coefficient. The drag coefficient of the clean configuration airfoil increased with angle of attack at a greater rate than the spoiler equipped configurations. In all configurations, once the airfoil entered pre-stall, the drag coefficient increased rapidly. In the clean configuration case, the airfoil experienced pre-stall at a lower angle of attack than the spoiler configurations. Thus at high angles of attack the spoiler equipped airfoils produced less drag than the clean configuration airfoil. 


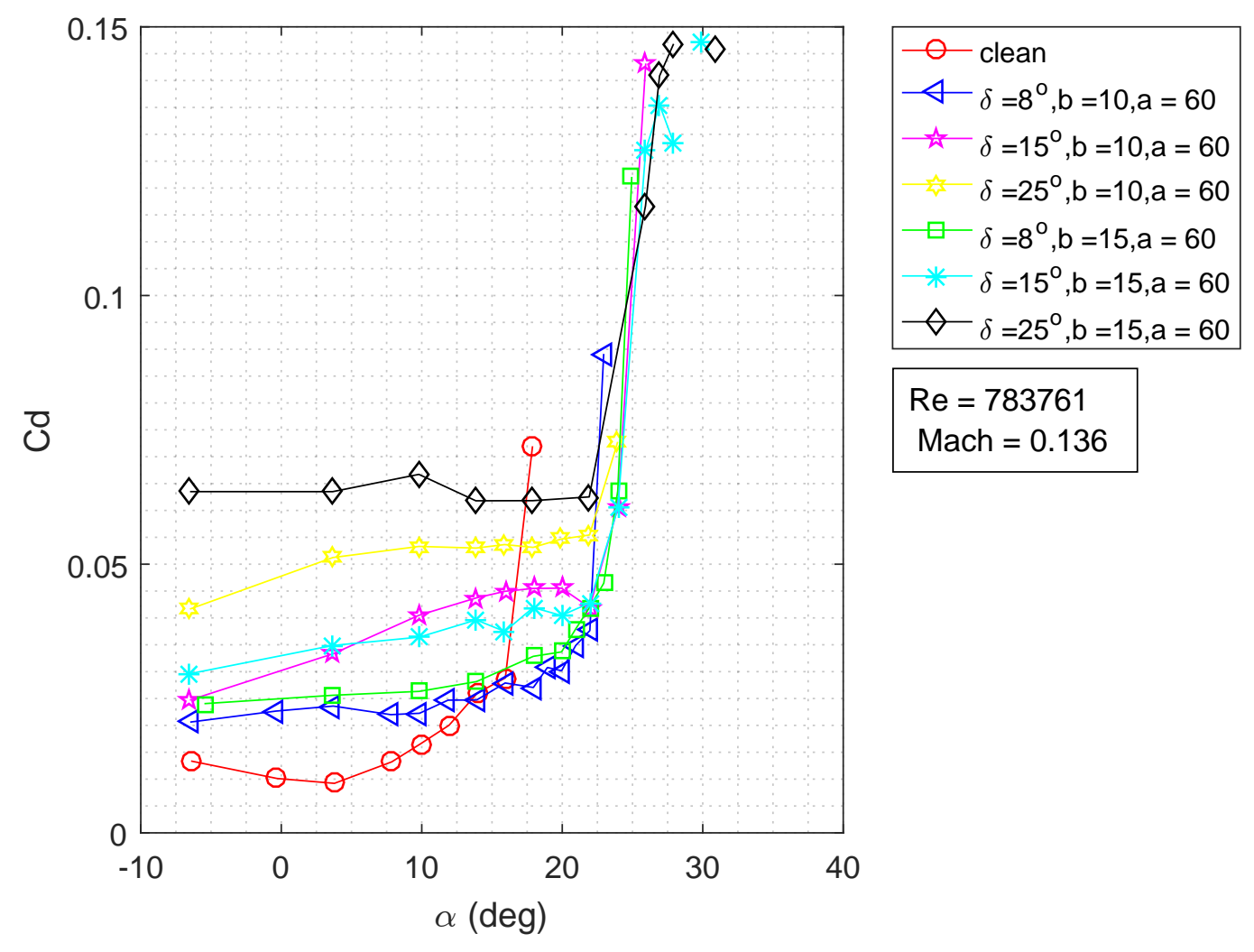

Figure 2.8: Wind tunnel $C_{d}-\alpha$ comparison between clean and spoiler configurations.

The combined effects of a reduced $C_{d}$ and a higher $C_{l}$ resulted in a higher aerodynamic efficiency at high angles of attack (defined as $C_{l} / C_{d}$ ). This is evident in Figure 2.9 The clean configuration was more efficient at low and mid-range angles of attack, however at higher angles of attack the spoiler-equipped airfoil became more efficient. The $\delta=8^{\circ}, \mathrm{b}=10, \mathrm{a}=60$ spoiler configuration was the most efficient in this range. 


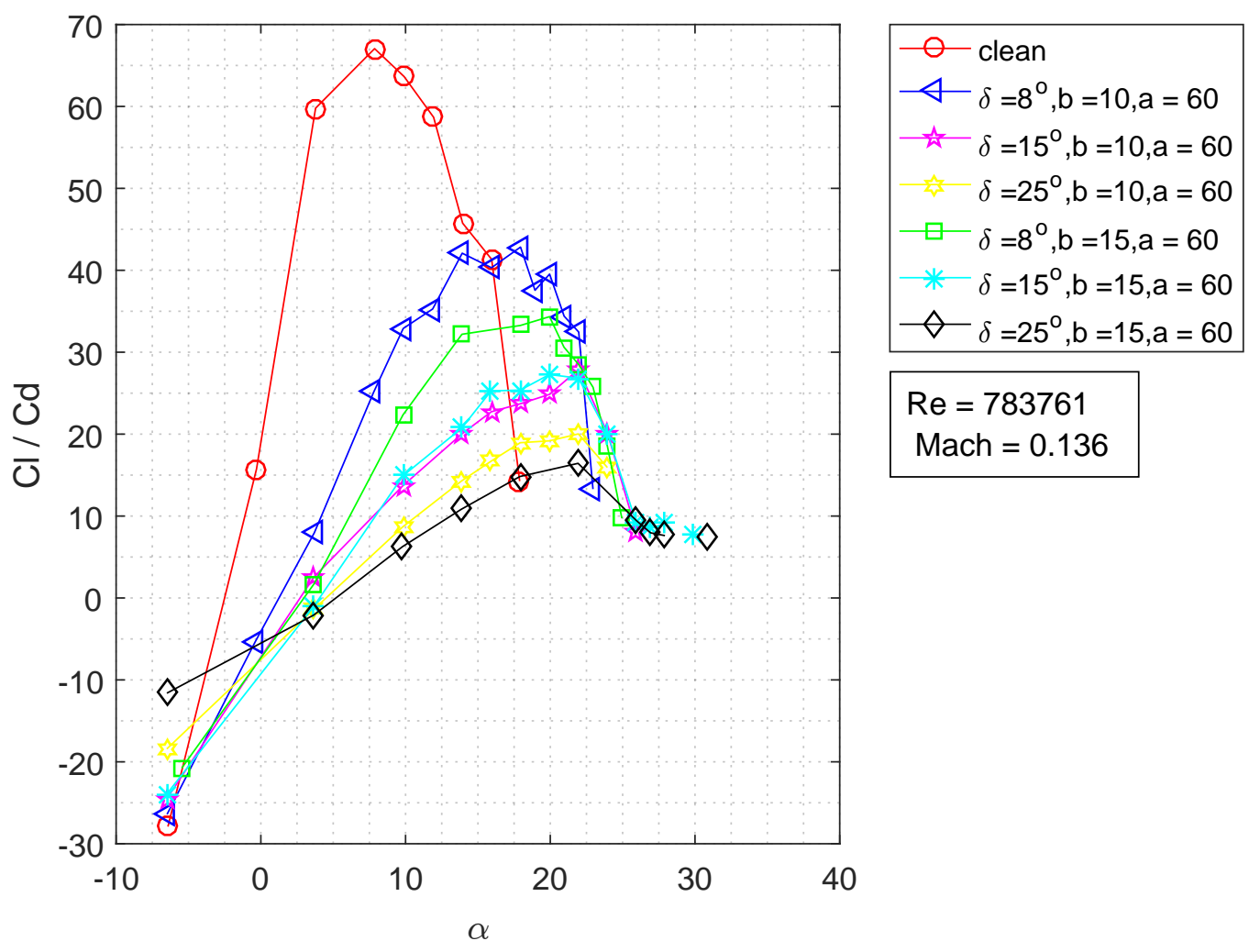

Figure 2.9: Wind tunnel $C_{l} / C_{d}-\alpha$ comparison between clean and spoiler configurations. 


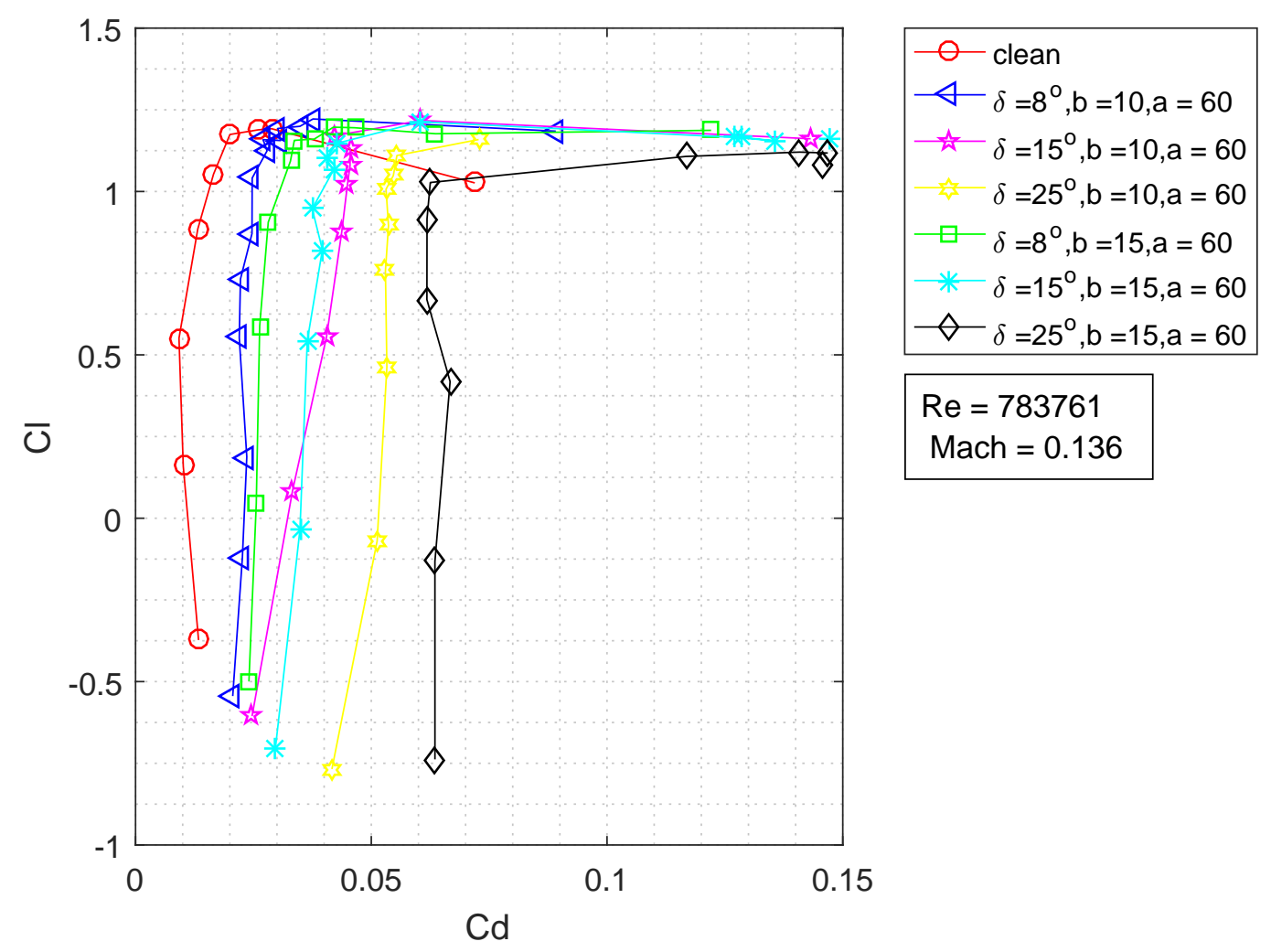

Figure 2.10: Wind tunnel $C_{l}$ vs $C_{d}$ comparison between clean and spoiler configurations.

The $C_{m_{\frac{1}{4} c}}-\alpha$ curve for each of the configurations is shown in Figure 2.11 The $C_{m_{\frac{1}{4}} c}$ tends to drop sharply once the airfoil stalls. The spoiler increases $\alpha_{\text {stall }}$, thus delaying the onset of moment coefficient drop off, and delaying the potential control issues associated with a sudden change in moment coefficient. The $C_{m_{\frac{1}{4} c}}-\alpha$ curve can be used to identify possible control issues if the lifting spoiler effect is to be implemented, since any increase to the moment coefficient must be trimmed by the aircraft's tail. Therefore any large jumps in moment coefficient between the clean configuration and a given spoiler configuration could prevent the lifting spoiler from being used. However an analysis on the impact of a changing moment coefficient on aircraft control and stability is beyond the scope of this study. 


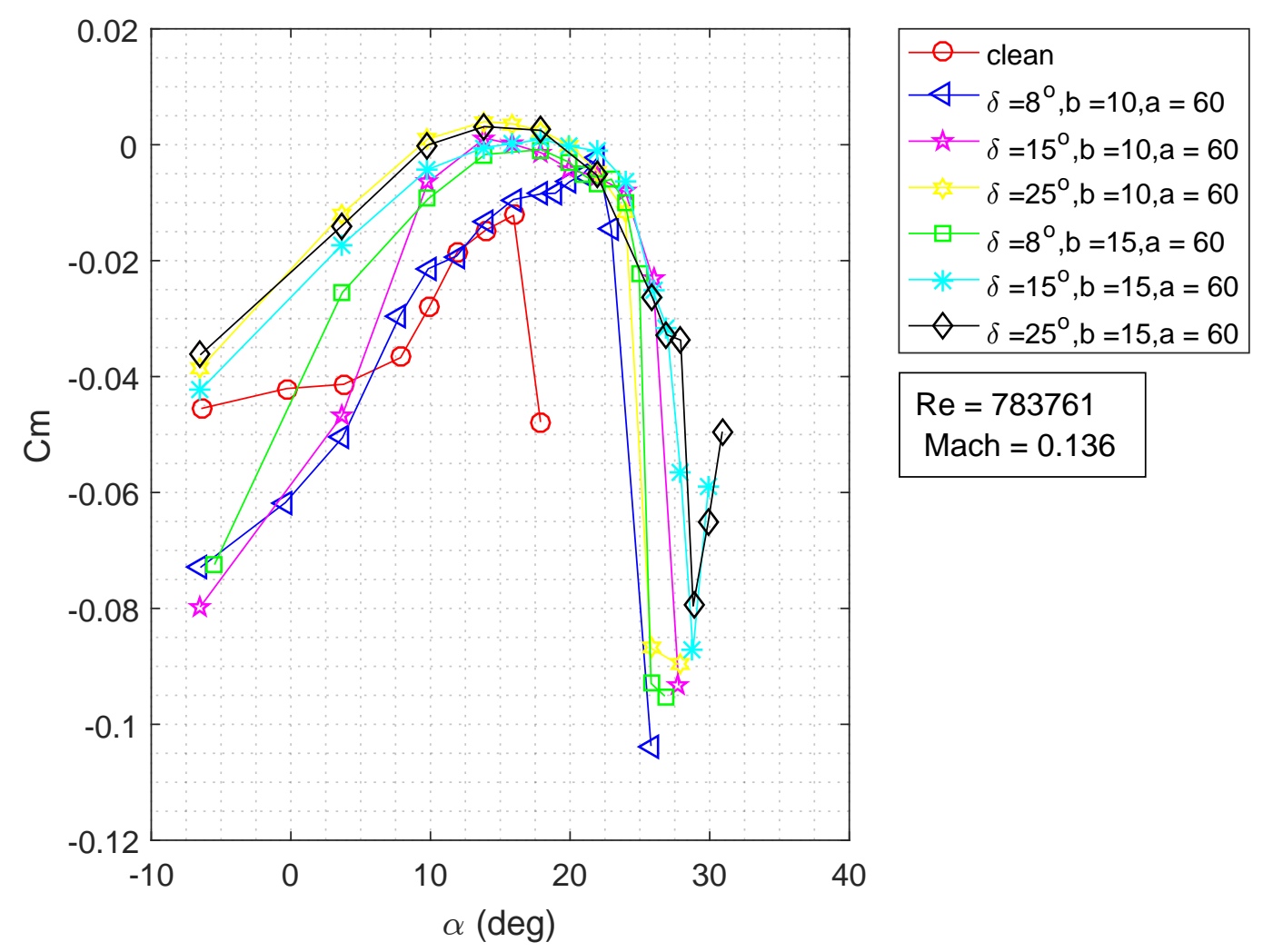

Figure 2.11: Wind tunnel $C_{m_{\frac{1}{4}} c}-\alpha$ comparison between clean and spoiler configurations.

Figure 6.75 and figure 6.78 compare the differences in pressure distribution between the clean configuration and spoiler configuration airfoils at low and high angles of attack. These figures are representative of other spoiler configurations at additional $\alpha$. For a complete list of pressure distributions from each configuration and $\alpha$, see Appendix 6. The figures contain the non-dimensionalized pressure data gathered from the airfoil pressure taps. Two spline interpolations were fitted to each set of pressure data, one for the upper surface, and a second for the lower surface. For full details on this procedure, see Appendix 3

Figure 6.75 shows the pressure distribution of the clean configuration airfoil, and the $\delta=8^{\circ}, b=$ $10, a=60$ spoiler configuration at $\alpha \approx 3.7^{\circ}$ before streamline curvature effects have been accounted for. Due to the correction for streamline curvature, these two configurations are at slightly different $\alpha$, however they are sufficiently similar to be compared with each other. At $\alpha \approx 3.7^{\circ}$, the spoiler caused the pressure to be higher on the upper surface upstream of the spoiler. The spoiler configuration generated a lower pressure on the lower surface than the clean configuration. These two factors acted to reduce the overall lift generated by the airfoil. The spoiler created a large discontinuity in pressure on the upper surface where the spoiler is located $(x / c=0.6)$. 
Figure 6.78 shows the pressure distribution of the same airfoil configurations at $\alpha \approx 17.7^{\circ}$. The spoiler created a pressure recovery step at the spoiler location, resulting in increased suction upstream of the spoiler, and an increase in pressure downstream of the spoiler. The increased suction upstream of the spoiler is greater than the increase in pressure downstream, resulting in a net lift gain generated by the airfoil. The lower surface also experienced slightly higher pressure, which also slightly contributed to the lift increase.

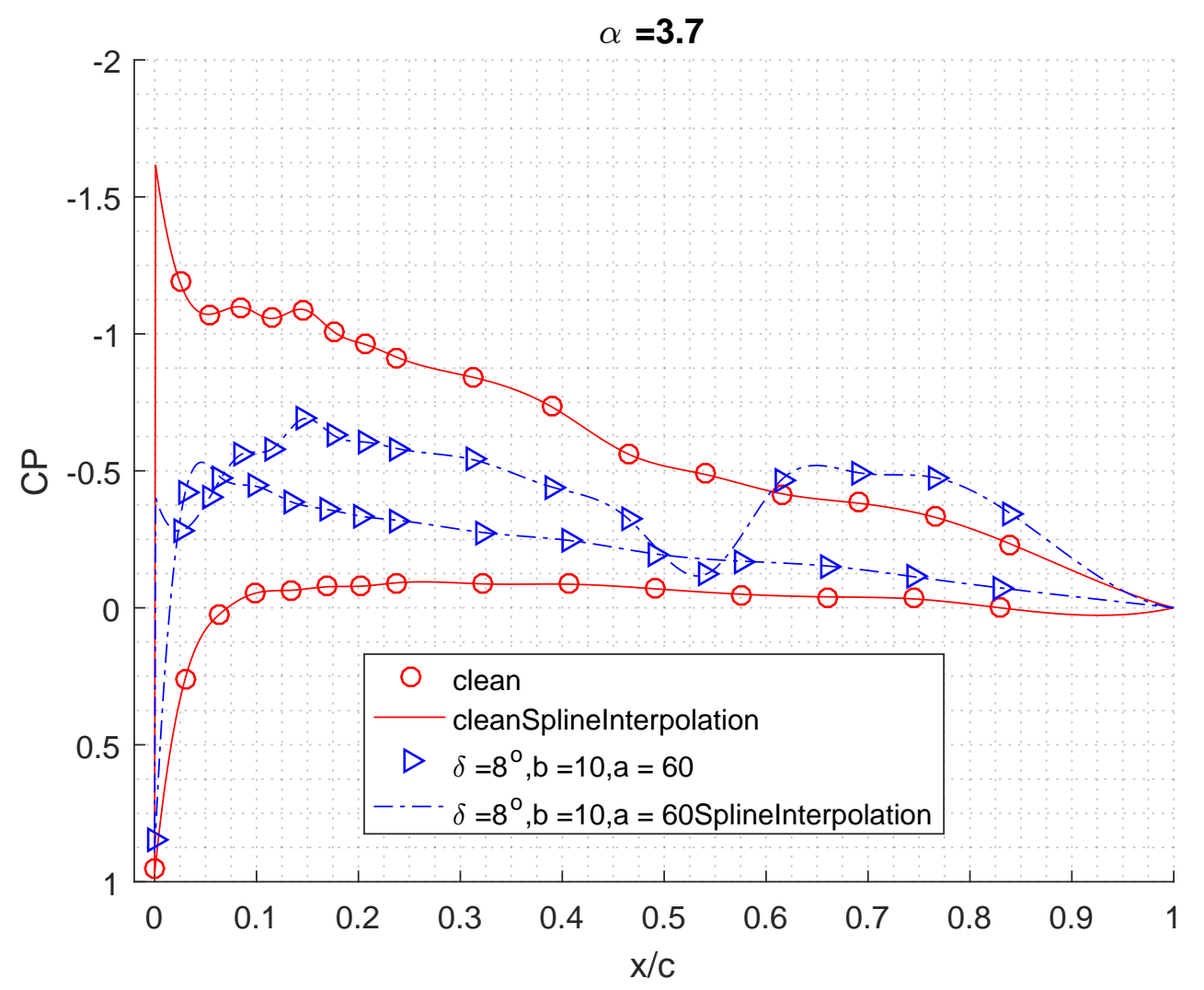

Figure 2.12: $C_{p}$ plot comparison of clean and spoiler equipped airfoil at $\alpha \approx 3.7^{\circ}$ 


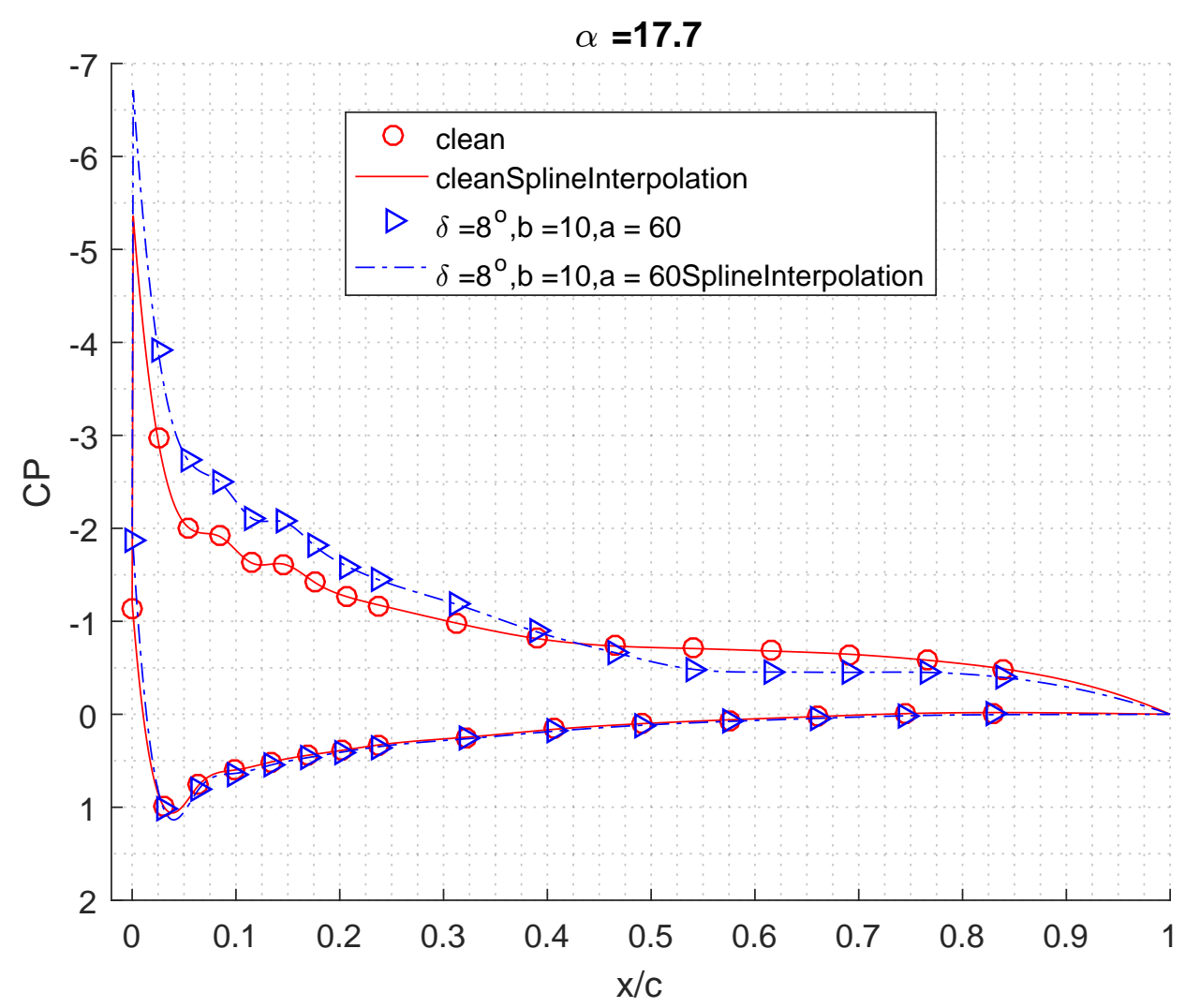

Figure 2.13: $C_{p}$ plot comparison of clean and spoiler equipped airfoil at $\alpha \approx 17.7^{\circ}$

Figure 7.75 and figure 7.64 depict the wake profile behind the airfoil. These wake plots are representative of the typical wakes produced at low angles of attack (figure 7.75) and high angles of attack 7.64. For a complete list of wake profiles from each configuration and $\alpha$, see Appendix 7 Each data point represents a measurement taken by the wake rake. The y-axis indicates the y-coordinate of the wake measurement, and the $\mathrm{x}$-axis indicates the wake velocity non-dimensionalized with the free stream velocity. The data was fitted with a spline interpolation. For full details on this procedure, see Appendix 4

Figure 7.75 compares the wakes of the clean configuration and $\delta=8^{\circ}, b=10, a=60$ spoiler configuration at $\alpha \approx 3.7^{\circ}$. The spoiler airfoil created a thicker wake than the clean configuration, thus resulting in the higher $C_{d}$ values represented in figure 2.8. At higher angles of attack such as $\alpha \approx 15.7^{\circ}$ shown in figure 7.64 the clean configuration airfoil generates a deeper wake than the spoiler airfoil, thus the spoiler airfoil creates less drag at this angle of attack. 


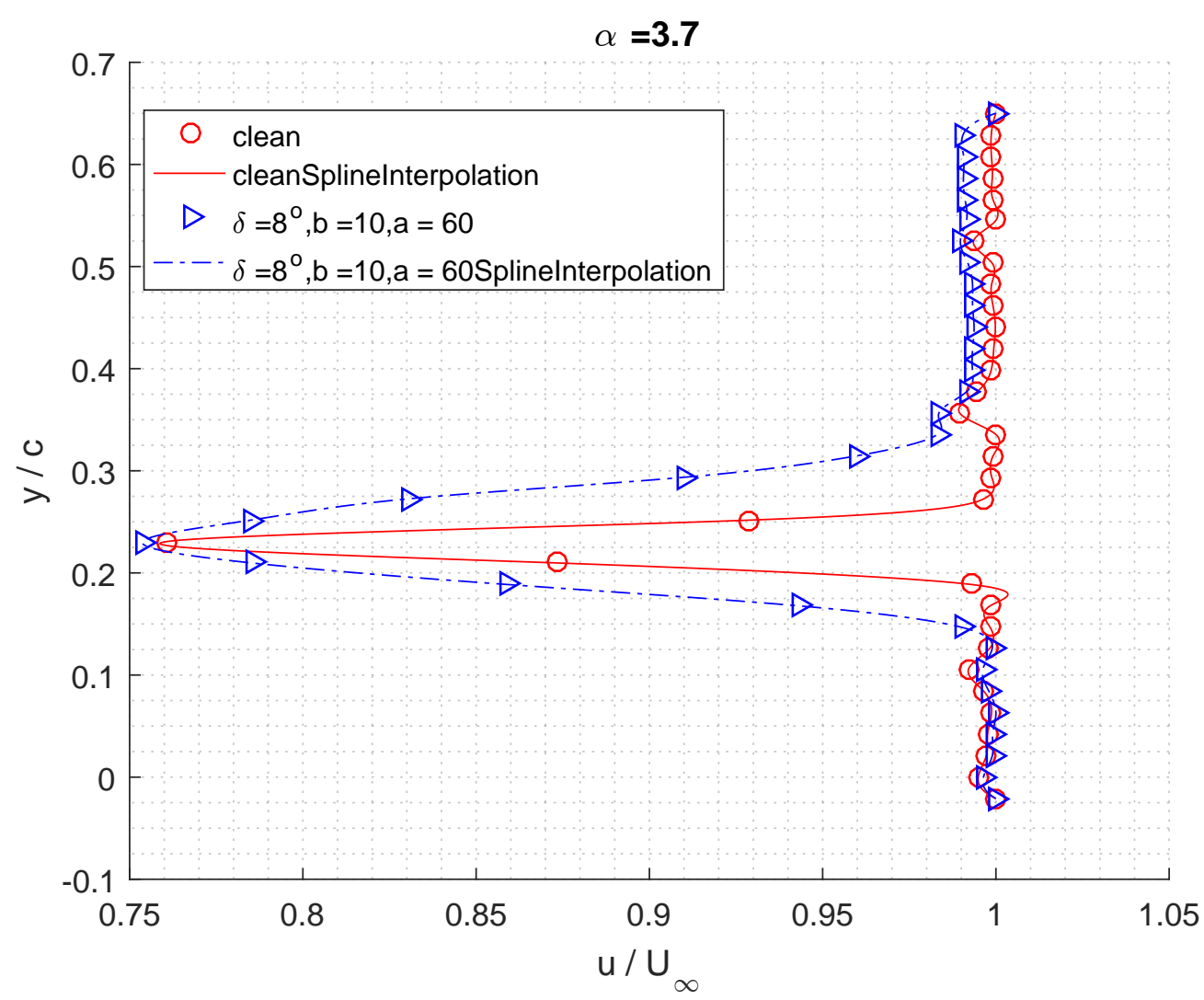

Figure 2.14: Wake plot comparison of clean and spoiler equipped airfoil at $\alpha \approx 3.7^{\circ}$ 


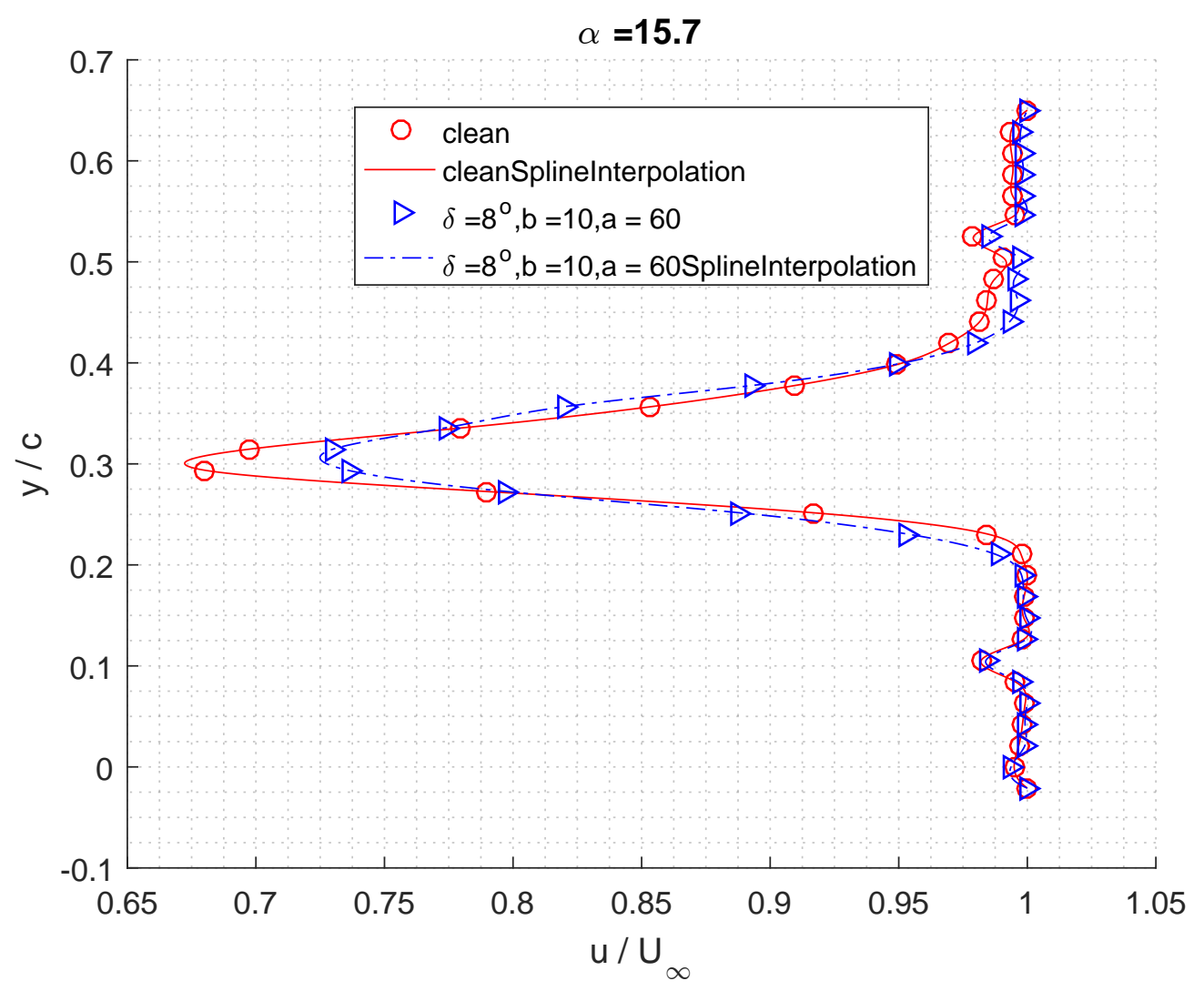

Figure 2.15: Wake plot comparison of clean and spoiler equipped airfoil at $\alpha \approx 15.7^{\circ}$

\subsection{Comments on Repeatability}

During the setup process, the clean configuration cases were run multiple times to test the equipment. During this time, the experimental setup produced consistent pressure distributions and wake profiles for a given angle of attack. Therefore the author is confident in the replicability of these results. 


\section{Chapter 3}

\section{Computational Study}

\subsection{Computational Setup}

The CFD solution was set to replicate the conditions of the Ryerson Low-Speed Wind tunnel as best as possible. For this reason, the airfoil geometry was set to match the geometry of the wind tunnel model as closely as possible (sharp tip NACA 2412 airfoil, chord $=307.626 \mathrm{~mm}$ ) A free-stream velocity of 47 $\mathrm{m} / \mathrm{s}$ was selected as this was the target velocity of the wind tunnel experiments. At sea-level conditions, this results in a Reynolds number of $1,017,697$. The wind tunnel wall effects were not studied in the computational model since they were corrected for in the wind tunnel experiment.

The computational process was completed using the ANSYS 15.0 suite of programs. The airfoil geometry and fluid domain was created using CATIA V5 and imported into ANSYS Design modeler. ICEM was used to generate the mesh. ANSYS Fluent (Version 15.0) solver was selected due to it's power, long history of wide-spread use, and it's availability. Fluent includes well-validated physical modeling capabilities to deliver accurate results across a wide range of CFD applications. A transient model was used rather than a steady-state model to accurately capture the shedding vorticies associated with aircraft spoilers, and to more accurately capture airfoil behavior post-stall.

The transition SST turbulence model was selected due to it's use in Pabla's computational study on spoilers [9]. Pabla performed an analysis of the Spalart-Allmaras model, $k-\omega$ transitional model, $k-\epsilon$ model, and the transition SST model. The conclusions of this analysis was that the transition SST model generated the most accurate predictions of a flow-field heavily influenced by viscous flow-separation. All turbulence models showed comparable lift and drag coefficient results at angles of attack between $4^{\circ}$ and $12^{\circ}$. At angles of attack $16^{\circ}$ and above, $k-\omega$ transitional model, $k-\epsilon$ did not agree with experiment. The Spalart-Alamaras model, and the Transition SST model had similar lift predictions at angles of attack at $16^{\circ}-18^{\circ}$. However the Spalart-Alamaras produced drag coefficients at least $10 \%$ higher than the Transition SST model at angles of attack between $16^{\circ}$ and $18^{\circ}$, due to the Spalart-Alamaras model over predicting the size of the flow separated region. Since this study's main focus was airfoil stall, which is dominated by viscous flow separation, the transition SST was selected. 
The pressure-velocity coupling scheme was selected, and the SIMPLE solution algorithm was used. Spatial discretization of pressure and moment was second-order, and spatial discretization of turbulent kinetic energy, specific dissipation rate, intermittency, and momentum thickness was first order upwind.

Each airfoil model was run for a full angle of attack sweep starting from zero degrees, and proceeding well into the post-stall region. The results of the previous angle of attack was used to initialize the solution for the next angle of attack. Each individual time step was considered converged once all residuals fell below $1 e-5$. The time to run was determined by the settling of $C_{l}, C_{d}$, and $C_{m_{\frac{1}{4}}}$. A CFD-Post data file file was written after a determined interval of time steps, and $C_{l}, C_{d}, C_{m_{\frac{1}{4}} c}$, pressure coefficient plot, and a wake profile plot were extracted from each output file. The pressure coefficient plots, and wake profile plots for each angle of attack were computed from the average of the final 5 output files in the settled region. In order to process the large volume of output files, the process described above was handled with the use of a CFD-Post macro written in Perl, and a MATLAB 2016a script to compute the force coefficients $C_{l}, C_{d}, C_{m_{\frac{1}{4}}}$ by taking a moving average of the coefficients' change with time and taking the last value. A diagram of the process is shown in figure 3.1 below. 


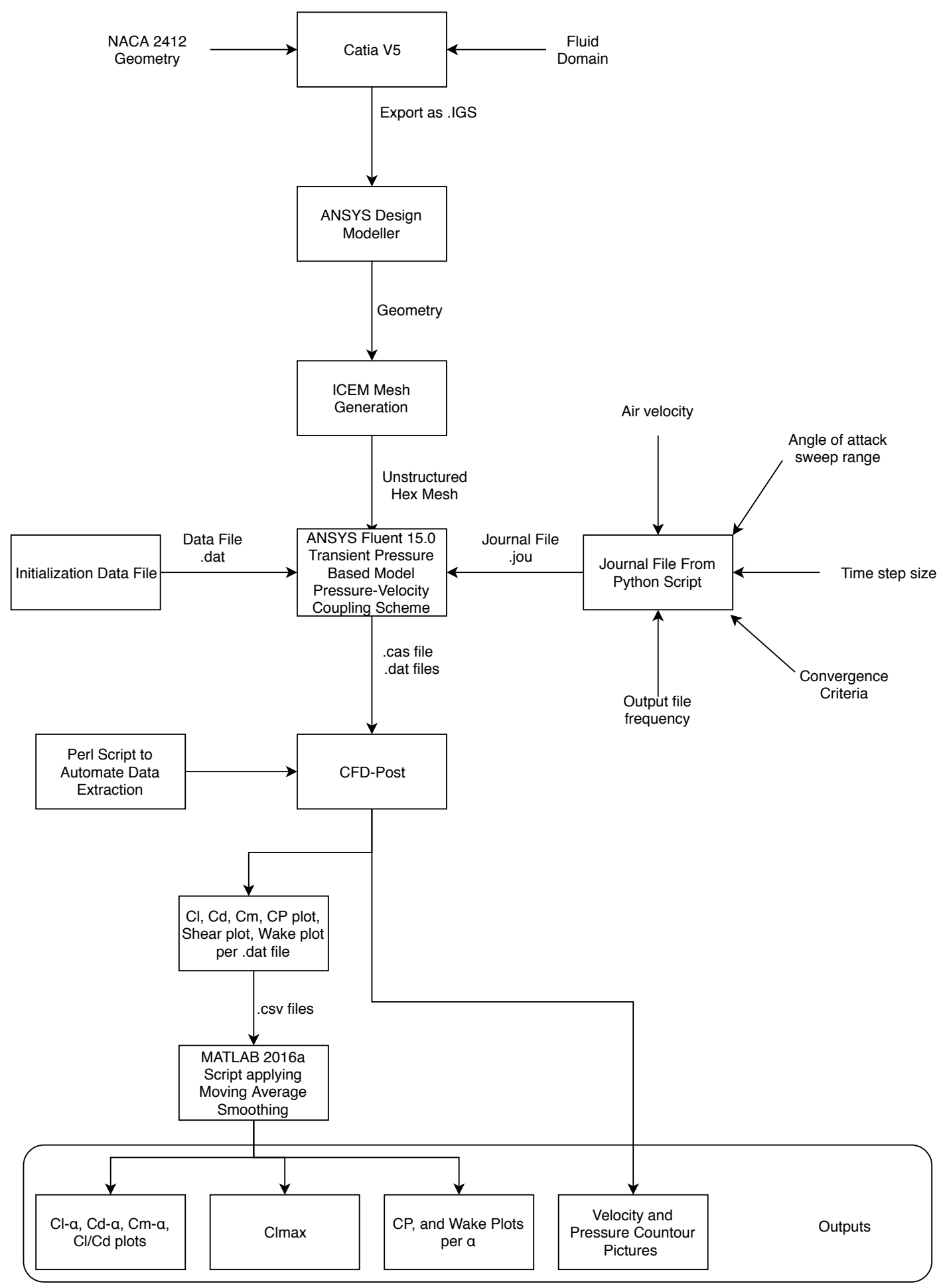

Figure 3.1: CFD Process Flow 


\subsection{Grid Independent Study}

\subsubsection{Method}

A grid independent study was used to determine a suitable mesh density and error estimation associated with the nodal distribution. To determine grid convergence, it was necessary to generate multiple grids that were systematically refined. The objective was to ensure that the error band associated with grid spacing was small enough to yield meaningful results. Three meshes (coarse, medium and fine) for each case were created. The Richardson Extrapolation method [14 was used to determine grid convergence. The theory states that discrete grid solutions $f$ are assumed to have a series representation in the grid spacing $h$ of

$$
f=f_{\text {exact }}+g_{1} h+g_{2} h^{2}+g_{3} h^{3} \ldots
$$

The quantity $f$ is considered second-order if $g_{1}=0.0$. The $f_{\text {exact }}$ is the continuum value at zero grid spacing. The functions $g_{1}, g_{2}$, and $g_{3}$ do not depend on the discretization. $f_{1}$ represents the solution for the finely-spaced grid ( $f_{2}$ represents the medium grid, and so on)

The grid refinement ratio, represented by $r$, is the grid spacing in any given dimension. The grid refinement ratio is defined by

$$
r=h_{2} / h_{1}
$$

Where $h_{1}$ is the grid spacing for the finely-spaced grid in one dimension $\left(h_{2}\right.$ represents the medium grid, and so on).

A Richardson extrapolated value of $f_{\text {exact }}$ can then be estimated:

$$
f_{\text {exact }} \approx f_{1}+\frac{f_{1}-f_{2}}{r^{k}-1}
$$

where $k$ is the order of convergence. Without an exact solution for the problem, it is necessary to have three grids (fine, medium, coarse) in order to extract $k$. If the grid refinement is performed with constant $r$, then the order can be taken from the solution to the three grids by the following:

$$
p=\ln \left(\frac{f_{3}-f_{2}}{f_{2}-f_{1}}\right) / \ln (r)
$$

The grid convergence index $(G C I)$ is a measure of the percentage the solution value $f$ is away from the value of the asymptotic numerical value. For a three grid procedure, a grid convergence index is computed between the fine and medium mesh $\left(G C I_{12}\right)$, and a between the medium and coarse mesh $\left(G C I_{23}\right)$

$$
G C I_{12}=F_{s}\left|\frac{f_{2}-f_{1}}{f_{1}}\right| /\left(r^{k}-1\right)
$$




$$
G C I_{23}=F_{s}\left|\frac{f_{3}-f_{2}}{f_{2}}\right| /\left(r^{k}-1\right)
$$

$F_{s}$ is a factory of safety. It is recommended that $F_{s}=1.25$ for Richardson extrapolations over three grids [15. It is important to check that the solutions from all three grids fall within the asymptotic range. This is accomplished by checking that

$$
G C I_{23} \approx r^{k} G C I_{12}
$$

An important aspect of Richardson extrapolation is that it applies not only to point-by-point solution values but also to solution functionals such as $C_{l}, C_{d}$, and $C_{m_{\frac{1}{4}} c}\left[15 . C_{l}\right.$ can be computed with a high degree of fidelity with computational fluid dynamics, however, it is largely independent of the boundary effects unlike $C_{d}$. In order to properly capture the grid refinement effects on adequately resolving the boundary layer, $C_{d}$ was used as the value for $f$.

\subsubsection{Grids}

A grid convergence study on the clean configuration airfoil, and one spoiler configuration airfoil was performed. It was assumed that the grids of each individual spoiler configuration would be similar enough to not require a separate grid convergence study. The grids for the clean configuration airfoil are shown in the figure below. 

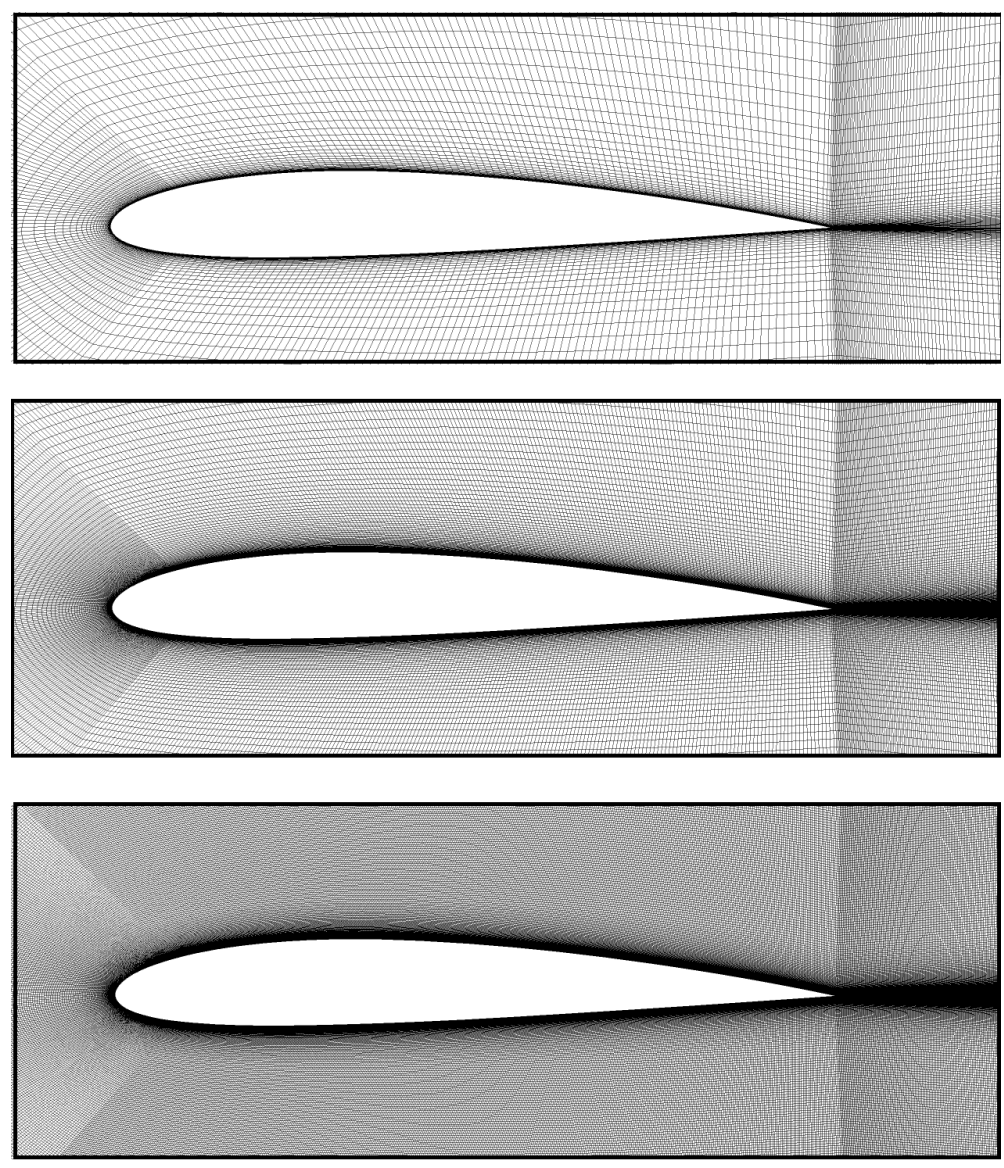

Figure 3.2: Clean Configuration Airfoil Grids

The table below indicates the number of cells present in each grid. Each successive grid refinement contains approximately four times the number of cells as the previous grid, resulting in a grid refinement ratio of two $(r \approx 2)$.

\begin{tabular}{l|l} 
Mesh & Cells \\
\hline Fine & $1,012,910$ \\
Medium & 266,310 \\
Coarse & 65,660
\end{tabular}

Table 3.1: Clean Configuration Grid Information

The grids for the spoiler configuration airfoil are shown in the figure 3.3 below. The spoiler meshes are less refined than the corresponding clean configuration mesh. The large separation bubble that occurs behind spoiler requires a smaller time step to adequately resolve, thereby increasing the number 
of time-steps required for values to settle. In order to keep the computational time within an acceptable range, the number of cells was reduced. The configuration that was selected for the grid convergence study is as follows:

$$
\begin{aligned}
& a=60 \\
& b=10 \\
& \delta=8^{\circ}
\end{aligned}
$$
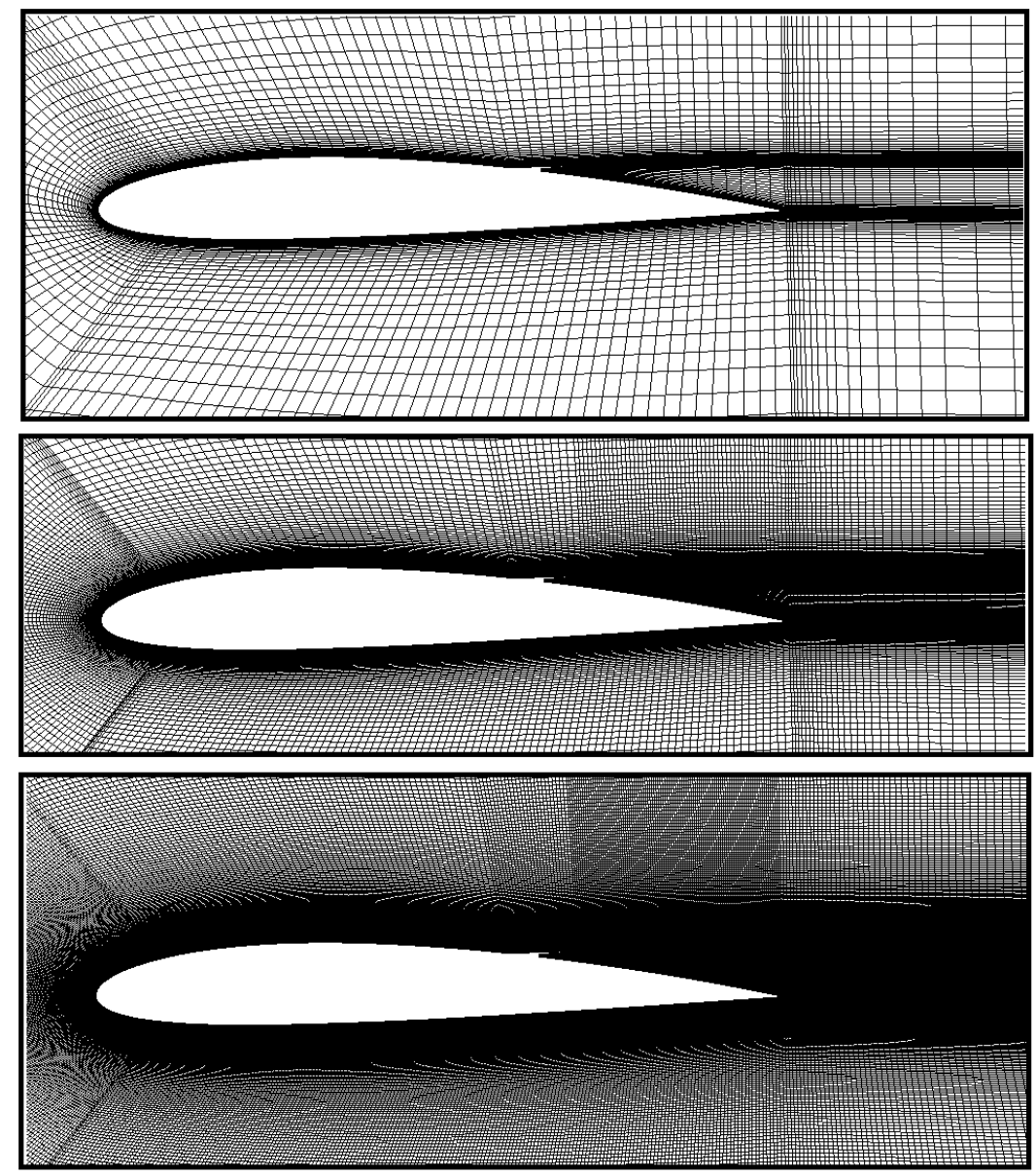

Figure 3.3: Spoiler Airfoil Grids

The table below indicates the number of cells present in each grid. Each successive grid refinement contains approximately four times the number of cells as the previous grid, resulting in a grid refinement ratio of two $(r \approx 2)$. 


\begin{tabular}{l|l} 
Mesh & Cells \\
\hline Fine & 443,804 \\
Medium & 109,700 \\
Coarse & 26,472
\end{tabular}

Table 3.2: Spoiler Configuration Grid Information

\subsubsection{Clean Configuration Grid Study Results}

Table 3.3 and table 3.4 below contains the results of the clean configuration grid convergence study. Table 3.3 contains the extracted values of $C_{d}$ at $\alpha=14^{\circ}$ for each mesh. Table 3.4 contains the calculated average grid refinement ratio, order of convergence, grid convergence indexes, and the asymptotic check value.

\begin{tabular}{l|l|l} 
Mesh & Cells & fvalue $\left(C_{d}\right)$ \\
\hline Fine & $1,012,910$ & 0.035483696 \\
Medium & 266,310 & 0.035265128 \\
Coarse & 65,660 & 0.033137404
\end{tabular}

Table 3.3: Clean Configuration Grid Convergence Study Results

\begin{tabular}{l|l} 
Parameter & Value \\
\hline$r$ & 1.964848 \\
$k$ & 3.369353376 \\
$G C I_{12}$ & 0.000881479 \\
$G C I_{23}$ & 0.008634249 \\
Asymptotic Check Value & 0.993840331
\end{tabular}

Table 3.4: Clean Configuration Grid Convergence Study Results

The asymptotic check value is approximately equal to one, therefore the values of $C_{d}$ are in the asymptotic range. The grid convergence index of mesh 2 and $3\left(G C I_{23}\right)$ is less than one percent, thus indicating that the medium refined mesh is adequate, and therefore was used to extract more useful data over a wide range of $\alpha$.

\subsubsection{Spoiler Configuration Grid Study Results}

Table 3.5 and table 3.6 below contains the results of the spoiler configuration grid convergence study. Table 3.5 contains the extracted values of $C_{d}$ at $\alpha=14^{\circ}$ for each mesh. Table 3.6 contains the calculated 
average grid refinement ratio, order of convergence, grid convergence indexes, and the asymptotic check value.

\begin{tabular}{l|l|l} 
Mesh & Cells & fvalue $\left(C_{d}\right)$ \\
\hline Fine & 443,804 & 0.040018464 \\
Medium & 109,700 & 0.040364687 \\
Coarse & 26,472 & 0.045392524
\end{tabular}

Table 3.5: Spoiler Configuration Grid Convergence Study Results

\begin{tabular}{l|l} 
Parameter & Value \\
\hline$r$ & 2.047404 \\
$k$ & 3.733968 \\
$G C I_{12}$ & 0.000800 \\
$G C I_{23}$ & 0.011514656 \\
Asymptotic Check Value & 1.008651601
\end{tabular}

Table 3.6: Spoiler Configuration Grid Convergence Study Results

The grid convergence index for the spoiler configuration of mesh 1 and $2\left(G C I_{12}\right)$ was less than one percent, and the grid convergence index between mesh 2 and $3\left(G C I_{23}\right)$ was not. Therefore the fine mesh was used to extract more useful data over a wide range of $\alpha$. The asymptotic check value is approximately equal to one, therefore the values of $C_{d}$ are in the asymptotic range.

\subsection{Computational Results}

Using the procedure detailed in Section 3.1. computational results were obtained. Due constraints on time and available computational power, only 3 spoiler configurations and the clean configuration were able to be tested. The chaotic nature of the airflow behind the spoiler required a small time step (1e- 6 seconds) for adequate convergence, which caused the number of time steps for adequate settling to be high. Configurations 1 (clean), 2, 4 and 5 (see table 1.1) were studied.

\subsubsection{Force Curves}

Figure 3.4 below shows the $C_{l}$ vs $\alpha$ graph for the different configurations. Unlike in the wind tunnel study (see figure 2.7), the lifting spoiler phenomenon was less apparent. The $\delta=8^{\circ}, \mathrm{b}=10$, a $=60$ configuration did not experience a lift drop off typically associated with stall. The $\delta=15^{\circ}, \mathrm{b}=10$, a $=60$ configuration had a very sharp drop off in lift after $C_{l_{\max }}$ was reached, and had a maximum lift coefficient $4.652 \%$ less than the clean configuration. These results did not align with the results from the wind tunnel study shown in figure 2.7 

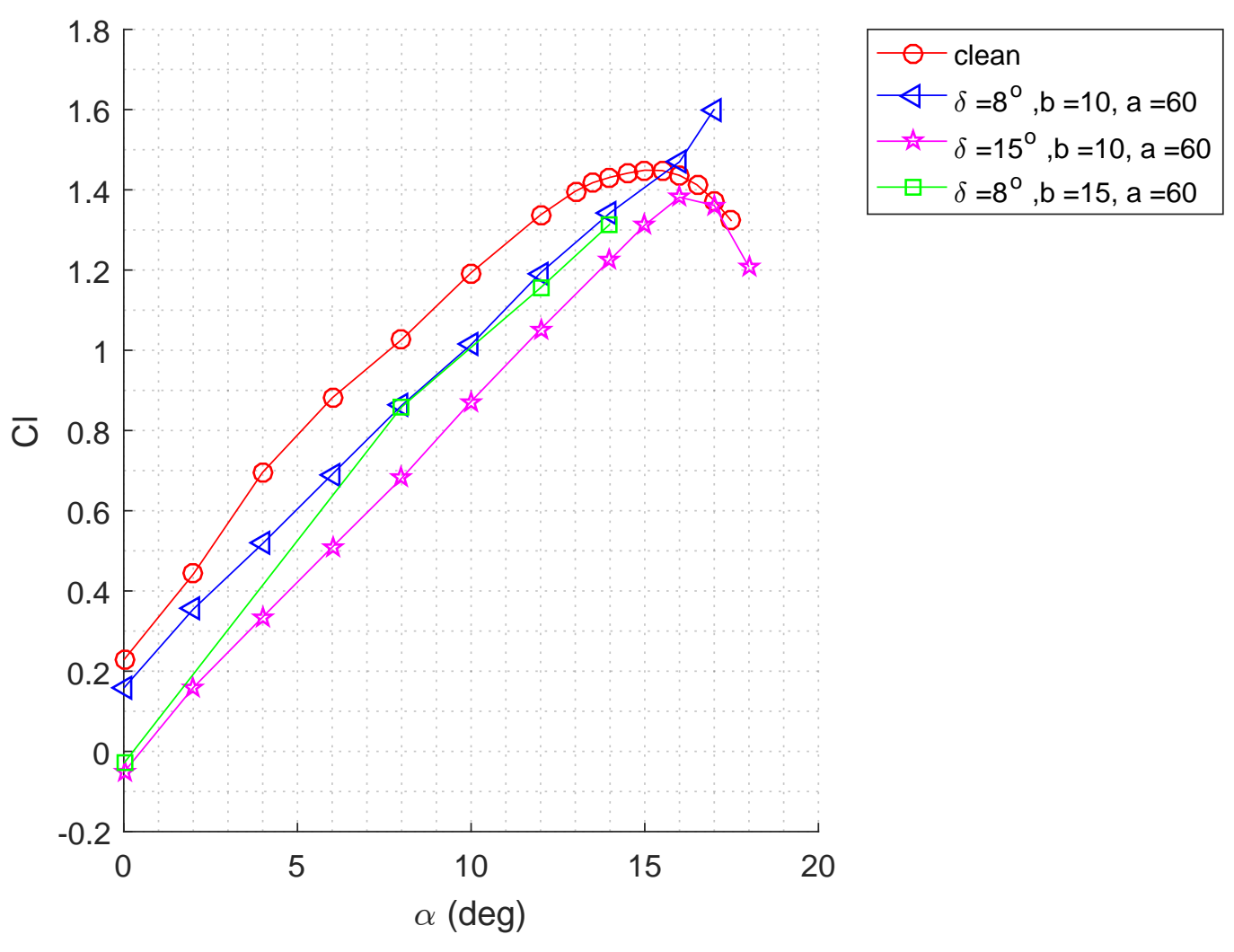

Figure 3.4: $\mathrm{CFD} \mathrm{Cl}-\alpha$ curve

\begin{tabular}{c|c|c|c|c} 
Configuration & $C_{l}$ Max & $C_{l}$ Max $\%$ Difference & Stall $\alpha$ & Stall $\alpha \%$ Difference \\
\hline clean & 1.449 & - & 15.0 & - \\
$\delta=8^{\circ}, \mathrm{b}=10, \mathrm{a}=60$ & 1.601 & 10.508 & 17.0 & 13.333 \\
$\delta=15^{\circ}, \mathrm{b}=10, \mathrm{a}=60$ & 1.382 & -4.652 & 16.0 & 6.667 \\
$\delta=8^{\circ}, \mathrm{b}=15, \mathrm{a}=60$ & 1.315 & -9.273 & 14.0 & -6.667
\end{tabular}

Table 3.7: CFD $C_{l_{\max }}$ and $\alpha_{\text {stall }}$ change with spoiler configuration

Figure 3.5 below shows the $C_{d}$ vs $\alpha$ graph for the different configurations. The results shown in figure 3.5 differ from the trend shown in the wind tunnel drag data in figure 2.8 . In the wind tunnel study, the drag coefficient of each configuration rose sharply after the stall angle was reached. Since the spoiler configurations reached stall at a higher angle of attack than the clean configuration, the spoiler configurations produced less drag at high angles of attack than the clean configuration. This is not evident in the CFD drag results. The drag coefficient of the clean configuration case was lower than the 
spoiler configurations at each angle of attack.

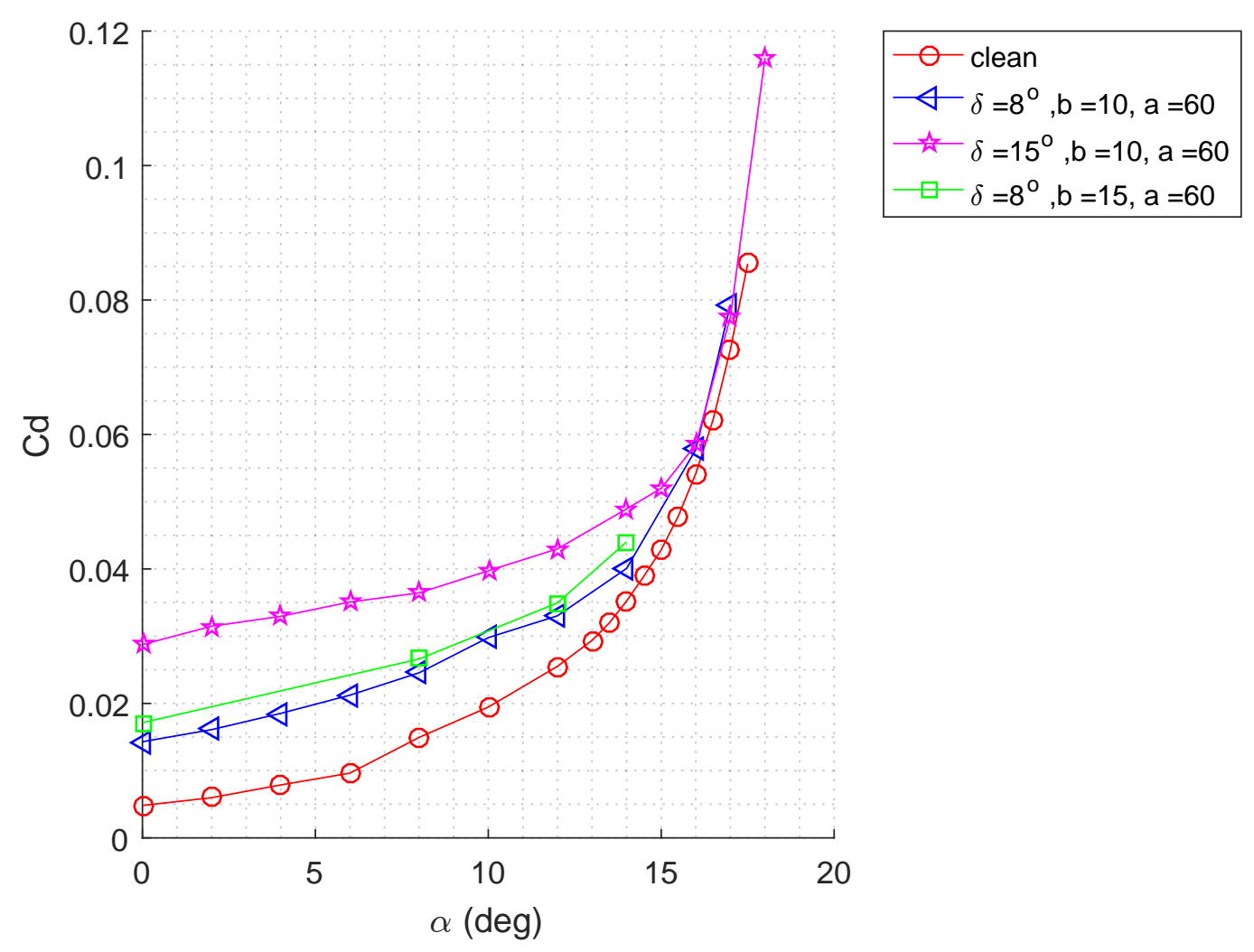

Figure 3.5: CFD Cd - $\alpha$ curve

The higher drag coefficient generated by the spoiler configurations resulted in lower aerodynamic efficiency compared with the clean configuration. In the wind tunnel study, the aerodynamic efficiency (see figure 2.9p of the spoiler configurations were substantially higher than the clean configuration at angles of attack above $18^{\circ}$. In the CFD study, only the $\delta=8^{\circ}, \mathrm{b}=10, \mathrm{a}=60$ showed improvements to aerodynamic efficiency $\left(6.786 \%\right.$ increase at $\left.\alpha=17.0^{\circ}\right)$. 


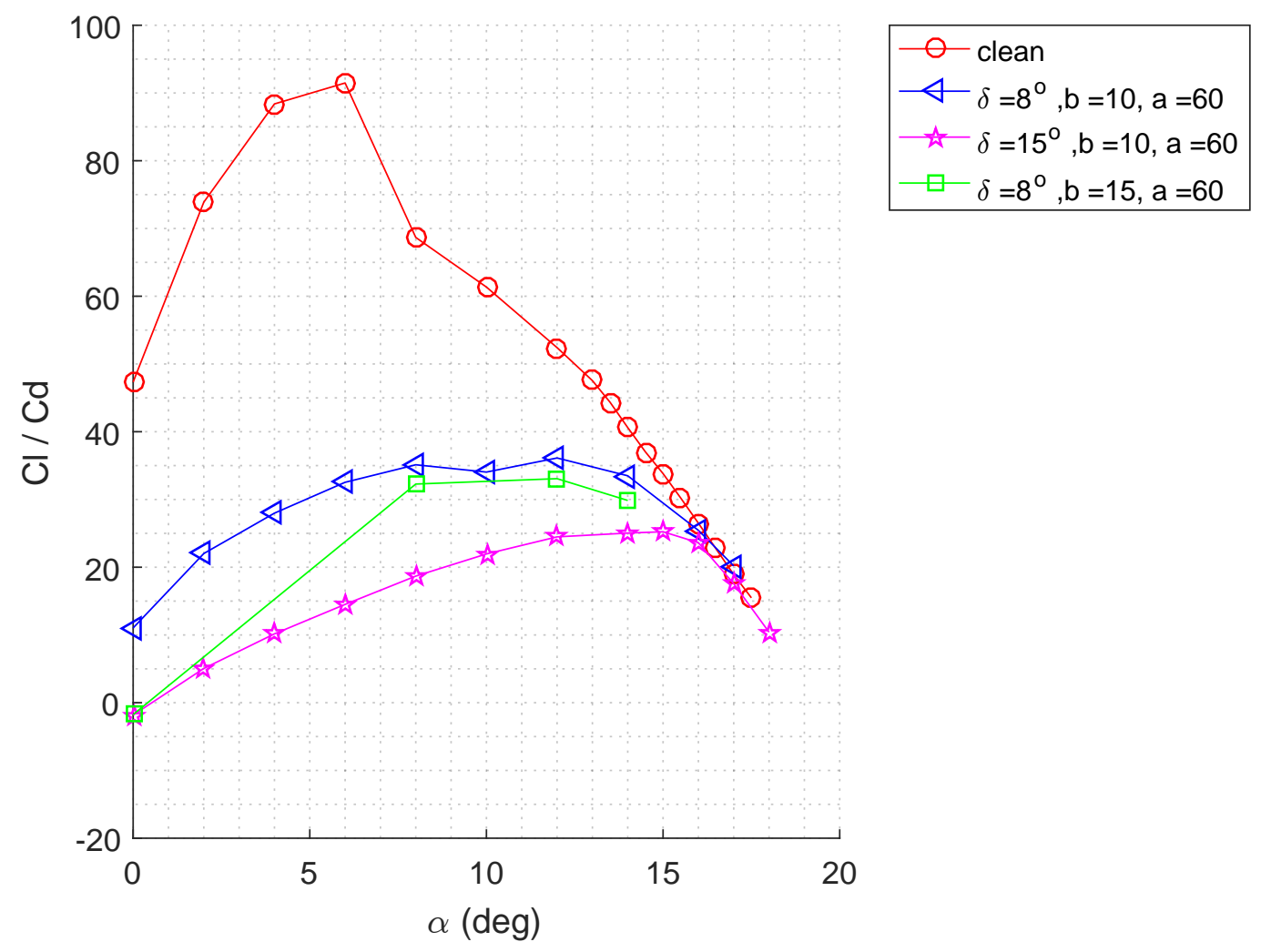

Figure 3.6: $\mathrm{CFD} \mathrm{Cl} / \mathrm{Cd}-\alpha$ curve 


\begin{tabular}{c|c|c|c|c|} 
& \multicolumn{4}{|c|}{ Aerodynamic Efficiency of Each Configuration } \\
\hline$\alpha(\mathrm{deg})$ & clean & $\delta=8^{\circ}, \mathrm{b}=10, \mathrm{a}=60$ & $\delta=15^{\circ}, \mathrm{b}=10, \mathrm{a}=60$ & $\delta=8^{\circ}, \mathrm{b}=15, \mathrm{a}=60$ \\
\hline 0.0 & 47.339 & 11.038 & -1.823 & -1.749 \\
2.0 & 73.885 & 22.020 & 5.052 & - \\
4.0 & 88.386 & 27.981 & 10.164 & - \\
6.0 & 91.490 & 32.535 & 14.512 & - \\
8.0 & 68.679 & 35.121 & 18.687 & 32.275 \\
10.0 & 61.354 & 34.042 & 21.957 & - \\
12.0 & 52.410 & 36.123 & 24.482 & 33.070 \\
13.0 & 47.542 & - & - & - \\
13.5 & 44.345 & - & - & - \\
14.0 & 40.611 & 33.496 & 25.040 & - \\
14.5 & 37.025 & - & - & - \\
15.0 & 33.816 & - & 25.236 & - \\
15.5 & 30.213 & - & - & - \\
16.0 & 26.498 & 25.441 & 23.618 & - \\
16.5 & 22.707 & - & - & - \\
17.0 & 18.910 & 20.193 & 17.564 & - \\
17.5 & 15.485 & - & - &
\end{tabular}

Table 3.8: CFD Aerodynamic Efficiency Values of Each Configuration 


\begin{tabular}{c|c|c|c|c|} 
& \multicolumn{3}{|c|}{ Aerodynamic Efficiency in Percent Difference Compared with Clean Configuration } \\
\hline$\alpha(\mathrm{deg})$ & clean & $\delta=8^{\circ}, \mathrm{b}=10, \mathrm{a}=60$ & $\delta=15^{\circ}, \mathrm{b}=10, \mathrm{a}=60$ & $\delta=8^{\circ}, \mathrm{b}=15, \mathrm{a}=60$ \\
\hline 0.0 & - & -76.684 & -103.851 & -103.696 \\
2.0 & - & -70.197 & -93.162 & - \\
4.0 & - & -68.342 & -88.500 & - \\
6.0 & - & -64.439 & -84.138 & - \\
8.0 & - & -48.862 & -72.791 & -53.006 \\
10.0 & - & -44.516 & -64.213 & - \\
12.0 & - & -31.077 & -53.288 & -36.903 \\
13.0 & - & - & - & - \\
13.5 & - & - & - & -26.488 \\
14.0 & - & -17.520 & -38.342 & - \\
14.5 & - & - & - & - \\
15.0 & - & - & -25.374 & - \\
15.5 & - & - & - & - \\
16.0 & - & -3.989 & -10.870 & - \\
16.5 & - & - & - & - \\
17.0 & - & 6.786 & -7.114 & - \\
17.5 & - & - & - &
\end{tabular}

Table 3.9: CFD Aerodynamic Efficiency Compared with Clean Configuration 


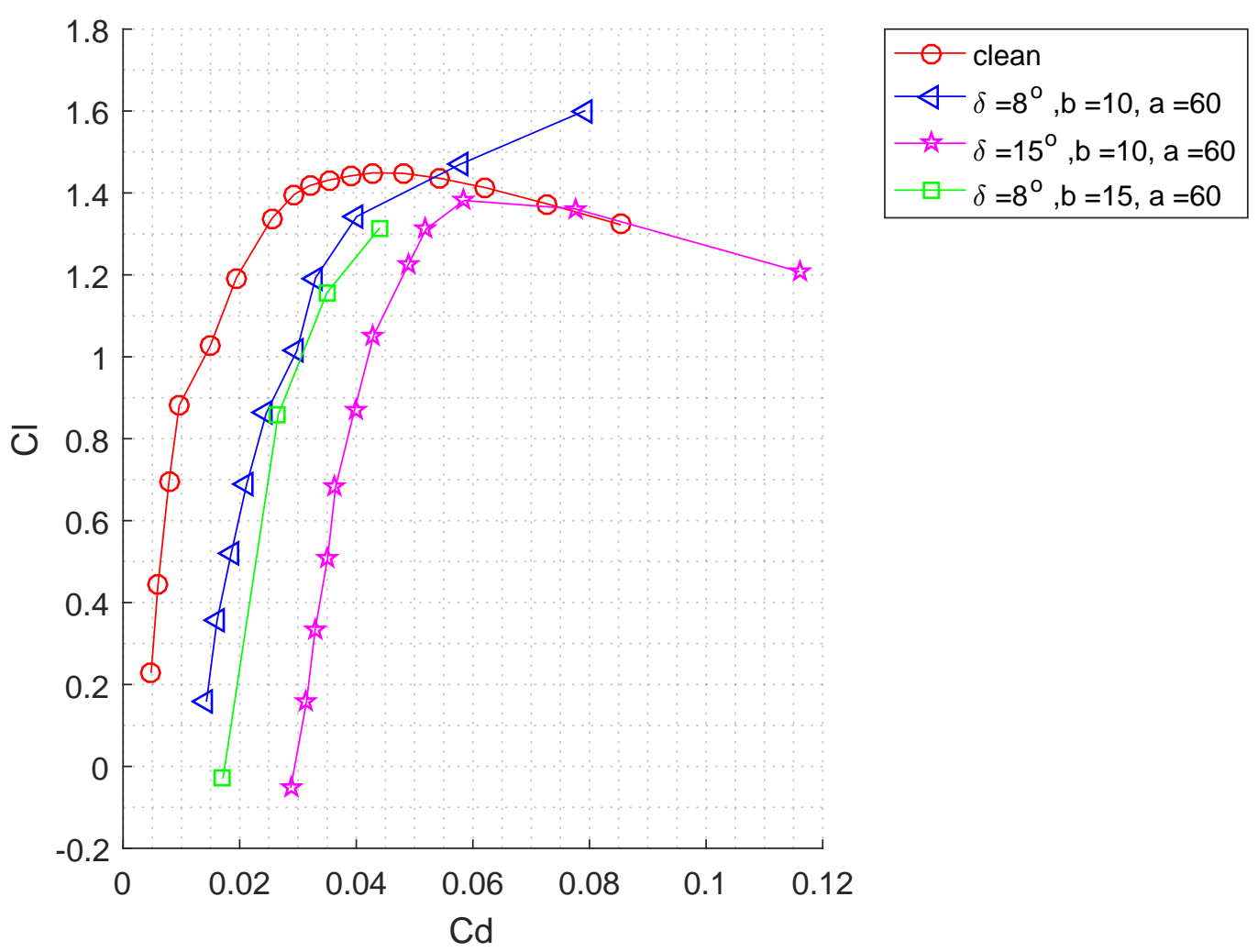

Figure 3.7: $\mathrm{CFD} \mathrm{Cl}$ vs Cd curve 


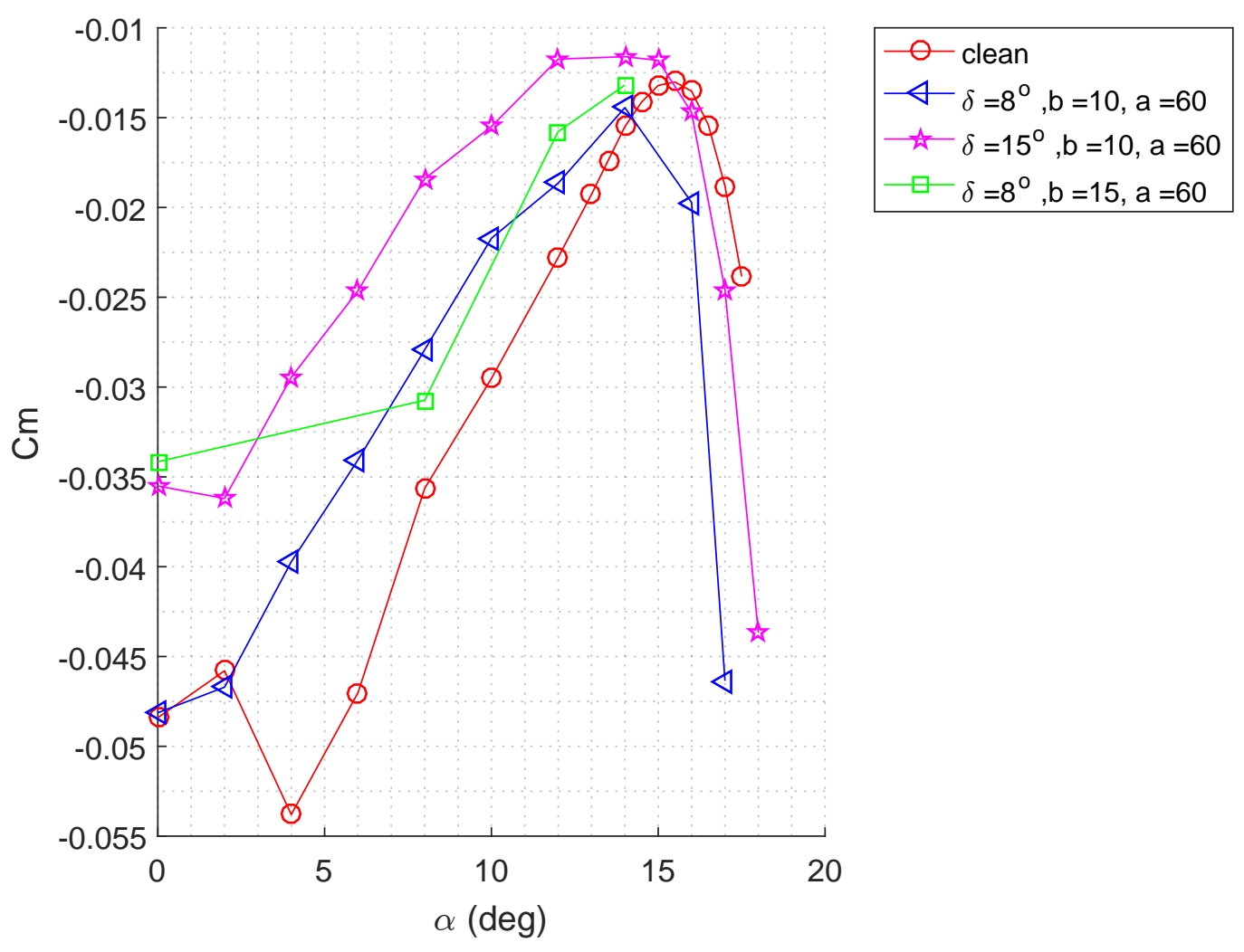

Figure 3.8: CFD Cm - $\alpha$ curve

\subsubsection{Time History of Force Coefficients}

The steady state force coefficients were taken by applying a moving average over the time history of each force coefficient, and taking the average in the settled region. The following section details the time history of the force coefficients for each configuration.

The time history of the clean configuration $C_{l}, C_{d}$, and $C_{m_{\frac{1}{4}} c}$ are shown in figure 3.9 below. Each large jump is caused by a change in angle of attack. After the angle of attack is changed, the simulation was allowed to run until no significant oscillations in value were observed. The coefficient values shown in figures 3.4 3.5, and 3.8 above are taken from a moving average within the converged regions. 

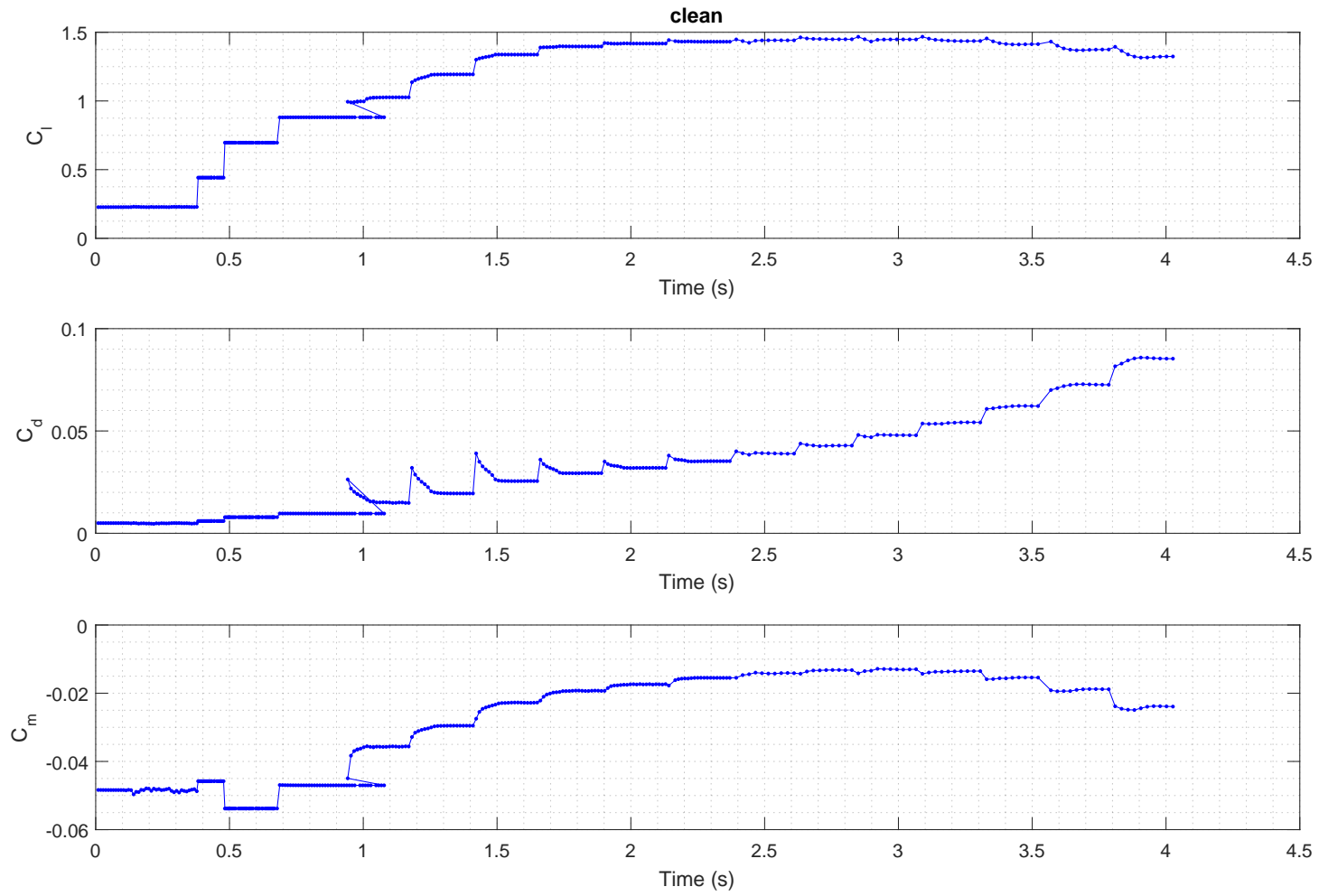

Figure 3.9: CFD Force coefficient time history - clean configuration

The time history of the $b=10, \delta=8^{\circ}$ case is shown in figure 3.10 below. This configuration reaches a steady value in the low to mid range angles of attack. At the higher angles of attack, the force coefficients fluctuate considerably with time. This calls into question the validity of the steady state values at $\alpha=16^{\circ}$ and $\alpha=17^{\circ}$. 

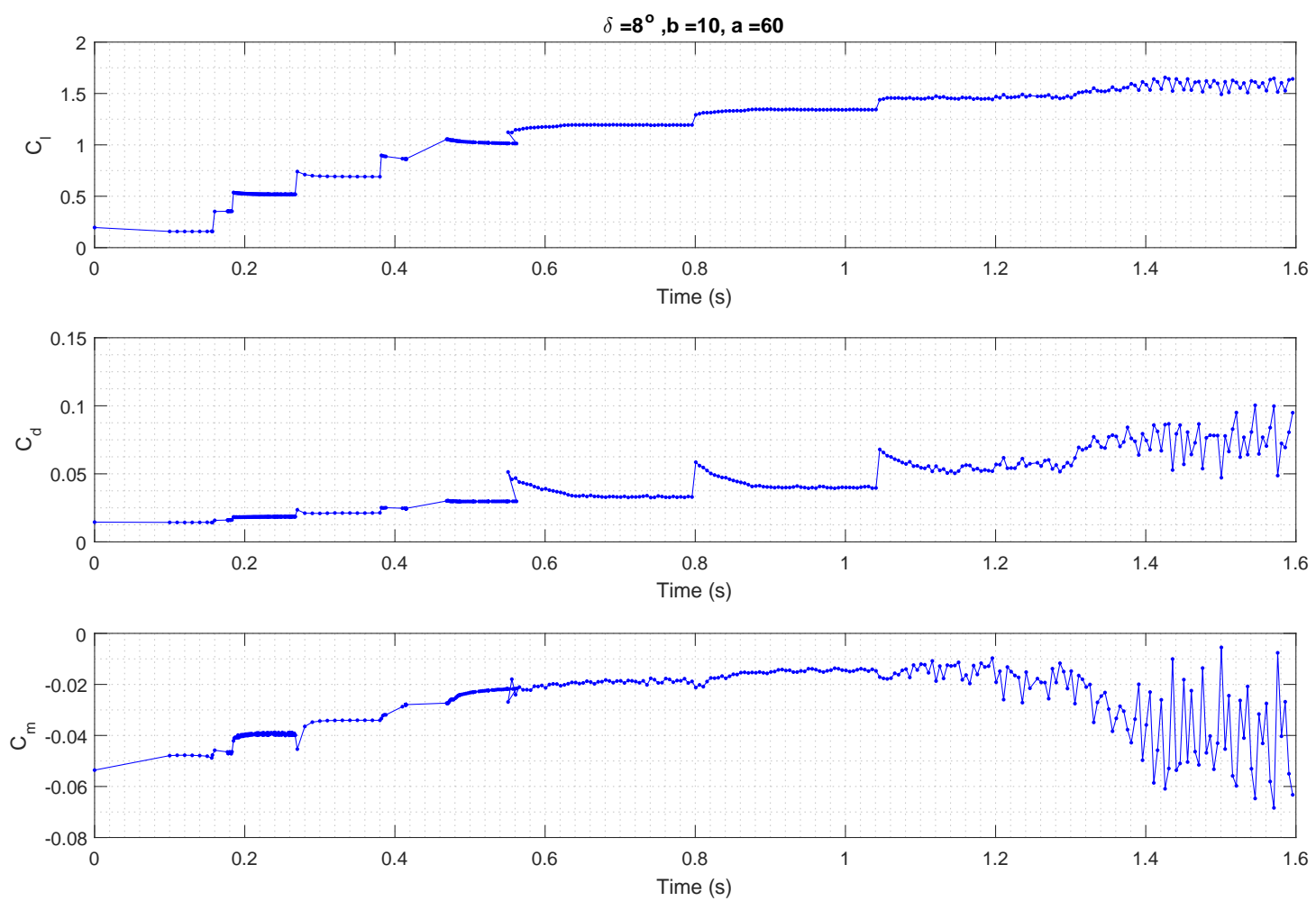

Figure 3.10: CFD Force coefficient time history - configuration 2

The time history of the $b=10, \delta=15^{\circ}$ case is shown in figure 3.11 below. This configuration tends to produce values that are more steady than the $b=10, \delta=8^{\circ}$ case. 

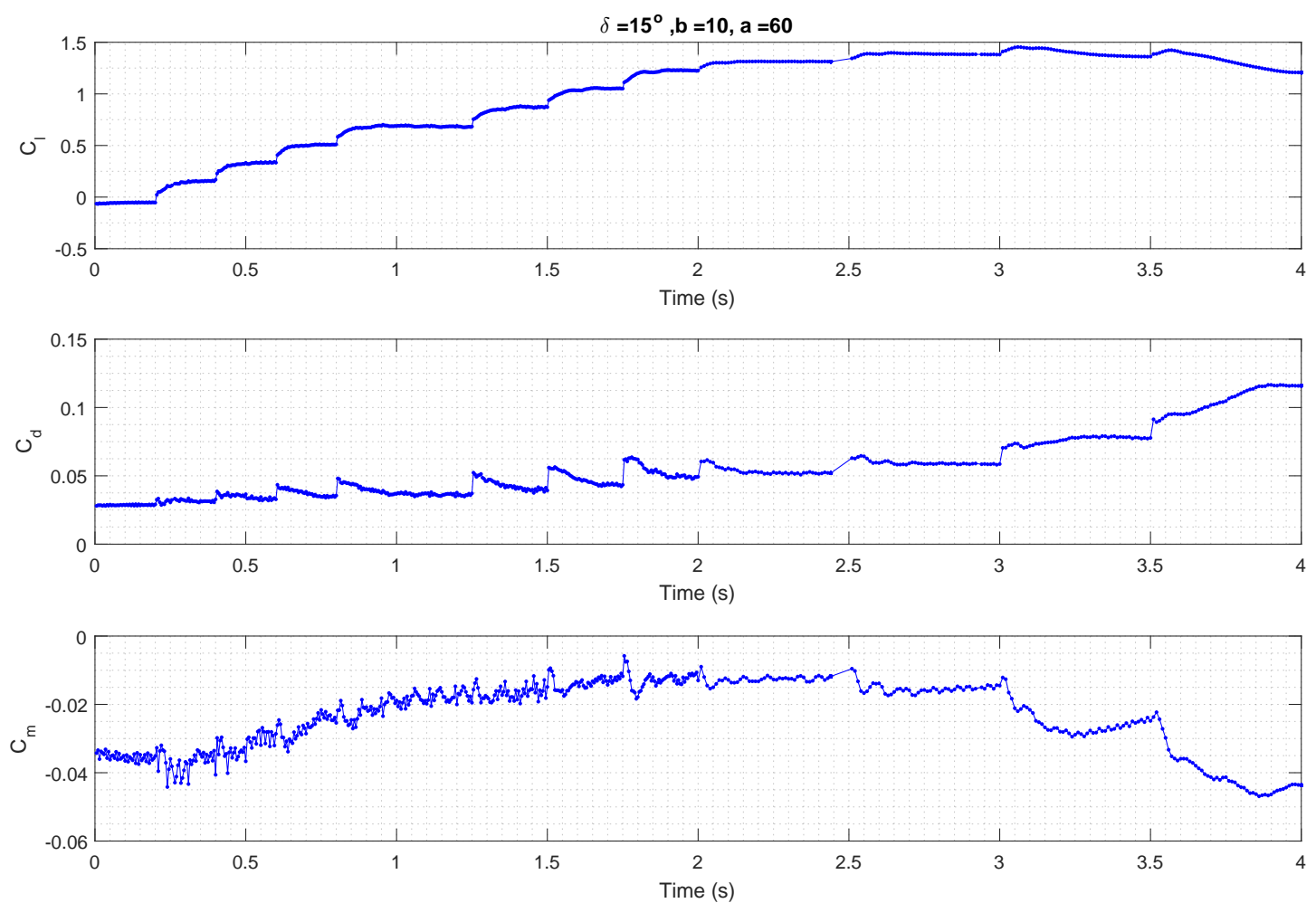

Figure 3.11: CFD Force coefficient time history - configuration 3

The time history of the $b=15, \delta=8^{\circ}$ case is shown in figure 3.12 below. This configuration tends to produce values that are relatively steady. However no data points at higher angles of attack were able to be studied due to constraints on available time. 

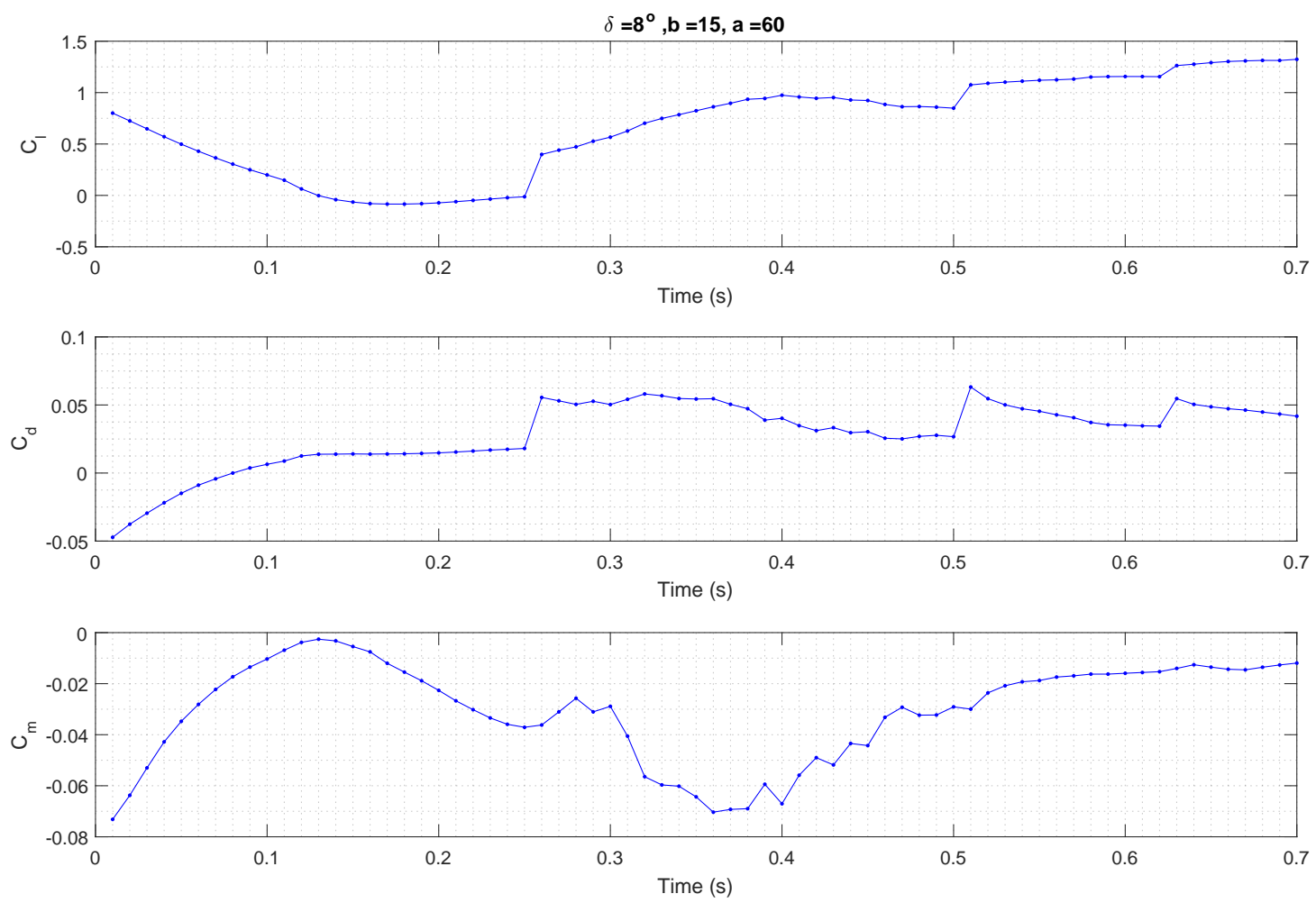

Figure 3.12: CFD Force coefficient time history - configuration 5

\subsubsection{Pressure Coefficient Distribution}

The pressure coefficient curves from the computational study show similar patterns to that of the wind tunnel experiment. Figure 3.13 below shows the pressure coefficient plot of the clean configuration and the $\delta=15^{\circ}, b=10, a=60$ spoiler configuration at $\alpha=4^{\circ}$. At this low angle of attack, the spoiler reduces the lift of the airfoil by decreasing the upper surface suction peak, and increasing the pressure on the upper surface downstream of the spoiler. The airfoil lower surface also generates less pressure, thus furthering reducing lift. This pattern is consistent with the results from the wind tunnel study shown in figure 6.75. Figure 3.14 below shows the pressure coefficient plot of the clean configuration, the $\delta=18^{\circ}, b=10, a=60$ spoiler configuration, and the $\delta=15^{\circ}, b=10, a=60$ spoiler configuration

at $\alpha=17^{\circ}$. The $\delta=8^{\circ}$ configuration creates a small increase to the airfoil suction peak, thus resulting in a net increase in lift coefficient. This is consistent with the pressure coefficient distributions at high $\alpha$ from the wind tunnel experiment (see figure 6.78, , however the effect was greater in the wind tunnel experiment. The $\delta=15^{\circ}$ configuration creates a slight reduction in the airfoil suction peak, thus creating 
a slight decrease in lift coefficient. This pressure coefficient distribution is not consistent with the wind tunnel experimental results (see figure 6.78).

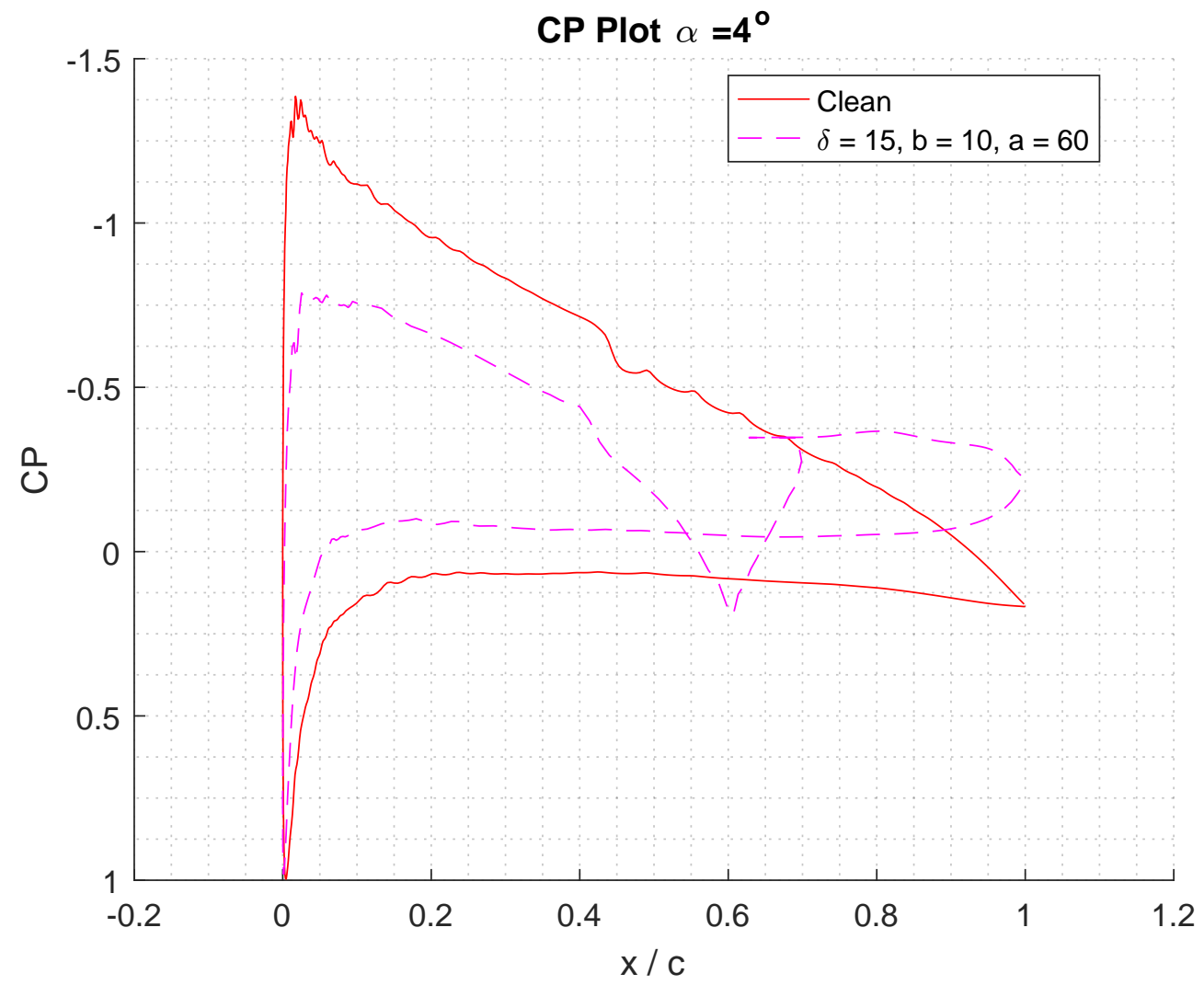

Figure 3.13: CFD Pressure Coefficient Curve Comparing Configuration 3 and Clean at $\alpha=4^{\circ}$ 


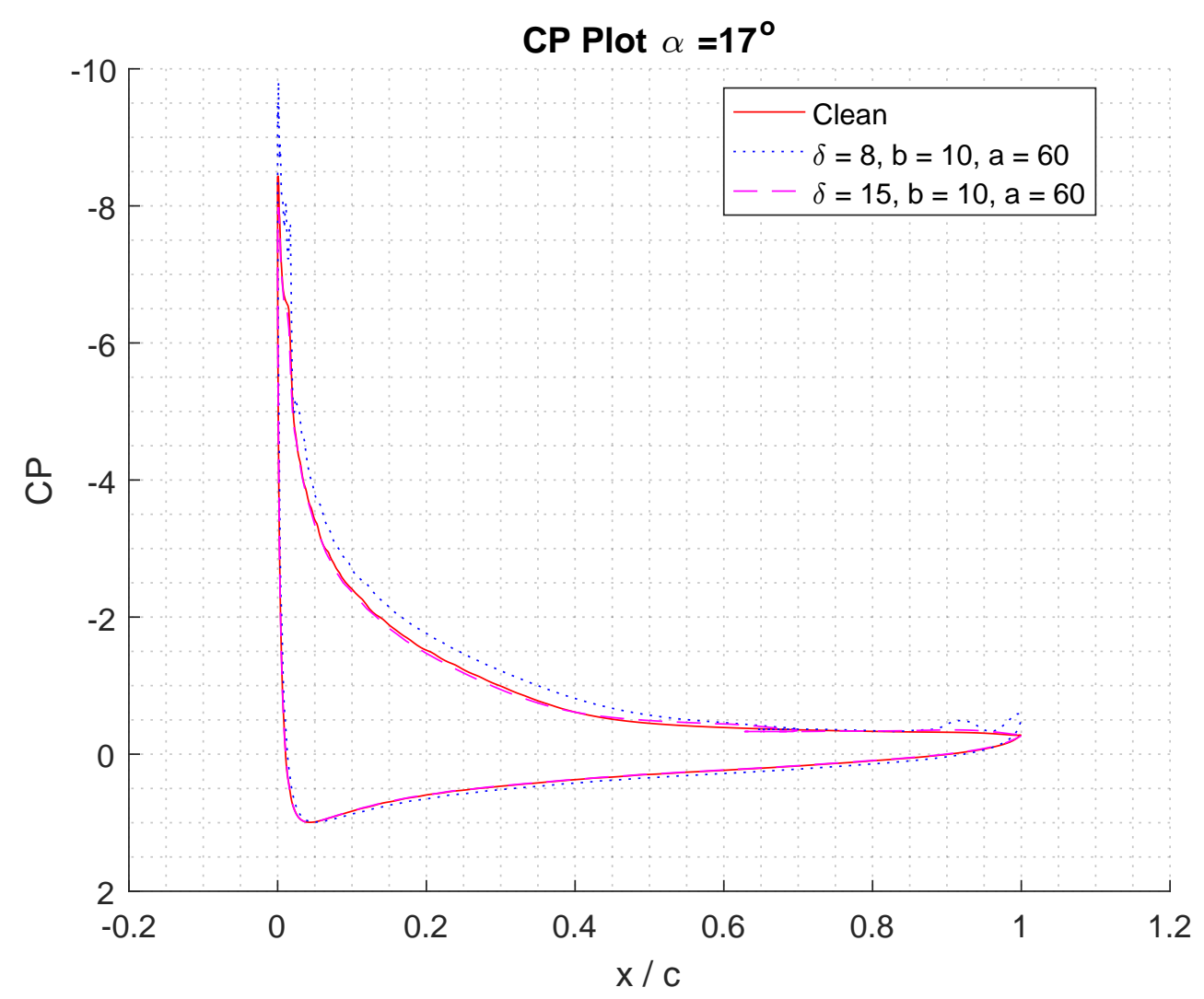

Figure 3.14: CFD Pressure Coefficient Curve Comparing Configuration 3 and Clean at $\alpha=17^{\circ}$

\subsubsection{Streamline Plots}

The velocity and pressure contour plots, and the streamline plot demonstrated the effect of spoiler configuration on airfoil performance. At low angles of attack, the spoiler created a separation bubble downstream of the spoiler (see figure 3.15). The separation bubble increased in size with an increased deflection angle, and increased spoiler length. The flow separation created a pressure increase on the airfoil upper surface, which reduced lift.

At high angles of attack approaching stall, the clean configuration airfoil produced a flow separation bubble on the upper surface. The results from the wind tunnel experiment suggest that the flow separation bubble produced by the spoiler configuration airfoils would be smaller than that of the clean configuration airfoil, since the spoiler airfoils produce higher lift coefficients at high angles of attack. This would also be consistent with the results from Pabla's computational study on spoiler configurations at high angles of attack [9] (see figure 1.14). However a reduction in separation bubble size was not apparent (see figure 3.16 below). The three configurations depicted in figure 3.16 appear to be undergoing stall, despite the $\delta=8^{\circ}, b=10, a=60$ spoiler configuration having not reached maximum 
lift. The lifting spoiler phenomeon does not appear to be present in figure 3.16

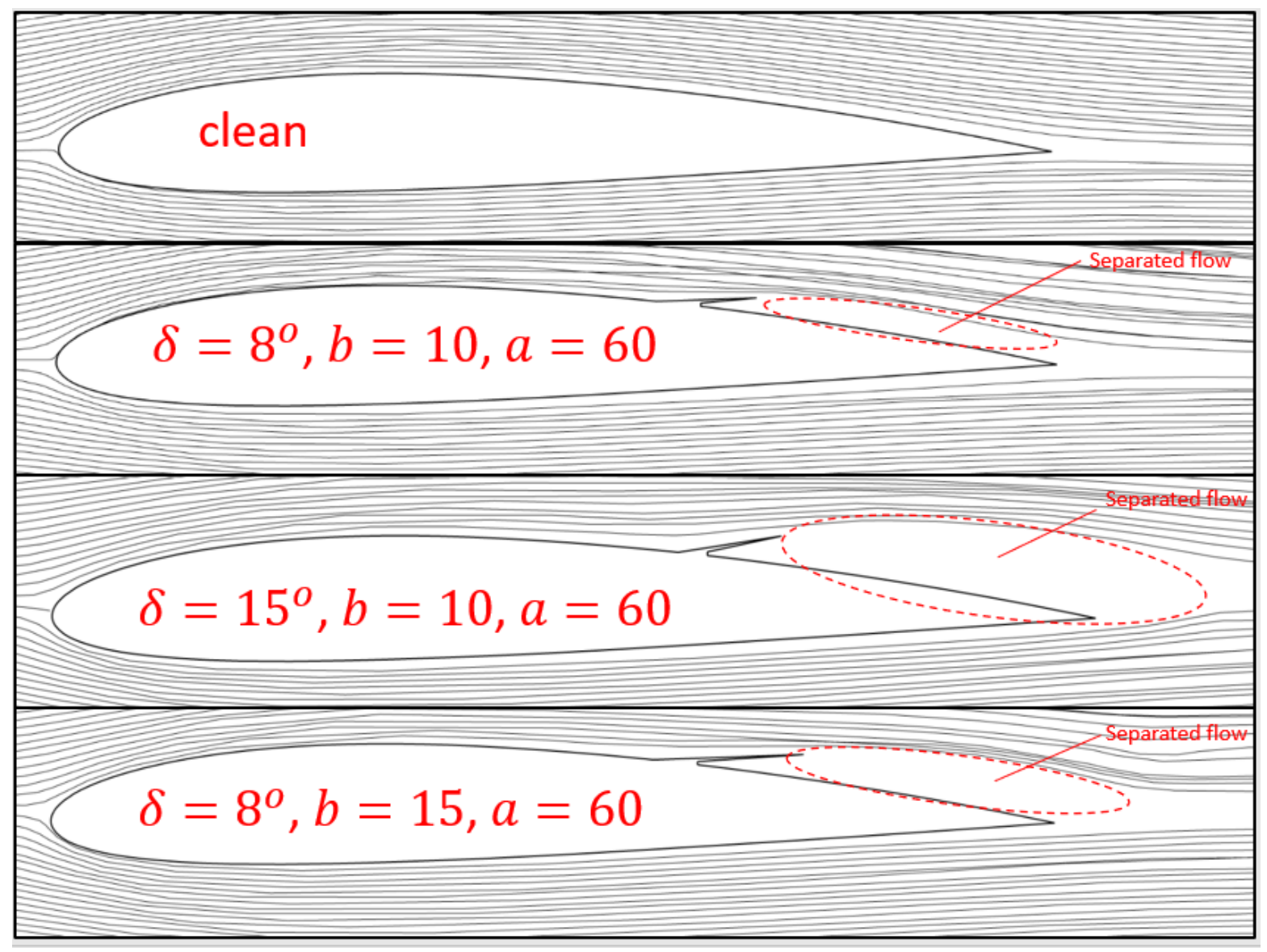

Figure 3.15: Streamline Plot Comparison at $\alpha=0^{\circ}$ 


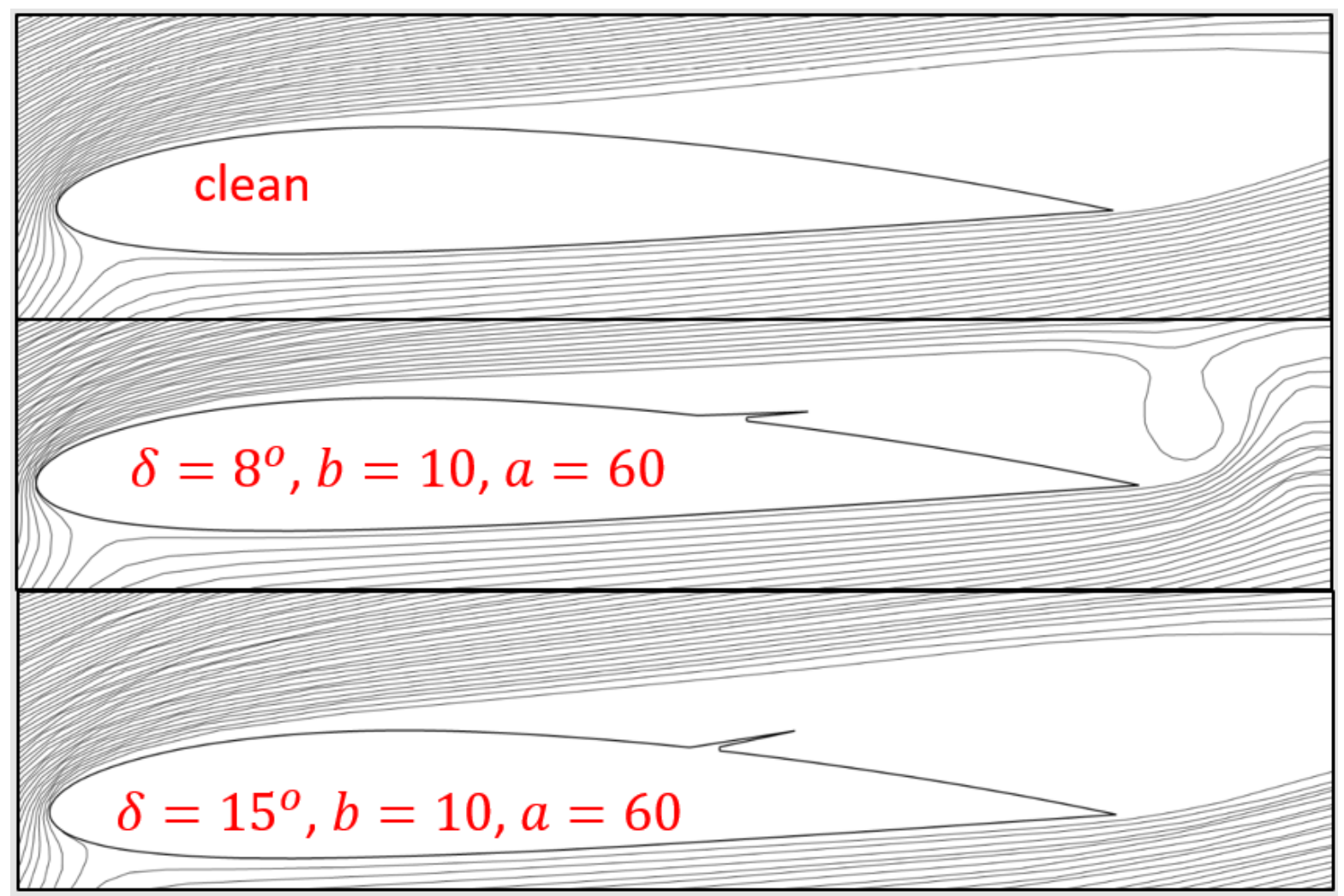

Figure 3.16: Streamline Plot Comparison at $\alpha=17^{\circ}$ 


\section{Chapter 4}

\section{Wind Tunnel and CFD Comparison}

This chapter compares the differences between the CFD and Wind Tunnel results.

\subsection{Lift Coefficient Curves}

The four figures below (figure 4.1 figure 4.2 figure 4.3 figure 4.4 compare the $C_{l}-\alpha$ curves of the computational study and the wind tunnel study. The computational study consistently over predicts lift compared with the wind tunnel. In the low to mid angle of attack region, the computational study predicts a similar lift slope compared with the wind tunnel.

The stall behaviour of the computational clean configuration was similar to that of the wind tunnel clean configuration. Both exhibited a gentle stall behaviour where maximum lift coefficient occurred at roughly $15^{\circ}$. The stall behaviour of Configuration 2 and 3 did not match the stall behaviour of the wind tunnel experimental data. Based on the wind tunnel data, the spoiler configurations tended to flatten out the peak of the $C_{l}$ - $\alpha$ curve, however the stall beahviour of configuration 3 was sharp. Configuration 2 did not experience a lift reduction, despite the streamline plot at this angle of attack (see figure 3.16) indicating that the flow had stalled.

It is plausible that the high amounts of vorticies and turbulence behind the spoiler-equipped airfoils at high angles of attack were not adequately captured by the computational study, thus resulting in the inconsistency between the computational stall beahviour and the wind tunnel stall behaviour. Shrinking the time-step size could potentially solve this problem, however this would increase the number of iterations required for adequate convergence. The computational resources required to investigate this were not available at the time of this study. It is also likely that using a different turbulence model would have resolved this problem, however the computational resources required to experiment with different models was not adequate given the time-frame of the study. 


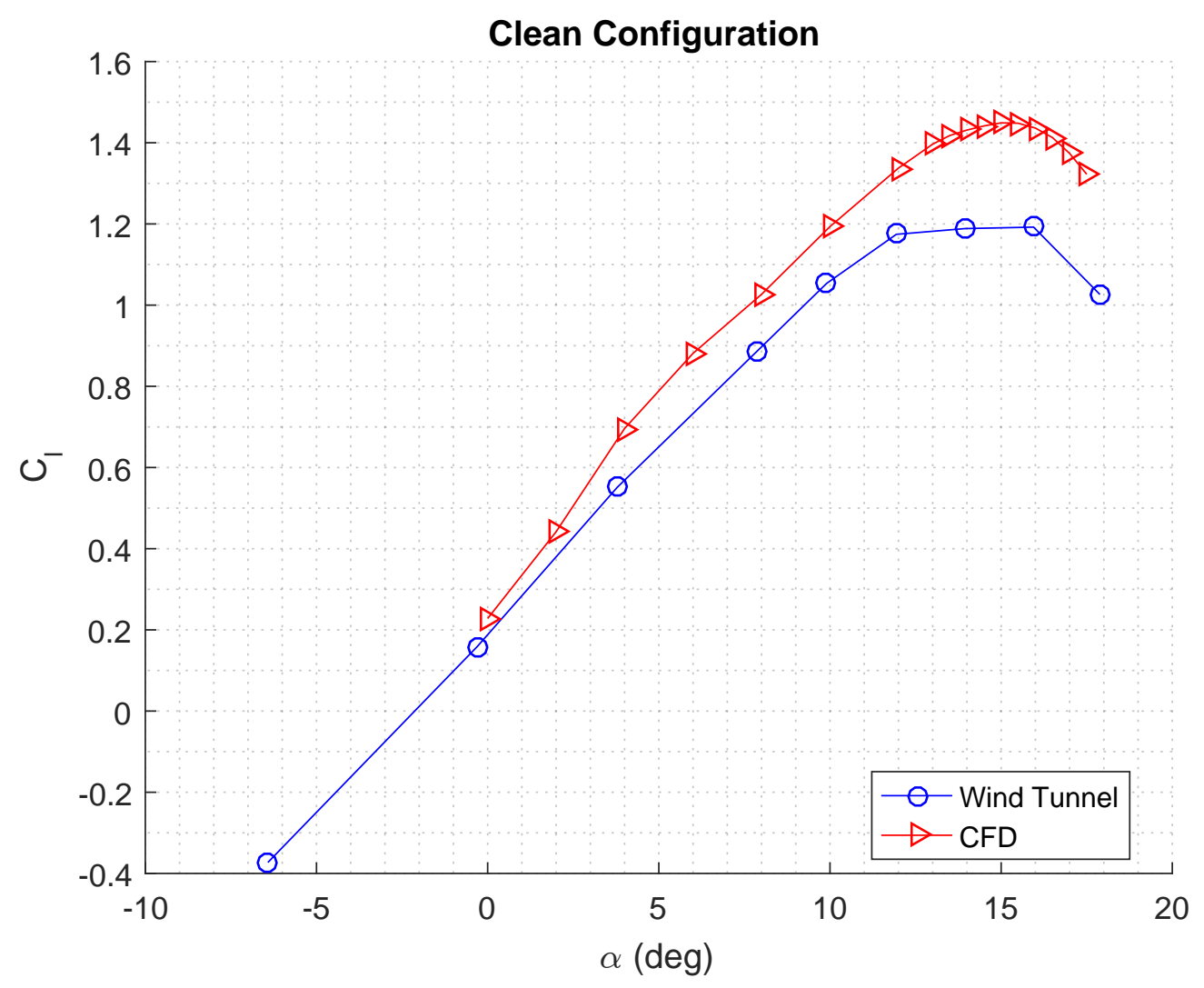

Figure 4.1: CFD and Wind Tunnel Comparison $\mathrm{Cl}-\alpha$ curve Configuration 1 


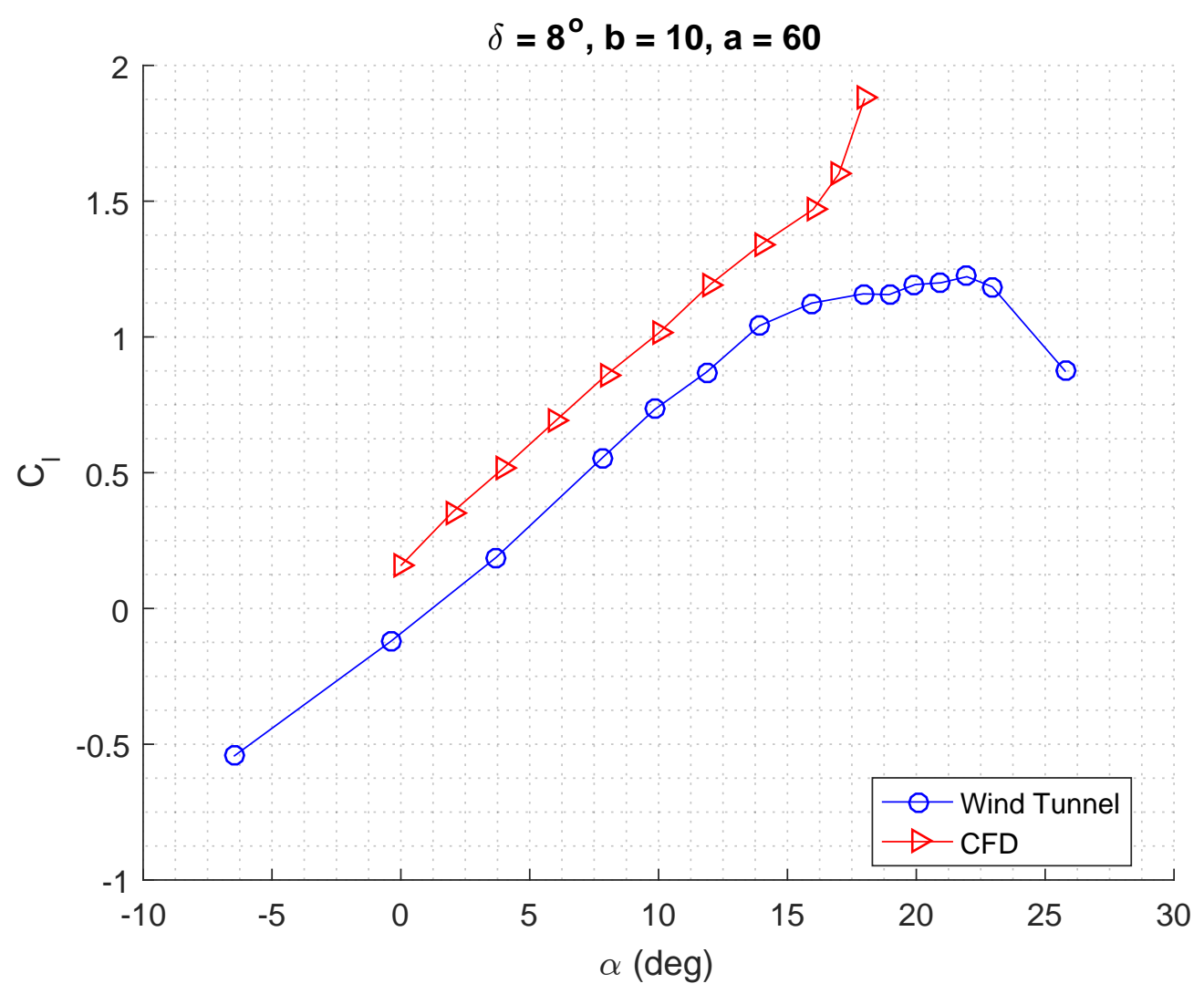

Figure 4.2: CFD and Wind Tunnel Comparison $\mathrm{Cl}-\alpha$ curve Configuration 2 


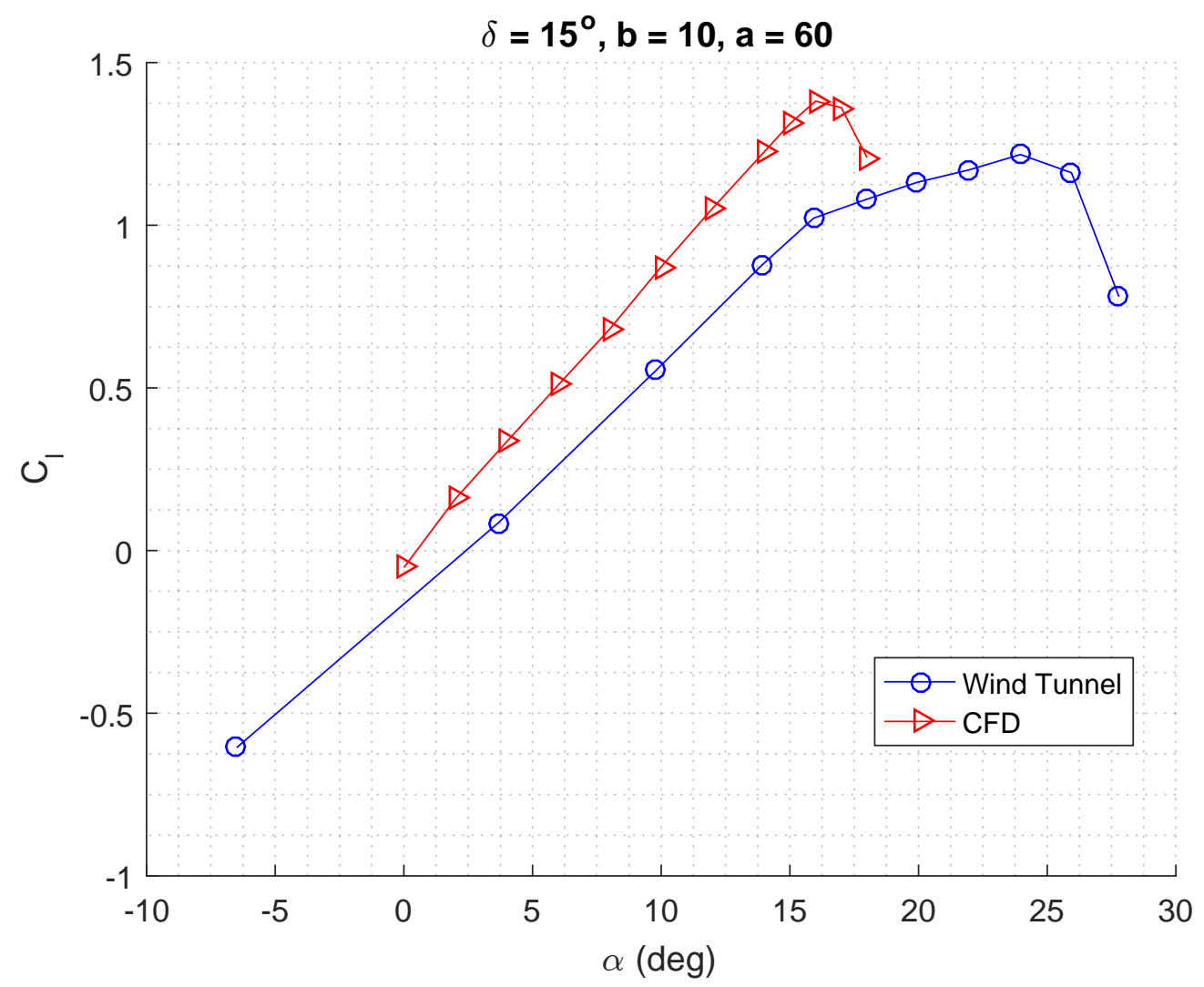

Figure 4.3: CFD and Wind Tunnel Comparison $\mathrm{Cl}-\alpha$ curve Configuration 3 


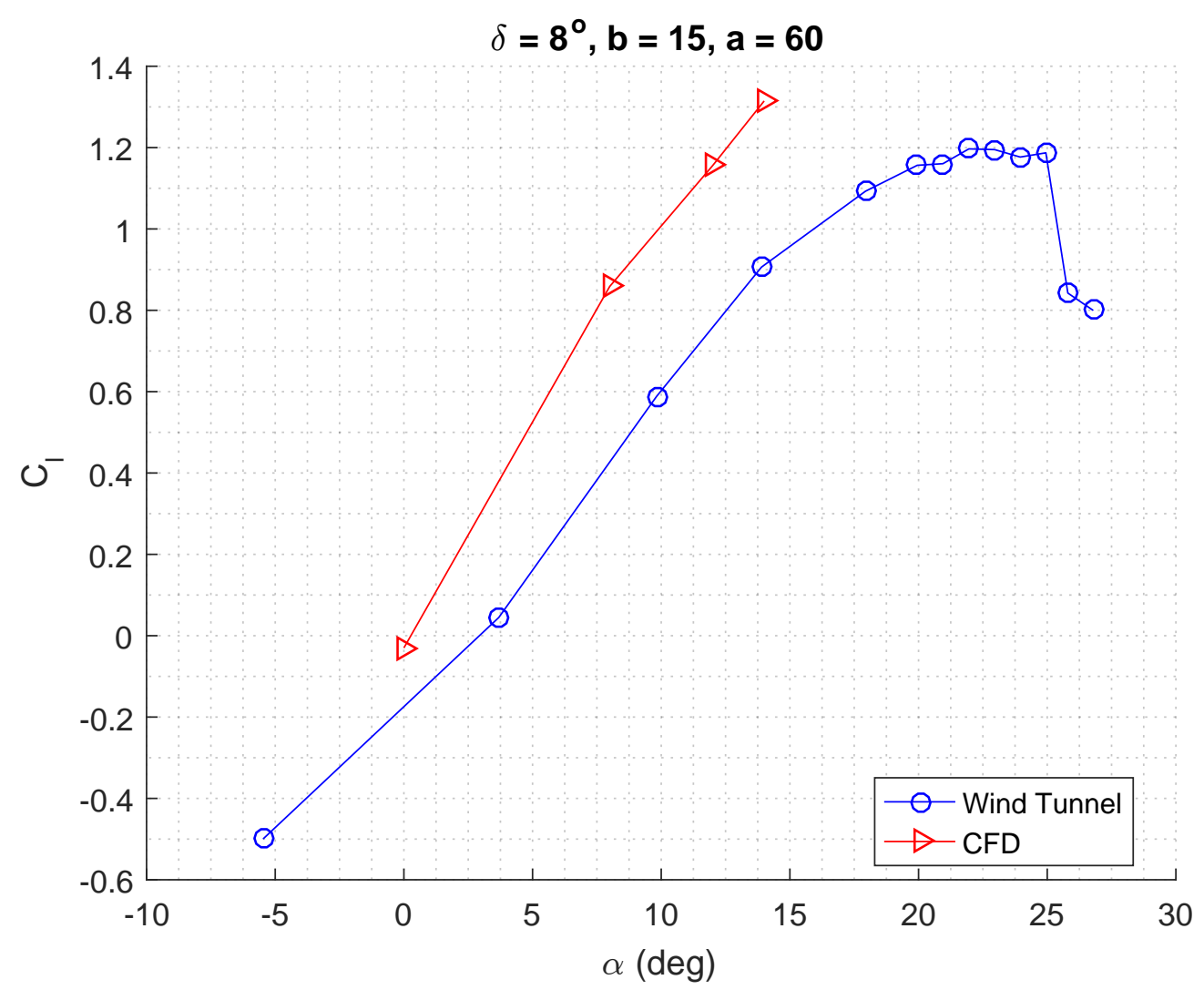

Figure 4.4: CFD and Wind Tunnel Comparison $\mathrm{Cl}-\alpha$ curve Configuration 5

\subsection{Drag Coefficient Curves}

The four figures below (figure 4.5 figure 4.6 figure 4.7 figure 4.8) compare the $C_{d}-\alpha$ curves of the computational study and the wind tunnel study. At low to moderate angles of attack, the computational study showed good agreement with the wind tunnel study. However the sudden drag increase associated with the onset of stall occurred at lower angles of attack in the computational study than in the wind tunnel study. This indicates that the computational model has difficultly accurately predicting drag once the viscous effects of stall have a greater influence on the flow. 


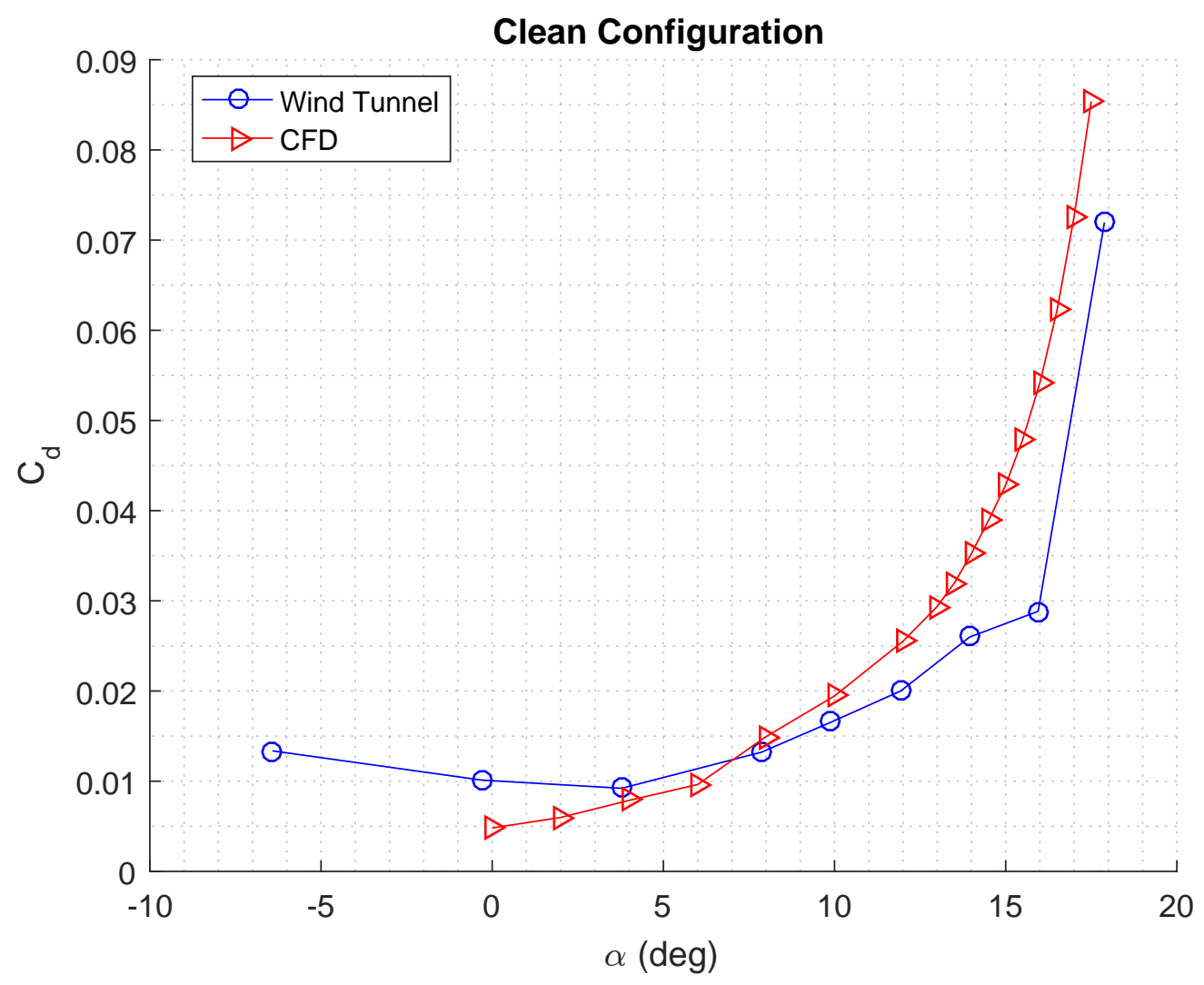

Figure 4.5: CFD and Wind Tunnel Comparison Cd - $\alpha$ curve Configuration 1 


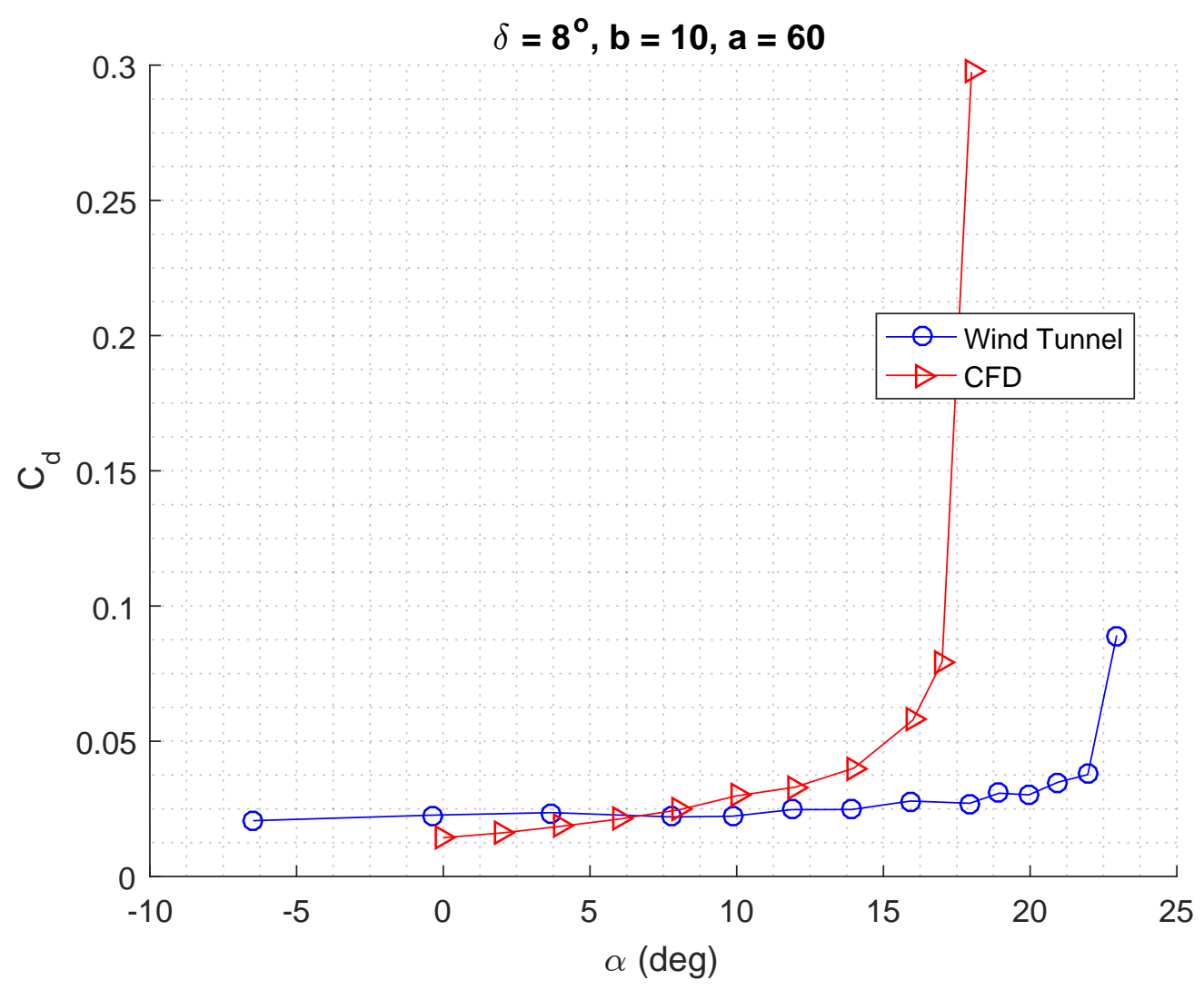

Figure 4.6: CFD and Wind Tunnel Comparison Cd - $\alpha$ curve Configuration 2 


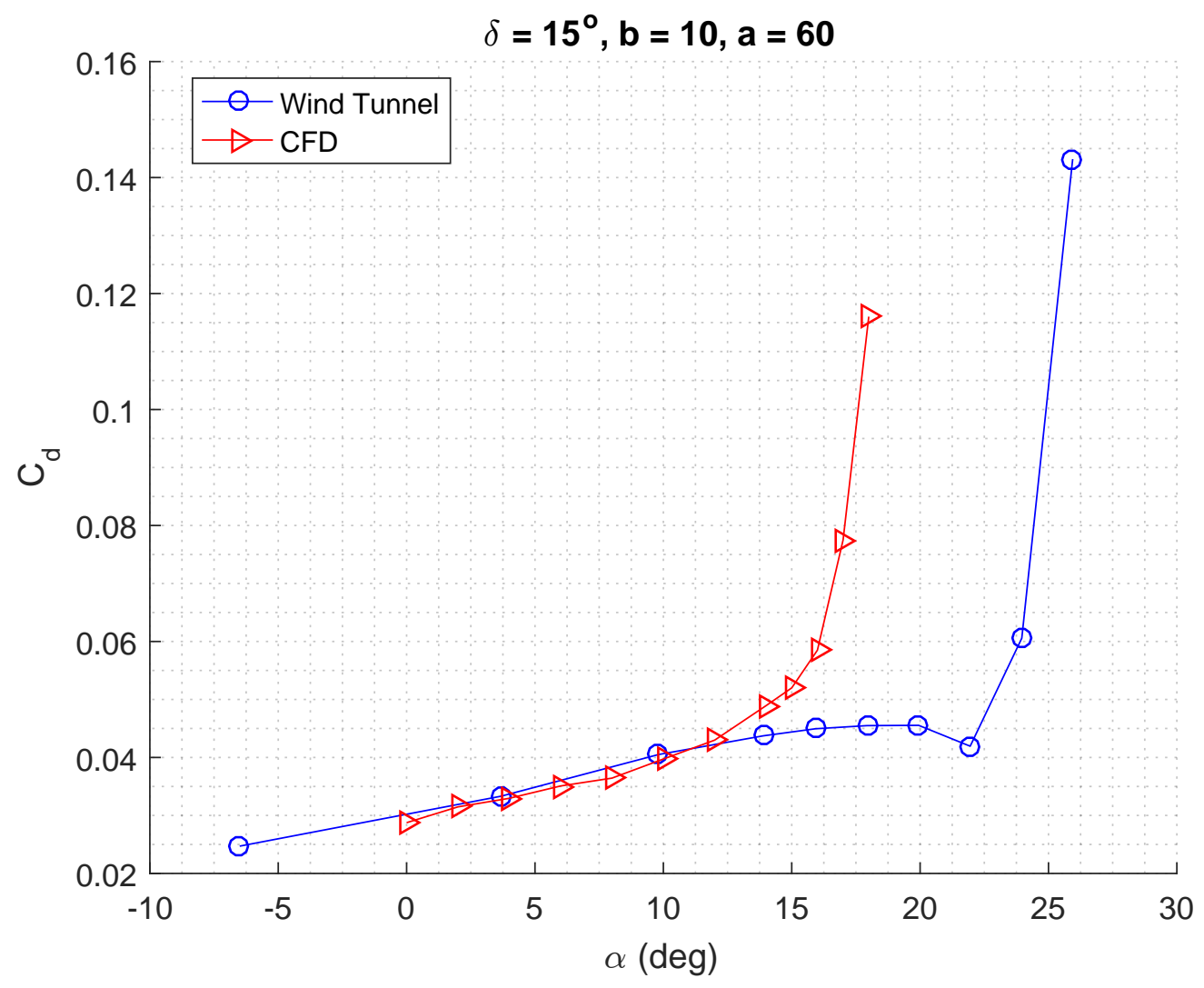

Figure 4.7: CFD and Wind Tunnel Comparison Cd - $\alpha$ curve Configuration 3 


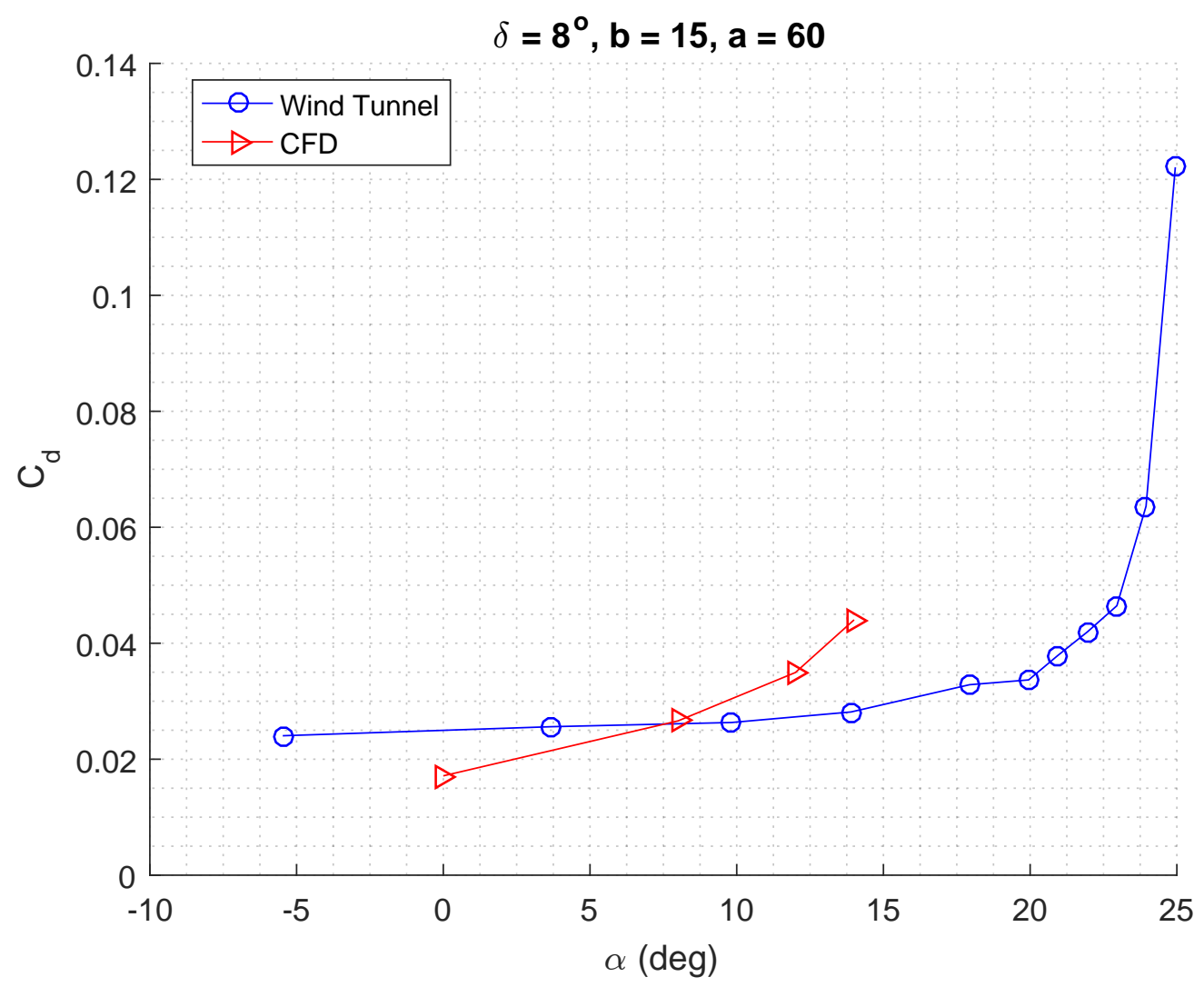

Figure 4.8: CFD and Wind Tunnel Comparison Cd - $\alpha$ curve Configuration 5 


\section{Chapter 5}

\section{Conclusion}

The results of the wind tunnel study demonstrate that the maximum lift coefficient and aerodynamic efficiency of a NACA 2412 airfoil was increased by deploying spoilers at high angles of attack. The maximum lift coefficient was increased by $0.393 \%-2.497 \%$ depending on the spoiler configuration. A spoiler of $10 \%$ chord length increased the maximum lift coefficient by $2.497 \%$ when deflected $8^{\circ}$, by $2.110 \%$ when deflected $15^{\circ}$, and reduced the maximum lift coefficient by $2.783 \%$ when deflected $25^{\circ}$. A spoiler of $15 \%$ chord length produced smaller maximum lift coefficient gains; $0.393 \%$ when deflected $8^{\circ}$, by $1.760 \%$ when deflected $15^{\circ}$, and reduced the maximum lift coefficient by $4.475 \%$ when deflected $25^{\circ}$. Deflecting the spoiler increased the stall angle between $37.658 \%$ and $87.544 \%$ when compared with the clean configuration. The lift gains produced by the spoiler at high angles of attack are caused by an increased suction peak on the airfoil upper surface upstream of the spoiler, and a slight increase in pressure on the lower surface. The upper surface pressure downstream of the spoiler was increased, however the effect of upstream suction increase was greater, thus resulting in a net lift increase. The drag coefficient of spoiler configurations was lower than the clean configuration at angles of attack above $18^{\circ}$. The combination of the increased lift and reduced drag at angles of attack above $18^{\circ}$ created by the spoiler configurations resulted in a higher aerodynamic efficiency than the clean configuration case. A $10 \%$ chord length spoiler deflected at $8^{\circ}$ produced the highest aerodynamic efficiency gains.

The results of the computational study were less conclusive than the wind tunnel experiment. At low angles of attack, the computational study produced consistently higher lift coefficients compared with the the wind tunnel experiment. However the lift-slope was consistent with the wind tunnel experiment lift-slope. The $C_{l}-\alpha$ curve of the $\delta=8^{\circ}, b=10, a=60$ configuration does not experience a peak and fall once stalled. The lift coefficient continues to increase despite the streamline plot suggesting a stalled airfoil. No additional data points were able to be gathered due to convergence problems at the higher angles of attack. The $C_{l}-\alpha$ curve of the $\delta=15^{\circ}, b=10, a=60$ configuration stalls sharply at a lower $C_{l_{\max }}$ than the clean configuration, which is inconsistent with the results from the wind tunnel experiment, which indicate a $C_{l_{\max }}$ increase. The $\delta=8^{\circ}, b=15, a=60$ configuration began experiencing problems with convergence when attempting to gather data at angles of attack beyond $14^{\circ}$. The drag coefficient results were consistent with the wind tunnel experiment at low angles of 
attack. In both the wind tunnel and computational studies, the drag coefficient sharply increased with an increasing angle of attack. This sharp increase occurs at a lower angle of attack in the computational study than in the wind tunnel experiment. This also suggests convergence issues with the higher angles of attack. The convergence problems became more pronounced with higher angles of attack, higher spoiler deflection angles, and a longer spoiler lengths. This indicates that the large amount of recirculation and turbulence effects created by the spoiler were unable to be adequately captured by the computational model. Therefore, no meaningful conclusions can be made regarding the lifting spoiler phenomenon from the computational study until this issue is investigated. This problem could be alleviated by experimenting with different higher-fidelity turbulence models, or by shrinking the timestep size. However the computational resources were not available to perform this task in the required time-frame. 


\section{Appendix 1}

\section{Detailed Geometry Information}

\section{$1.1 \quad$ Airfoil Coordinates}

The coordinates for the NACA 2412 airfoil are listed in 1.1 below. The NACA coordinates are joined together with a spline interpolation from the upper surface trailing edge coordinate (Number 1) to the lower surface trailing edge coordinate (Number 35). A sharp trailing edge was added to the airfoil by the inclusion of one additional coordinate (Number 36). This coordinate is determine so that a line drawn from Number 36 to Number 1 will be tangent with the spline curve, and a line drawn from Number 36 to Number 35 will also be tangent with the spline curve. A plot of this airfoil is shown in 1.1 below. 


\begin{tabular}{c|l|l||c|l|l}
\multicolumn{3}{c||}{ Upper Surface } & \multicolumn{3}{c}{ Lower Surface } \\
\hline Number & $\mathrm{x}(\mathrm{mm})$ & $\mathrm{y}(\mathrm{mm})$ & Number & $\mathrm{x}(\mathrm{mm})$ & $\mathrm{y}(\mathrm{mm})$ \\
\hline 1 & 304.800 & 0.396 & 19 & 3.810 & -5.029 \\
2 & 289.560 & 3.475 & 20 & 7.620 & -6.919 \\
3 & 274.320 & 6.340 & 21 & 15.240 & -9.174 \\
4 & 243.840 & 11.430 & 22 & 22.860 & -10.546 \\
5 & 213.360 & 15.789 & 23 & 30.480 & -11.430 \\
6 & 182.880 & 19.385 & 24 & 45.720 & -12.497 \\
7 & 152.400 & 22.068 & 25 & 60.960 & -12.893 \\
8 & 121.920 & 23.774 & 26 & 76.200 & -12.863 \\
9 & 91.440 & 24.018 & 27 & 91.440 & -12.558 \\
10 & 76.200 & 23.378 & 28 & 121.920 & -11.582 \\
11 & 60.960 & 22.128 & 29 & 152.400 & -10.180 \\
12 & 45.720 & 20.147 & 30 & 182.880 & -8.412 \\
13 & 30.480 & 17.160 & 31 & 213.360 & -6.523 \\
14 & 22.860 & 15.118 & 32 & 243.840 & -4.572 \\
15 & 15.240 & 12.588 & 33 & 274.320 & -2.499 \\
16 & 7.620 & 9.114 & 34 & 289.560 & -1.463 \\
17 & 3.810 & 6.553 & 35 & 304.800 & -0.396 \\
18 & 0.000 & 0.000 & 36 & 307.626 & -0.194
\end{tabular}

Table 1.1: NACA 2412 airfoil coordiantes $(c=0.307626$ with sharp tip) 


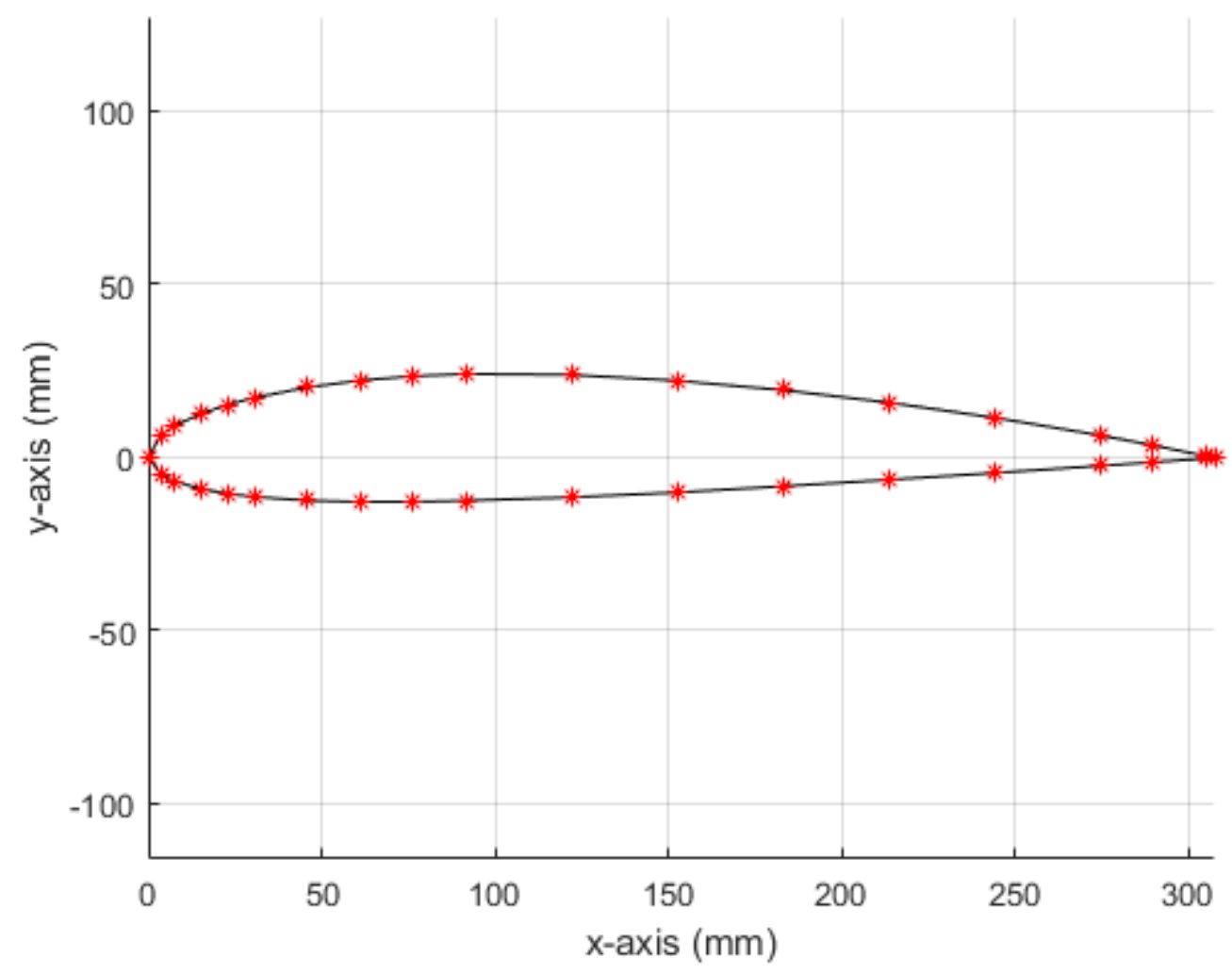

Figure 1.1: NACA 2412 Airfoil Coordinates from Table 1.1

\subsection{Spoiler Geometry}

Figure 1.2 indicates the configuration of the sharp tipped, NACA 2412 airfoil equipped with a spoiler that was tested in this study. 


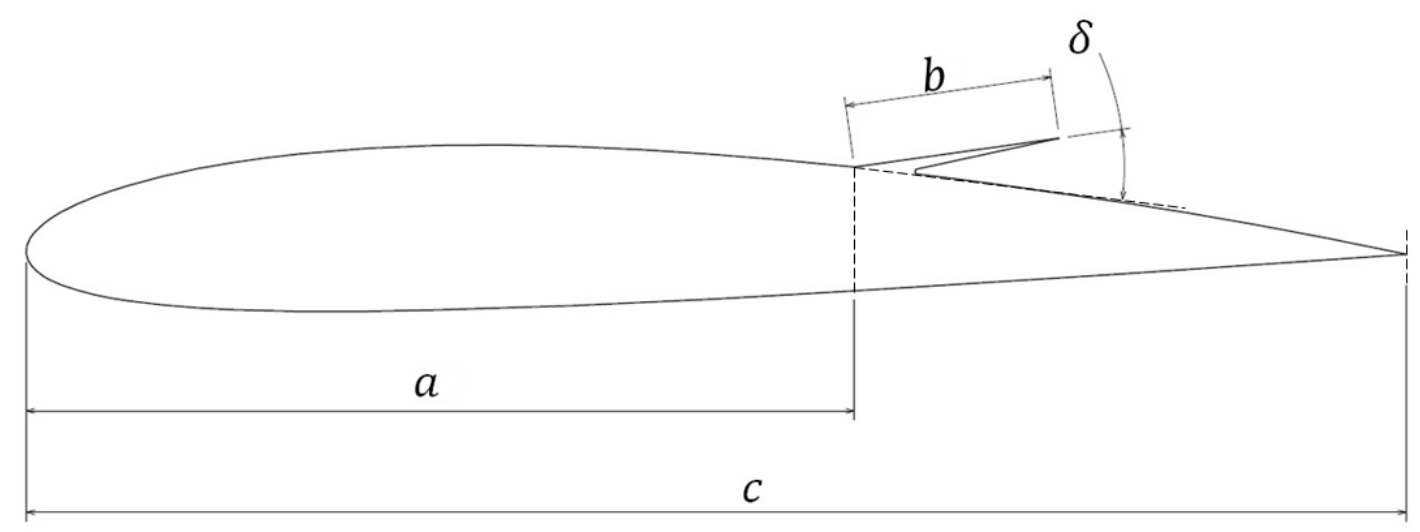

Figure 1.2: Geometry of a sharp tip NACA 2412 airfoil equipped with a spoiler

Where $c$ indicates the airfoil chord (horizontal line from airfoil leading edge to trailing edge). $a$ indicates the spoiler position (distance from the leading edge to the spoiler hinge) in percent chord. $b$ indicates the spoiler length (distance from the spoiler hinge to the spoiler tip) in percent chord.

$\delta$ indicates the spoiler deflection angle (angle measured between upstream surface of the spoiler and an imaginary straight line tangent with the airfoil curve at the spoiler hinge, shown as a dashed line in 1.3 .

The spoiler geometry is defined by three straight lines drawn between four coordinates as shown in Figure 1.3 below. A spoiler equipped airfoil is identical to the clean configuration airfoil with the addition of these three lines. The x-coordinate of Point (1) is equal to $a c$ where $a$, and the y-coordinate of Point (1) is equal to the y-coordinate of the clean airfoil upper surface at $x=a c$. Point (2) is located $b c$ distance away from Point (1) in the direction of $\delta$. Point (4) lays coincident with the clean configuration airfoil's upper surface, and Point (3) is placed in vertical alignment with Point (4). The exact location of Point (3) and (4) are arbitrarily placed for each spoiler configuration at coordinates that aid in creating a high quality mesh. 


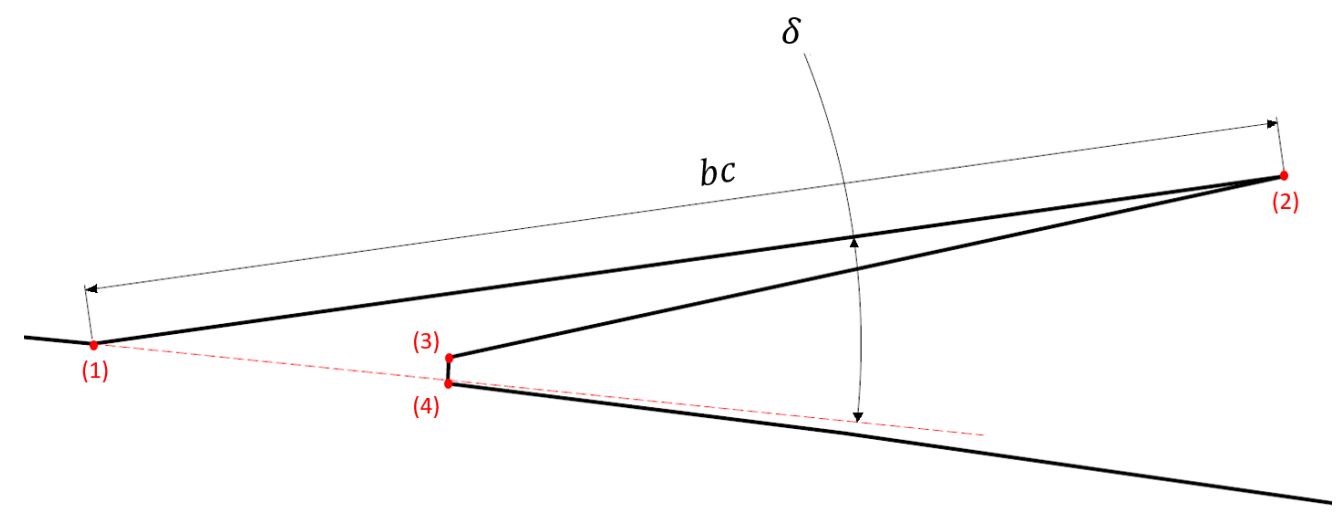

Figure 1.3: Spoiler Section

The coordinates of Point (1), (2), (3) and (4) for the following configurations are shown in Table 1.2 below:

\begin{tabular}{c|c|c|c|c|c|c|c|c}
\multirow{2}{*}{ Configuration Number } & \multicolumn{2}{|c|}{ Point $(1)$} & \multicolumn{2}{c|}{ Point $(2)$} & \multicolumn{2}{c|}{ Point $(3)$} & \multicolumn{2}{c}{ Point $(4)$} \\
\cline { 2 - 9 } & $\mathrm{x}(\mathrm{mm})$ & $\mathrm{y}(\mathrm{mm})$ & $\mathrm{x}(\mathrm{mm})$ & $\mathrm{y}(\mathrm{mm})$ & $\mathrm{x}(\mathrm{mm})$ & $\mathrm{y}(\mathrm{mm})$ & $\mathrm{x}(\mathrm{mm})$ & $\mathrm{y}(\mathrm{mm})$ \\
\hline 1 & - & - & - & - & - & - & - & - \\
2 & 184.576 & 19.209 & 215.32 & 20.281 & 198.22 & 18.684 & 198.22 & 17.683 \\
3 & 184.576 & 19.209 & 214.96 & 24.02 & 193.179 & 19.355 & 193.179 & 18.268 \\
4 & 184.576 & 19.209 & 213.663 & 29.223 & 193.179 & 19.355 & 193.179 & 18.268 \\
5 & 184.576 & 19.209 & 230.692 & 20.818 & 198.22 & 18.684 & 198.22 & 17.683 \\
6 & 184.576 & 19.209 & 230.152 & 26.426 & 193.179 & 19.355 & 193.179 & 18.268 \\
7 & 184.576 & 19.209 & 228.206 & 34.23 & 193.179 & 19.355 & 193.179 & 18.268
\end{tabular}

Table 1.2: Spoiler Coordinates by Configuration 


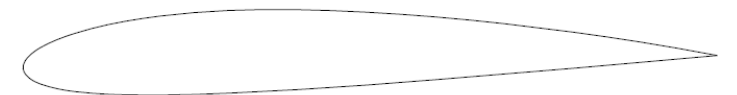

(1)

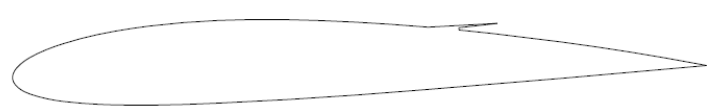

(2)

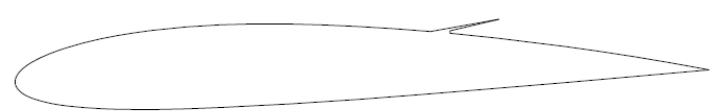

(3)

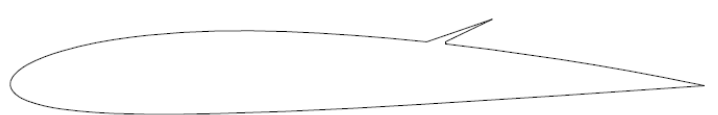

(4)

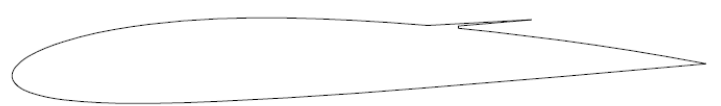

(5)

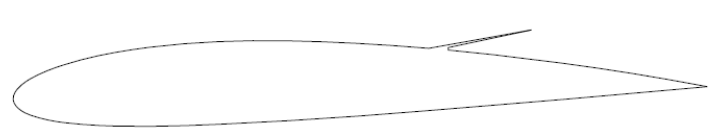

(6)

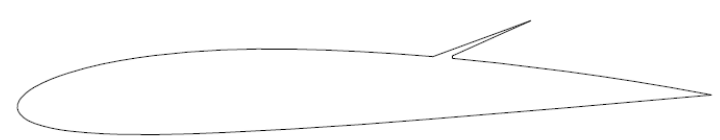

(7)

Figure 1.4: Sketch of the Configurations Described in Table 1.2 


\section{Appendix 2}

\section{Wind Tunnel Model Details}

This appendix section details the design and construction of the wind tunnel model used to gather all necessary data outlined in Chapter 1.2 .3 . 


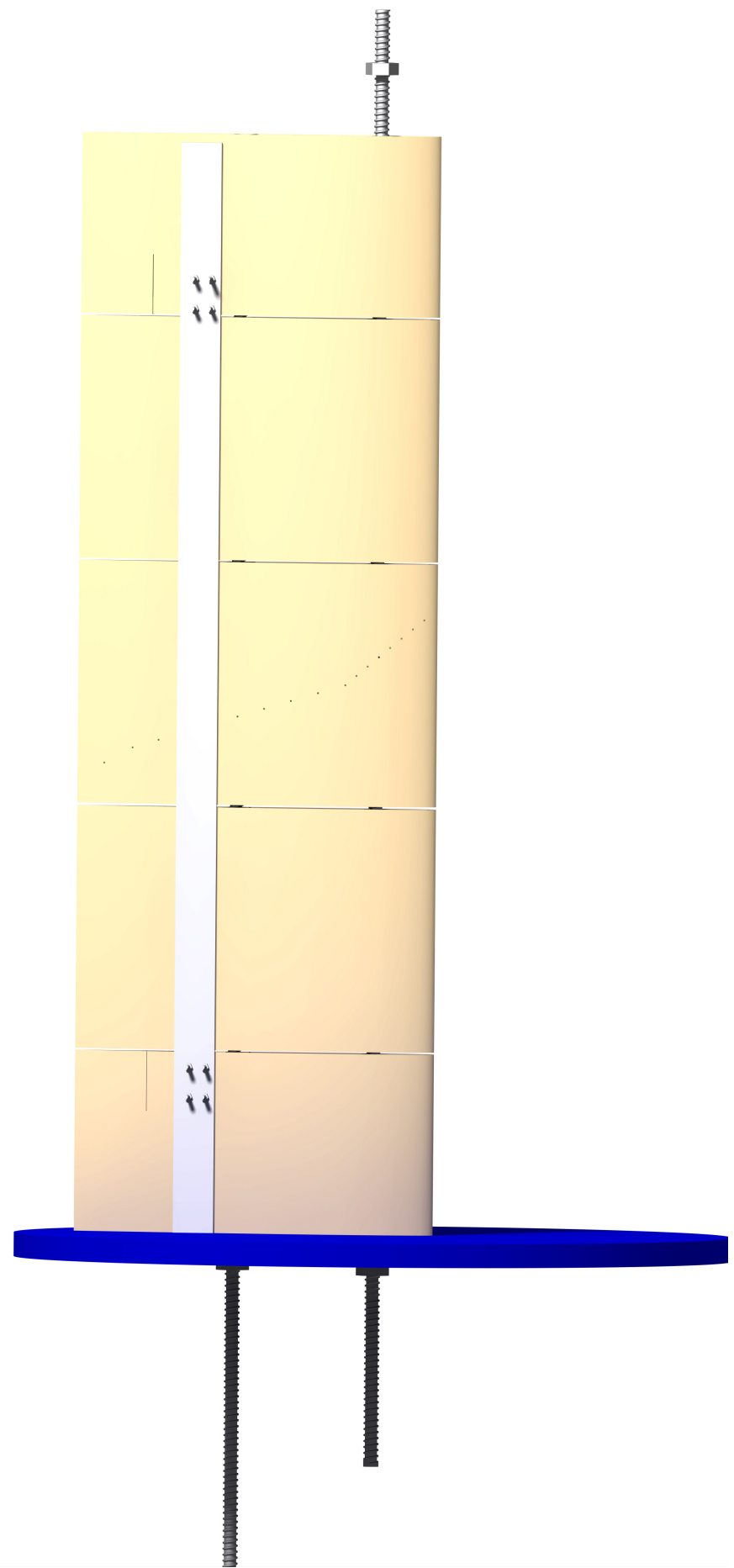

Figure 2.1: CATIA V5 Render of Wind Tunnel Model 


\subsection{Requirements}

The following is a list of requirements imposed on the wind tunnel model design.

1. High as possible Reynolds Number.

At low Reynolds numbers, the flow is initially laminar and is prone to separate even under mild adverse pressure gradient. Under certain flow conditions, the separated flow reattaches and forms a laminar separation bubble while transitioning from laminar to turbulent state. Laminar separation bubbles can modify the effective shape of the airfoil and consequently influence the aerodynamic performance [16]. The objective of this study is to assess the performance of airfoil-spoiler configurations, not to study the behaviour of laminar separation bubbles. It was deemed important to raise the Reynolds number sufficiently such that these bubbles cannot form, thus eliminating a variable from consideration.

2. Quickly and easily change angle of attack

The airfoil must be able to change angle of attack easily without needing to turn the wind tunnel off to do so.

3. Adequately capture airfoil pressure distribution

The model must be able to adequately capture the pressure distribution around the airfoil. The pressure distribution gives useful insight into how the spoiler influences the flow-field, and is used to calculate lift (see Appendix ??).

4. Adequately capture airfoil wake profile

The model must be able to adequately capture the wake profile created by the airfoil. The wake profile is used to gain insight to the airfoil behaviour, and to calculate drag (see Appendix 4 ).

5. Reduce the influence of wind tunnel wall effects

The wind tunnel wall effects outlined in Appendix 5 must be minimized as much as possible. According to Barlow et al [13, excessive errors caused by wall interference can occur in $C_{l_{\text {max }}}$, a critical test value for this experiment, if the chord length exceeds $0.4 h$ where $h$ is the wind tunnel height. The $3 \mathrm{ft} \times 3 \mathrm{ft}(0.9144 \mathrm{~m} \times 0.9144 \mathrm{~m})$ test section of the tunnel limits the maximum possible chord to approximately $1.2 \mathrm{ft}(0.3658 \mathrm{~m})$.

6. Reduce three-dimensional aerodynamic effects as much as possible

The purpose of this study is to determine the spoiler effects on a two-dimensional airfoil. Therefore the model should have minimal span-wise variations, and should have no gaps between the model and the tunnel wall in order to eliminate the need to correct for down-wash effects.

7. Quickly be able to switch from one configuration to another

The model must quickly and easily be changed from one spoiler configuration to another without fully disassembling the model. The model must also be able to put into a clean configuration. 
8. Able to be fabricated with the available $3 \mathrm{D}$ printer

It was desired to fabricate the main body of the model out of ABS plastic using the 3D printer available in the Ryerson Fabrication Lab. This printer has a footprint size of 12 in $\times 12$ in $(0.3048$ $\mathrm{m} \times 0.3048 \mathrm{~m}$ ), however placing the airfoil chord on the diagonal yields some extra space for a slightly longer airfoil.

9. Compatibility with the Ryerson Low-Speed Wind Tunnel

The Ryerson Low-Speed Wind Tunnel test section has a turntable mounted on the lower floor of the tunnel, and an opening mounted above. All support structure must be mounted in these areas. The center of the turntable is aligned with a hole in the top of the wind tunnel.

\section{High structural strength}

The model must be able to bear the aerodynamic loads without breaking.

\subsection{Model Design}

The model sizing was influenced by the objective to have a Reynolds number as large as possible, and by the constraints from the wind tunnel size, and 3D printer footprint. The model was designed with a $0.3048 \mathrm{~m}(1 \mathrm{ft})$ chord length. With the addition of the sharp tip to the NACA 2412 airfoil, the chord length was increased to $0.307626 \mathrm{~m}$. This combined with a tunnel speed of approximately $47 \mathrm{~m} / \mathrm{s}$ resulted in a Reynolds number of roughly $1.0 \times 10^{6}$ at standard atmospheric conditions (however heating from the wind tunnel fan lowered this significantly). To eliminate 3D aerodynamic effects, the model was designed to span the entire wind tunnel $(3 \mathrm{ft}$ or $0.9144 \mathrm{~m})$. However tall 3D printed parts tend to warp due to uneven cooling of the plastic. The model was printed with a vertical orientation to ensure a smooth surface, thus the span was limited in size to 8 inches (as per guidance from Mr. Peter Bradley). To achieve a $3 \mathrm{ft}$ long span, the model was broken up into 5 separate primary airfoil parts. Three 8 inch parts, and two 6 inch parts. The seems in the 5 pieces were covered up using Tuck Tape. The two pieces located on either end contained gaps for a spoiler holder insert piece. The center airfoil piece contained 31 holes for pressure taps. The airfoil pieces were hollow to allow tubing to run through the span of the model and out the wind tunnel. Two rib sections were added to increase the structural strength of the model. Each rib contained a $\frac{1}{2}$ inch hole for a threaded steel rod. 


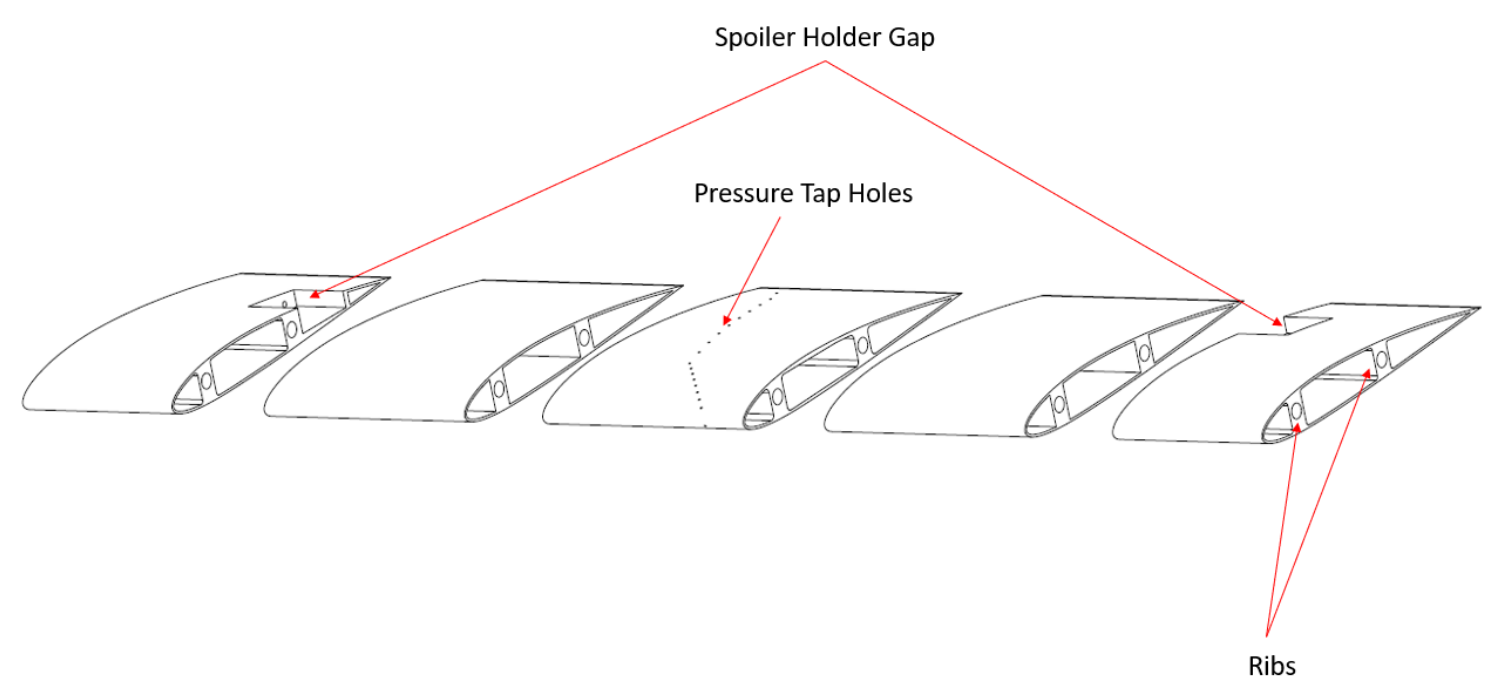

Figure 2.2: Drawing of Airfoil Section Pieces

The steel rods provided structural support to resist the aerodynamic loads, and were the connection points between the model and the turntable. Both rods were mounted to the turntable, and one rod went through the model and the hole in the top of the tunnel. The other rod terminated inside the model and was held in place by a nut. This allowed the model to be rotated with the turntable, thus allowing the angle of attack to be easily changed. A diagram of how the model mounts to the wind tunnel is shown in the figure below: 


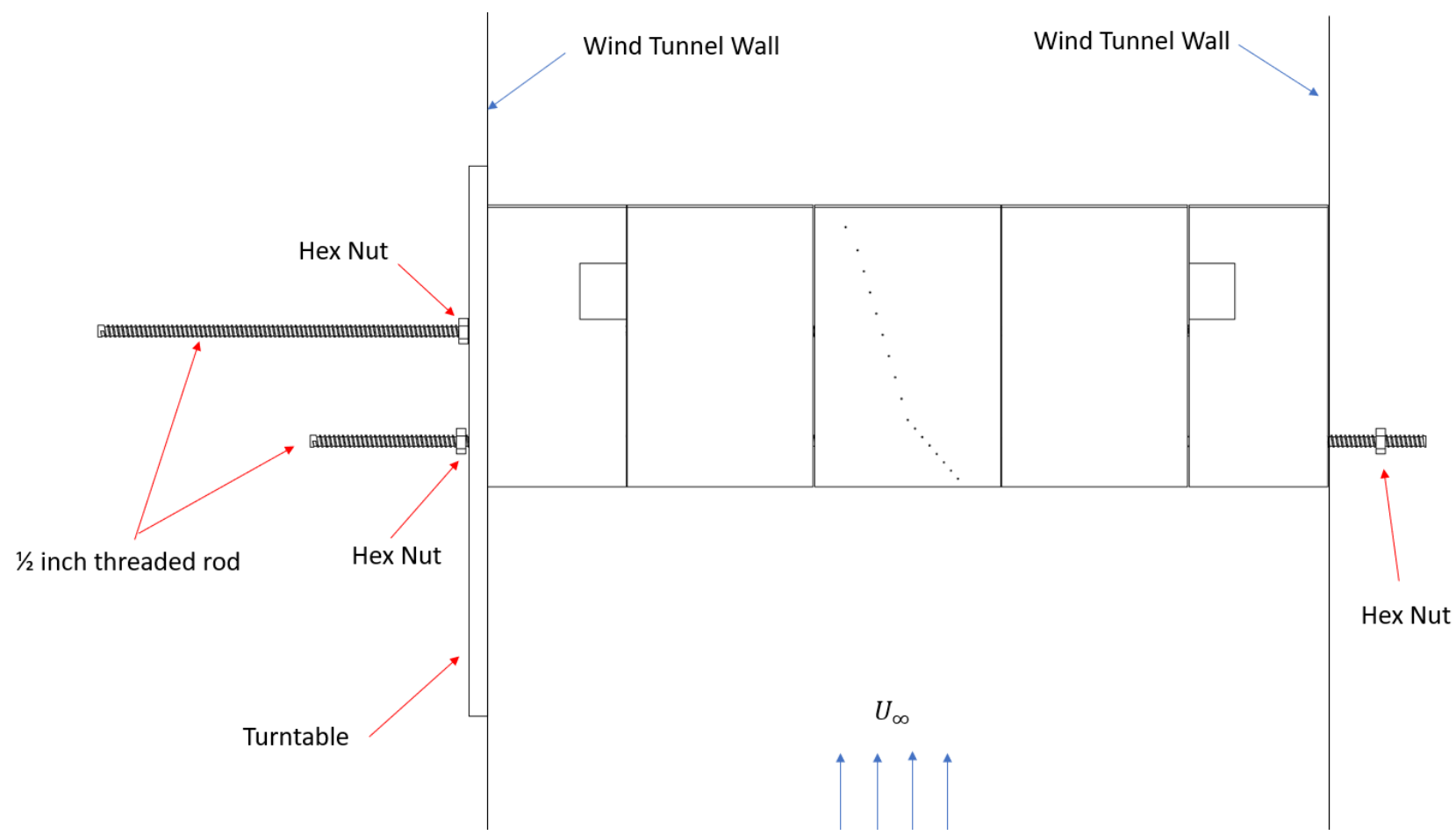

Figure 2.3: Diagram of Model Mount to Wind Tunnel

Brass tubes $\left(\frac{1}{16}\right.$ inch) bent into elbow joints were inserted into the pressure tap holes with epoxy resin. Care was taken to ensure that the tubing was flush with the outside airfoil surface. Poly-urethane tubing was connected to the ends of the brass tubes and ran through the span of the model to the pressure transducers located outside of the wind tunnel. A higher density of pressure taps was placed near the leading edge of the airfoil to better capture the large changes in pressure expected in this region. The pressure taps were staggered in the span-wise direction (z-direction) so that airflow disturbances caused by a pressure tap would have a minimal impact on other taps. Since this experiment was twodimensional, the z-coordinate of the pressure taps were not involved with any calculations. The pressure tap locations are recorded in the table below: 


\begin{tabular}{c|c|c|c|} 
Tap Number & $\mathrm{x}(\mathrm{mm})$ & $\mathrm{y}(\mathrm{mm})$ & $\mathrm{z}(\mathrm{mm})$ \\
\hline 1 & 258.367 & 9.105 & 46.786 \\
2 & 235.377 & 12.709 & 53.592 \\
3 & 212.335 & 15.923 & 60.413 \\
4 & 189.241 & 18.708 & 67.25 \\
5 & 166.095 & 20.976 & 74.102 \\
6 & 142.907 & 22.715 & 80.967 \\
7 & 119.686 & 23.851 & 87.842 \\
8 & 96.441 & 24.11 & 94.723 \\
9 & 73.212 & 23.186 & 101.6 \\
10 & 63.701 & 22.405 & 109.621 \\
11 & 54.205 & 21.343 & 117.628 \\
12 & 44.731 & 19.991 & 125.617 \\
13 & 35.294 & 18.258 & 133.575 \\
14 & 25.925 & 15.982 & 141.476 \\
15 & 16.652 & 13.115 & 149.296 \\
16 & 7.631 & 9.12 & 156.903 \\
17 & 0.164 & -0.788 & 163.2 \\
18 & 9.215 & -7.483 & 155.567 \\
19 & 19.735 & -10.074 & 146.696 \\
20 & 30.395 & -11.422 & 137.707 \\
21 & 41.086 & -12.26 & 128.691 \\
22 & 51.791 & -12.717 & 119.664 \\
23 & 62.501 & -12.907 & 110.632 \\
24 & 73.212 & -12.895 & 101.6 \\
25 & 99.251 & -12.343 & 93.891 \\
26 & 125.282 & -11.45 & 86.185 \\
27 & 151.302 & -10.239 & 78.482 \\
28 & 177.308 & -8.752 & 70.783 \\
29 & 203.307 & -7.149 & 63.086 \\
30 & 229.306 & -5.519 & 55.389 \\
31 & 255.299 & -3.798 & 47.694
\end{tabular}

Table 2.1: Pressure Tap Locations

The spoilers were made using 12 gauge cold rolled sheet metal cut with to a length of $3 \mathrm{ft} \times 0.1 \mathrm{ft}$ $(0.1 \mathrm{c}$ length spoiler $)$ and $3 \mathrm{ft} \times 0.15 \mathrm{ft}(0.15 \mathrm{c}$ length spoiler $)$. The spoiler was attached to the model using two spoiler-holder pieces (fabricated out of 3D printed plastic) that fit into gaps in the two airfoil end pieces. The spoiler attached to the holder pieces using four bolts. The spoiler holders set the spoiler 
at a given deflection angle. Spoiler holder pieces were fabricated that set the spoiler at $8^{\circ}, 15^{\circ}$, and $25^{\circ}$. Inserts were also printed that sealed the gap in the airfoil end piece to create a clean configuration model. This system allows for quick transition from one spoiler configuration to another.
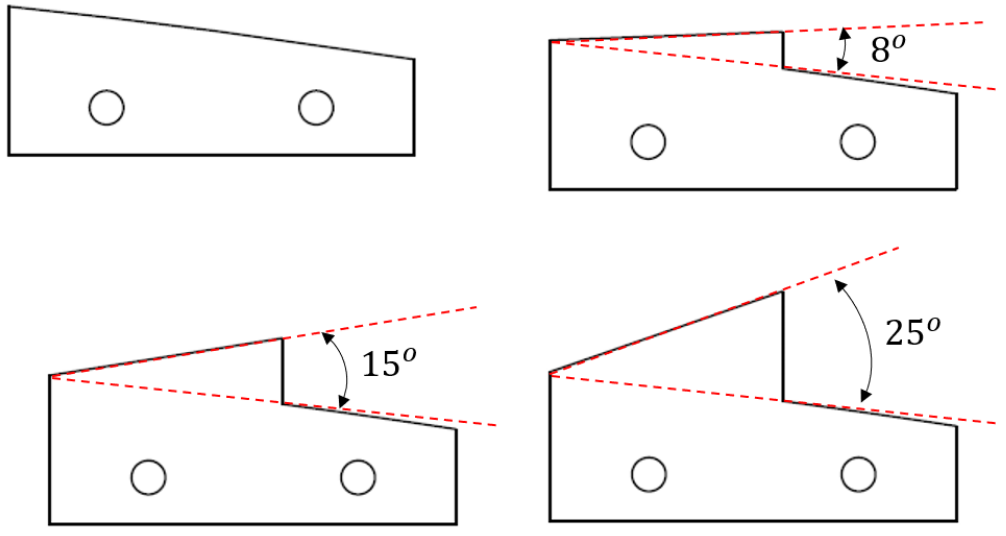

Figure 2.4: Front view drawing of four different spoiler holder insert parts (clean, $8^{\circ}, 15^{\circ}, 25^{\circ}$ ). 

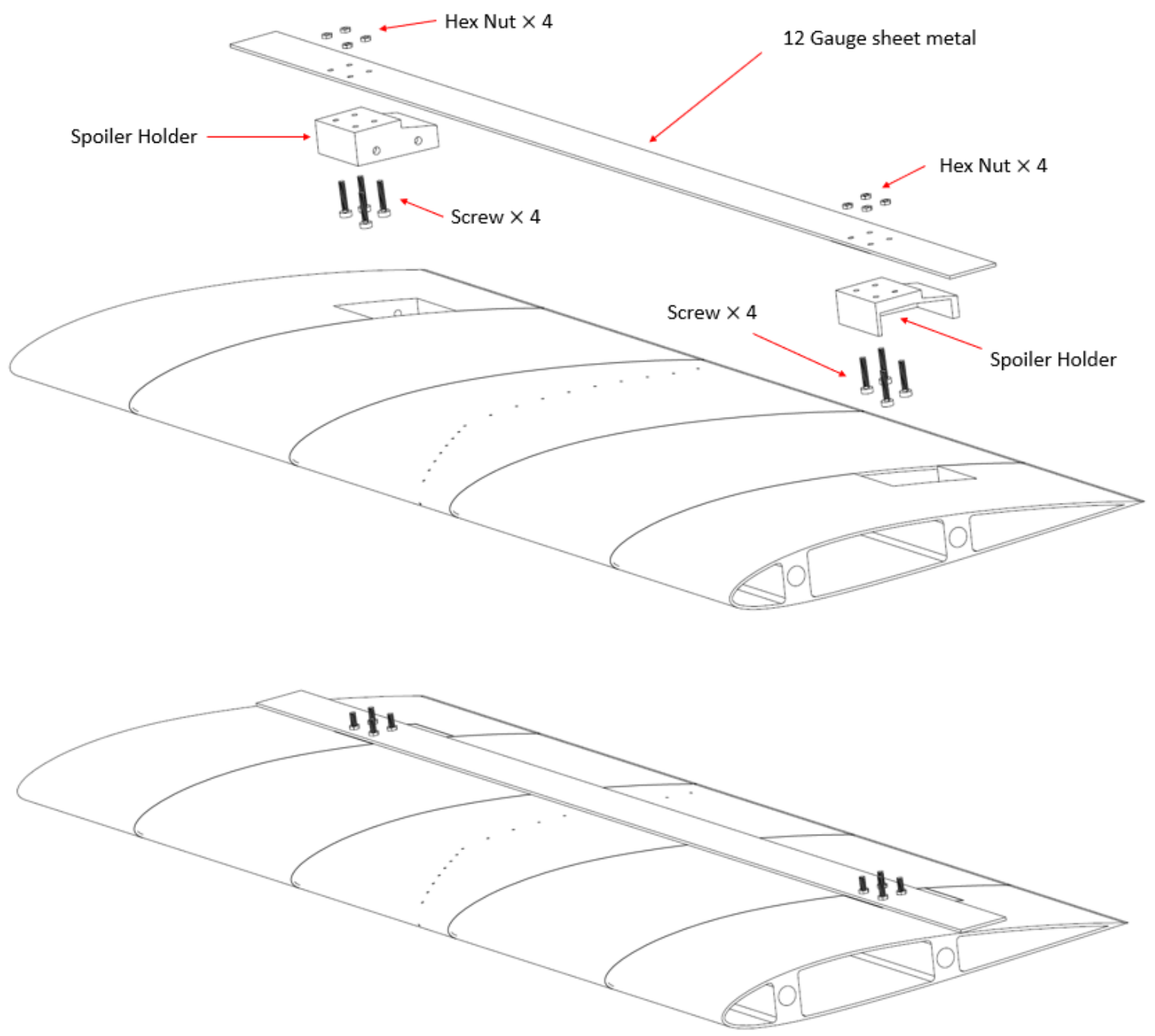

Figure 2.5: Drawing of spoiler system assembly.

To capture the wake profile, a wake rake device was constructed using two stacked brass plates, $\frac{1}{16}$ inch copper tubing, and polyurethane tubing. The lower brass plate was $10 \mathrm{inch} \times 4$ inch, and the upper brass plate was 10 inch $\times 1$ inch. The two brass plates were soldered together to create a ledge. The copper tubes cut into 4 inch long pieces were soldered to this ledge. A $\frac{1}{4}$ of copper tube overhung the edge, thus allowing the polyurethane to be affixed to the copper tube with epoxy resin. $2 \frac{3}{4}$ inch of copper tubing extended into the airflow ahead of the brass plates. The wake rake was directly connected to the airfoil model using two aluminum arm pieces which had been constructed for a previous experiment. The arm pieces contained a $\frac{3}{8}$ hole. A $\frac{3}{8}$ steel threaded rod was put through these holes, through a $\frac{3}{8}$ hole placed in the lower brass plate, and affixed with 4 hex nuts. Four different spar holes were placed 
in the wake rake so that the rake location could be changed to be aligned with the wake.

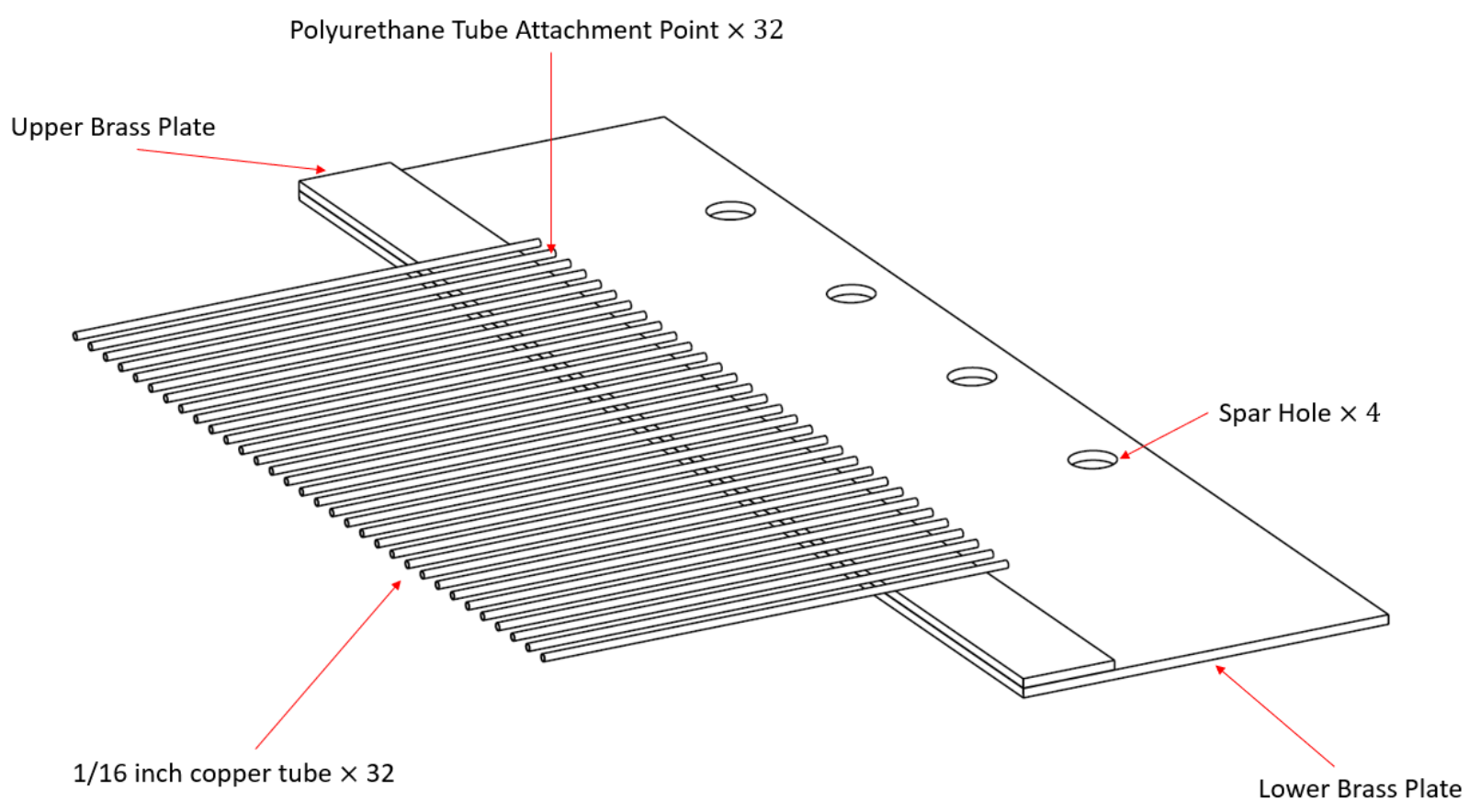

Figure 2.6: Wake rake drawing 


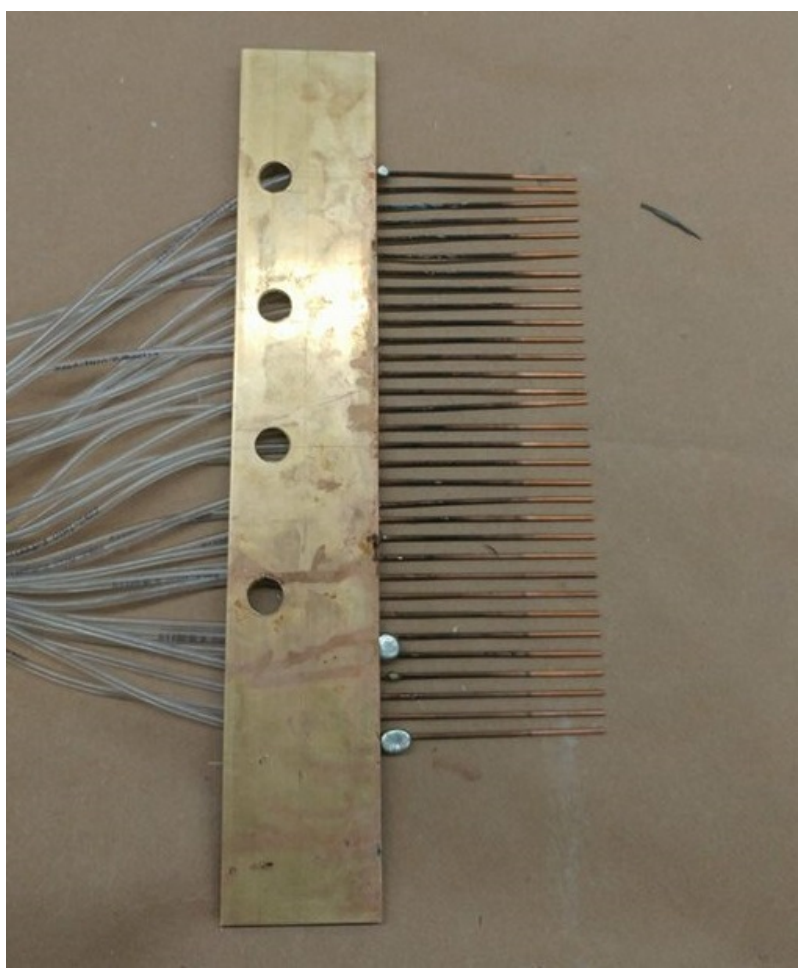

Figure 2.7: Wake rake with polyurethane tubing attached.

The completed model mounted in the Ryerson Large Wind Tunnel is shown in 2.8 below. 


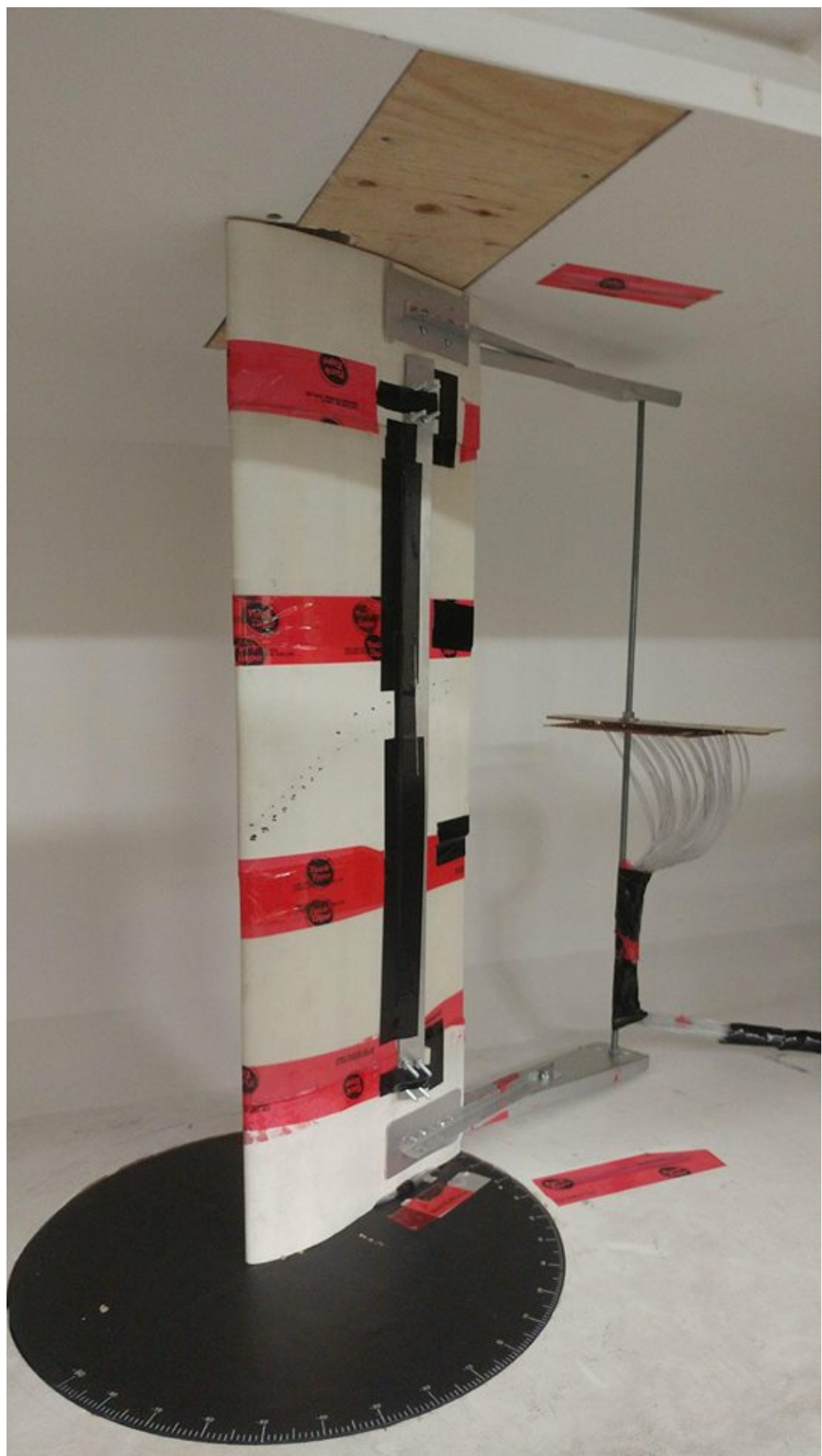

Figure 2.8: Wind tunnel model fully assembled and mounted in the Ryerson Large Wind Tunnel. 


\section{Appendix 3}

\section{Pressure Taps Error Analysis}

Equation 2.12 is more accurate with a larger number of pressure taps. Since physical constraints limit the number of pressure taps to 31 , a certain degree of error is present in the calculation. The CFD solution of the clean configuration airfoil was used to quantify this source of error.

CFX5Post (part of the ANSYS 15.0 suite) was used to extract the lift coefficient and pressure distribution of the clean configuration NACA 2412 airfoil over angles of attack ranging from 0 to 17.5. The pressure distribution extracted from CFX5Post was then reduced to 31 pressure points located at the the same x-coordinate of the real-world model's pressure taps (and $C_{P}=0$ at the trailing edge). Lift was then calculated using equation 2.12, and compared with the lift calculated from the force calculator in CFX5Post.

Using the above process, it was found that the amount of error could be reduced by using a spline interpolation of the pressure points, and then integrating the spline curve to find lift. The lift coefficients outputted by CFX5Post, lift coefficients calculated from a pressure integration using only 32 data points, and the lift coefficients calculated from a numerical integration of a spline curve fitted to the 32 data points are shown in Table 3.1 below. The percent difference between the integrated lift coefficient values and the CFX5Post values are shown in Table 3.2 


\begin{tabular}{c|c|c|c|}
$\alpha(\mathrm{deg})$ & $C_{l}$ From CFX5Post & $\begin{array}{c}C_{l} \text { From Pressure Integration } \\
\text { (No Spline Interpolation) }\end{array}$ & $\begin{array}{c}C_{l} \text { From Pressure Integration } \\
\text { (With Spline) }\end{array}$ \\
\hline 0.0 & 0.229 & 0.235 & 0.216 \\
2.0 & 0.442 & 0.450 & 0.419 \\
4.0 & 0.696 & 0.692 & 0.639 \\
8.0 & 1.027 & 0.995 & 0.933 \\
10.0 & 1.193 & 1.169 & 1.076 \\
12.0 & 1.337 & 1.331 & 1.210 \\
13.0 & 1.397 & 1.346 & 1.244 \\
13.5 & 1.418 & 1.355 & 1.256 \\
14.0 & 1.431 & 1.361 & 1.263 \\
14.5 & 1.441 & 1.367 & 1.268 \\
15.0 & 1.449 & 1.373 & 1.271 \\
15.5 & 1.448 & 1.369 & 1.266 \\
16.0 & 1.437 & 1.355 & 1.252 \\
16.5 & 1.414 & 1.334 & 1.229 \\
17.0 & 1.375 & 1.295 & 1.191 \\
17.5 & 1.324 & 1.246 & 1.143
\end{tabular}

Table 3.1: $C_{l}$ values calculated from trapezodial integration of pressure, with and without spline interpolation compared with values from CFX5Post 


\begin{tabular}{c|c|c}
$\alpha(\mathrm{deg})$ & $\begin{array}{c}\text { \% Difference } \\
\text { (No Spline Interpolation) }\end{array}$ & $\begin{array}{c}\text { \% Difference } \\
\text { (With Spline) }\end{array}$ \\
\hline 0.0 & 5.743 & 2.813 \\
2.0 & 5.054 & 1.783 \\
4.0 & 8.226 & 0.578 \\
8.0 & 9.135 & 3.071 \\
10.0 & 9.858 & 2.033 \\
12.0 & 9.554 & 0.486 \\
13.0 & 10.923 & 3.617 \\
13.5 & 11.387 & 4.456 \\
14.0 & 11.754 & 4.908 \\
14.5 & 12.058 & 5.191 \\
15.0 & 12.268 & 5.227 \\
15.5 & 12.544 & 5.443 \\
16.0 & 12.834 & 5.659 \\
16.5 & 13.081 & 5.678 \\
17.0 & 13.382 & 5.801 \\
17.5 & 13.678 & 5.897
\end{tabular}

Table 3.2: Trapezodial integration lift coefficient percent difference (compared with CFX5Post value) with and without spline interpolation 


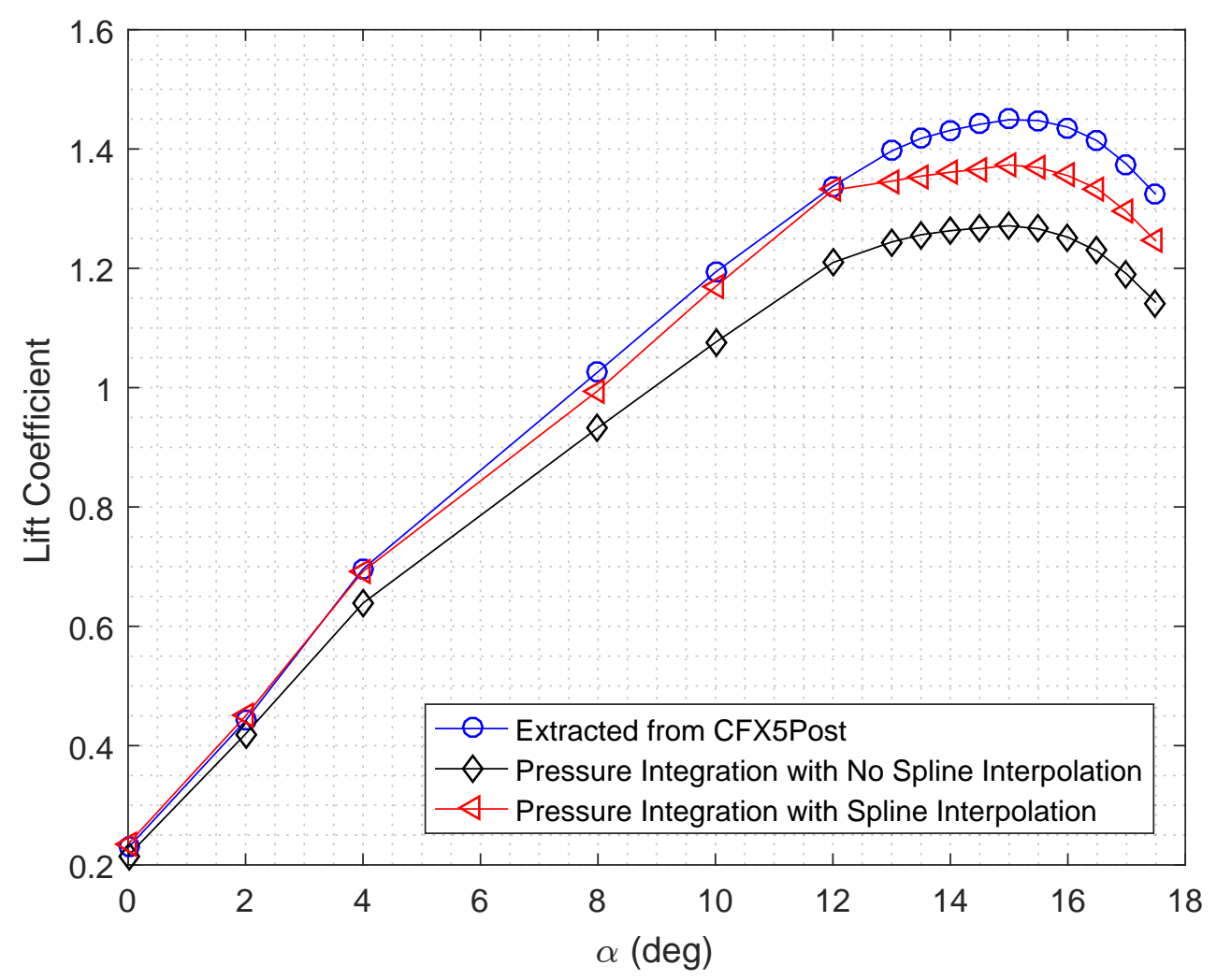

Figure 3.1: $C_{l}-\alpha$ curve containing data from Table 3.1.

Table 3.2 and Figure 3.1 indicate that integrating a spline-fit curve decreased the discrepancy between the integrated lift coefficient and the CFX5Post output lift coefficient. Therefore this method was selected for use. This method shows good agreement with the CFX5Post lift coefficient at lower angles of attack. At higher angles, the pressure integration method under predicts lift. The cause of this difference is evident when comparing the full pressure distribution exported from CFX5Post with the spline interpolation curve at low and at high angles of attack. At high angles of attack, a large, narrow suction peak develops near the leading edge of the airfoil as shown in Figure 3.2 This narrow suction peak is not present at lower angles of attack as shown in Figure 3.3 resulting in better agreement at lower angles of attack. This source of error could be mitigated by placing more pressure taps in the leading edge of the airfoil, however constraints on the model size prevented placing more pressure taps in this region.

In conclusion, a maximum error of approximately $5.897 \%$ is expected when calculating the lift coefficient from the 32 pressure taps located on the surface of the wind tunnel model by using a trapezoidal integration scheme of a spline interpolation curve of the 32 pressure taps. At higher angles of attack, more of the lift is generated by an increasingly large, narrow suction peak. This suction peak is difficult 
to capture given the pressure tap spacing. Therefore error is expected to increase with higher angle of attack.

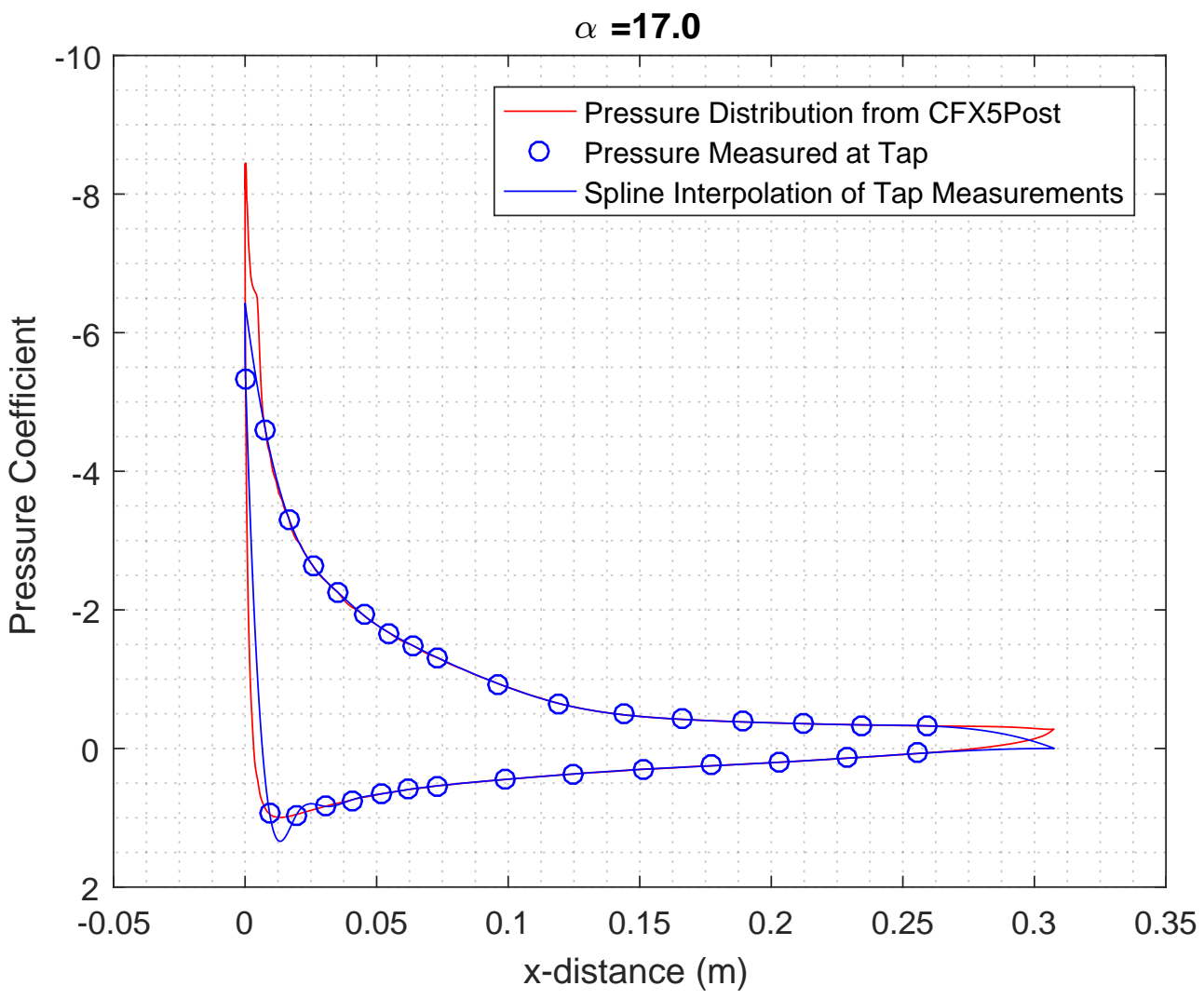

Figure 3.2: Comparison between $C_{p}$ plot extracted CFX5Post and spline interpolation of 32 pressure tap locations $\left(\alpha=17^{\circ}\right)$ 


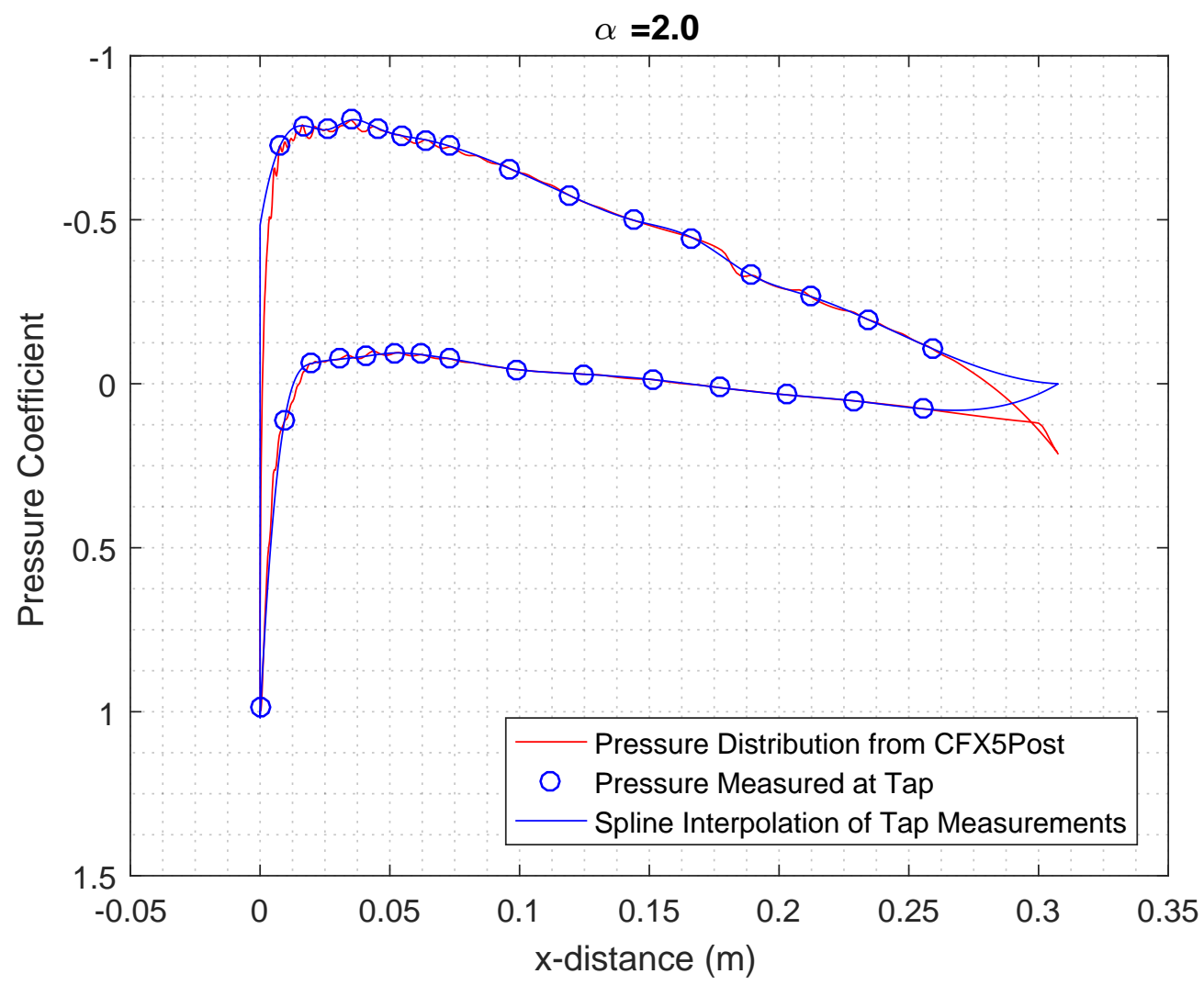

Figure 3.3: Comparison between $C_{p}$ plot extracted CFX5Post and spline interpolation of 32 pressure tap locations $\left(\alpha=2^{o}\right)$ 


\section{Appendix 4}

\section{Wake Rake Error Analysis}

Equation 2.16 is more accurate with more pressure tubes to better capture the wake. Since the number of pressure tubes was limited to 32, a certain degree of error is present in the calculation. The CFD solution of the clean configuration airfoil was used to quantify this source of error.

CFX5Post (part of the ANSYS 15.0 suite) was used to extract the drag coefficient and the wake profile of the clean configuration NACA 2412 airfoil over angles of attack ranging from 0 to 17.5. The wake profile extracted from CFX5Post was then reduced to 32 velocity measurements behind the airfoil. Drag coefficient was then calculated using equation 2.16, and compared with the drag calculated from the force calculator in CFX5Post. The results of this comparison are shown in figure 4.1 and table 4.1 The largest errors occurred at lower $\alpha$. The wake was thinner at these angles and thus contained fewer data points to define the wake shape. At higher $\alpha$, there were more data points inside the wake, which created a more accurate picture of the wake shape, and therefore enabled more accurate drag coefficient calculation. 


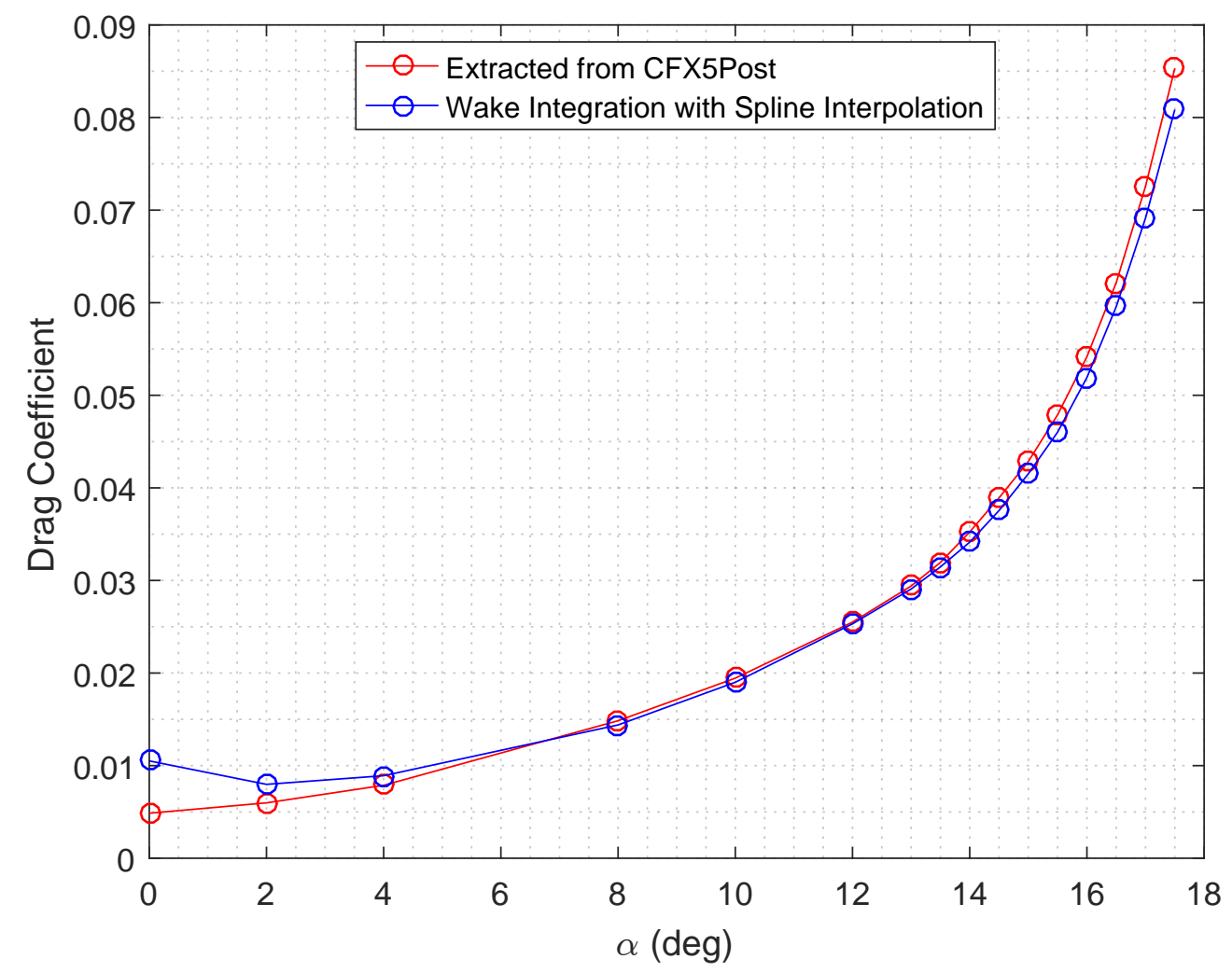

Figure 4.1: $C_{d}-\alpha$ curve containing data from Table 4.1 


\begin{tabular}{c|c|c|c}
$\alpha(\mathrm{deg})$ & $C_{d}$ from CFX5Post & $C_{d}$ From Wake Integration of Spline Fit & Percent Difference \\
\hline 0.0 & 0.00486 & 0.01051 & 116.305 \\
2.0 & 0.00598 & 0.00798 & 33.435 \\
4.0 & 0.00788 & 0.00891 & 13.160 \\
8.0 & 0.01485 & 0.01439 & 3.070 \\
10.0 & 0.01945 & 0.01900 & 2.293 \\
12.0 & 0.02550 & 0.02532 & 0.713 \\
13.0 & 0.02940 & 0.02907 & 1.105 \\
13.5 & 0.03194 & 0.03146 & 1.505 \\
14.0 & 0.03524 & 0.03414 & 3.141 \\
14.5 & 0.03892 & 0.03755 & 3.511 \\
15.0 & 0.04285 & 0.04152 & 3.094 \\
15.5 & 0.04789 & 0.04602 & 3.910 \\
16.0 & 0.05416 & 0.05192 & 4.140 \\
16.5 & 0.06217 & 0.05957 & 4.178 \\
17.0 & 0.07258 & 0.06914 & 4.733 \\
17.5 & 0.08532 & 0.08085 & 5.237
\end{tabular}

Table 4.1: Trapezodial integration drag coefficient percent difference (compared with CFX5Post value) 


\section{Appendix 5}

\section{Wind Tunnel Correction Factors}

Wind tunnel experiments are performed to determine how a geometry behaves in an unbounded air stream, however, the presence of the solid tunnel boundaries causes variations in the flow field from a true free-air case. This can result in differences in the aerodynamic forces acting on the geometry. Standard correction factors described in Barlow et. al 's textbook Low-Speed Wind Tunnel Testing [13. are commonly used to make these corrections. The presence of the wind tunnel walls creates four phenomena that effect the accuracy of a two-dimensional, tunnel spanning, wind tunnel experiment. These phenomena are

1. Horizontal Buoyancy

2. Solid Blockage

3. Wake Blockage

4. Streamline Curvature

Horizontal buoyancy is caused by boundary layer formation on the wind tunnel walls, which creates a static pressure gradient in the flow direction. This leads to higher pressure forming upstream of the model than downstream. This pressure gradient is independent of the effects of the model itself, causing the force measurements in flow direction to be larger than in a pure free-stream case. This effect increases in severity the larger the model is in the flow direction. This effect is described with the equation:

$$
\frac{d p}{d l}=\frac{k(\rho / 2) U^{2}}{B}
$$

where $l$ is the jet length

$B$ is the jet width

$k$ is a non-dimensional factor that must be determined experimentally for the tunnel. 
The total drag increment acting on an airfoil spanning a wind tunnel can be estimated using the following relation determined by Allen and Vincenti [3], assuming that the pressure gradient is linear.

$$
\begin{gathered}
\sigma=\frac{\pi^{2}}{48}\left(\frac{c}{h}\right)^{2} \\
D_{B}=\frac{6 h^{2}}{\pi} \Lambda \sigma p^{\prime} \\
p^{\prime}=\frac{d p}{d x}
\end{gathered}
$$

where $D_{B}$ is the drag increment due to horizontal buoyancy

$c$ is the airfoil chord length

$h$ is the wind tunnel height

$\Lambda$ is a body shape factor determined from Figure 1

$p^{\prime}$ is the linear pressure gradient

\begin{tabular}{l|l}
$t / c$ & $\Lambda$ \\
\hline 0.06 & 0.111 \\
0.09 & 0.172 \\
0.12 & 0.237 \\
0.15 & 0.305 \\
0.18 & 0.376 \\
0.21 & 0.450
\end{tabular}

Table 5.1: Body Shape Factor change with Thickness ratio for NACA 4-Digit Airfoils [3]

Solid blockage is caused by the constriction of the flow of air around the model. As per the principles of continuity, this causes an increase in air velocity in the region of the model more than would be anticipated if the model were in free-stream. The velocity increase is approximated to be constant over the model. The effect is dependent on the model thickness, and is independent of camber. Solid blockage is quantified by the parameter $\epsilon_{s b}$ and is defined by the following equation:

$$
\epsilon_{s b}=\frac{\Delta V}{V_{u}}
$$

where $\Delta V$ is the change in velocity

$V_{u}$ is the uncorrected velocity

The blockage effects due to a two-dimensional airfoil spanning a wind tunnel is defined by the 
following equation from Allen and Vincenti [3]:

$$
\epsilon_{s b}=\Lambda \sigma
$$

Any body in a fluid flow will create a wake downstream that will have a mean velocity that is lower than that of the free-steam. In a free-stream case, the velocity magnitude outside of the wake would be the same as the velocity magnitude in the oncoming airflow. However, due to the principles of continuity and the tunnel walls, the airflow outside of the wake must be moving at higher speeds than the oncoming air. This results in a higher airspeed at the model surface. This phenomenon is called wake blockage.

This effect can be accounted for using the following relation:

$$
\epsilon_{w b}=\frac{\Delta V}{V}=\frac{c / h}{2} C_{d u}
$$

where $C_{d u}$ is the uncorrected drag coefficient.

The wind tunnel walls prevent the curvature of air that would occur due to a lifting body in freestream conditions. This causes the lifting body to have a larger effective camber, called streamline curvature. This causes an increase in lift, moment about the quarter-chord, and angle of attack. Allen and Vincenti [3] describe this effect using the following equations:

$$
\begin{gathered}
\Delta C_{l_{s c}}=\sigma C_{l_{u}} \\
\Delta C_{m_{1 / 4 s c}}=\frac{\sigma}{4} \Delta C_{l_{s c}} \\
\Delta \alpha_{s c}=\frac{57.3 \sigma}{2 \pi}\left(C_{l_{u}}+4 C_{m_{1 / 4}}\right)
\end{gathered}
$$

where $\Delta C_{l_{s c}}$ is the lift coefficient increment due to streamline curvature $C_{l_{u}}$ is the uncorrected lift coefficient $\Delta C_{m_{1 / 4 s c}}$ is the moment coefficient about the quarter chord increment due to streamline curvature $\Delta \alpha_{s c}$ is the angle of attack increase increment due to streamline curvature $C_{m_{1 / 4 u}}$ is the uncorrected moment coefficient about the quarter chord.

The effects of solid blockage, wake blockage and streamline curvature can be combined into a single set of equations (horizontal bouncy must be dealt with separately) for a two-dimensional wind tunnel experiment.

$$
\epsilon=\epsilon_{s b}+\epsilon_{w b}
$$

The velocity increasing effects of the combined wake and solid blockages must be applied to every 
aerodynamic property involving velocity.

$$
V=V_{u}(1+\epsilon) q=q_{u}(1+\epsilon) \operatorname{Re}=\operatorname{Re}_{u}(1+\epsilon)
$$

Combining the blockage effects with streamline curvature yields the following equations:

$$
\begin{gathered}
\alpha=\alpha_{u}+\frac{57.3 \sigma}{2 \pi}\left(C_{l_{u}}+C_{m_{1 / 4}}\right) \\
C_{l}=C_{l_{u}}(1-\sigma-2 \epsilon) \\
C_{m_{1 / 4}}=C_{m_{1 / 4 u}}(1-2 \epsilon)+\frac{1}{4} \sigma C_{l} \\
C_{d_{o}}=C_{d_{o_{u}}}\left(1-3 \epsilon_{s b}-2 \epsilon_{w b}\right)
\end{gathered}
$$




\section{Appendix 6}

\section{Pressure Coefficient Plots from Wind Tunnel Experiment}

\subsection{Clean Configuration}

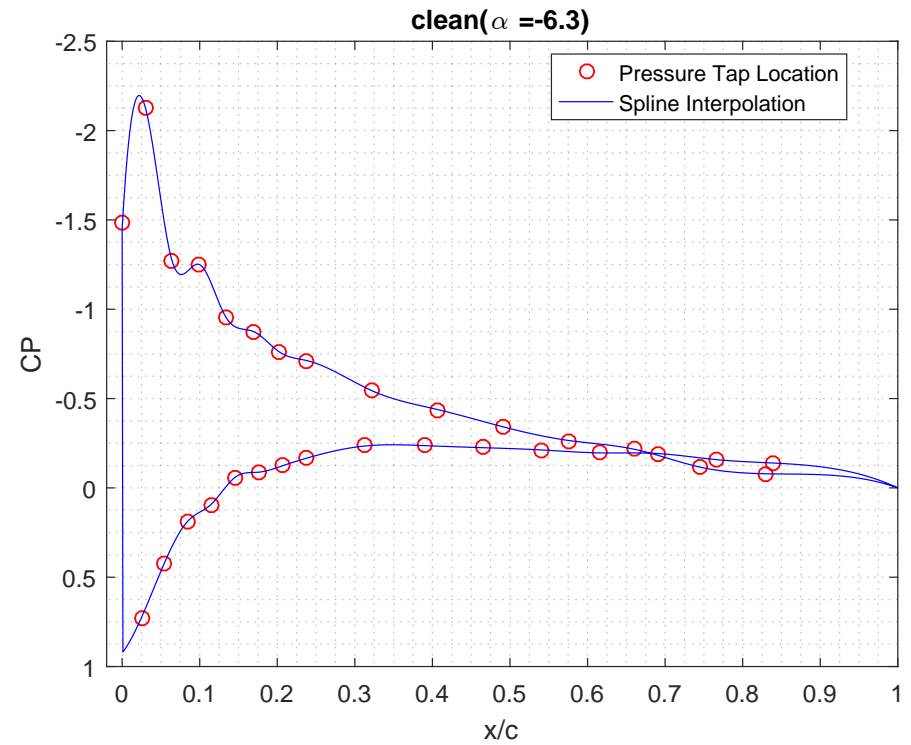

Figure 6.1: $C_{p}$ plot clean airfoil at $\alpha \approx-6.3^{\circ}$ 


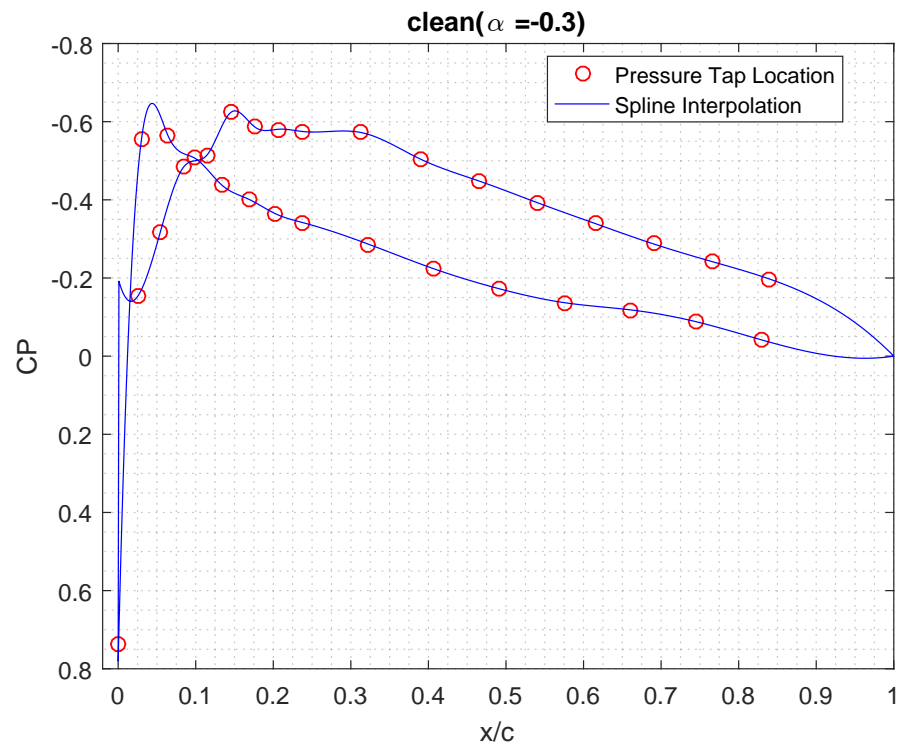

Figure 6.2: $C_{p}$ plot clean airfoil at $\alpha \approx-0.3^{\circ}$

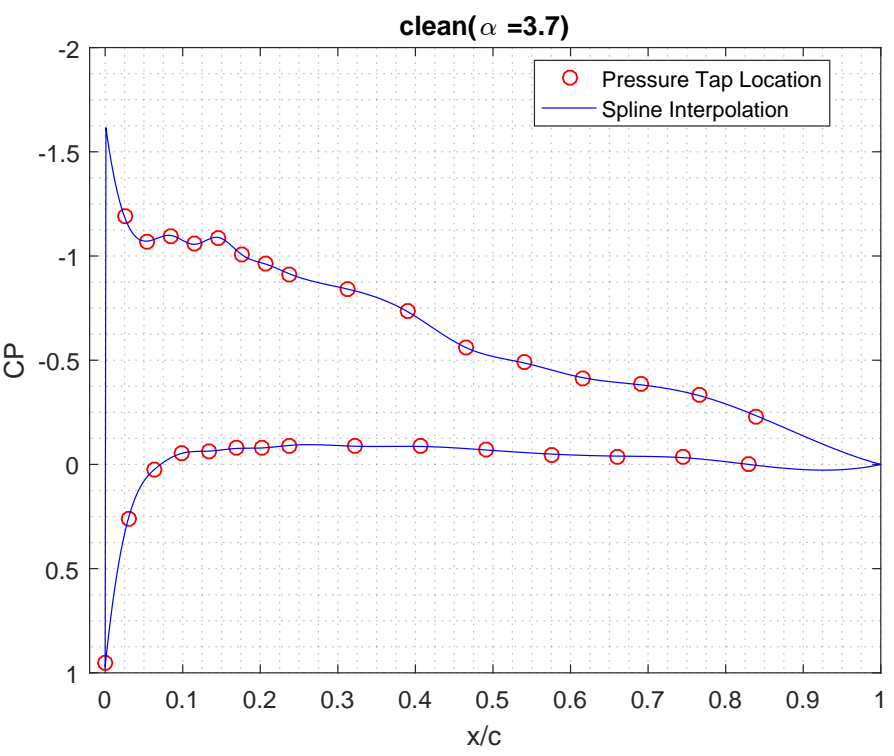

Figure 6.3: $C_{p}$ plot clean airfoil at $\alpha \approx 3.7^{\circ}$ 


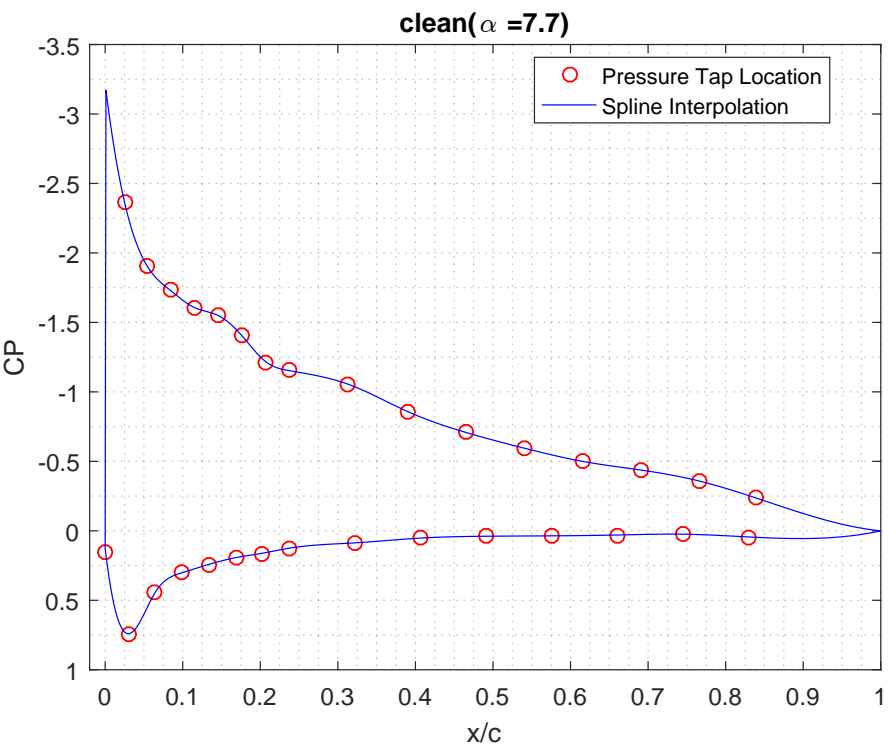

Figure 6.4: $C_{p}$ plot clean airfoil at $\alpha \approx 7.7^{\circ}$

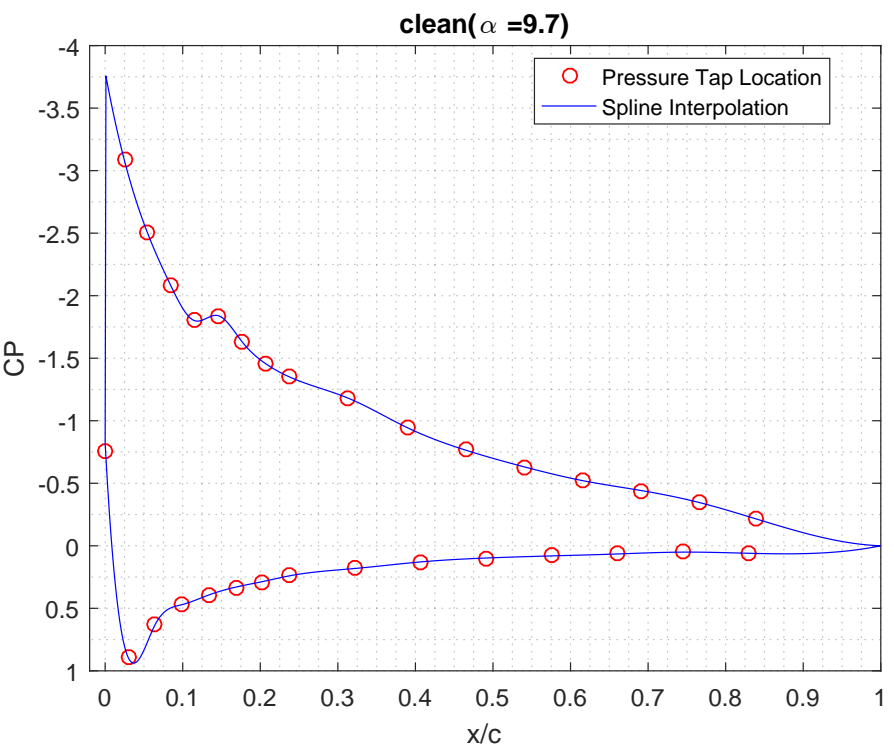

Figure 6.5: $C_{p}$ plot clean airfoil at $\alpha \approx 9.7^{\circ}$ 


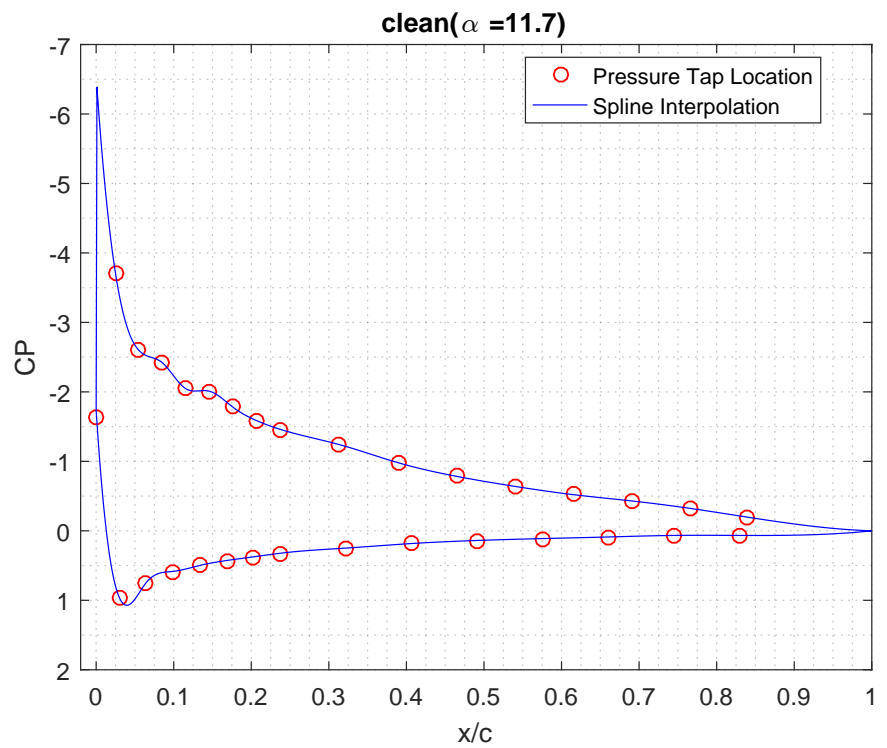

Figure 6.6: $C_{p}$ plot clean airfoil at $\alpha \approx 11.7^{\circ}$

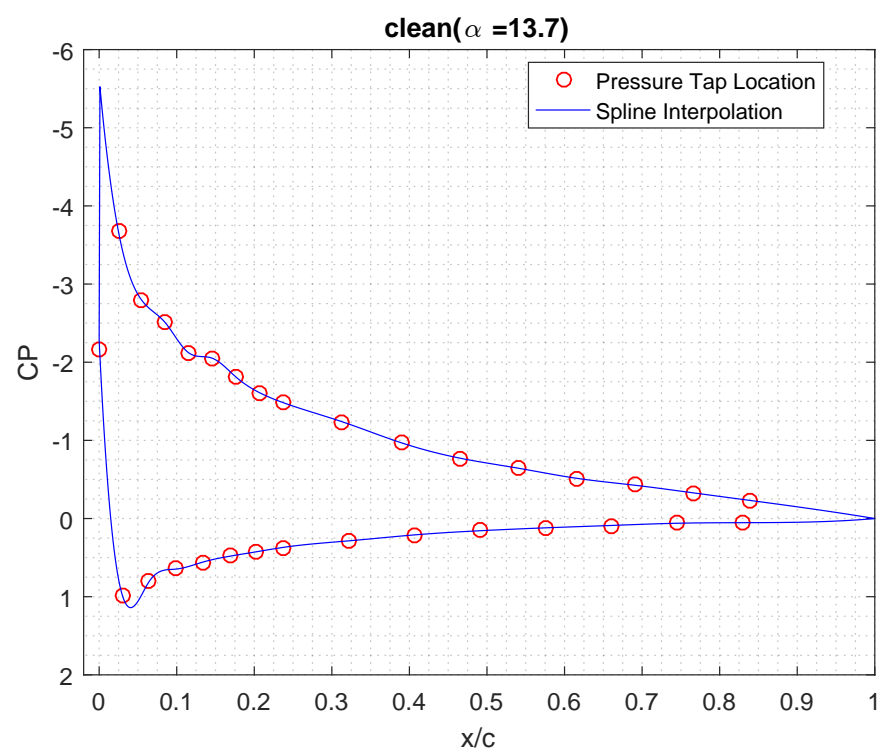

Figure 6.7: $C_{p}$ plot clean airfoil at $\alpha \approx 13.7^{\circ}$ 


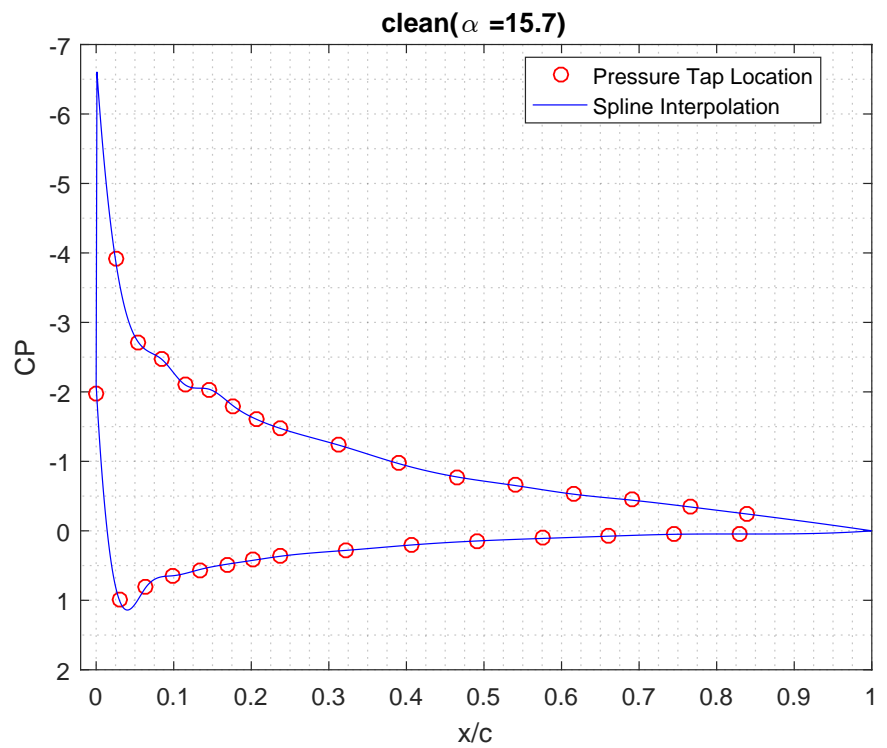

Figure 6.8: $C_{p}$ plot clean airfoil at $\alpha \approx 15.7^{\circ}$

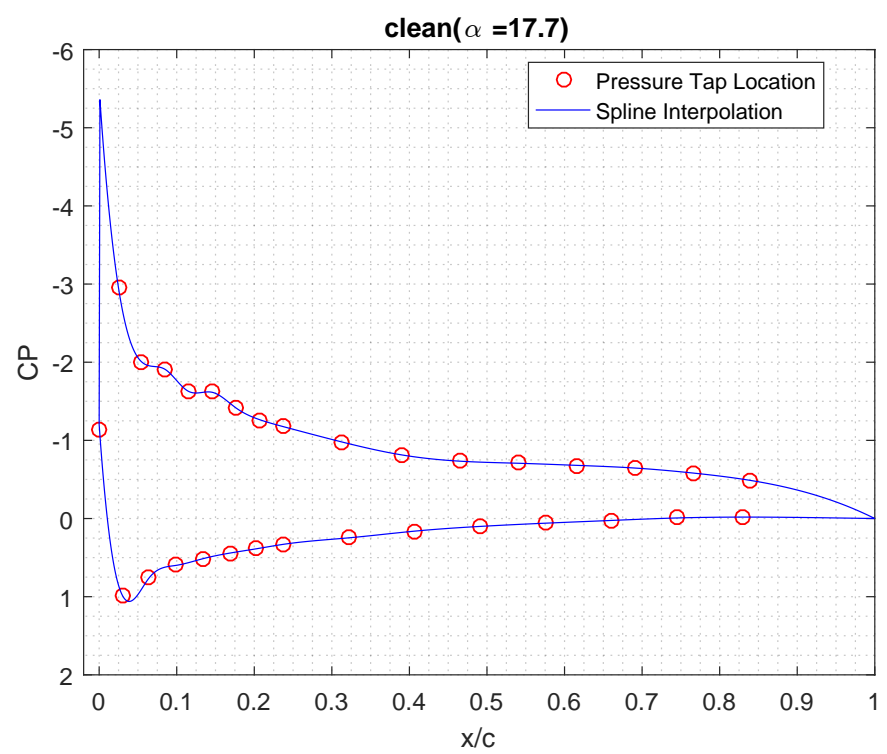

Figure 6.9: $C_{p}$ plot clean airfoil at $\alpha \approx 17.7^{\circ}$ 


\section{$6.2 \delta=8^{\circ}, b=10, a=60$ Configuration}

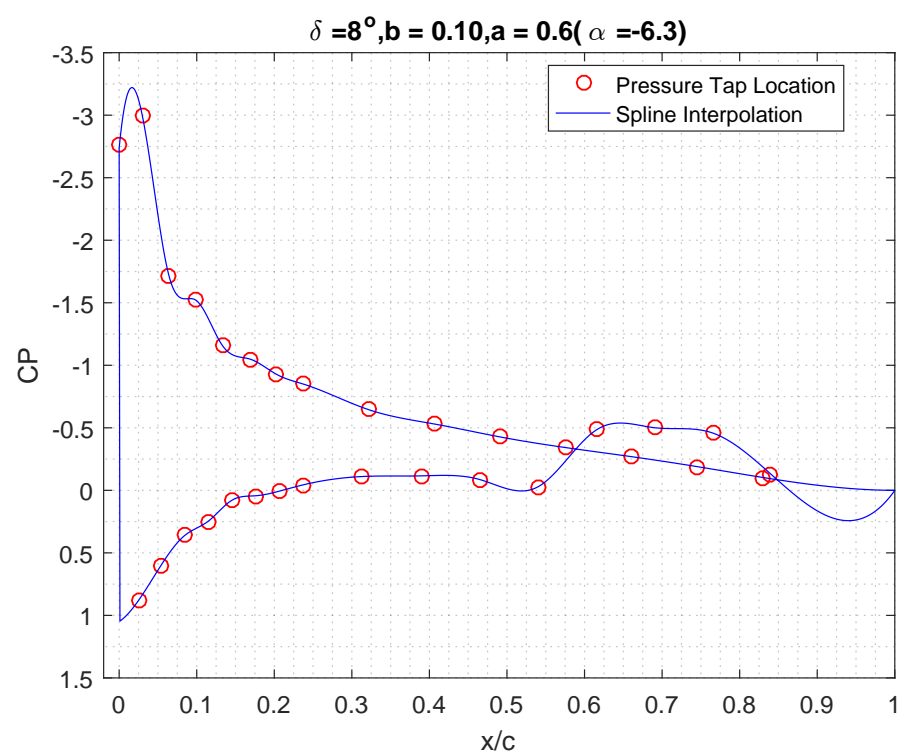

Figure 6.10: $C_{p}$ plot clean airfoil at $\alpha \approx-6.3^{\circ}$

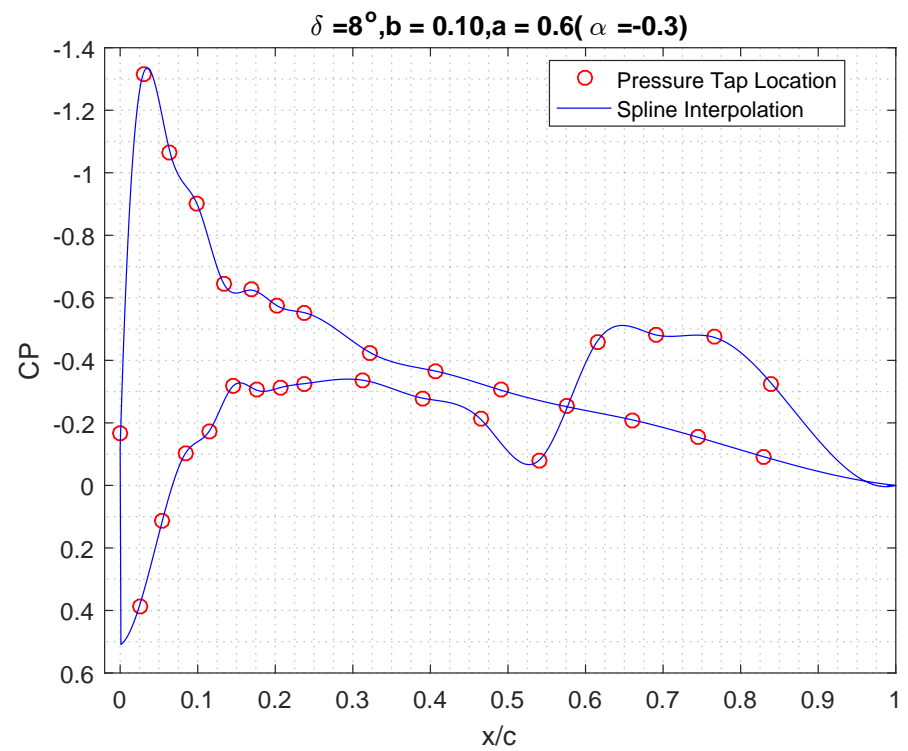

Figure 6.11: $C_{p}$ plot clean airfoil at $\alpha \approx-0.3^{\circ}$ 


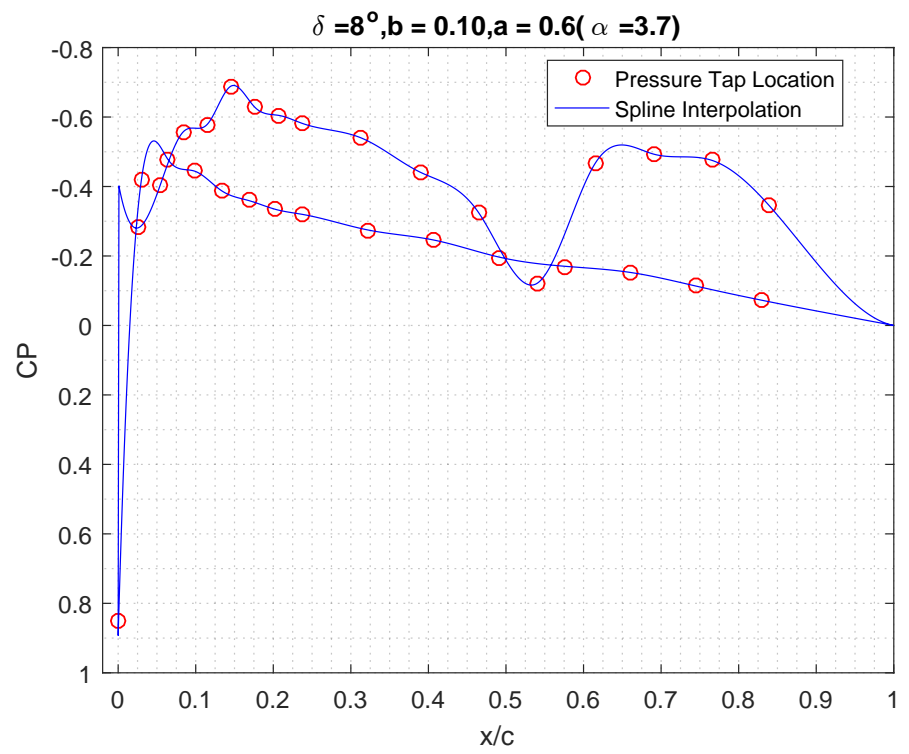

Figure 6.12: $C_{p}$ plot clean airfoil at $\alpha \approx 3.7^{\circ}$

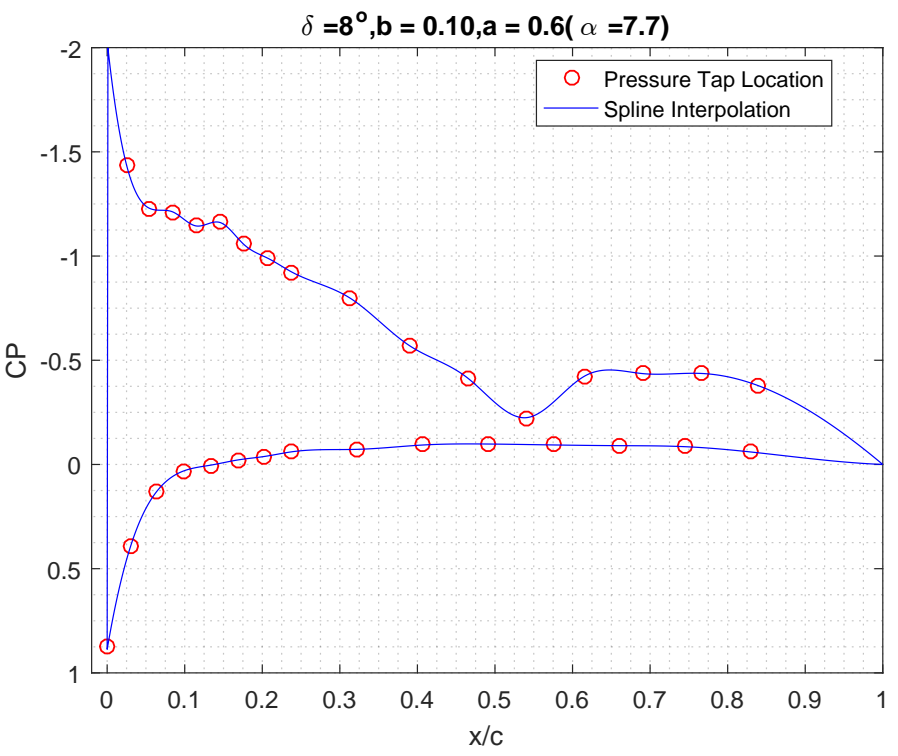

Figure 6.13: $C_{p}$ plot clean airfoil at $\alpha \approx 7.7^{\circ}$ 


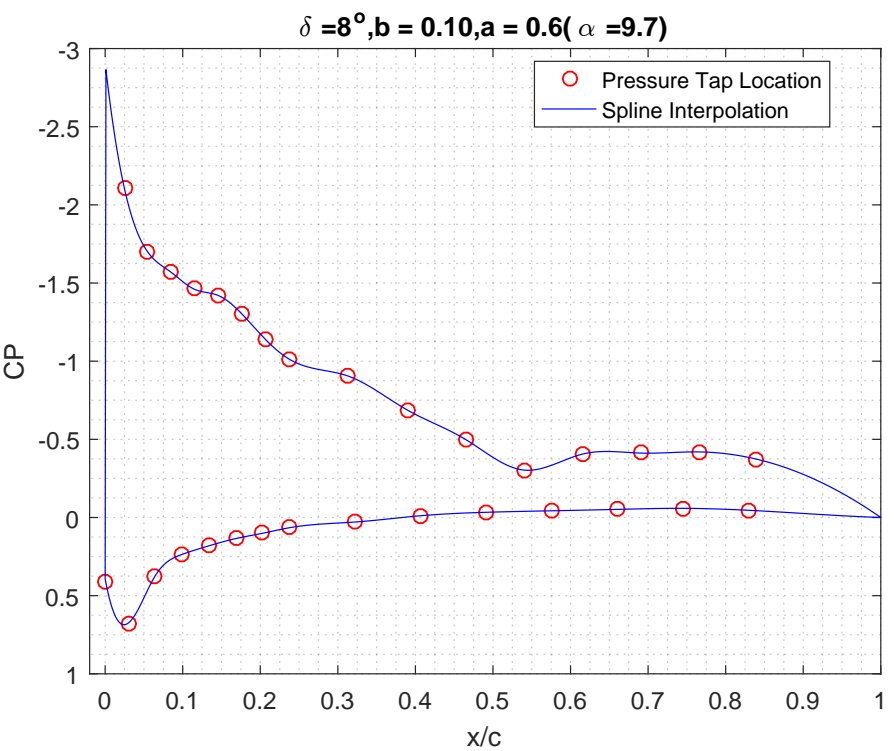

Figure 6.14: $C_{p}$ plot clean airfoil at $\alpha \approx 9.7^{\circ}$

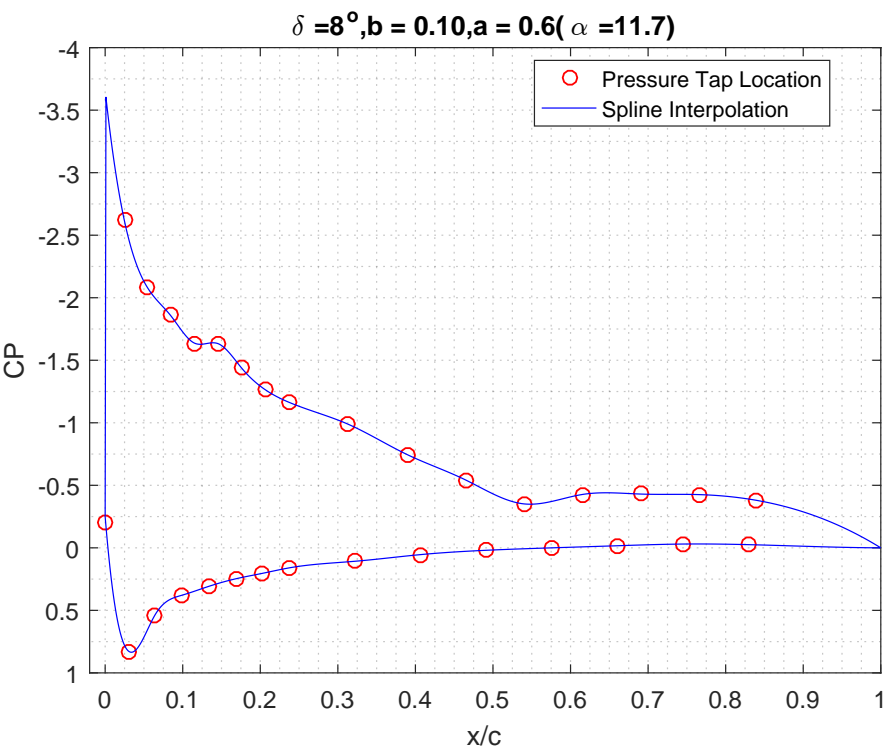

Figure 6.15: $C_{p}$ plot clean airfoil at $\alpha \approx 11.7^{\circ}$ 


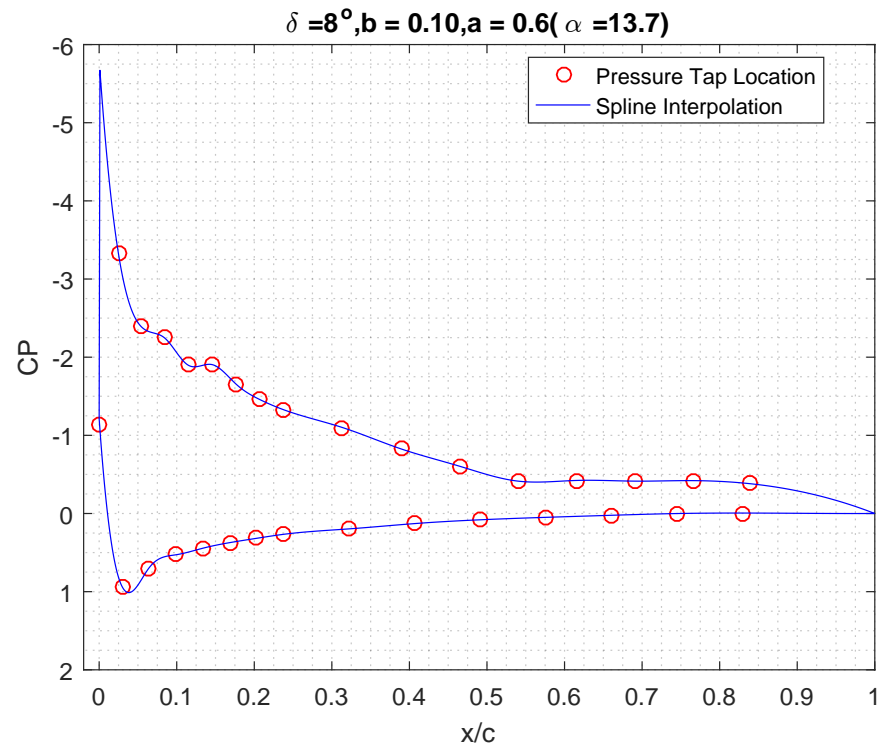

Figure 6.16: $C_{p}$ plot clean airfoil at $\alpha \approx 13.7^{\circ}$

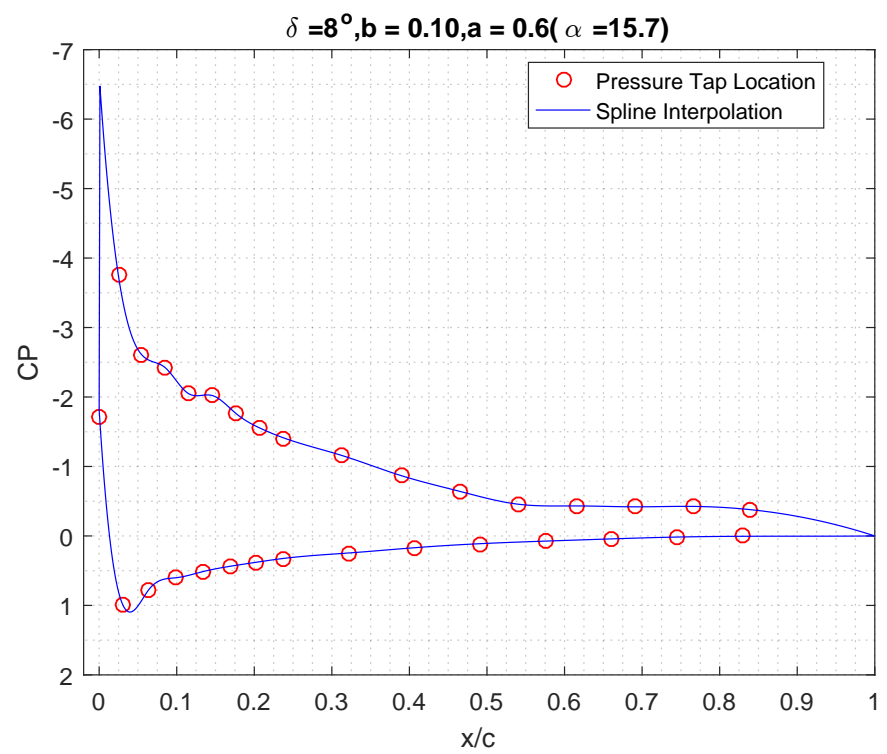

Figure 6.17: $C_{p}$ plot clean airfoil at $\alpha \approx 15.7^{\circ}$ 


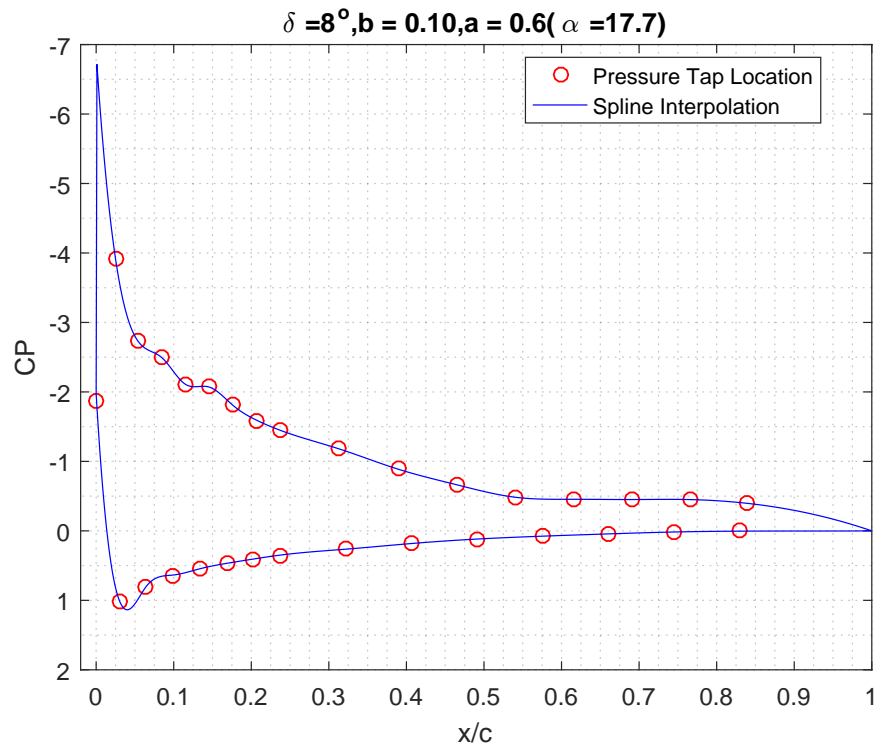

Figure 6.18: $C_{p}$ plot clean airfoil at $\alpha \approx 17.7^{\circ}$

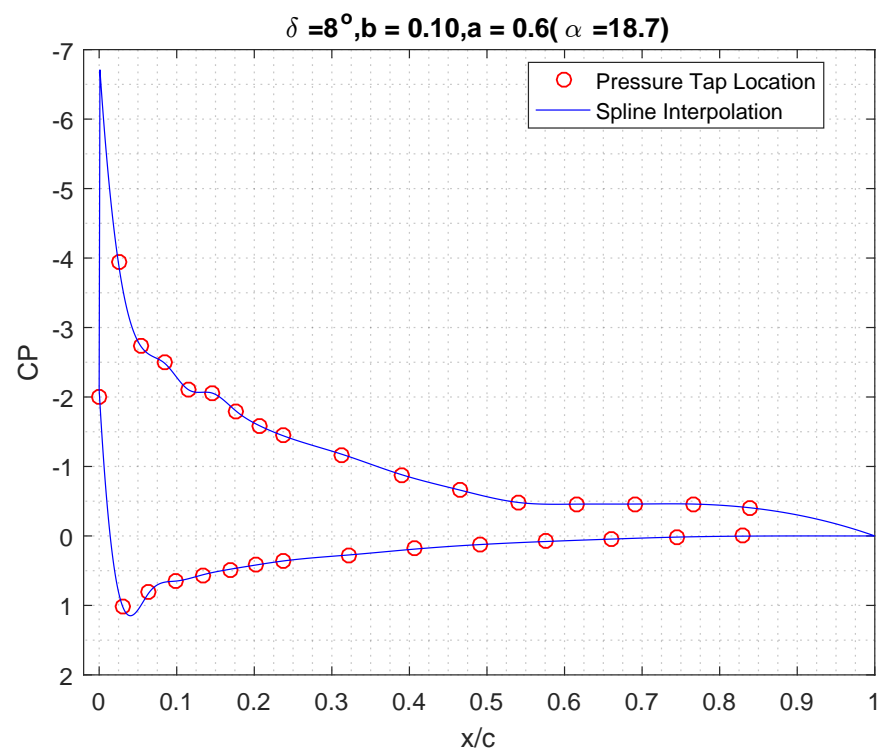

Figure 6.19: $C_{p}$ plot clean airfoil at $\alpha \approx 18.7^{\circ}$ 


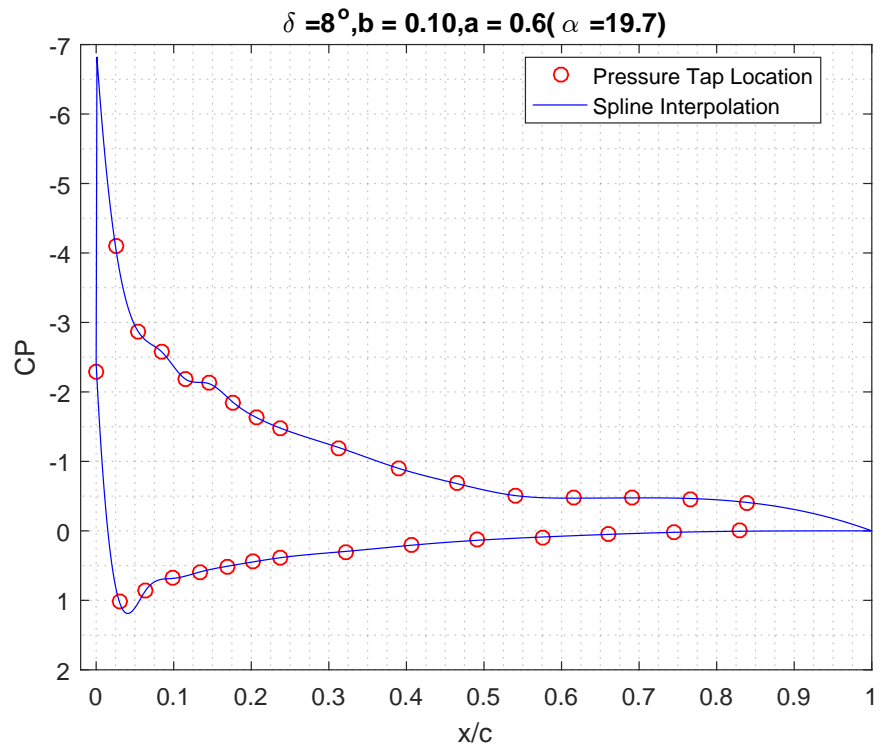

Figure 6.20: $C_{p}$ plot clean airfoil at $\alpha \approx 19.7^{\circ}$

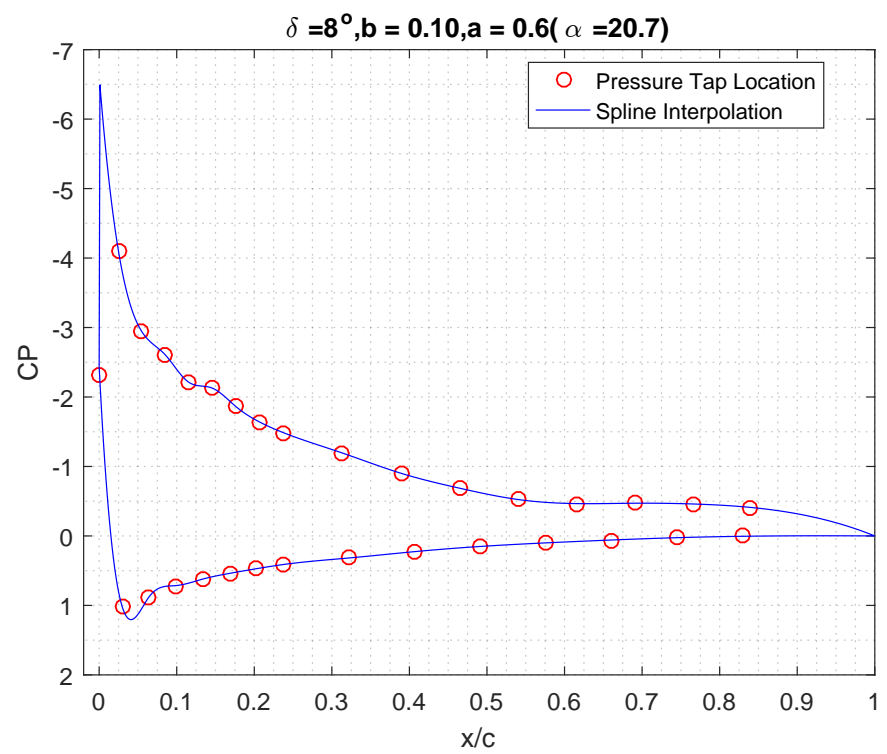

Figure 6.21: $C_{p}$ plot clean airfoil at $\alpha \approx 20.7^{\circ}$ 


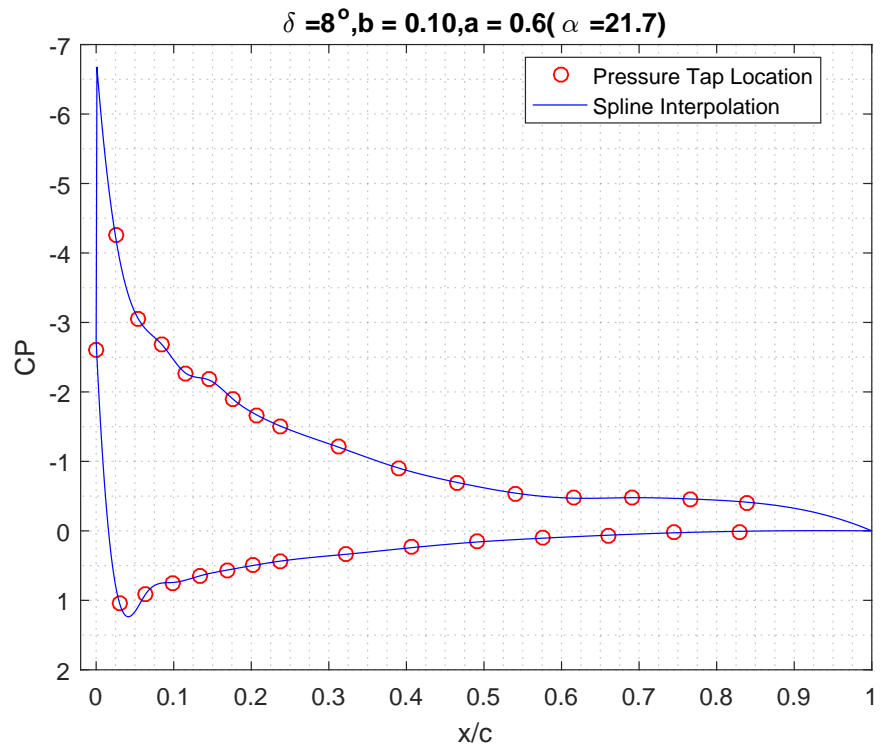

Figure 6.22: $C_{p}$ plot clean airfoil at $\alpha \approx 21.7^{\circ}$

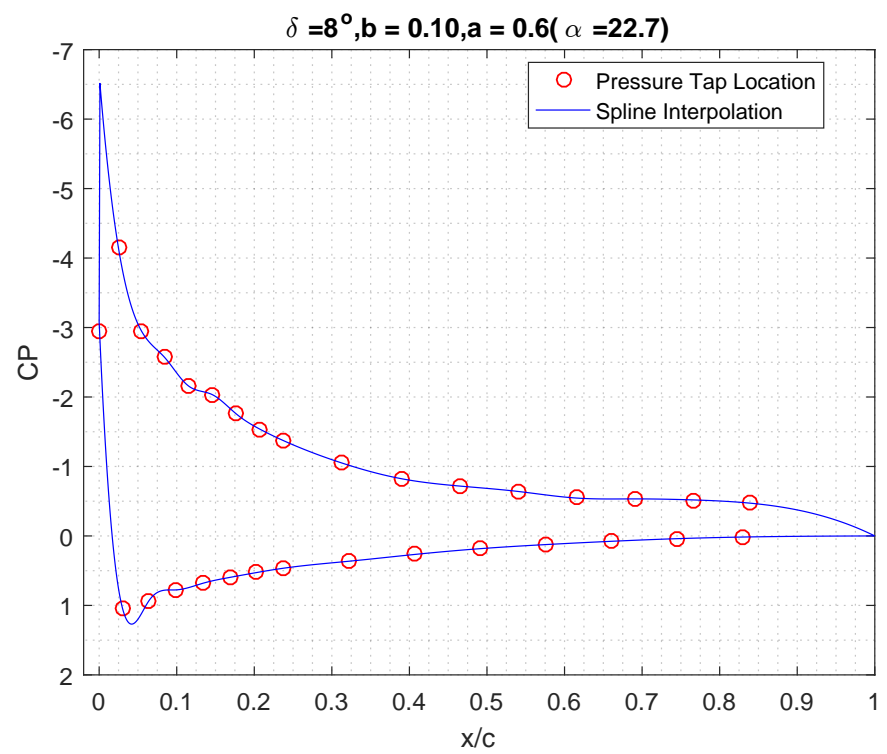

Figure 6.23: $C_{p}$ plot clean airfoil at $\alpha \approx 22.7^{\circ}$ 


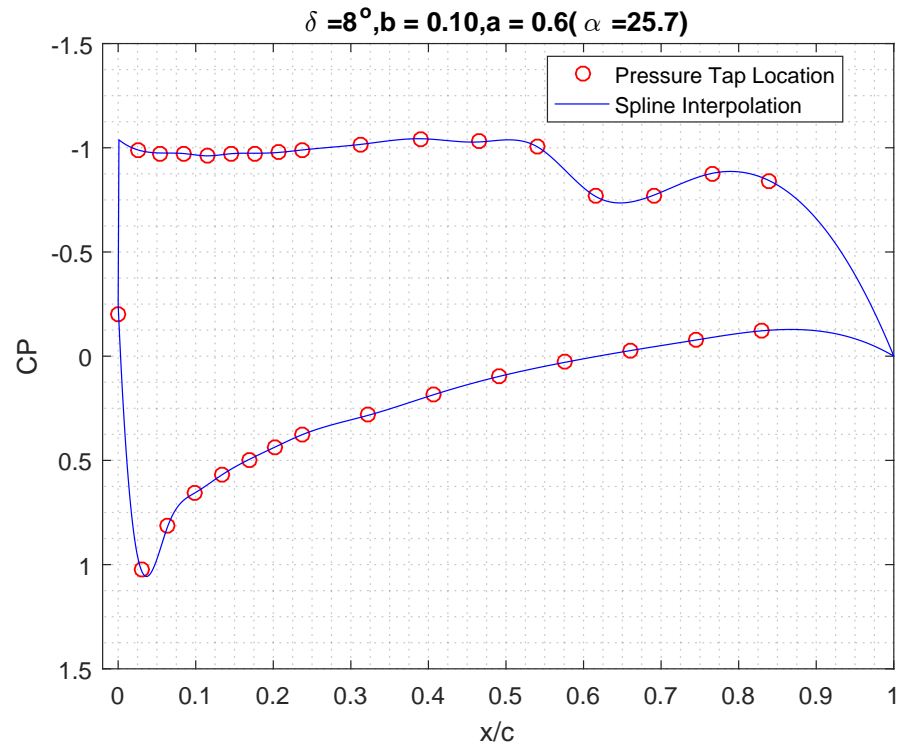

Figure 6.24: $C_{p}$ plot clean airfoil at $\alpha \approx 25.7^{\circ}$

\section{3 $\delta=15^{\circ}, b=10, a=60$ Configuration}

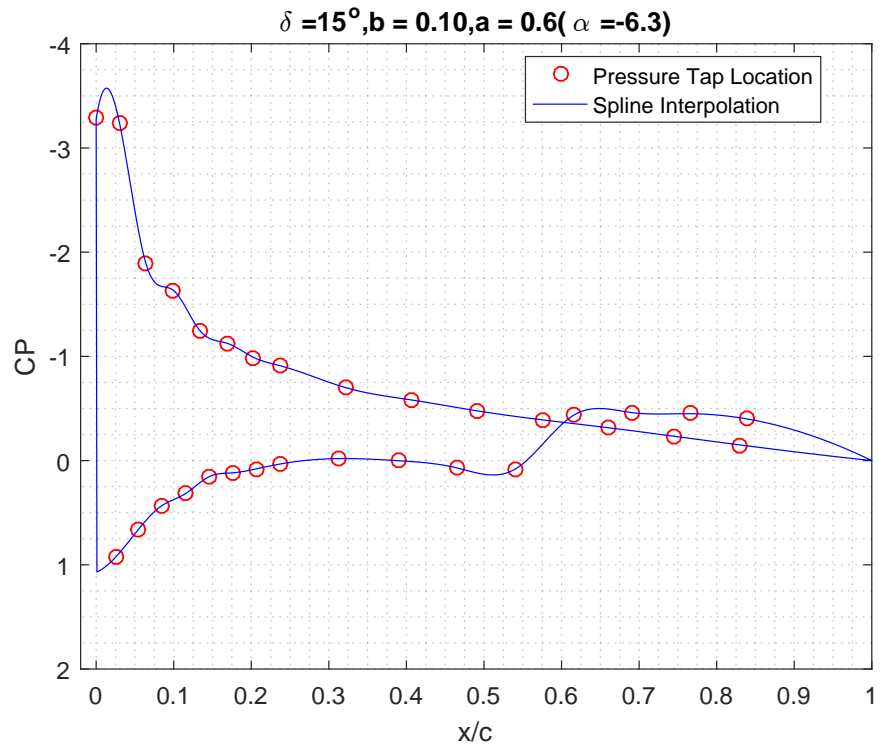

Figure 6.25: $C_{p}$ plot clean airfoil at $\alpha \approx-6.3^{\circ}$ 


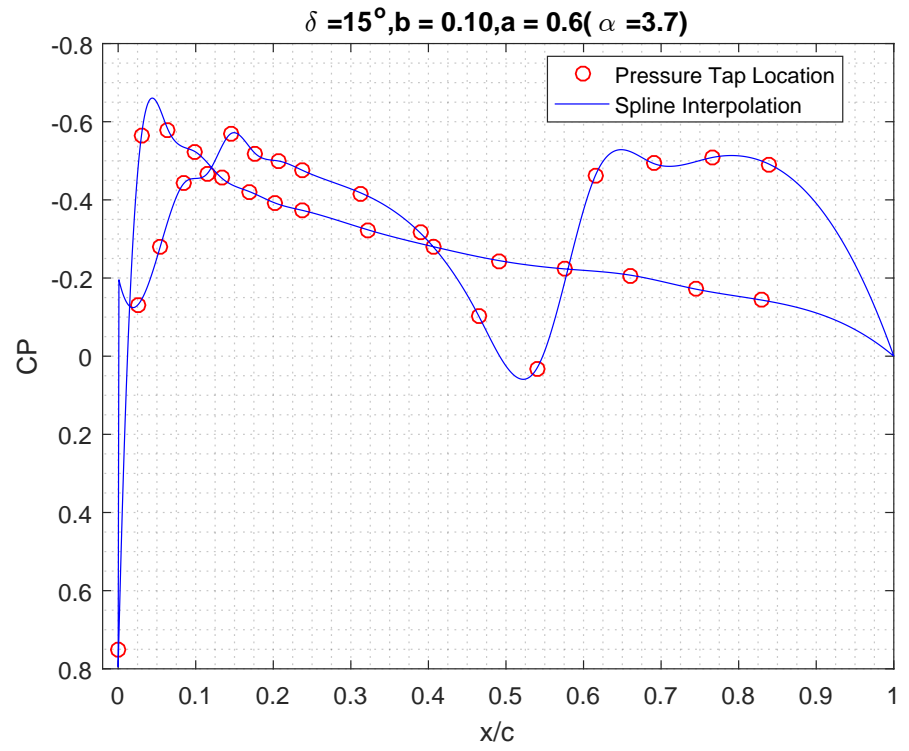

Figure 6.26: $C_{p}$ plot clean airfoil at $\alpha \approx 3.7^{\circ}$

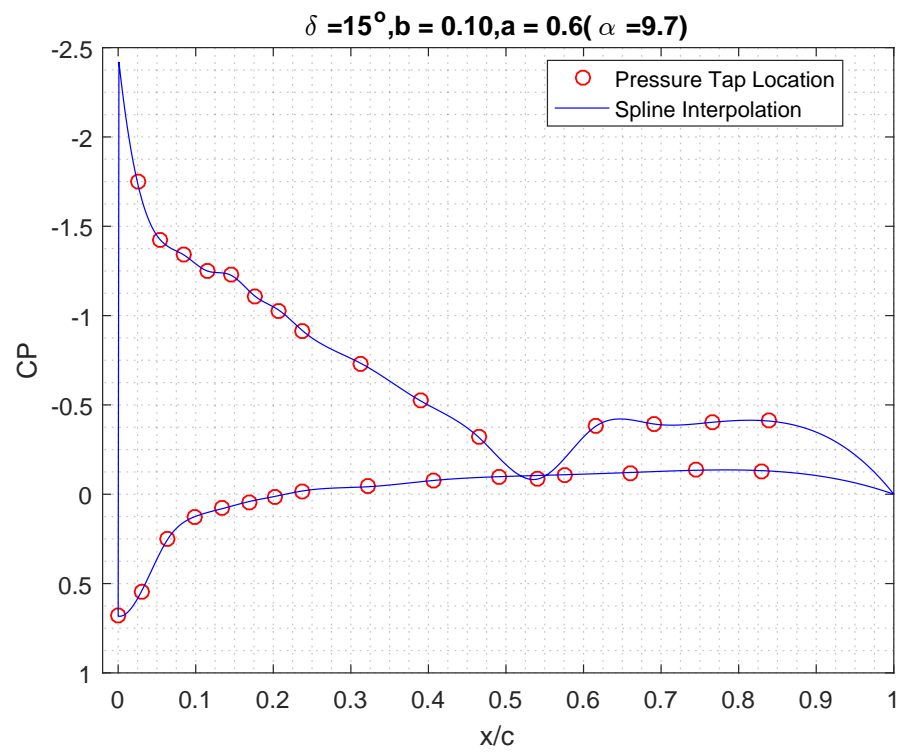

Figure 6.27: $C_{p}$ plot clean airfoil at $\alpha \approx 9.7^{\circ}$ 


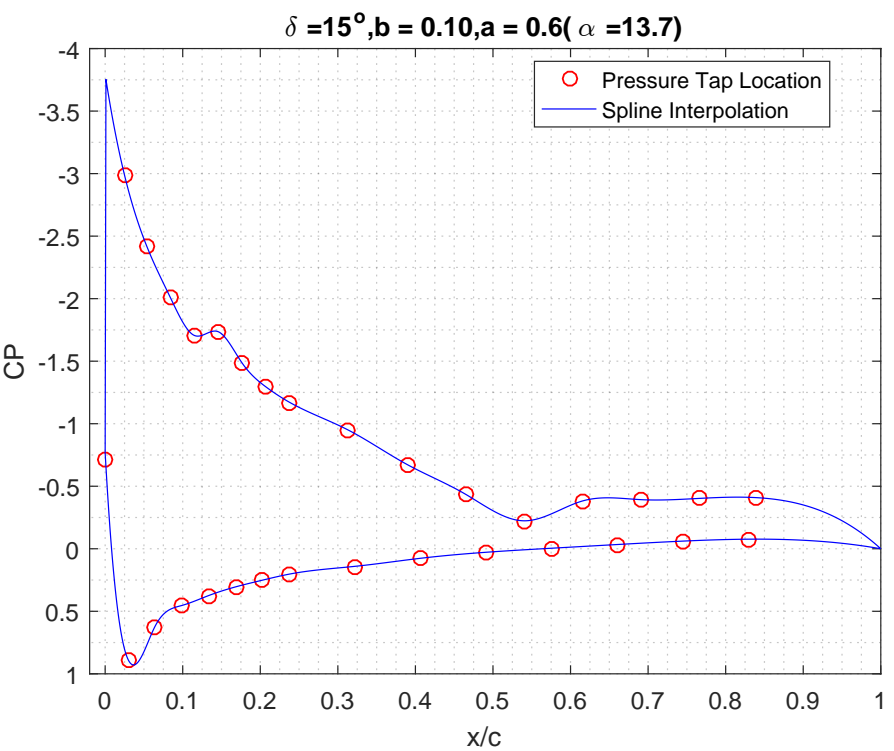

Figure 6.28: $C_{p}$ plot clean airfoil at $\alpha \approx 13.7^{\circ}$

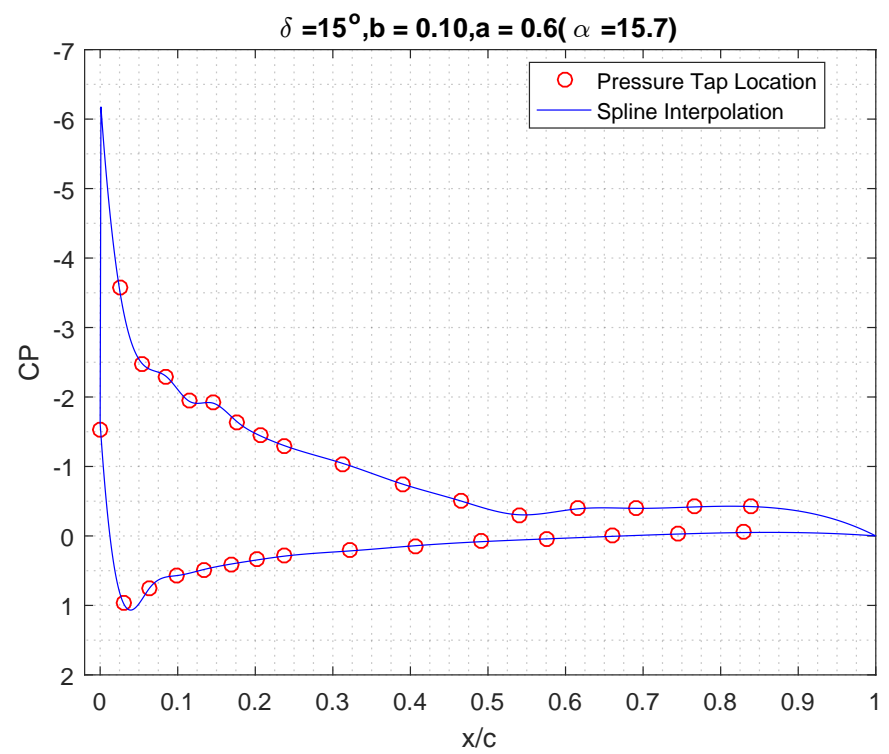

Figure 6.29: $C_{p}$ plot clean airfoil at $\alpha \approx 15.7^{\circ}$ 


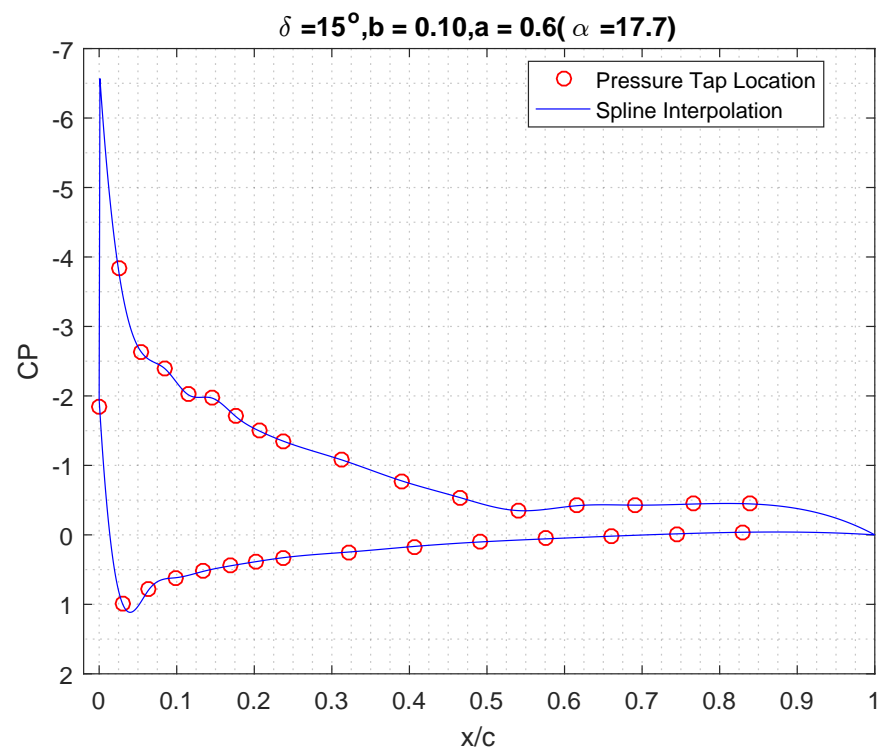

Figure 6.30: $C_{p}$ plot clean airfoil at $\alpha \approx 17.7^{\circ}$

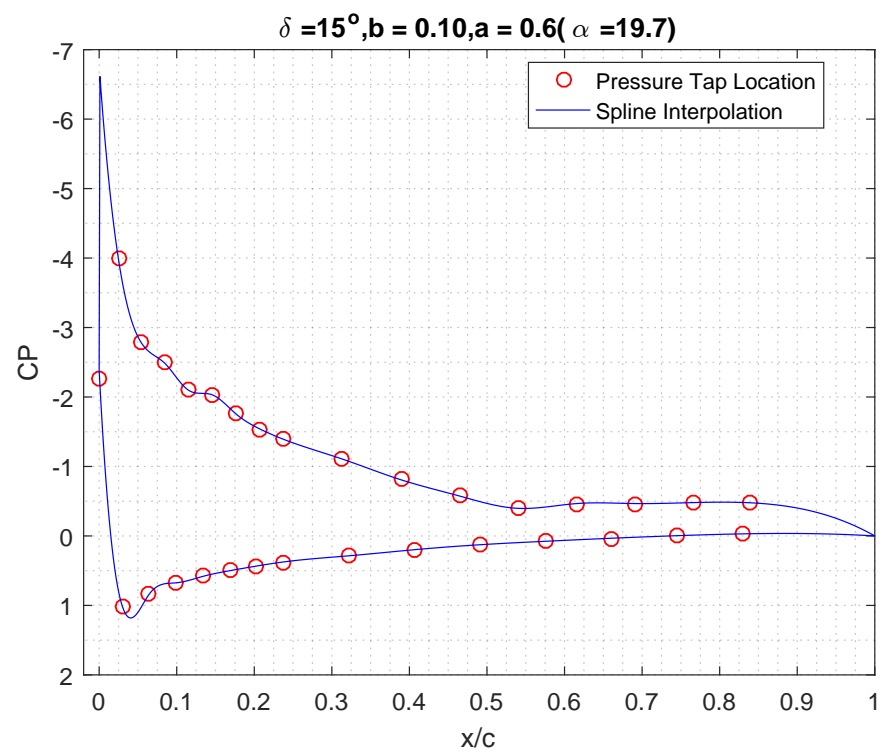

Figure 6.31: $C_{p}$ plot clean airfoil at $\alpha \approx 19.7^{\circ}$ 


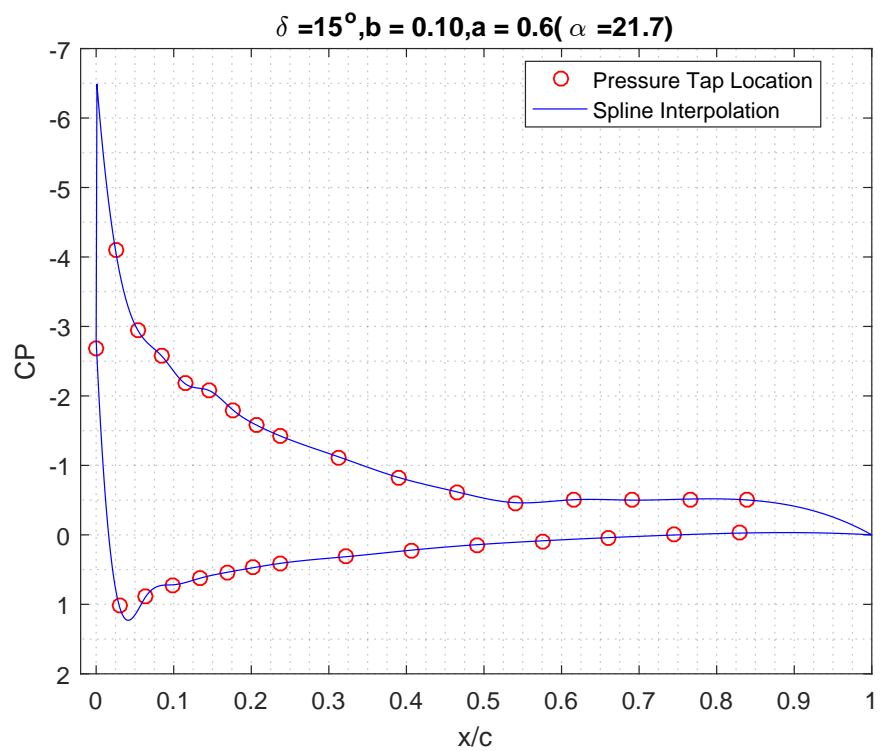

Figure 6.32: $C_{p}$ plot clean airfoil at $\alpha \approx 21.7^{\circ}$

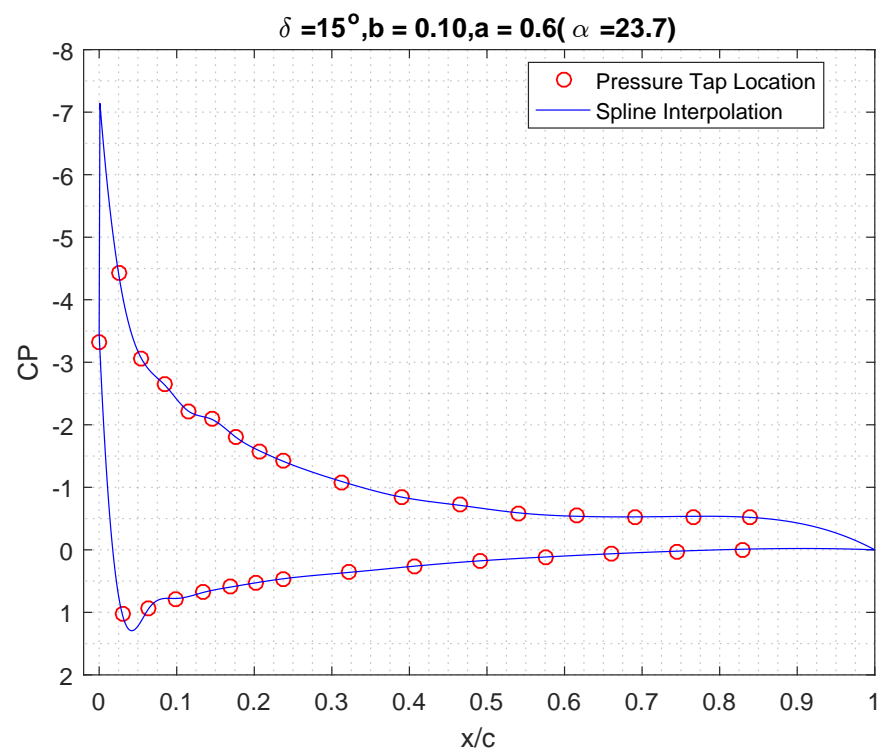

Figure 6.33: $C_{p}$ plot clean airfoil at $\alpha \approx 23.7^{\circ}$ 


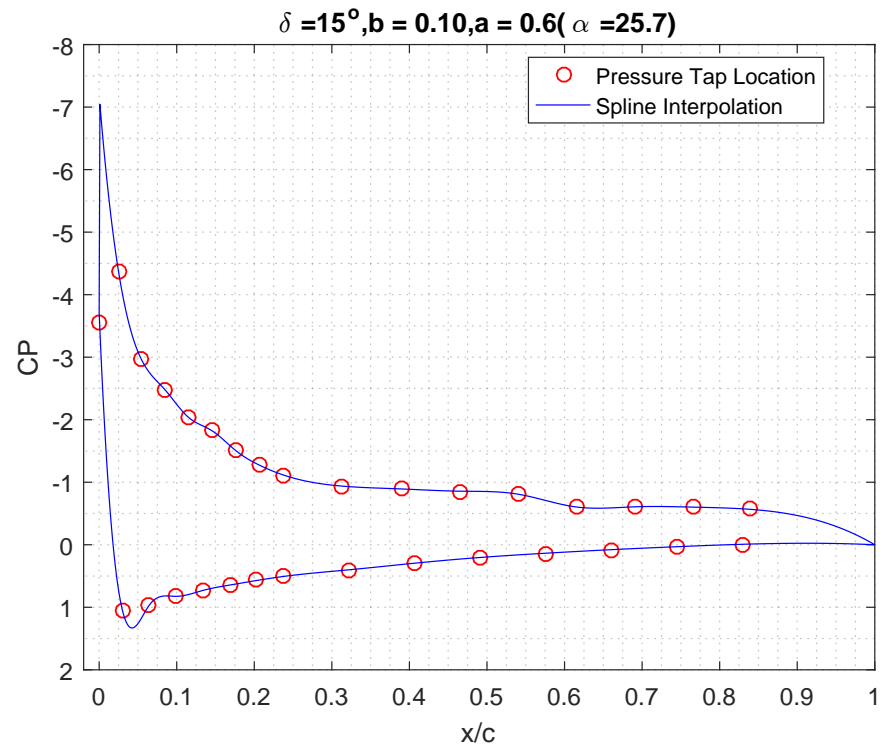

Figure 6.34: $C_{p}$ plot clean airfoil at $\alpha \approx 25.7^{\circ}$

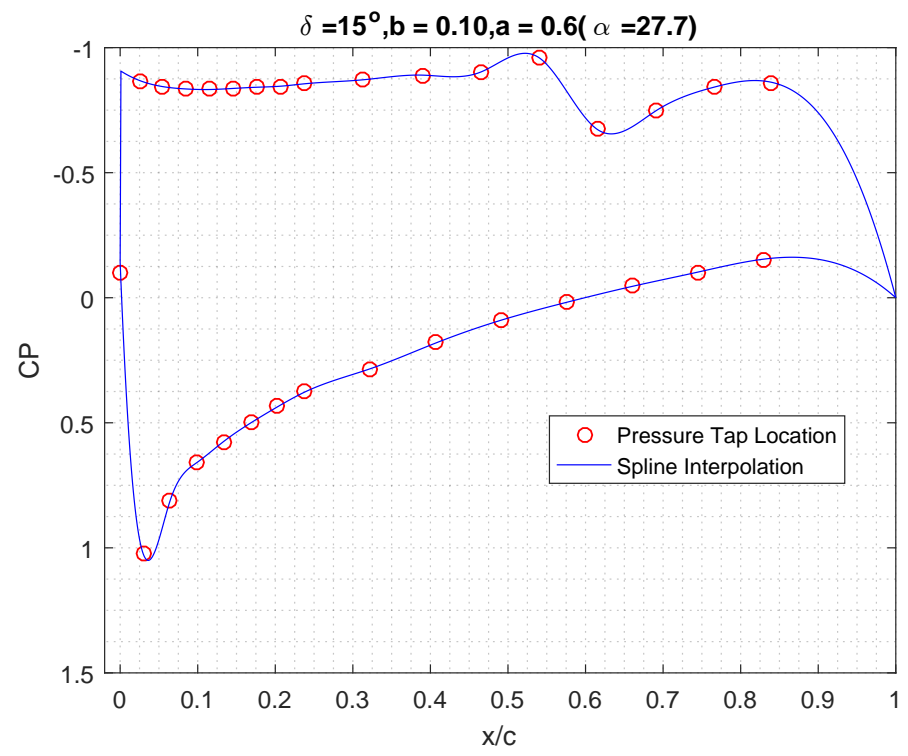

Figure 6.35: $C_{p}$ plot clean airfoil at $\alpha \approx 27.7^{\circ}$ 


\section{4 $\delta=25^{\circ}, b=10, a=60$ Configuration}

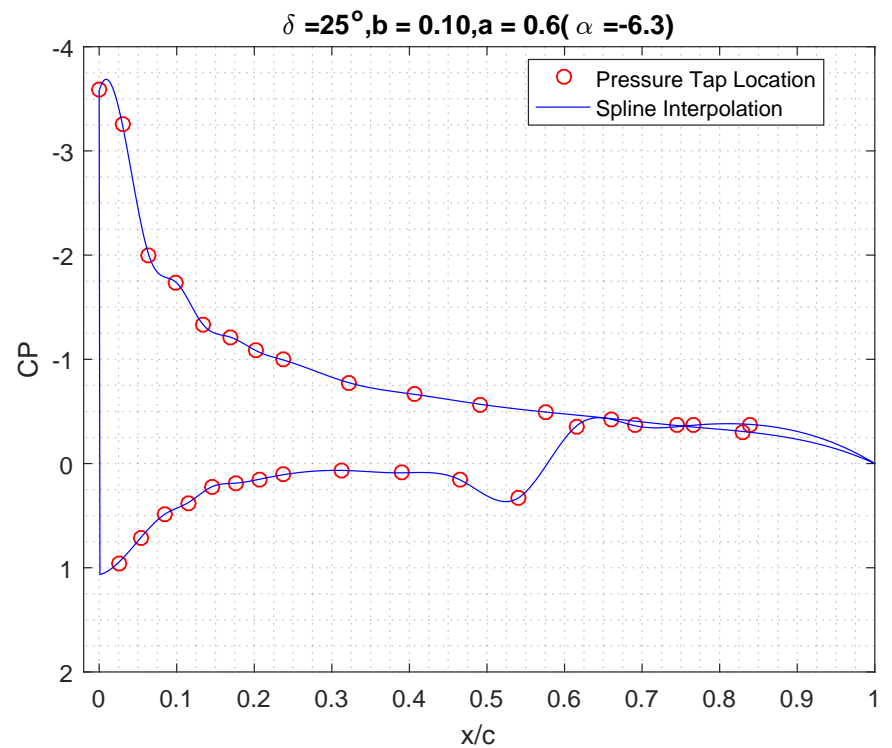

Figure 6.36: $C_{p}$ plot clean airfoil at $\alpha \approx-6.3^{\circ}$

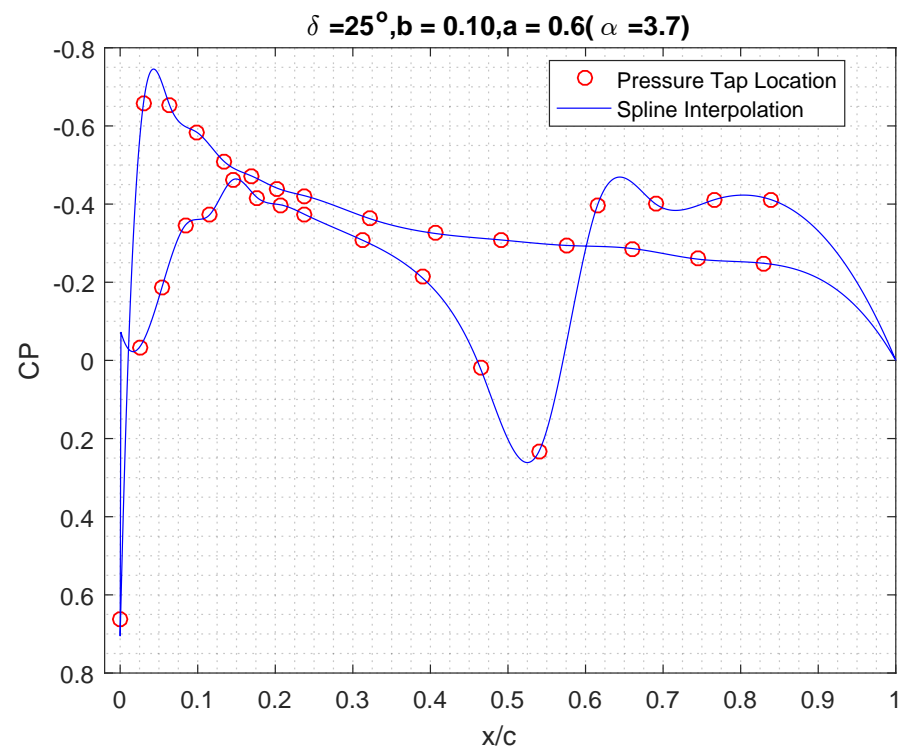

Figure 6.37: $C_{p}$ plot clean airfoil at $\alpha \approx 3.7^{\circ}$ 


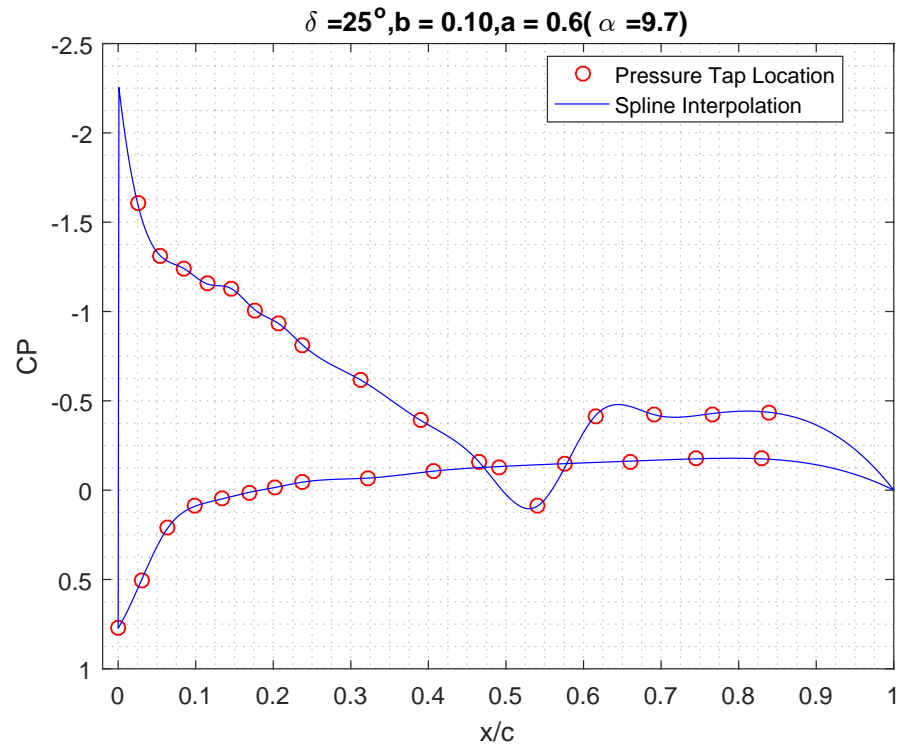

Figure 6.38: $C_{p}$ plot clean airfoil at $\alpha \approx 9.7^{\circ}$

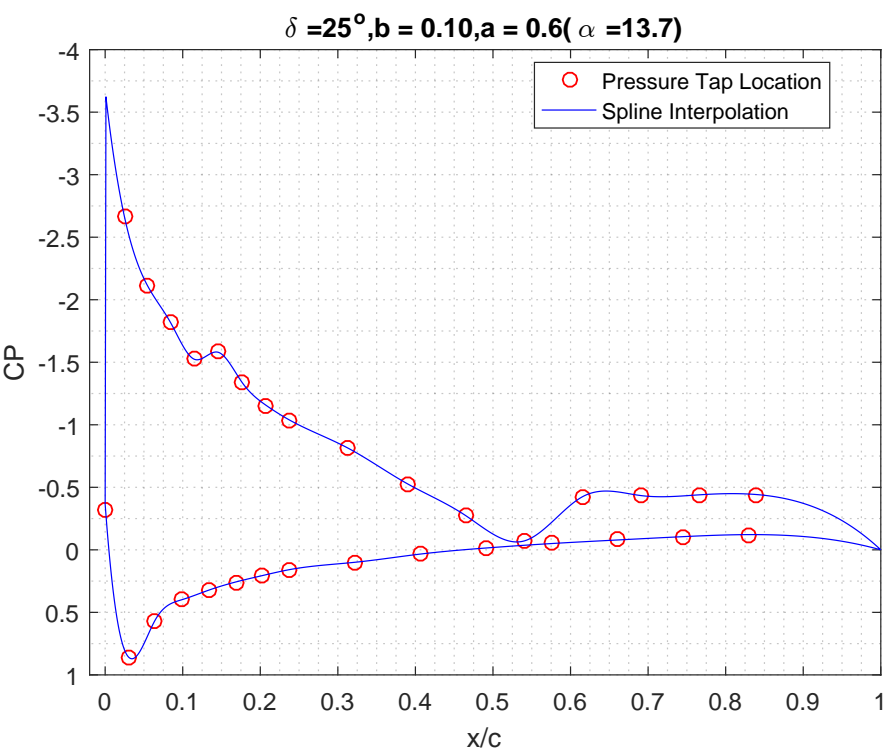

Figure 6.39: $C_{p}$ plot clean airfoil at $\alpha \approx 13.7^{\circ}$ 


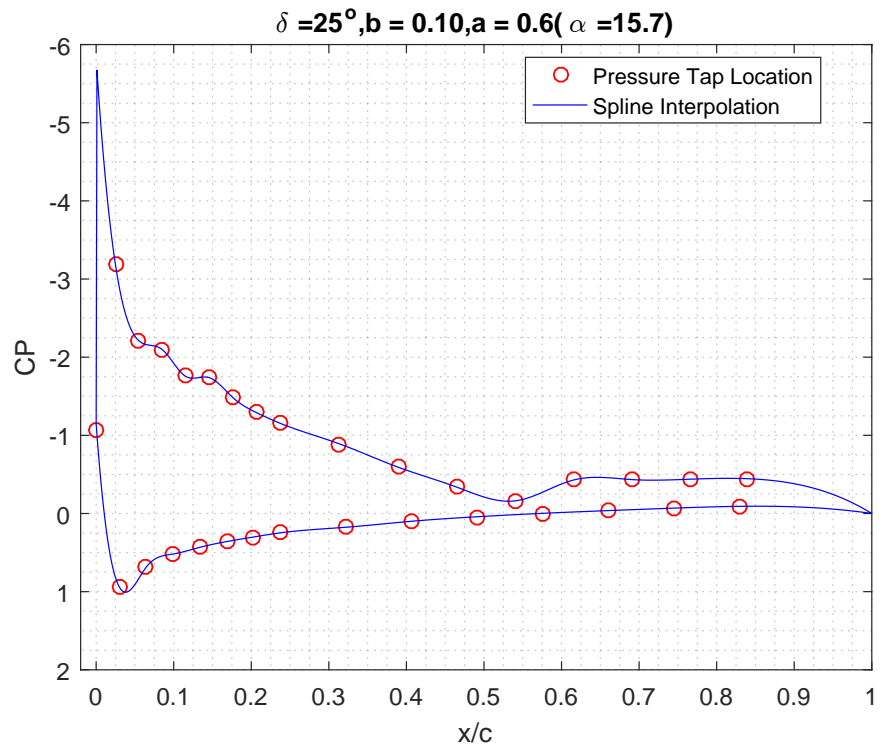

Figure 6.40: $C_{p}$ plot clean airfoil at $\alpha \approx 15.7^{\circ}$

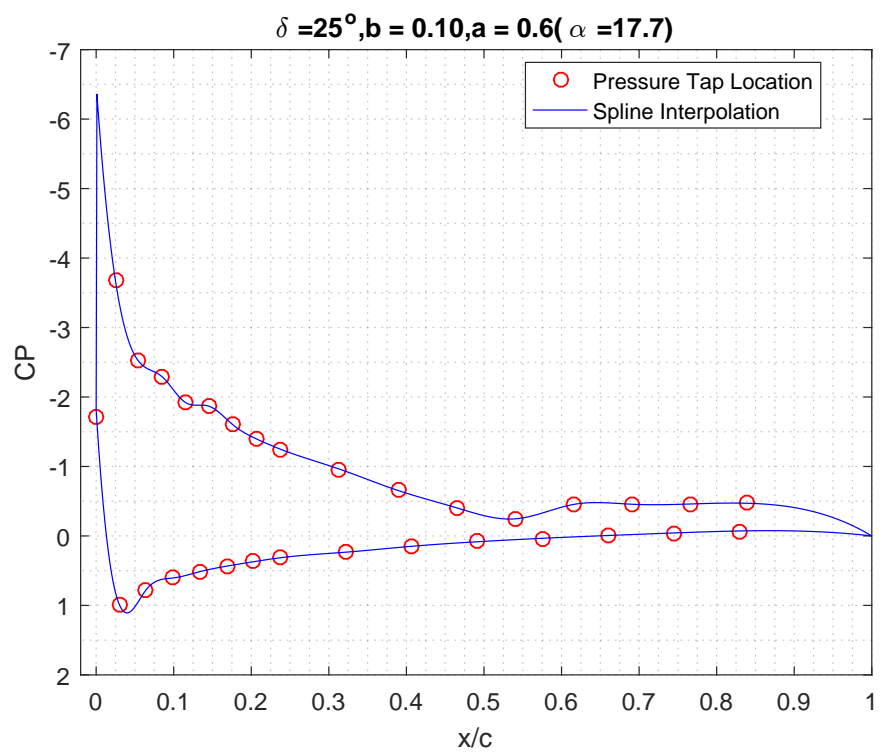

Figure 6.41: $C_{p}$ plot clean airfoil at $\alpha \approx 17.7^{\circ}$ 


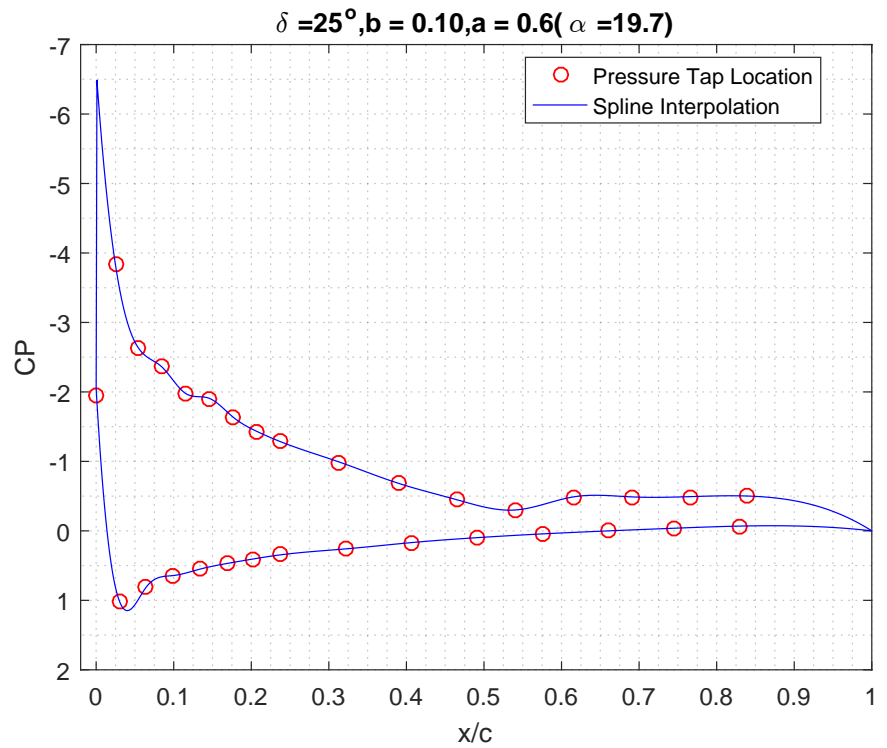

Figure 6.42: $C_{p}$ plot clean airfoil at $\alpha \approx 19.7^{\circ}$

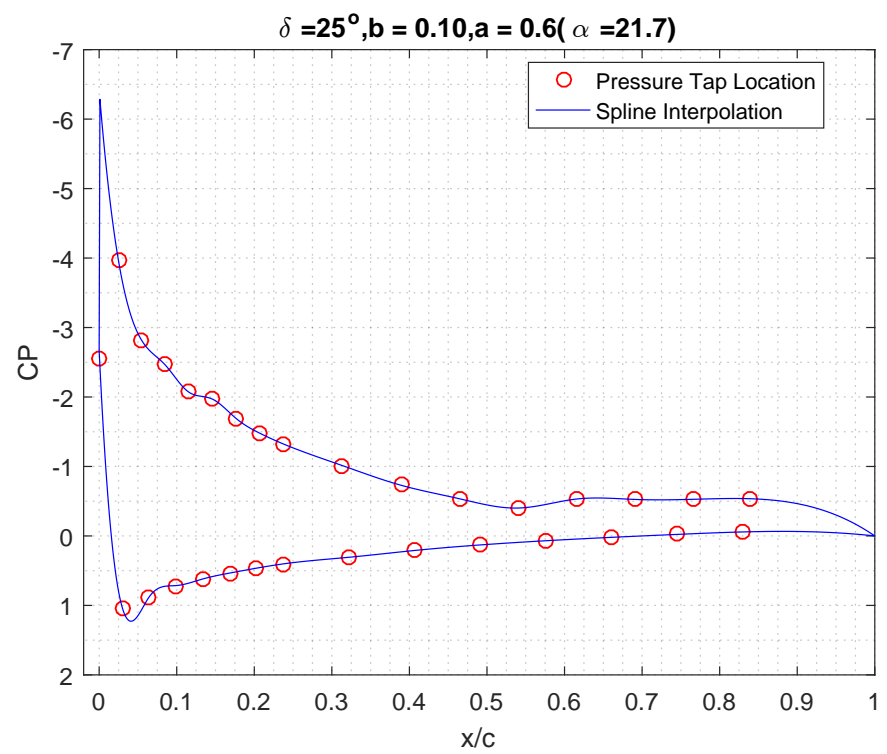

Figure 6.43: $C_{p}$ plot clean airfoil at $\alpha \approx 21.7^{\circ}$ 


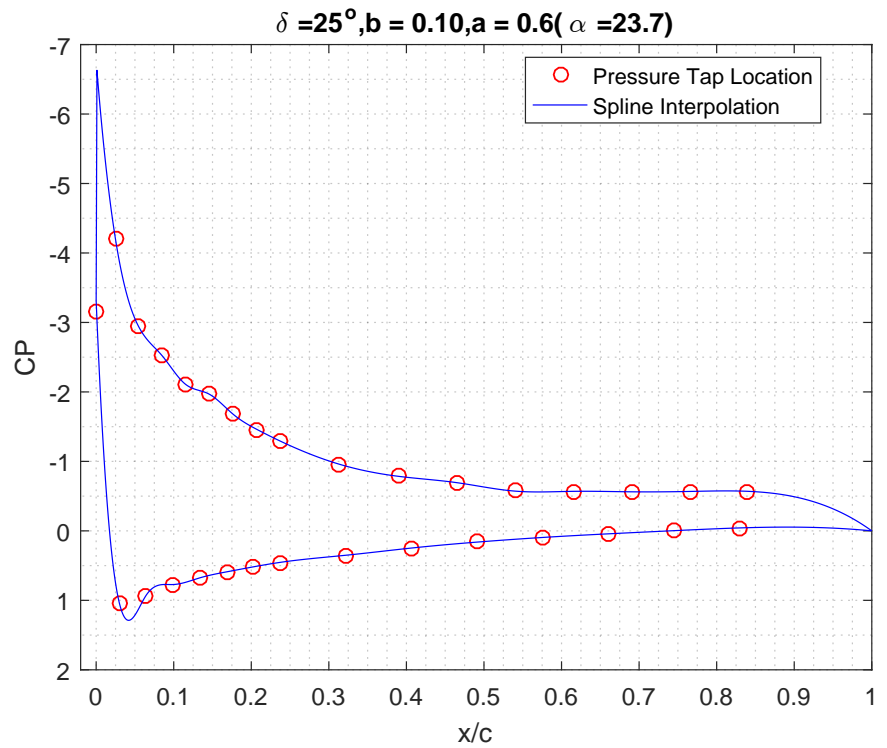

Figure 6.44: $C_{p}$ plot clean airfoil at $\alpha \approx 23.7^{\circ}$

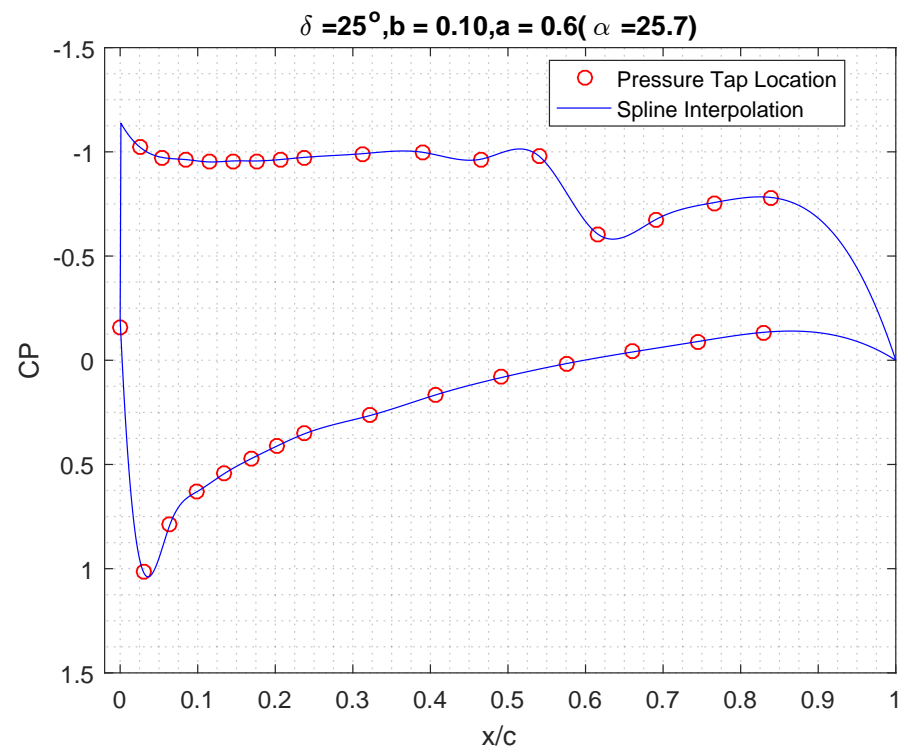

Figure 6.45: $C_{p}$ plot clean airfoil at $\alpha \approx 25.7^{\circ}$ 


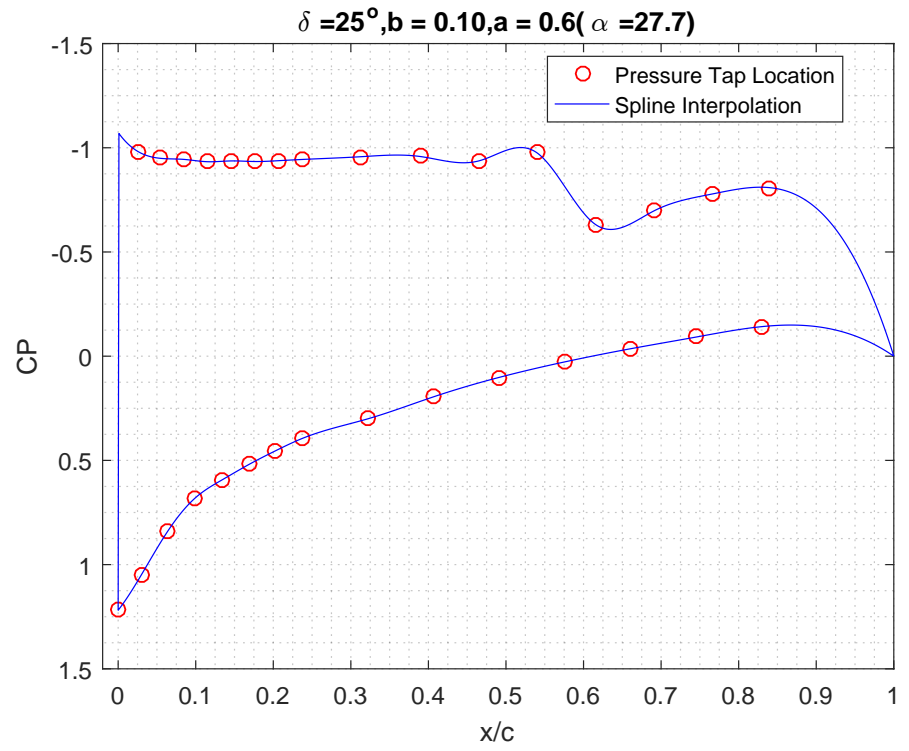

Figure 6.46: $C_{p}$ plot clean airfoil at $\alpha \approx 27.7^{\circ}$

\section{5 $\delta=8^{o}, b=15, a=60$ Configuration}

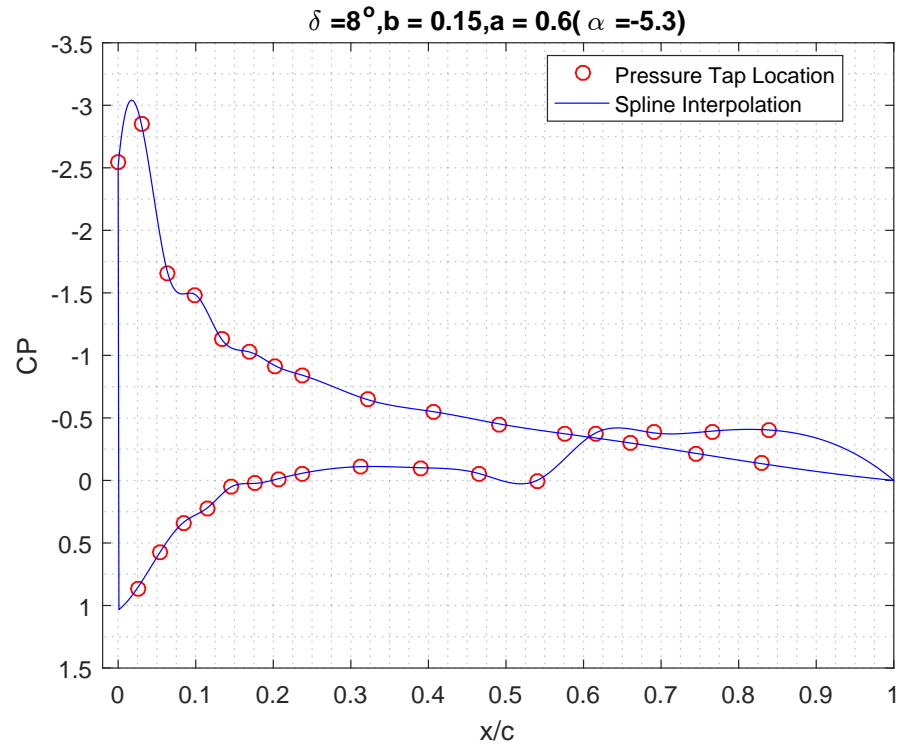

Figure 6.47: $C_{p}$ plot clean airfoil at $\alpha \approx-5.3^{\circ}$ 


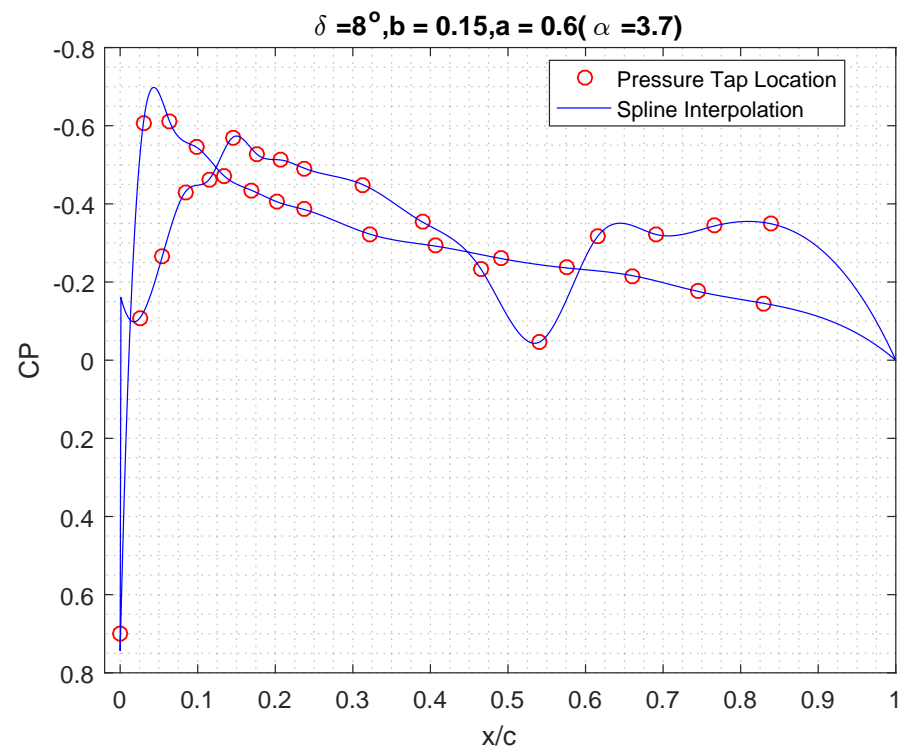

Figure 6.48: $C_{p}$ plot clean airfoil at $\alpha \approx 3.7^{\circ}$

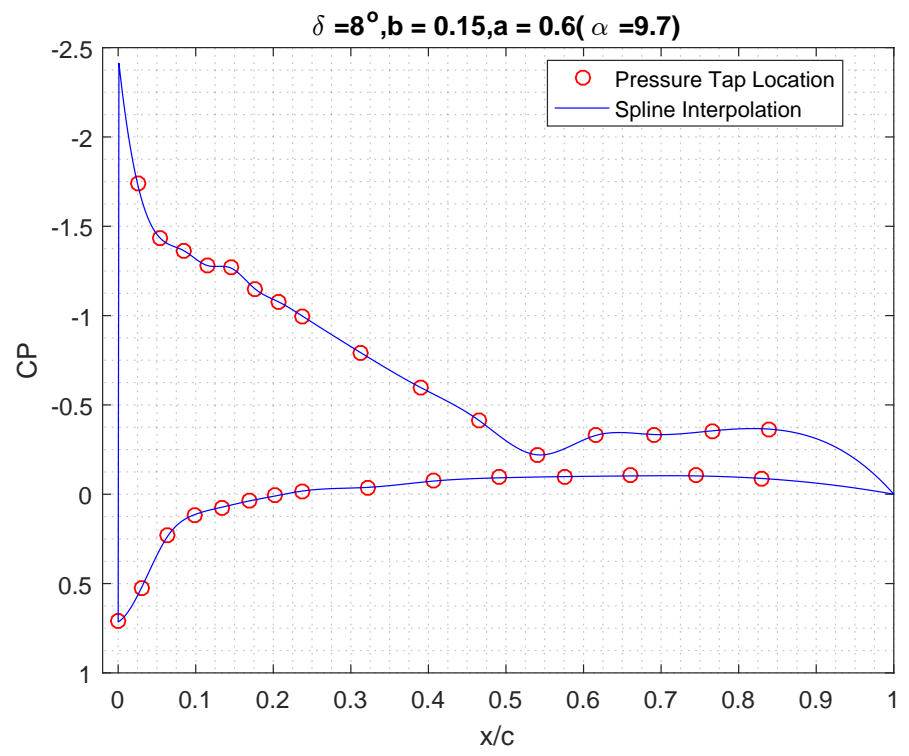

Figure 6.49: $C_{p}$ plot clean airfoil at $\alpha \approx 9.7^{\circ}$ 


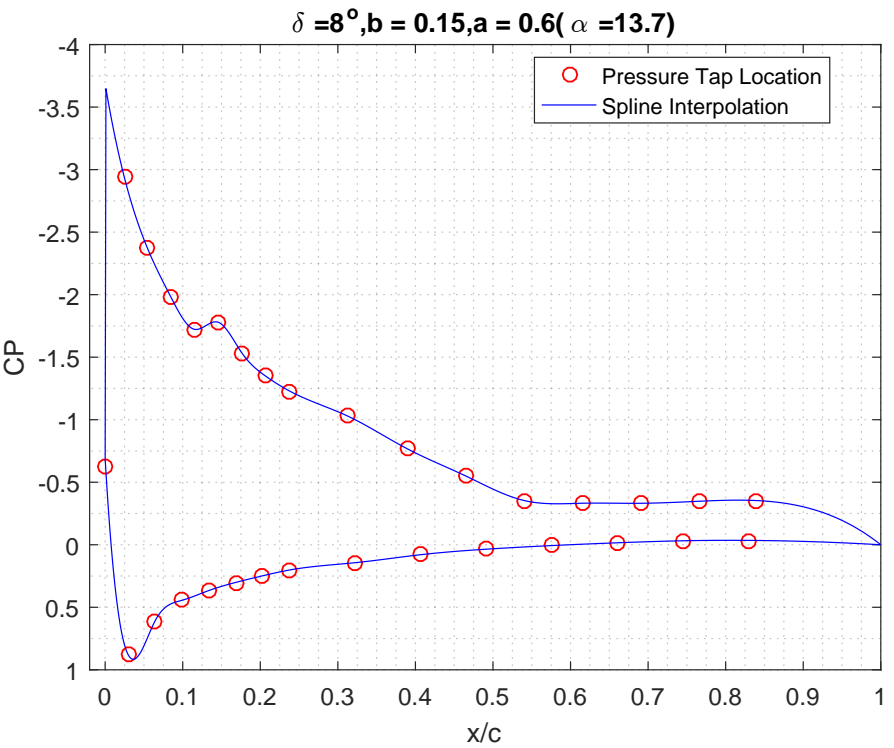

Figure 6.50: $C_{p}$ plot clean airfoil at $\alpha \approx 13.7^{\circ}$

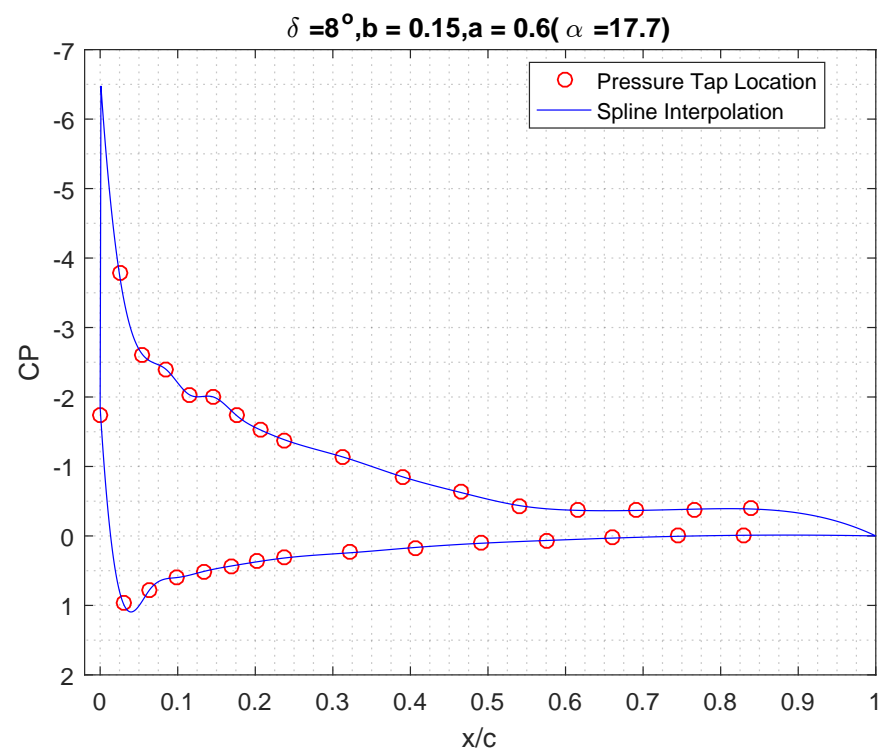

Figure 6.51: $C_{p}$ plot clean airfoil at $\alpha \approx 17.7^{\circ}$ 


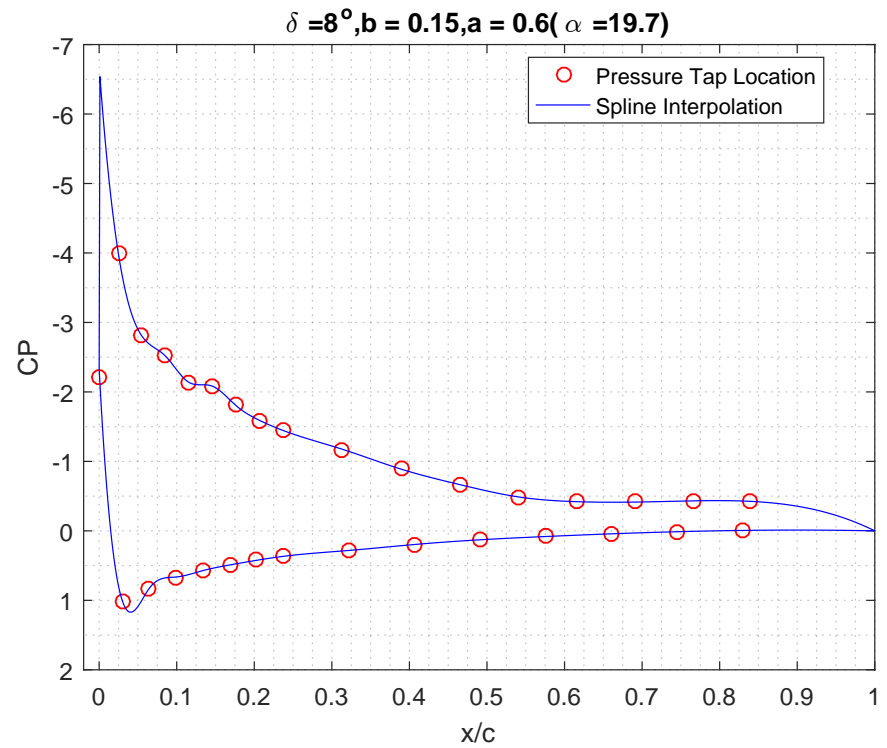

Figure 6.52: $C_{p}$ plot clean airfoil at $\alpha \approx 19.7^{\circ}$

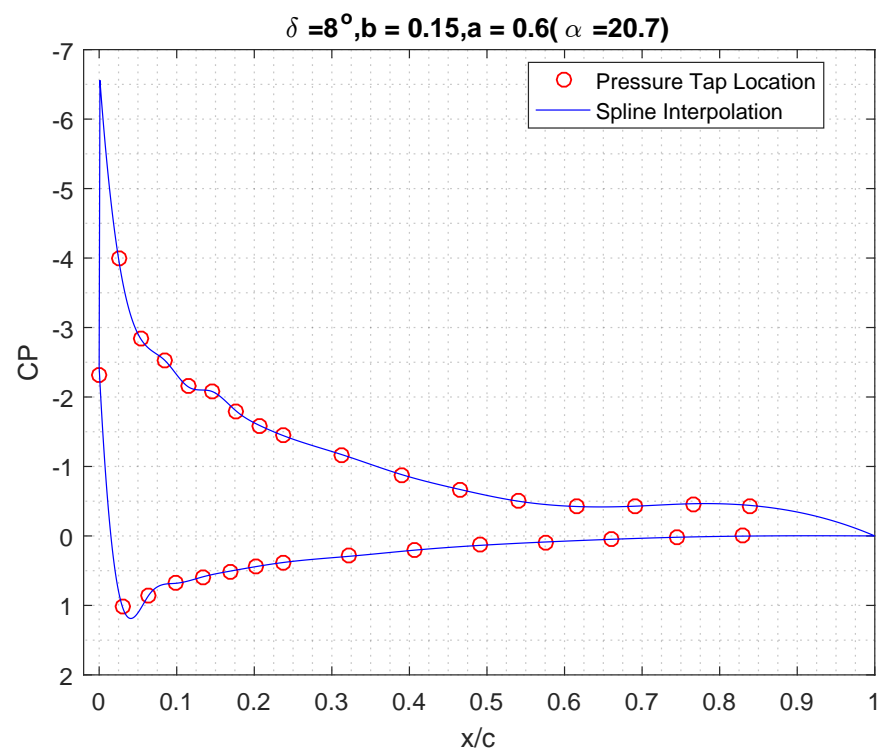

Figure 6.53: $C_{p}$ plot clean airfoil at $\alpha \approx 20.7^{\circ}$ 


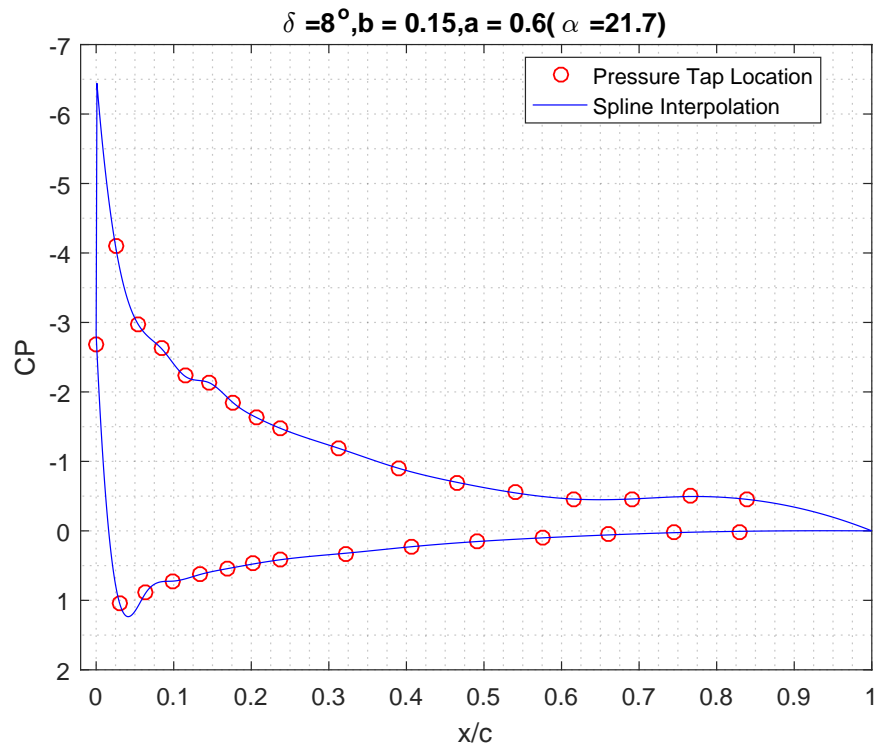

Figure 6.54: $C_{p}$ plot clean airfoil at $\alpha \approx 21.7^{\circ}$

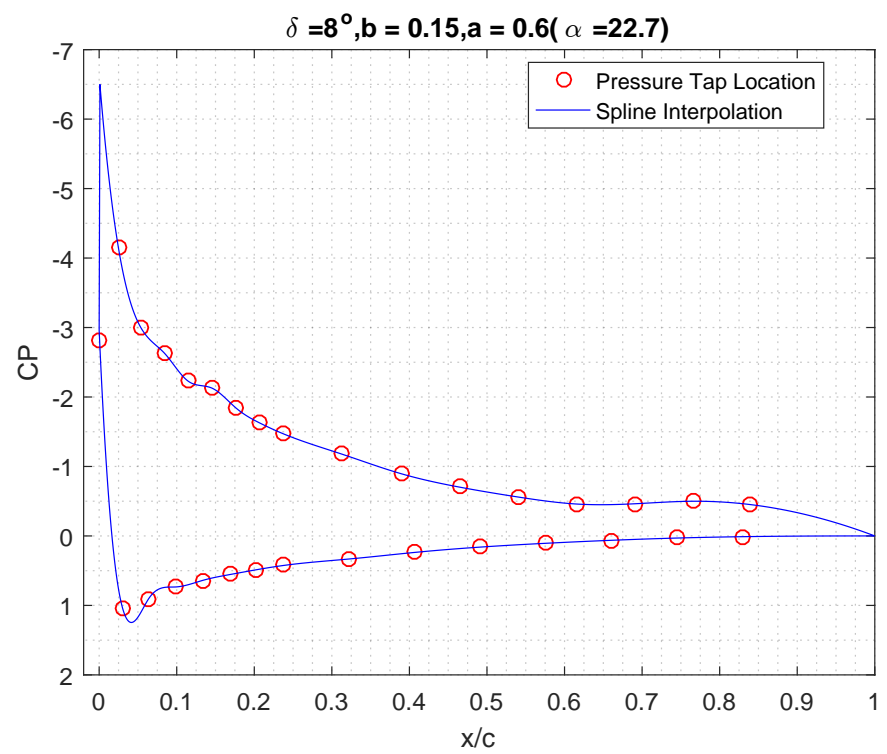

Figure 6.55: $C_{p}$ plot clean airfoil at $\alpha \approx 22.7^{\circ}$ 


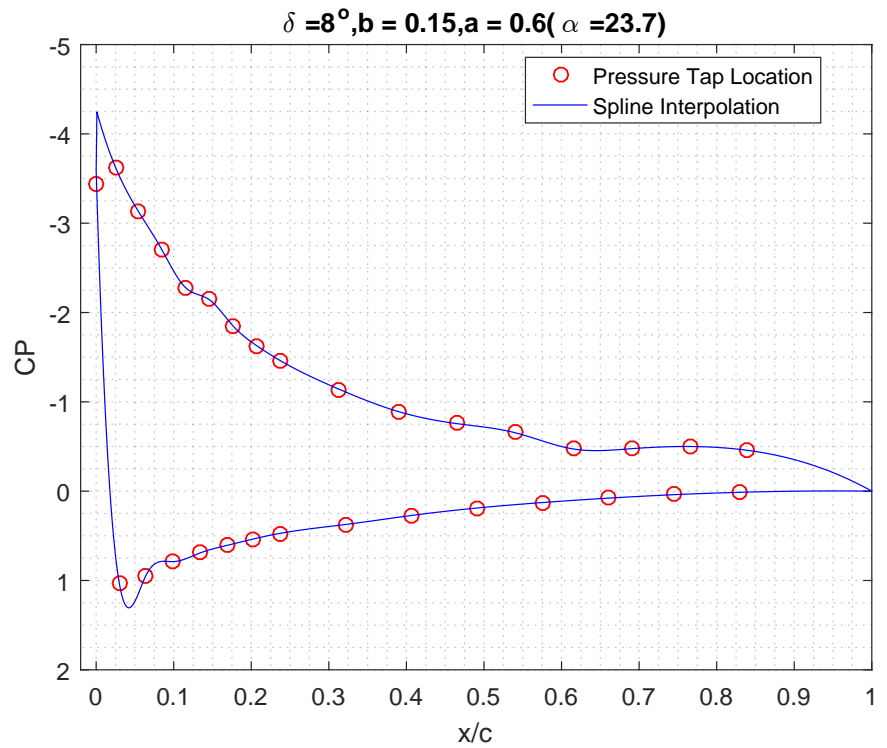

Figure 6.56: $C_{p}$ plot clean airfoil at $\alpha \approx 23.7^{\circ}$

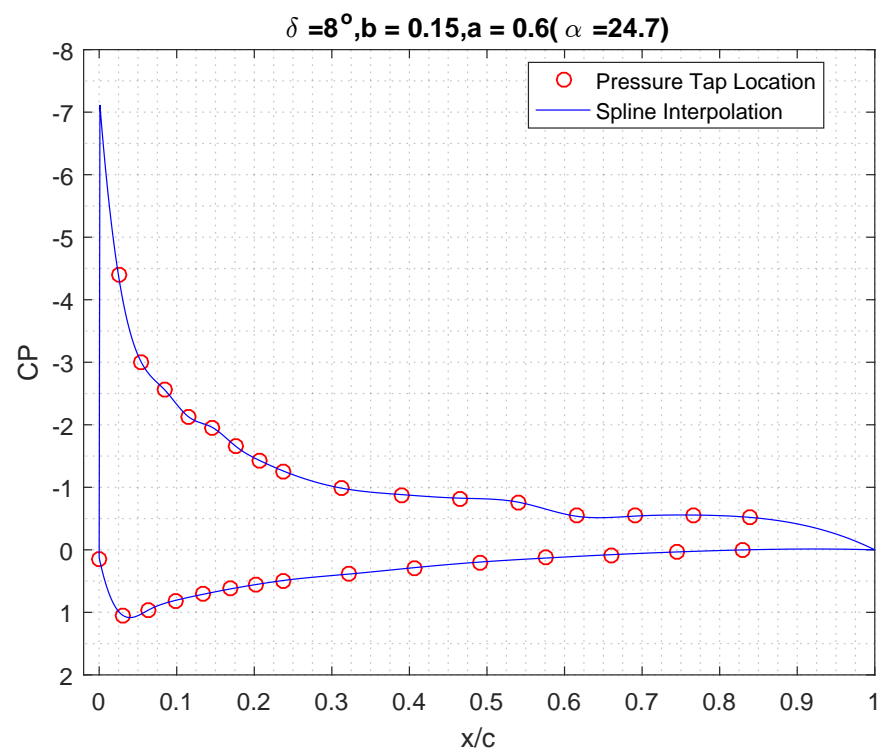

Figure 6.57: $C_{p}$ plot clean airfoil at $\alpha \approx 24.7^{\circ}$ 


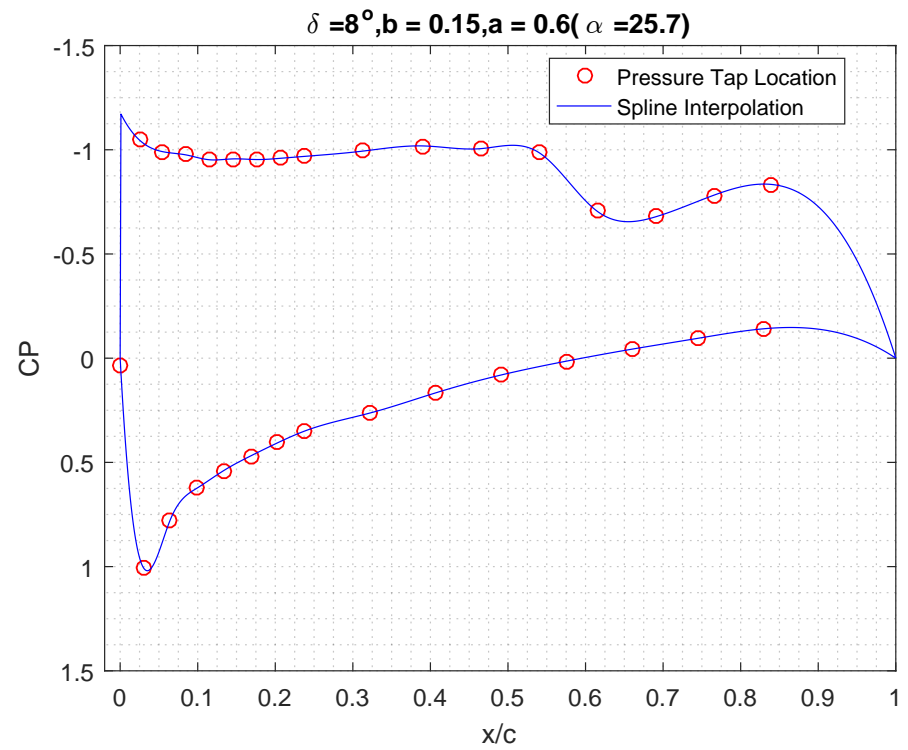

Figure 6.58: $C_{p}$ plot clean airfoil at $\alpha \approx 25.7^{\circ}$

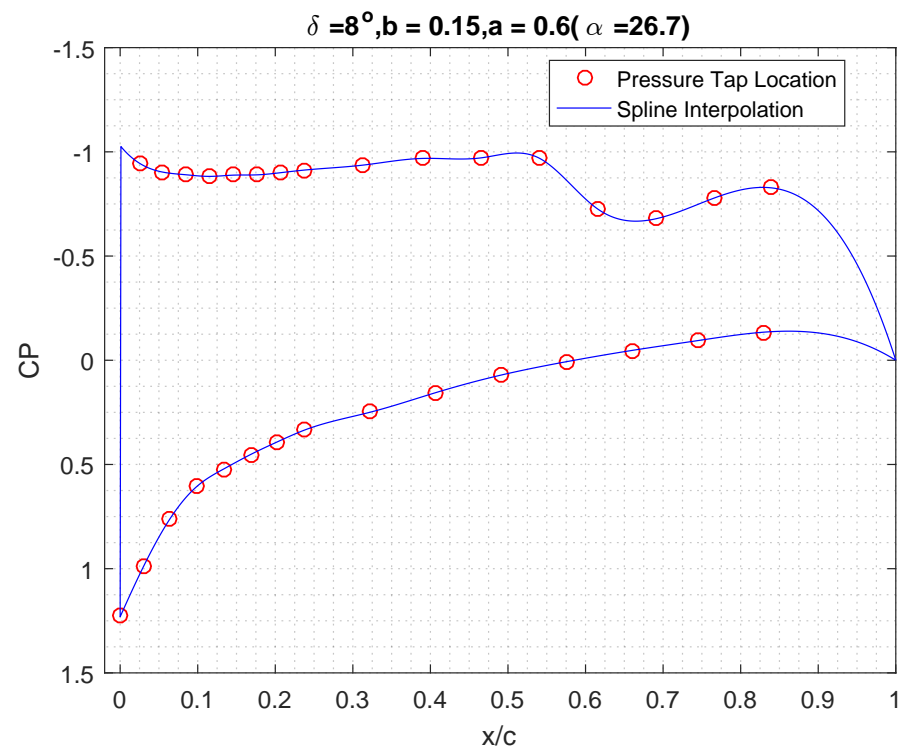

Figure 6.59: $C_{p}$ plot clean airfoil at $\alpha \approx 26.7^{\circ}$ 


\section{6 $\delta=15^{\circ}, b=15, a=60$ Configuration}

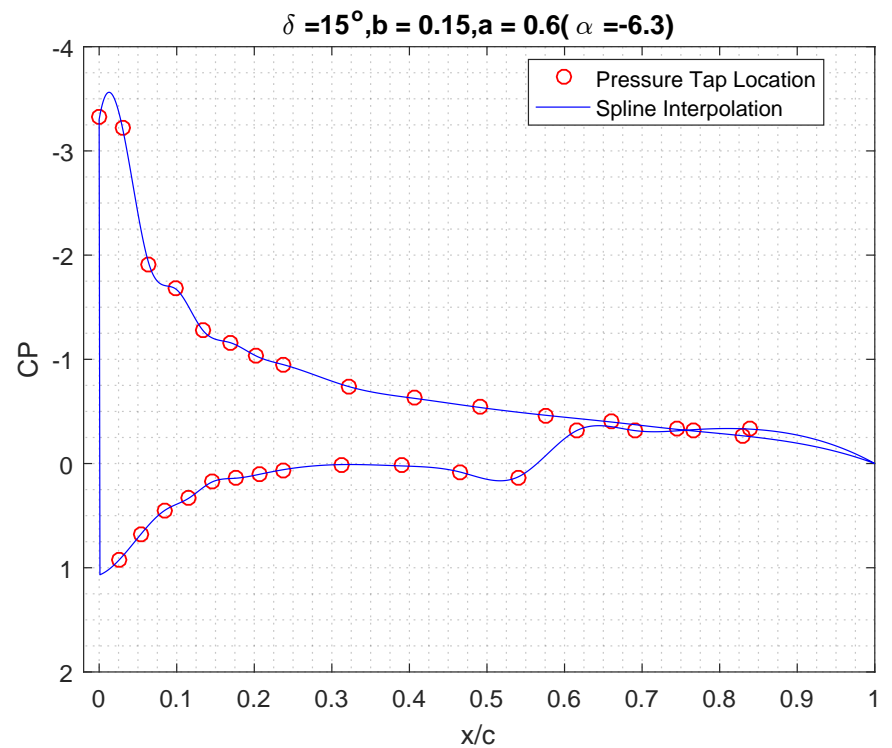

Figure 6.60: $C_{p}$ plot clean airfoil at $\alpha \approx-6.3^{\circ}$

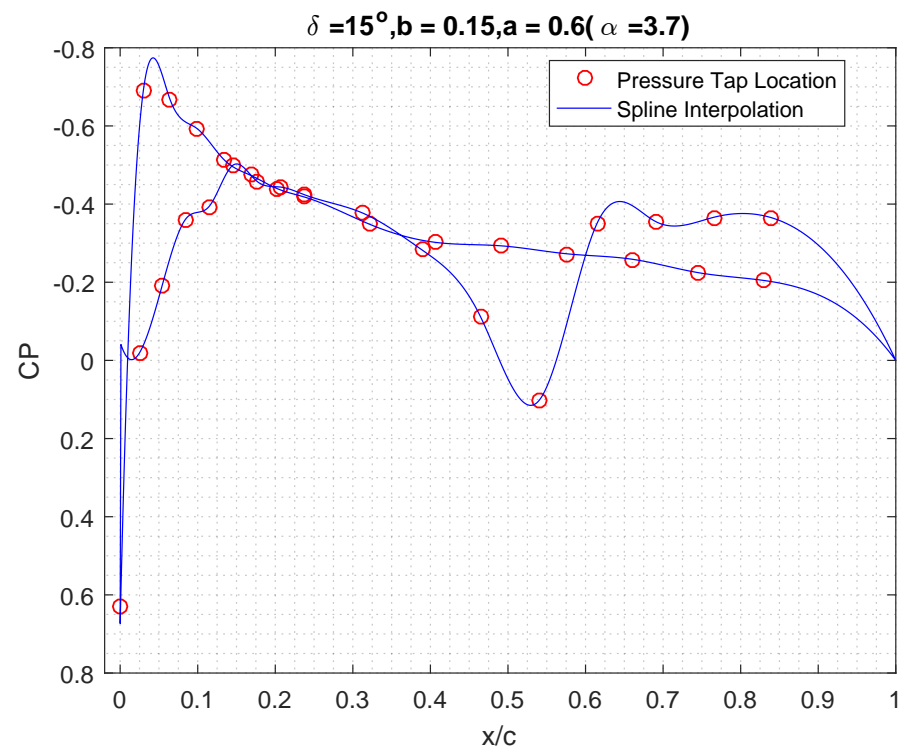

Figure 6.61: $C_{p}$ plot clean airfoil at $\alpha \approx 3.7^{\circ}$ 


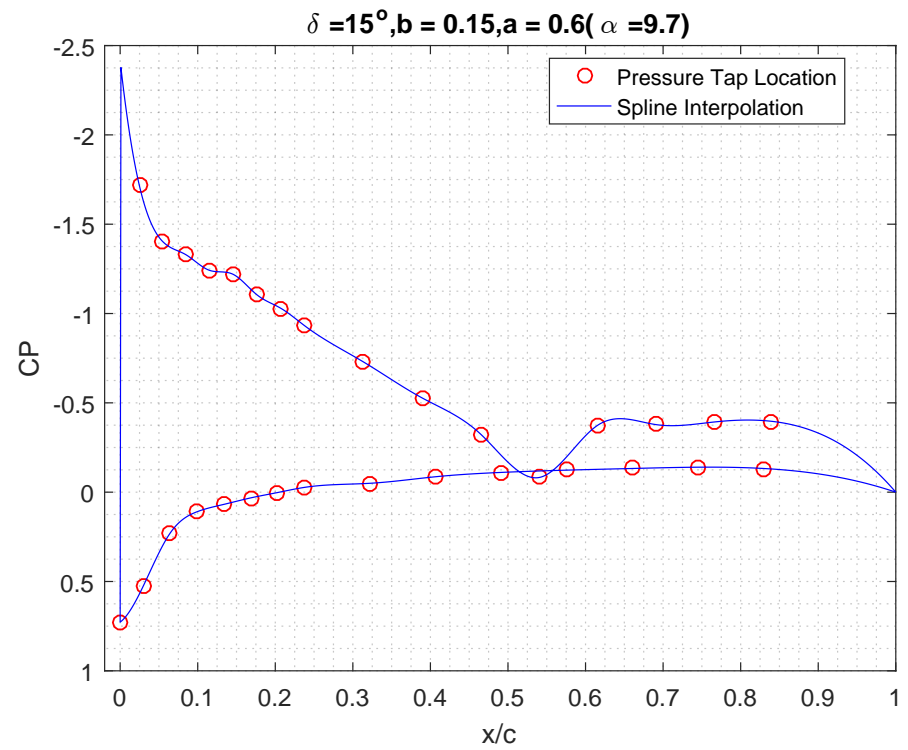

Figure 6.62: $C_{p}$ plot clean airfoil at $\alpha \approx 9.7^{\circ}$

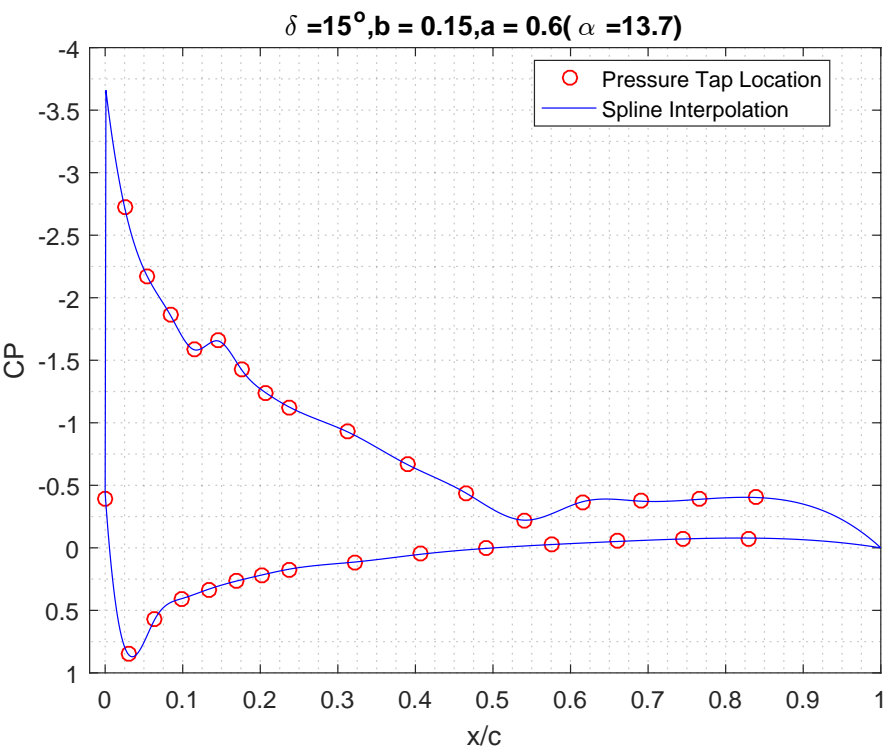

Figure 6.63: $C_{p}$ plot clean airfoil at $\alpha \approx 13.7^{\circ}$ 


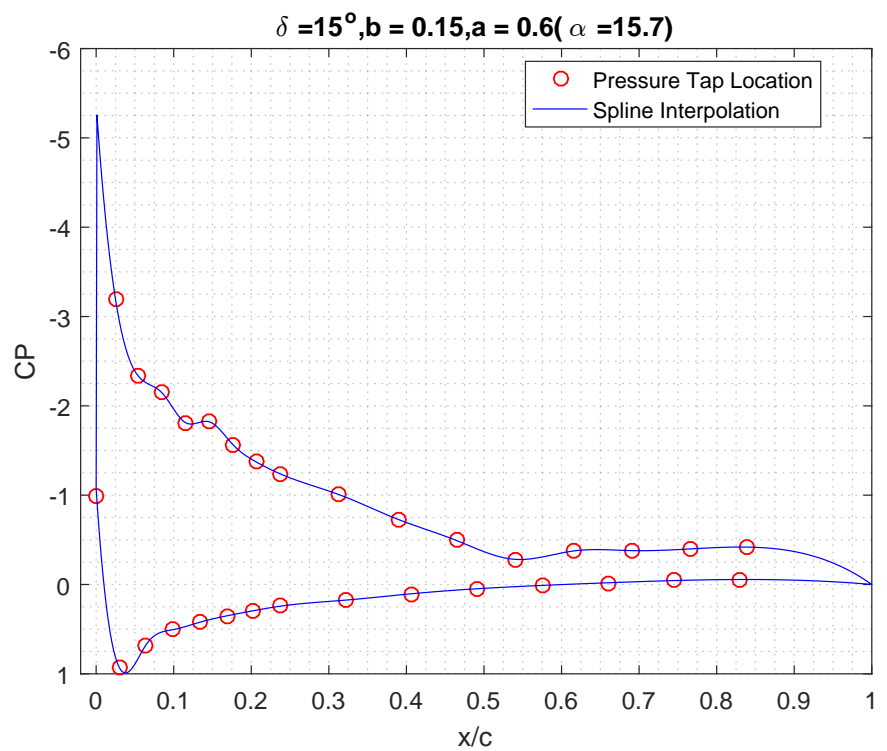

Figure 6.64: $C_{p}$ plot clean airfoil at $\alpha \approx 15.7^{\circ}$

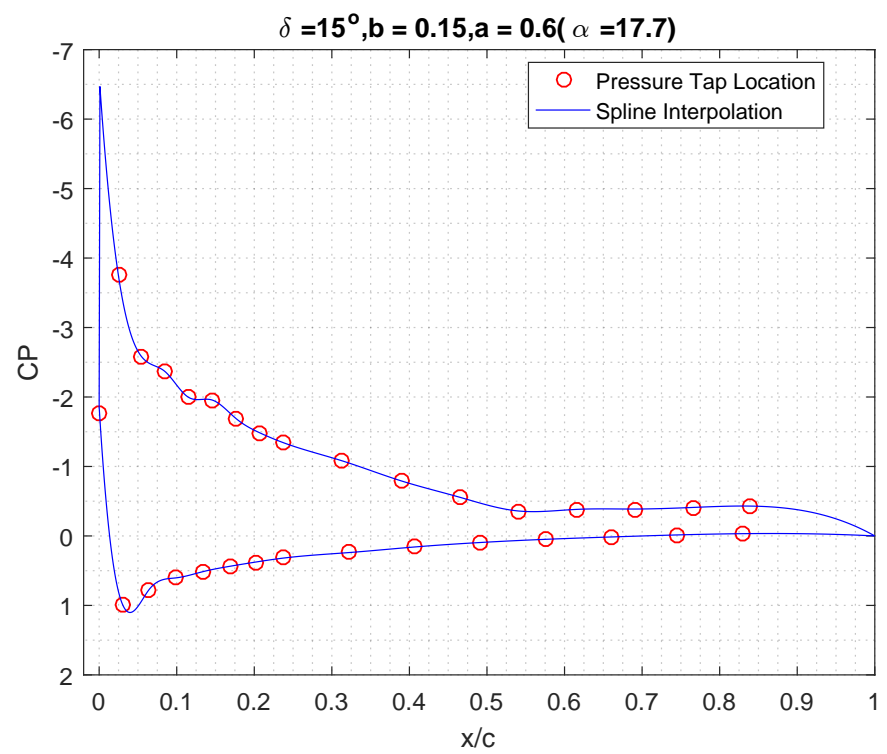

Figure 6.65: $C_{p}$ plot clean airfoil at $\alpha \approx 17.7^{\circ}$ 


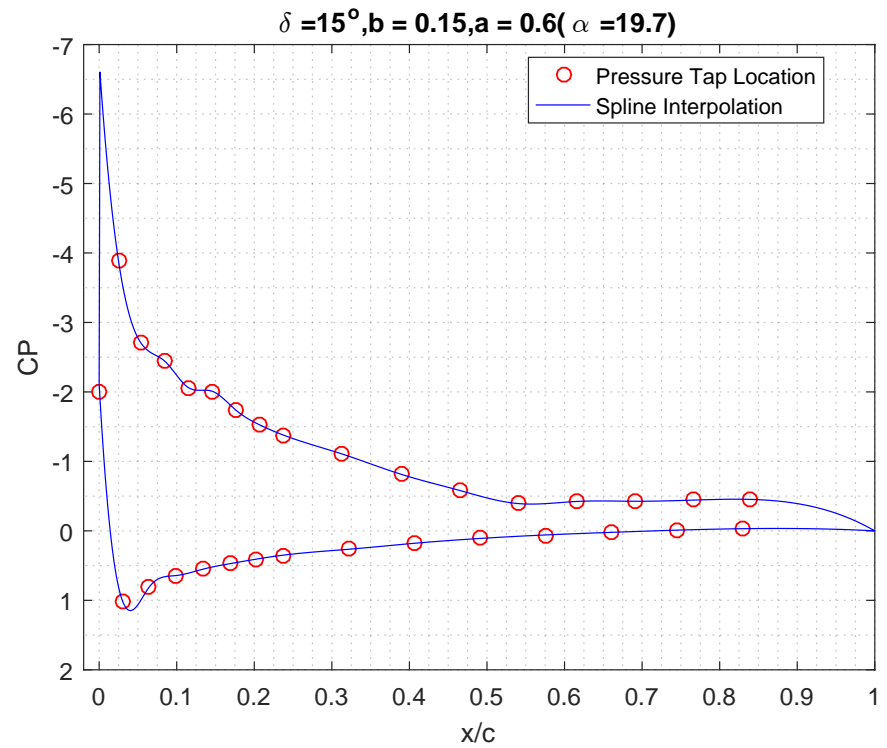

Figure 6.66: $C_{p}$ plot clean airfoil at $\alpha \approx 19.7^{\circ}$

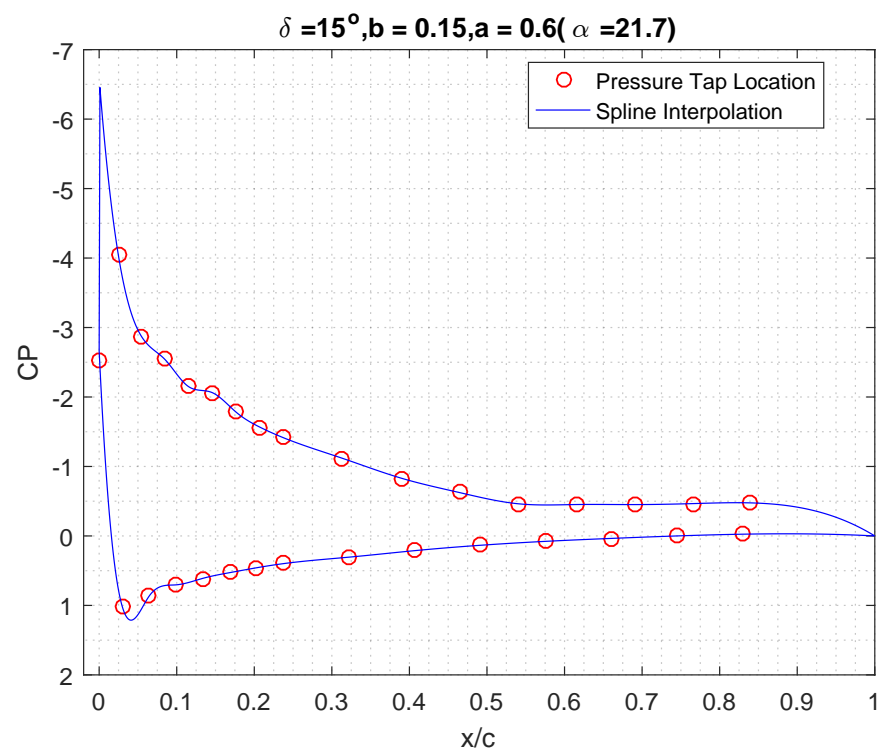

Figure 6.67: $C_{p}$ plot clean airfoil at $\alpha \approx 21.7^{\circ}$ 


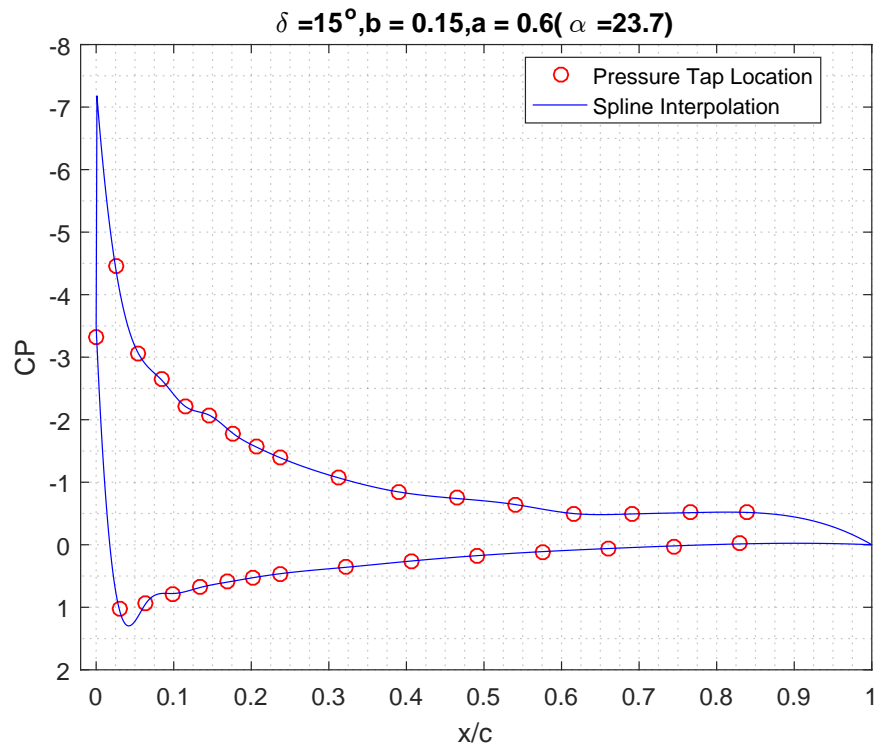

Figure 6.68: $C_{p}$ plot clean airfoil at $\alpha \approx 23.7^{\circ}$

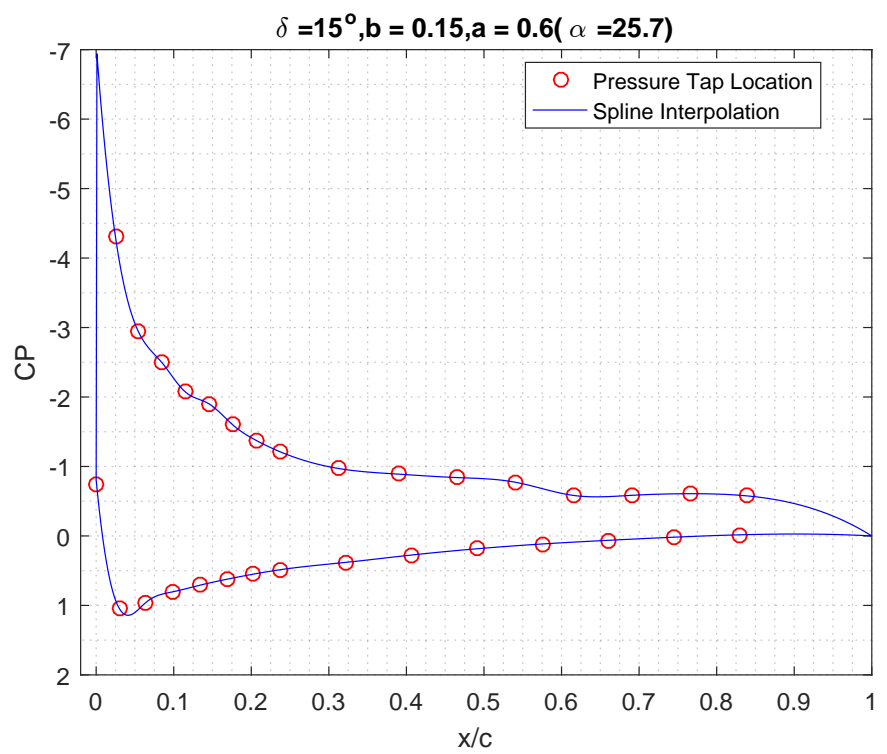

Figure 6.69: $C_{p}$ plot clean airfoil at $\alpha \approx 25.7^{\circ}$ 


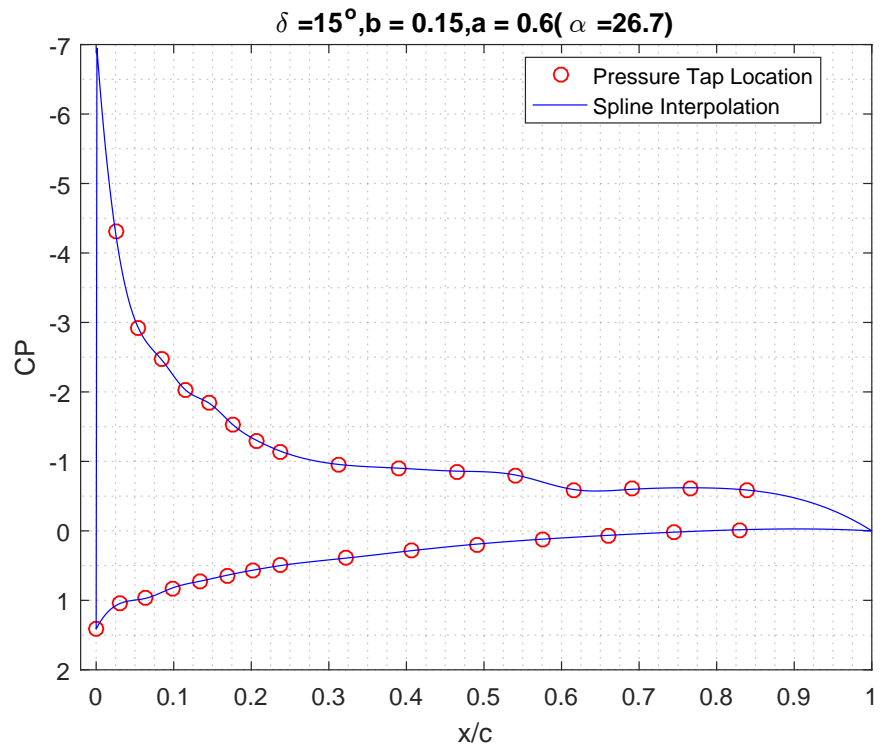

Figure 6.70: $C_{p}$ plot clean airfoil at $\alpha \approx 26.7^{\circ}$

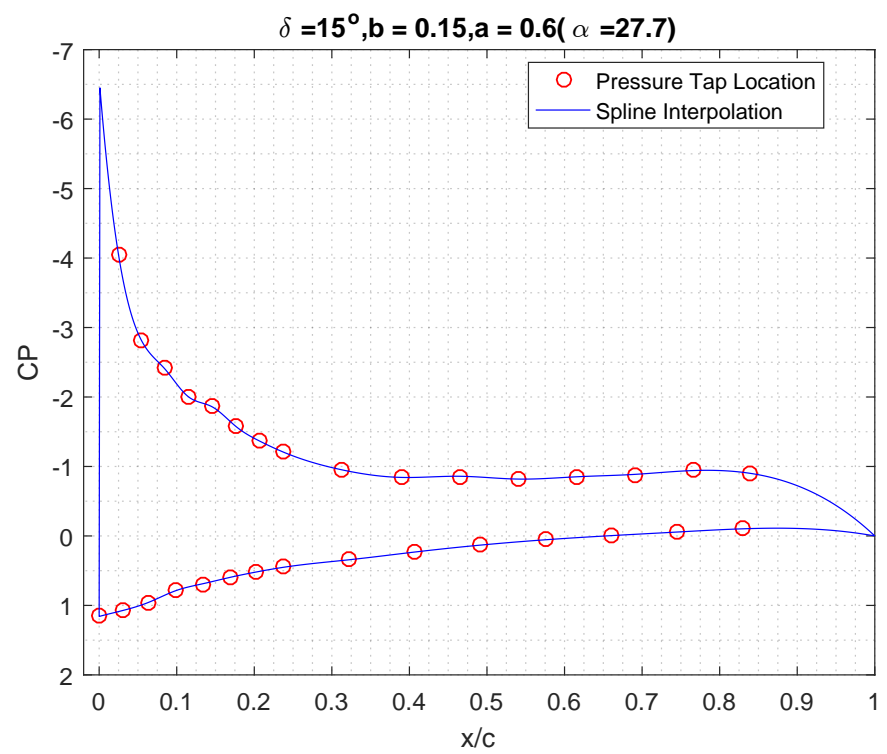

Figure 6.71: $C_{p}$ plot clean airfoil at $\alpha \approx 27.7^{\circ}$ 


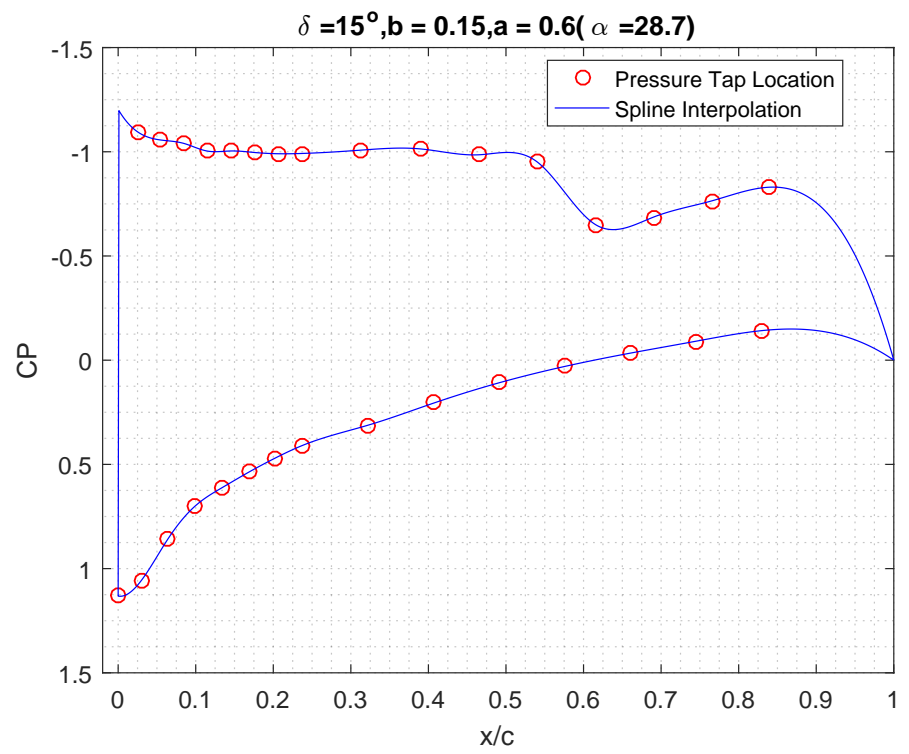

Figure 6.72: $C_{p}$ plot clean airfoil at $\alpha \approx 28.7^{\circ}$

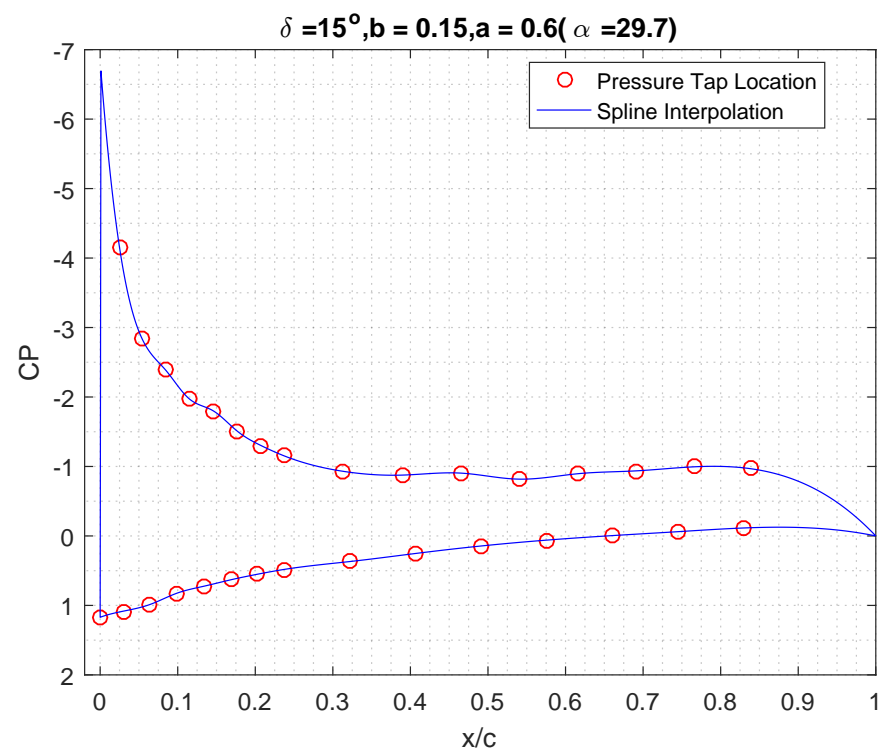

Figure 6.73: $C_{p}$ plot clean airfoil at $\alpha \approx 29.7^{\circ}$ 


\section{7 $\delta=25^{\circ}, b=15, a=60$ Configuration}

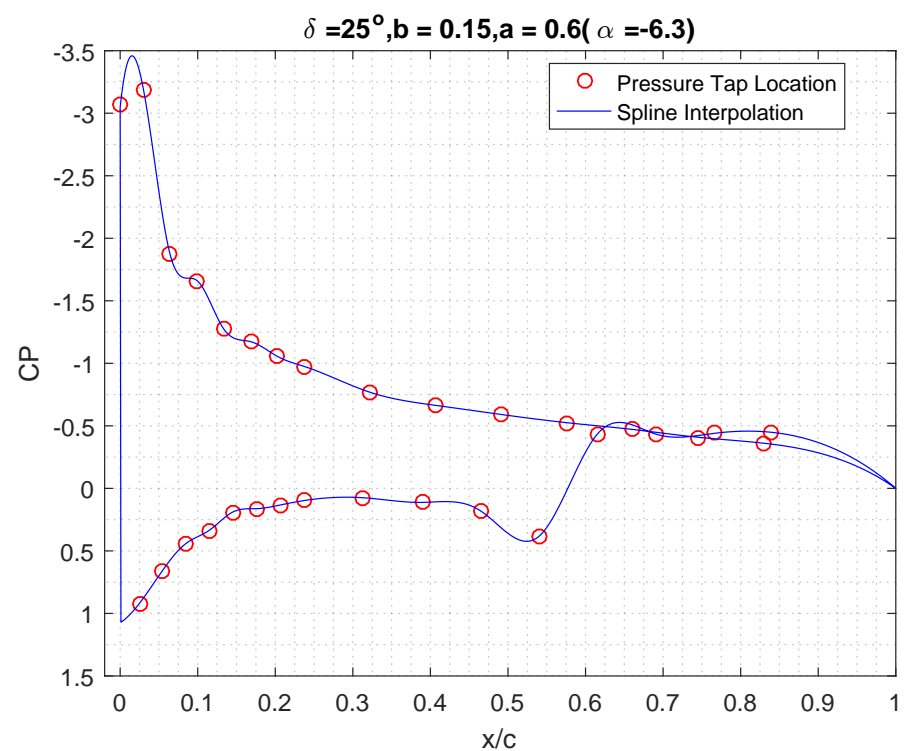

Figure 6.74: $C_{p}$ plot clean airfoil at $\alpha \approx-6.3^{\circ}$

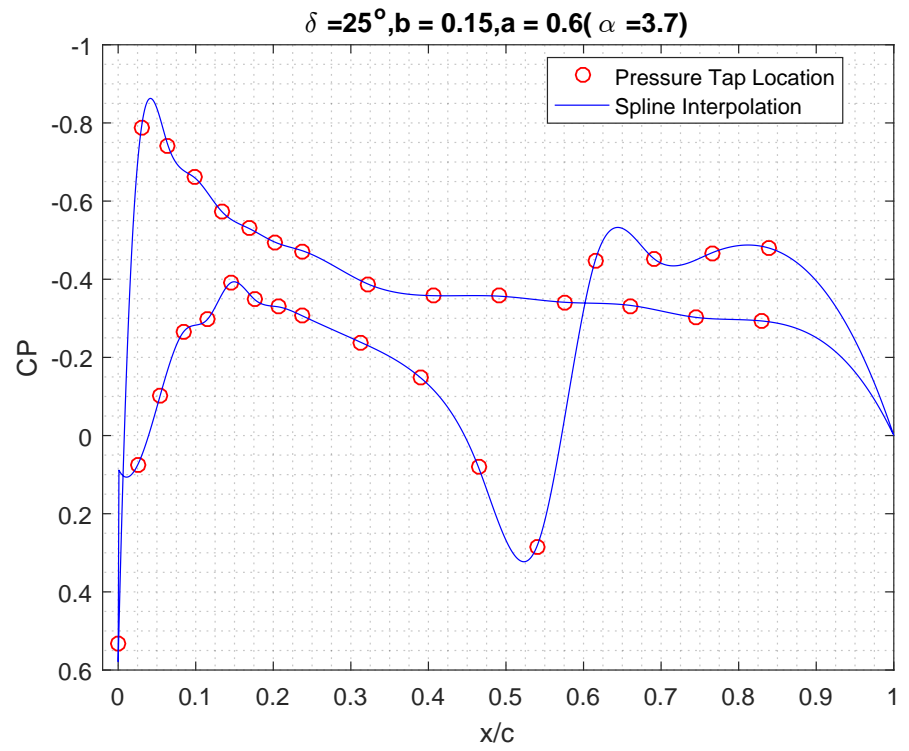

Figure 6.75: $C_{p}$ plot clean airfoil at $\alpha \approx 3.7^{\circ}$ 


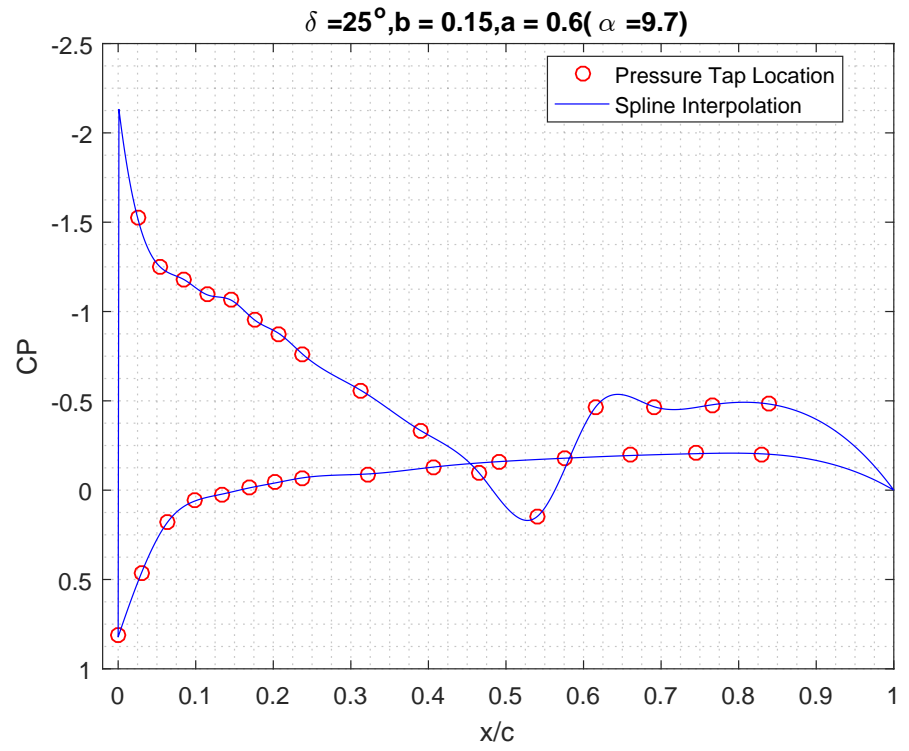

Figure 6.76: $C_{p}$ plot clean airfoil at $\alpha \approx 9.7^{\circ}$

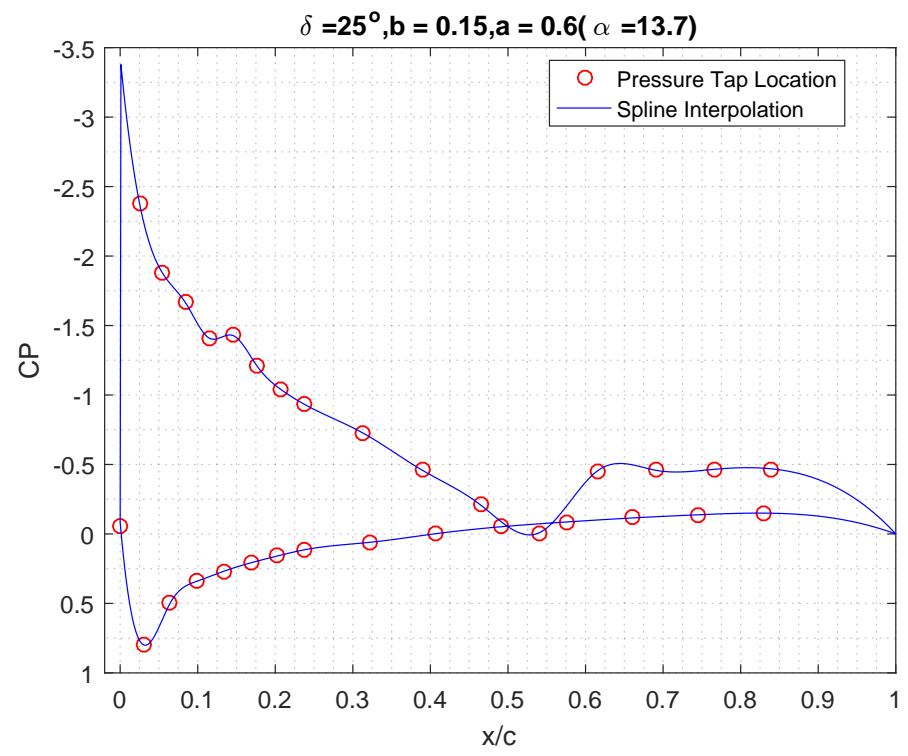

Figure 6.77: $C_{p}$ plot clean airfoil at $\alpha \approx 13.7^{\circ}$ 


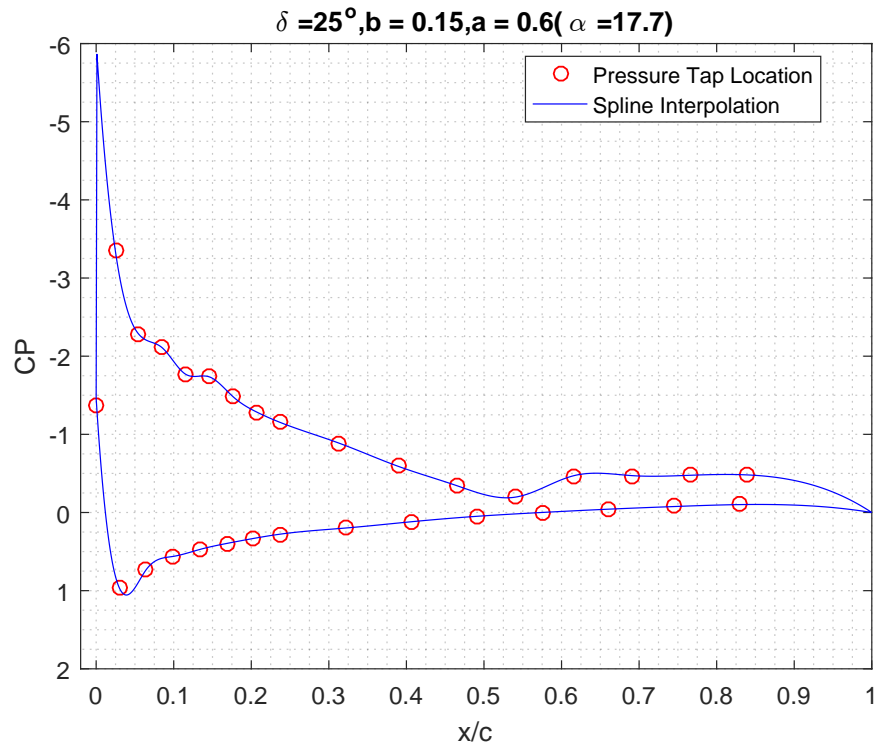

Figure 6.78: $C_{p}$ plot clean airfoil at $\alpha \approx 17.7^{\circ}$

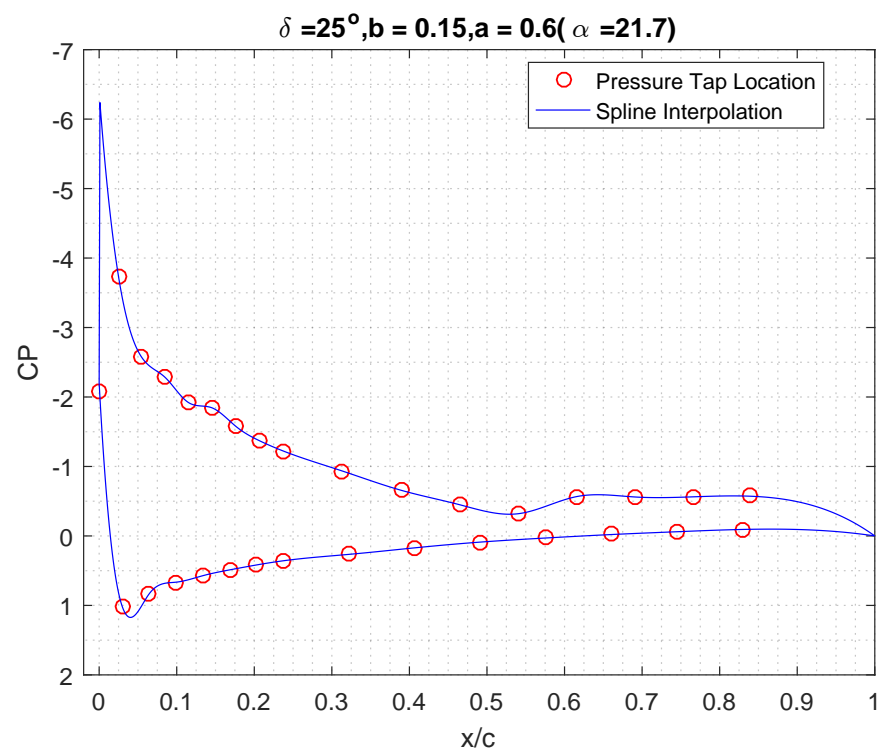

Figure 6.79: $C_{p}$ plot clean airfoil at $\alpha \approx 21.7^{\circ}$ 


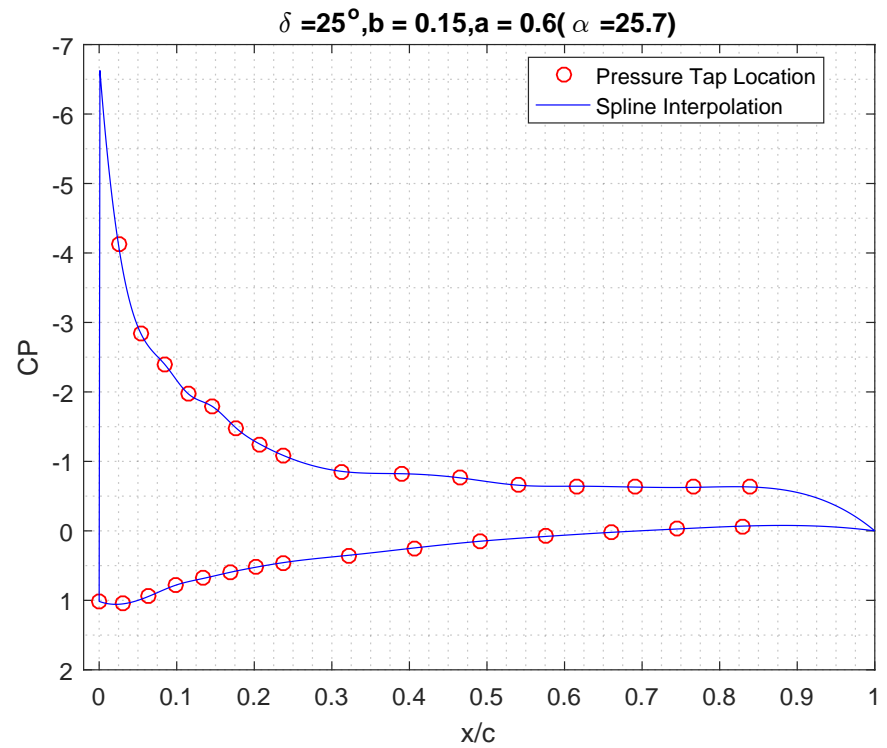

Figure 6.80: $C_{p}$ plot clean airfoil at $\alpha \approx 25.7^{\circ}$

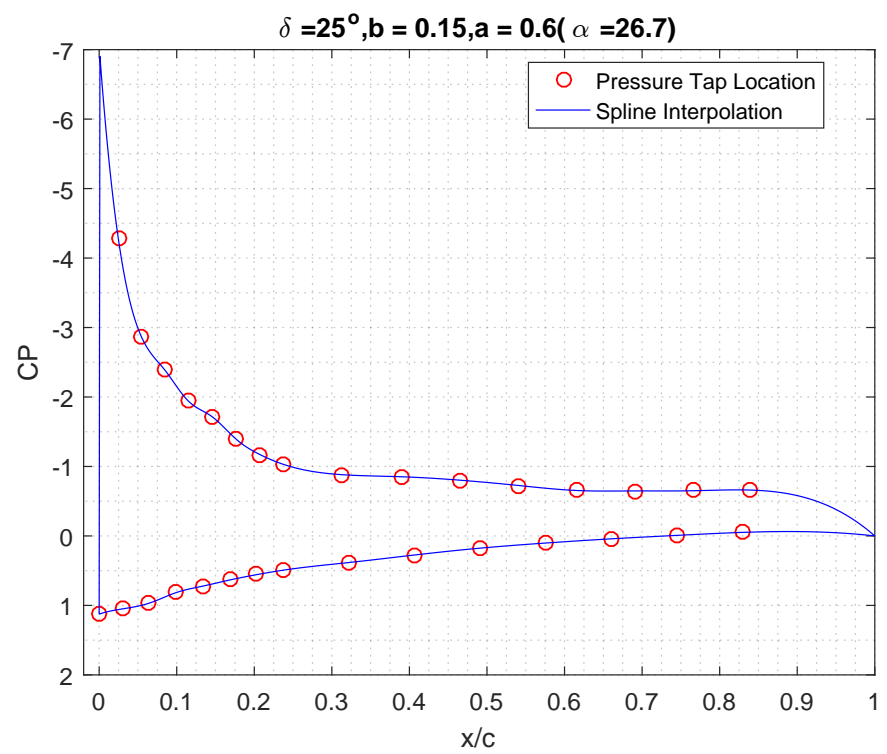

Figure 6.81: $C_{p}$ plot clean airfoil at $\alpha \approx 26.7^{\circ}$ 


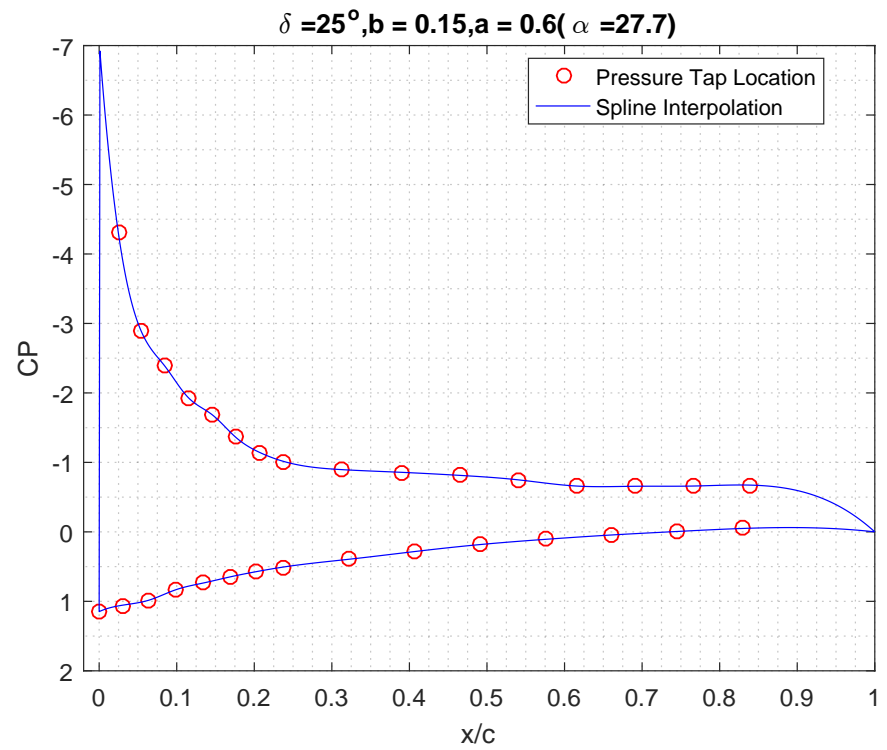

Figure 6.82: $C_{p}$ plot clean airfoil at $\alpha \approx 27.7^{\circ}$

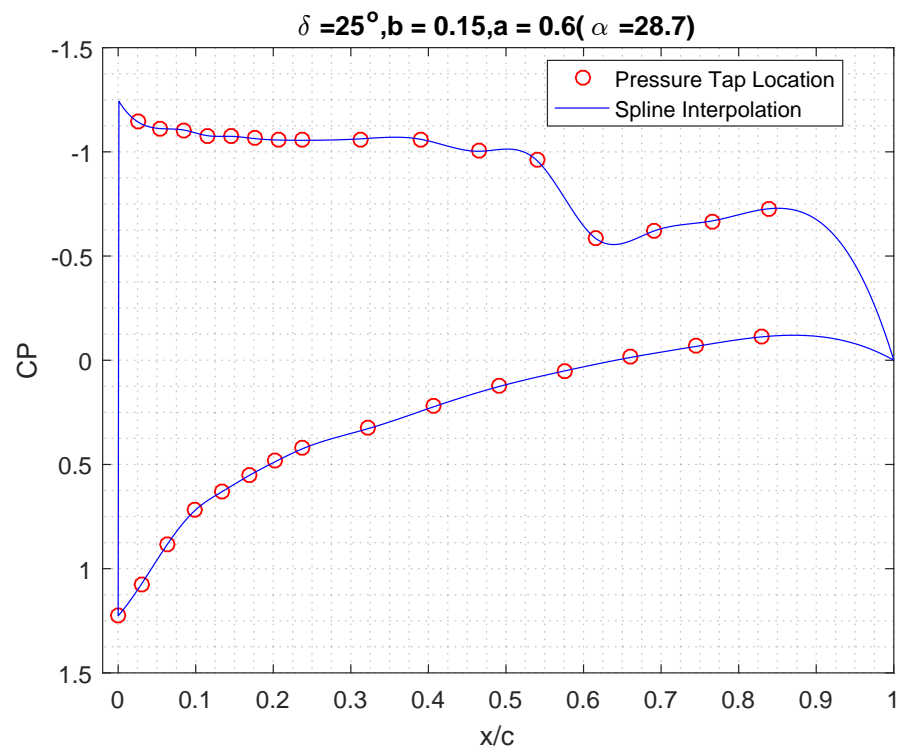

Figure 6.83: $C_{p}$ plot clean airfoil at $\alpha \approx 28.7^{\circ}$ 


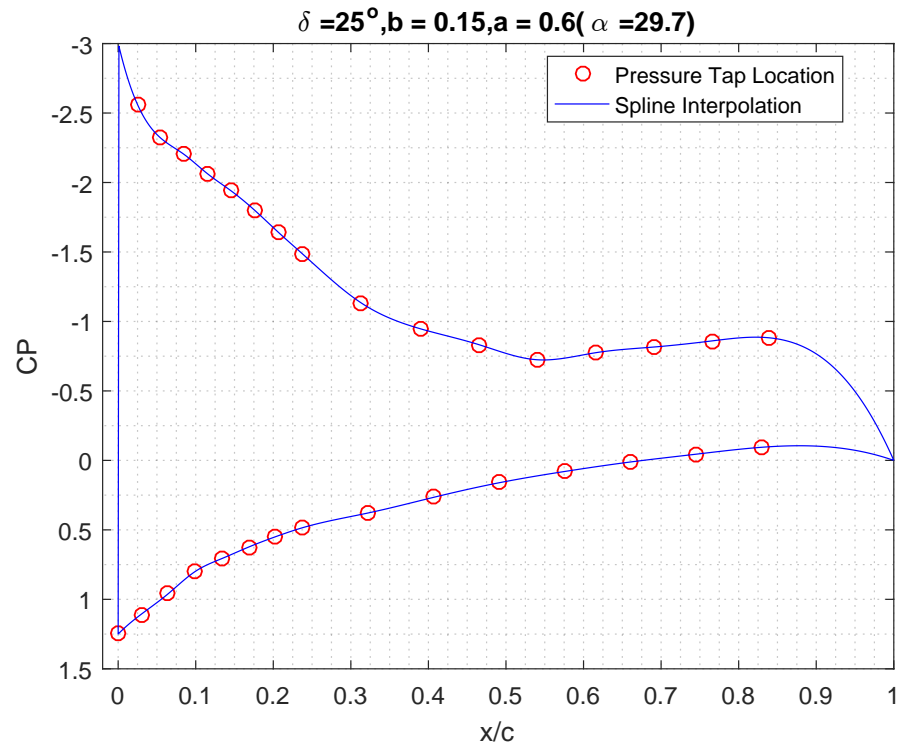

Figure 6.84: $C_{p}$ plot clean airfoil at $\alpha \approx 29.7^{\circ}$

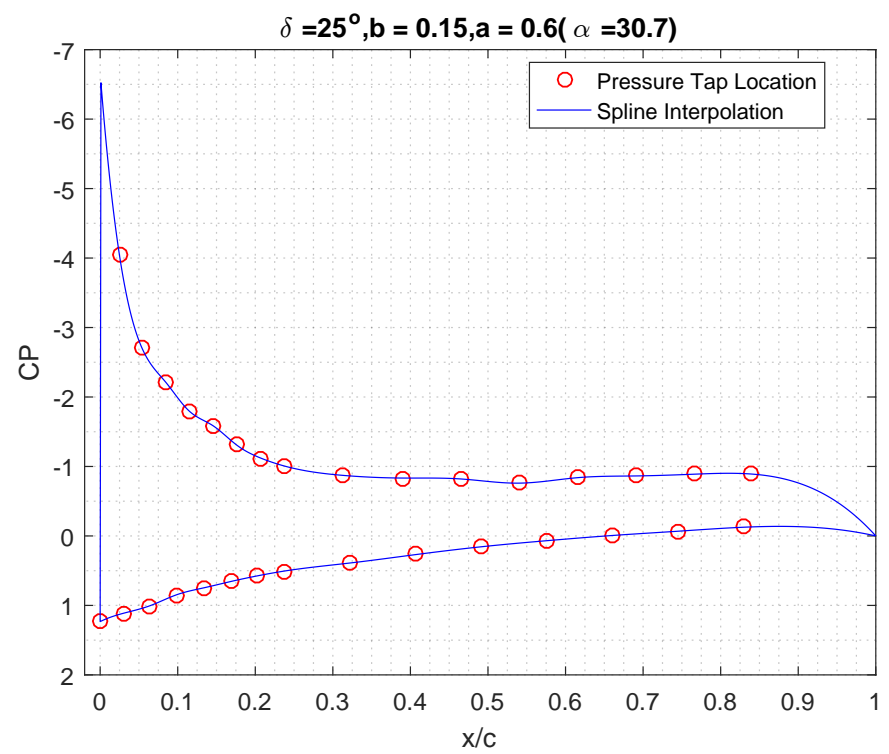

Figure 6.85: $C_{p}$ plot clean airfoil at $\alpha \approx 30.7^{\circ}$ 


\section{Appendix 7}

\section{Wake Profile Plots from Wind Tunnel Experiment}

\subsection{Clean Configuration}

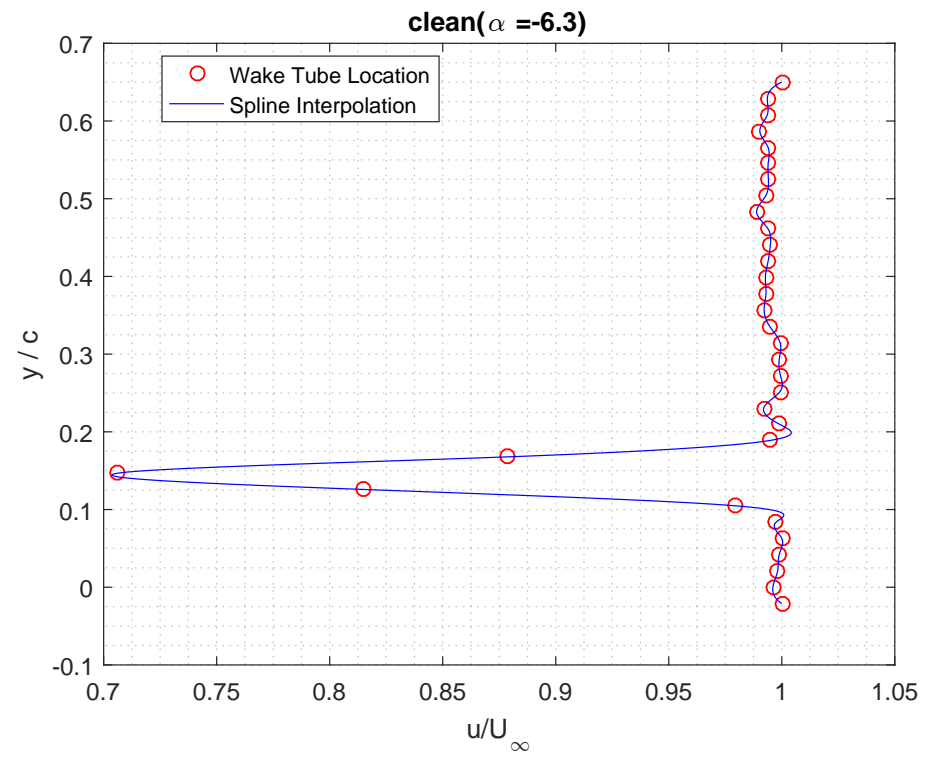

Figure 7.1: Wake plot clean airfoil at $\alpha \approx-6.3^{\circ}$ 


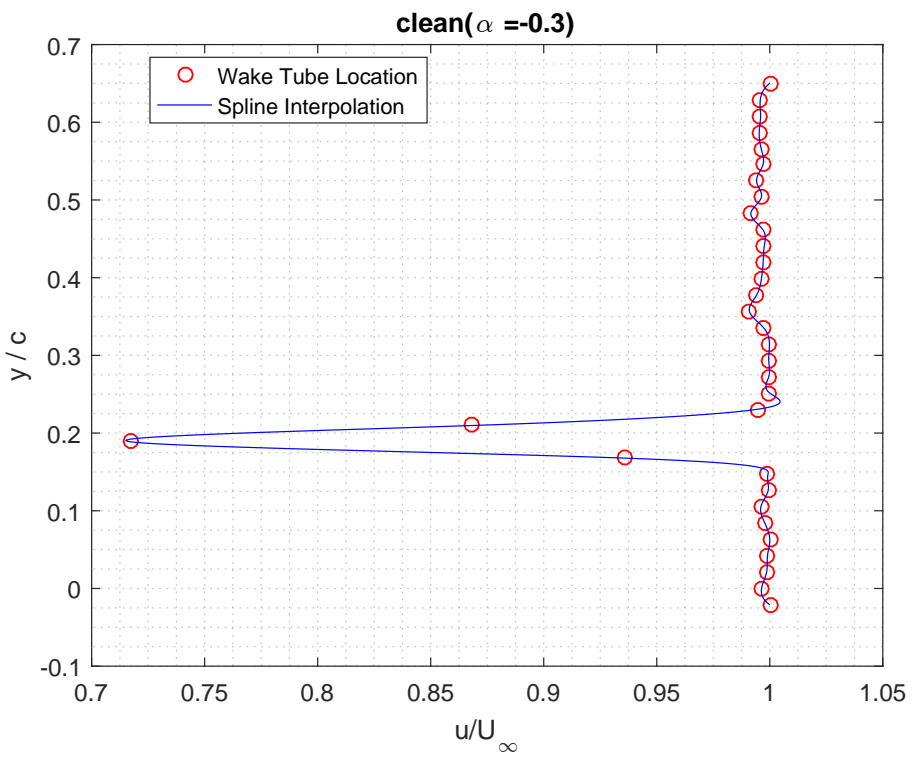

Figure 7.2: Wake plot clean airfoil at $\alpha \approx-0.3^{\circ}$

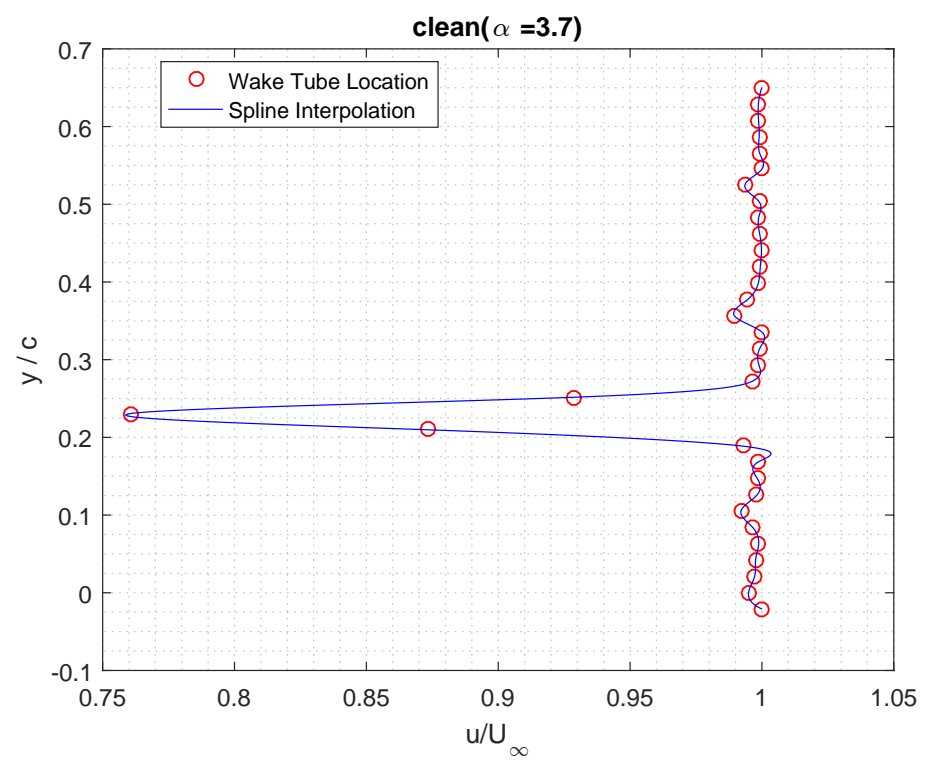

Figure 7.3: Wake plot clean airfoil at $\alpha \approx 3.7^{\circ}$ 


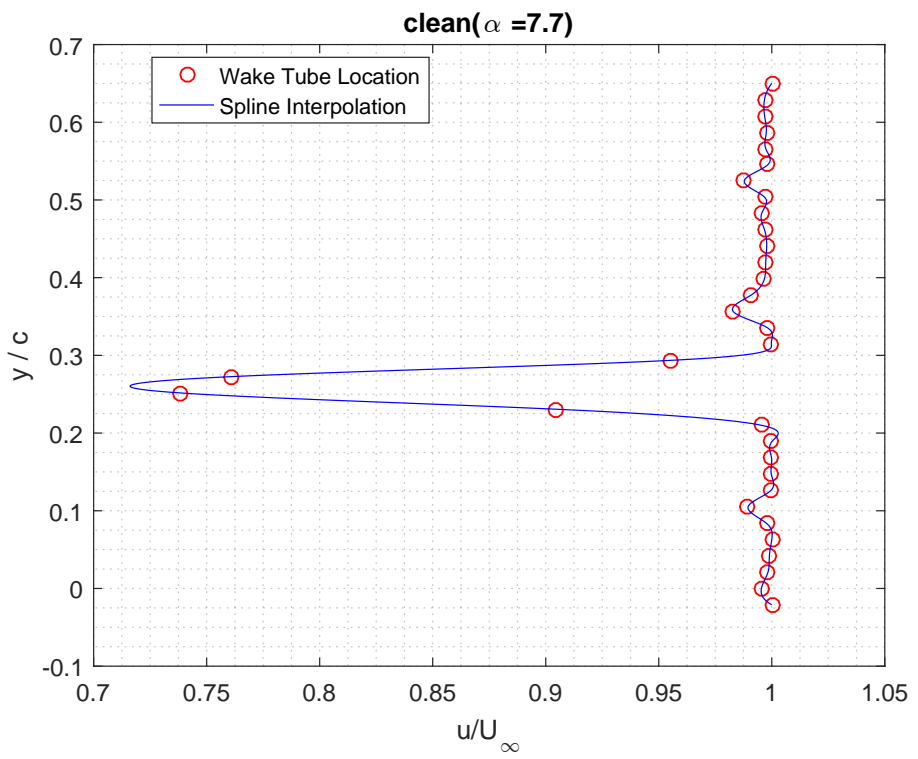

Figure 7.4: Wake plot clean airfoil at $\alpha \approx 7.7^{\circ}$

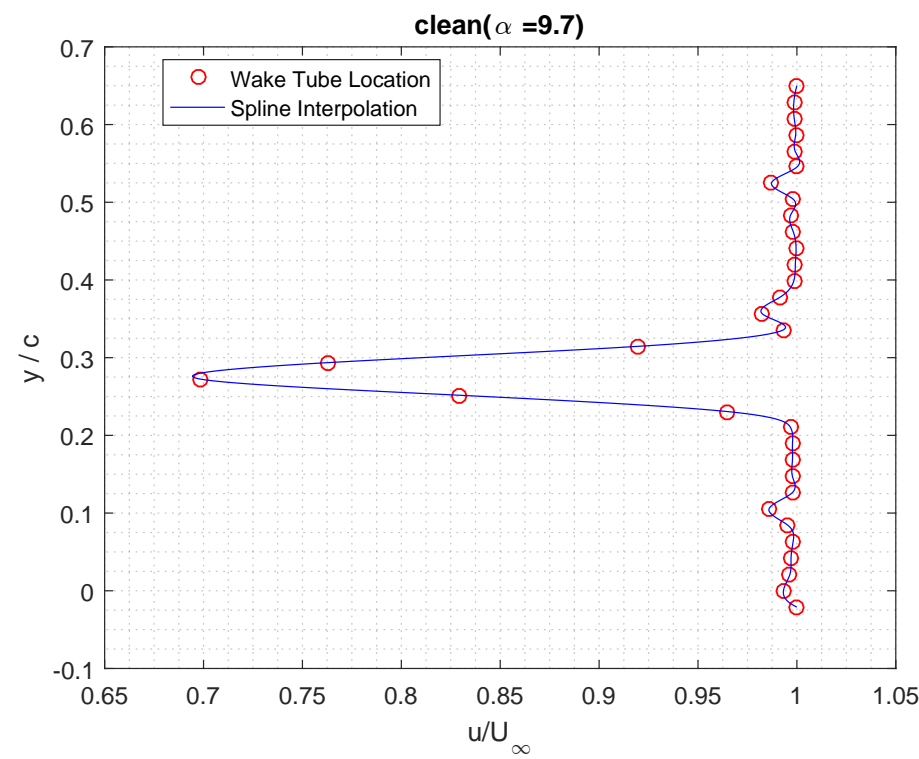

Figure 7.5: Wake plot clean airfoil at $\alpha \approx 9.7^{\circ}$ 


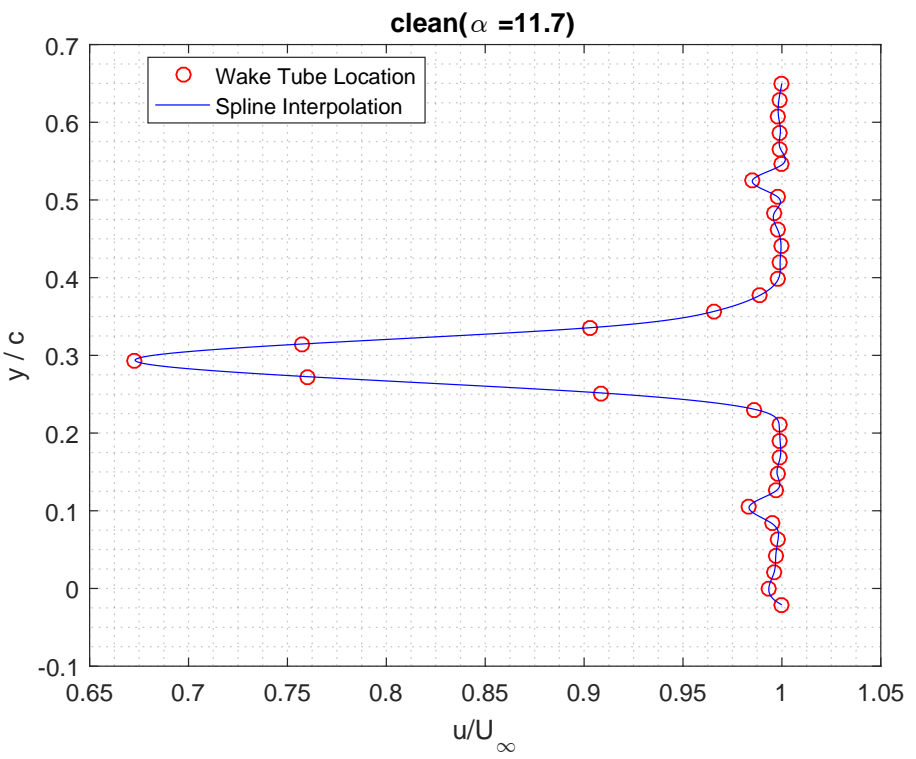

Figure 7.6: Wake plot clean airfoil at $\alpha \approx 11.7^{\circ}$

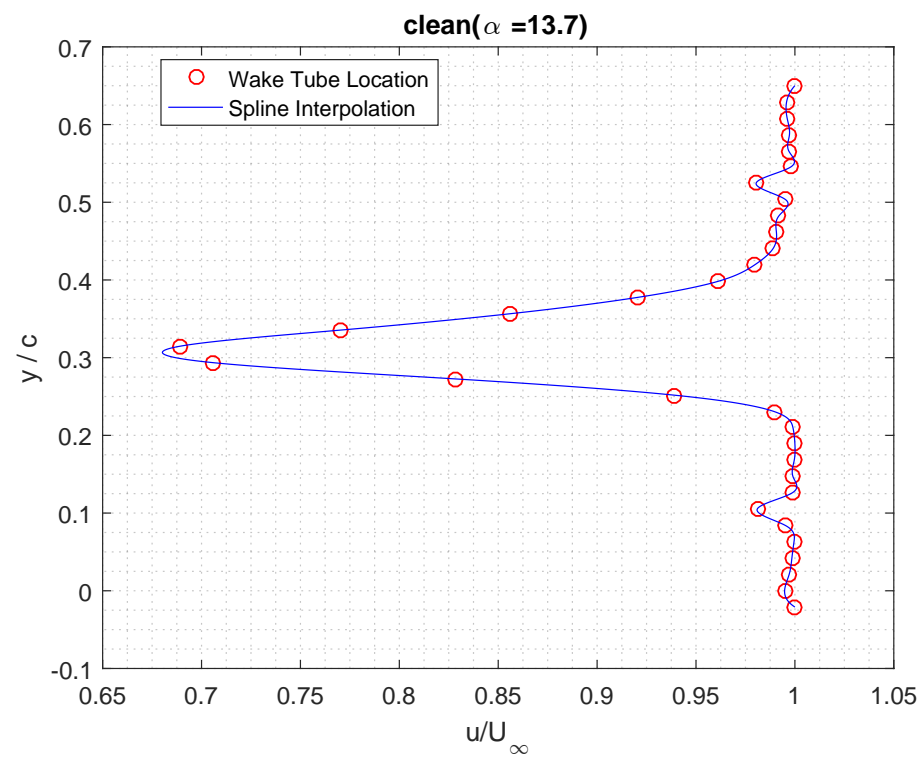

Figure 7.7: Wake plot clean airfoil at $\alpha \approx 13.7^{\circ}$ 


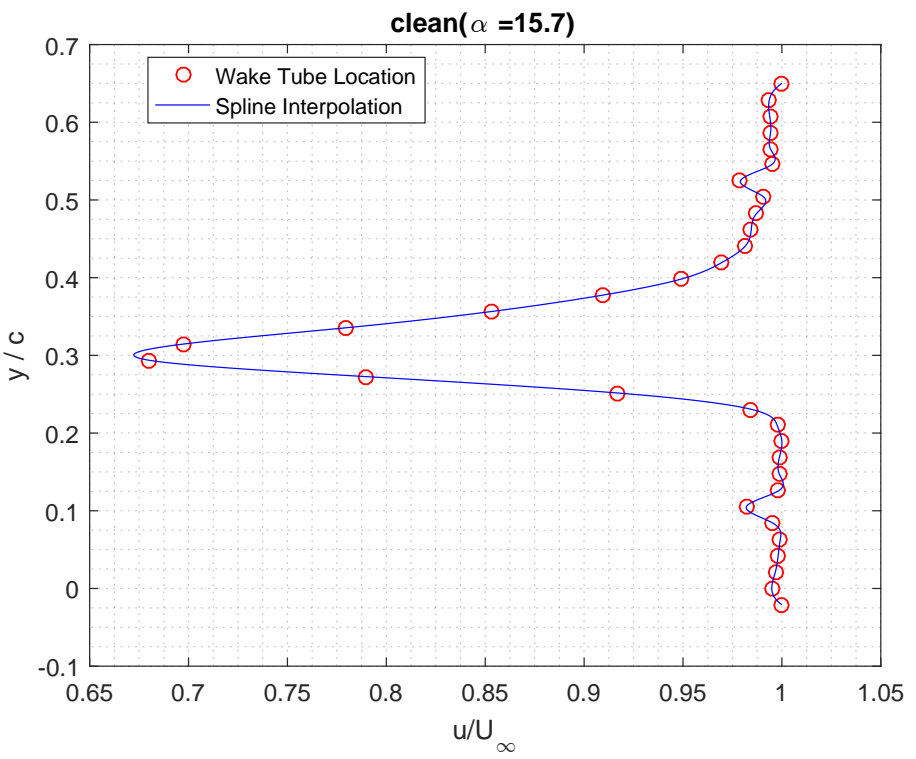

Figure 7.8: Wake plot clean airfoil at $\alpha \approx 15.7^{\circ}$

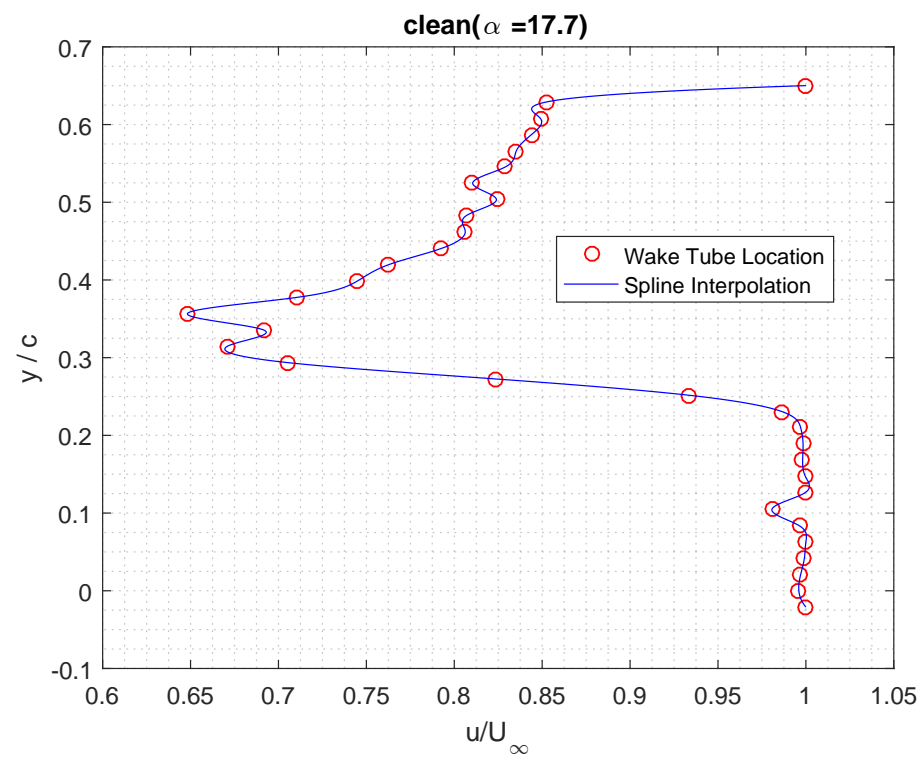

Figure 7.9: Wake plot clean airfoil at $\alpha \approx 17.7^{\circ}$ 
$7.2 \delta=8^{\circ}, b=10, a=60$ Configuration

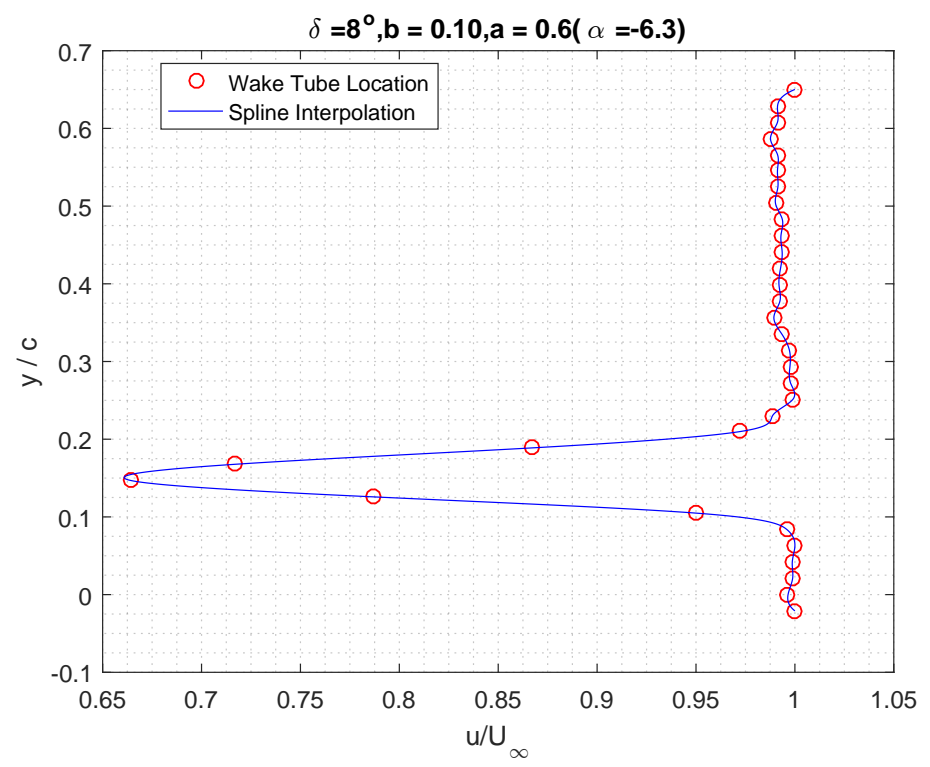

Figure 7.10: Wake plot clean airfoil at $\alpha \approx-6.3^{\circ}$

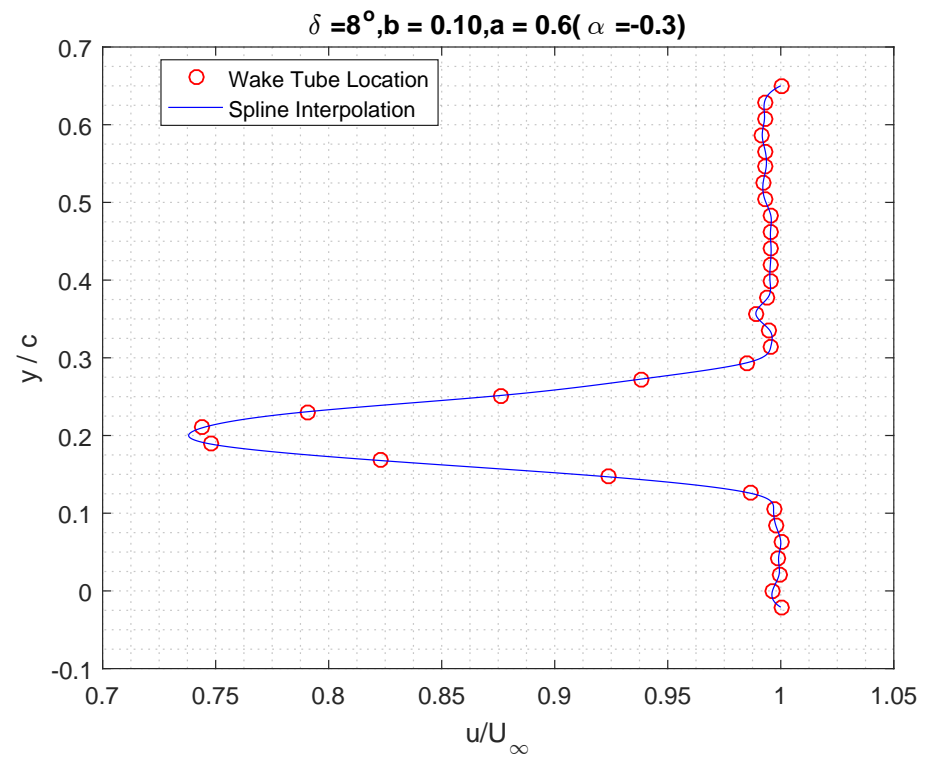

Figure 7.11: Wake plot clean airfoil at $\alpha \approx-0.3^{\circ}$ 


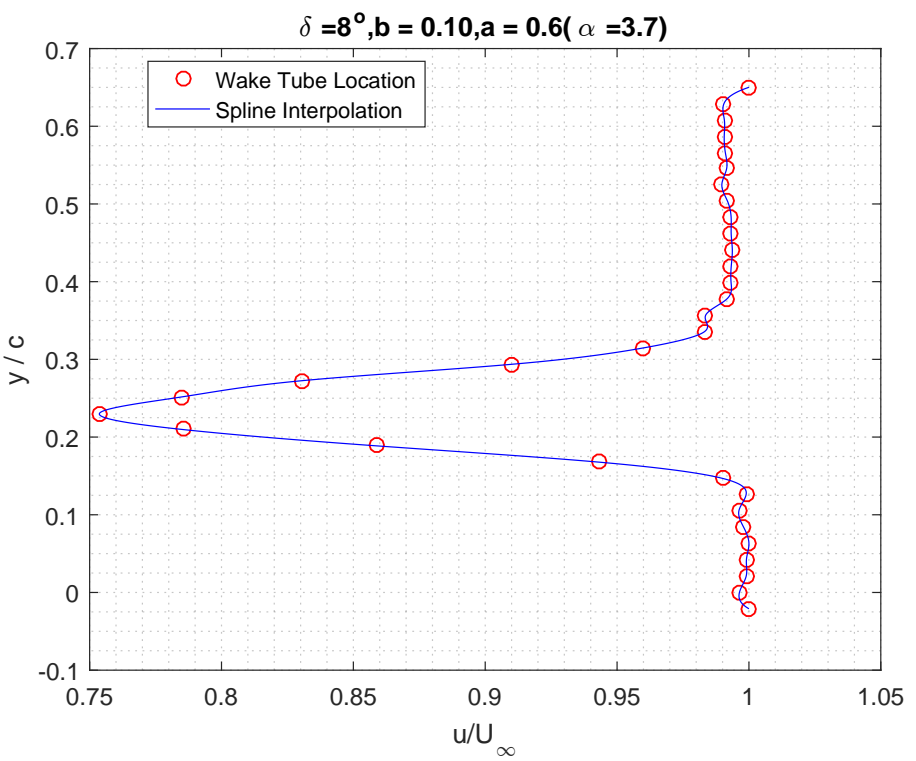

Figure 7.12: Wake plot clean airfoil at $\alpha \approx 3.7^{\circ}$

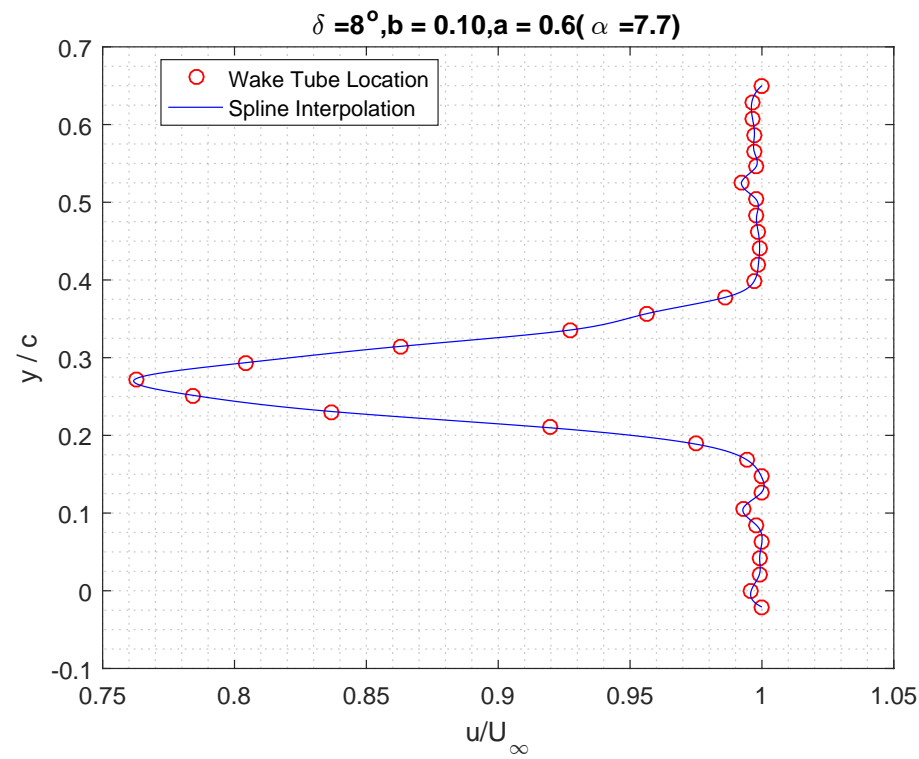

Figure 7.13: Wake plot clean airfoil at $\alpha \approx 7.7^{\circ}$ 


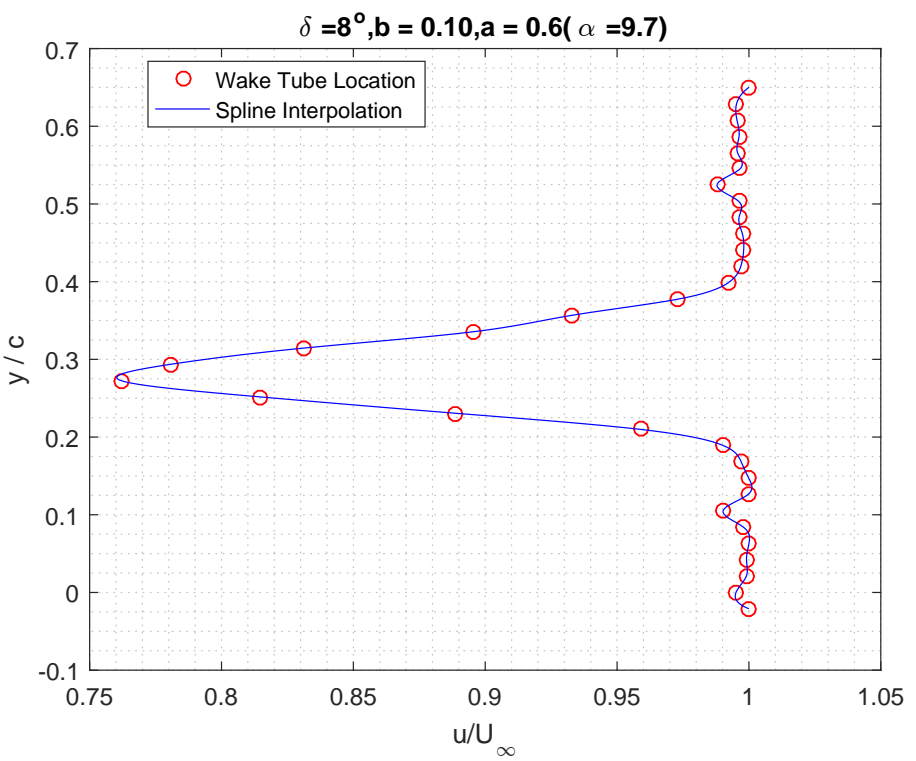

Figure 7.14: Wake plot clean airfoil at $\alpha \approx 9.7^{\circ}$

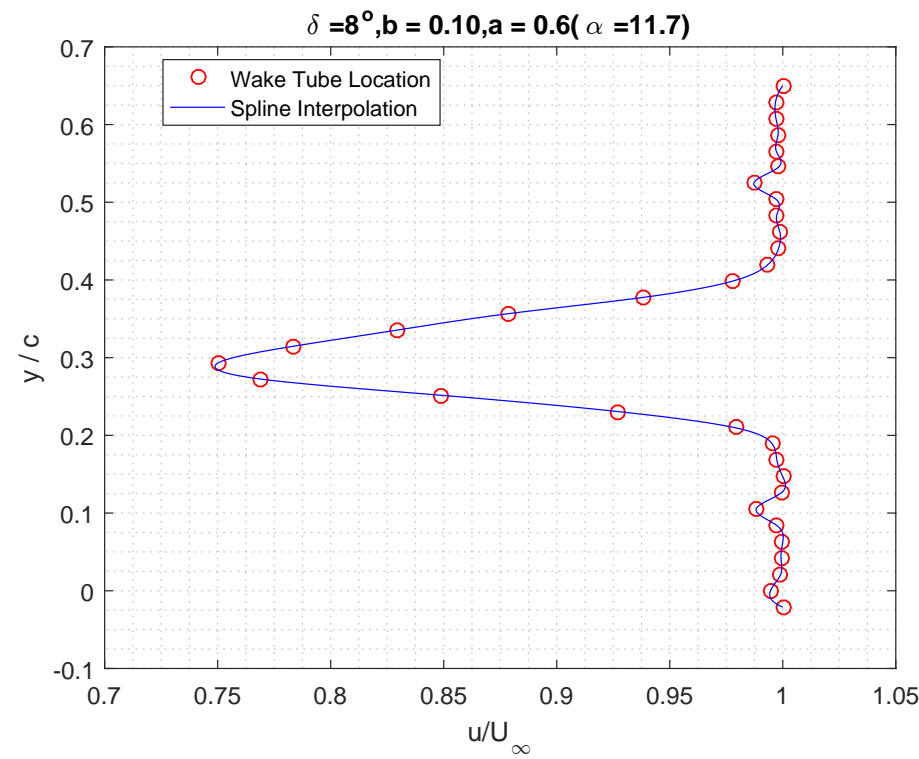

Figure 7.15: Wake plot clean airfoil at $\alpha \approx 11.7^{\circ}$ 


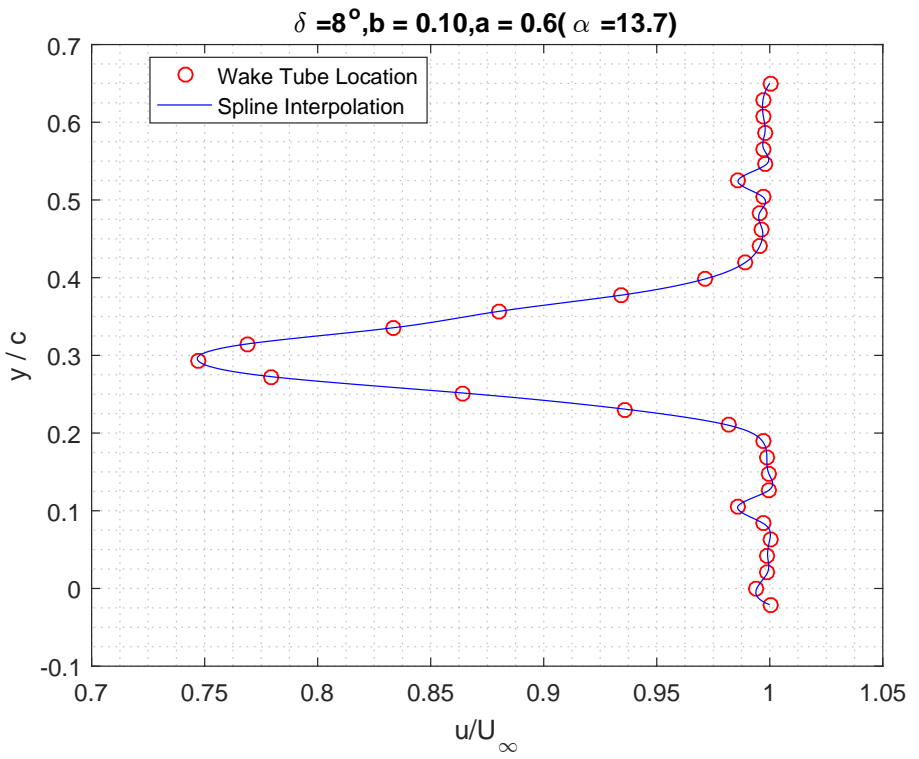

Figure 7.16: Wake plot clean airfoil at $\alpha \approx 13.7^{\circ}$

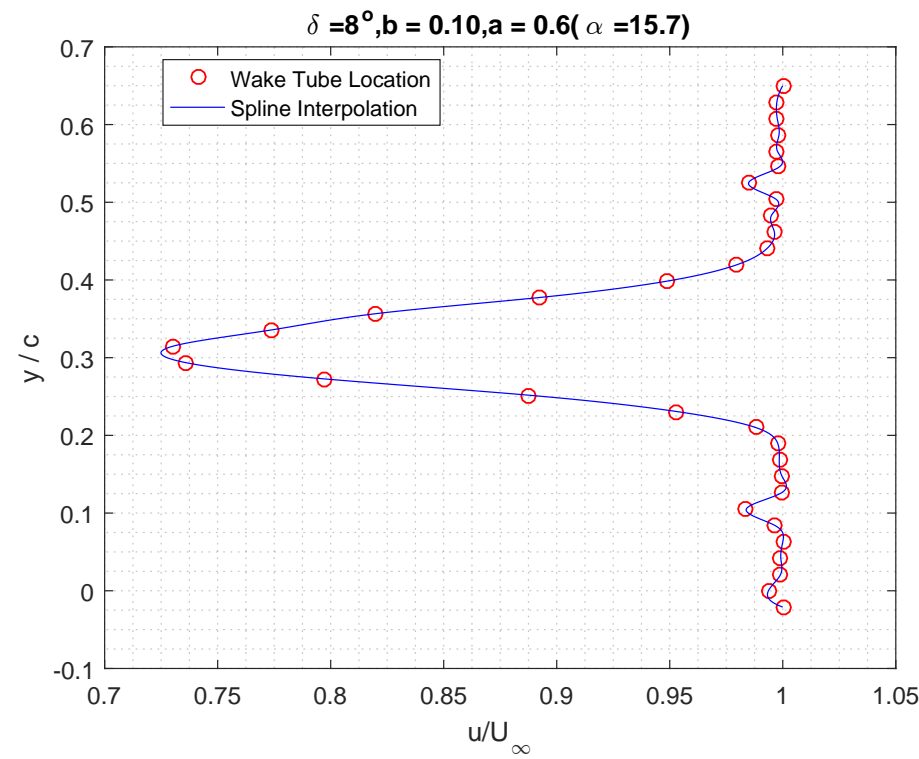

Figure 7.17: Wake plot clean airfoil at $\alpha \approx 15.7^{\circ}$ 


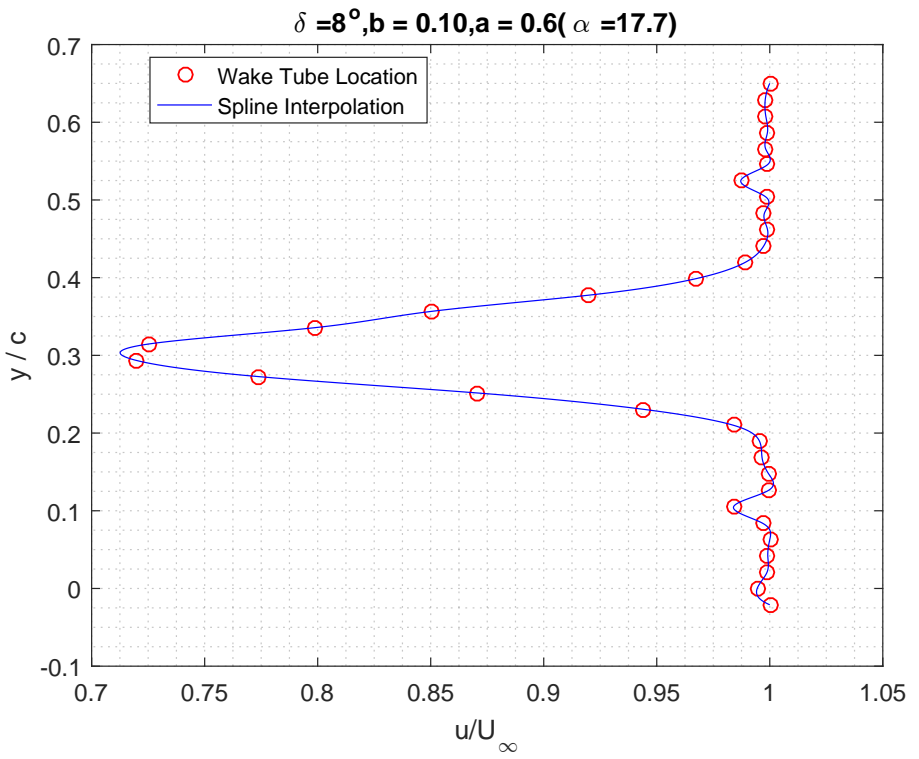

Figure 7.18: Wake plot clean airfoil at $\alpha \approx 17.7^{\circ}$

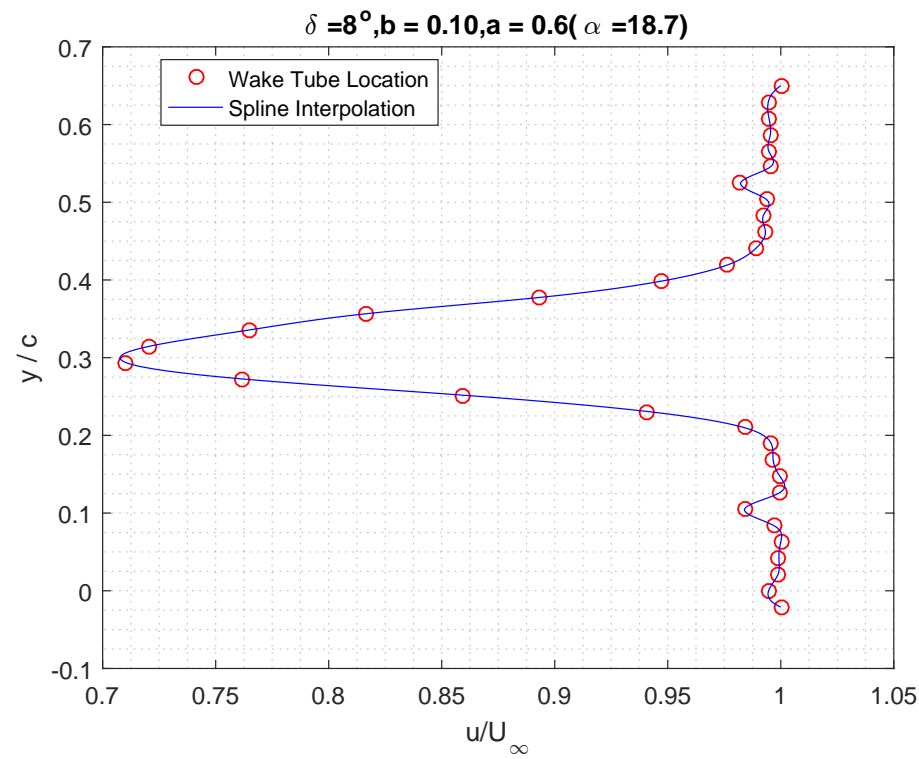

Figure 7.19: Wake plot clean airfoil at $\alpha \approx 18.7^{\circ}$ 


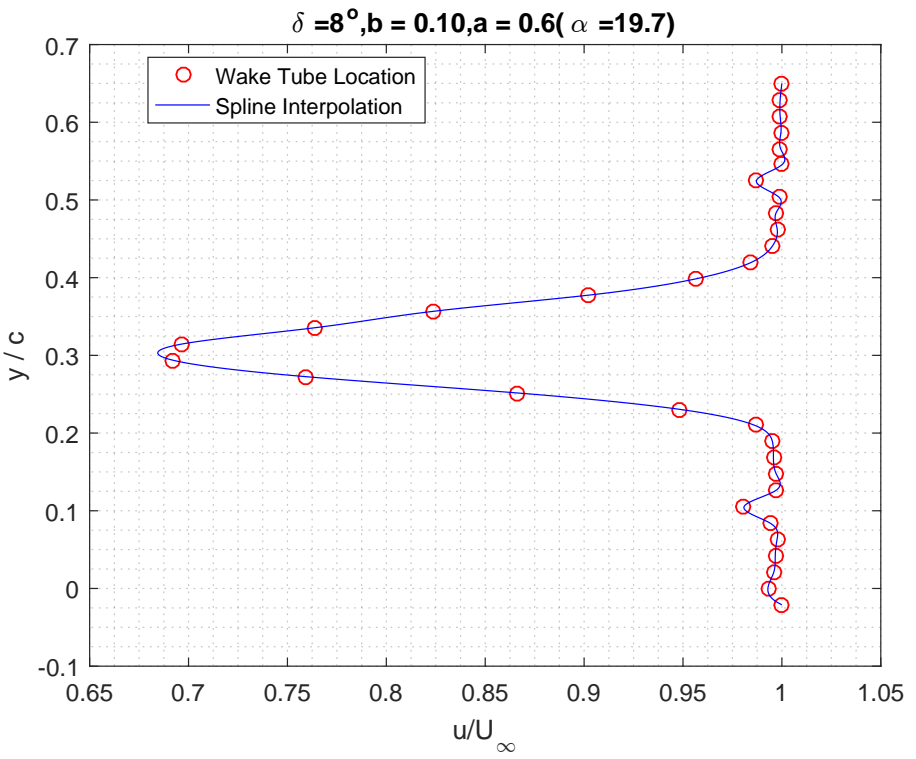

Figure 7.20: Wake plot clean airfoil at $\alpha \approx 19.7^{\circ}$

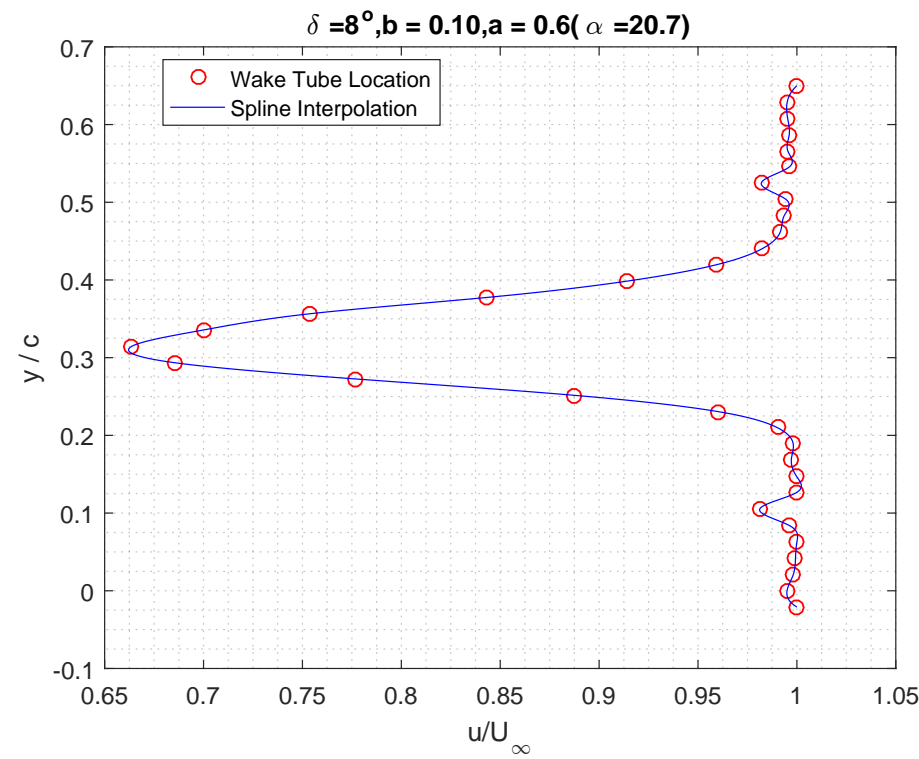

Figure 7.21: Wake plot clean airfoil at $\alpha \approx 20.7^{\circ}$ 


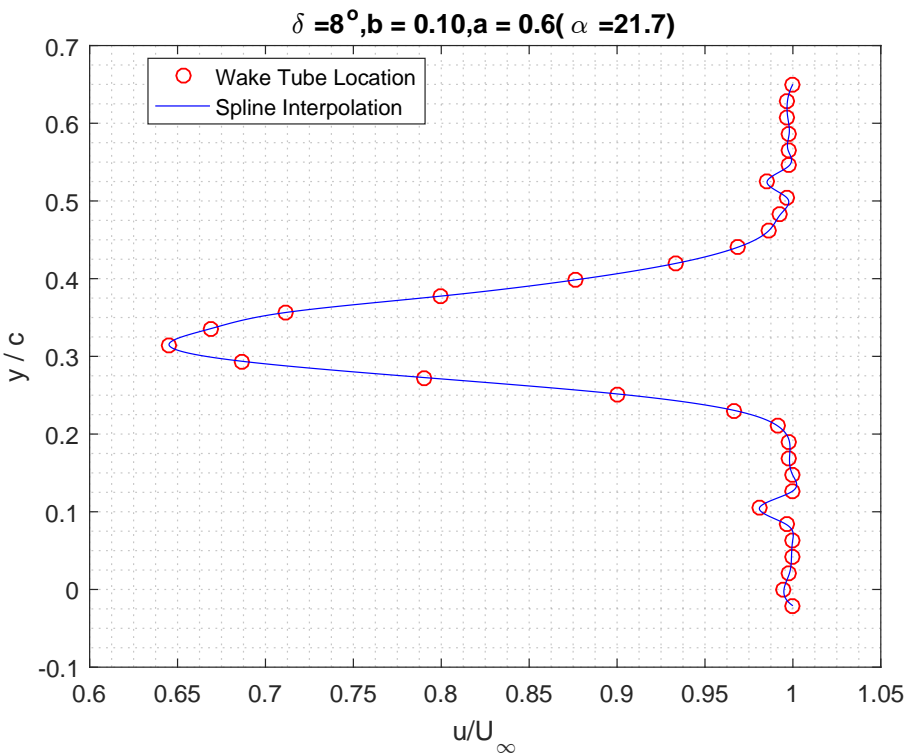

Figure 7.22: Wake plot clean airfoil at $\alpha \approx 21.7^{\circ}$

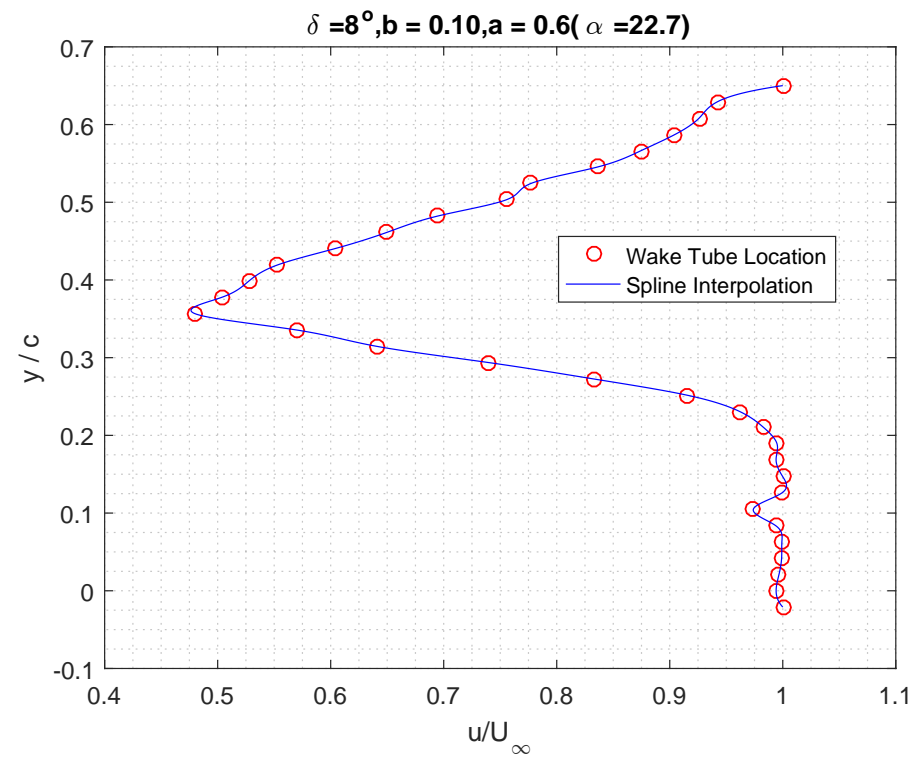

Figure 7.23: Wake plot clean airfoil at $\alpha \approx 22.7^{\circ}$ 


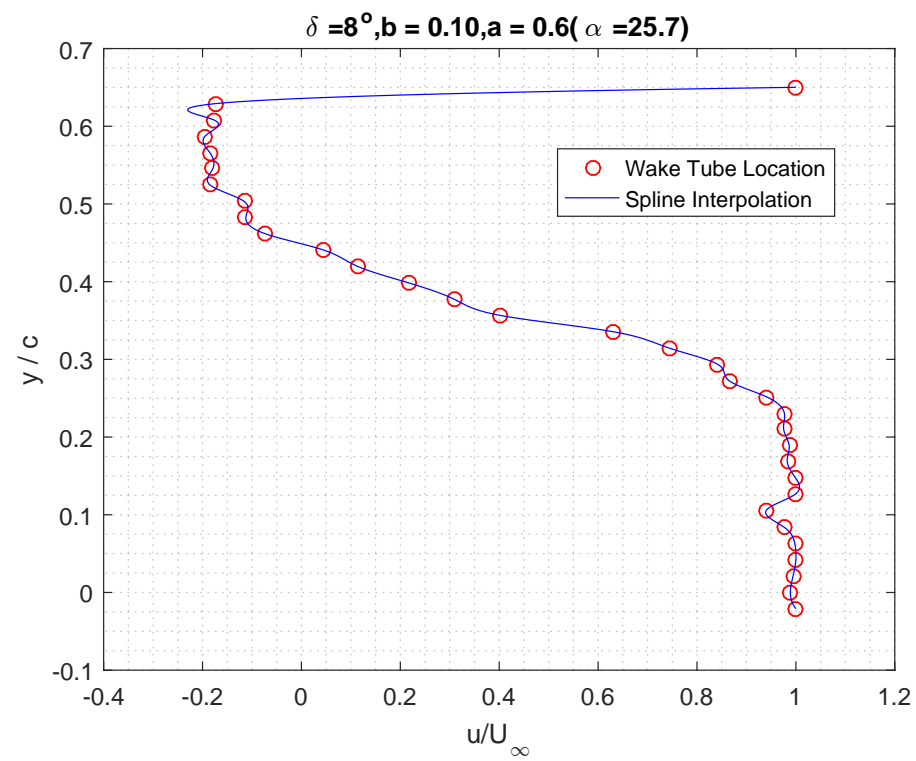

Figure 7.24: Wake plot clean airfoil at $\alpha \approx 25.7^{\circ}$

7.3 $\delta=15^{\circ}, b=10, a=60$ Configuration

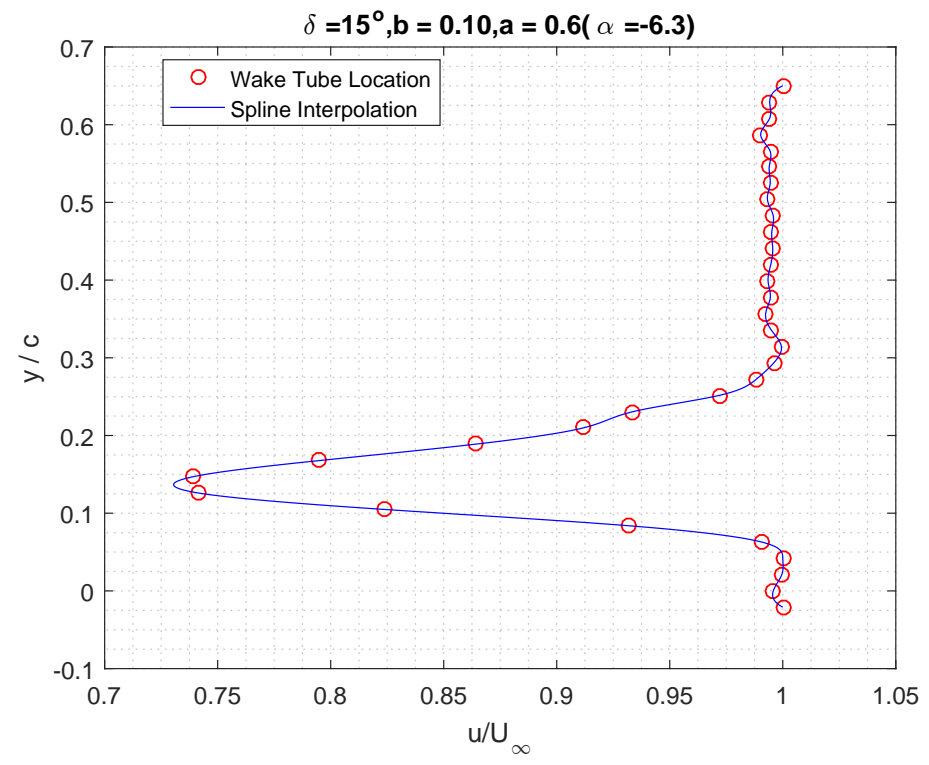

Figure 7.25: Wake plot clean airfoil at $\alpha \approx-6.3^{\circ}$ 


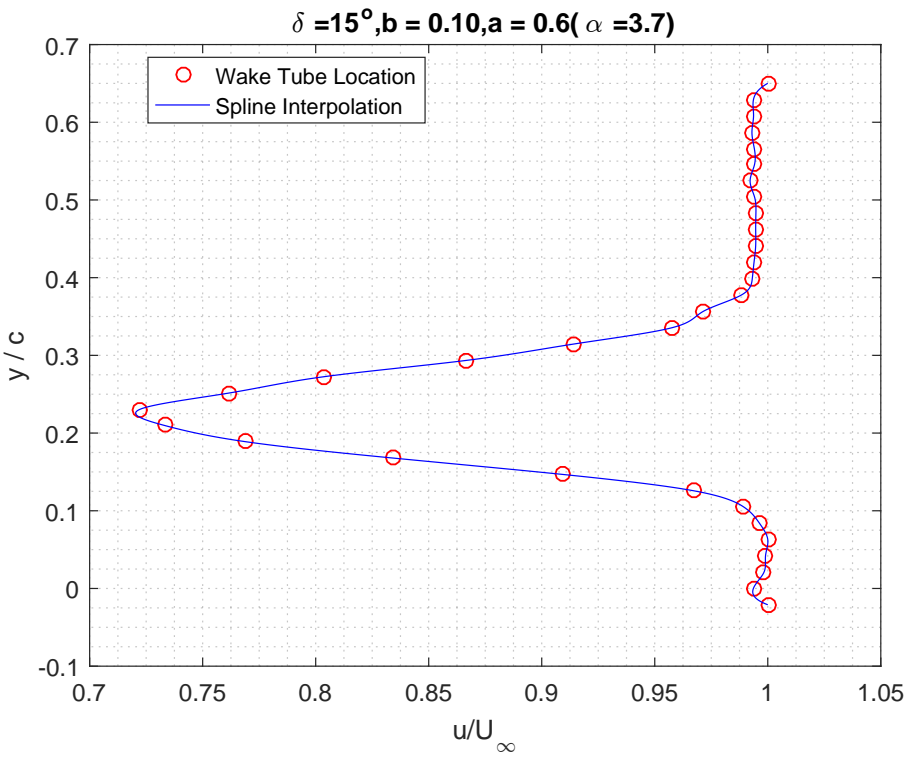

Figure 7.26: Wake plot clean airfoil at $\alpha \approx 3.7^{\circ}$

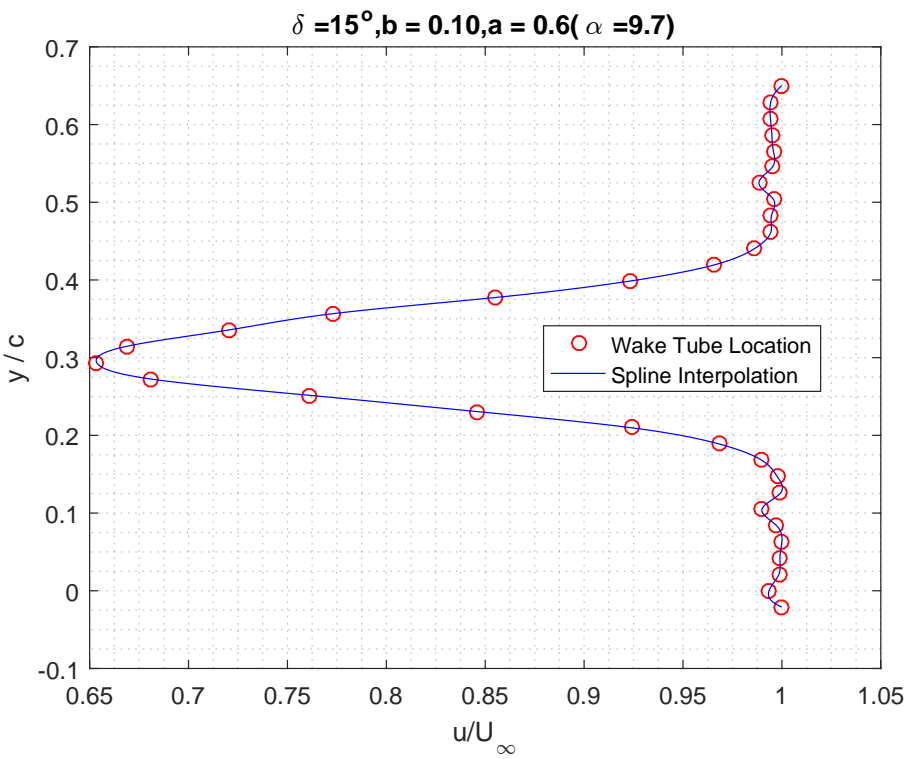

Figure 7.27: Wake plot clean airfoil at $\alpha \approx 9.7^{\circ}$ 


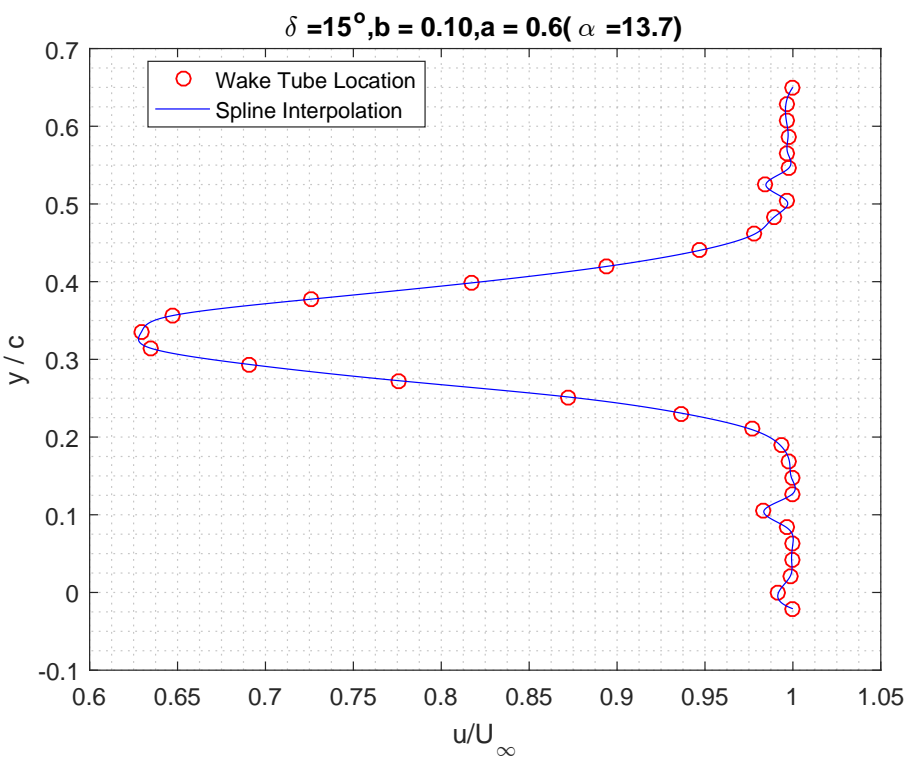

Figure 7.28: Wake plot clean airfoil at $\alpha \approx 13.7^{\circ}$

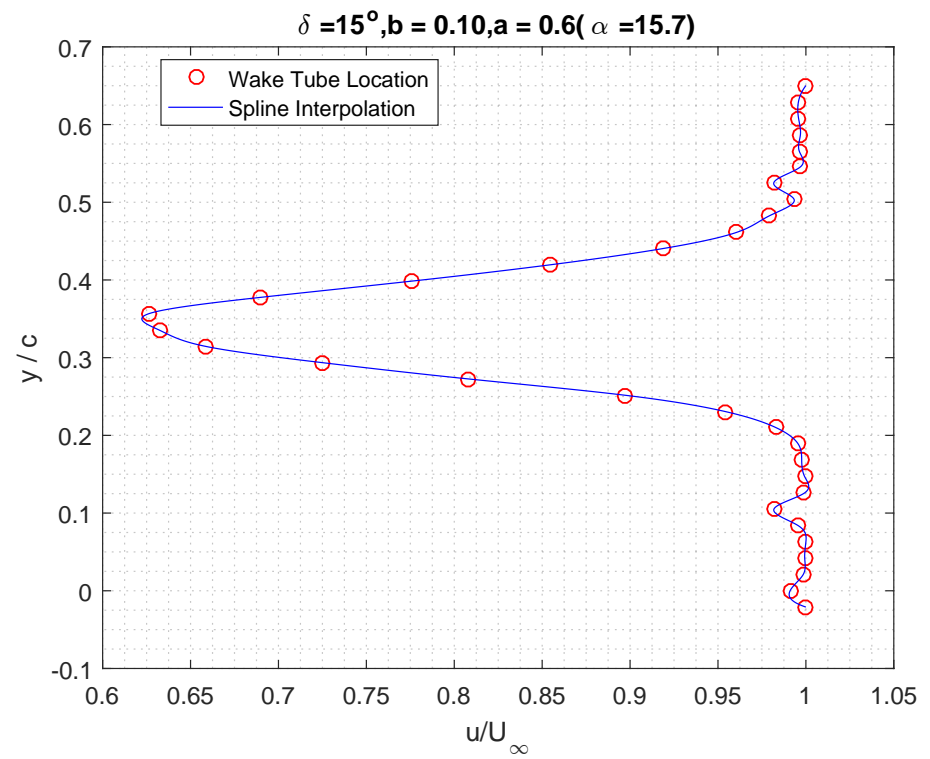

Figure 7.29: Wake plot clean airfoil at $\alpha \approx 15.7^{\circ}$ 


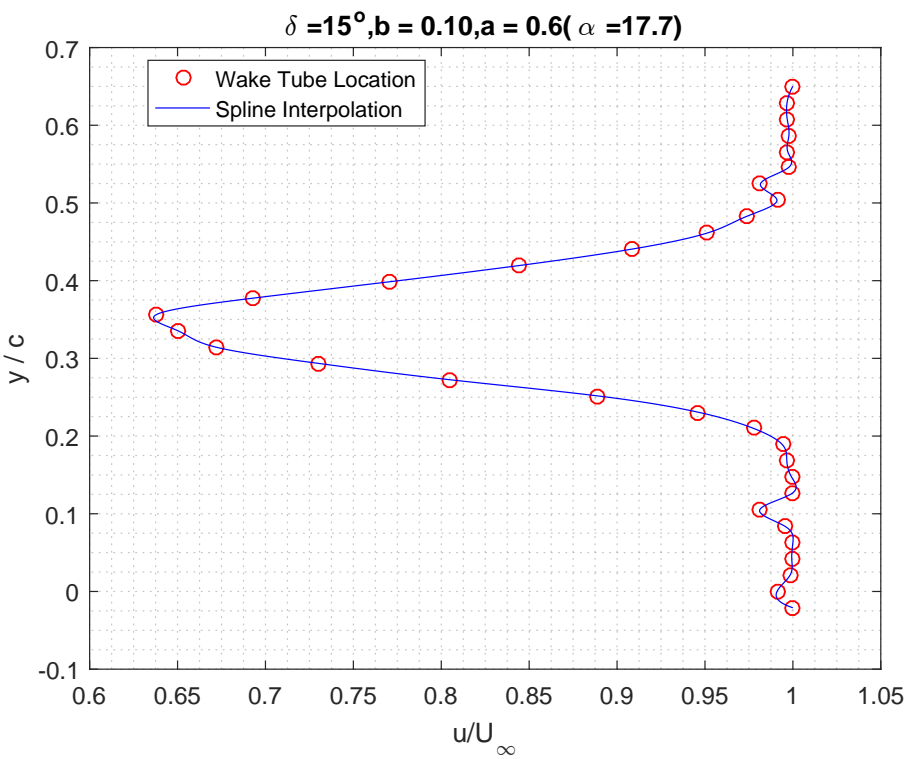

Figure 7.30: Wake plot clean airfoil at $\alpha \approx 17.7^{\circ}$

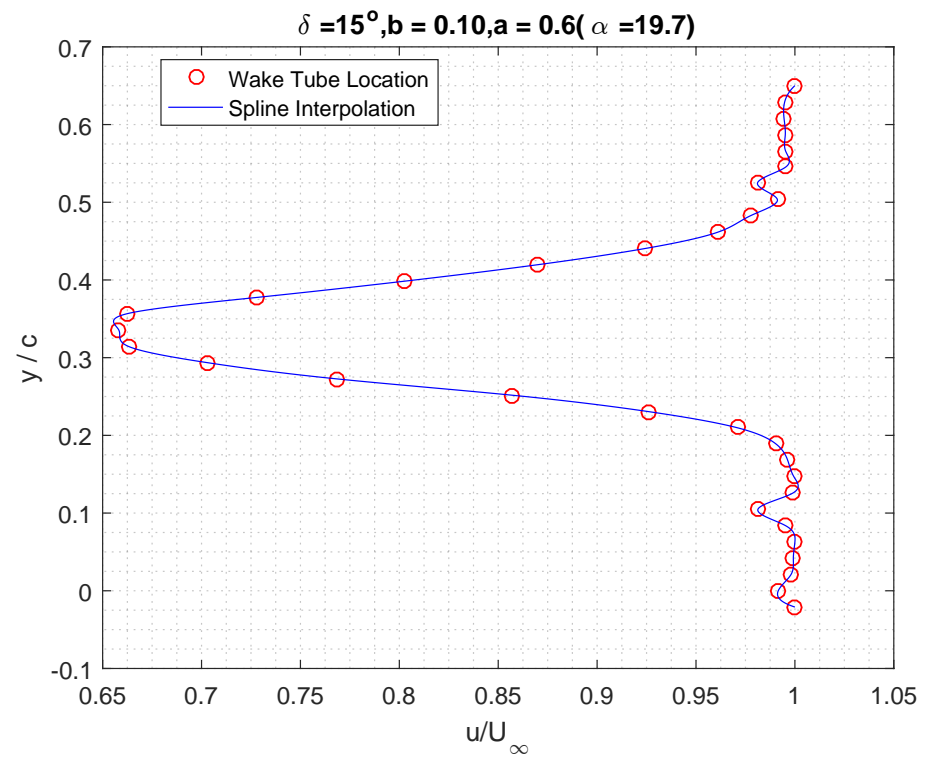

Figure 7.31: Wake plot clean airfoil at $\alpha \approx 19.7^{\circ}$ 


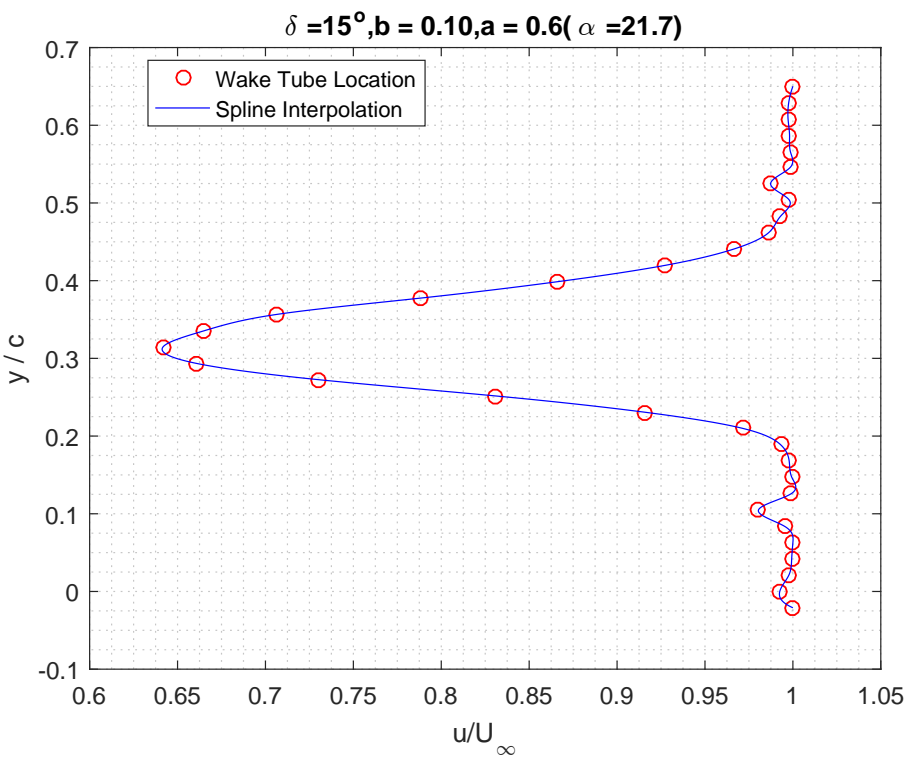

Figure 7.32: Wake plot clean airfoil at $\alpha \approx 21.7^{\circ}$

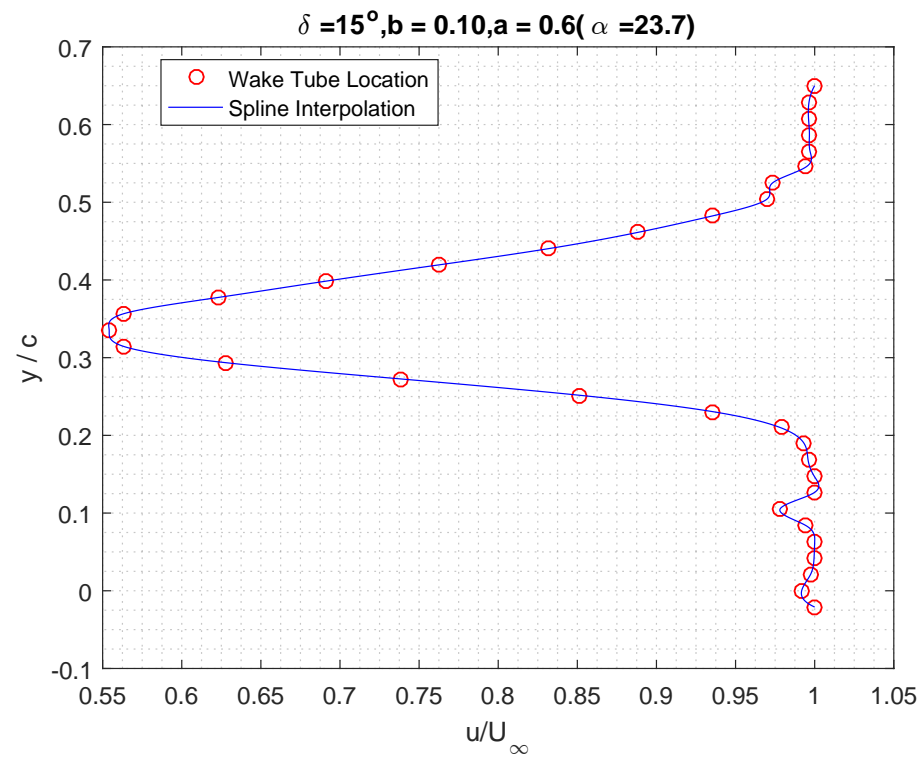

Figure 7.33: Wake plot clean airfoil at $\alpha \approx 23.7^{\circ}$ 


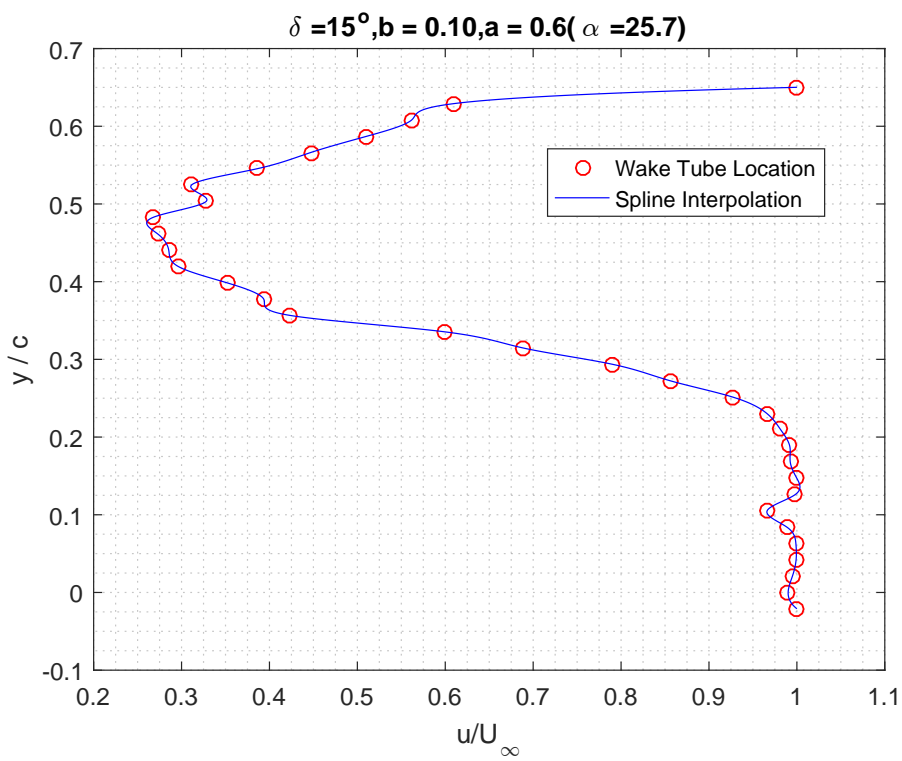

Figure 7.34: Wake plot clean airfoil at $\alpha \approx 25.7^{\circ}$

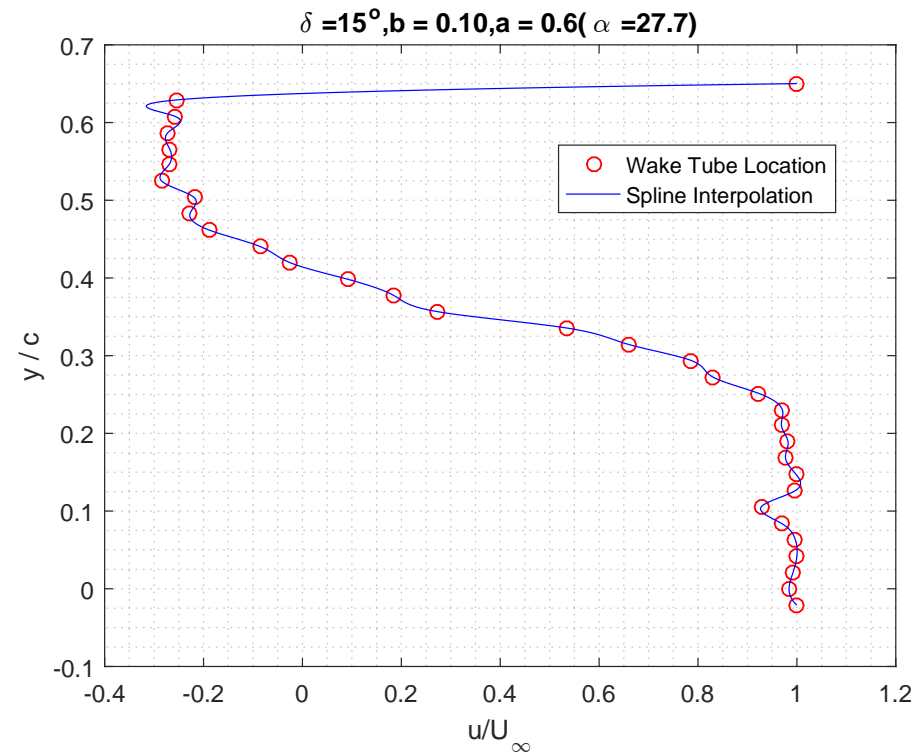

Figure 7.35: Wake plot clean airfoil at $\alpha \approx 27.7^{\circ}$ 
$7.4 \delta=25^{\circ}, b=10, a=60$ Configuration

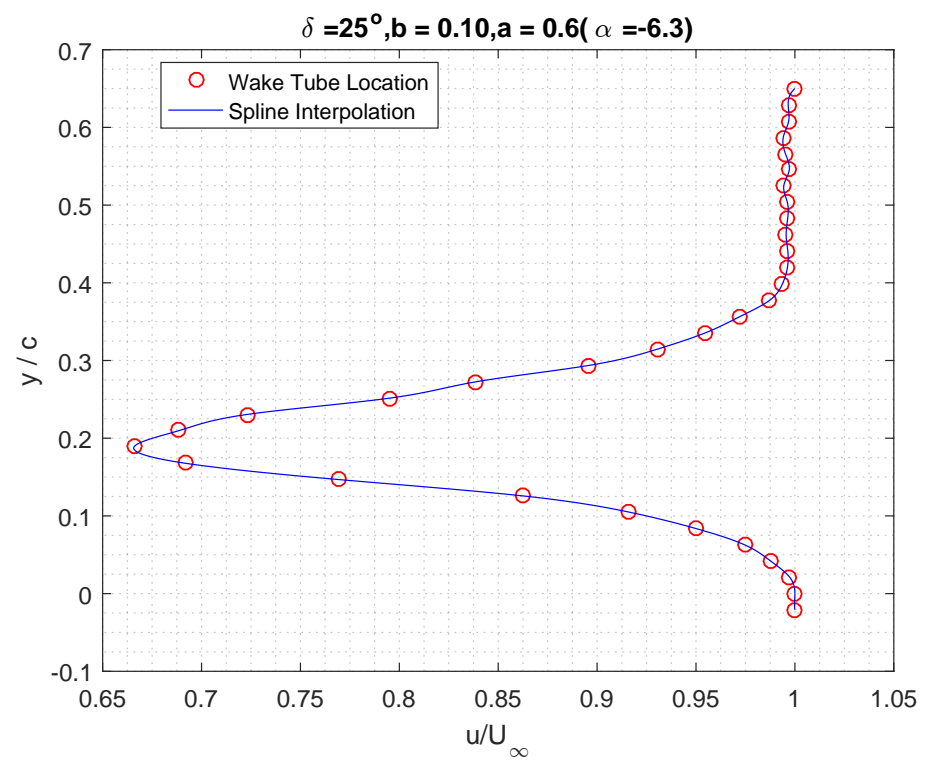

Figure 7.36: Wake plot clean airfoil at $\alpha \approx-6.3^{\circ}$

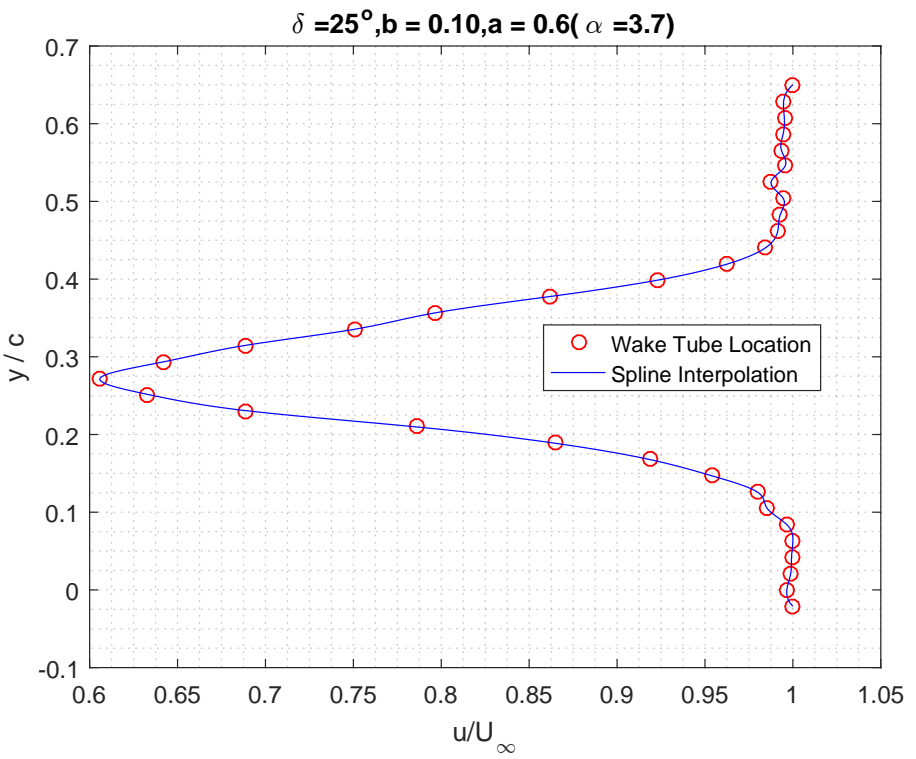

Figure 7.37: Wake plot clean airfoil at $\alpha \approx 3.7^{\circ}$ 


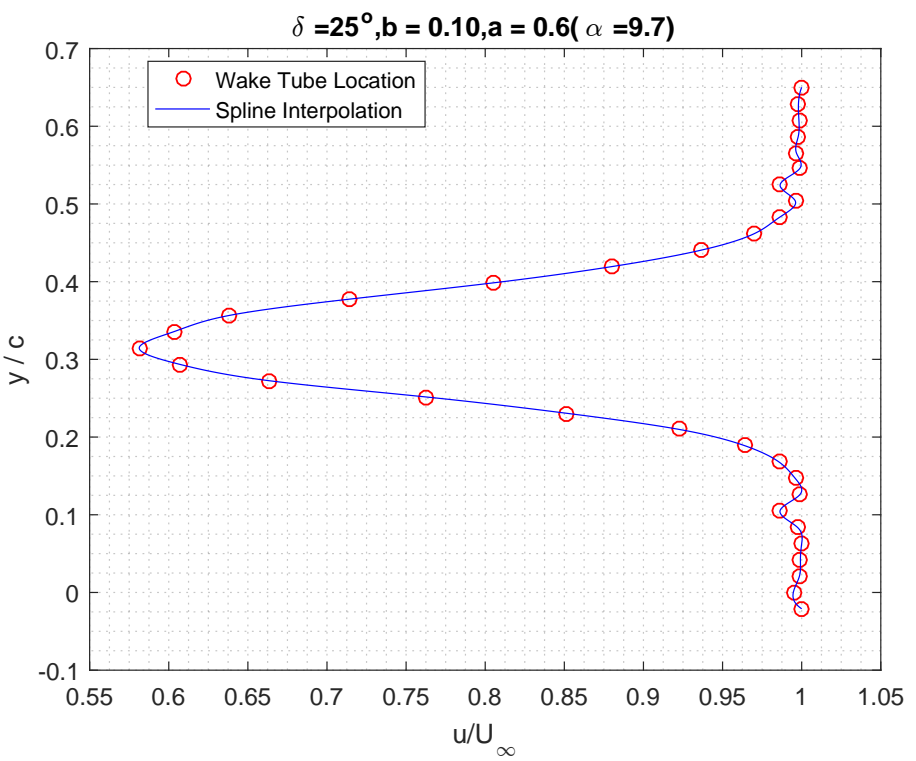

Figure 7.38: Wake plot clean airfoil at $\alpha \approx 9.7^{\circ}$

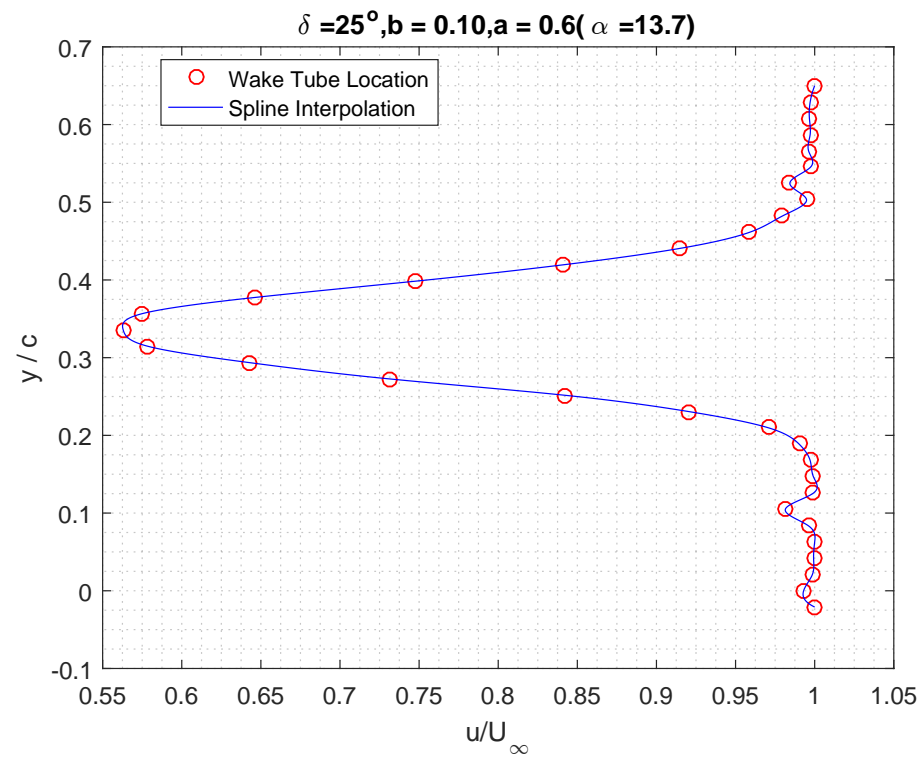

Figure 7.39: Wake plot clean airfoil at $\alpha \approx 13.7^{\circ}$ 


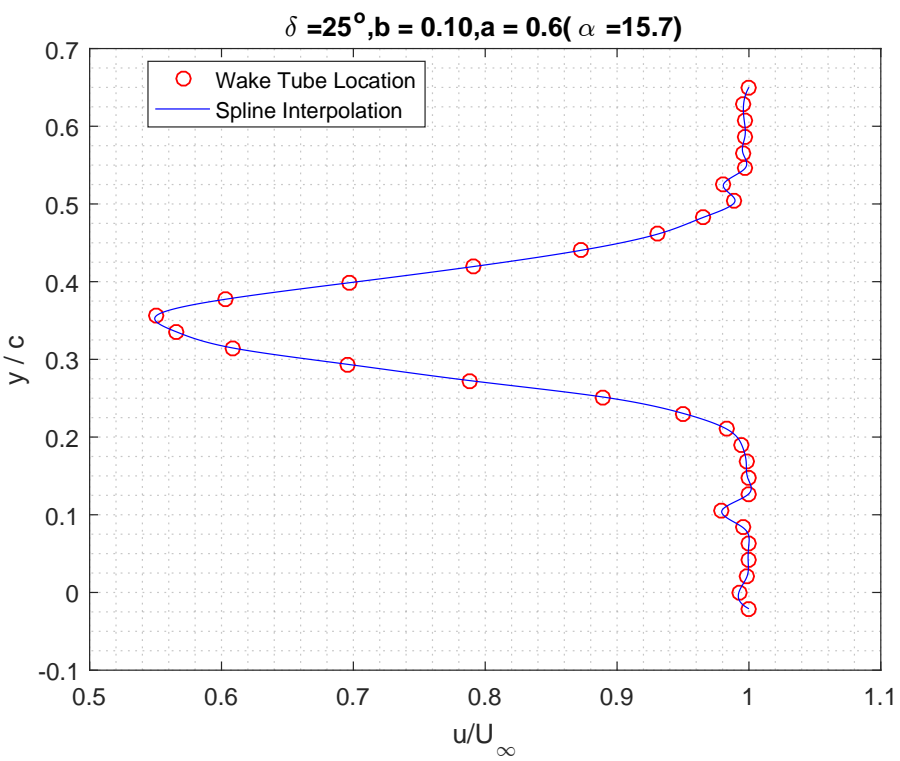

Figure 7.40: Wake plot clean airfoil at $\alpha \approx 15.7^{\circ}$

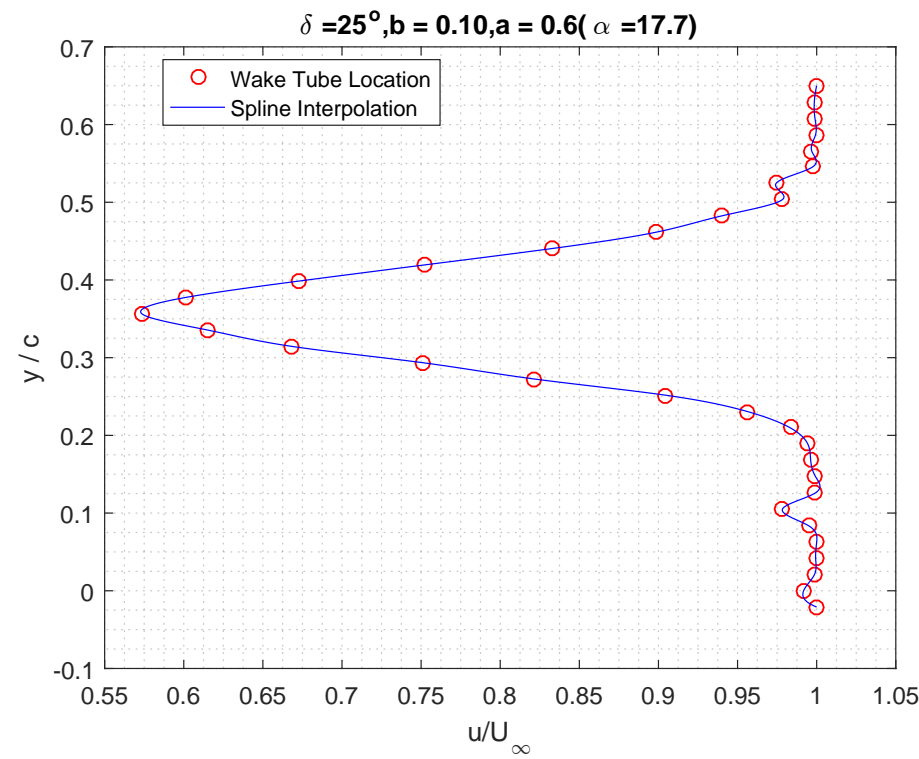

Figure 7.41: Wake plot clean airfoil at $\alpha \approx 17.7^{\circ}$ 


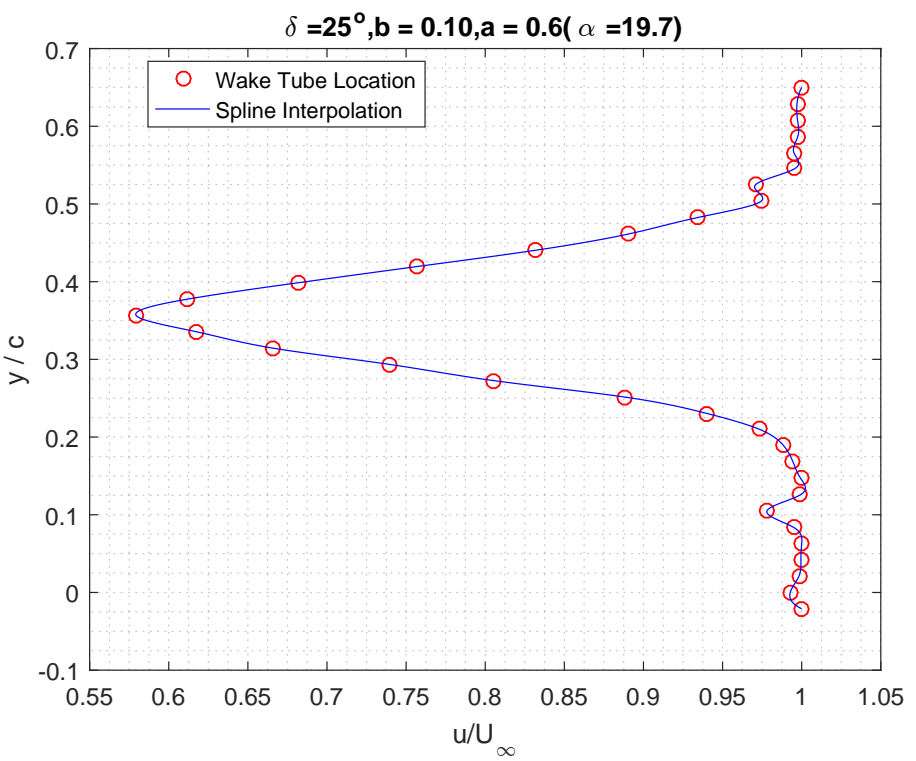

Figure 7.42: Wake plot clean airfoil at $\alpha \approx 19.7^{\circ}$

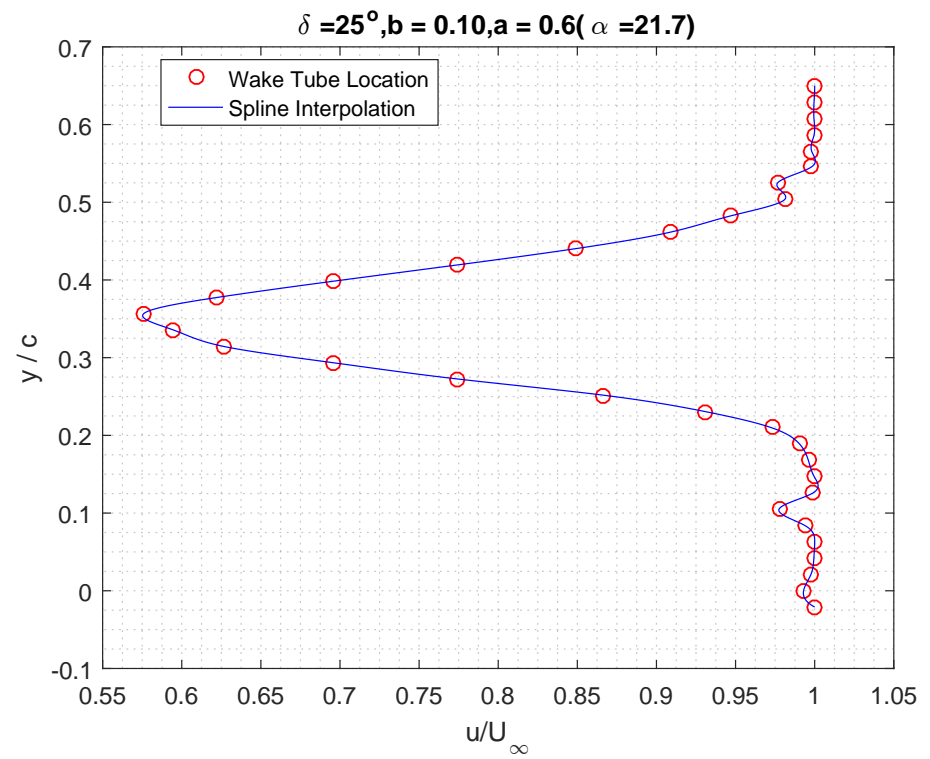

Figure 7.43: Wake plot clean airfoil at $\alpha \approx 21.7^{\circ}$ 


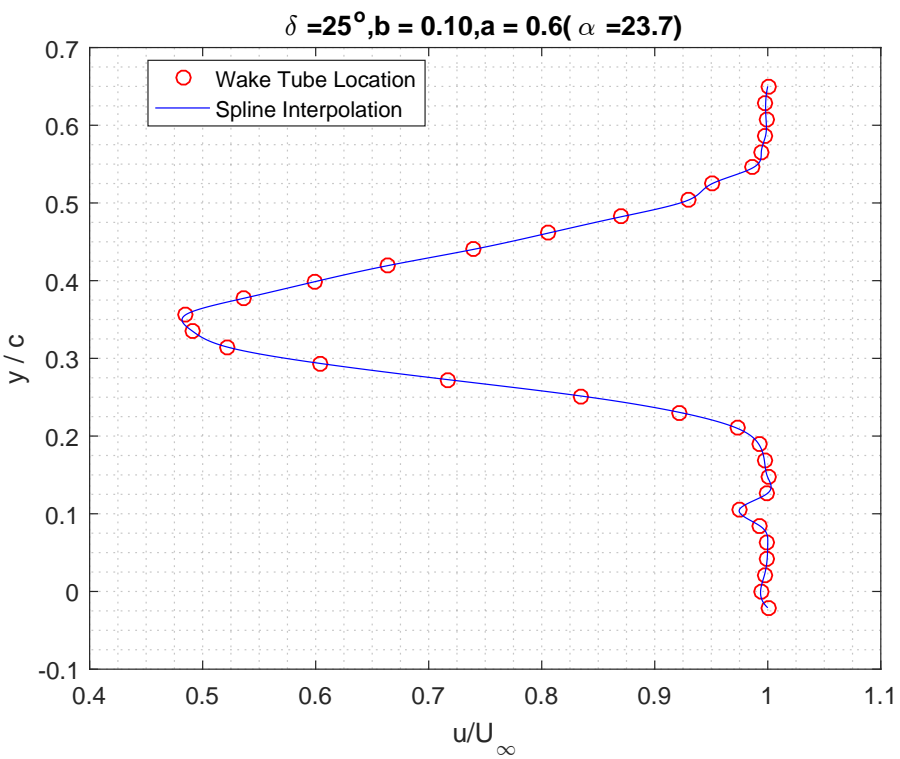

Figure 7.44: Wake plot clean airfoil at $\alpha \approx 23.7^{\circ}$

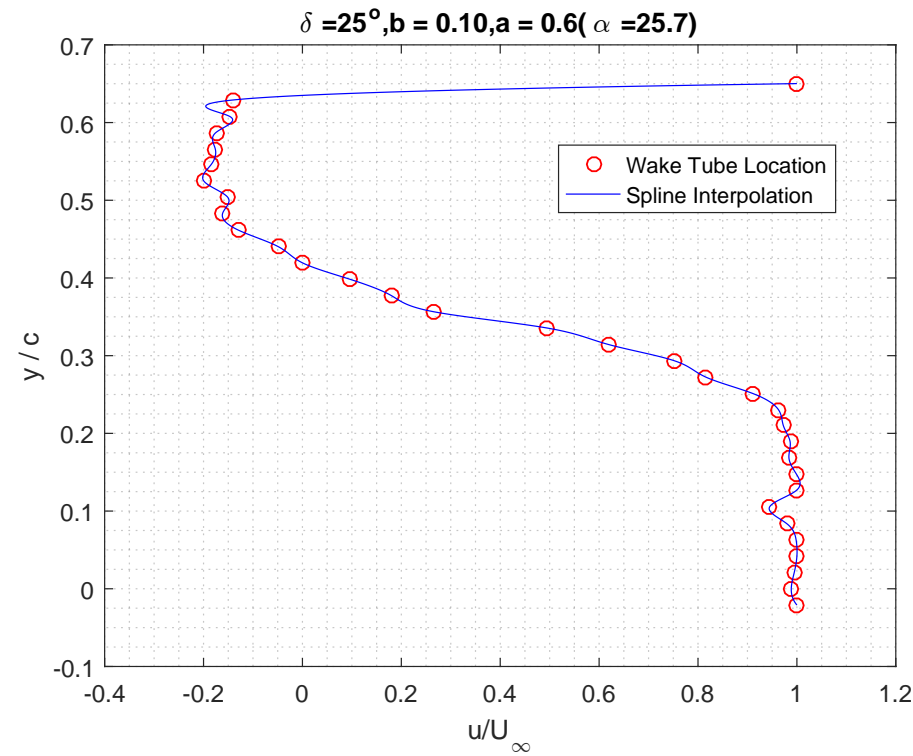

Figure 7.45: Wake plot clean airfoil at $\alpha \approx 25.7^{\circ}$ 


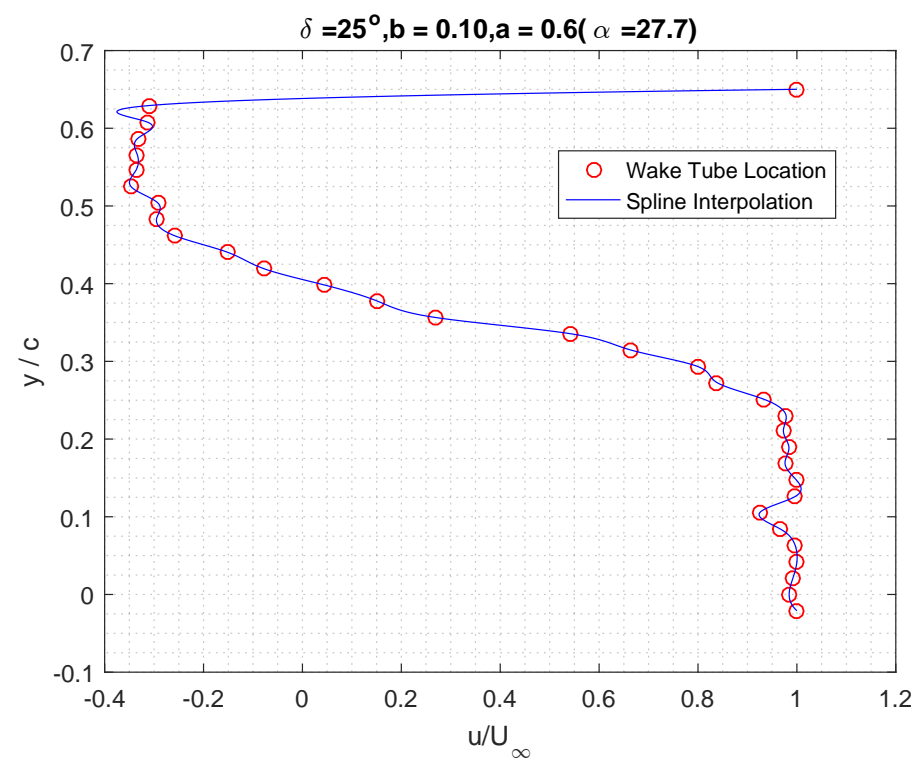

Figure 7.46: Wake plot clean airfoil at $\alpha \approx 27.7^{\circ}$

\section{5 $\delta=8^{\circ}, b=15, a=60$ Configuration}

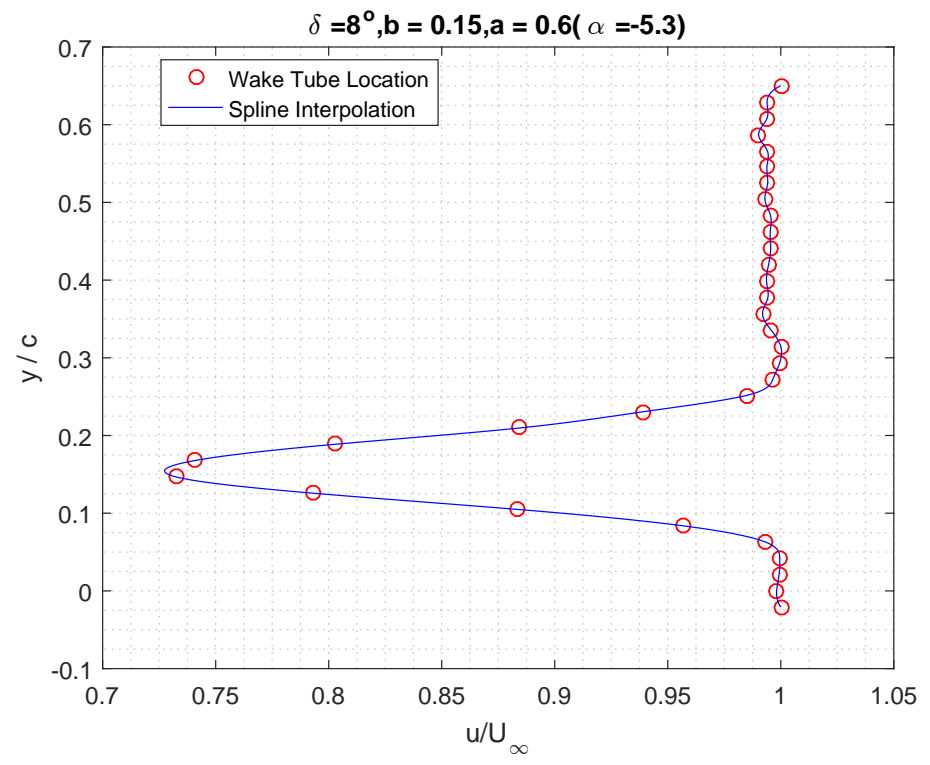

Figure 7.47: Wake plot clean airfoil at $\alpha \approx-5.3^{\circ}$ 


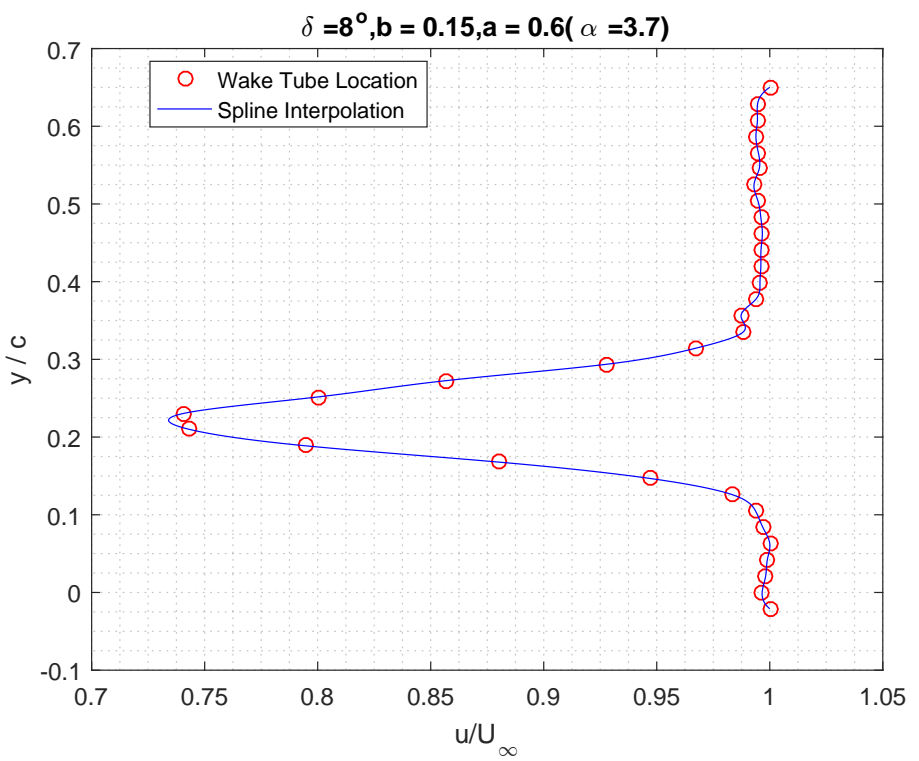

Figure 7.48: Wake plot clean airfoil at $\alpha \approx 3.7^{\circ}$

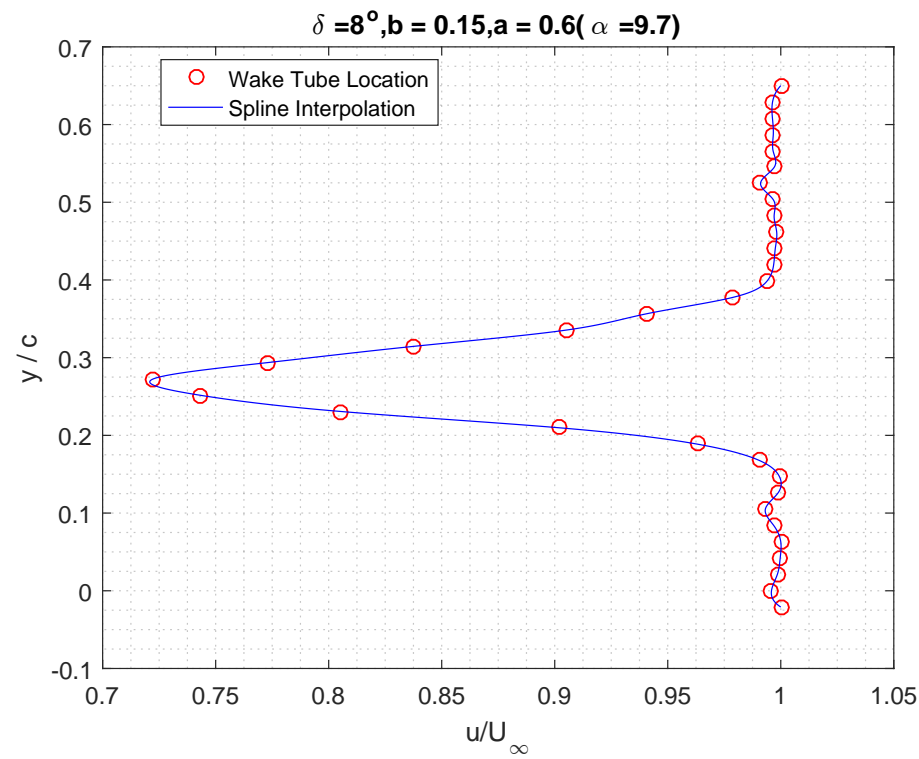

Figure 7.49: Wake plot clean airfoil at $\alpha \approx 9.7^{\circ}$ 


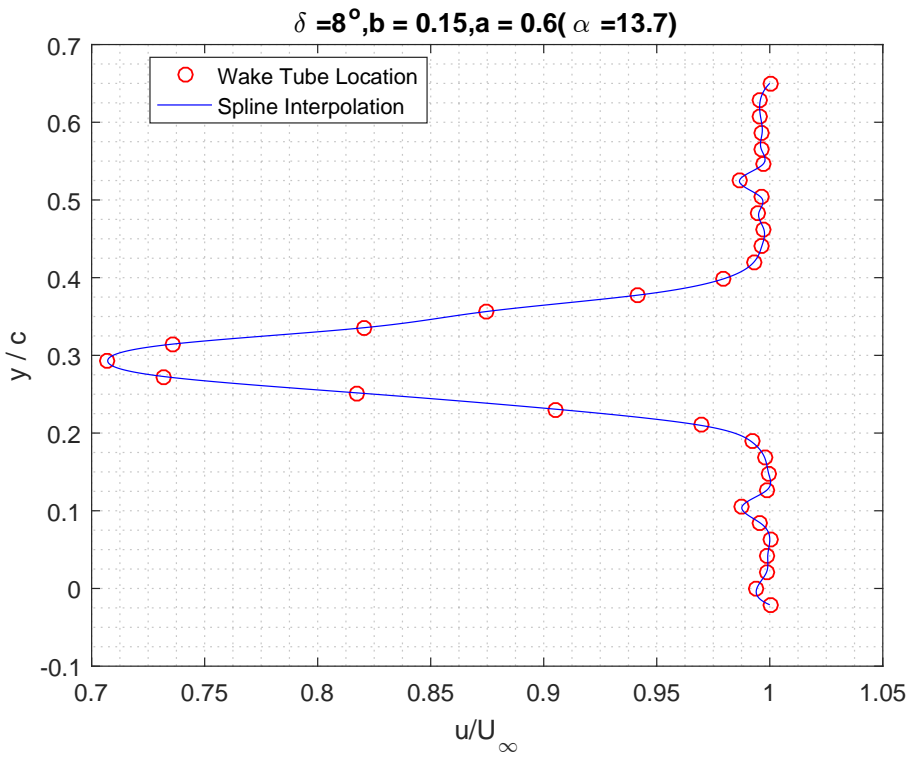

Figure 7.50: Wake plot clean airfoil at $\alpha \approx 13.7^{\circ}$

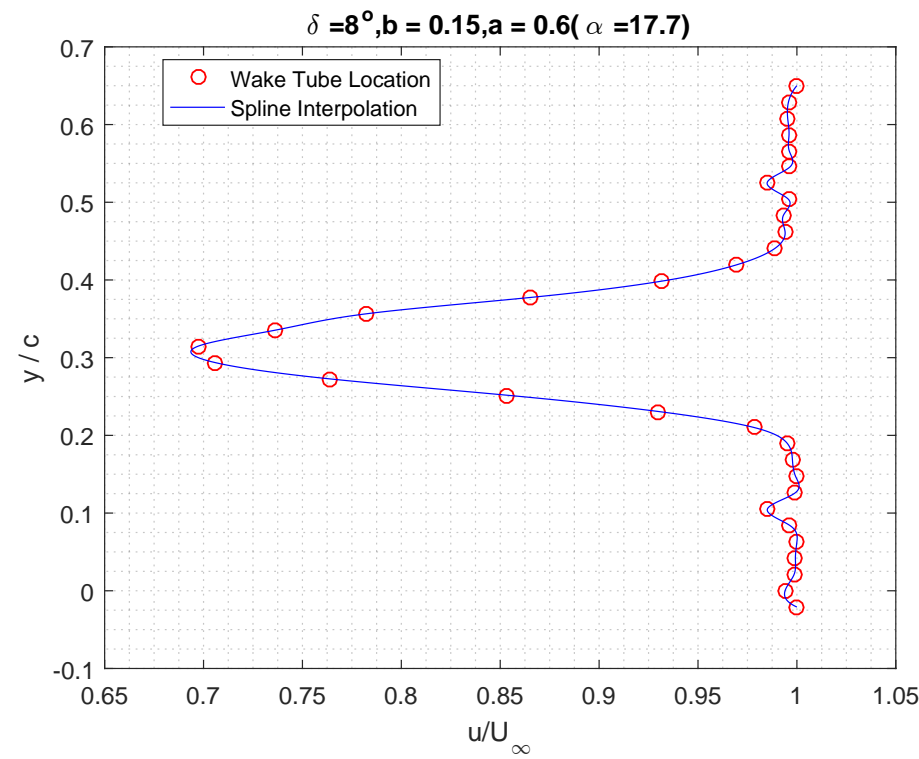

Figure 7.51: Wake plot clean airfoil at $\alpha \approx 17.7^{\circ}$ 


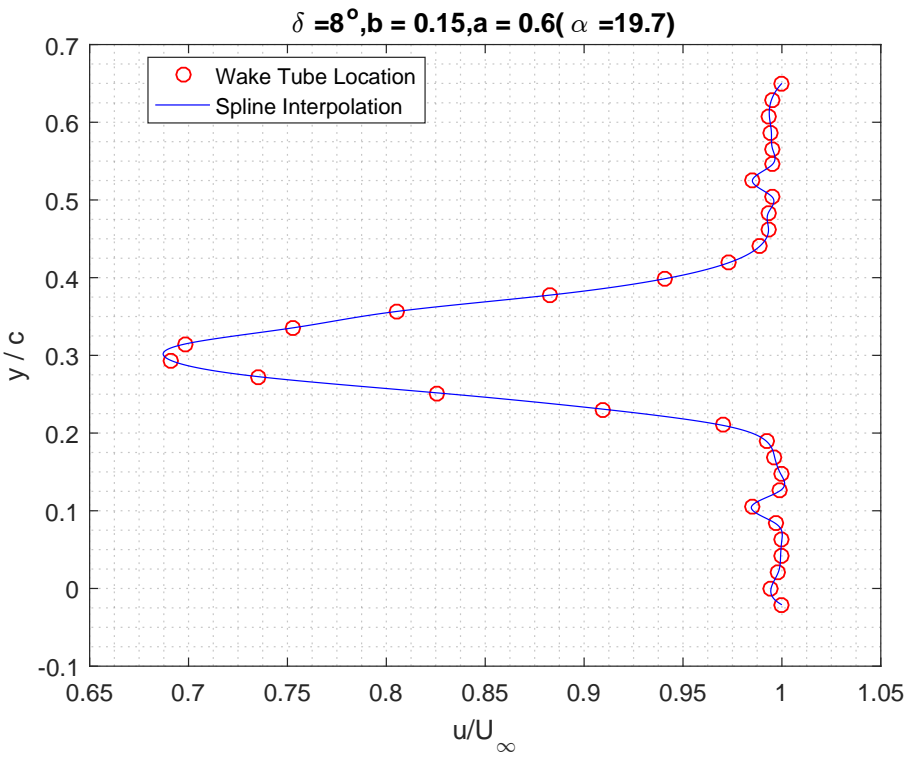

Figure 7.52: Wake plot clean airfoil at $\alpha \approx 19.7^{\circ}$

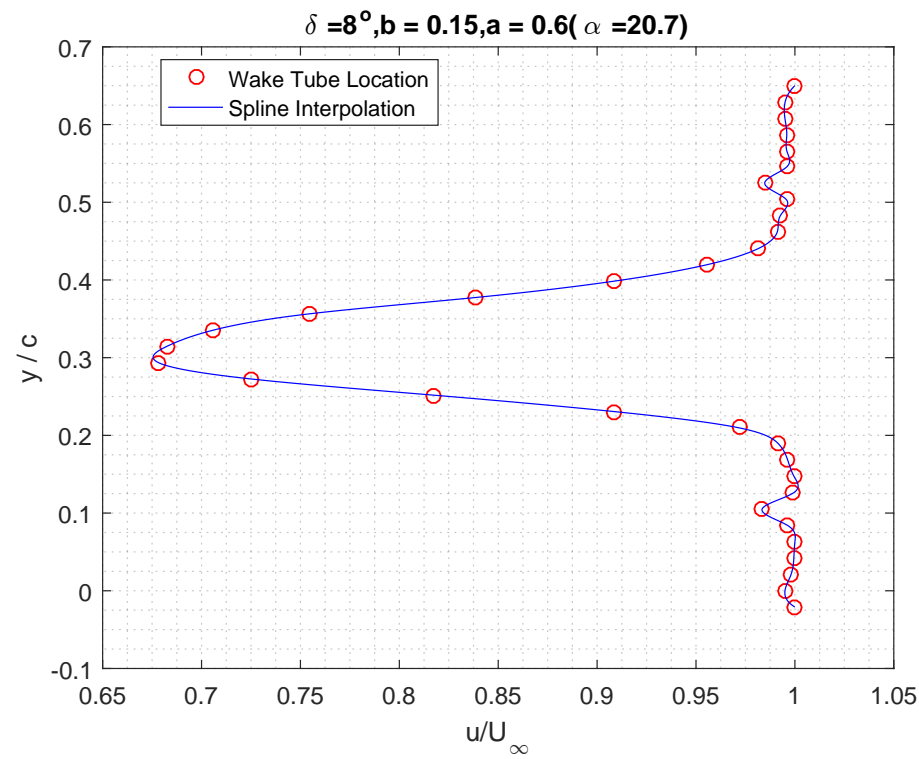

Figure 7.53: Wake plot clean airfoil at $\alpha \approx 20.7^{\circ}$ 


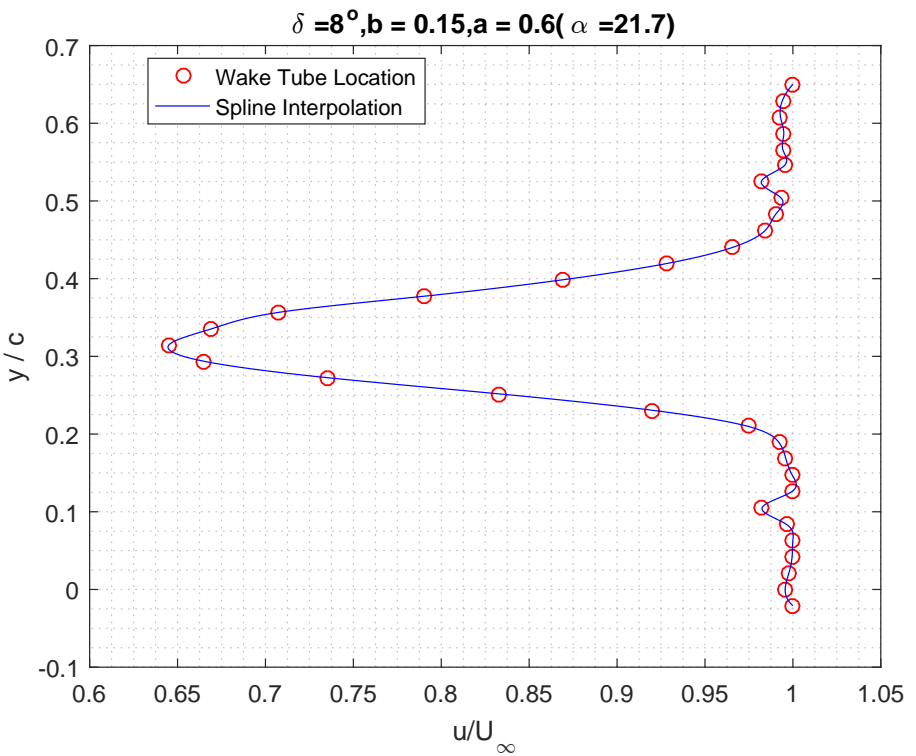

Figure 7.54: Wake plot clean airfoil at $\alpha \approx 21.7^{\circ}$

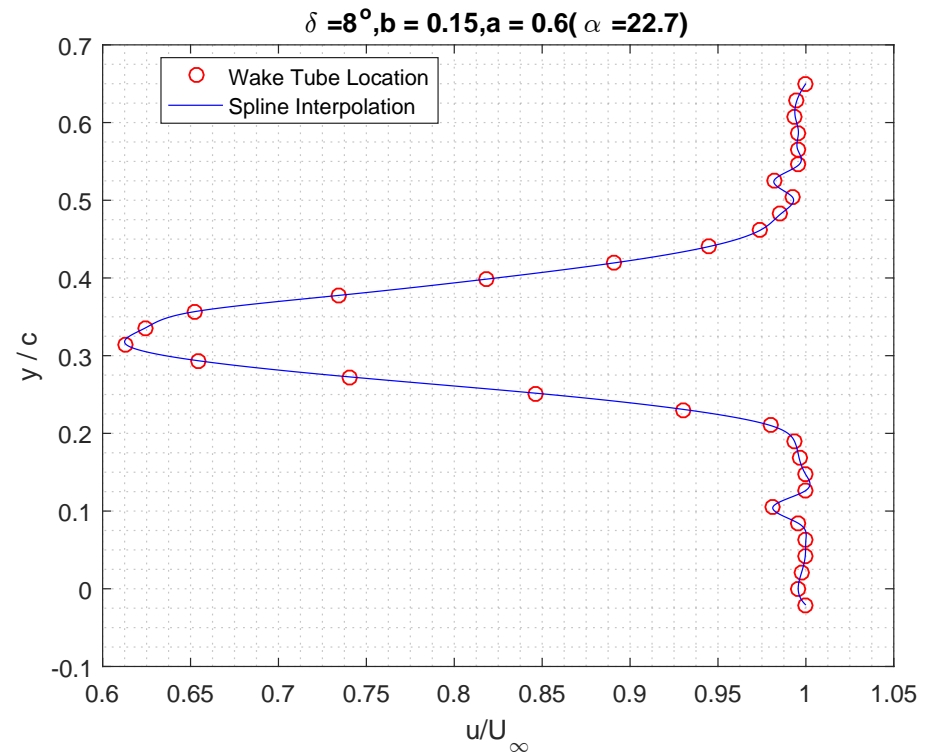

Figure 7.55: Wake plot clean airfoil at $\alpha \approx 22.7^{\circ}$ 


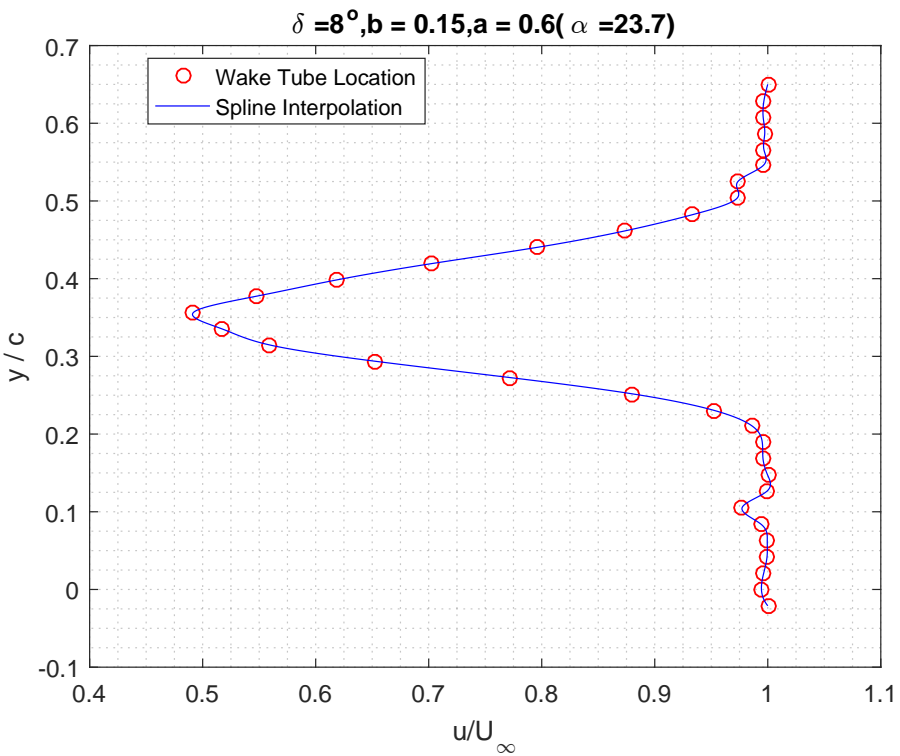

Figure 7.56: Wake plot clean airfoil at $\alpha \approx 23.7^{\circ}$

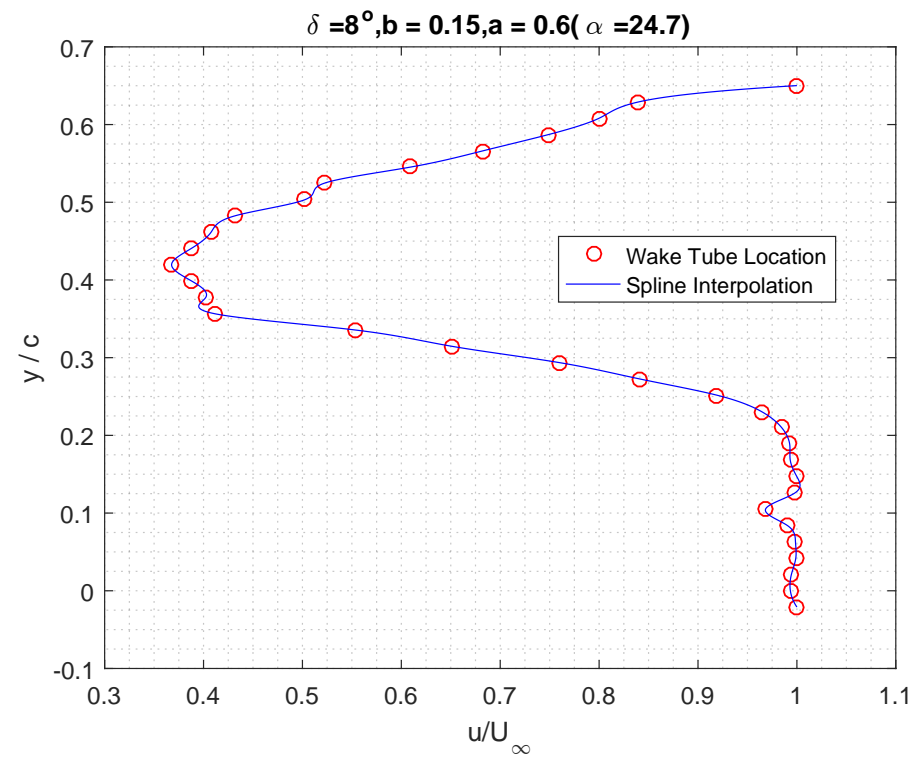

Figure 7.57: Wake plot clean airfoil at $\alpha \approx 24.7^{\circ}$ 


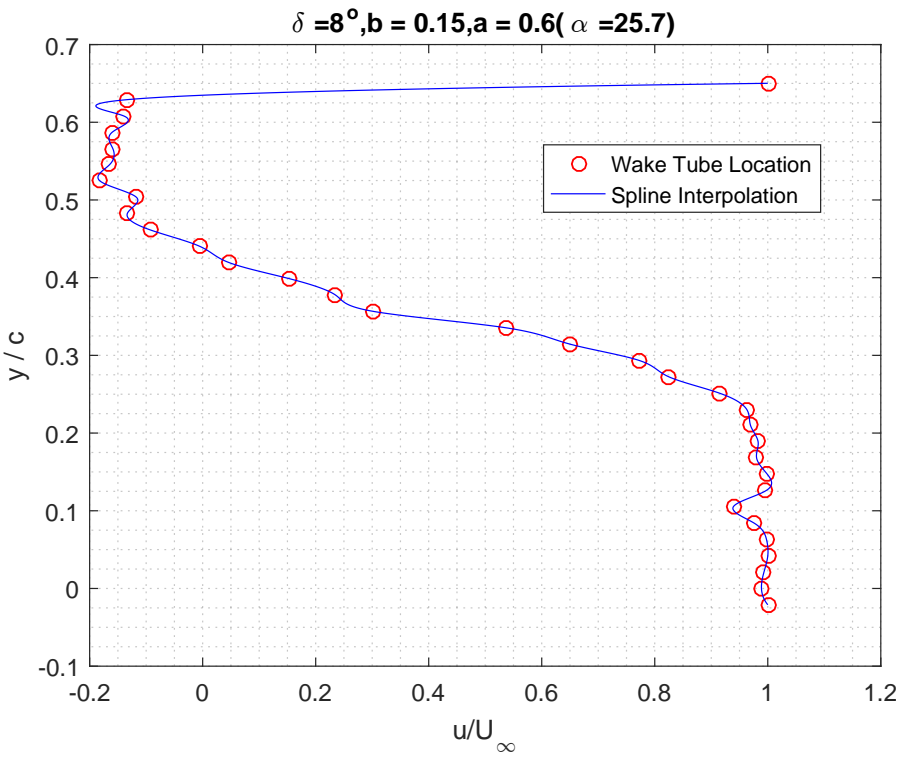

Figure 7.58: Wake plot clean airfoil at $\alpha \approx 25.7^{\circ}$

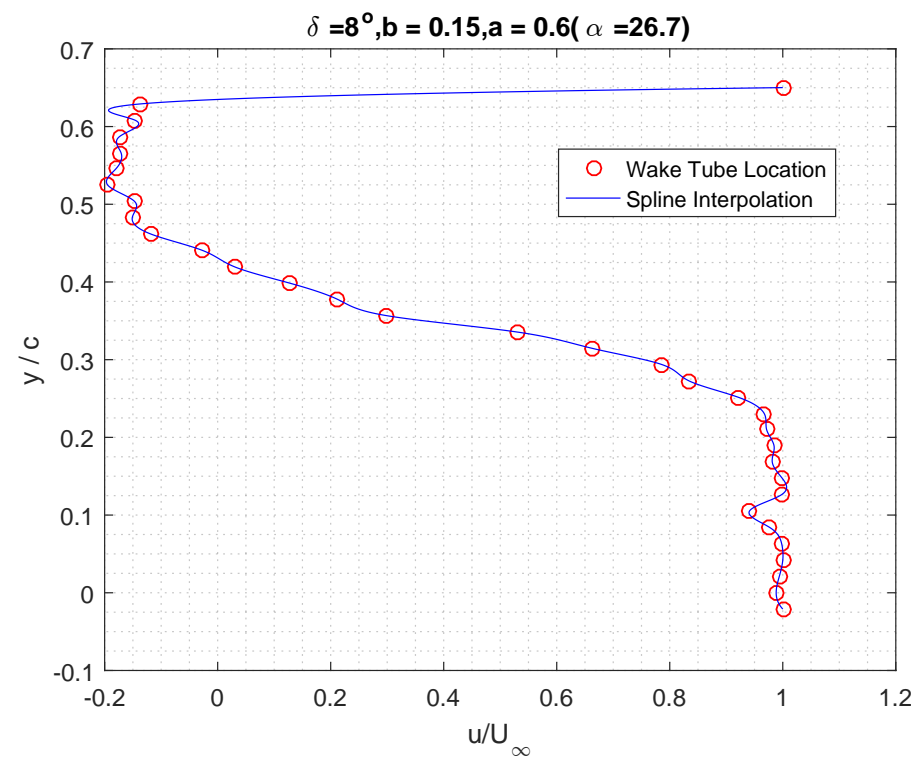

Figure 7.59: Wake plot clean airfoil at $\alpha \approx 26.7^{\circ}$ 


\section{6 $\delta=15^{\circ}, b=15, a=60$ Configuration}

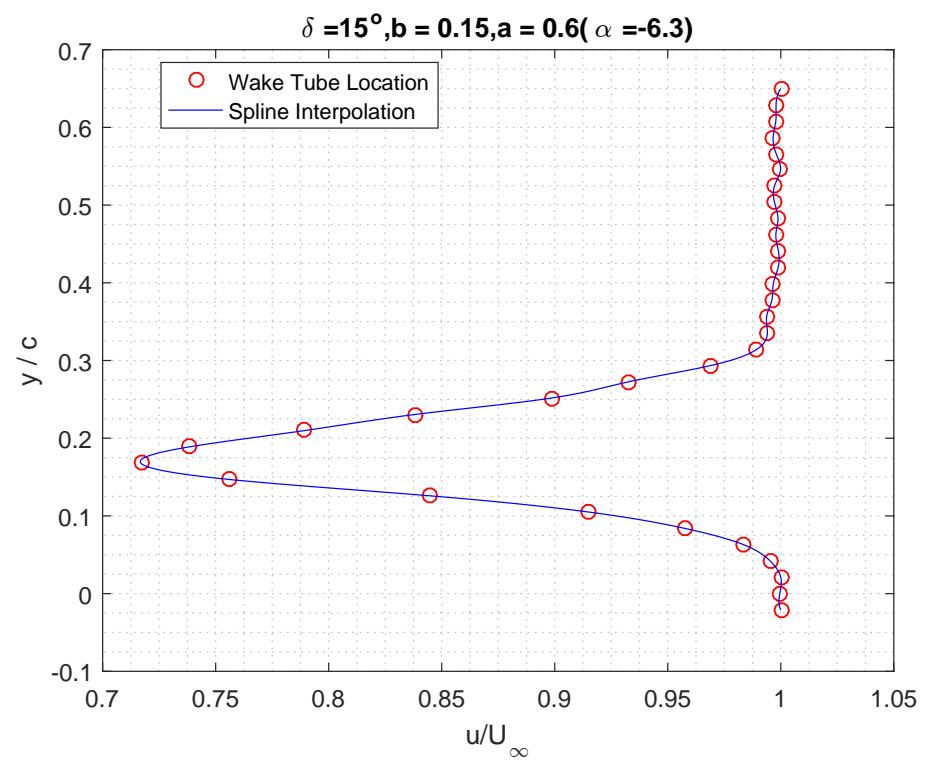

Figure 7.60: Wake plot clean airfoil at $\alpha \approx-6.3^{\circ}$

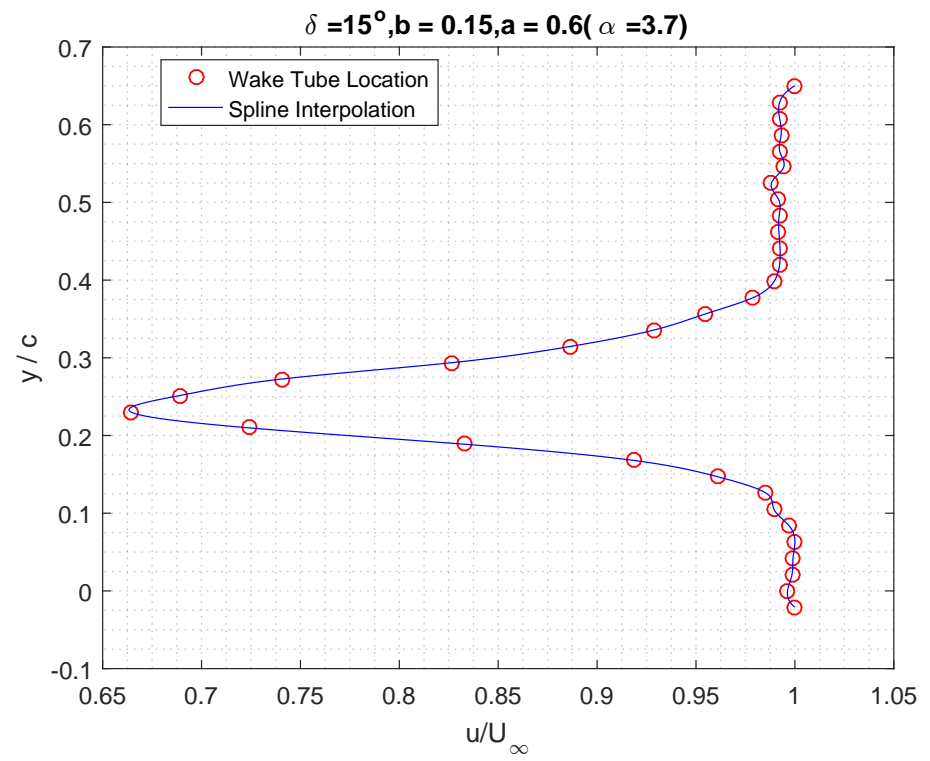

Figure 7.61: Wake plot clean airfoil at $\alpha \approx 3.7^{\circ}$ 


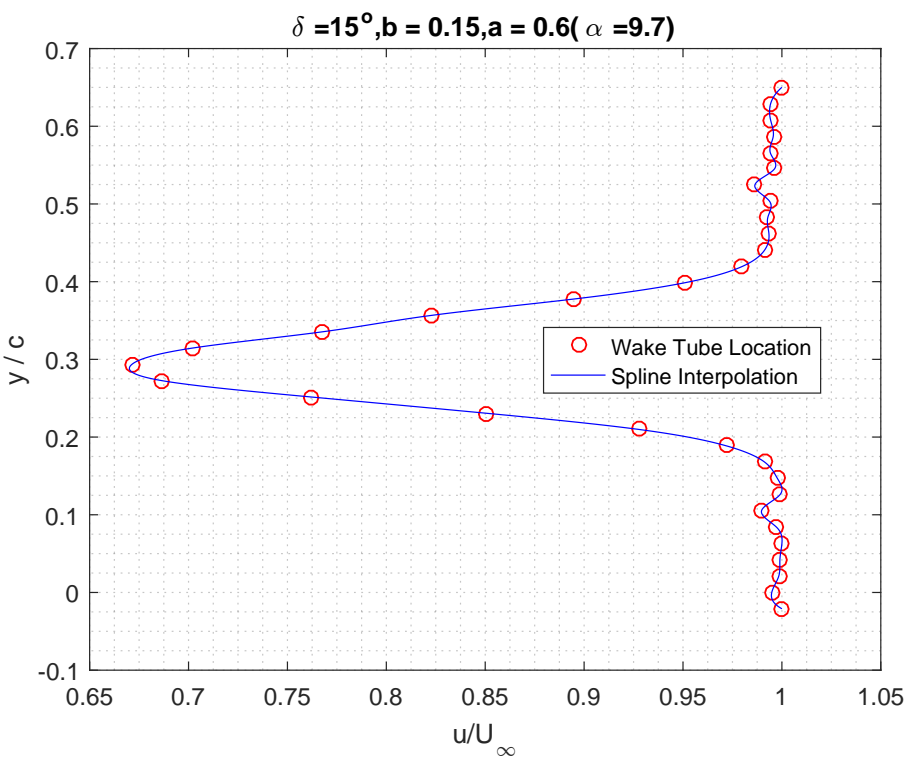

Figure 7.62: Wake plot clean airfoil at $\alpha \approx 9.7^{\circ}$

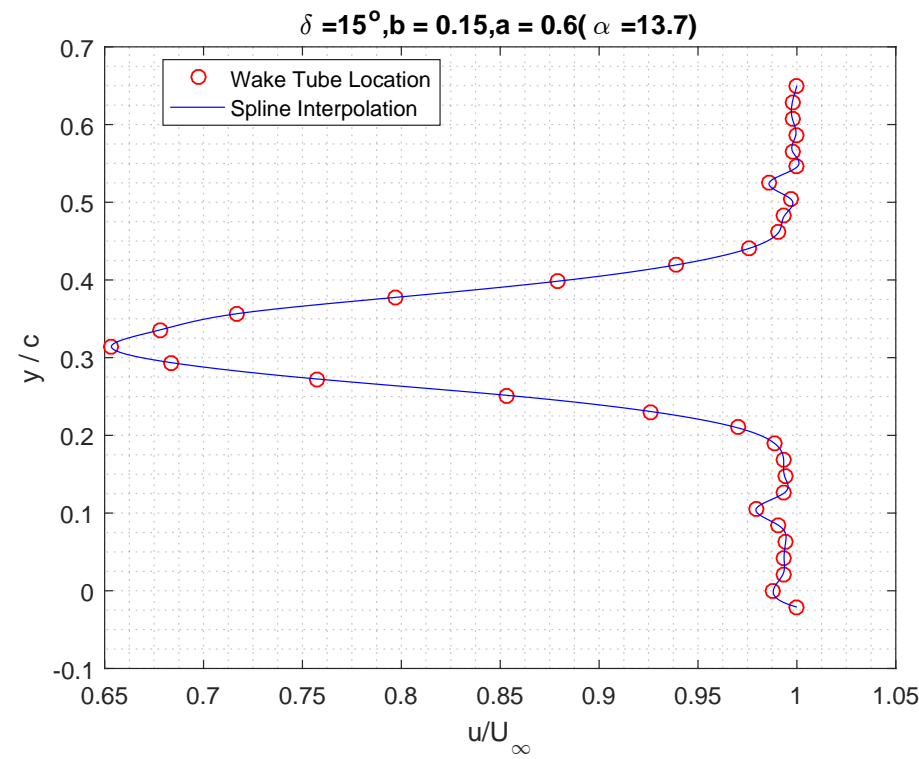

Figure 7.63: Wake plot clean airfoil at $\alpha \approx 13.7^{\circ}$ 


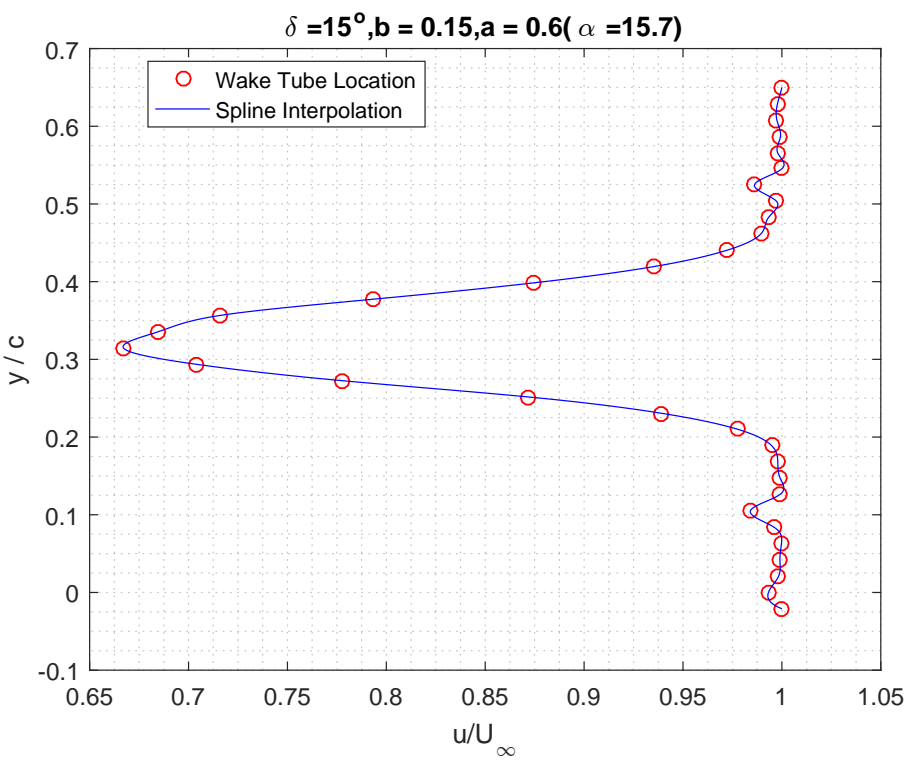

Figure 7.64: Wake plot clean airfoil at $\alpha \approx 15.7^{\circ}$

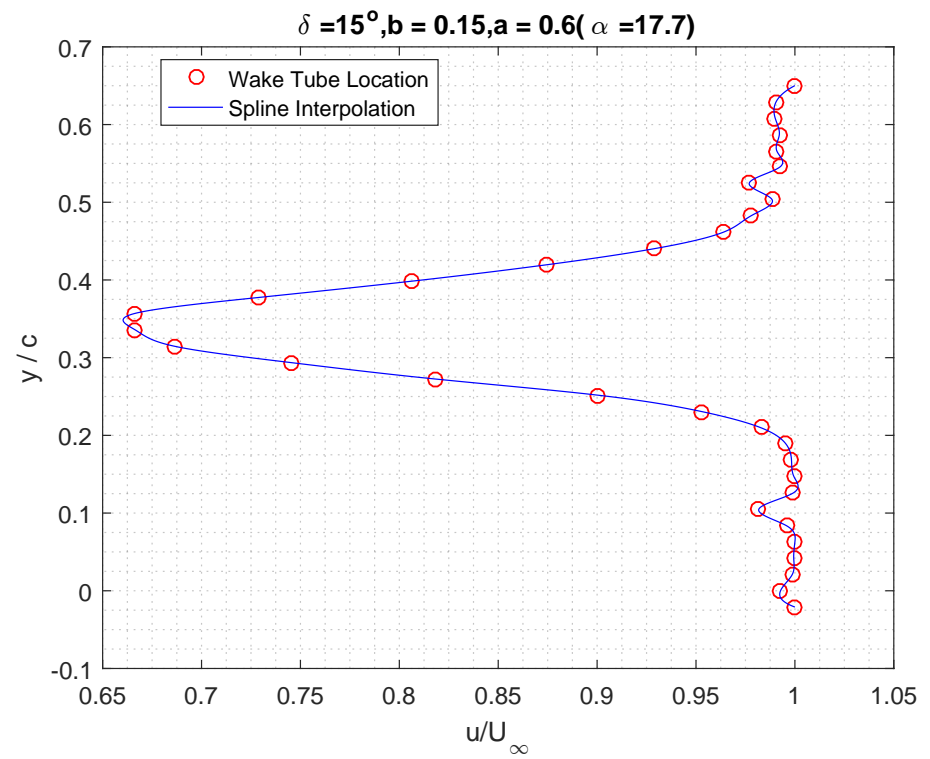

Figure 7.65: Wake plot clean airfoil at $\alpha \approx 17.7^{\circ}$ 


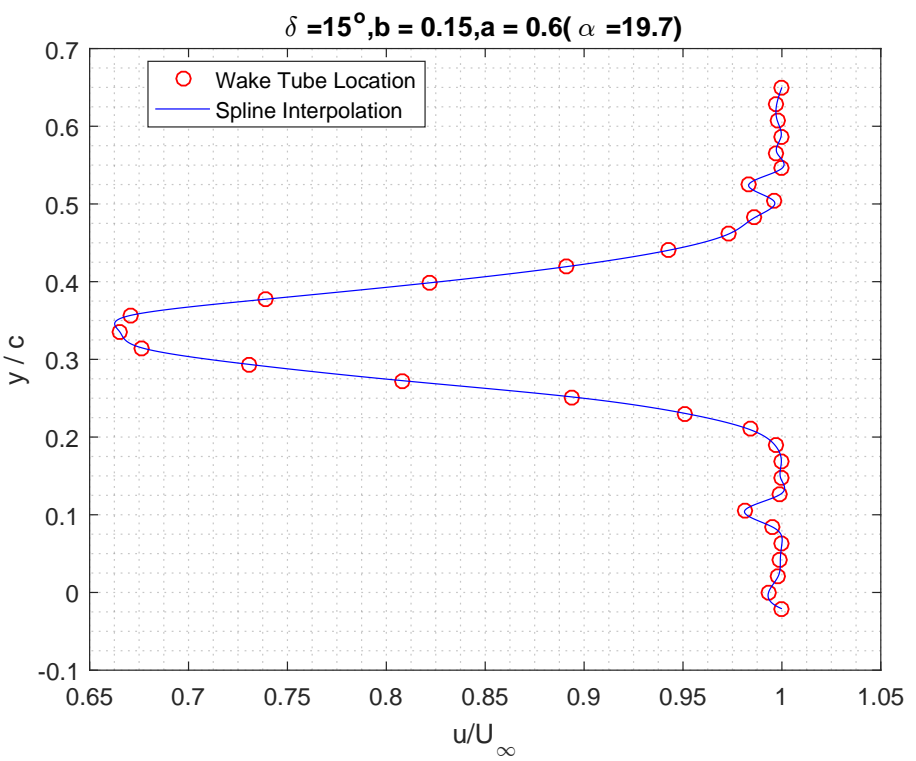

Figure 7.66: Wake plot clean airfoil at $\alpha \approx 19.7^{\circ}$

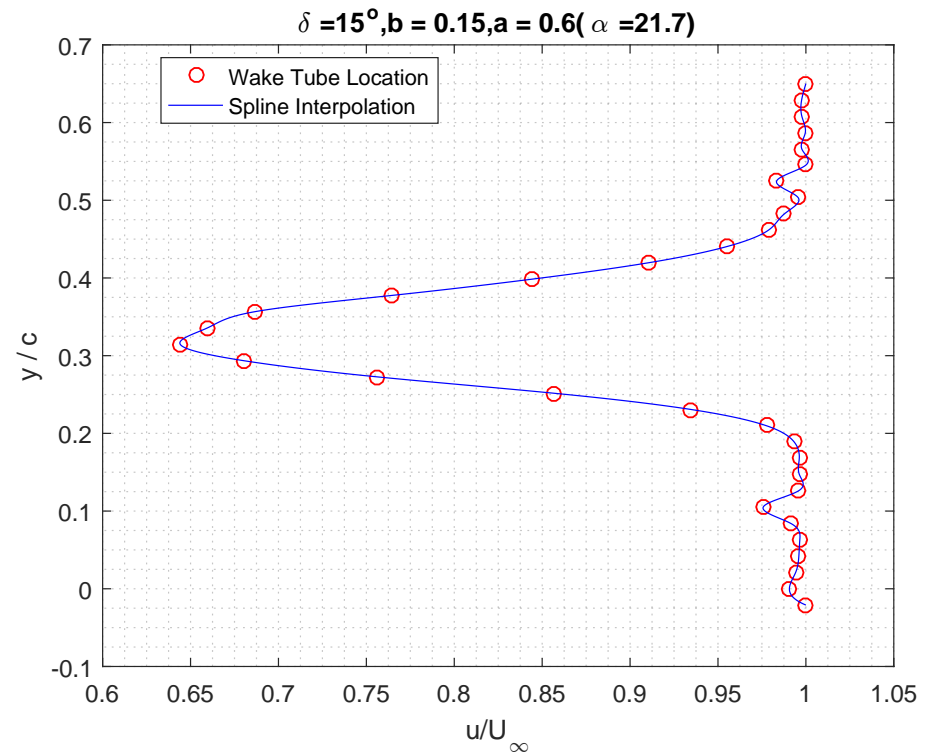

Figure 7.67: Wake plot clean airfoil at $\alpha \approx 21.7^{\circ}$ 


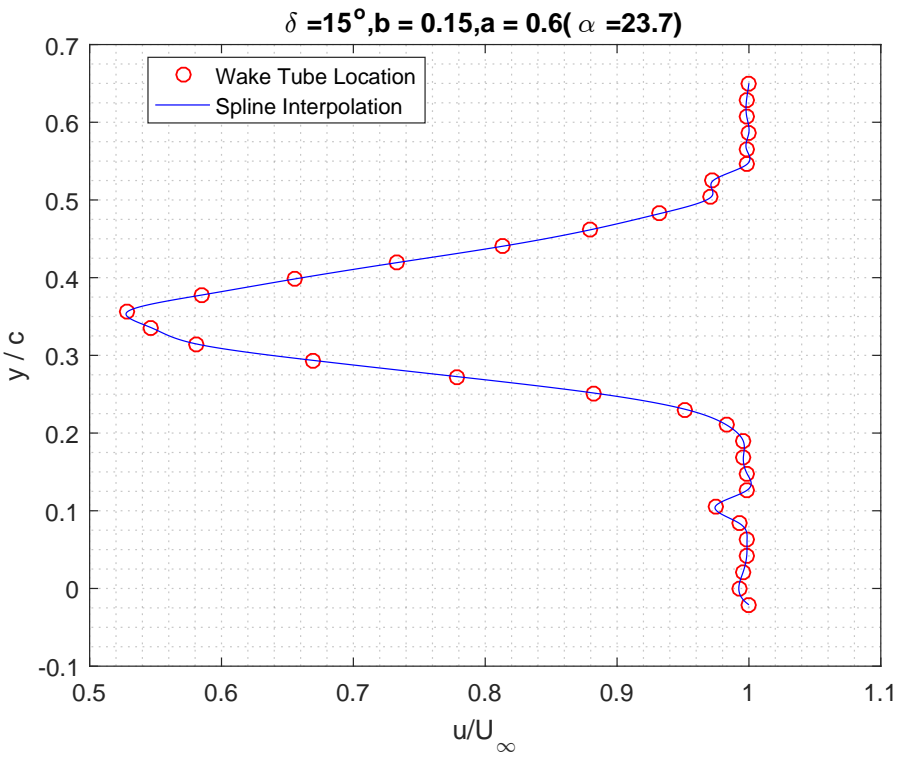

Figure 7.68: Wake plot clean airfoil at $\alpha \approx 23.7^{\circ}$

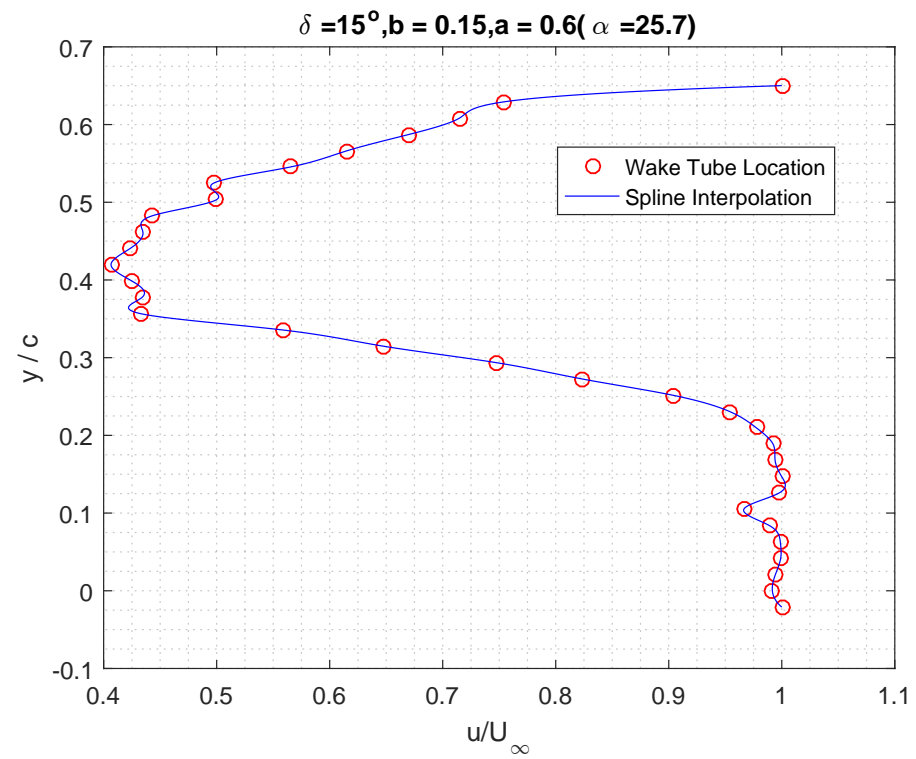

Figure 7.69: Wake plot clean airfoil at $\alpha \approx 25.7^{\circ}$ 


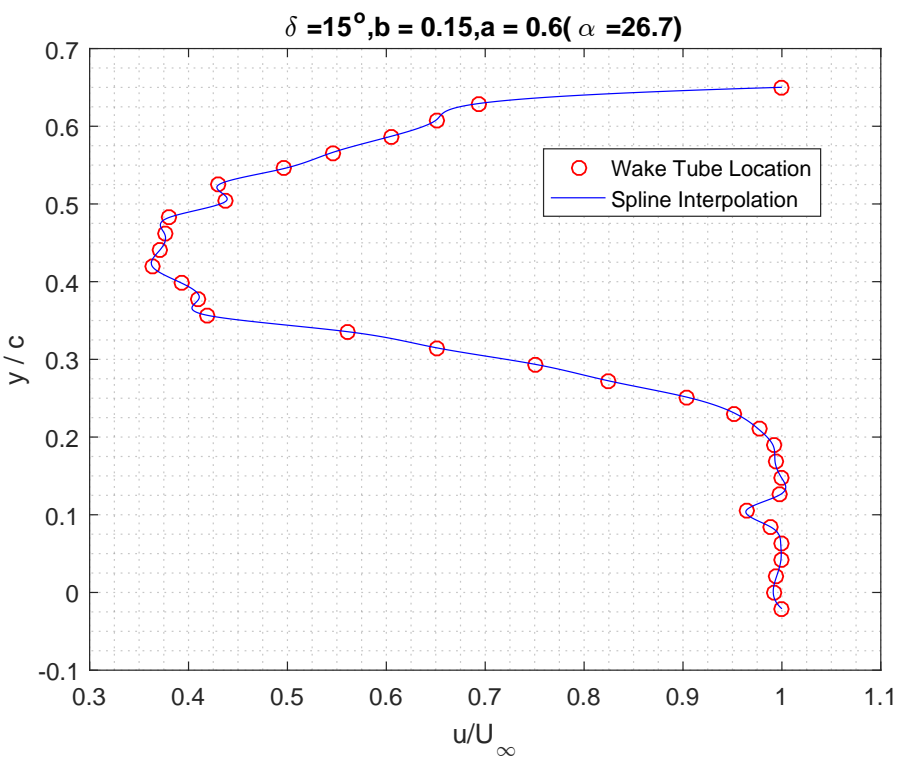

Figure 7.70: Wake plot clean airfoil at $\alpha \approx 26.7^{\circ}$

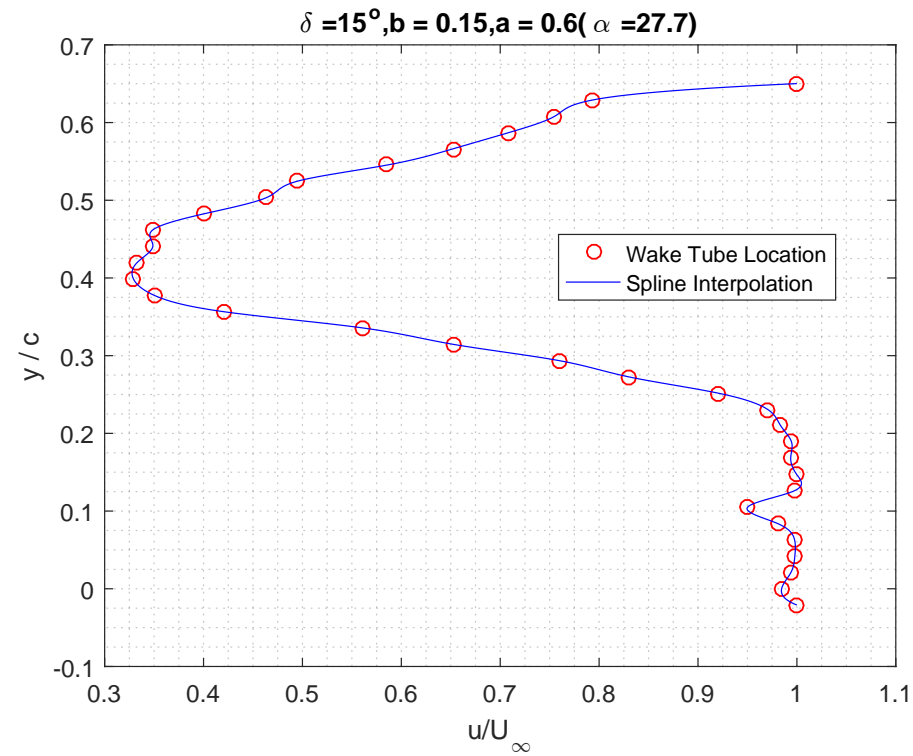

Figure 7.71: Wake plot clean airfoil at $\alpha \approx 27.7^{\circ}$ 


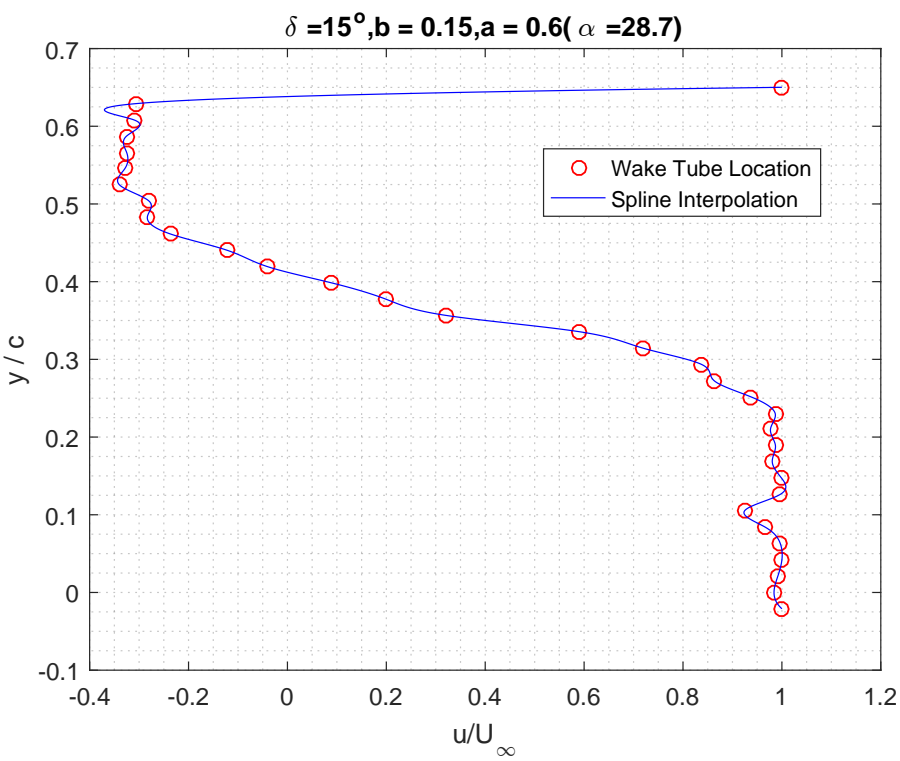

Figure 7.72: Wake plot clean airfoil at $\alpha \approx 28.7^{\circ}$

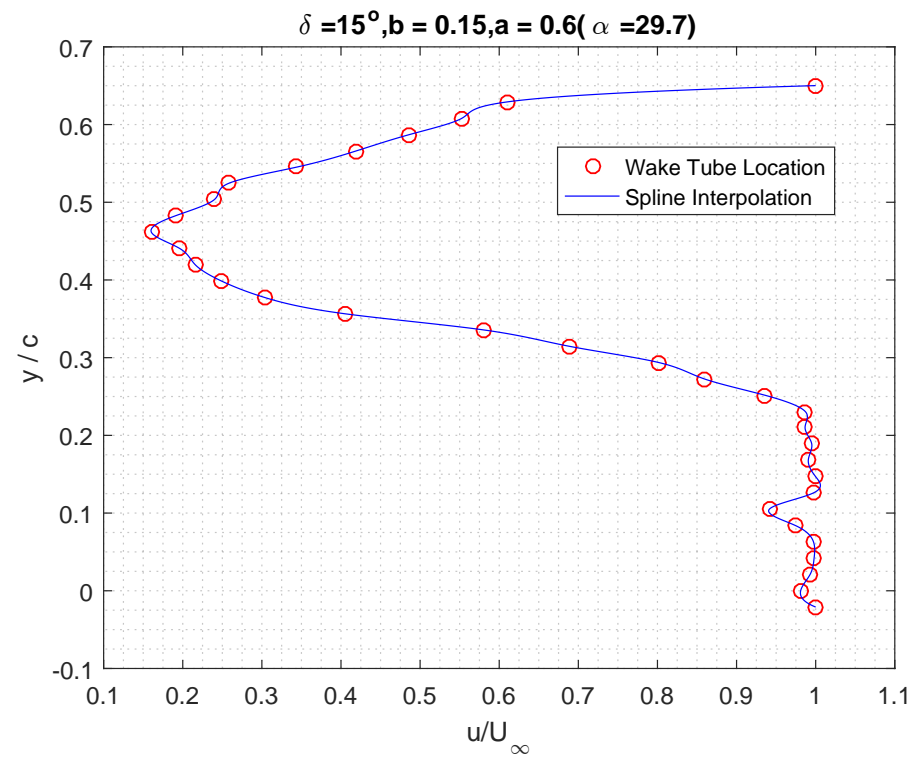

Figure 7.73: Wake plot clean airfoil at $\alpha \approx 29.7^{\circ}$ 
7.7 $\delta=25^{\circ}, b=15, a=60$ Configuration

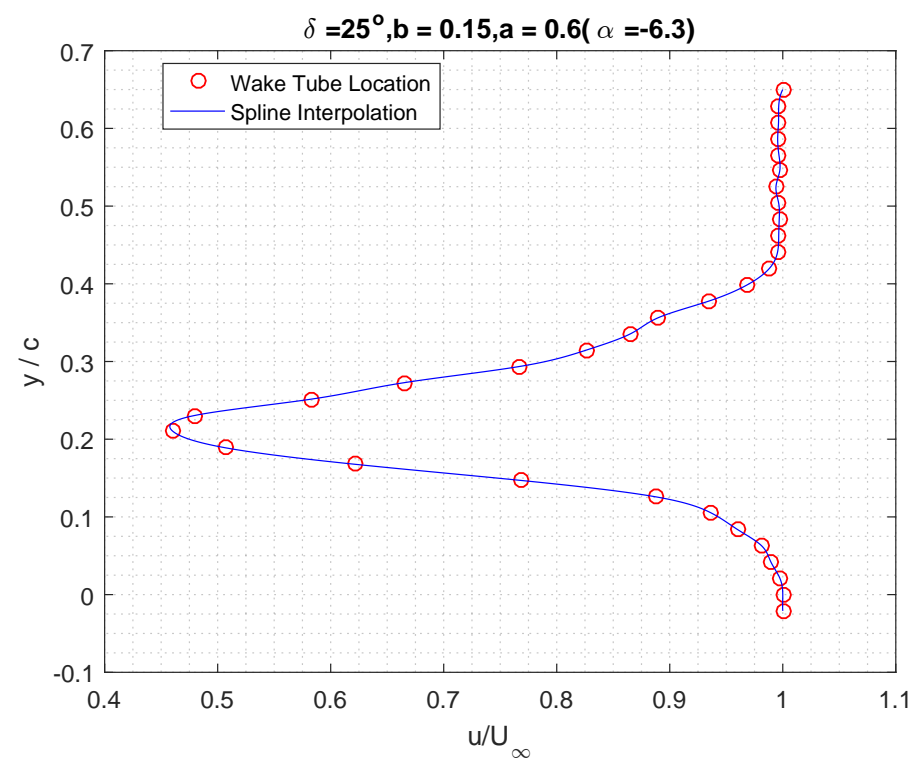

Figure 7.74: Wake plot clean airfoil at $\alpha \approx-6.3^{\circ}$

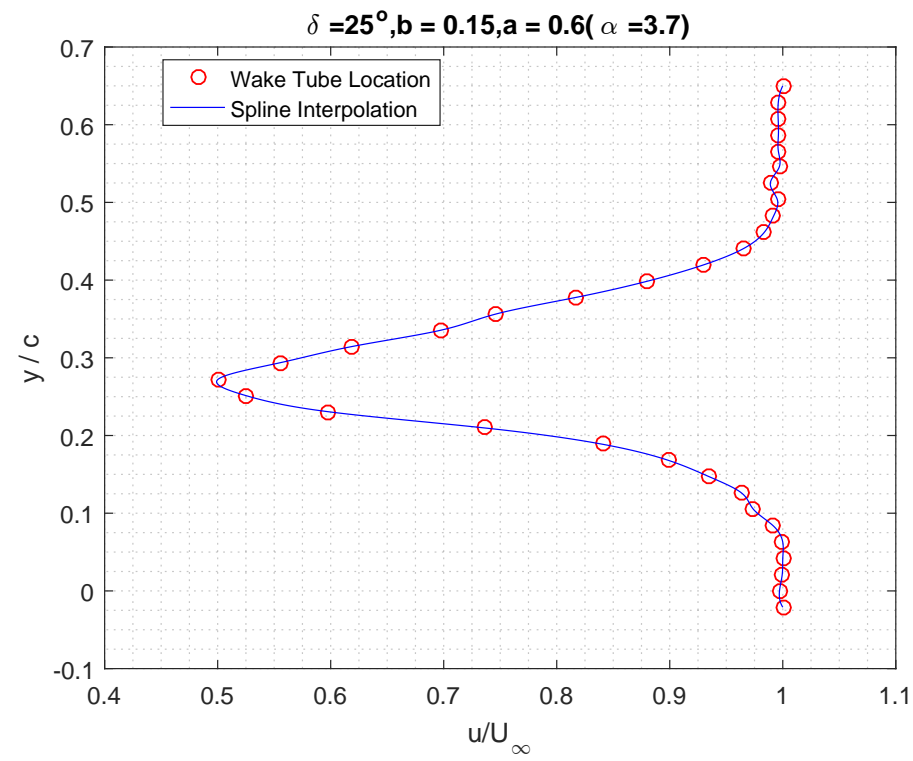

Figure 7.75: Wake plot clean airfoil at $\alpha \approx 3.7^{\circ}$ 


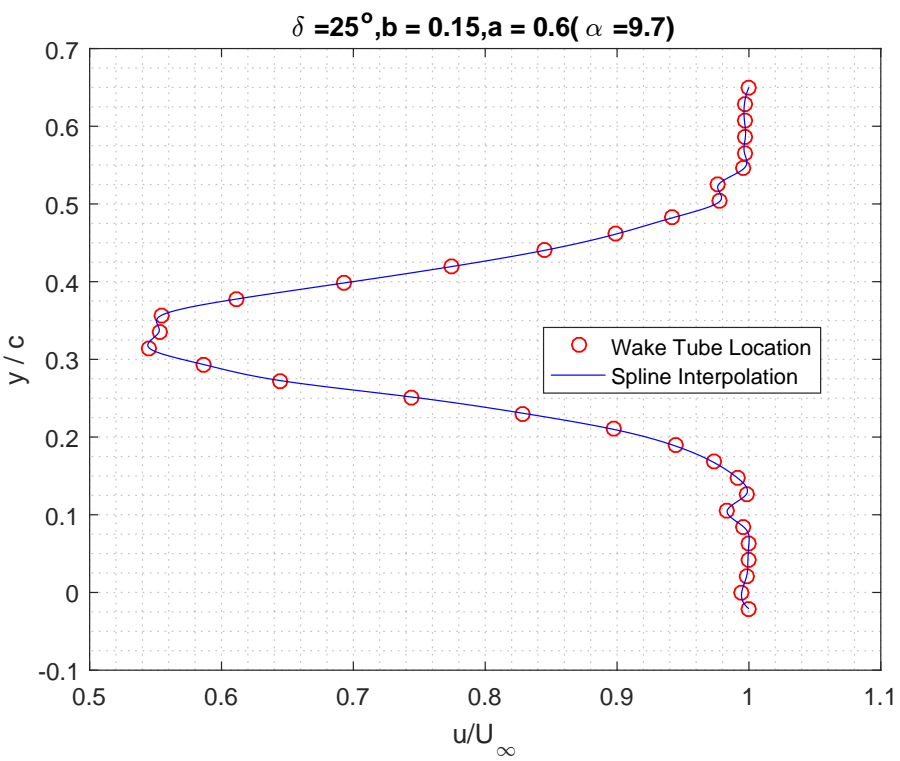

Figure 7.76: Wake plot clean airfoil at $\alpha \approx 9.7^{\circ}$

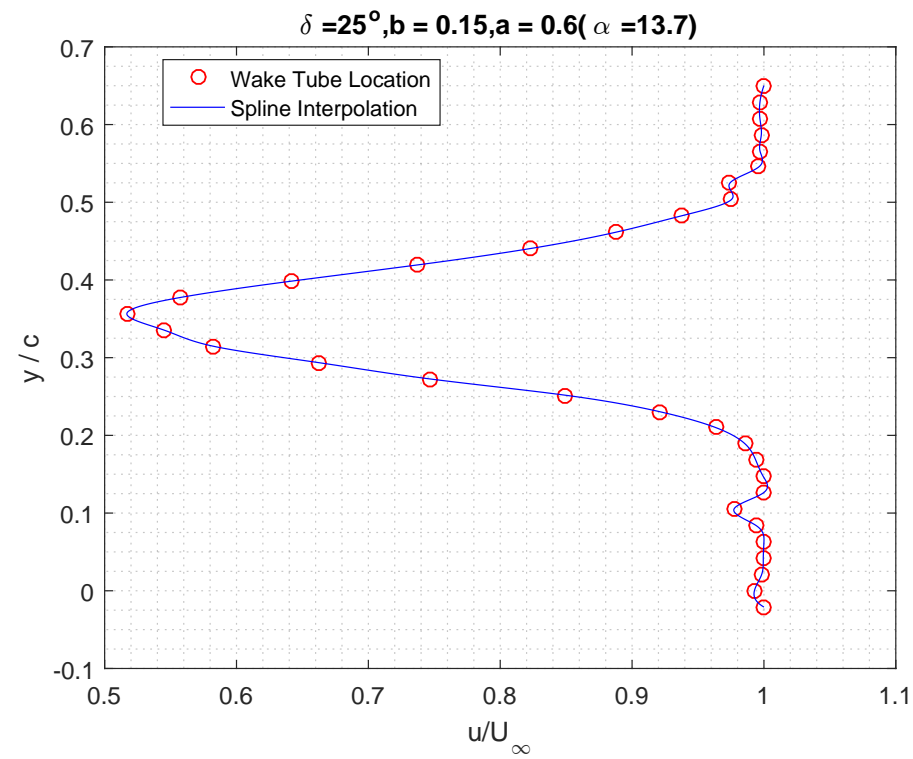

Figure 7.77: Wake plot clean airfoil at $\alpha \approx 13.7^{\circ}$ 


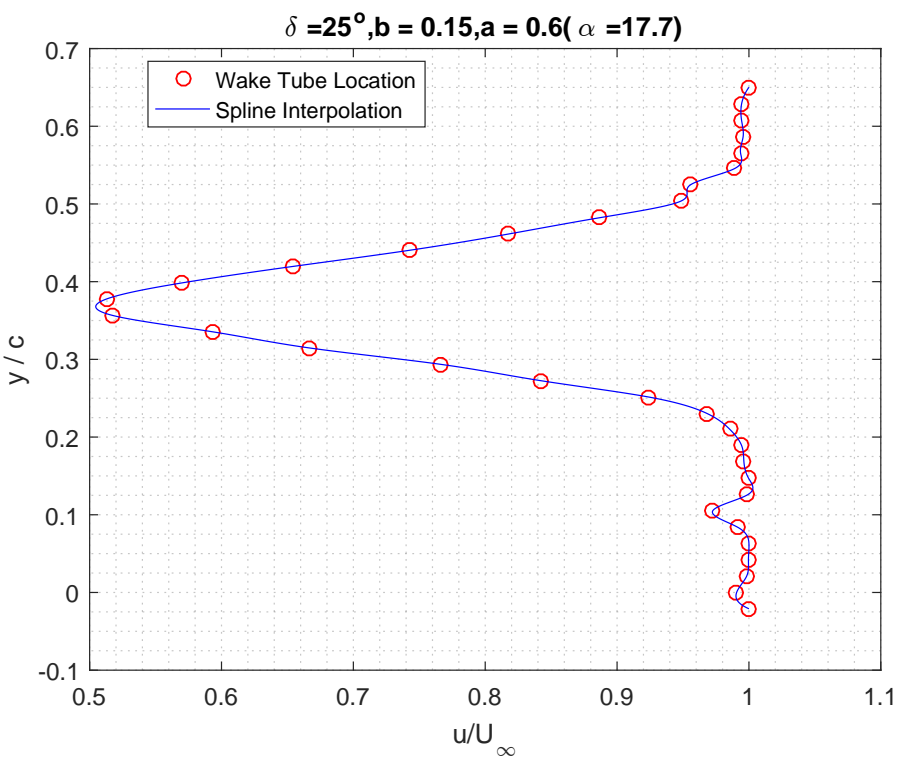

Figure 7.78: Wake plot clean airfoil at $\alpha \approx 17.7^{\circ}$

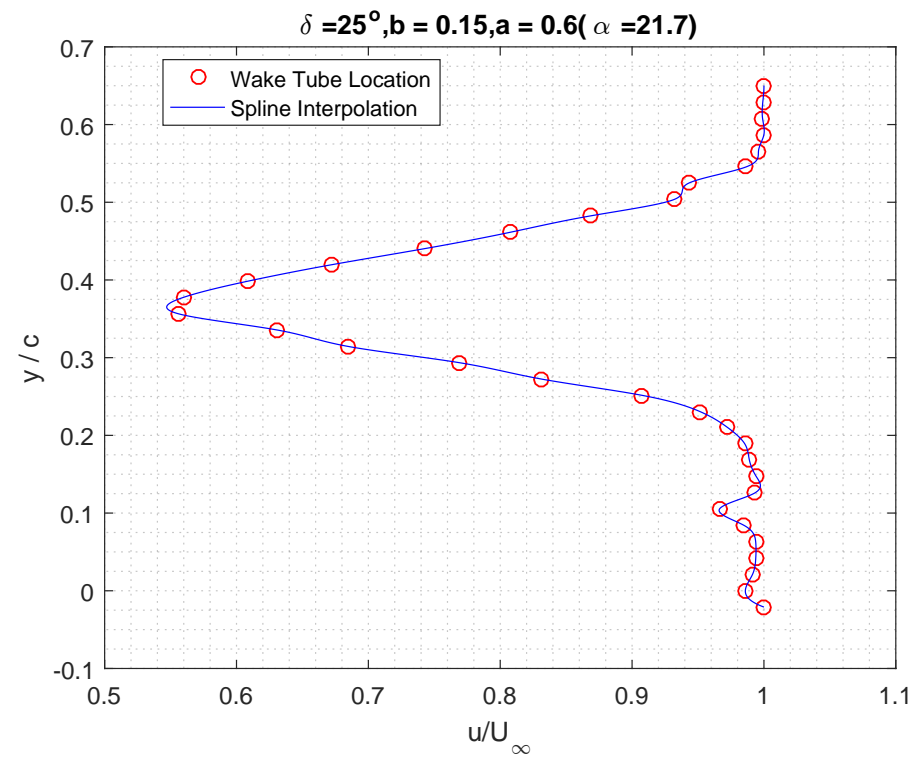

Figure 7.79: Wake plot clean airfoil at $\alpha \approx 21.7^{\circ}$ 


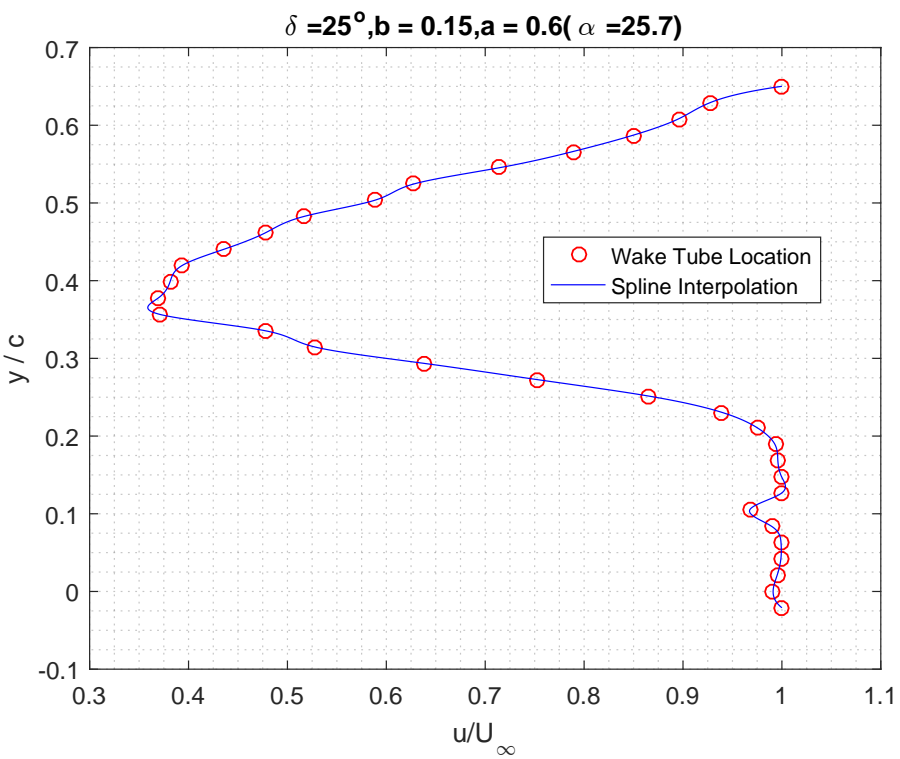

Figure 7.80: Wake plot clean airfoil at $\alpha \approx 25.7^{\circ}$

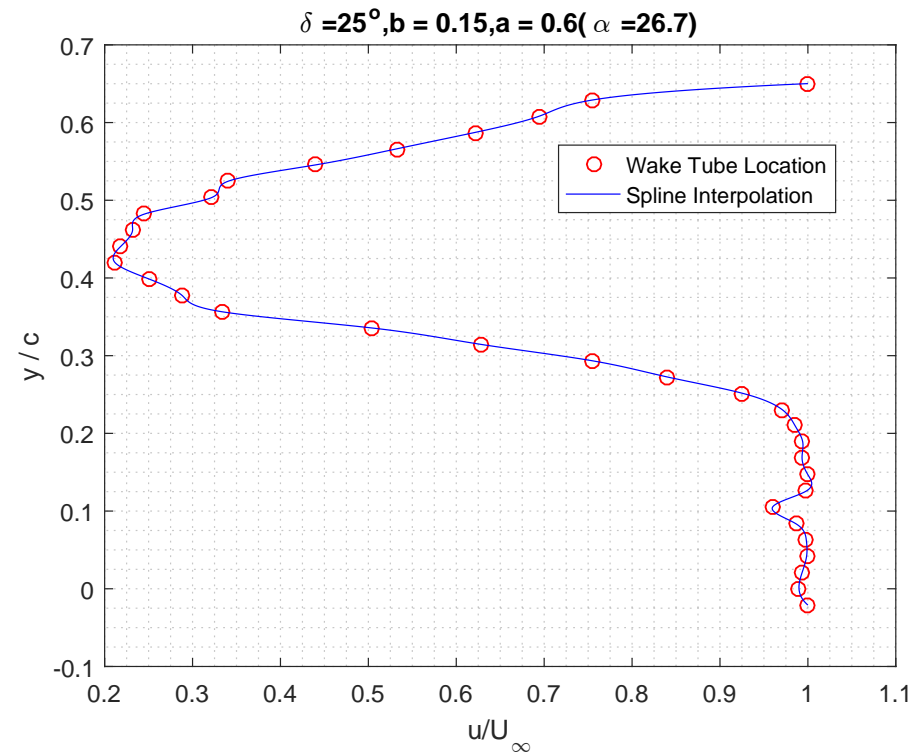

Figure 7.81: Wake plot clean airfoil at $\alpha \approx 26.7^{\circ}$ 


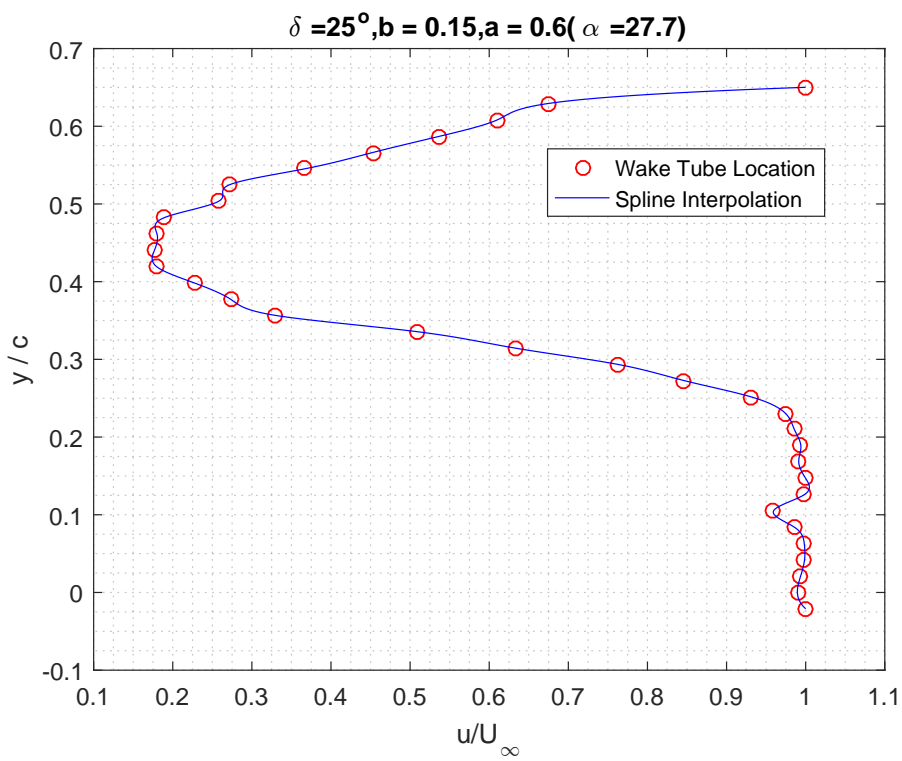

Figure 7.82: Wake plot clean airfoil at $\alpha \approx 27.7^{\circ}$

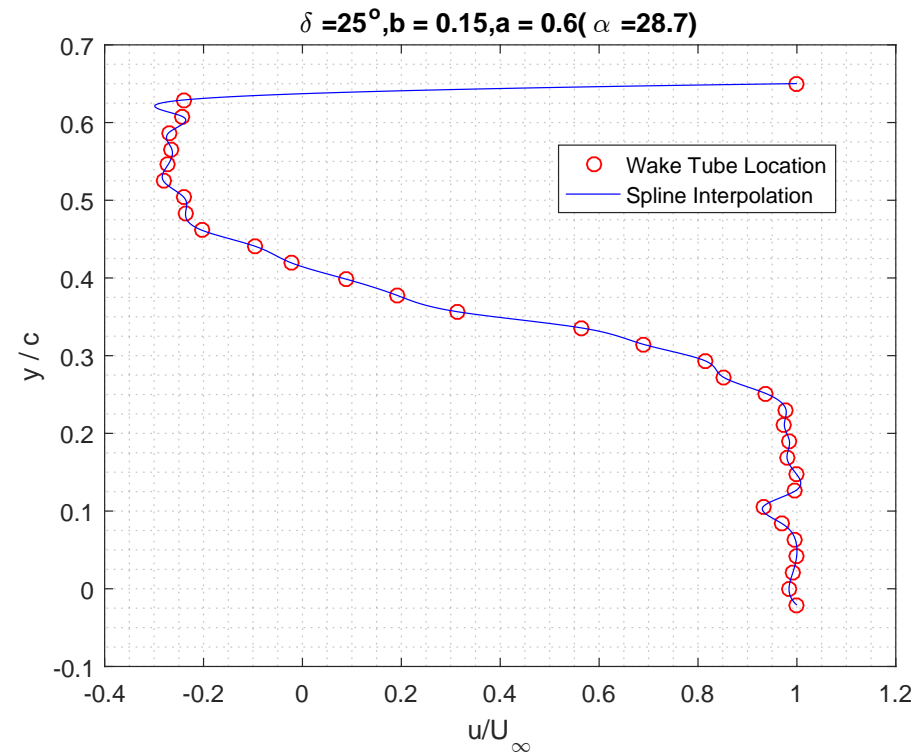

Figure 7.83: Wake plot clean airfoil at $\alpha \approx 28.7^{\circ}$ 


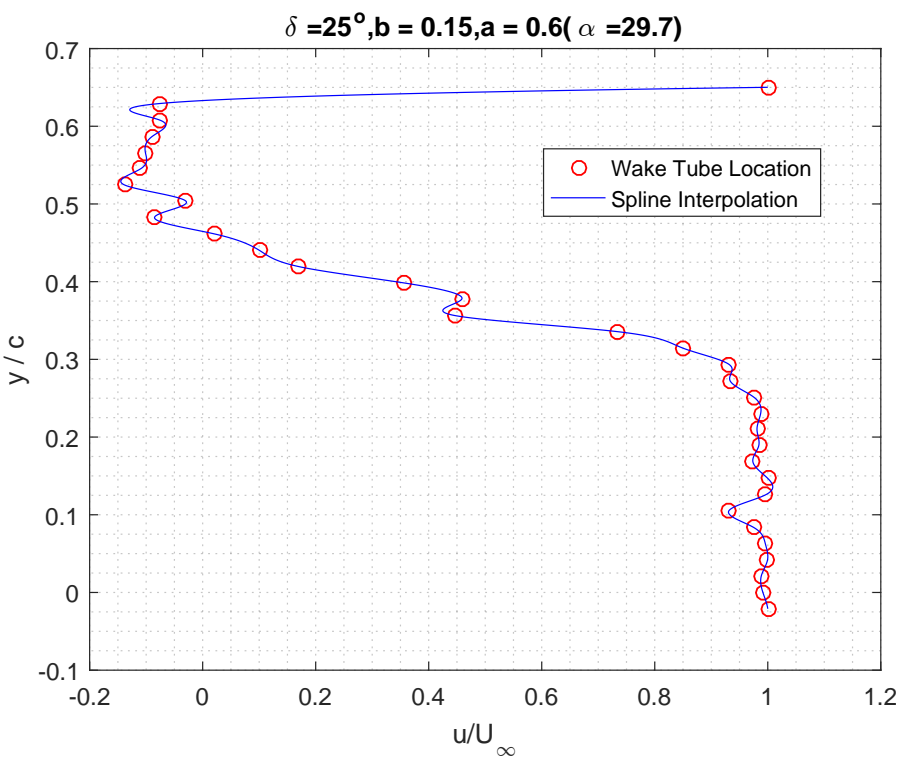

Figure 7.84: Wake plot clean airfoil at $\alpha \approx 29.7^{\circ}$

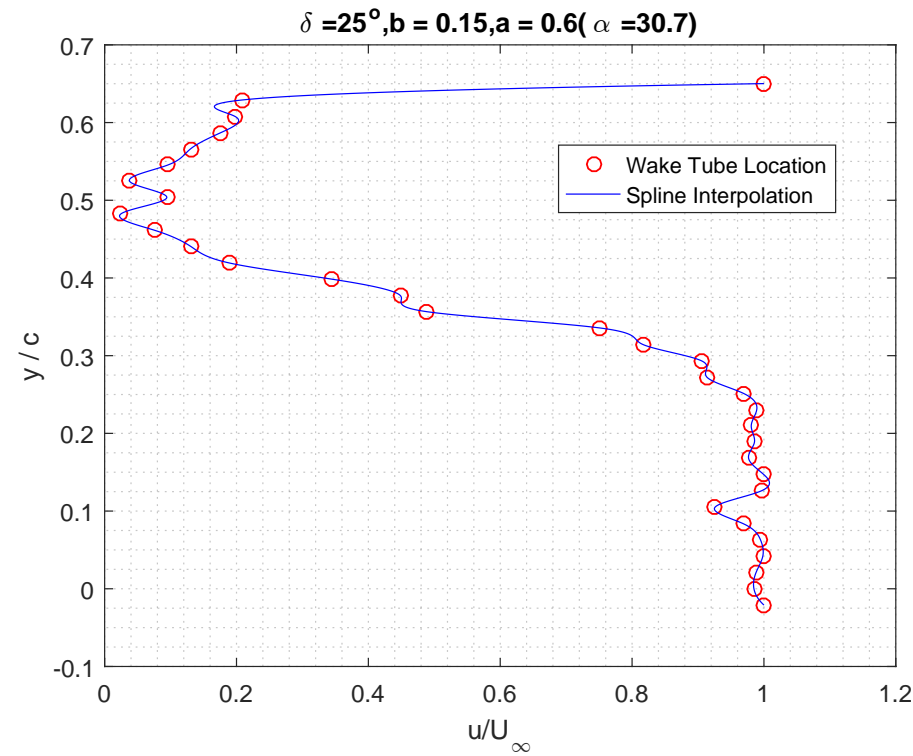

Figure 7.85: Wake plot clean airfoil at $\alpha \approx 30.7^{\circ}$ 


\section{Appendix 8}

\section{Computational Study Plots}

\subsection{Computational Pressure Coefficient Plots}

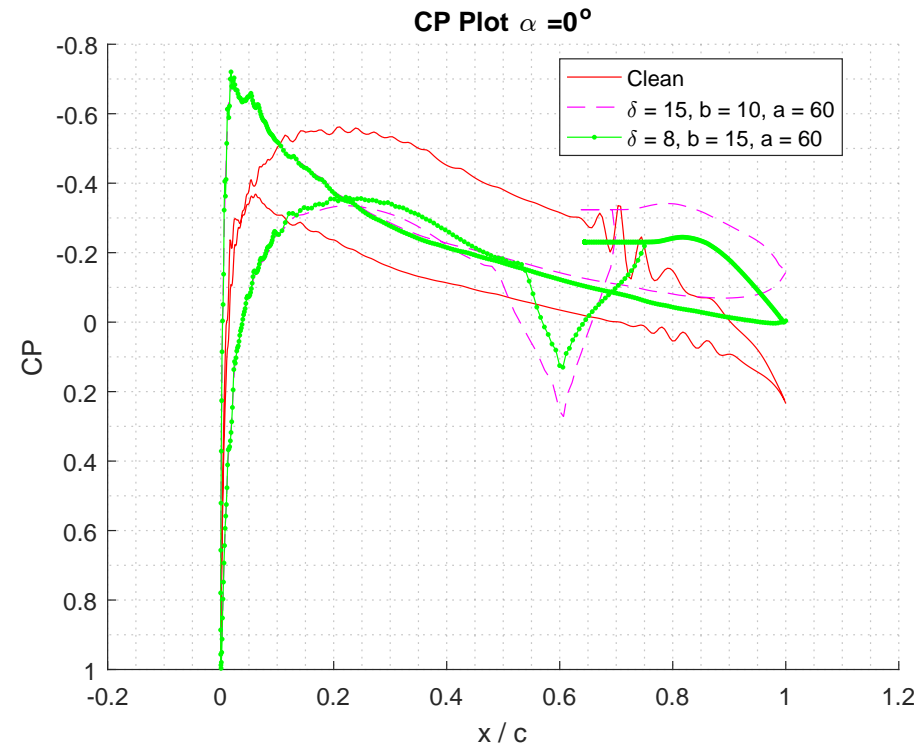

Figure 8.1: CFD CP Plot at $\alpha=0.0^{\circ}$ 


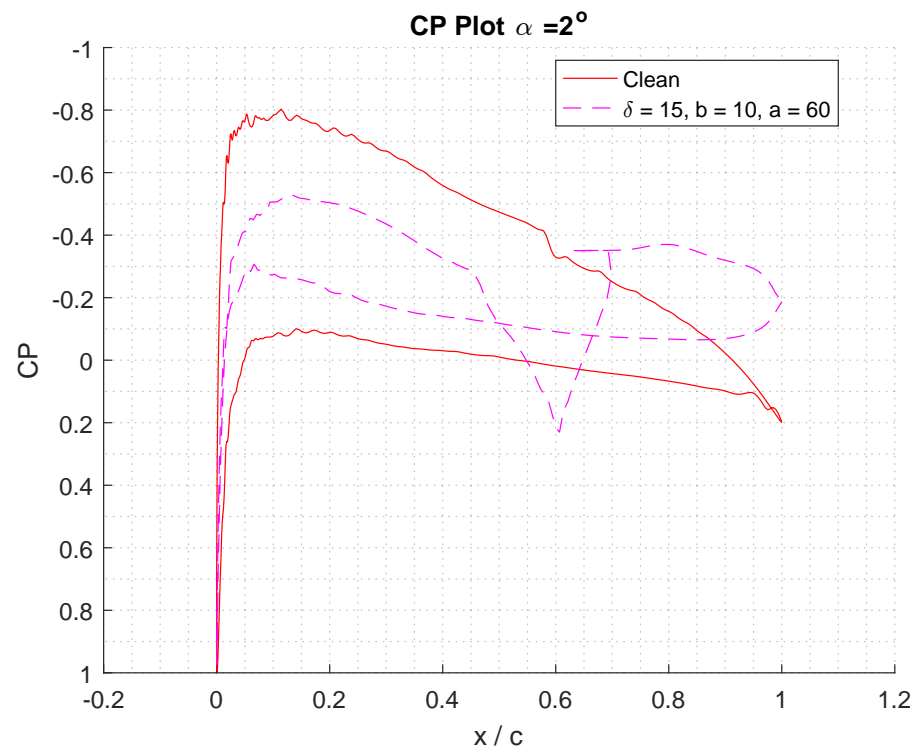

Figure 8.2: CFD CP Plot at $\alpha=2.0^{\circ}$

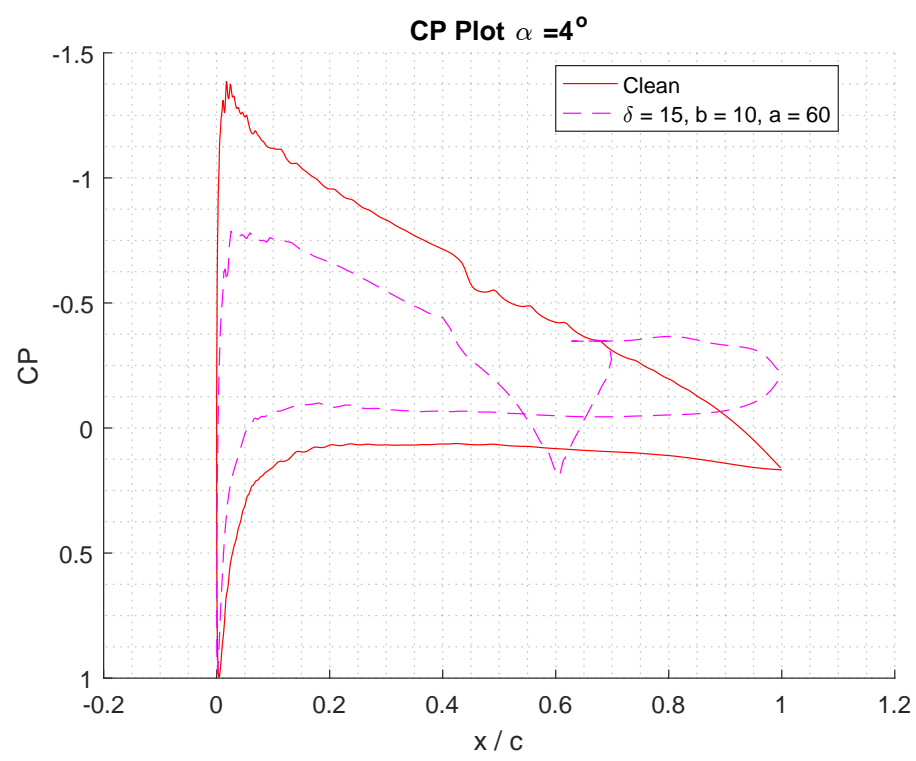

Figure 8.3: CFD CP Plot at $\alpha=4.0^{\circ}$ 


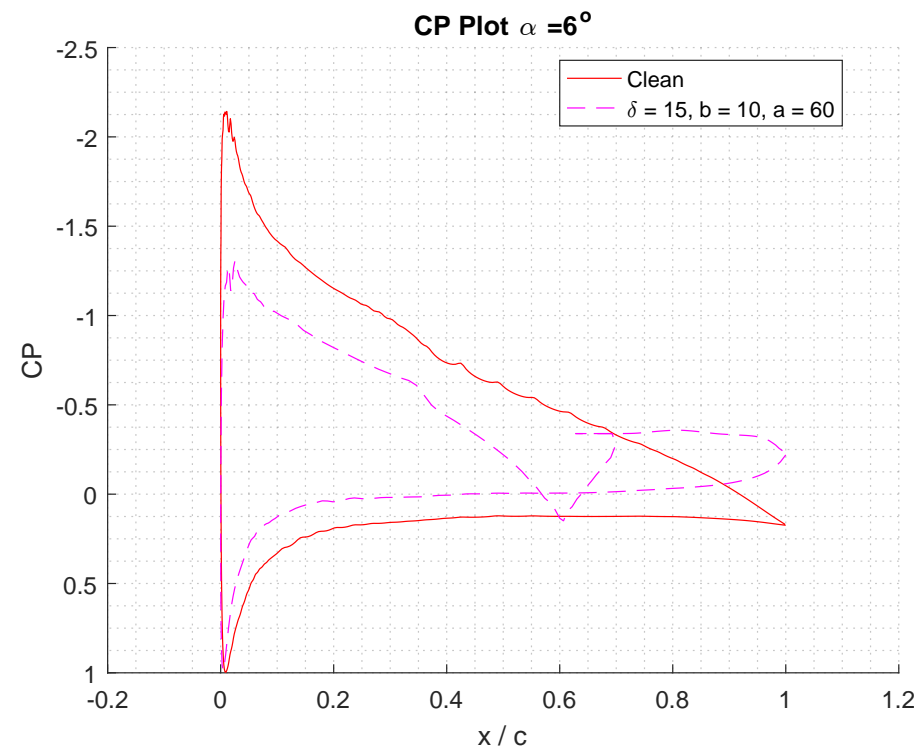

Figure 8.4: CFD CP Plot at $\alpha=6.0^{\circ}$

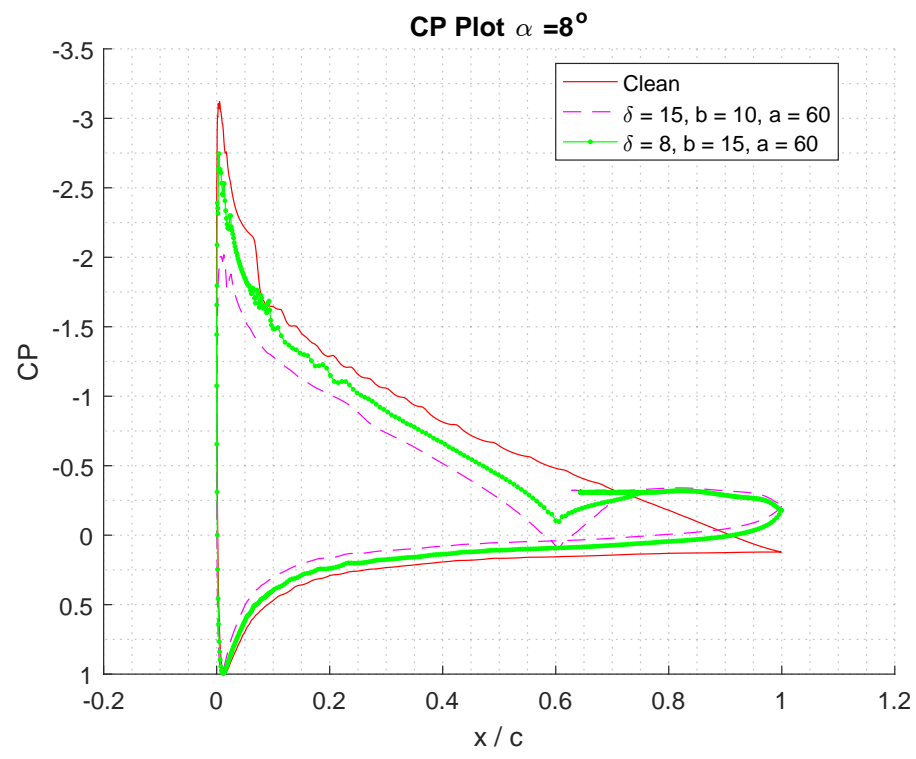

Figure 8.5: CFD CP Plot at $\alpha=8.0^{\circ}$ 


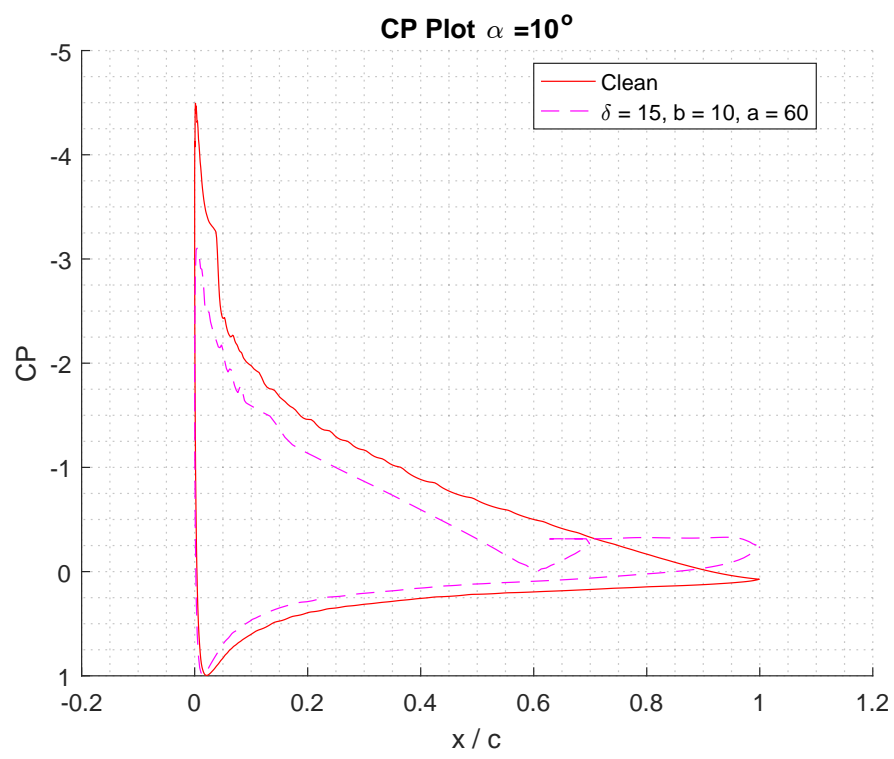

Figure 8.6: CFD CP Plot at $\alpha=10.0^{\circ}$

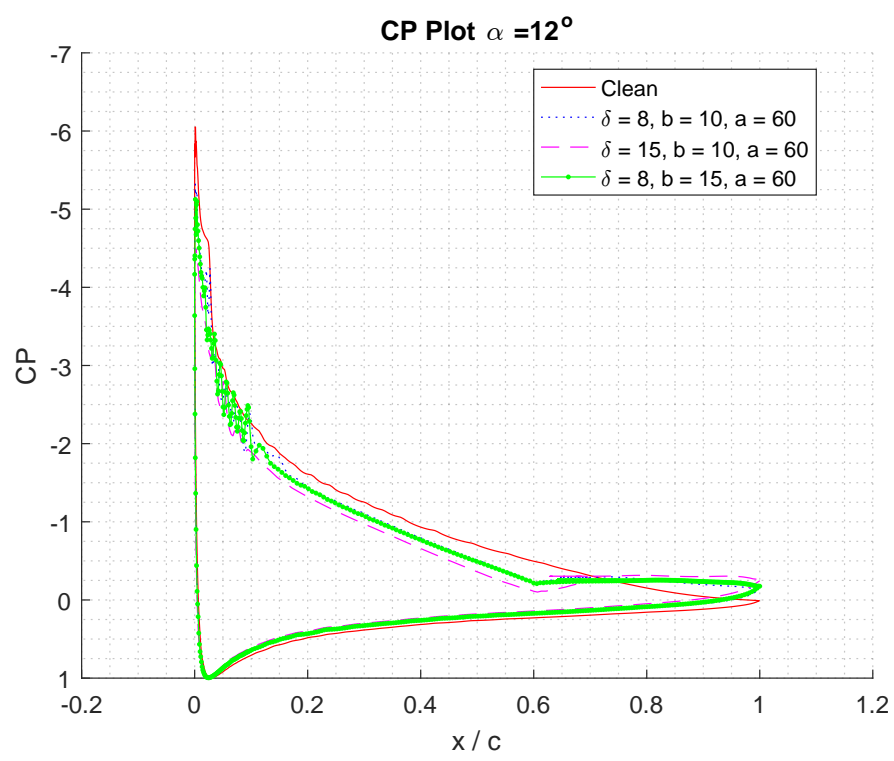

Figure 8.7: CFD CP Plot at $\alpha=12.0^{\circ}$ 


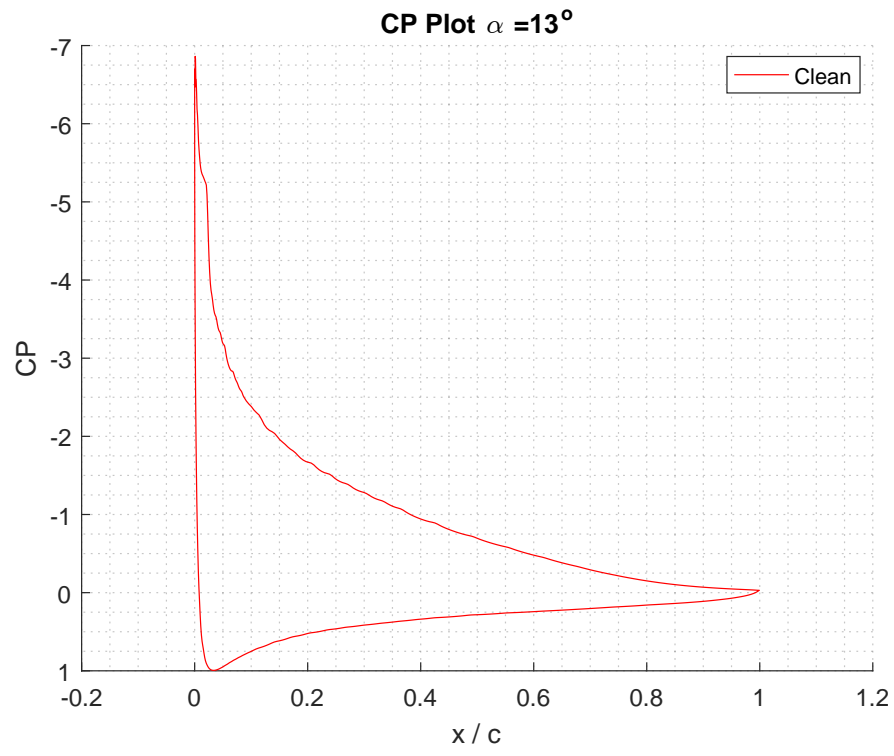

Figure 8.8: CFD CP Plot at $\alpha=13.0^{\circ}$

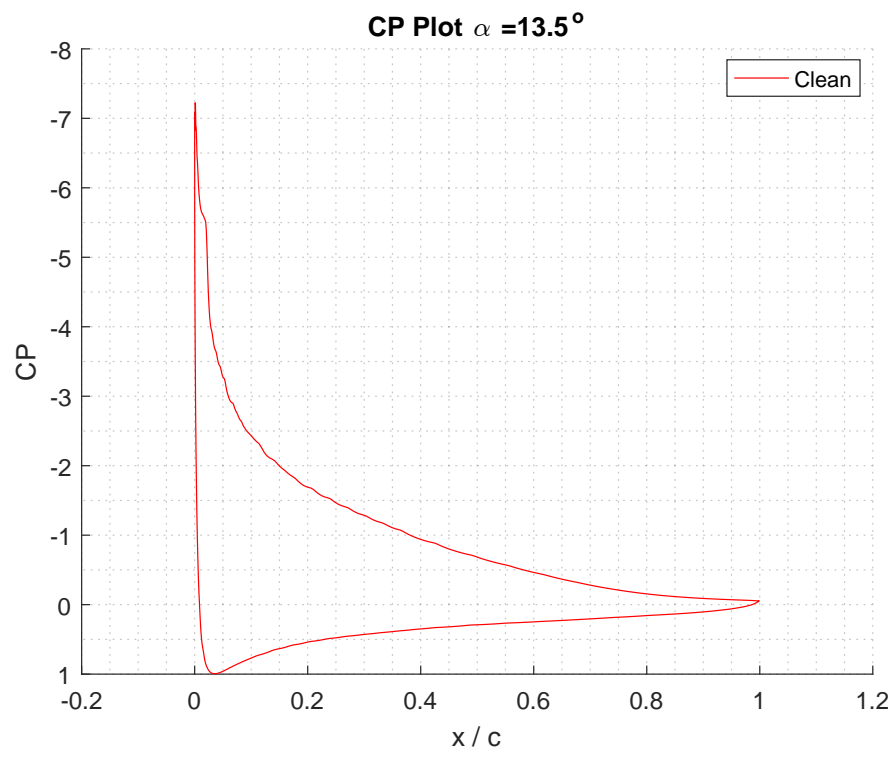

Figure 8.9: CFD CP Plot at $\alpha=13.5^{\circ}$ 


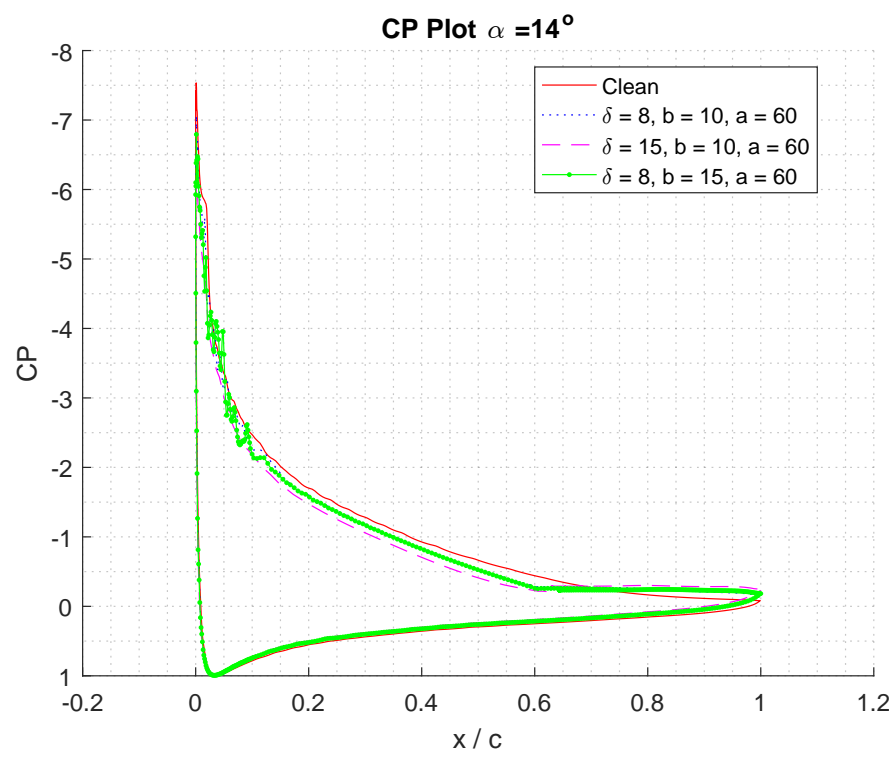

Figure 8.10: CFD CP Plot at $\alpha=14.0^{\circ}$

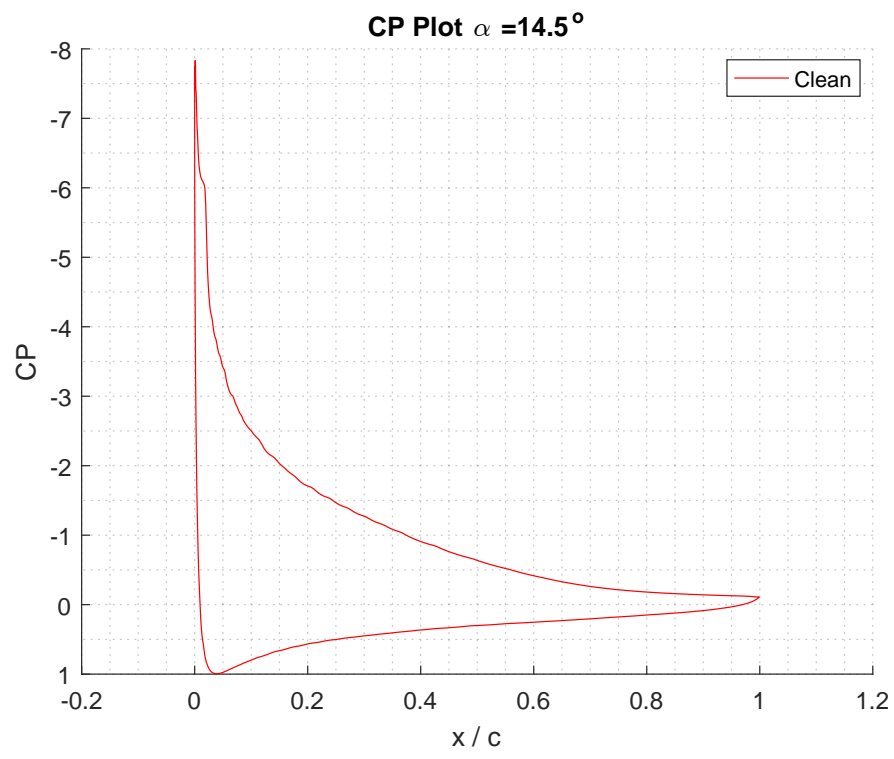

Figure 8.11: CFD CP Plot at $\alpha=14.5^{\circ}$ 


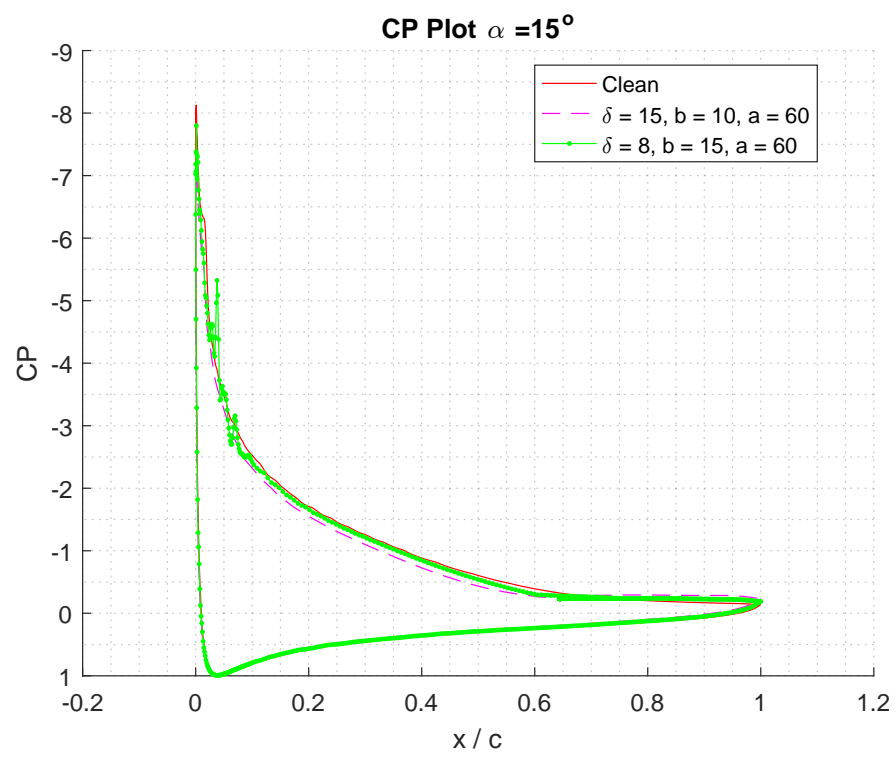

Figure 8.12: CFD CP Plot at $\alpha=15.0^{\circ}$

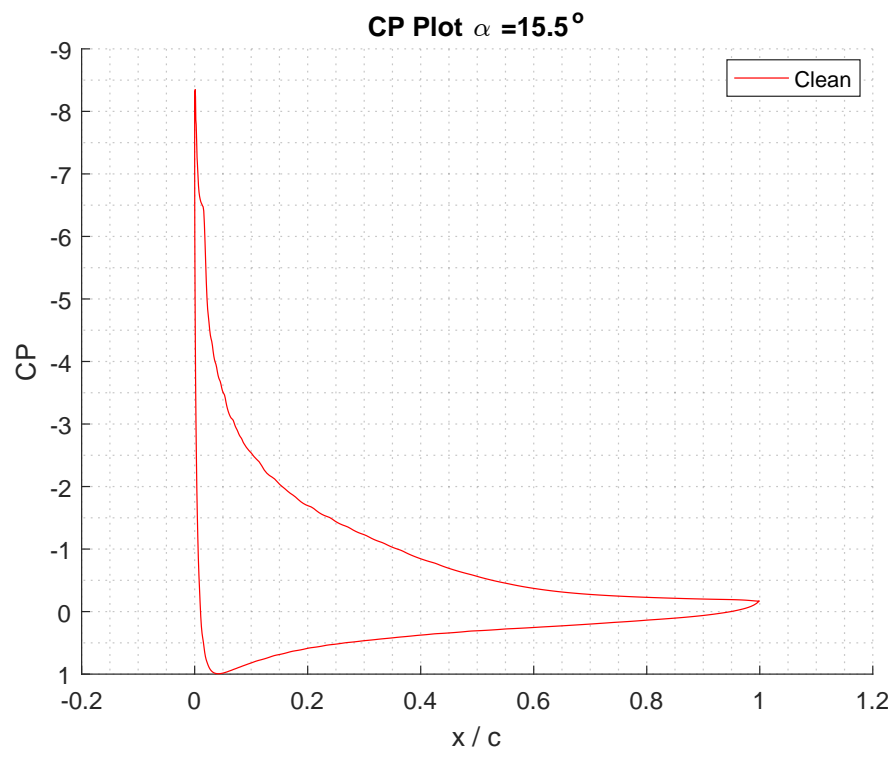

Figure 8.13: CFD CP Plot at $\alpha=15.5^{\circ}$ 


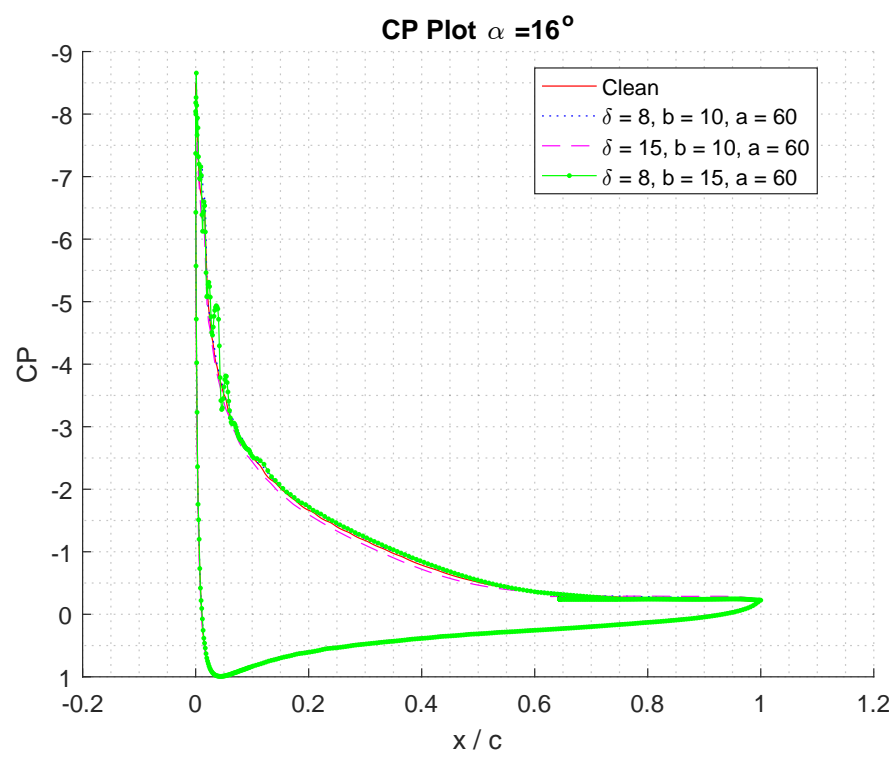

Figure 8.14: CFD CP Plot at $\alpha=16.0^{\circ}$

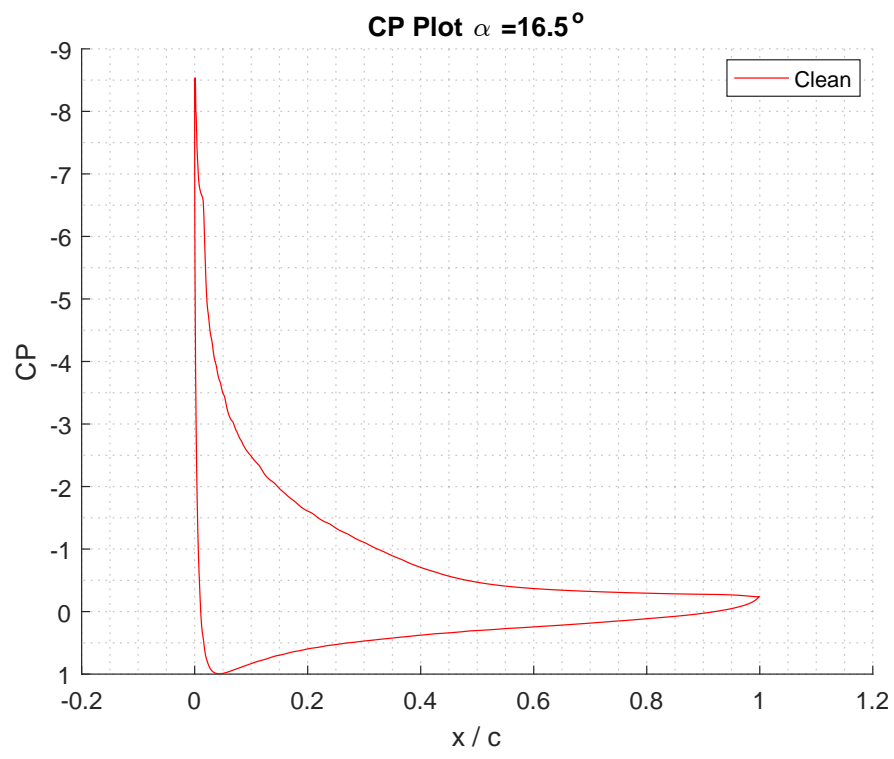

Figure 8.15: CFD CP Plot at $\alpha=16.5^{\circ}$ 


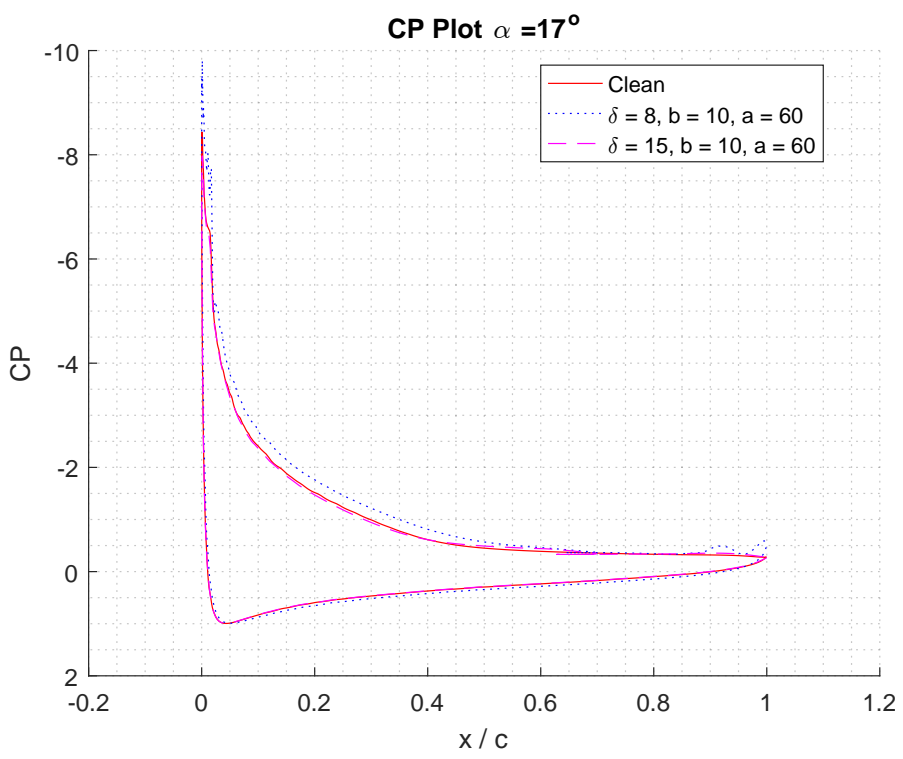

Figure 8.16: CFD CP Plot at $\alpha=17.0^{\circ}$

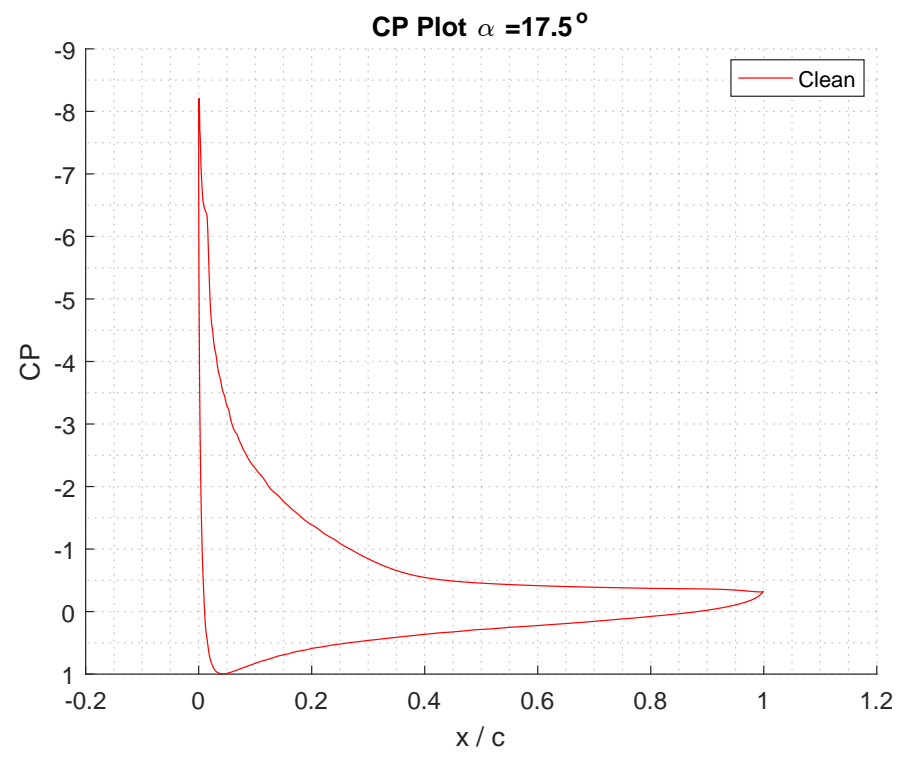

Figure 8.17: CFD CP Plot at $\alpha=17.5^{\circ}$ 


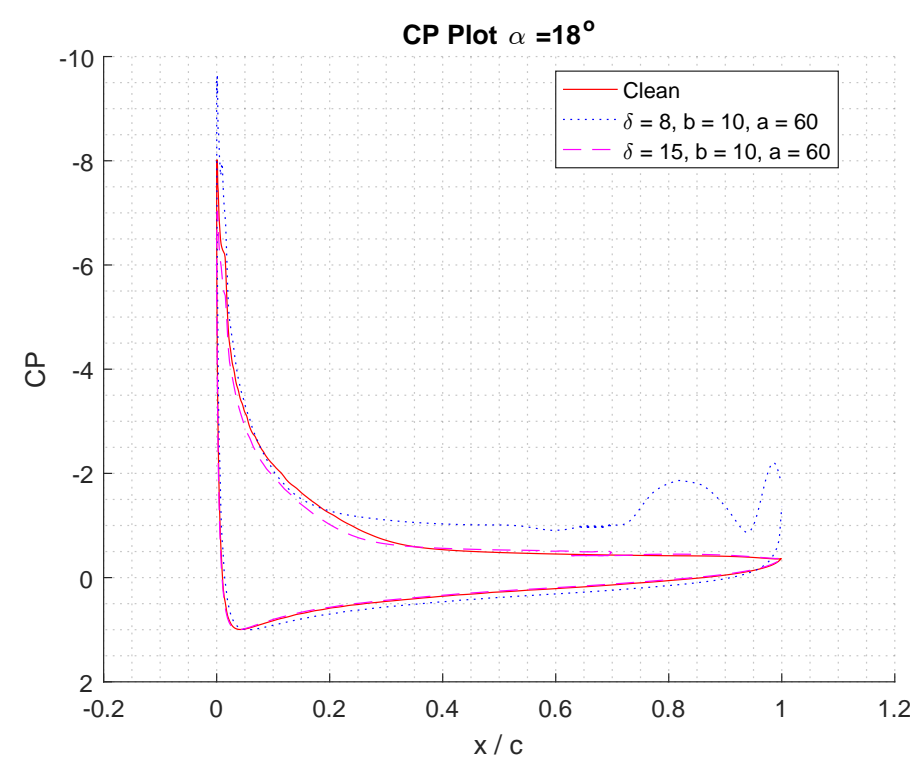

Figure 8.18: CFD CP Plot at $\alpha=18.0^{\circ}$

\subsection{Computational Wake Plots}

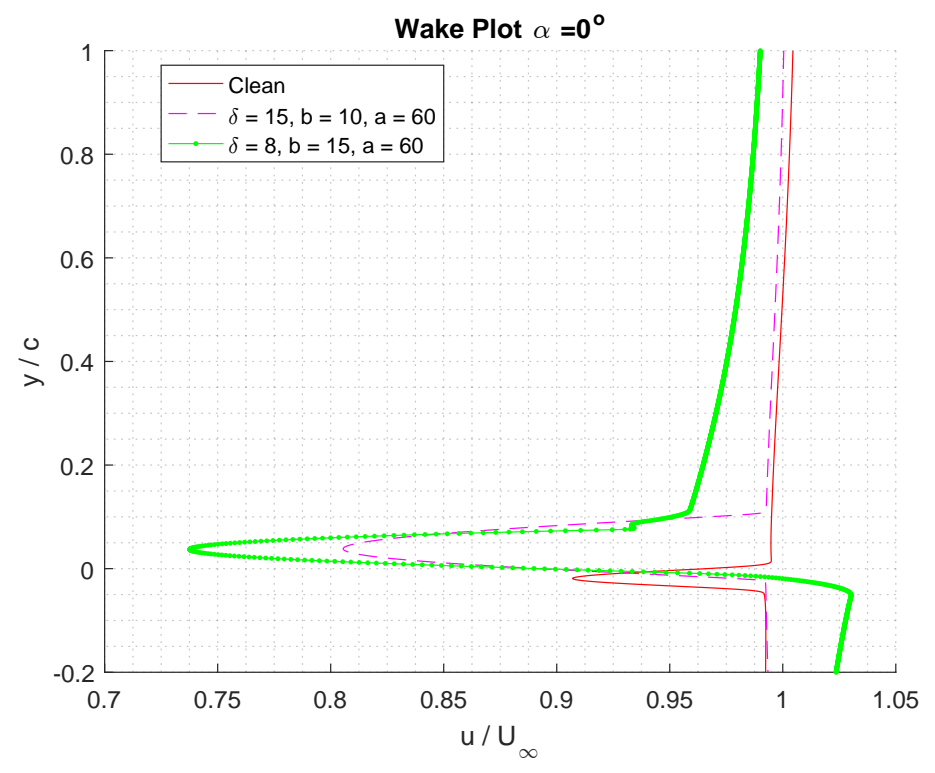

Figure 8.19: CFD Wake Plot at $\alpha=0.0^{\circ}$ 


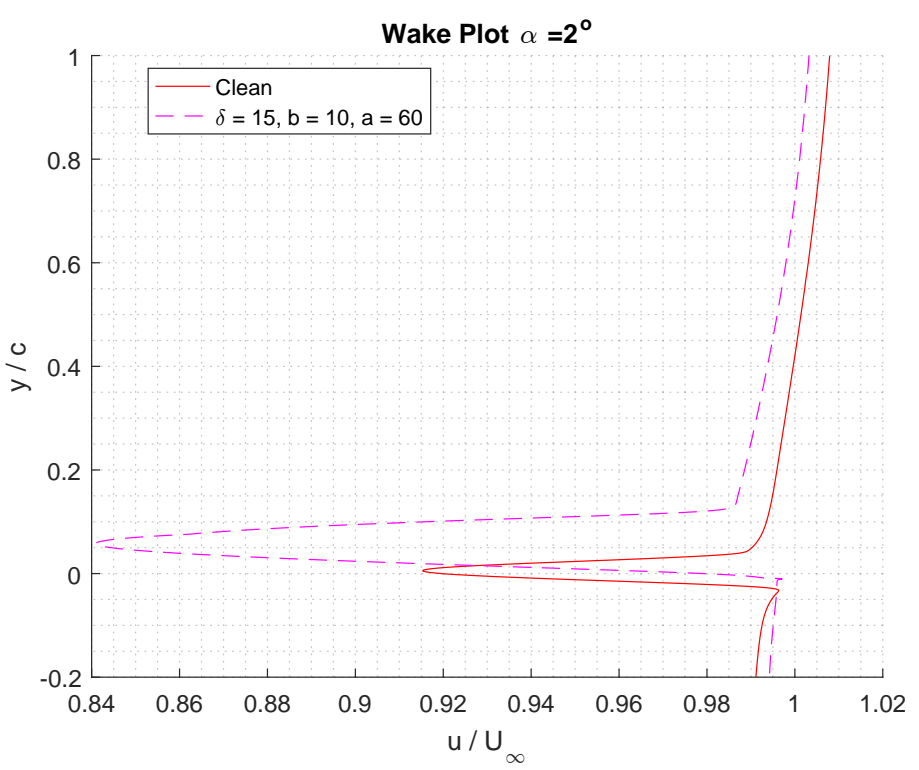

Figure 8.20: CFD Wake Plot at $\alpha=2.0^{\circ}$

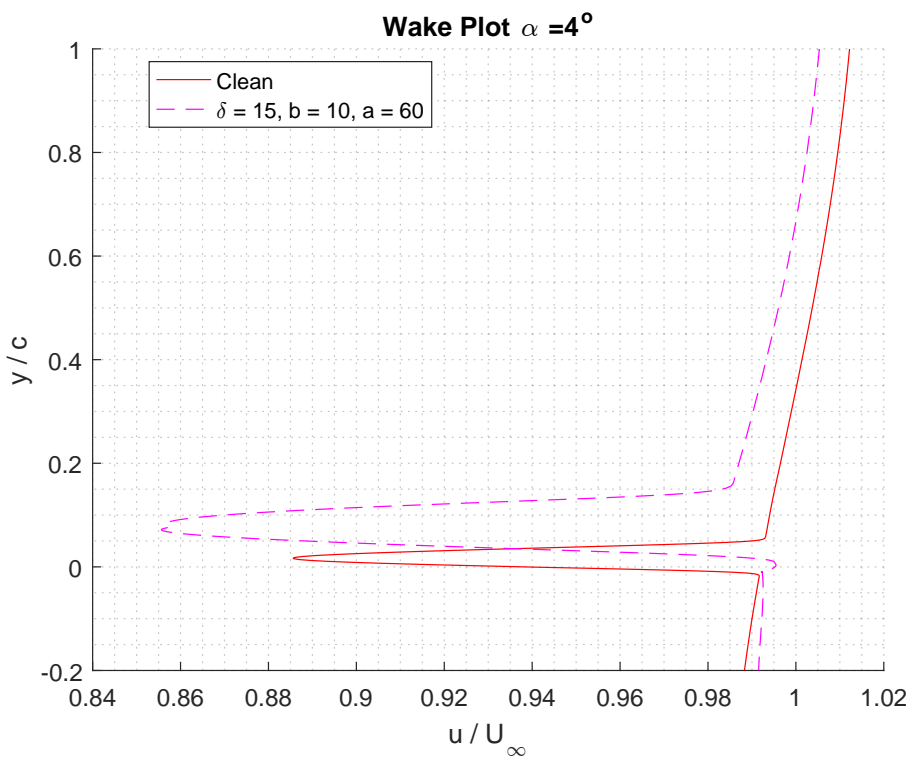

Figure 8.21: CFD Wake Plot at $\alpha=4.0^{\circ}$ 


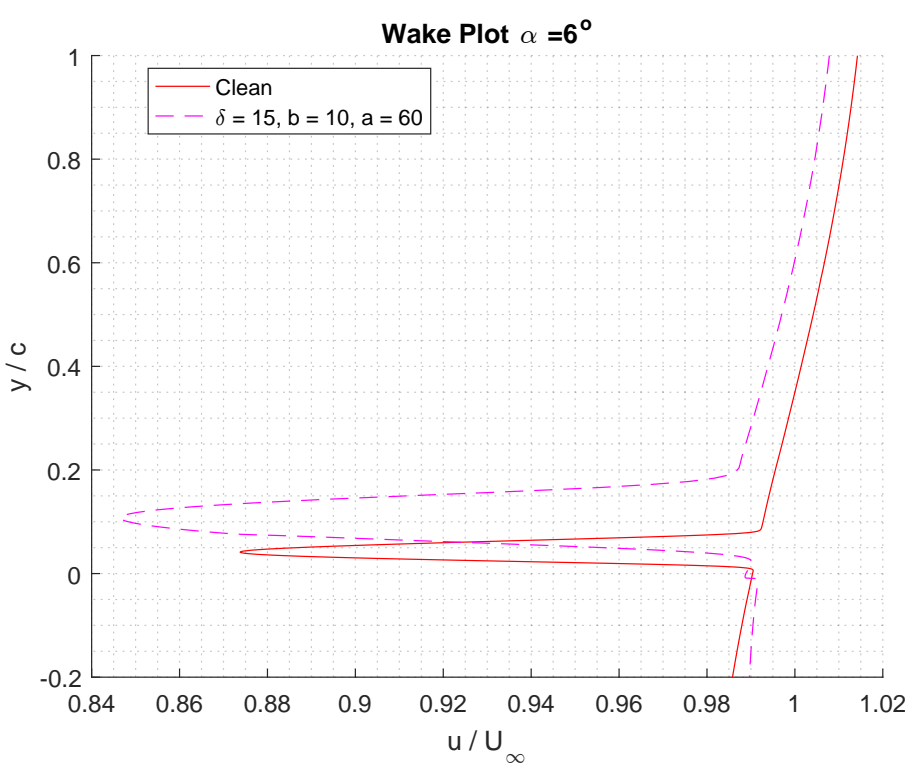

Figure 8.22: CFD Wake Plot at $\alpha=6.0^{\circ}$

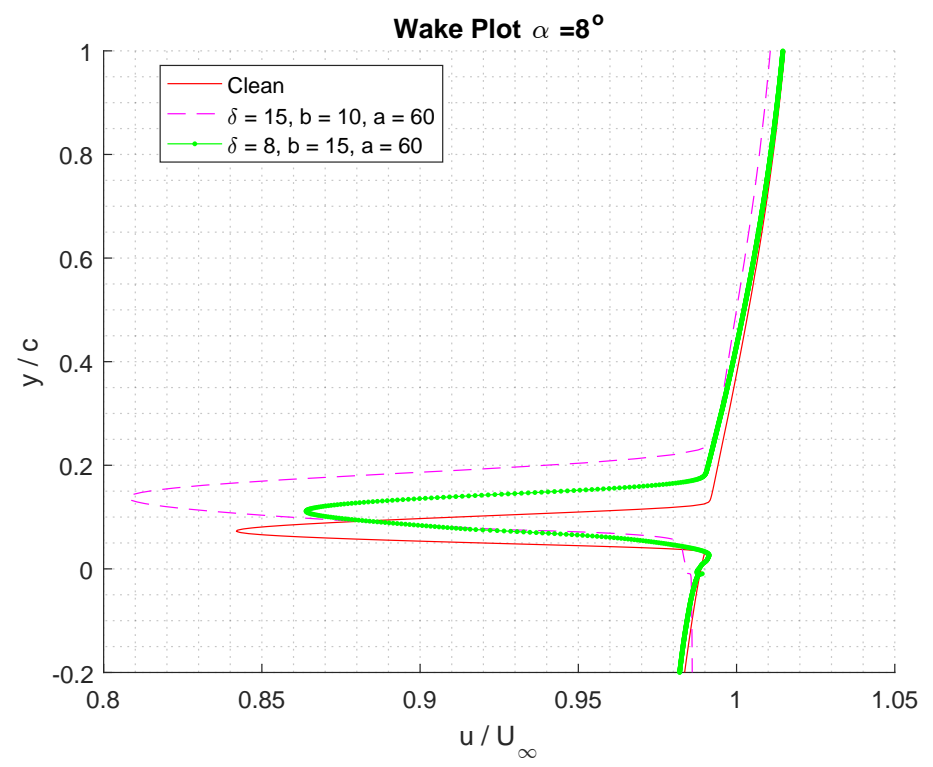

Figure 8.23: CFD Wake Plot at $\alpha=8.0^{\circ}$ 


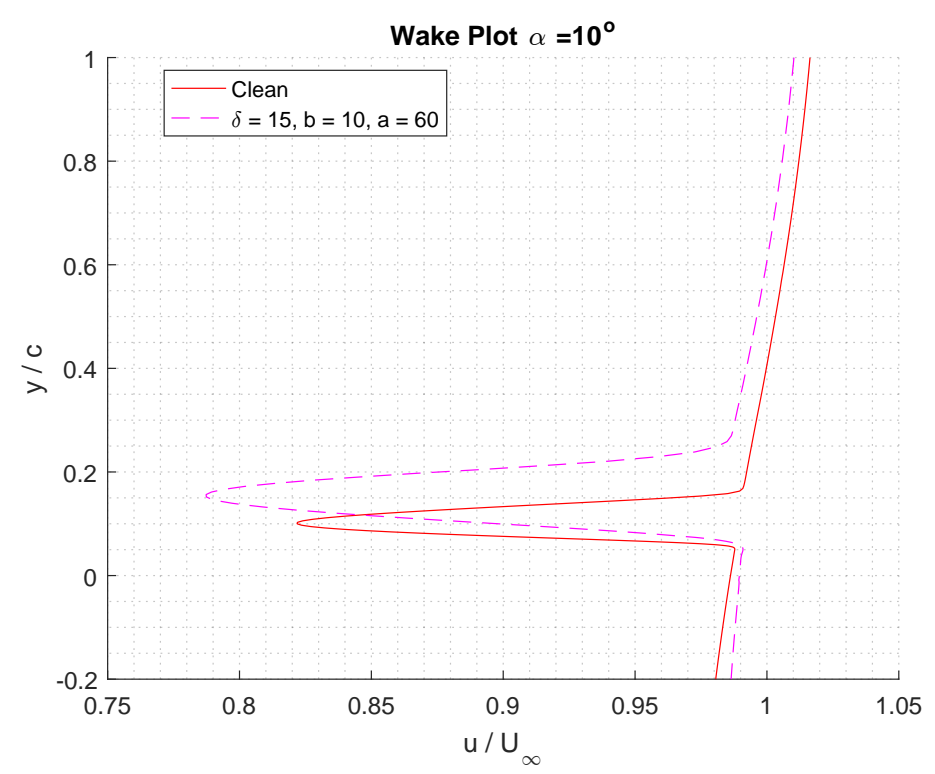

Figure 8.24: CFD Wake Plot at $\alpha=10.0^{\circ}$

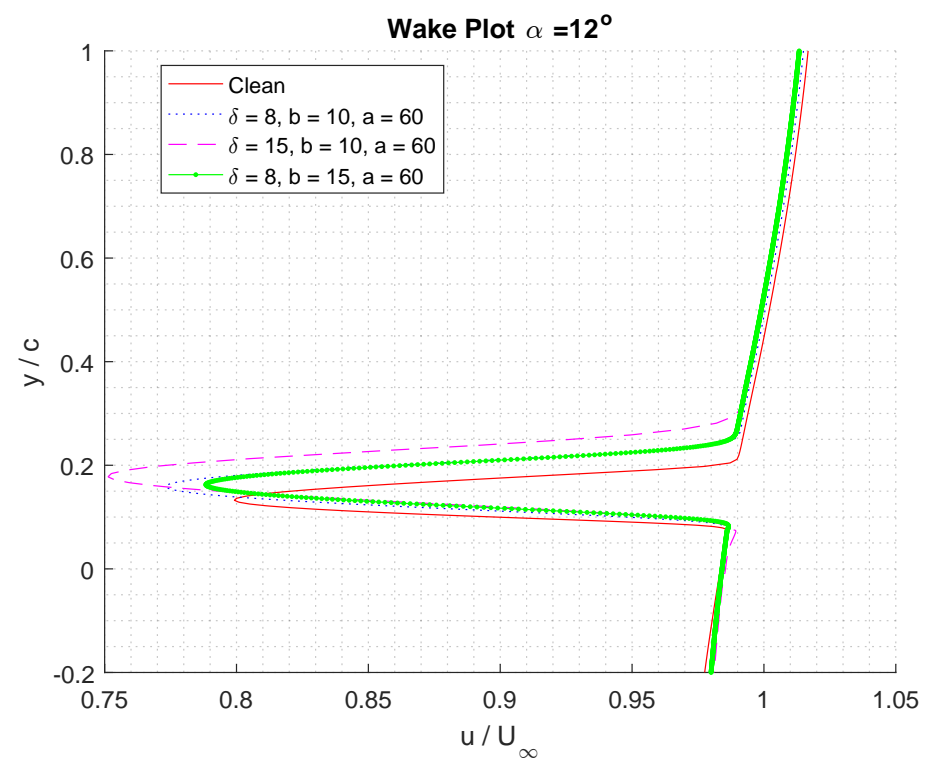

Figure 8.25: CFD Wake Plot at $\alpha=12.0^{\circ}$ 


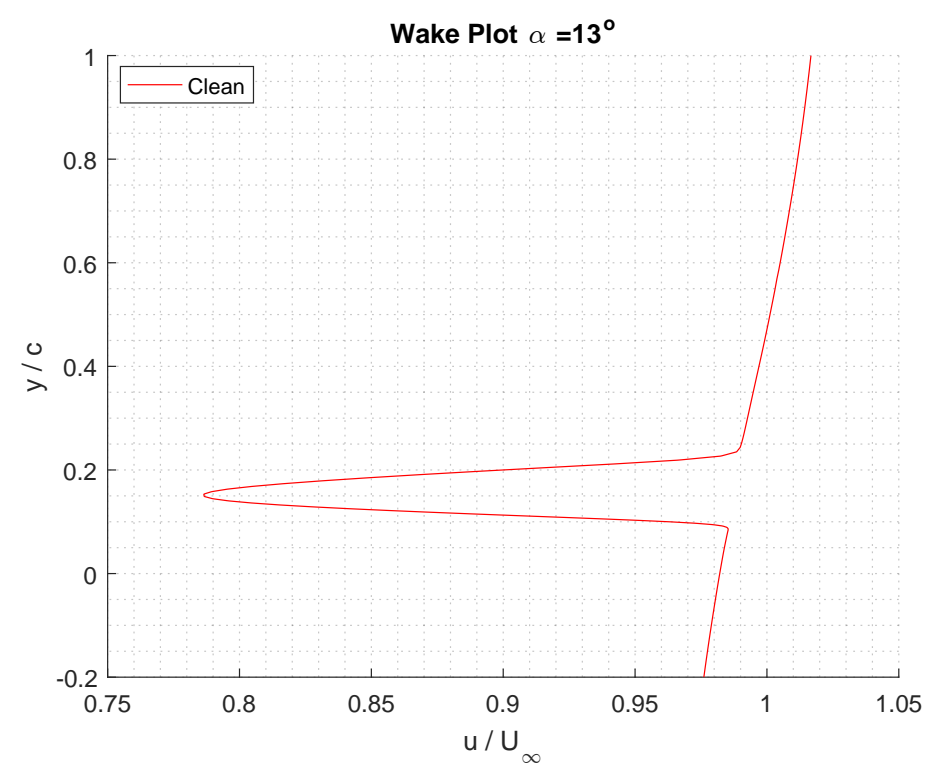

Figure 8.26: CFD Wake Plot at $\alpha=13.0^{\circ}$

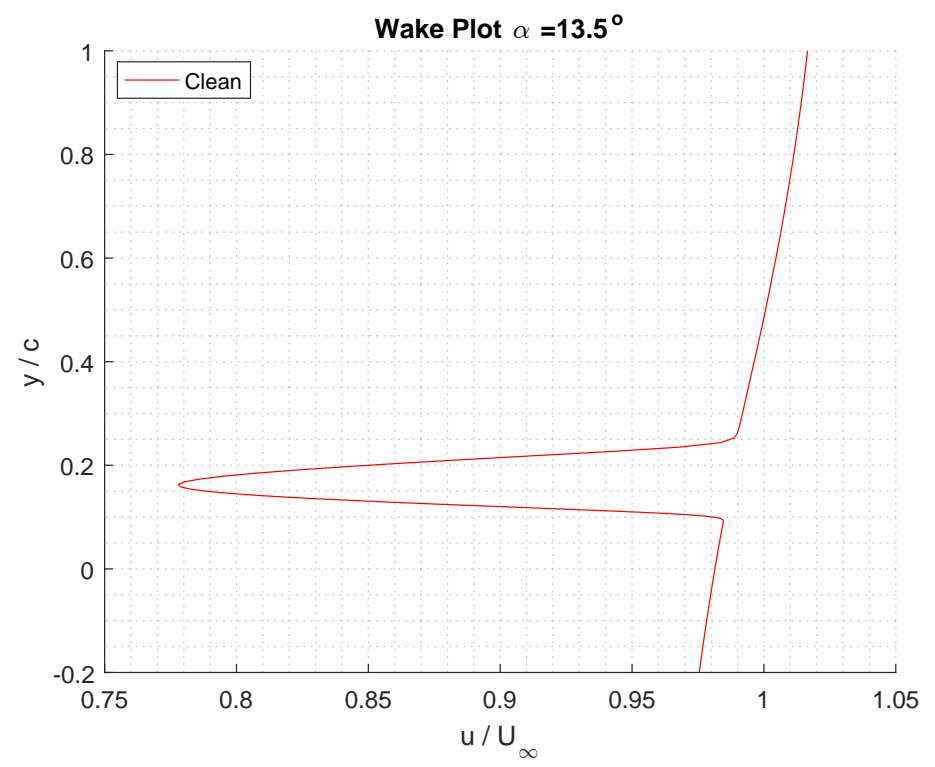

Figure 8.27: CFD Wake Plot at $\alpha=13.5^{\circ}$ 


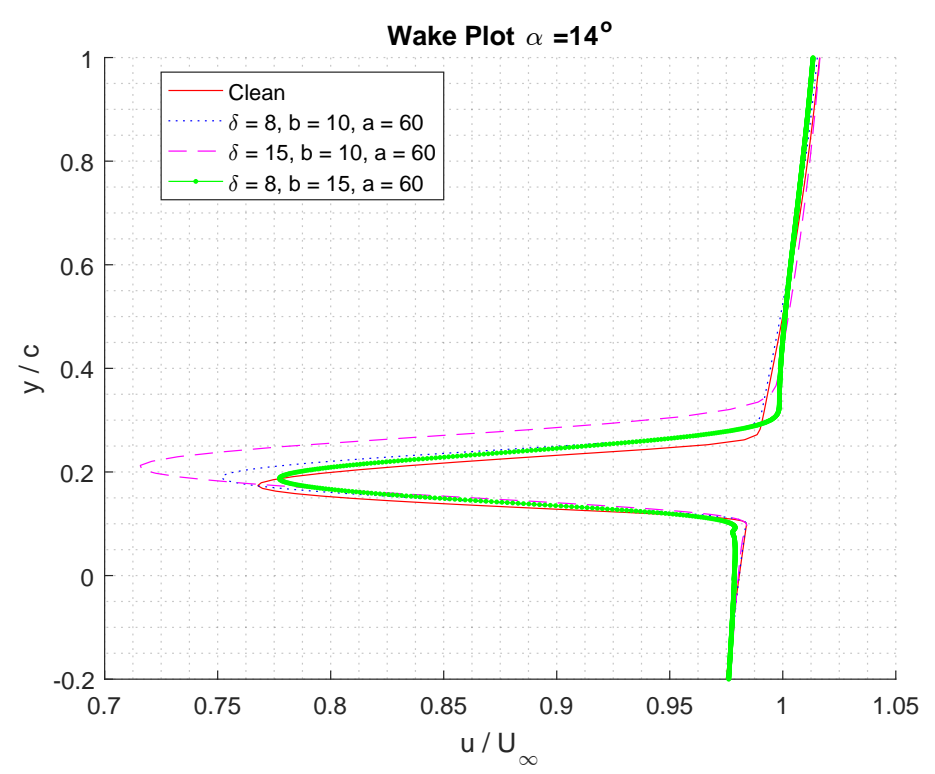

Figure 8.28: CFD Wake Plot at $\alpha=14.0^{\circ}$

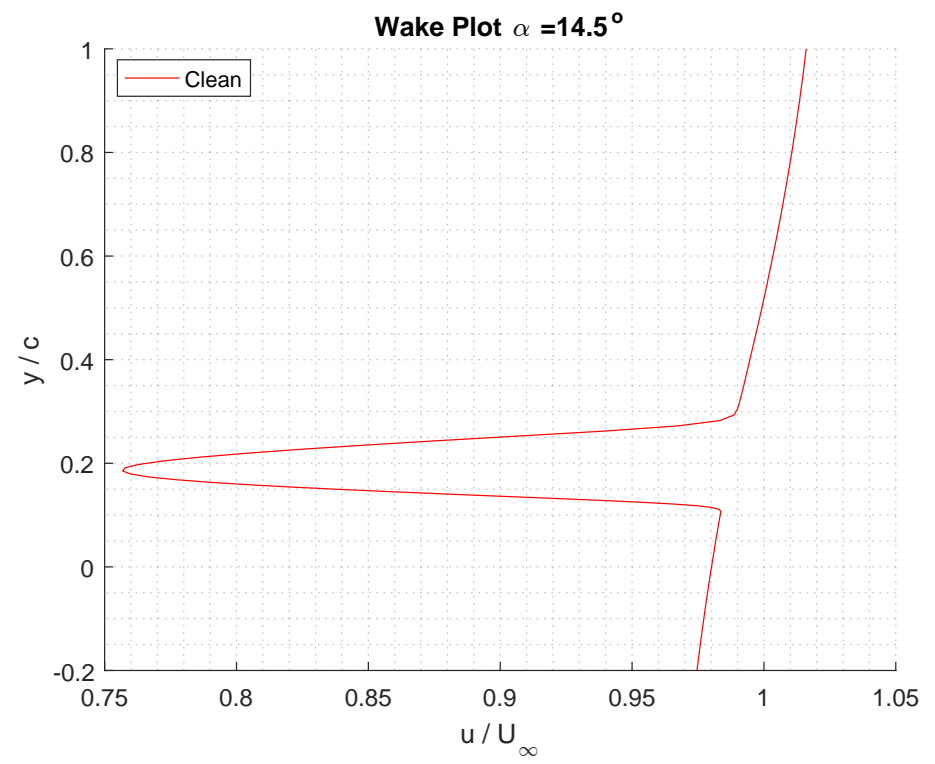

Figure 8.29: CFD Wake Plot at $\alpha=14.5^{\circ}$ 


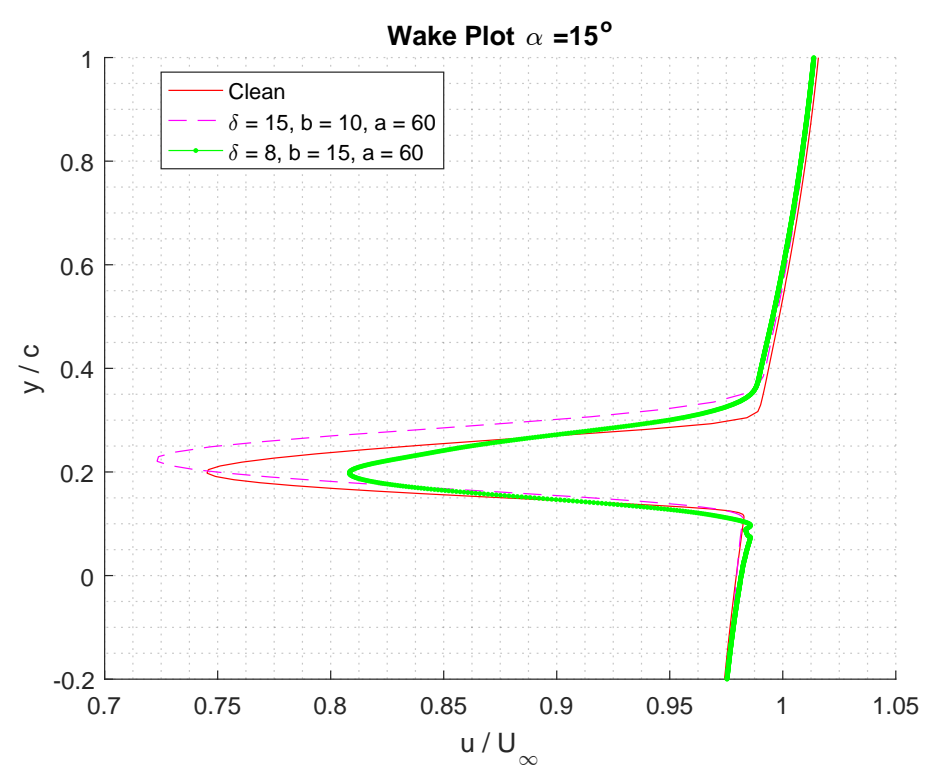

Figure 8.30: CFD Wake Plot at $\alpha=15.0^{\circ}$

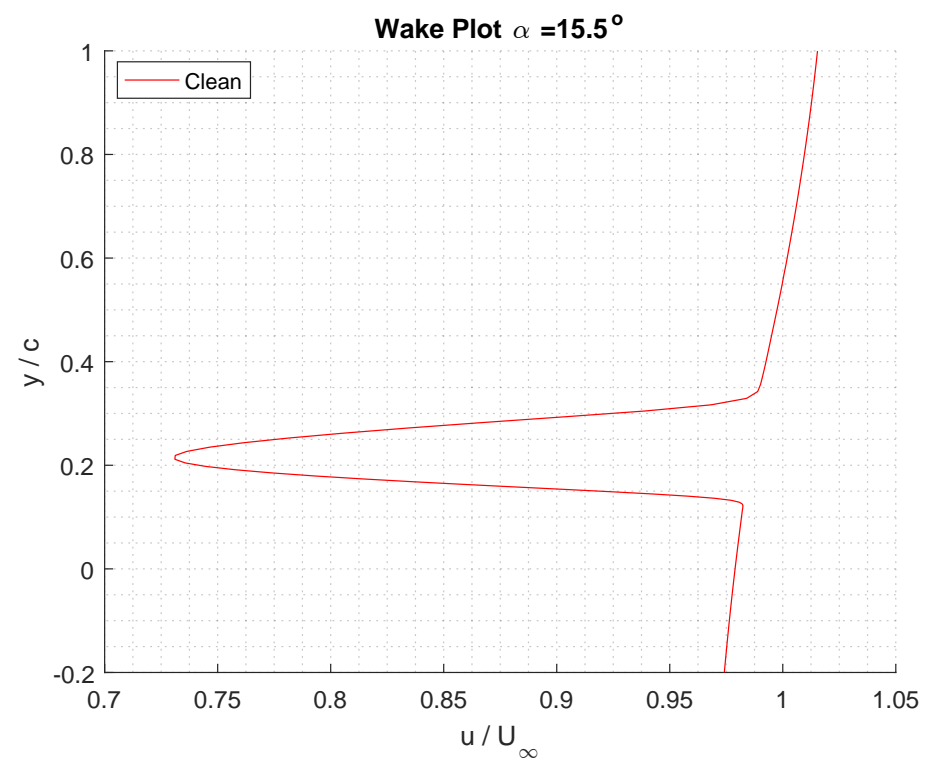

Figure 8.31: CFD Wake Plot at $\alpha=15.5^{\circ}$ 


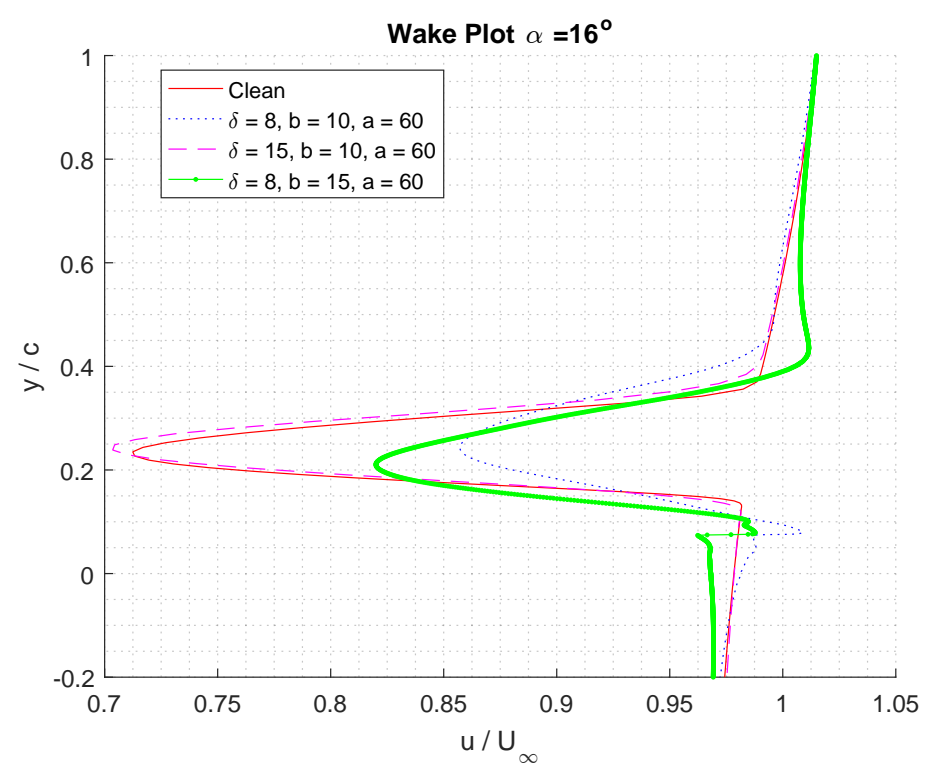

Figure 8.32: CFD Wake Plot at $\alpha=16.0^{\circ}$

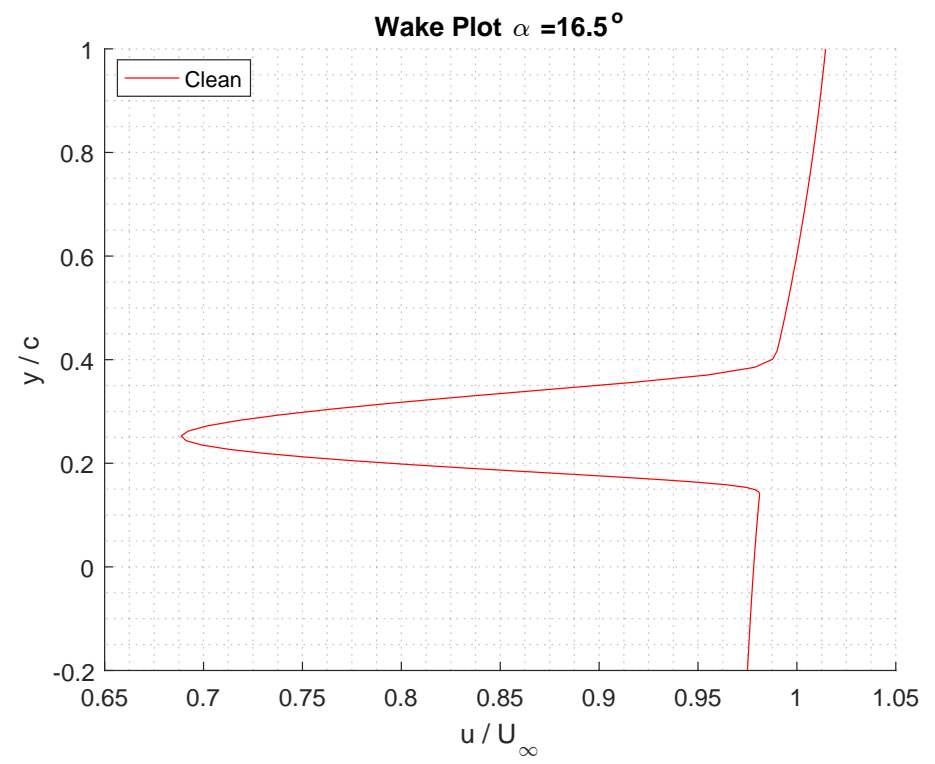

Figure 8.33: CFD Wake Plot at $\alpha=16.5^{\circ}$ 


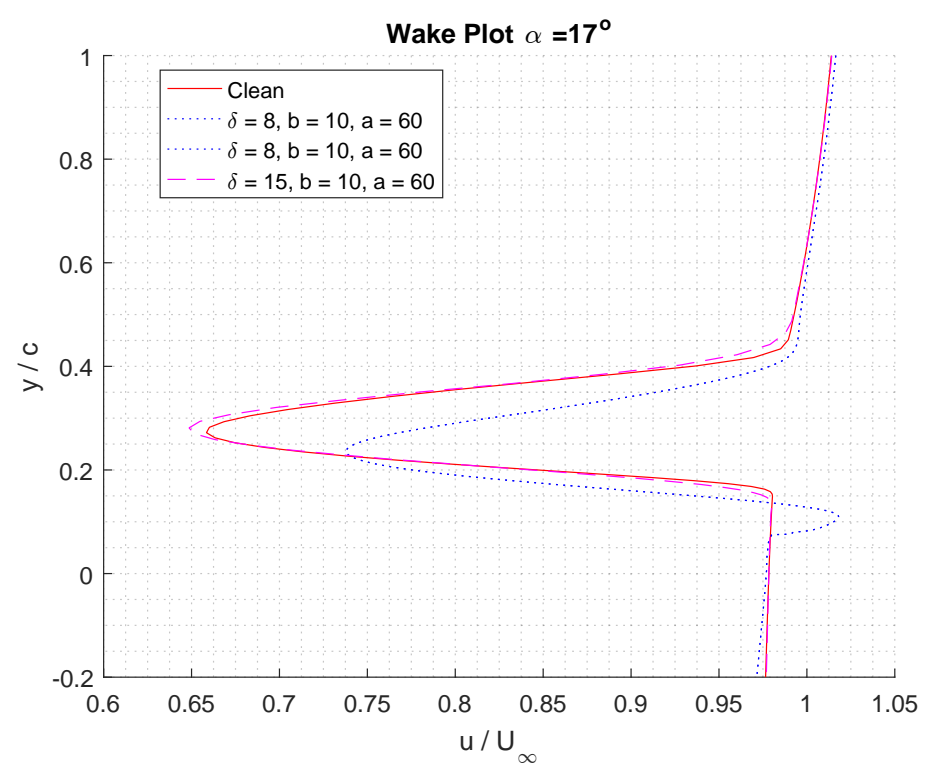

Figure 8.34: CFD Wake Plot at $\alpha=17.0^{\circ}$

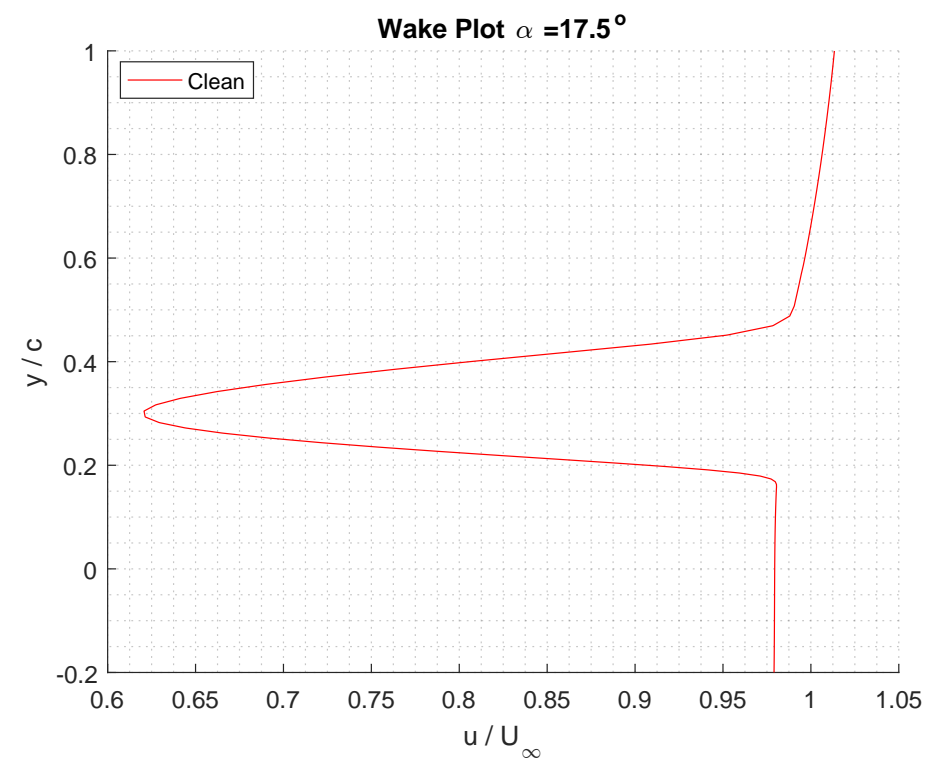

Figure 8.35: CFD Wake Plot at $\alpha=17.5^{\circ}$ 


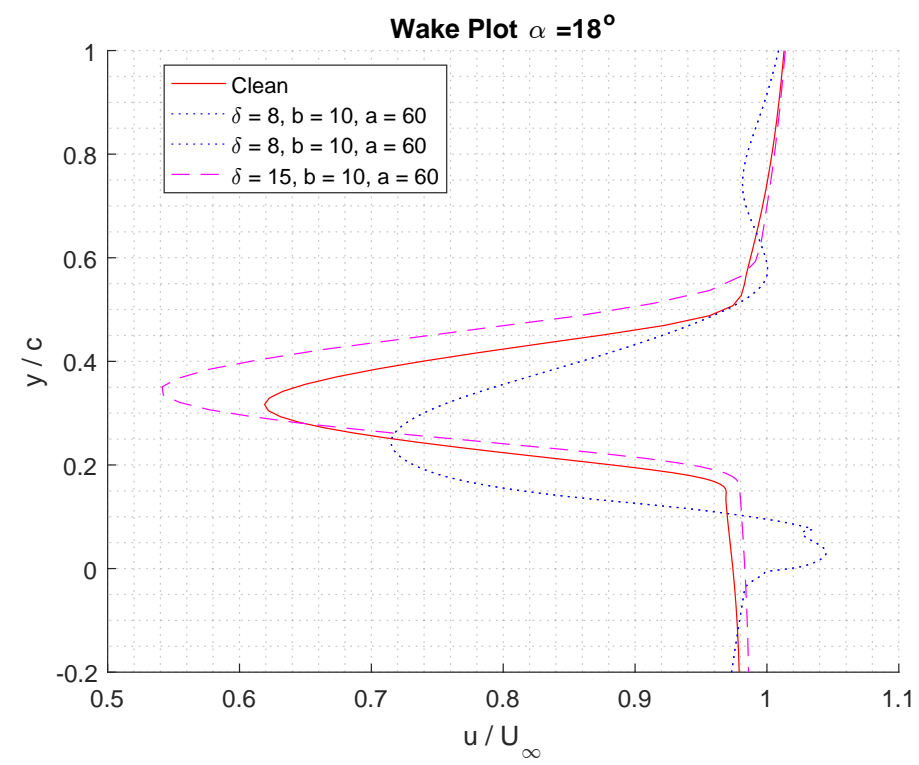

Figure 8.36: CFD Wake Plot at $\alpha=18.0^{\circ}$ 


\section{References}

[1] LM34 Precision Fahrenheit Temperature Sensors.

[2] PX274, PX275, PX277, PX278 Series.

[3] Allen, J. and Vincenti, W. G., "Wall Interference in a Two-Dimensional-Flow Wind Tunnel, With Consideration of the Effect of Compressibility," NACA-TR-782, 1944.

[4] Lee, C. S. and Bodapati, S., "Experimental Investigations of the Flowfield of an Airfoil with Spoiler," Vol. 25, No. 11, 1987, pp. 1411-1416.

[5] Alhawwary, M. A., Owis, F. M., and Abdelrahman, M. M., "Numerical Simulation of the Flowfield around Airfoil with Spoiler using the Higher Order Spectral Difference Method," 53rd AIAA Aerospace Sciences Meeting 2015, 2015, pp. 11098-11115.

[6] Bramesfeld, G. and Maughmer, M. D., "Experimental Investigation of Self-Actuating, UpperSurface, High-Lift-Enhancing Effectors," Journal of Aircraft, Vol. 39, No. 1, 2002, pp. 120-124.

[7] Traub, L. W. and Jaybush, L., "Experimental Investigation of Separation Control Using UpperSurface Spoilers," Journal of Aircraft, Vol. 47, No. 2, 2010, pp. 714-717.

[8] Johnston, J. and Gopalarathnam, A., "Investigation of a bio-inspired lift-enhancing effector on a 2D airfoil," AIAA Applied Aerodynamics Conference, 2011.

[9] Pabla, H. K., Stall Mitigation using Wing Spoilers, Ph.D. thesis, Ryerson University, 2012.

[10] Model DSA3217/3218 Digital Sensor Array Data Sheet No.G511.

[11] Silverstein, A. and Katzoff, S., "A Simplified Method for Determining Wing Profile Drag in Flight," Journal of the Aeronautical Sciences, Vol. 7, No. 7, 1940, pp. 295-301.

[12] Plaisance, C., The development of an integrating wake rake for in-flight measurements of profile drag, Masters thesis, Pennsylvania State University, 1997.

[13] Barlow, Jewel, B., Rae, W. H., and Pope, A., Low Speed Wind Tunnel Testing, 3rd Edition, John Wiley \& Sons, New York, 3rd ed., 1999. 
[14] Oberkampf, W. L. and Trucano, T. G., "Verification and validation in computational fluid dynamics," Progress in Aerospace Sciences, Vol. 38, 2002, pp. 209-272.

[15] Roache, P. J., "Discussion : " Factors of Safety for Richardson Extrapolation " ( Xing , T ., Eng ., 132 , p. 061403 )," Journal of Fluids Engineering, Vol. 133, No. 11, 2011, pp. 1-4.

[16] Jahanmiri, M., "Laminar Separation Bubble : Its Structure, Dynamics and Control," Tech. rep., Chalmers University of Technology, Göteborg, Sweden, 2011. 
